\title{
Elementos da teoria algébrica das formas quadráticas e de seus anéis graduados
}

\author{
Duílio Ferreira Santos
}

\author{
DisSERTAÇÃO APRESENTADA \\ $\mathrm{AO}$ \\ Instituto DE MATEMÁticA E EstatísticA \\ DA \\ Universidade DE SÃo Paulo \\ PARA \\ OBTENÇÃO DO TÍTULO \\ $\mathrm{DE}$ \\ Mestre em CiênCIAS
}

\author{
Área de Concentração: Matemática \\ Orientador: Prof. Dr. Hugo Luiz Mariano
}

Data da defesa: 27 de novembro de 2015 


\title{
Elementos da teoria algébrica das formas quadráticas e de seus anéis graduados
}

\author{
Esta versão da dissertação contém as correções e alterações sugeridas \\ pela Comissão Julgadora durante a defesa da versão original do trabalho, \\ realizada em 27/11/2015. Uma cópia da versão original está disponível no \\ Instituto de Matemática e Estatística da Universidade de São Paulo.
}

Comissão Julgadora:

- Prof. Dr. Hugo Luiz Mariano (orientador) - IME-USP

- Prof. Dr. Francisco Miraglia Neto - IME-USP

- Prof. Dr. Vinícius Cifú Lopes - UFABC

- Prof. Dr. Paulo Agozzini Martin - IME-USP (Suplente)

- Prof. Dr. Odilon Otavio Luciano - IME-USP (Suplente)

- Prof ${ }^{\mathrm{a}}$. Dr ${ }^{\mathrm{a}}$. Ires Dias - ICMC-USP (Suplente) 


\section{Agradecimentos}

Agradeço ao fundamento de toda existência por permitir-me chegar até aqui, sem o qual, nada do que foi feito estaria de pé.

Aos meus pais, Israel e Lourdes, aos meus irmãos Isaías, Abraão, André e à minha companheira de todas as horas Iara pelo amor e apoio incondicionais nos momentos difíceis.

Ao meu orientador e amigo Hugo Mariano por acreditar em mim nos momentos dramáticos e por passar confiança nos momentos em que o espírito duvidava de si mesmo. Hugo nunca esquecerei das lições que aprendi com você, da amizade e de toda motivação que sempre emanou de você. Obrigado pela parceria no mestrado e nas iniciações científicas. Sem a sua imensa ajuda este trabalho não seria possível.

Aos professores da graduação e pós graduação. Em especial, ao professor Odilon Luciano (e Leo) por participar e influenciar decisivamente em minha formação ao longo desses oito anos. Aos professores Manuel e Sônia pelo trabalho desenvolvido em duas iniciações científicas.

A professora Zara pela confiança em meus vários trabalhos de monitoria dentro e fora do instituto.

Aos professores Francisco Miraglia e Vinícius por aceitarem participar da banca, pelas sugestões e correções que ajudaram a aperfeiçoar esse trabalho.

A todos os amigos que fiz no instituto : Everton, Jéssica, Júlio, Gilberto, Clayton, Jean, Gervázio, Max, Arlane, André Zaidan, André Porto, Gílson, Luan, Dimi, Caio Mendes, Roberto, Darllan, Camila, Walter,Fernando, Marcelo, Thiago, Ulisses e Francisco.

Ao Kaique pelo exímio trabalho executado com os arquivos do latex da presente dissertação, a saber, revisão técnica e concatenação dos arquivos. Estou em dívida com você meu amigo. Ao pessoal da biblioteca : Bete, Priscila e Ronaldo. Vocês foram anjos em minha vida. 


\section{Resumo}

SANTOS, D. F. Elementos da teoria algébrica das formas quadráticas e de seus anéis graduados. 2015. 220 f. Dissertação (Mestrado) - Instituto de Matemática e Estatística, Universidade de São Paulo, São Paulo, 2015.

Neste trabalho procuramos realizar uma apresentação autocontida sobre os conceitos da teoria algébrica de formas quadráticas e sobre os anéis graduados que surgiram no desenvolvimento desta teoria. Iniciamos procurando esclarecer o sentido da equivalência entre as várias acepções do conceito de forma quadrática. Após a apresentação de ingredientes e resultados geométricos, fazemos um extrato da teoria dos anéis de Witt, conceito que originou a moderna teoria algébrica de formas quadráticas. Disponibilizamos os elementos fundamentais para a formulação das teorias de cohomologia, nos concentrado no desenvolvimento da teoria de cohomologia profinita e, sobretudo, galoisiana. Descrevemos os funtores $K_{0}, K_{1}$ e $K_{2}$ da K-teoria clássica e também a K-teoria de Milnor, que é mais adequada para formular questões sobre formas quadráticas. Finalizamos o trabalho com a apresentação de alguns conceitos da Teoria dos Grupos Especiais, uma codificação em primeira-ordem da teoria algébrica das formas quadráticas e exemplificamos sua importância, fornecendo um extrato da prova realizada por Dickmann-Miraglia da conjectura de Marshall sobre assinaturas, que se baseia fortemente nesta teoria.

Palavras-chave: corpos; formas quadráticas; anel de Witt; cohomologia galoisiana, K-teoria de Milnor; conjectura de Marshall. 


\section{Abstract}

SANTOS, D. F. Elements of the algebraic theory of quadratic forms and its graded rings. 2015. 220 f. Dissertação (Mestrado) - Instituto de Matemática e Estatística, Universidade de São Paulo, São Paulo, 2015.

In this work I try to provide a self-contained presentation on the concepts of algebraic theory of quadratic forms and on the graded rings that have emerged in the development of this theory. I started trying to clarify the meaning of "equivalence"between the various meanings of the concept of quadratic form. After the presentation of geometrical ingredients and results, we make an extract of the theory of Witt rings, a concept that originated the modern algebraic theory of quadratic forms. It is provided the key elements for the formulation of cohomology theories, focusing on the development of profinite cohomology theory and, especially, on galoisian cohomology. Are described the functors $K_{0}, K_{1}$ and $K_{2}$ of classical K-theory and also the Milnor K-theory, which is more appropriate to formulate questions about quadratic forms. The dissertation is finished with the presentation of some concepts of the Theory of Special Groups, a first-order encoding of algebraic theory of quadratic forms, and with an example its importance by providing an extract of proof by Dickmann-Miraglia of the Marshall's conjecture on signatures, which relies heavily on this theory.

Keywords: fields; quadratic forms; Witt rings; Galois cohomology; Milnor K-theory; Marshall's conjecture. 


\section{Sumário}

Introdução 1

$\begin{array}{|ll|}1 & \text { Ingredientes da teoria algébrica de formas quadráticas }\end{array}$

1.1 Categorias Quadráticas . . . . . . . . . . . . . . . . . . 9

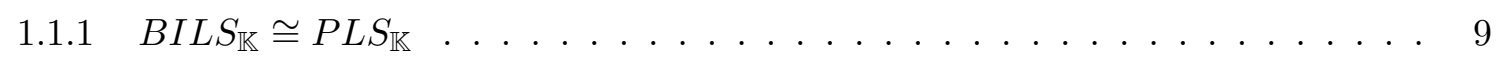

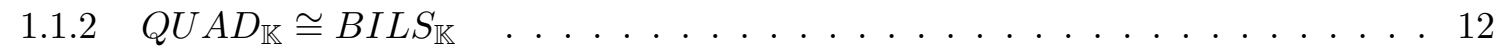

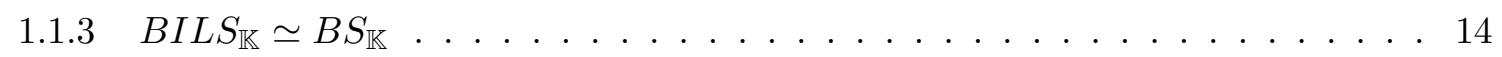

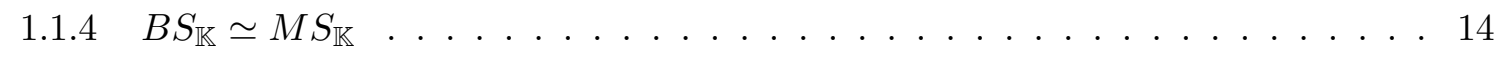

1.1 .5 Resultados centrais sobre categorias quadráticas . . . . . . . . . . . . 17

1.2 Operações e resultados básicos sobre formas quadráticas $\ldots \ldots \ldots \ldots \ldots$. . . . . 17

$1.2 .1 \quad$ Soma direta e Produto tensorial em $B I L S_{\mathbb{K}} \ldots \ldots \ldots \ldots \ldots$. . . . . . . . . . . . . . . .

1.2 .2 Espaços regulares e diagonalização de EBS $\ldots \ldots \ldots$. . . . . . . . . 20

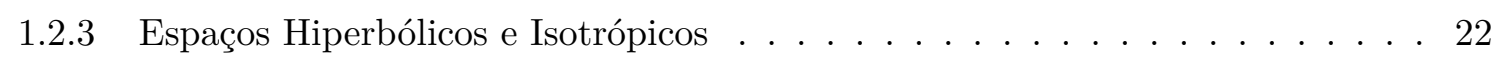

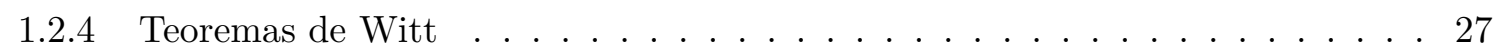

1.2 .5 Formas de Pfister . . . . . . . . . . . . . . . . . . . . 29

$1.2 .6 \quad$ Formas quadráticas e os Quatérnios $\ldots \ldots \ldots$. . . . . . . . . . . 31

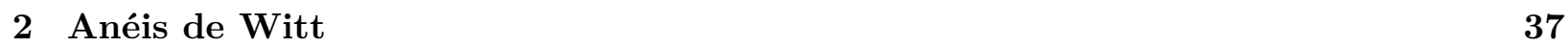

2.1 Semi-anéis de formas quadráticas $\ldots \ldots \ldots \ldots \ldots \ldots \ldots$

2.2 Anéis de Witt-Grothendieck e de Witt de corpos . . . . . . . . . . . . . . . 38

2.3 Extensão de escalares: Funtor Anel de Witt - Grothendieck e Funtor Anel de Witt . 42

2.4 Particularizações do anel de Witt . . . . . . . . . . . . . . . . . . . . . . . 45

2.4 .1 Corpos quadraticamente fechados . . . . . . . . . . . . . . . . . . . . 45

2.4 .2 Corpos ordenados e corpos pitagóricos $\ldots \ldots \ldots \ldots$. . . . . . . . 46

2.4 .3 Corpos euclidianos e corpos reais fechados . . . . . . . . . . . . . . . . . . . . . . . . . . . . . .

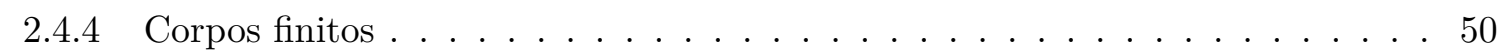

$2.5 \quad$ Apresentações de $W G(\mathbb{K})$ e $W(\mathbb{K}) \ldots \ldots \ldots \ldots \ldots \ldots \ldots \ldots$

$2.6 \quad$ O espaço de ordens de um corpo e assinaturas $\ldots \ldots \ldots \ldots \ldots \ldots \ldots$

$2.7 \quad \mathrm{O}$ princípio local-global de Pfister $\ldots \ldots \ldots \ldots \ldots \ldots \ldots$

2.8 O grupo de classes de quadrado $\ldots \ldots \ldots \ldots \ldots$. . . . . . . . . . . . . . 61

2.9 Invariantes de Hasse e de Witt $\ldots \ldots \ldots \ldots$. . . . . . . . . . . . . . . . 66 
2.10 Os Funtores Anel Filtrado e Anel Graduado de Witt . . . . . . . . . . . . . . . . . 70

\begin{tabular}{|lll}
\hline 3 & Elementos de cohomologia galoisiana & $\mathbf{7 3}$
\end{tabular}

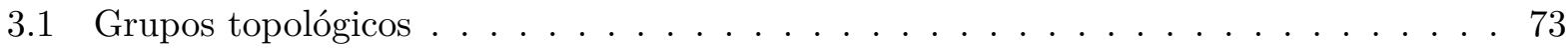

3.2 Limites projetivos e Grupos profinitos $\ldots \ldots \ldots$. . . . . . . . . . . 74

3.3 Grupos de Galois . . . . . . . . . . . . . . . . . . . . . . . . . . 77

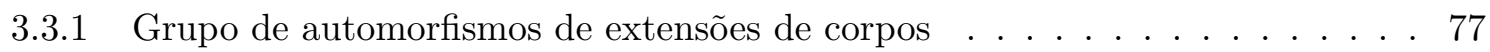

$3.3 .2 \quad$ O grupo de Galois absoluto $\ldots \ldots \ldots \ldots \ldots$. . . . . . . . . . . 83

3.4 Cohomologia profinita $\ldots \ldots \ldots \ldots \ldots \ldots \ldots \ldots$

$3.4 .1 \quad$ G-módulos profinitos . . . . . . . . . . . . . . . . . . . . . 84

3.4 .2 Cadeias não-homogêneas . . . . . . . . . . . . . . . . . . 85

$3.4 .3 \quad$ A sequência exata longa de cohomologia . . . . . . . . . . . . . . . . . 92

3.4 .4 Funtores cohomológicos . . . . . . . . . . . . . . . . . . . . 94

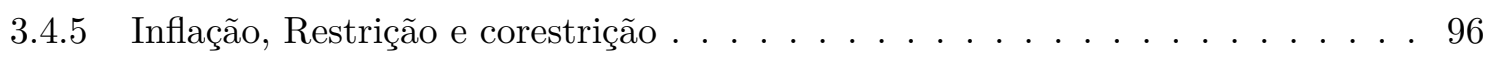

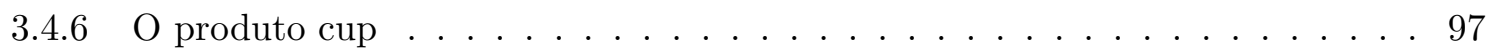

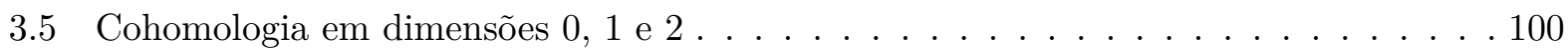

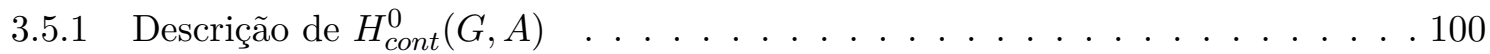

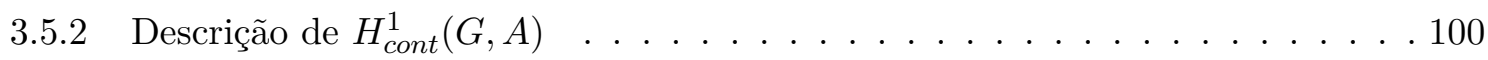

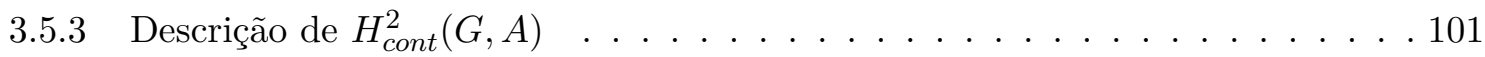

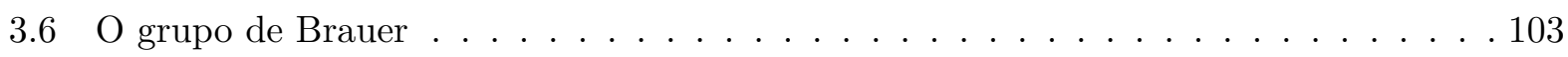

3.6 .1 R-álgebras . . . . . . . . . . . . . . . . . . . . . . . . . 103

$3.6 .2 \quad$ Álgebras simples e centrais simples . . . . . . . . . . . . . . . . . . . . . . . . . . . . . . . . . . . . . . .

3.6 .3 Definição do grupo de Brauer . . . . . . . . . . . . . . . . . . . . . . 111

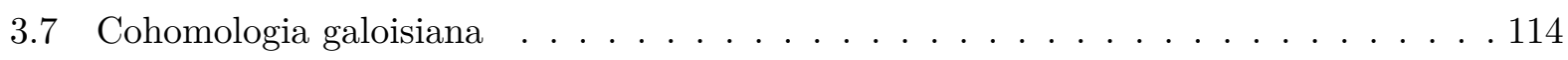

3.8 Classes de Stiefel-Whitney $\ldots \ldots \ldots$. . . . . . . . . . . . . . . . . . . . . . . . . . . . 119

4 Os funtores $\mathrm{K}_{0}, \mathrm{~K}_{1}$ e $\mathrm{K}_{2}$ da K-teoria algébrica clássica $\quad 121$

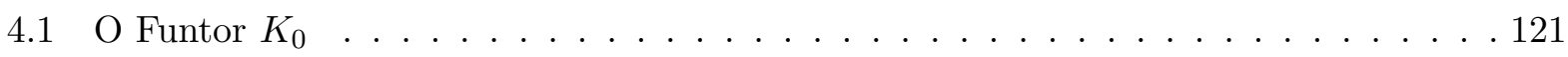

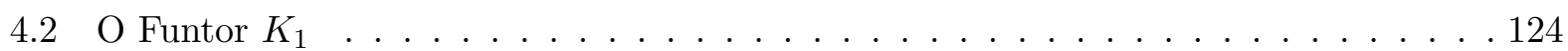

$4.3 \quad \mathrm{O}$ Funtor $K_{2} \ldots \ldots \ldots \ldots \ldots \ldots$

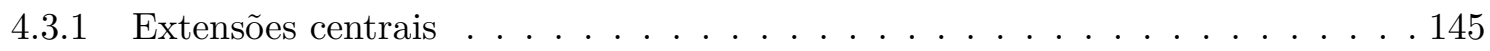

$4.3 .2 \quad \mathrm{St}(\mathrm{R})$ como uma extensão central universal de $\mathrm{E}(\mathrm{R}) \quad \ldots \ldots \ldots$. . . . . . . 150

$4.3 .3 \quad$ O teorema de Matsumoto . . . . . . . . . . . . . . . . . . . . . . . . 154

$4.4 \mathrm{~K}_{1}(\mathrm{R})$ e $\mathrm{K}_{2}(\mathrm{R})$ como grupos de $($ co)homologia $\ldots \ldots \ldots \ldots \ldots \ldots \ldots$

$\begin{array}{|lll|}5 & \text { A K-teoria de Milnor e a Conjectura de Marshall } & 163\end{array}$

5.1 A K-teoria de Milnor . . . . . . . . . . . . . . . . . . . . . . . . . . 163

5.1 .1 A K-teoria de Milnor de um corpo . . . . . . . . . . . . . . . . . . . 163

$5.1 .2 \quad \mathrm{O}$ cálculo de $K_{\star}$ para corpos residuais por valorações $\ldots$. . . . . . . . . . . . 170

5.1 .3 Stiefel-Whitney de um espaço quadrático . . . . . . . . . . . . . . . 175

$5.1 .4 \quad \mathrm{O}$ triângulo incompleto de Milnor . . . . . . . . . . . . . . . . . . 179 
$5.2 \quad$ Anéis graduados, Teoria algébrica de formas quadráticas e Grupos especiais . . . . . 185

$5.2 .1 \quad$ A conjectura Lam . . . . . . . . . . . . . . . . . 185

$5.2 .2 \quad$ A conjectura Marshall $\ldots \ldots \ldots$. . . . . . . . . . . . . . 187

$5.2 .3 \quad$ Alguns detalhes sobre a resolução da conjectura de Marshall . . . . . . . . . 191

\begin{tabular}{|ll}
\hline A O Grupo de Grothendieck & 195
\end{tabular}

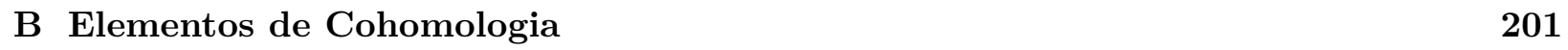

B.1 Preliminares. . . . . . . . . . . . . . . . . . . . . . . . . . . 201

B.1.1 A categoria de complexos . . . . . . . . . . . . . . . . . 201

B.1.2 Objetos injetivos e projetivos . . . . . . . . . . . . . . . . . 202

B.1.3 Resoluções injetivas e projetivas . . . . . . . . . . . . . . . . . 203

B.2 Funtores derivados à direita . . . . . . . . . . . . . . . . . . . . . . . . 205

B.2.1 Funtores Ext . . . . . . . . . . . . . . . . . . . . . 207

B.3 G-módulos . . . . . . . . . . . . . . . . . . . . . . . . . . 208

B.3.1 Módulos induzidos . . . . . . . . . . . . . . . . . . . . . . . 209

B.4 Cohomologia ordinária de grupos . . . . . . . . . . . . . . . . . . . . . 212

B.4.1 Grupos de cohomologia via cocadeias . . . . . . . . . . . . . . . . 216

\begin{tabular}{ll}
\hline Referências Bibliográficas & 219
\end{tabular} 


\section{Introdução}

Formas quadráticas surgiram, historicamente, no seio da teoria dos números, mais precisamente no estudo de equações diofantinas quadráticas sobre o anel dos números inteiros. Nos anos de 1880, o matemático alemão H. Minkowski $(1864$ - 1909) matematizou a ideia de que o entendimento global de soluções de certas equações sobre um domínio de integridade, passa pelo entendimento das soluções sobre o corpo de frações.

Herman Minkowski: No período de 1884 - 1890, Minkowski desenvolveu os fundamentos de uma teoria geral das formas quadráticas sobre os números racionais e os números inteiros.

Em 1884 ele publica um excelente trabalho ([Min1]), onde desenvolve a teoria de classificação local para formas quadráticas sobre o anel dos inteiros.

Minkowski prossegue suas investigações em sua dissertação ([Min2]), sob o título : Investigações sobre formas quadráticas. Determinação do número de formas distintas que estão contidas em um determinado gênero.

Em Min3 ele dá um resumo muito claro e legível de sua dissertação e também descreve as contribuições de Henry J. S. Smith. Esse trabalho é uma referência importante para os interessados no tema "somas de quadrados".

Nos anos de 1890 Minkowski recoloca em (Min4) uma questão devida a Hilbert e Hurwitz, a saber : Sobre quais condições duas formas quadráticas, com coeficientes racionais, podem ser transformadas uma na outra?

Essa questão pode ser colocada da seguinte forma: Como classificar formas quadráticas sobre $o$ corpos dos números racionais?

Seja $V$ um $\mathbb{Q}$ - espaço vetorial de dimensão finita e $q: V \longrightarrow \mathbb{Q}$ uma forma quadrática regular. A dimensão de $V$, o discriminante de $q$ (visto como um elemento de $\dot{\mathbb{Q}} / \dot{\mathbb{Q}}^{2}$ ), e a assinatura de $q$ são invariantes da classe de isomorfismo de $q$. Em adição a estes invariantes clássicos, Minkoswki introduziu um novo invariante: para cada primo $p$, ele definiu $C_{p}(q)= \pm 1$ e mostrou que $C_{p}(q)$ é também um invariante para $q$. Além disso, provou que dessa forma obtém-se um sistema completo de invariantes. Em outras palavras, se duas formas quadráticas não-degeneradas sobre o corpo dos números racionais têm a mesma dimensão, mesmo discriminante, mesma assinatura e os invariantes $C_{p}(q)$ coincidem, para todo primo $p$, então elas são isomorfas.

Leonard Eugene Dickson: Entre os anos de 1850 e 1890 os trabalhos dos matemáticos : Karl von Staudt (1887), Richard Dedekind (1871), Leopold Kronecker (1881); era o prenúncio de um desenvolvimento (sem volta) da álgebra abstrata. Muitas questões de álgebra e álgebra linear foram clarificadas. Este era um momento propício para que a seguinte questão:

"Quando duas formas quadráticas sobre um a dado corpo são isométricas?"

se tornasse central em teoria de formas quadráticas. Minkowski resolveu essa questão para o corpo dos números racionais. Em 1899, L. E. Dickson resolveu o problema de classificação para corpos finitos. Em 1907, Dickson publicou um artigo com o título: On quadratic forms in a general field, um 
título muito parecido com o de um trabalho que viria a aparecer 30 anos depois, realizado por Ernest Witt. Nele Dickson inicia com as palavras: "Nós investigamos a equivalência, sob transformação linear em um corpo geral $F$, de duas formas quadráticas...". Em terminologia moderna o paper de Dickson contém os seguintes resultados :

1. Toda forma quadrática sobre o um corpo de característica $\neq 2$ pode ser diagonalizada.

2. Se $q=\left\langle a_{1}, \ldots, a_{n}\right\rangle$ representa $b$, então $q \cong\langle b\rangle \oplus\left\langle c_{2}, \ldots, c_{n}\right\rangle$

3. Toda isometria entre subespaços regulares pode ser estendida para uma isometria do espaço quadrático.

4. Vale a seguinte propriedade de cancelamento: se $\left\langle a, a_{2}, \ldots, a_{n}\right\rangle \cong\left\langle a, b_{2}, \ldots, b_{n}\right\rangle$, então $\left\langle a_{2}, \ldots, a_{n}\right\rangle \cong\left\langle b_{2}, \ldots, b_{n}\right\rangle$

Helmut Hasse: O próximo passo na teoria de formas quadráticas foi dado por Helmut Hasse. Em maio de 1921, Hasse tinha completado sua tese ([Has1]), introduzindo e provando, um famoso Princípio Local-Global para a representação de um número racional por uma dada forma quadrática com coeficientes racionais. Explicitamente:

Sejam $a \in \mathbb{Q}$ e $f\left(x_{1}, \ldots, x_{n}\right)$ uma forma quadrática sobre $\mathbb{Q}$. Como encontrar condições necessárias e suficientes de modo que a seja representado por $f\left(x_{1}, \ldots, x_{n}\right)$, ou seja, para que exista $x \in \mathbb{Q}^{n}$ tal que $f(x)=a$

O Princípio Local-Global de Hasse diz que a é representável por $f\left(x_{1}, \ldots, x_{n}\right)$ se, e somente se, é representável sobre os completamentos $p$-ádicos para todos primos $p$ (inclusive $\mathbb{R}$, tomando $p=\infty)$. Em outras palavras, a fórmula

$$
\exists x_{1}, \ldots, \exists x_{n}, a=f\left(x_{1}, \ldots, x_{n}\right)
$$

vale em $\mathbb{Q}$ se, e somente se vale sobre $\mathbb{R}$ e sobre $\mathbb{Q}_{p}$, para todo primo $p$. O ponto fundamental é que nesses corpos completos, existem critérios aritméticos explícitos disponíveis para saber se uma dada forma representa ou não $a$.

Após sua tese, Hasse publicou uma rápida sucessão de seis artigos (sendo um deles em conjunto com o seu orientador Hensel). No primeiro desses papers ([Has2]), ele desenvolve um Princípio Local-Global forte para formas quadráticas equivalentes sobre $\mathbb{Q}$. A noção de equivalência entre duas formas quadráticas no paper de Hasse ([Has2]) é como segue:

Duas formas quadráticas $f_{1}\left(x_{1}, \ldots, x_{n}\right)$ e $f_{2}\left(x_{1}, \ldots, x_{n}\right)$ sobre o corpo dos números racionais $\mathbb{Q}$, são ditas equivalentes (resp. sobre qualquer completamento $\mathbb{Q}_{p}$ ), quando existe uma transformação linear não-singular das variáveis que transforma uma na outra.

Dadas duas formas quadráticas, Hasse provou que elas são equivalentes sobre $\mathbb{Q}$ se, e somente se são equivalentes sobre $\mathbb{Q}_{p}$, para todo primo $p$ (inclusive, $p=\infty$ ). Vale ressaltar que critérios de decisão acerca da equivalência de duas formas quadráticas foram exaustivamente estudados por $\mathrm{H}$. Minkowski nos anos de $\mathbf{1 8 8 0}$ e, por esse motivo, o teorema de Hasse é frequentemente chamado de teorema de Hasse-Minkowski. Não obstante, Minkowski não trabalhava com completamentos p-ádicos e esse fato não o habilitou a formular o princípio local-global forte. Hasse em ([Has2] $)$ cita Minkowski; mas não usa o resultado de Minkowski porque, segundo ele, Minkowski depende de sua teoria sistemática sobre o anel dos inteiros $\mathbb{Z}$ e ele (Hasse), por sua vez, não necessitaria pois era inadequada para o presente propósito, uma vez que o problema era de natureza racional e não integral. Em vez disso, Hasse introduz invariantes locais que são suficientes para caracterizar formas quadráticas sobre $\mathbb{Q}_{p}$, a menos de equivalência. Além do número de variáveis $n$ e do discriminante $d \neq 0$ (módulo quadrado) ele introduz o que atualmente é chamado de "Invariante de Hasse" de uma forma quadrática. Se a forma $f$ é transformada em uma forma diagonal: 


$$
f\left(x_{1}, \ldots, x_{n}\right)=\sum_{i=1}^{n} a_{i} x_{i}^{2}, a_{i} \in \dot{\mathbb{Q}}_{1}
$$

(o que é sempre possível) então o invariante de Hasse é dado por

$$
C_{p}(q)=\prod_{i \leq j}\left(a_{i}, a_{j}\right)_{p}
$$

onde $\left(a_{i}, a_{j}\right)_{p}= \pm 1$ denota o símbolo quadrático de Hilbert. Este invariante é atualmente chamado invariante de Hasse-Witt, invariante de Hasse ou invariante de Clifford. Este último nome vem do fato de que este objeto também pode ser definido utilizando-se a álgebra de Clifford associada a forma $q$.

Na década de 1920, a teoria dos corpos locais e globais associados a números algébricos estava suficientemente desenvolvida. Hasse dá um passo importante e não-trivial concernente a generalização daqueles princípios locais-globais a partir do corpo $\mathbb{Q}$ para um corpo numérico algébrico de grau finito. Isso foi feito em ([Has6], Has7]), depois de ter trabalhado com teoremas preparatórios sobre símbolos de resíduos quadráticos em corpos numéricos ([Has3] , [Has4]).

Ernest Witt: Em 1937, o matemático alemão Ernest Witt publica um trabalho fundamental (Witt]), considerado por muitos, como o começo da teoria algébrica das formas quadráticas moderna. Não é fácil mensurar a importância do paper de Witt. No entanto, em minha opinião, um trabalho é tanto importante quanto maior for o seu grau de universalidade. Além disso, as influências que exerce na sequência nos ajudam a fazer esse julgamento. Nesse sentido, podemos dizer que:

1. A teoria de Witt contém o trabalho de seus antecessores.

2. Plasma de maneira elegante uma boa dose de geometria no âmago da teoria algébrica, dose essa, que Dickson não havia notado.

3. Codifica de forma adequada a totalidade de formas quadráticas ao introduzir um anel cujos elementos estão em correspôndencia bijetiva com as classes de isomorfismo de formas não singulares do corpo em questão.

4. Influencia decisivamente quase todos os trabalhos posteriores na teoria algébricas de formas quadráticas.

Devido a importância do trabalho de Witt (de fato, um divisor de águas), entendemos que aqui está a primeira revolução na teoria algébrica de formas quadráticas.

Albrecht Pfister: Os trabalhos de Pfister ([Pfi1, [Pfi2, [Pfi3]) foram cruciais para o entendimento do funcionamento interno do anel de Witt e futuras comparações funtoriais.

1. Pfister introduz a noção de forma de Pfister. Essa noção é sem dúvida um conceito central na teoria algébrica de formas quadráticas. Formas de Pfister são formas de dimensão $2^{n} \mathrm{e}$ podem ser escritas como um produto de formas do tipo $\langle 1,-a\rangle$. Precisamente:

$$
\left\langle\left\langle a_{1}, \ldots, a_{n}\right\rangle\right\rangle=\otimes_{i=1}^{n}\left\langle 1,-a_{i}\right\rangle
$$

2. Pfister mostrou, usando o conceito de forma de Pfister, resultados sobre a composição de Formas quadráticas. 
3. Pfister mostrou que o ideal fundamental do anel de Witt é gerado por formas de Pfister.

4. Formas de Pfister estão fundamentalmente ligadas a teoremas de estruturas do anel de Witt.

5. Se valendo de todo o espaço de ordens de um corpo, Pfister prova um princípio local-global que generaliza vastamente o teorema de Sylvester sobre inércia de formas quadráticas com coeficientes reais.

6. A noção de forma de Pfister permite provar um caso de índice baixo da conjectura Milnor.

7. Formas de Pfister permitem estabelecer comparações naturais com cohomologia de Galois e com K-teoria.

Devido a importância dos trabalhos de Pfister, em particular da introdução de um conceito instrumental decisivo para o avanço da teoria algébrica de formas quadráticas, nós entendemos que ele realizou a segunda revolução na teoria algébrica de formas quadráticas.

John Milnor: Problemas fundamentais do trabalhos de Pfister ecoariam no trabalho revolucionário de Jonh Milnor. Pfister havia demonstrado em Pfi3 que o determinante de uma forma quadrática $\left(\operatorname{det}(\phi) \in \dot{\mathbb{K}} / \dot{\mathbb{K}}^{2}\right)$ induzia um isomorfismo canônico $e_{1}: I^{1}(\mathbb{K}) / I^{2}(\mathbb{K}) \longrightarrow H^{1}(\mathbb{K})$, onde $I(\mathbb{K})$ é o ideal fundamental do anel de Witt. Em seguida, o próprio perguntou se o invariante de Clifford $\left(I^{2}(\mathbb{K}) / I^{3}(\mathbb{K}) \longrightarrow B r(\mathbb{K}) \cong H^{2}(\mathbb{K})\right)$ era injetivo. Com essa filosofia, um problema central em teoria algébrica de formas quadráticas é o seguinte:

Como definir invariantes cohomológicos de grau superior?

Esse problema mostrou-se extremamente complicado.

Inspirado em invariantes da Topologia Algébrica, A. Delzant define em ([Del $]$ ) um sistema de invariantes de Stiefel - Whitney de uma forma quadrática. No entanto, esse sistema não é um sistema completo de invariantes. A redução do problema, passa então a ser a legitimidade de um (iso)morfismo

$$
e_{n}: I^{n}(\mathbb{K}) / I^{n+1}(\mathbb{K}) \longrightarrow H^{n}(\mathbb{K}) .
$$

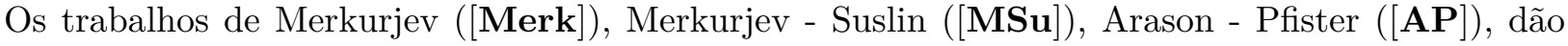
um caráter ainda mais sério ao problema da existência dos invariantes cohomológicos superiores.

O matemático americano John Milnor havia sido atraído para teoria algébrica de formas quadráticas, provavelmente devido a importância da teoria algébrica de formas quadráticas para a Geometria e Topologia Algébrica. Continuando o trabalho de Whitehead ([Whi2]), Milnor (1958) discute em que medida uma 4-variedade é determinada por sua forma quadrática. Ele mostra que duas tais variedades têm o mesmo tipo de homotopia se, e somente se suas formas quadráticas são isométricas. De modo que, para uma classificação de tipos de homotopia, necessita-se de uma classificação de formas unimodulares integrais. Em 1961, Milnor discute a seguinte questão:

Quando a forma intersecção é uma forma anisotrópica?

como uma aplicação do teorema de Hasse-Minkowski, ele prova que este é o caso se, e somente se, ela é indefinida.

Nas palavras de H. Bass :

Milnor tinha um interesse profundo e carinhoso pela teoria de formas quadráticas, como foi evidente de seu belo livro escrito com Husemoller ([MH]$)$, baseado em suas notas de aula. Seu paper : Algebraic K-Theory and quadratic forms ([Miln3]), continua a guiar boa parte da pesquisa moderna em teoria de formas quadráticas. 
O trabalho de Milnor de 1970 ([Miln3]) é sem dúvida o seu trabalho mais importante com relação a teoria de formas quadráticas. Baseado em um ideal moderno de comparação funtorial de estruturas, Milnor interpola, genialmente, o problema da extensão dos invariantes cohomológicos superiores através de um objeto (de notável pedigree) com grau adequado de genericidade entre $I^{n}(\mathbb{K}) / I^{n+1}(\mathbb{K})$ e $H^{n}(\mathbb{K})$. Em linhas gerais:

1. Estende técnicas do trabalho de Gauss para dar evidências de uma conjectura.

2. Dá evidências para questões fundamentais iniciadas por Pfister.

3. Contém alguns aspectos do trabalho de Pfister.

4. Põe em movimento a teoria algébrica de formas quadráticas, estabelecendo relações com outras áreas fundamentais da Matemática.

5. É um marco para as novas pesquisas na teoria de formas quadráticas.

Do exposto acima, entendemos que Milnor é responsável por uma terceira revolução na teoria algébrica de formas quadráticas.

Dickmann - Miraglia : No seio da teoria de formas quadráticas repousava, ainda sem forma, a ideia de quão universal e importante a teoria das álgebras booleana seria para as formas quadráticas. De fato, entreve-se algo no princípio local-global de Pfister, devido a importância do espaço booleano formado por todas as ordens possíveis em um corpo. Em outros cálculos - estava lá quase vagando - a também a noção de anel booleano. Evocava-se, mais uma vez, aquele princípio de comparação funtorial de estruturas.

1. Isometria de $n$ - formas são essencialmente isometria de 2-formas ([Witt]).

2. Seja $\mathbb{K}$ um corpo de característica $\neq 2$. Informações do anel de Witt-Grothendieck de $\mathbb{K}$ estão codificadas, essencialmente, nos grupos quociente $I(\mathbb{K}) / I^{2}(\mathbb{K})$ e $I^{2}(\mathbb{K}) / I^{3}(\mathbb{K})([\mathbf{D e l}])$.

3. A noção de anel booleano ligado a um corpo aparece quando se trata de entender a conjectura Milnor para a parte de torsão do anel de Witt ([Aras $]$ ).

4. Milnor mostrou que a $\mathrm{n}$ - forma de Pfister $\left\langle\left\langle a_{1}, \ldots, a_{n}\right\rangle\right\rangle$ determina um invariante $l(-1)^{t-n} l\left(a_{1}\right) \ldots l\left(a_{n}\right)$, onde $t=2^{n-1}$. Os matemáticos R. Elman e T.Y. Lam estabeleceram em $([\mathbf{E L 1}])$ que $l\left(a_{1}\right) \ldots l\left(a_{n}\right) \in k_{n}(\mathbb{K})$ é um tipo de invariante completo para a classe de isometria de $n$ - formas de Pfister.

Ao lado dos matemáticos M. Marshall ([Mar2] $)$ e Kula, Szczepanik, Szymiczek ([KSS $]$ ), os latino-americanos Maximo Dickmann e Francisco Miraglia desenvolvem uma teoria algébrica de formas quadráticas em base axiomática, por estes denominada Teoria dos Grupos Especiais. Todos esses tratamentos axiomáticos são (em algum sentido), essencialmente os mesmos. Por outro lado, pela teoria dos grupos especiais ser uma axiomática em primeira ordem, ficam facilitados ou em mais evidência, outros aspectos estruturais dessas teorias abstratas. Dentre seus trabalhos mais importantes em conexão com a teoria de formas quadráticas tem-se a resolução da conjectura Milnor para a parte de torsão do anel de Witt e a resolução da conjectura de Marshall sobre assinaturas e potências do ideal fundamental. Em linhas gerais eles:

1. Sintetizaram muitas ideias, até então vagas, da teoria algébrica de formas quadráticas. 
2. Introduziram vários métodos novos na teoria das formas quadráticas, pondo em relevo a importância da teoria das álgebras booleanas. Em particular, introduzem os invariantes de Horn-Tarski aditivo, que não possuem um análogo conhecido no caso de um corpo.

3. Introduziram, em analogia com os invariantes de Stiefel-Whitney, os invariantes de Horn -Tarski multiplicativo.

4. Apresentaram uma prova alternativa (usando invariantes booleanos) do seguinte resultado, devido a Elman - Lam ([EL2]):

Seja $F$ um corpo. Então, dimensão e o invariante total de Stiefel-Whitney caracterizam isometria de formas quadráticas em $F$ se, e somente se $I^{3}(F)$ é livre de torsão.

5. Usando os métodos desenvolvidos em ([DM2]) forneceram uma resposta positiva para Conjectura Marshall sobre assinaturas, para o caso dos corpos pitágoricos e formalmente reais.

6. Responderam afirmativamente a Conjectura Lam ([DM3 $)$.

Percebe-se assim o quanto é alto o grau de universalidade dos trabalhos de Dickmann e Miraglia. Além disso, seus trabalhos têm norteado pesquisas na direção dos fundamentos da teoria de formas quadráticas. Dessa forma, entendemos que eles vem realizando uma quarta revolução na teoria algébrica de formas quadráticas.

Após este breve histórico da teoria das formas quadráticas, delineamos a estrutura de nossa dissertação.

Capítulo 1: Iniciamos este capítulo procurando responder de forma precisa a seguinte questão : $O$ que é uma forma quadrática? Para responder a essa questão lançamos mão de cinco categorias (chamadas genericamente de categorias quadráticas) associadas a cada corpo $\mathbb{K}$ tal que $\operatorname{char}(\mathbb{K}) \neq 2$ e mostramos que elas são duas a duas equivalentes. Na sequência, apresentamos as definições e resultados básicos acerca desses objetos de natureza geométrica, entre estes destacamos os teoremas de equivalência de cadeias, de cancelamento e de classificação, todos obtidos por E. Witt em [Witt].

Capítulo 2: Neste capítulo apresentamos um dos conceitos centrais da teoria algébrica de formas quadráticas, a saber, o anel de Witt de um corpo, objeto de natureza algébrica introduzido em Witt. Destacamos também o caráter funtorial das construções de anel de Witt-Grothendieck e de anel de Witt. Fornecemos apresentações (canônicas) desses anéis e fazemos o seu cálculo em alguns casos particulares. Apresentamos o anel graduado de Witt e analisamos suas primeiras componentes. Explicitamos a relação entre ordens de um corpo e suas assinaturas e descrevemos a propriedade local - global de Pfister. Para complementar este capítulo, desenvolvemos um apêndice (Apêndice 1), onde consideramos de um ponto de vista mais genérico a noção de grupo de Grothendieck, que será útil também no capítulo 4.

Capítulo 3 Neste capítulo consideramos as informações essenciais sobre cohomologia galoisiana, com o objetivo de explicitar (no capítulo 5) a sua conexão funtorial com a teoria algébrica de formas quadráticas. Iniciamos desenvolvendo idéias gerais sobre grupos profinitos, fatos básicos sobre teoria de corpos e teoria de Galois infinita. O principal objetivo deste capítulo é usar a teoria Kummer, o teorema 90 de Hilbert e o grupo de Brauer para computar alguns grupos de cohomologia. Anexamos ainda a este capítulo um apêndice (Apêndice 2) para apresentar questões gerais 
de cohomologia e funtores derivados e para complementar a computação da cohomologia com base no grupo multiplicativo do corpo.

Capítulo 4: Neste capítulo fazemos uma construção dos três primeiros funtores da K-teoria algébrica clássica com a intenção de naturalizar a introdução ad hoc da K-teoria de Milnor - que é muito adequada para atacar problemas postos em teoria algébrica de formas quadráticas. Fazemos um pouco mais que isso, pois mostramos que, nos níveis 0,1 e 2, esses funtores são, na realidade, funtores de cohomologia.

Capítulo 5: Neste capítulo final, fechamos esta dissertação através de duas seções que correlacionam definições, resultados e questões postos nos outros capítulos. Entendemos que este é o principal capítulo da dissertação, pois expomos alguns dos principais resultados da teoria algébrica de formas quadráticas apresentados nos últimos 45 anos. Na primeira seção fazemos um extrato do artigo clássico do, medalhista Fields, John Milnor Algebraic K-theory and quadratic formas(Miln3]) assim como uma discussão sobre algumas conjecturas colocadas neste trabalho, que foram posteriormente resolvidas como consequência do trabalho de Vladimir Voevodski e outros ([Voe1], OVV]), culminando com o recebimento da medalha Fields em 2002. Na seção final, apresentamos a conjectura de Murray Marshall sobre assinaturas e esboçamos a solução desta para os corpos pitagóricos e formalmente reais, realizada por Max Dickmann e Francisco Miraglia em outro artigo revolucionário ([DM2 $)$, por meio da introdução do conceito de grupo especial ([DM1]) e de interligações entre os anéis graduados: de Witt, de k-teoria reduzida de Milnor e de cohomologia.

Finalizamos assim nossa dissertação no ponto onde pretendemos iniciar nosso desenvolvimento em outra etapa acadêmica, procurando estabelecer relações gerais entre os constituintes do tripé: anéis graduados, teorias abstratas de formas quadráticas, grupos profinitos. Referências: [AKM], [DM4, [Efr3], [Mrn], MS].

subfile 


\section{Capítulo 1}

\section{Ingredientes da teoria algébrica de formas quadráticas}

Este capítulo inicial tem por objetivos: (i) descrever o que pode ser entendido como "forma quadrática", desenvolvido na seção 1; (ii) estabelecer operações e resultados básicos sobre estes objetos matemáticos, apresentados na seção 2 .

\subsection{Categorias Quadráticas}

Nesta seção procuramos responder, de forma precisa, a indagação: o que é uma forma quadrática sobre um corpo? Esta questão se põe naturalmente pois na literatura específica (e.g., [Lam1, [Lam3 ], há diversas alternativas possíveis postas como definição, e no máximo alguma indicação sobre algum tipo de equivalência entre essas. Chamaremos genericamente, pelo nome de categoria quadrática (sobre um corpo $\mathbb{K}$ ), às categorias formadas por objetos de diferentes naturezas que poderiam servir como base para a teoria algébrica de formas quadráticas - são cinco categorais, no nosso texto - que demonstraremos serem duas a duas equivalentes, quando $\operatorname{char}(\mathbb{K}) \neq 2$.

Nesta seção, está fixado um corpo arbitrário $\mathbb{K}$.

\subsection{1 $B I L S_{\mathbb{K}} \cong P L S_{\mathbb{K}}$}

Seja $V$ um espaço vetorial sobre um corpo $\mathbb{K}$. Uma aplicação $B: V \times V \rightarrow \mathbb{K}$ é dita bilinear simétrica quando satisfaz as seguintes propriedades

$\left(B_{1}\right) \quad B(u+v, w)=B(u, w)+\beta(v, w), \forall u, v, w \in V ;$

$\left(B_{2}\right) \quad B(u, \lambda v)=\lambda B(u, v), \forall u, v \in V, \forall \lambda \in \mathbb{K} ;$

$\left(B_{3}\right) \quad B(u, v)=B(v, u), \forall u, v \in V$

I.e., para cada $u, v \in V$, as funções $B(u,-), B(-, v): V \longrightarrow \mathbb{K}$ são $\mathbb{K}$-lineares e $B(u,-)=$ $B(-, u)$.

Observação 1. Segue diretamente dos axiomas acima que $B(0,-)=0 \in V^{*}$ e $B(-v,-)=$ $-B(v,-) \in V^{*}$.

Definição 1. Um espaço bilinear simétrico (EBS) é um par $(V, B)$, onde $V$ é um $\mathbb{K}$ - espaço vetorial de dimensão finita e $B$ uma forma bilinear simétrica. Um morfismo entre dois $E B S(V, B)$ 
$e\left(V^{\prime}, B^{\prime}\right)$ é uma tripla $\left(B, T, B^{\prime}\right)$, onde $T: V \longrightarrow V^{\prime}$ é uma aplicação $\mathbb{K}$ - linear que faz o seguinte diagrama comutar.

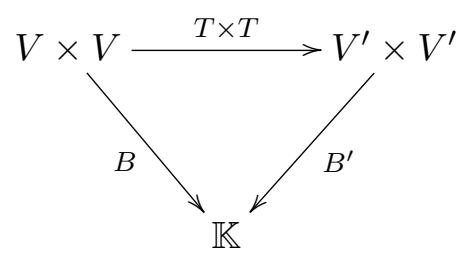

Observação 2. Note que, se $\left(V^{\prime}, B^{\prime}\right)$ é um EBS e $T: V \longrightarrow V^{\prime}$ é uma aplicação $\mathbb{K}$ - linear, então $B^{\prime} \circ(T \times T): V \times V \longrightarrow \mathbb{K}$ é uma forma bilinear simétrica, sempre que $\operatorname{dim}(V) \in \mathbb{N}$.

O símbolo $\operatorname{Hom}_{E B S}\left((V, B),\left(V^{\prime}, B^{\prime}\right)\right)$ é usado para designar o conjunto dos morfismos entre os EBS $(V, B)$ e $\left(V^{\prime}, B^{\prime}\right)$. Claramente $\left(B, I d_{V}, B\right) \in H_{o m} m_{E S}((V, B),(V, B))$. É fácil ver que se $\left(B, T, B^{\prime}\right) \in H_{\operatorname{Hom}}\left((V, B),\left(V^{\prime}, B^{\prime}\right)\right)$ e $\left(B^{\prime}, T^{\prime}, B^{\prime \prime}\right) \in H^{\prime} o_{E B S}\left(\left(V^{\prime}, B^{\prime}\right),\left(V^{\prime \prime}, B^{\prime \prime}\right)\right)$, então $\left(B, T^{\prime} \circ T, B^{\prime \prime}\right) \in \operatorname{Hom}_{E B S}\left((V, B),\left(V^{\prime \prime}, B^{\prime \prime}\right)\right)$.

Definição 2. Definimos BILS $S_{\mathbb{K}}$ para ser a categoria que tem como classe de objetos

$$
O b\left(B I L S_{\mathbb{K}}\right)=\{(V, B):(V, B) \in E B S(\mathbb{K})\} .
$$

E como classe de morfismos

$$
\operatorname{Mor}\left(B I L S_{\mathbb{K}}\right)=\bigcup\left\{\operatorname{Hom}_{E B S}\left((V, B),\left(V^{\prime}, B^{\prime}\right)\right):(V, B),\left(V^{\prime}, B^{\prime}\right) \in O b\left(B I L S_{\mathbb{K}}\right)\right\}
$$

Observação 3. Uma isometria entre dois espaços bilineares $(V, B)$ e $\left(V^{\prime}, B^{\prime}\right)$ é um morfismo $\sigma: V \longrightarrow V^{\prime}$ que é um isomorfismo. Nesse caso, simbolizamos $(V, B) \simeq\left(V^{\prime}, B^{\prime}\right)$. Note que $\simeq \dot{e}$ uma relação de equivalência em $O b\left(B I L S_{\mathbb{K}}\right)$.

Consideramos agora uma nova classe de objetos. Seja $V$ um espaço vetorial sobre $\mathbb{K}$ de dimensão finita, denotamos $\varepsilon_{V}: V \longrightarrow V^{* *}$ a transformação linear de avaliação e fixamos $\varphi: V \longrightarrow V^{*}$ uma transformação $\mathbb{K}$-linear . Um tal par $(V, \varphi)$ é pareamento linear simétrico (PLS) quando o diagrama abaixo for comutativo

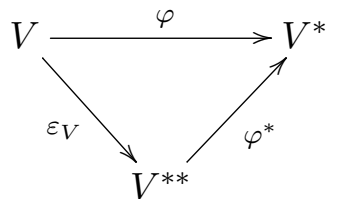

Como para cada $u, v \in V,\left(\varphi^{*} \circ \varepsilon_{V}\right)(v)(u)=\varepsilon_{V}(v)(\varphi(u))=\varphi(u)(v)$, a condição de comutatividade do diagrama equivale a $\varphi(v)(u)=\varphi(u)(v), \forall u, v \in V$.

Definição 3. Um PLS-morfismo de um objeto $(V, \varphi)$ para outro objeto $\left(V^{\prime}, \varphi^{\prime}\right)$ é uma tripla $\left(\varphi, T, \varphi^{\prime}\right)$, onde $T$ é uma transformação linear de $V$ em $V^{\prime}$, tal que o seguinte diagrama é comutativo

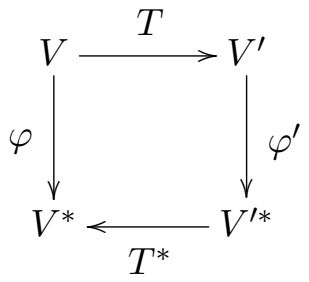


Como $\forall v \in V \quad \varphi(v)=T^{*}\left(\varphi^{\prime}(T(v))\right)=\varphi^{\prime}(T(v) \circ T$, a condição de comutatividade do diagrama equivale a $\varphi(v)(u)=\varphi^{\prime}(T(v))(T(u)), \forall u, v \in V$.

Lema 1. Se $T:(V, \varphi) \longrightarrow\left(V^{\prime}, \varphi^{\prime}\right)$ e $T^{\prime}:\left(V^{\prime}, \varphi^{\prime}\right) \longrightarrow\left(V^{\prime \prime}, \varphi^{\prime \prime}\right)$ são PLS-morfismos, então o seguinte diagrama é comutativo

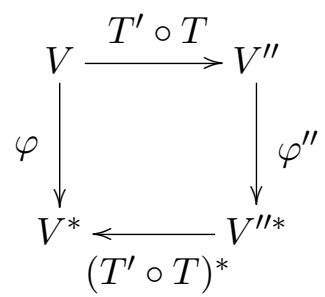

Ou seja, $T^{\prime} \circ T:(V, \varphi) \longrightarrow\left(V^{\prime \prime}, \varphi^{\prime \prime}\right)$ é um PLS-morfismo.

Demonstração. $\left(T^{\prime} \circ T\right)^{*} \circ \varphi^{\prime \prime} \circ\left(T^{\prime} \circ T\right)=\left(T^{*} \circ T^{*}\right) \circ \varphi^{\prime \prime} \circ\left(T^{\prime} \circ T\right)=T^{*} \circ\left(T^{*} \circ \varphi^{\prime \prime} \circ T^{\prime}\right) \circ T=$ $T^{*} \circ \varphi^{\prime} \circ T=\varphi$

Assim claramente podemos fazer a seguinte

Definição 4. Seja $P L S_{\mathbb{K}}$ a categoria que tem por objetos os pares $(V, \varphi)$ e morfismos $(V, \varphi) \longrightarrow$ $\left(V^{\prime}, \varphi^{\prime}\right)$ como acima.

Consideramos agora, para cada tripla de conjuntos $X, Y, Z$, as bijeções (naturais) inversas:

$$
\begin{array}{r}
\operatorname{Set}(X \times Y, Z) \underset{\stackrel{\cong}{\cong}}{\stackrel{\check{\cong}}{\rightleftarrows}} \operatorname{Set}(X, \operatorname{Set}(Y, Z)) \\
(f: X \times Y \longrightarrow Z) \leadsto(\check{f}: X \longrightarrow \operatorname{Set}(Y, Z)), \check{f}(x)(y):=f(x, y) \\
(F: X \longrightarrow \operatorname{Set}(Y, Z)) \leftrightarrow(\hat{F}: X \times Y \longrightarrow Z), \quad \hat{F}(x, y):=F(x)(y)
\end{array}
$$

Proposição 1. A correspondência natural descrita acima origina um isomorfismo de categorias $B I L S_{\mathbb{K}} \cong P L S_{\mathbb{K}}$.

Demonstração. Para cada $\mathbb{K}$ - espaço vetorial $V$ temos bijeções naturais

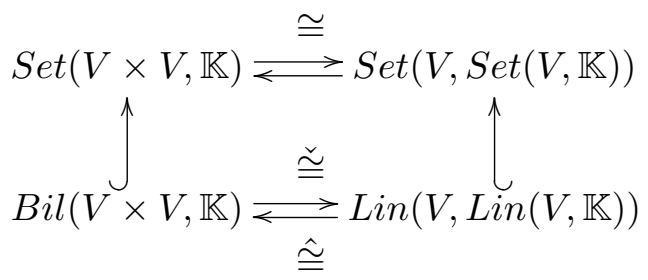

De fato, verifica-se diretamente que: (i) para cada $B: V \times V \longrightarrow \mathbb{K}, B$ é bilinear se e somente se $\check{B} \in \operatorname{Lin}(V, \operatorname{Lin}(V, \mathbb{K}))$; (ii) para cada $\varphi: V \longrightarrow V^{*}, \varphi$ é linear se e só se $\hat{\varphi}$ é bilinear.

A partir daí, vemos que para cada par $(B, \varphi)$ em correspondência (i.e. $\breve{B}=\varphi$, e/ou $\hat{\varphi}=B$ ), vale $B(u, v)=B(v, u)$ se e só se $\varphi(u)(v)=\varphi(v)(u)$, para cada $u, v \in V$. Desta forma, estabelecemos bijeção entre $O b\left(B I L S_{\mathbb{K}}\right)$ e $O b\left(P L S_{\mathbb{K}}\right)$.

Seja $T: V \longrightarrow V^{\prime}$ uma transformação linear entre $\mathbb{K}$-espaços de dimensão finita. $T$ determina um $E B S$-morfismo $T:(V, B) \longrightarrow\left(V, B^{\prime}\right)$ se e só se, para cada $u, v \in V B(u, v)=$ 
$B^{\prime}\left(T(u), T(v)\right.$, mas isto ocorre se e só se $\check{B}(u)(v)=\check{B}^{\prime}(T(u))(T(v)), \forall u, v \in V$. i.e., precisamente quando $T:(V, \check{B}) \longrightarrow\left(V^{\prime}, \check{B}^{\prime}\right)$ é um $P L S$-morfismo. Desta forma, estabelecemos bijeção entre $H_{\operatorname{Hom}}{ }_{E S}\left((V, B),\left(V^{\prime}, B^{\prime}\right)\right)$ e $\operatorname{Hom}_{P L S}\left((V, \check{B}),\left(V^{\prime}, \check{B}^{\prime}\right)\right)$.

Assim, claramente temos funtores :

- $F: B I L S_{\mathbb{K}} \longrightarrow P L S_{\mathbb{K}}$,

$\left((V, B) \stackrel{\left(B, T, B^{\prime}\right)}{\longrightarrow}\left(V^{\prime}, B^{\prime}\right)\right) \mapsto\left((V, \check{B}) \stackrel{\left(\check{B}, T, \check{B}^{\prime}\right)}{\longrightarrow}\left(V^{\prime}, \check{B}^{\prime}\right)\right)$;

- $G: P L S_{\mathbb{K}} \longrightarrow B I L S_{\mathbb{K}}$

$\left((V, \varphi) \stackrel{\left(\varphi, T, \varphi^{\prime}\right)}{\longrightarrow}\left(V^{\prime}, \varphi^{\prime}\right)\right) \mapsto\left((V, \hat{\varphi}) \stackrel{\left(\hat{\varphi}, T, \hat{\varphi}^{\prime}\right)}{\longrightarrow}\left(V^{\prime}, \hat{\varphi}^{\prime}\right)\right)$

e estes estabelecem isomorfismos inversos de categorias.

\subsection{2 $Q U A D_{\mathbb{K}} \cong B I L S_{\mathbb{K}}$}

Seja $\mathbb{K}$ uma corpo de característica $\neq 2$ e seja $V$ um $\mathbb{K}$ - espaço vetorial de dimensão finita. Uma $\mathbb{K}$ - forma quadrática (ou forma quadrática sobre $\mathbb{K}$ ) é uma aplicação $q: V \longrightarrow \mathbb{K}$ que cumpre os seguintes axiomas:

$\left(q_{1}\right) q(\lambda u)=\lambda^{2} q(u), \forall \lambda \in \mathbb{K}, \forall u \in V ;$

$\left(q_{2}\right) q(u+v)+q(u-v)=2(q(u)+q(v)), \forall u, v \in V ;$

$\left(q_{3}\right) q(u+\lambda v)-q(u-\lambda v)=\lambda(q(u+v)-\lambda q(u-v)), \forall \lambda \in \mathbb{K}, \forall u, v \in V$.

Observação 4. Segue diretamente de $\left(q_{1}\right)$ que $q(0)=0$ e $q(-u)=q(u), \forall u \in V$. Da "lei do paralelogramo" $\left(i . e ., q_{2}\right)$ notamos que $q(u+v)-(q(u)+q(v))=(q(u+v)-q(u-v)) / 2, \forall u, v \in V$.

Definição 5. Um espaço quadrático é um par $(V, q)$ constando de um $\mathbb{K}$ - espaço vetorial $V$ de dimensão finita e uma forma quadrática $q$.

Um morfismo entre espaços quadráticos $(V, q)$ e $\left(V^{\prime}, q^{\prime}\right)$ é uma tripla $\left(q, \sigma, q^{\prime}\right)$, onde $\sigma: V \longrightarrow V$ é uma aplicação linear que faz o seguinte diagrama comutar

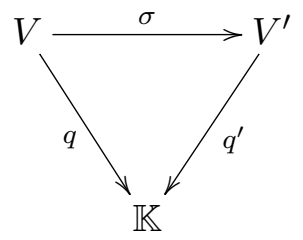

ou seja, $q^{\prime}(\sigma(v))=q(v), \forall v \in V$.

Esses objetos e morfismos nos permitem definir uma categoria, a qual denotamos por $Q U A D_{\mathbb{K}}$.

Para cada forma bilinear $B: V \times V \longrightarrow \mathbb{K}$ associamos uma forma quadrática $q_{B}(v) \doteq B(v, v)$ : segue imediatamente dos axiomas de forma bilinear simétrica que $q_{B}$ é, de fato, uma forma quadrática. Reciprocamente, dada uma forma quadrática $q: V \longrightarrow \mathbb{K}$, associamos uma forma bilinear simétrica $B_{q}(u, v)=(q(u+v)-q(u-v)) / 4$. A simetria de $B_{q}$ segue do axioma $\left(q_{1}\right)$, pois $q(v-u)=q(u-v)$; o axioma $\left(q_{3}\right)$ nos fornece

$$
B_{q}(u, \lambda v)=\lambda B_{q}(u, v), \forall \lambda \in \mathbb{K}, \forall u, v \in U .
$$

Finalmente, fazemos em $\left(q_{2}\right) ; u=w_{1} \pm w$ e $v=w_{2} \pm w$ e obtemos

$$
(*) q\left(w_{1}+w_{2} \pm 2 w\right)+q\left(w_{1}-w_{2}\right)=2\left(q\left(w_{1} \pm w\right)+q\left(w_{2} \pm w\right)\right)
$$


e assim

$$
(* *) B_{q}\left(w_{1}+w_{2}, 2 w\right)=2 B_{q}\left(w_{1}, w\right)+2 B_{q}\left(w_{2}, w\right)
$$

fazendo $w_{2}=0$ na relação anterior vem

$$
B_{q}\left(w_{1}, 2 w\right)=2 B_{q}\left(w_{1}, w\right)
$$

logo, como $\operatorname{char}(\mathbb{K}) \neq 2$, obtemos de $\left(^{*}\right)$ e $(* *)$

$$
B_{q}\left(w_{1}+w_{2}, w\right)=B_{q}\left(w_{1}, w\right)+B_{q}\left(w_{2}, w\right), \forall w_{1}, w_{2}, w \in U
$$

Note agora que:

- $B_{q_{B}}(u, v)=(B(u+v, u+v)-B(u-v, u-v)) / 4=B(u, v), \forall u, v \in V$;

- $q_{B_{q}}(u)=(q(u+u)-q(u-u)) / 4=q(u), \forall u \in V$.

Precisamos isso, dizendo que as aplicações (bem definidas) abaixo, são bijeções inversas uma da outra

$$
\begin{gathered}
Q: O b\left(B I L S_{\mathbb{K}}\right) \longrightarrow O b\left(Q U A D_{\mathbb{K}}\right) \\
(V, B) \longmapsto\left(V, q_{B}\right) \\
B: O b\left(Q U A D_{\mathbb{K}}\right) \longrightarrow O b\left(B I L S_{\mathbb{K}}\right) \\
(V, q) \longmapsto\left(V, B_{q}\right)
\end{gathered}
$$

Se $\left(q, \sigma, q^{\prime}\right):(V, q) \longrightarrow\left(V^{\prime}, q^{\prime}\right)$ for um morfismo entre espaços quadráticos, então $\left(B_{q}, \sigma, B_{q^{\prime}}\right)$ é um morfismo entre os espaços bilineares simétricos $\left(V, B_{q}\right)$ e $\left(V^{\prime}, B_{q^{\prime}}\right)$. De fato,

$$
\begin{aligned}
B_{q^{\prime}} \circ(\sigma \times \sigma)(u, v) & =B_{q^{\prime}}(\sigma(u), \sigma(v)) \\
= & \left(q^{\prime}(\sigma(u)+\sigma(v))-q^{\prime}(\sigma(u)-\sigma(v))\right) / 4 \\
= & \left(q^{\prime}(\sigma(u+v))-q^{\prime}(\sigma(u-v))\right) / 4 \\
= & (q(u+v)-q(u-v)) / 4 \\
= & B_{q}(u, v)
\end{aligned}
$$

Por outro lado, se $\left(B, \varphi, B^{\prime}\right) \in \operatorname{Mor}\left(B I L S_{\mathbb{K}}\right)$, então $\left(Q_{B}, \varphi, Q_{B^{\prime}}\right) \in \operatorname{Mor}\left(Q U A D_{\mathbb{K}}\right)$, pois $q_{B}(u)=B(u, u)=B^{\prime}(T(u), T(u))=q_{B^{\prime}}(T(u)), \forall u \in V$.

Em suma, temos a seguinte

Proposição 2. Os seguintes funtores

$$
\begin{gathered}
B: Q U A D_{\mathbb{K}} \longrightarrow B I L S_{\mathbb{K}} \\
(V, q) \in O b\left(Q U A D_{\mathbb{K}}\right) \longmapsto\left(V, B_{q}\right) \in O b\left(B I L S_{\mathbb{K}}\right) \\
\left(q, \sigma, q^{\prime}\right) \in \operatorname{Hom}\left((V, q),\left(V^{\prime}, q^{\prime}\right)\right) \longmapsto\left(B_{q}, \sigma, B_{q^{\prime}}\right) \in \operatorname{Hom}\left(\left(V, B_{q}\right),\left(V^{\prime}, B_{q^{\prime}}\right)\right) \\
Q: B I L S_{\mathbb{K}} \longrightarrow Q U A D_{\mathbb{K}}
\end{gathered}
$$




$$
\begin{gathered}
(V, B) \in O b\left(B I L S_{\mathbb{K}}\right) \longmapsto\left(V, Q_{B}\right) \in O b\left(Q U A D_{\mathbb{K}}\right) \\
\left(B, \sigma, B^{\prime}\right) \in \operatorname{Hom}\left((V, B),\left(V^{\prime}, B^{\prime}\right)\right) \longmapsto\left(Q_{B}, \sigma, Q_{B}^{\prime}\right) \in \operatorname{Hom}\left(\left(V, Q_{B}\right)\left(V^{\prime}, Q_{B^{\prime}}\right)\right)
\end{gathered}
$$

são tais que $B \circ Q=I_{B I L S_{\mathbb{K}}}$ e $Q \circ B=I_{Q U A D_{\mathbb{K}}}$. Em outros termos, o par de funtores $(B, Q)$ estabelece um isomorfismo entre as categorias $Q U A D_{\mathbb{K}}$ e $B I L S_{\mathbb{K}}$.

\subsection{3 $B I L S_{\mathbb{K}} \simeq B S_{\mathbb{K}}$}

Definição 6. Sejam $\varepsilon$ e $\varepsilon^{\prime}$ bases ordenadas, respectivamente, dos $\mathbb{K}$-espaços bilineares simétricos $(V, B)$ e $\left(V, B^{\prime}\right)$. Um morfismo de $(V, B, \varepsilon)$ para $\left(V^{\prime}, B^{\prime}, \varepsilon^{\prime}\right)$ é uma tripla $\left(B, T, B^{\prime}\right)$ tal que $B=$ $B^{\prime} \circ(T \times T)$, onde $T: V \longrightarrow V^{\prime}$ é uma aplicação $\mathbb{K}$ - linear. Isso define uma categoria, a qual denotamos por $B S_{\mathbb{K}}$.

Proposição 3. O Funtor de esquecimento $\mathcal{U}: B S_{\mathbb{K}} \longrightarrow B I L S_{\mathbb{K}}$ determina uma equivalência de categorias.

Demonstração. Um resultado fundamental da Teoria das Categorias, afirma que ${ }^{1}$ um funtor determina uma equivalência de categorias se e só se este funtor for pleno, fiel e tiver imagem repleta (i.e., cada objeto da categoria alvo é isomorfo a algum objeto da imagem do funtor). É imediato que o funtor de esquecimento $\mathcal{U}$ é pleno, fiel e sobrejetor nos objetos, logo determina equivalência de categorias $B I L S_{\mathbb{K}} \simeq B S_{\mathbb{K}}$.

\subsection{4 $B S_{\mathbb{K}} \simeq M S_{\mathbb{K}}$}

Seja $\mathcal{S}(\mathbb{K})=\bigcup_{n \in \mathbb{N}} \mathcal{S}_{n}(\mathbb{K})$, onde $\mathcal{S}_{n}(\mathbb{K})$ é o conjunto das matrizes simétricas de ordem n com entradas no corpo $\mathbb{K}$. Um morfismo entre pares $(n, M),\left(n^{\prime}, M^{\prime}\right) \in \mathbb{N} \times \mathcal{S}(\mathbb{K})$ é uma matriz $U \in$ $M_{n^{\prime} \times n}(\mathbb{K})$ tal que $M=U^{t} \cdot M^{\prime} \cdot U$.

Observação 5. (1) Note que se $M^{\prime} \in \mathcal{S}_{n^{\prime}}(\mathbb{K})$ e $U \in M_{n^{\prime} \times n}(\mathbb{K})$, então $U^{t} \cdot M^{\prime} . U \in \mathcal{S}_{n}(\mathbb{K})$, pois esta é uma matriz $n \times n$ e $\left(U^{t} \cdot M^{\prime} \cdot U\right)^{t}=(U)^{t} \cdot\left(M^{\prime}\right)^{t} \cdot\left(U^{t}\right)^{t}=U^{t} \cdot M^{\prime} \cdot U$.

(2) Se $U:(n, M) \longrightarrow\left(n^{\prime}, M^{\prime}\right)$ e $U^{\prime}:\left(n^{\prime}, M^{\prime}\right) \longrightarrow\left(n^{\prime \prime}, M^{\prime \prime}\right)$ são morfismos, então $U^{\prime} \cdot U$ é um morfismo de $(n, M)$ para $\left(n^{\prime \prime}, M^{\prime \prime}\right)$ : pois, como por hipótese $M=U^{t} M^{\prime} U$ e $M^{\prime}=\left(U^{\prime}\right)^{t} M^{\prime \prime}\left(U^{\prime}\right)$, segue que $\left(U^{\prime} U\right)^{t} M^{\prime \prime}\left(U^{\prime} U\right)=U^{t}\left(U^{\prime t} M^{\prime \prime} U^{\prime}\right) U=U^{t} M^{\prime} U=M$.

\section{Definição 7.}

(i) O conjunto de morfismos de $(n, M)$ para $\left(n^{\prime}, M^{\prime}\right)$ é representado por $\operatorname{Hom}\left((n, M),\left(n^{\prime}, M^{\prime}\right)\right)$.

(ii) Denotamos por $\mathrm{Ob}\left(M S_{\mathbb{K}}\right)$ o conjunto $\mathbb{N} \times \mathcal{S}(\mathbb{K})$ e por $\operatorname{Mor}\left(M S_{\mathbb{K}}\right)$ a coleção de morfismos

$$
\operatorname{Mor}\left(M S_{\mathbb{K}}\right)=\left\{U:(n, M) \longrightarrow\left(n^{\prime}, M^{\prime}\right): U \in M_{n^{\prime} \times n}(\mathbb{K}), M=U^{t} \cdot M^{\prime} \cdot U\right\}
$$

(iii) Como observado acima, podemos definir a seguinte operação associativa de composição

$$
\begin{aligned}
& \circ: \operatorname{Hom}\left((n, M),\left(n^{\prime}, M^{\prime}\right)\right) \times \operatorname{Hom}\left(\left(n^{\prime}, M^{\prime}\right),\left(n^{\prime \prime}, M^{\prime \prime}\right)\right) \longrightarrow \operatorname{Hom}\left((n, M),\left(n^{\prime \prime}, M^{\prime \prime}\right)\right) \\
& \left(U, U^{\prime}\right) \quad \longmapsto \quad U^{\prime} U
\end{aligned}
$$

e é fácil ver que $\left(O b\left(M S_{\mathbb{K}}\right), M o r\left(M S_{\mathbb{K}}\right)\right.$, o) é uma categoria. Denotamos tal categoria por $M S_{\mathbb{K}}$.

\footnotetext{
${ }^{1}$ Mediante uma versão suficientemente forte do Axioma da Escolha.
} 
Observação 6. (1) Note que $M S_{\mathbb{K}}$ é uma categoria pequena, i.e., a classe de objetos é um conjunto $e$ a classe dos morfismos também é um conjunto.

(2) Seguirá de resultados apresentados na próxima seção que toda matriz simétrica é $M S_{\mathbb{K}^{-}}$ isomorfa a alguma matriz diagonal.

(3) Seja $\mathbb{K}\left[X_{1}, \cdots, X_{n}\right]$ um anel de polinômios em $n$ indeterminadas. Temos uma clara bijeção entre polinômios homogêneos de grau $2, h\left(X_{1}, \cdots, X_{n}\right)=\sum_{1 \leq i \leq j \leq n} a_{i j} X_{i} X_{j}$ e matrizes triangulares superiores $T \in M_{n \times n}(\mathbb{K})$. Se char $(\mathbb{K}) \neq 2$, temos correspondência bijetiva entre matrizes triangulares superiores $T \in M_{n \times n}(\mathbb{K})$ e matrizes simétricas $M \in M_{n \times n}(\mathbb{K})$.

Listaremos abaixo algumas associações:

(A) A cada transformação $\mathbb{K}$-linear $T: V \longrightarrow V^{\prime}$ e a cada escolha de bases ordenadas, $\varepsilon=\left(v_{1}, \cdots, v_{n}\right)$ de $V$ e $\varepsilon^{\prime}=\left(v_{1}^{\prime}, \cdots, v_{n^{\prime}}^{\prime}\right)$ de $V^{\prime}$, denotaremos ${ }_{\varepsilon}[T]_{\varepsilon^{\prime}} \in M_{n \times n^{\prime}}(\mathbb{K})$ a matriz correspondente.

(B) A cada forma bilinear simétrica $B: V \times V \longrightarrow \mathbb{K}$ e a cada base ordenada $\varepsilon=\left(v_{1}, \cdots, v_{n}\right)$ de $V$ denotaremos a matriz simétrica correspondente por ${ }_{\varepsilon}[B]_{\varepsilon} \in \mathcal{S}_{n}(\mathbb{K})$, i.e. $\left({ }_{\varepsilon}[B]_{\varepsilon}\right)_{i j}:=B\left(v_{i}, v_{j}\right)$, $1 \leq i, j \leq n$.

Note que, se $B^{\prime}: V^{\prime} \times V^{\prime} \longrightarrow K$ é bilinear e simétrica e $T: V \longrightarrow V^{\prime}$ é linear, então

$$
\left({ }_{\varepsilon}\left[B^{\prime} \circ(T \times T)\right]_{\varepsilon}\right)_{i j}:=B^{\prime}\left(T\left(v_{i}\right), T\left(v_{j}\right)\right)=\left(\left({ }_{\varepsilon}[T]_{\varepsilon^{\prime}}\right)^{t}\left(\varepsilon_{\varepsilon^{\prime}}\left[B^{\prime}\right]_{\varepsilon^{\prime}}\right)\left({ }_{\varepsilon}[T]_{\varepsilon^{\prime}}\right)\right)_{i j} .
$$

(C) A cada matriz $U \in M_{n^{\prime} \times n}(\mathbb{K})$ associamos uma aplicação $\mathbb{K}$-linear $L_{U}: \mathbb{K}^{n} \longrightarrow \mathbb{K}^{n^{\prime}}$ mediante o seguinte diagrama comutativo

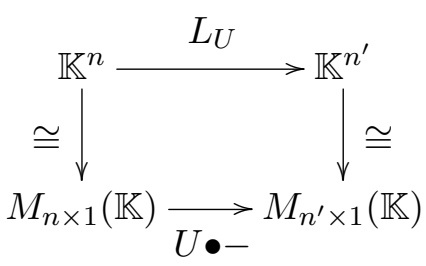

onde

Para cada $j \in \mathbb{N}, \mathbb{K}^{j} \stackrel{\cong}{\longrightarrow} M_{j \times 1}(\mathbb{K})$ indica o $\mathbb{K}$-isomorfismo que identifica uplas com vetores coluna e

$$
\begin{aligned}
& U \bullet-: M_{n \times 1}(\mathbb{K}) \quad \longrightarrow \quad M_{n^{\prime} \times 1}(\mathbb{K}) \\
& {\left[a_{1} \ldots a_{n}\right]^{t} \longmapsto U \cdot\left[a_{1} \ldots a_{n}\right]^{t}}
\end{aligned}
$$

Note que $\operatorname{Can(\mathbb {K}^{n})}\left[L_{U}\right]_{\operatorname{Can}\left(\mathbb{K}^{n^{\prime}}\right)}=U$.

(D) A cada matriz $M \in \mathcal{S}_{n}(\mathbb{K})$ associamos uma forma bilinear simétrica $\hat{M}: \mathbb{K}^{n} \times \mathbb{K}^{n} \longrightarrow \mathbb{K}$ através do seguinte diagrama comutativo

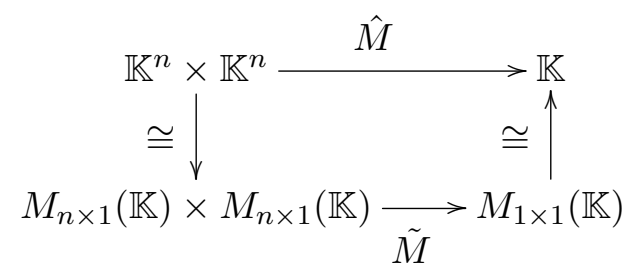


com

$$
\begin{array}{cl}
\tilde{M}: M_{n \times 1}(\mathbb{K}) \times M_{n \times 1}(\mathbb{K}) & \longrightarrow M_{1 \times 1}(\mathbb{K}) \\
(a, b) & \longmapsto a^{t} M b
\end{array}
$$

Note que:

- $\operatorname{Can}\left(\mathbb{K}^{n}\right)[\hat{M}]_{\operatorname{Can}\left(\mathbb{K}^{n}\right)}=M$;

- Se $M^{\prime} \in \mathcal{S}_{n^{\prime}}(\mathbb{K})$ e $U \in M_{n^{\prime} \times n}(\mathbb{K})$, então $U^{t} M^{\prime} U \in \mathcal{S}_{n}(\mathbb{K})$ e $\left(\widehat{U^{t} M^{\prime} U}\right)=\widehat{M^{\prime}} \circ\left(L_{U} \times L_{U}\right)$.

Proposição 4. As seguintes aplicações

$$
\begin{gathered}
F: B S_{\mathbb{K}} \longrightarrow M S_{\mathbb{K}} \\
(V, B, \varepsilon) \in O b\left(B S_{\mathbb{K}}\right) \longmapsto F(V, B, \varepsilon)=\left(\operatorname{dim}_{\mathbb{K}} V,_{\varepsilon}[B]_{\varepsilon}\right) \\
\left(B, T, B^{\prime}\right) \in \operatorname{Hom}\left((V, B, \varepsilon),\left(V^{\prime}, B^{\prime}, \varepsilon^{\prime}\right)\right) \longmapsto F\left(\left(B, T, B^{\prime}\right)\right)={ }_{\varepsilon}[T]_{\varepsilon^{\prime}} \\
G: M S_{\mathbb{K}} \longrightarrow B S_{\mathbb{K}} \\
(n, M) \in O b\left(M S_{\mathbb{K}}\right) \longmapsto G(n, M)=\left(\mathbb{K}^{n}, \hat{M}, \operatorname{Can}\left(\mathbb{K}^{n}\right)\right) \\
U \in \operatorname{Hom}\left((n, M),\left(n^{\prime}, M^{\prime}\right)\right) \longmapsto G(U)=\left(\hat{M}, L_{U}, \hat{M}^{\prime}\right)
\end{gathered}
$$

definem funtores tais que $F \circ G=I d e G \circ F \cong I d$. Em particular, determinamos uma equivalência de categorias $B S_{\mathbb{K}} \simeq M S_{\mathbb{K}}$.

Demonstração. • Pelo que vimos acima, $F$ e $G$ determinam, de fato, funções bem definidas nos objetos e flechas.

- Se $U \in M_{n^{\prime} \times n}(\mathbb{K})$ e $U^{\prime} \in M_{n^{\prime \prime} \times n^{\prime}}(\mathbb{K})$, como $U^{\prime}\left(U\left[a_{1} \cdots a_{n}\right]^{t}\right)=\left(U^{\prime} U\right)\left[a_{1} \cdots a_{n}\right]^{t}$, segue que $L_{U^{\prime} U}=L_{U^{\prime}} \circ L_{U}$; claramente $L_{I d_{n}}=I d_{\mathbb{K}^{n}}$. Logo $G$ é um funtor.

- Se $\varepsilon=\left(v_{1}, \cdots, v_{n}\right)$ é base ordenada de $V$ temos que $i d_{V}:(V, B, \varepsilon) \longrightarrow(V, B, \varepsilon)$ é tal que $F\left(\left(B, i d_{V}, B\right)\right)={ }_{\varepsilon}\left[i d_{V}\right]_{\varepsilon}=I d_{n}$. Se $\left(B, T, B^{\prime}\right) \in \operatorname{Hom}\left((V, B, \varepsilon),\left(V^{\prime}, B^{\prime}, \varepsilon^{\prime}\right)\right)$ e $\left(B^{\prime}, T^{\prime}, B^{\prime \prime}\right) \in$ $\operatorname{Hom}\left(\left(V^{\prime}, B^{\prime}, \varepsilon^{\prime}\right),\left(V^{\prime \prime}, B^{\prime \prime}, \varepsilon^{\prime \prime}\right)\right)$, temos

$$
F\left(B, T^{\prime} \circ T, B^{\prime \prime}\right)={ }_{\varepsilon}\left[T^{\prime} \circ T\right]_{\varepsilon^{\prime \prime}}={ }_{\varepsilon^{\prime}}\left[T^{\prime}\right]_{\varepsilon^{\prime \prime} \cdot \varepsilon}[T]_{\varepsilon^{\prime}}=F\left(B^{\prime}, T^{\prime}, B^{\prime \prime}\right) \cdot F\left(B, T, B^{\prime}\right)
$$

Assim $F$ é funtor.

- Para $(n, M) \in O b\left(M S_{\mathbb{K}}\right) \operatorname{temos} F G(n, M)=F\left(\mathbb{K}^{n}, \hat{M}, \operatorname{Can}\left(\mathbb{K}^{n}\right)\right)=\left(n, \operatorname{Can}\left(\mathbb{K}^{n}\right)[\hat{M}]_{\operatorname{Can}\left(\mathbb{K}^{n}\right)}\right)=$ $(n, M)$. Se $U \in \operatorname{Hom}\left((n, M),\left(n^{\prime}, M^{\prime}\right)\right)$,então

$$
F G(U)=F\left(T_{U}:\left(\mathbb{K}^{n}, \hat{M}, \operatorname{Can}\left(\mathbb{K}^{n}\right)\right) \longrightarrow\left(\mathbb{K}^{n^{\prime}}, \hat{M}^{\prime}, \operatorname{Can}\left(\mathbb{K}^{n^{\prime}}\right)\right)\right)=_{\operatorname{Can}\left(\mathbb{K}^{n}\right)}\left[L_{U}\right]_{\operatorname{Can}\left(\mathbb{K}^{n^{\prime}}\right)}=U
$$

- Se $(V, B, \varepsilon) \in O b\left(B S_{\mathbb{K}}\right)$, então $G F(V, B, \varepsilon)=G\left(\operatorname{dim}_{\mathbb{K}} V_{,_{\varepsilon}}[B]_{\varepsilon}\right)=$ $\left(\mathbb{K}^{\operatorname{dim}_{\mathbb{K}} V}, \widehat{\varepsilon[B]_{\varepsilon}}, \operatorname{Can}\left(\mathbb{K}^{d i m_{K} V}\right)\right)$. A base ordenada $\varepsilon=\left(v_{1}, \cdots, v_{n}\right)$ de $V$ determina um único isomorfismo $\mathbb{K}$-linear $E_{\varepsilon}: V \longrightarrow \mathbb{K}^{n}$ que faz corresponder o vetor $v_{i} \in V$ com o vetor $e_{i} \in \mathbb{K}^{n}$ da base canônica. Como $\widehat{\varepsilon[B]_{\varepsilon}} \circ\left(E_{\varepsilon} \times E_{\varepsilon}\right)\left(v_{i}, v_{j}\right)=\widehat{\varepsilon_{[B]}}\left(e_{i}, e_{j}\right)=B\left(v_{i}, v_{j}\right)$, temos que $E_{\varepsilon}:(V, B, \varepsilon) \stackrel{\cong}{\longrightarrow}$ $\left(\mathbb{K}^{n}, \widehat{\varepsilon[B]_{\varepsilon}}, C a n\left(\mathbb{K}^{n}\right)\right)$ é $B S_{\mathbb{K}^{-}}$isomorfismo. Denotamos $\eta_{(V, B, \varepsilon)}:(V, B, \varepsilon) \stackrel{\cong}{\longrightarrow} G(F(V, B, \varepsilon))$ o $B S_{\mathbb{K}^{-}}$ isomorfismo $E_{\varepsilon}$.

- Resta verificar que a família dos isomorfismos $\eta_{(V, B, \varepsilon)}$, para $(V, B, \varepsilon) \in O b\left(B S_{\mathbb{K}}\right)$, descrita logo acima, determina isomorfismo natural $\eta: I d \stackrel{\cong}{\longrightarrow} G \circ F$. Seja $\left(B, T, B^{\prime}\right) \in \operatorname{Hom}\left((V, B, \varepsilon),\left(V^{\prime}, B^{\prime}, \varepsilon^{\prime}\right)\right)$. 
$G F\left(B, T, B^{\prime}\right)=G\left({ }_{\varepsilon}[T]_{\varepsilon^{\prime}}\right)=\left(\widehat{{ }_{\varepsilon}[B]_{\varepsilon}}, L_{\varepsilon[T]_{\varepsilon^{\prime}},}, \widehat{\varepsilon^{\prime}\left[B^{\prime}\right]_{\varepsilon^{\prime}}}\right)$. Temos $G F\left(B, T, B^{\prime}\right) \circ \eta_{(V, B, \varepsilon)}=\left(B, L_{\varepsilon[T]_{\varepsilon^{\prime}}} \circ\right.$

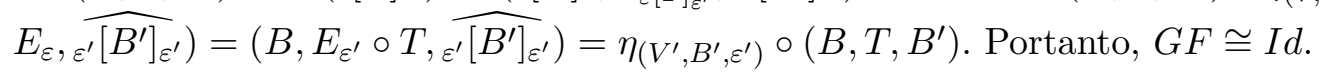

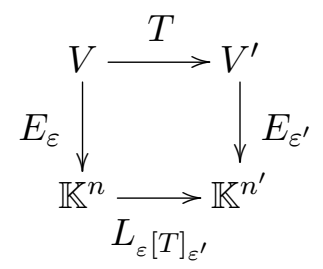

\subsubsection{Resultados centrais sobre categorias quadráticas}

Devido as Proposições 1, 2, 3, 4 obtemos o:

Teorema 2. Se $\mathbb{K}$ é um corpo com característica $\neq 2$, as categorias quadráticas

$$
B I L S_{K}, P L S_{\mathbb{K}}, Q U A D_{\mathbb{K}}, B S_{K}, M S_{\mathbb{K}}
$$

são categorias duas a duas equivalentes.

Como a categoria $M S_{\mathbb{K}}$ é uma categoria pequena, obtemos do Teorema o seguinte:

Corolário 1. Se $\mathbb{K}$ é um corpo com característica $\neq 2$, as categorias quadráticas

$$
B I L S_{K}, P L S_{\mathbb{K}}, Q U A D_{\mathbb{K}}, B S_{K}, M S_{\mathbb{K}}
$$

possuem esqueletos pequenos e dois a dois isomorfos. Em particular, para cada par de categorias quadráticas, temos bijeção entre suas classes de isomorfismo (ou de isometria) de objetos e, se tomamos representantes para estas classes de isomorfismo, obtemos uma bijeções entre cada par de totalidades de representantes, sendo que estas totalidades formam conjuntos.

\subsection{Operações e resultados básicos sobre formas quadráticas}

Já tendo identificado as apresentações alternativas do conceito geométrico de "forma quadrática", apresentamos nesta seção as definições e resultados centrais necessários para uma adequada codificação algébrica deste conceito - através da noção de anel de Witt- que será apresentada no Capítulo 2. Adicionamos também ligação entre formas quadráticas e álgebras de quatérnions e o conceito de forma de Pfister. A referência central sobre o material desenvolvido nesta seção é o livro [Lam3], contudo procuramos apresentar provas alternativas ou em ordenação diferente da apresentada neste importante livro, para benefício de um futuro interessado no tema.

Nesta seção, fixamos um corpo $\mathbb{K}$ tal que $\operatorname{char}(\mathbb{K}) \neq 2$.

\subsubsection{Soma direta e Produto tensorial em $B I L S_{\mathbb{K}}$}

É bem conhecido que a noção de soma direta de espaços vetoriais tem uma caracterização em termos de propriedades universais que a descreve como coproduto na categoria dos espaços vetoriais sobre um corpo fixado. Esta construção pode ser "levantada" para formas bilineares, mais precisamente: para determinados pares de morfismos em $B I L S_{\mathbb{K}}$ podemos construir o coproduto nesta categoria e este tem como espaço vetorial subjacente a soma direta dos espaços originais. 
Definição 8. Sejam $T \in \operatorname{Hom}\left((V, B),\left(V^{\prime \prime}, B^{\prime \prime}\right)\right)$ e $T^{\prime} \in \operatorname{Hom}\left(\left(V^{\prime}, B^{\prime}\right),\left(V^{\prime \prime}, B^{\prime \prime}\right)\right)$. Dizemos que $T$ é ortogonal a $T^{\prime}$ quando $\operatorname{Im}(T) \perp \operatorname{Im}\left(T^{\prime}\right)$.

Sejam $(V, B)$ e $\left(V^{\prime}, B^{\prime}\right)$ EBS. Definimos um novo EBS $V^{\prime \prime} \doteq\left(V \oplus^{\perp} V^{\prime}, B \oplus^{\perp} B^{\prime}\right)$, onde $B \oplus^{\perp} B^{\prime}$ é a forma bilinear simétrica em $V \oplus V^{\prime}$ definida por "justaposição", i.e. $B \oplus^{\perp} B^{\prime}\left(\left(u_{1}, u_{1}^{\prime}\right),\left(u_{2}, u_{2}^{\prime}\right)\right):=$ $B\left(u_{1}, u_{2}\right)+B^{\prime}\left(u_{1}^{\prime}, u_{2}^{\prime}\right)$, para cada $u_{1}, u_{2} \in V$ e cada $u_{1}^{\prime}, u_{2}^{\prime} \in V^{\prime}$ (bem definida). Nesse caso, se $\iota_{V}$ e $\iota_{V^{\prime}}$ denotam as inclusões naturais em $V \oplus V^{\prime}$, então claramente $\iota_{V}$ e $\iota_{V^{\prime}}$ são ortogonais. Além disso:

Proposição 5. $\left(V^{\prime \prime}, \iota_{V}, \iota_{V^{\prime}}\right)$ é o coproduto dos objetos $(V, B)$ e $\left(V^{\prime}, B^{\prime}\right)$ em relação a classe dos EBS-morfismos ortogonais.

Demonstração. Se $\left(\tilde{V}, t, t^{\prime}\right)$ é uma tripla , onde $(\tilde{V}, \tilde{B})$ é um espaço bilinear simétrico e os morfismos $t \in \operatorname{Hom}((V, B),(\tilde{V}, \tilde{B}))$ e $t^{\prime} \in \operatorname{Hom}\left(\left(V^{\prime}, B^{\prime}\right),(\tilde{V}, \tilde{B})\right)$ são ortogonais, então existe um único EBSmorfismo $\phi$ que torna o seguinte diagrama comutativo

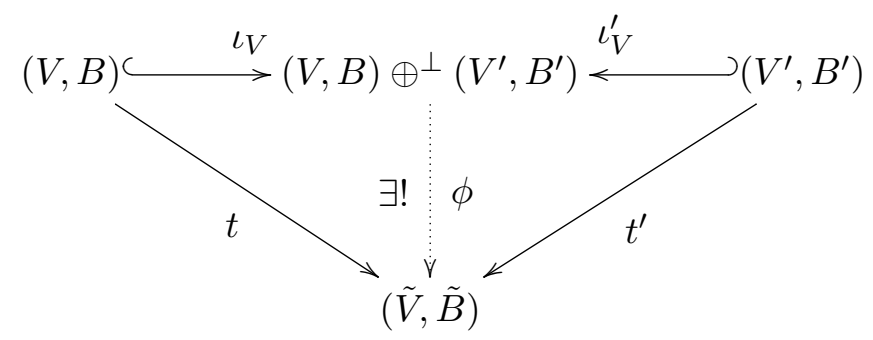

De fato, definindo $\phi\left(x, x^{\prime}\right):=t(x)+t^{\prime}\left(x^{\prime}\right)$ é fácil ver que $\phi$ é linear e faz o diagrama comutar e também que é único com estas propriedades. Basta então verificar que $\phi$ é um EBS morfismo. Mas:

$$
\begin{gathered}
\tilde{B}\left(\phi\left(x_{1}, x_{1}^{\prime}\right), \phi\left(x_{2}, x_{2}^{\prime}\right)\right)=\tilde{B}\left(t\left(x_{1}\right)+t^{\prime}\left(x_{1}^{\prime}\right), t\left(x_{2}\right)+t^{\prime}\left(x_{2}^{\prime}\right)\right)= \\
\tilde{B}\left(t\left(x_{1}\right), t\left(x_{2}\right)\right)+\tilde{B}\left(t^{\prime}\left(x_{1}^{\prime}\right), t^{\prime}\left(x_{2}^{\prime}\right)\right)+\tilde{B}\left(t\left(x_{1}\right), t^{\prime}\left(x_{2}^{\prime}\right)\right)+\tilde{B}\left(t^{\prime}\left(x_{1}^{\prime}\right), t\left(x_{2}\right)\right)= \\
B\left(x_{1}, x_{2}\right)+B^{\prime}\left(x_{1}^{\prime}, x_{2}^{\prime}\right)+0+0=\left(B \oplus^{\perp} B^{\prime}\right)\left(\left(x_{1}, x_{1}^{\prime}\right),\left(x_{2}, x_{2}^{\prime}\right)\right) .
\end{gathered}
$$

Observação 7. $O$ coproduto de dois $E B S(V, B),\left(V^{\prime}, B^{\prime}\right)$ é também denotado por $\left(V \perp V^{\prime}, B \perp\right.$ $\left.B^{\prime}\right)$.

Definição 9. (Produto tensorial de EBS) O produto tensorial de dois $E B S(V, B)$ e $\left(V^{\prime}, B^{\prime}\right)$ é um $E B S\left(V^{\prime \prime}, B^{\prime \prime}\right)$ tal que o seguinte diagrama é comutativo

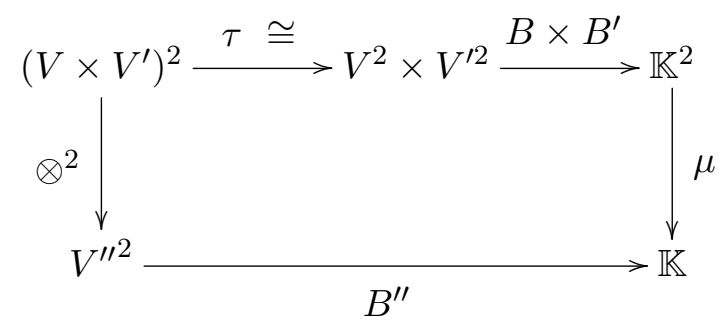

Onde $\left(V^{\prime \prime}, \otimes\right)$ é um produto tensorial para $V$ e $V^{\prime}$ e $\mu$ é a multiplicação no corpo $\mathbb{K}$, i.e. $B^{\prime \prime}$ é uma função bilinear simétrica tal que $B^{\prime \prime}\left(u_{1} \otimes u_{1}^{\prime}, u_{2} \otimes u_{2}^{\prime}\right)=B\left(u_{1}, u_{2}\right) \cdot B^{\prime}\left(u_{1}^{\prime}, u_{2}^{\prime}\right)$, para cada $u_{1}, u_{2} \in V$ e cada $u_{1}^{\prime}, u_{2}^{\prime} \in V^{\prime}$. 
Proposição 6. O produto tensorial de EBS existe é unico a menos de isomorfismo.

Demonstração. Unicidade: Sejam $\left(W_{1}, \otimes_{1}\right),\left(W_{2}, \otimes_{2}\right)$ produtos tensoriais para os espaços $V$ e $V^{\prime}$ e $B_{1}: W_{1} \times W_{1} \longrightarrow \mathbb{K}, B_{2}: W_{2} \times W_{2} \longrightarrow \mathbb{K}$ bilineares simétricas tais que os seguintes diagramas
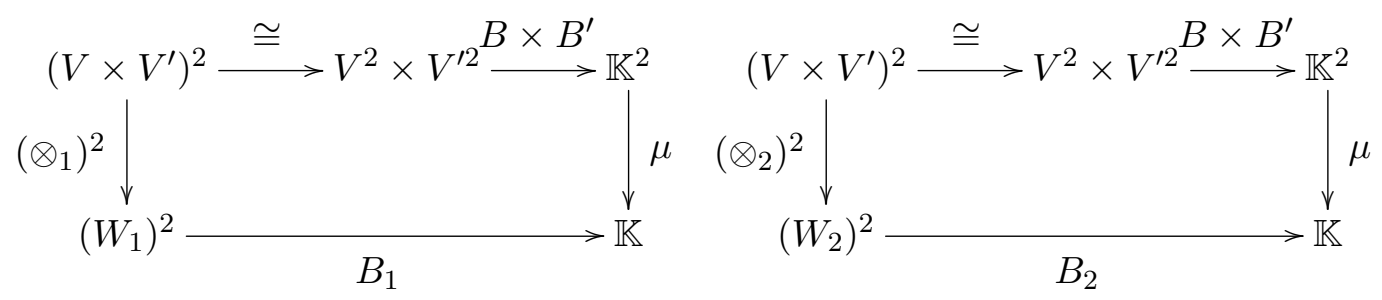

Seja $T: W_{1} \longrightarrow W_{2}, v \otimes_{1} v^{\prime} \mapsto v \otimes_{2} v^{\prime}$ o isomorfismo linear canônico entre $W_{1}$ e $W_{2}$. Assim, como $\operatorname{Im}\left(\otimes_{i}^{2}\right)$ gera $W_{i}^{2}$, o próximo diagrama é comutativo

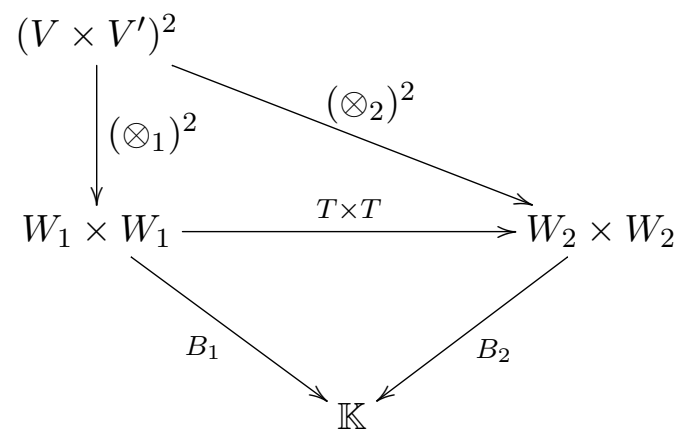

E portanto $\left(B_{1}, T, B_{2}\right)$ é uma isometria.

Existência: É uma consequência imediata da comutatividade do próximo diagrama

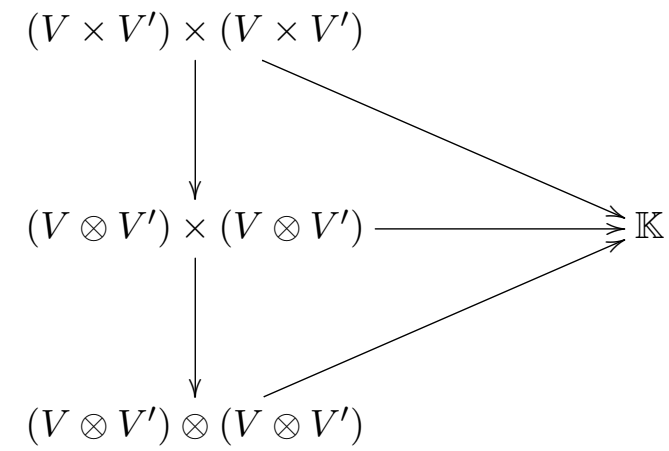

Observação 8. Sejam $\left.\left.(V, B, \varepsilon)=\left\{e_{i}: 1 \leq i \leq n\right\}\right),\left(V^{\prime}, B^{\prime}, \varepsilon^{\prime}\right)=\left\{e_{i}^{\prime}: 1 \leq i \leq n^{\prime}\right\}\right) \in$ $O b\left(B S_{\mathbb{K}}\right)$ e $\mathcal{U}: B I L S_{\mathbb{K}} \longrightarrow B S_{\mathbb{K}}$ o functor esquecimento. Mediante a equivalência de categorias $B S_{\mathbb{K}} \simeq_{\mathcal{F}} M S_{\mathbb{K}}$ nós temos $\mathcal{F}\left(\mathcal{U}(V, B, \varepsilon) \otimes \mathcal{U}\left(V^{\prime}, B^{\prime}, \varepsilon^{\prime}\right)\right)={ }_{\varepsilon}[B]_{\varepsilon} \otimes{ }_{\varepsilon^{\prime}}\left[B^{\prime}\right]_{\varepsilon^{\prime}}:=\oplus_{i, j=1}^{n} B\left(e_{i}, e_{j}\right)_{\varepsilon^{\prime}}\left[B^{\prime}\right]_{\varepsilon^{\prime}}$. Esta última matriz quadrada, de lados n.n', é chamada de produto de Kronecker da matrizes $\left(B\left(e_{i}, e_{j}\right)\right)_{i j} e\left(B^{\prime}\left(e_{k}^{\prime}, e_{l}^{\prime}\right)\right)_{k l}, 1 \leq i, j \leq n, 1 \leq k, l \leq n^{\prime}$. Em particular, para duas matrizes diagonais $\left\langle x_{1}, \ldots, x_{r}\right\rangle,\left\langle y_{1}, \ldots, y_{s},\right\rangle$ vale $\left\langle x_{1}, \ldots, x_{r}\right\rangle \otimes\left\langle y_{1}, \ldots, y_{s},\right\rangle \simeq\left\langle x_{1} y_{1}, \ldots, x_{1} y_{s}, \ldots, x_{r} y_{1}, \ldots, x_{r} y_{s}\right\rangle$.

Fato 1. Sejam $\phi, \phi^{\prime}, \psi, \psi^{\prime} E B S$. 
(1) $\phi \oplus \phi^{\prime} \simeq \phi^{\prime} \oplus \phi$

(2) $\left(\phi \oplus \phi^{\prime}\right) \oplus \psi \simeq \phi \oplus\left(\phi^{\prime} \oplus \psi\right)$

(3) $\phi \otimes \phi^{\prime} \simeq \phi^{\prime} \otimes \phi$

$(4)\left(\phi \otimes \phi^{\prime}\right) \otimes \psi \simeq \phi \otimes\left(\phi^{\prime} \otimes \psi\right)$

$(5) \phi \otimes\left(\psi \oplus \psi^{\prime}\right) \simeq(\phi \otimes \psi) \oplus\left(\phi \otimes \psi^{\prime}\right)$

$(6) \phi \otimes\langle 1\rangle \simeq \phi$

(7) $\phi \simeq \phi^{\prime}, \psi \simeq \psi^{\prime} \Longrightarrow \phi \oplus \psi \simeq \phi^{\prime} \oplus \psi^{\prime}, \phi \otimes \psi \simeq \phi^{\prime} \otimes \psi^{\prime}$

\subsubsection{Espaços regulares e diagonalização de EBS}

Definição 10. Seja $(V, B)$, um EBS.

(i) Se $W$ é subespaço de $V$ define-se o (subespaço) ortogonal $W^{\perp}=\{v \in V: B(v, w)=0, \forall w \in$ $W\}$.

(ii) $O$ radical de $V$ é $\operatorname{Rad}(V):=V^{\perp}$.

(iii) $(V, B)$ é dito um $E B S$ regular quando $\operatorname{Rad}(V)=\{0\}$.

\section{Observação 9.}

(i) $\operatorname{Rad}\left(\bigoplus_{i=1}^{n}\left(V_{i}, B_{i}\right)\right) \simeq \bigoplus_{i=1}^{n} \operatorname{Rad}\left(V_{i}, B_{i}\right)$;

(ii) $\bigoplus_{i=1}^{n}\left(V_{i}, B_{i}\right)$ é regular $\Longleftrightarrow\left(V_{i}, B_{i}\right)$ for regular, $\forall i \in I$.

Proposição 7. Para um espaço $(V, B) \in B I L S_{\mathbb{K}}$ as seguintes afirmações são equivalentes

(i) $(V, B)$ é regular.

(ii) $\operatorname{det}_{\varepsilon}[B]_{\varepsilon} \neq 0$ para alguma (ou para toda) base ordenada $\varepsilon$ de $V$.

(iii) $\check{B}: V \longrightarrow V^{*}$ é um isomorfismo linear.

Demonstração. $(i) \Longrightarrow($ ii $)$ Se $\varepsilon$ for uma base de $V$ tal que $\operatorname{det}_{\varepsilon}[B]_{\varepsilon}=0$,então existe $v \in V-\{0\}$ tal que $[B]_{\varepsilon}[v]_{\varepsilon}=0$ e assim $v \in \operatorname{Rad}(V)$.

$($ ii $) \Longrightarrow\left(\right.$ iii) Se $v \neq v^{\prime}$, então por hipótese $[v]_{\varepsilon}^{t}[B]_{\varepsilon} \neq\left[v^{\prime}\right]_{\varepsilon}^{t}[B]_{\varepsilon}$ para alguma base $\varepsilon$ de $V$. Assim ,$v \mapsto B(v,-)$ é injetora em dimensão finita e, portanto um isomorfismo.

(iii) $\Longrightarrow\left(\right.$ i) Seja $v \in V$ tal que $B\left(v, v^{\prime}\right)=0, \forall v^{\prime} \in V$,então $B(v,-)=0$ e por hipótese $v=0$, logo $\operatorname{Rad}(V)=\{0\}$.

Definição 11. Denotamos por $E B S R_{\mathbb{K}}$ as classes de isometria de espaços bilineares simétricos regulares. Seja $(V, B) \in E B S R_{\mathbb{K}}$. Definimos o discrimante de $(V, B)$ como sendo $\operatorname{det}(V, B)=$ $\operatorname{det}_{\varepsilon}[B]_{\varepsilon}$ para alguma base $\varepsilon$ de $V$.

Lema 3. A relação abaixo

$$
\begin{aligned}
& d: E B S R_{\mathbb{K}} \longrightarrow \quad \dot{\mathbb{K}} / \dot{\mathbb{K}}^{2} \\
& {[(V, B)] \longmapsto d(V, B)=\operatorname{det}(V, B) \cdot \dot{\mathbb{K}}^{2}}
\end{aligned}
$$

É uma função.

Demonstração. Seja $(V, B) \simeq\left(V^{\prime}, B^{\prime}\right)$. Sejam $\varepsilon, \varepsilon^{\prime}$ bases,respectivamente, de $V$ e $V^{\prime}$,então existe uma matriz invertível $\mathrm{M}$ tal que $\left[B^{\prime}\right]_{\varepsilon}=M^{t}[B]_{\varepsilon} M$ e assim $\operatorname{det}_{\varepsilon}^{\prime}\left[B^{\prime}\right]_{\varepsilon^{\prime}}=(\operatorname{det} M)^{2}{ }_{\varepsilon}[B]_{\varepsilon}$. 
Proposição 8. Seja $(V, B)$ um EBS regular e $W$ um subespaço de $V$, então:

(i) $\operatorname{dim}(W)+\operatorname{dim}\left(W^{\perp}\right)=\operatorname{dim}(V)$

(ii) $W^{\perp \perp}=W$

Demonstração. :

(i) Como $\breve{B}: V \longrightarrow V^{*}$ é um isomorfismo linear, temos que $W^{\perp}=\operatorname{Anulador}(\check{B}[W])$, logo

$$
\operatorname{dim}\left(W^{\perp}\right)=\operatorname{dim}\left(V^{*}\right)-\operatorname{dim}(\check{B}[W])=\operatorname{dim}(V)-\operatorname{dim}(W)
$$

(ii) A inclusão $W \subseteq W^{\perp \perp}$ vale em geral. Das condições e do item (i) temos

$$
\operatorname{dim}\left(W^{\perp}\right)^{\perp}=\operatorname{dim}(V)-\operatorname{dim}\left(W^{\perp}\right)=\operatorname{dim}(W)
$$

$\log W=W^{\perp \perp}$

Proposição 9. Para cada $(V, B) \in B I L S_{\mathbb{K}}$ as seguintes condições são equivalentes para um subespaço $W$ de $V$.

(i) $W$ é regular.

(ii) $W \cap W^{\perp}=0$

(iii) $V=W \oplus^{\perp} W^{\perp}$

Demonstração.

$(i) \Longleftrightarrow($ ii $)$ Basta notar que $\operatorname{Rad}\left(W,\left.B\right|_{W \times W}\right)=W \cap W^{\perp}$

$($ ii $) \Longleftrightarrow\left(\right.$ iii) Se $V=W \oplus^{\perp} W^{\perp}$, então $W \cap W^{\perp}=0$. Inversamente, $\operatorname{Ker}(w \mapsto B(w,-): W \longrightarrow$ $\left.W^{*}\right)=W \cap W^{\perp}=0$ e assim, $\left(w \mapsto B(w,-): W \longrightarrow W^{*}\right)$ é injetora e, portanto um isomorfismo linear, e assim, como para cada $v \in V$, o mapa $B(v,-): W \rightarrow \mathbb{K}$ é linear, existe um (único) $w \in W$ tal que $B(v,-)=B(w,-) \in W^{*}$; dessa forma, $B(v-w, x)=0, \forall x \in W$, portanto $v-w \in W^{\perp}$. Assim $V=W \oplus^{\perp} W^{\perp}$.

Lema 4. Seja $(V, B) \in B I L S_{\mathbb{K}} \operatorname{com} B \neq 0$. Então $q_{B} \neq 0$, i.e. existe $v \in V$ tal que $q_{B}(v)=$ $B(v, v) \neq 0$.

Demonstração. É uma consequência direta da fórmula

$$
B(u, v)=\left(q_{B}(u+v)-q_{B}(u)-q_{B}(v)\right) / 2
$$

Definição 12. Dado $(V, q)$ espaço quadrático, definimos o conjunto dos valores representados por $q$ como $D(q)=\left\{a \in \mathbb{K}^{*}: \exists v \in V-\{0\}, q(v)=a\right\}$.

Teorema 5. Cada $(V, B) \in B I L S_{\mathbb{K}}, \operatorname{com} \operatorname{dim}(V)=n$, admite alguma decomposição

$$
(V, B) \simeq\left(\mathbb{K} e_{0},\left.B\right|_{\mathbb{K} e_{0} \times \mathbb{K} e_{n-1}}\right) \oplus^{\perp} \cdots \oplus^{\perp}\left(\mathbb{K} e_{n-1},\left.B\right|_{\mathbb{K} e_{n-1} \times \mathbb{K} e_{n-1}}\right)
$$

onde $\left\{e_{0}, \cdots, e_{n-1}\right\}$ é uma base ortogonal de $V$. 
Demonstração. Por indução em $n=\operatorname{dim}(V)$. Se $B=0$, o resultado é óbvio. Se $B \neq 0$, o Lema 4 nos fornece $e_{1} \in V$ tal que $B\left(e_{0}, e_{1}\right) \neq 0$ e, em particular, $e_{1} \neq 0$. Assim, $\left(W=\mathbb{K} e_{1},\left.B\right|_{W \times W}\right)$ é regular e pela Proposição $9 V=W \oplus^{\perp} W^{\perp}$. Por hipótese de indução existe uma base ortogonal $e_{1}, \cdots, e_{n-1}$ de $W^{\perp}$ tal que

$$
\left(W^{\perp},\left.B\right|_{W^{\perp} \times W^{\perp}}\right) \simeq\left(\mathbb{K} e_{1},\left.B\right|_{\mathbb{K} e_{1} \times \mathbb{K} e_{1}}\right) \oplus^{\perp} \cdots \oplus^{\perp}\left(\mathbb{K} e_{n-1},\left.B\right|_{\mathbb{K} e_{n-1} \times \mathbb{K} e_{n-1}}\right)
$$

nesse caso, $\left\{e_{0}, e_{1}, \cdots, e_{n-1}\right\}$ é a base ortogonal de $(V, B)$ procurada.

Obtemos diretamente da prova deste teorema o seguinte:

Corolário 2. (Critério de Representação) $\operatorname{Sejam~}(V, B)$ um $E B S \operatorname{com} \operatorname{dim}(V)=n$ e a $\in \mathbb{K}^{*}$, então $a \in D\left(q_{B}\right)$ se e só se existem $b_{1}, \cdots, b_{n-1} \in \mathbb{K}$ tais que $(V, B) \simeq\left\langle a, b_{1}, \cdots, b_{n-1}\right\rangle$.

Corolário 3. Sejam $\alpha, \beta, \gamma, \delta \in \dot{\mathbb{K}}$. Então

$$
\langle\alpha, \beta\rangle \simeq\langle\gamma, \delta\rangle \Longleftrightarrow \alpha \beta \dot{\mathbb{K}}^{2}=\gamma \delta \dot{\mathbb{K}}^{2} \text { e } D_{\mathbb{K}}\langle\alpha, \beta\rangle \cap D_{\mathbb{K}}\langle\gamma, \delta\rangle \neq \emptyset .
$$

Demonstração. A implicação $(\Rightarrow)$ é clara. Para a reversa $(\Leftarrow)$ : se $\omega \in D\langle\alpha, \beta\rangle \cap D\langle\gamma, \delta\rangle$ e $\alpha \beta \dot{\mathbb{K}}^{2}=$ $\gamma \delta \dot{\mathbb{K}}^{2}$, então pelo critério logo acima $\langle\alpha, \beta\rangle \simeq\left\langle\omega, \omega^{\prime}\right\rangle$ e $\langle\gamma, \delta\rangle \simeq\left\langle\omega, \omega^{\prime \prime}\right\rangle$ para convenientes $\omega^{\prime}, \omega^{\prime \prime} \in \mathbb{K}$ (e estes são não nulos, por novas aplicações de $\Rightarrow$ ). Usando as informações sobre discrimantes obtemos $\langle\alpha, \beta\rangle \simeq\langle\omega, \alpha \beta \omega\rangle$ e $\langle\gamma, \delta\rangle \simeq\langle\omega, \gamma \delta \omega\rangle$ e, portanto $\langle\alpha, \beta\rangle \simeq\langle\gamma, \delta\rangle$.

\section{Fato 2.}

(i) Denotamos $\left(\mathbb{K} e_{1},\left.B\right|_{\mathbb{K} e_{1} \times \mathbb{K} e_{1}}\right) \oplus^{\perp} \cdots \oplus^{\perp}\left(\mathbb{K} e_{1},\left.B\right|_{\mathbb{K} e_{n} \times \mathbb{K} e_{n}}\right)$ simplesmente por $\left\langle B\left(e_{1}, e_{1}\right), \cdots, B\left(e_{n}, e_{n}\right)\right\rangle$ e diremos que ele é um espaço diagonal para $(V, B)$.

(ii) Se $M \in M S_{\mathbb{K}}$, então $M \simeq\left\langle a_{1}, \cdots, a_{n}\right\rangle$, onde $\left\langle a_{1}, \cdots, a_{n}\right\rangle$ indica as entradas de alguma matriz diagonal.

(iii) $\left\langle a_{1}, \cdots, a_{n}\right\rangle \simeq\left\langle a_{\sigma(1)}, \cdots, a_{\sigma(n)}\right\rangle$ para toda permutação $\sigma$ de $\{1, \cdots, n\}$.

(iv) $\left\langle a_{1}, \cdots, a_{n}\right\rangle \simeq\left\langle a_{1} x_{1}^{2}, \cdots, a_{n} x_{n}^{2}\right\rangle$, onde $x_{i} \neq 0, i=1, \cdots, n$.

(v) Todo espaço $(V, B) \in B I L S_{\mathbb{K}}$ admite pelo menos $n$ ! representações diagonais.

Os resultado (auxiliar) a seguir será útil mais adiante

Lema 6. Todo espaço $(V, B) \in B I L S_{\mathbb{K}}$ admite uma decomposição $(V, B) \simeq(\operatorname{Rad}(V), 0) \oplus^{\perp}\left(V^{\prime}, B^{\prime}\right)$, onde $\left(V^{\prime}, B^{\prime}\right)$ é subespaço regular (estes subespaços podem ser nulos, eventualmente).

Demonstração. Basta considerar a sequência exata

$$
0 \longrightarrow(\operatorname{Rad}(V), 0) \longrightarrow(V, B) \longrightarrow(V / \operatorname{Rad}(V), \tilde{B}) \longrightarrow 0
$$

onde $\tilde{B}(v+\operatorname{Rad}(V), w+\operatorname{Rad}(V))=B(v, w)$ e observar que $\operatorname{Rad}(V / \operatorname{Rad}(V))=0$.

\subsubsection{Espaços Hiperbólicos e Isotrópicos}

Definição 13. Um $\mathbb{K}$-espaço bilinear simétrico $(V, B)$ será chamado plano hiperbólico se existir uma isometria $(V, B) \simeq\left(\mathbb{K}^{2}, h\right)$,onde $h\left((x, y),\left(x^{\prime}, y^{\prime}\right)\right)=x x^{\prime}-y y^{\prime}$. Um espaço hiperbólico é um espaço que é isométrico a uma soma direta de planos hiperbólicos. 
Observação 10. Usaremos $\mathbb{H}$ para representar o espaço $\left(\mathbb{K}^{2}, h\right)$. A classe de planos hipérbólicos também será chamada de plano hiperbólico e usaremos, muitas vezes, a mesma letra. Além disso, denotamos por Hip $(\mathbb{K})$ a totalidade dos espaços hiperbólicos sobre $\mathbb{K}$.

\section{Proposição 10.}

i) ${ }_{\mathcal{E}} h_{\mathcal{E}}=\langle 1,-1\rangle$, se $\mathcal{E}$ for a base canônica de $\mathbb{K}^{2}$;

ii) $S e(V, B)$ for hiperbólico, então ${ }_{\mathcal{E}} B_{\mathcal{E}} \simeq\langle 1,-1, \ldots, 1,-1\rangle$ para qualquer base $\mathcal{E}$ de $V$;

iii) $\operatorname{dim}_{\mathbb{K}}(H) \in 2 \mathbb{Z}$, se $H$ for um espaço hiperbólico;

iv) $\langle 1,-1, \ldots, 1,-1\rangle \simeq\langle 1, \ldots, 1,-1, \ldots,-1\rangle$

Demonstração.

i) $h((1,0),(1,0))=1 \cdot 1-0 \cdot 0=1, h((1,0),(0,1))=1 \cdot 0-0 \cdot 1=0, h((0,1),(0,1))=0 \cdot 0-1 \cdot 1=$ $-1, h((0,1),(1,0))=0 \cdot 1-1 \cdot 0=0$.

ii) Seja $(V, B) \simeq \mathbb{H} \oplus^{\perp} \ldots \oplus^{\perp} \mathbb{H}$. Por $(\mathrm{i})(V, B) \simeq\langle 1,-1\rangle \oplus \ldots \oplus\langle 1,-1\rangle$.

iii) $(V, B)=\bigoplus_{i=1}^{n} \mathbb{H} \Longrightarrow \operatorname{dim}_{\mathbb{K}}(V, B)=n \operatorname{dim}_{\mathbb{K}} \mathbb{H}=n \cdot 2=2 n$

iv) Segue diretamente do Fato 2,(iii).

Proposição 11. Seja $R B I L S_{\mathbb{K}}$ a subcategoria de $B I l S_{\mathbb{K}}$ formada por espaços bilineares simétricos regulares e Vect $t_{\mathbb{K}}^{0}$ a categoria formada por espaços vetoriais com isomorfismos. Existe um funtor $\mathcal{H}: V e c t_{\mathbb{K}}^{0} \longrightarrow R B I L S_{\mathbb{K}}$

Demonstração. Para cada $V \in V e c t_{\mathbb{K}}^{0}$ associamos $\mathcal{H}(V)=\left(V \oplus V^{*}, h_{V}\right)$, onde $h_{V}\left(\left(v_{1}, f_{1}\right)\left(v_{2}, f_{2}\right)\right)=$ $f_{1}\left(v_{2}\right)+f_{2}\left(v_{1}\right)$ : claramente $\mathcal{H}(V)$ é um espaço bilinear simétrico. $\mathcal{H}(V)$ é regular pois, quando $v \neq 0$ tomamos $v^{*} \in V^{*}$ tal que $\hat{v}(v) \neq 0$ e quando $f \neq 0$ deve existir $v$ com $f(v) \neq 0$.

Agora, a cada isomorfismo linear $T: V \longrightarrow V^{\prime}$ associamos o isomorfismo linear $\mathcal{H}(T)=$ $T \oplus\left(T^{*}\right)^{-1}: V \oplus V^{*} \longrightarrow V^{\prime} \oplus V^{\prime *}$ : este define uma isometria $\mathcal{H}(T): \mathcal{H}(V) \longrightarrow \mathcal{H}\left(V^{\prime}\right)$, pois

$$
\begin{gathered}
h_{V^{\prime}}\left(T \oplus\left(T^{*}\right)^{-1}\left(v_{1}, f_{2}\right), T \oplus\left(T^{*}\right)^{-1}\left(v_{2}, f_{2}\right)\right)= \\
\left(T^{*}\right)^{-1}\left(f_{1}\right)\left(T\left(v_{2}\right)\right)+\left(T^{*}\right)^{-1}\left(f_{2}\right)\left(T\left(v_{1}\right)\right)=f_{1}\left(v_{2}\right)+f_{2}\left(v_{1}\right)=h_{V}\left(\left(v_{1}, f_{1}\right)\left(v_{2}, f_{2}\right)\right) .
\end{gathered}
$$

Por fim, observe que

$$
\begin{aligned}
\mathcal{H}\left(I_{V}\right)(v, f) & =\quad I_{V} \oplus I_{V^{*}}(v, f)=\left(I_{V}(v), I_{V^{*}}(f)\right) \\
& =(v, f)=I_{\mathcal{H}(V)}(v, f)
\end{aligned}
$$

e

$$
\begin{aligned}
& \mathcal{H}(S \circ T)(v, f)=(S \circ T) \oplus\left((S \circ T)^{*}\right)^{-1}(v, f) \quad=(S \circ T) \oplus\left(\left(S^{*}\right)^{-1} \circ\left(T^{*}\right)^{-1}\right)(v, f) \\
& =\left(S(T(v)),\left(S^{*}\right)^{-1}\left(\left(T^{*}\right)^{-1}(f)\right)\right)=\left(S \oplus\left(S^{*}\right)^{-1}\right)\left(\left(T \oplus\left(T^{*}\right)^{-1}\right)(v, f)\right) \\
& =\left(S \oplus\left(S^{*}\right)^{-1}\right) \circ\left(T \oplus\left(T^{*}\right)^{-1}\right)(v, f)=\quad \mathcal{H}(S) \circ \mathcal{H}(T)(v, f)
\end{aligned}
$$


Observação 11. Tomando $\mathcal{E}=\left(\left(v_{1}, 0\right), \cdots,\left(v_{n}, 0\right),\left(0, v_{1}^{*}\right), \cdots,\left(0, v_{n}^{*}\right)\right)$ uma base de $\mathcal{H}(V)$, é imediato ver que a matriz $\mathcal{E}\left(h_{V}\right)_{\mathcal{E}}$ é a matriz quadrada de lado $2 n$

$$
\left(\begin{array}{ll}
0_{n} & I_{n} \\
I_{n} & 0_{n}
\end{array}\right)
$$

Definição 14. Seja $(V, B)$ um EBS.

(i) Dizemos que $v \in V-\{0\}$ é um vetor isotrópico quando $B(v, v)=0$.

(ii Dizemos que $(V, B)$ é um EBS isotrópico quando possui algum vetor isotrópico, $(V, B)$ é anisotrópico caso contrário.

(iii) $O$ espaço $(V, B)$ será totalmente isotrópico se $B(v, v)=0, \forall v \in V$.

(iv) Denotamos por Iso( $\mathbb{K})$ todos os espaços isotrópicos e por Anis $(\mathbb{K})$ os espaços anisotrópicos.

Observação 12. Todo espaço anisotrópico é regular. De fato, se $(V, B)$ for singular, então existem $u, v \in V-\{0\}$ tais que $B(u, v)=0$, verificaremos que $(V, B)$ é isotrópico. Se $B(u, u)=0$ ou $B(v, v)=0$, já temos a isotropia; caso contrário consideremos $w:=u+v$, então $B(w, w)=$ $B(u, u)+2 B(u, v)+B(v, v)=0$ e $w \neq 0$ (senão $v=-u$ e $B(u,-u)=0$ mas $B(u, u) \neq 0$ : contradição).

Teorema 7. São equivalentes para um EBS regular $(V, B)$ de dimensão $2 n$

(i) $(V, B) \in \operatorname{Hip}(\mathbb{K})$;

(ii) $(V, B) \simeq \mathcal{H}(W) \operatorname{com}_{\operatorname{dim}}(W)=n$;

(iii) Existe $W \subseteq V$, subespaço totalmente isotrópico com $\operatorname{dim}_{\mathbb{K}}(W)=n$;

$($ iv $) B_{\mathcal{E}} \simeq\left(\begin{array}{cc}0 & M \\ M^{t} & N\end{array}\right)$ para alguma base $\mathcal{E}$ de $V$ com $M, N, 0$ matrizes quadradas de ordem $n$, $\operatorname{det}(M) \neq 0$ e $N=N^{t}$;

$(v) B_{\mathcal{E}^{\prime}} \simeq\left(\begin{array}{cc}0 & I_{n} \\ I_{n} & 0\end{array}\right)$ para alguma base $\mathcal{E}^{\prime}$ de $V$ onde $I_{n}$ é a matriz identidade de ordem $n$;

$(v i) B_{\mathcal{E}^{\prime \prime}} \simeq\left(\begin{array}{cc}A & 0 \\ 0 & -A\end{array}\right)$ para alguma base $\mathcal{E}^{\prime \prime}$ de $V \operatorname{com} \operatorname{det}(A) \neq 0$ e $A^{t}=A$.

Demonstração. $(v) \Longleftrightarrow($ ii $)$ Já observamos que $(i i) \Rightarrow(v)$. Seja agora $\mathcal{E}=\left(w_{1}, \cdots, w_{n}, u_{1}, \cdots, u_{n}\right)$ uma base de $V$ tal que $B_{\mathcal{E}} \simeq\left(\begin{array}{cc}0 & I_{n} \\ I_{n} & 0\end{array}\right)$

Seja $W$ o subespaço de $V$ com base $\left(w_{1}, \cdots, w_{n}\right)$. A aplicação linear $\theta: V \longrightarrow W \oplus W^{*}$ definida por $\theta\left(w_{i}\right)=w_{i}, \theta\left(u_{i}\right)=w_{i}^{*}$ é uma isometria de $(V, B)$ em $\mathcal{H}(W)$.

$(i) \Longleftrightarrow(v i)$ De fato, como $\langle 1,-1, \ldots, 1,-1\rangle \simeq\langle 1, \ldots, 1,-1, \ldots,-1\rangle$, o espaço $(V, B)$ é hiperbólico se e só se existe uma base $\mathcal{E}$ de $V$ tal que $B_{\mathcal{E}} \simeq\left(\begin{array}{cc}I_{n} & 0 \\ 0 & -I_{n}\end{array}\right)$

$($ iv $) \Longrightarrow($ iii $)$ é claro. $($ iii $) \Longrightarrow\left(\right.$ iv) Complete uma base $\varepsilon^{\prime}=\left(w_{1}, \cdots, w_{n}\right)$ de $W$ para uma base $\varepsilon=\left(w_{1}, \cdots, w_{n}, u_{1}, \cdots, u_{n}\right)$ de $V$. Por hipótese, $B\left(e, e^{\prime}\right)=0, \forall e, e^{\prime} \in \varepsilon^{\prime}$ e assim

$$
B_{\mathcal{E}}=\left(\begin{array}{cc}
0 & M \\
M^{t} & N
\end{array}\right)
$$

com N simétrica. Desde que, 


$$
\left|\begin{array}{cc}
0 & M \\
M^{t} & N
\end{array}\right|= \pm\left|\begin{array}{cc}
M & 0 \\
N & M^{t}
\end{array}\right|= \pm\left|\begin{array}{cc}
M^{t} & N^{t} \\
0 & M
\end{array}\right|= \pm\left|M^{t}\right||M|= \pm|M|^{2}
$$

segue por regularidade $|M|^{2} \neq 0$.

$(i v) \Longleftrightarrow(v)$ Segue da igualdade

$$
\left(\begin{array}{cc}
M & 0 \\
\frac{1}{2} N^{t} & I_{n}
\end{array}\right)\left(\begin{array}{cc}
0 & I_{n} \\
I_{n} & 0
\end{array}\right)\left(\begin{array}{cc}
M^{t} & \frac{1}{2} N \\
0 & I_{n}
\end{array}\right)=\left(\begin{array}{cc}
0 & M \\
M^{t} & \frac{1}{2}\left(N+N^{t}\right)
\end{array}\right)
$$

$(v) \Longleftrightarrow(v i)$ Segue da igualdade

$$
\left(\begin{array}{cc}
I_{n} & I_{n} \\
\left(\frac{1}{2} M^{-1}\right)^{t} & -\left(\frac{1}{2} M^{-1}\right)^{t}
\end{array}\right)\left(\begin{array}{cc}
M & 0_{n} \\
0_{n} & -M
\end{array}\right)\left(\begin{array}{cc}
I_{n} & \frac{1}{2} M^{-1} \\
I_{n} & -\frac{1}{2} M^{-1}
\end{array}\right)=\left(\begin{array}{cc}
0 & I_{n} \\
I_{n} & 0
\end{array}\right)
$$

notando que a matriz

$$
\left(\begin{array}{cc}
I_{n} & \frac{1}{2} M^{-1} \\
I_{n} & -\frac{1}{2} M^{-1}
\end{array}\right)
$$

é invertível.

Corolário 4. Seja $(V, B)$ um espaço regular de dimensão 2. As seguintes afirmações são equivalentes

(i) $(V, B)$ é hiperbólico;

(ii) $(V, B)$ é regular e isotrópico;

(iii)d $(V, B)=-1 . \dot{\mathbb{K}}^{2}$

$(i v)(V, B) \simeq\langle 1,-1\rangle \simeq\langle a,-a\rangle, \forall a \neq 0 ;$

(v) $B_{\mathcal{E}^{\prime}} \simeq\left(\begin{array}{ll}0 & 1 \\ 1 & 0\end{array}\right)$ para alguma base $\mathcal{E}^{\prime}$

(vi) $B_{\mathcal{E}} \simeq\left(\begin{array}{ll}0 & a \\ a & b\end{array}\right)$ para alguma base $\mathcal{E}$ e $a \neq 0$;

Corolário 5. $\phi \otimes \mathbb{H} \simeq \operatorname{dim}(\phi) . \mathbb{H}$ para $\phi$ regular.

Demonstração. Segue do item (ii) do Corolário acima. Tomando uma diagonalização de $\phi \simeq$ $\left\langle a_{1}, \cdots, a_{n}\right\rangle$, como $\phi$ é regular temos $a_{i}=\neq 0, \forall i \leq n$ e $\operatorname{assim} \phi \otimes \mathbb{H} \simeq\left\langle a_{1}, \cdots, a_{n}\right\rangle \otimes \mathbb{H} \simeq$ $\bigoplus_{i=1}^{n}\left\langle a_{i},-a_{i}\right\rangle \simeq \bigoplus_{i=1}^{n}\langle 1,-1\rangle \simeq \operatorname{dim}(\phi) . \mathbb{H}$.

Definição 15. Um EBS $(V, B)$ é dito universal quando $D_{\mathbb{K}}\left(q_{B}\right)=\{d \in \dot{K}: \exists v \in V ; B(v, v)=$ $d\}=\mathbb{K}$.

Observação 13. Segue imediatamente da fórmula

$$
a=\left(\frac{a+1}{2}\right)^{2}-\left(\frac{a-1}{2}\right)^{2}, \forall a \in \mathbb{K}
$$

que todo plano hiperbólico é universal. 
Proposição 12. Todo subespaço totalmente isotrópico $U$ de um espaço bilinear simétrico regular $(V, B)$ está contido em um espaço hiperbólico de dimensão $2 \operatorname{dim}_{\mathbb{K}} U$.

Demonstração. Seja $e_{1}, \ldots, e_{m}$ uma base de $U$ e consideremos o subespaço $W$ gerado por $\left\{e_{2}, \ldots, e_{m}\right\}$. Como $(V, B)$ é regular, segue da Proposiçao 8.(i) que $\operatorname{dim} U^{\perp}<\operatorname{dim} W^{\perp}$, logo existe $e_{1}^{\prime}$ tal que $B\left(e_{1}^{\prime}, e_{j}\right)=0, \forall j \in\{2, \ldots, m\}$ e $B\left(e_{1}^{\prime}, e_{1}\right) \neq 0$. O subespaço $\mathbb{K} e_{1}+\mathbb{K} e_{1}^{\prime}$ possui determinante $-B\left(e_{1}^{\prime}, e_{1}\right) \cdot B\left(e_{1}^{\prime}, e_{1}\right) \in-1 \dot{\mathbb{K}}^{2}$ e, portanto é um plano hiperbólico. Nesse caso, como $\mathbb{H}$ é regular, pela Proposição 9 temos $V \simeq \mathbb{H} \perp \mathbb{H}^{\perp}$, com $e_{2}, \ldots, e_{m} \in \mathbb{H}^{\perp}$. Desde que, $\mathbb{H}^{\perp}$ é também é regular (pelas Proposições 9 e 8.(ii)) a prova procede por indução na $\operatorname{dim}(U)$.

Corolário 6. Sejam $(V, B)$ e $\left(V^{\prime}, B^{\prime}\right)$ EBS regulares e $d \in \dot{\mathbb{K}}$. Então

(i) $(V, B)$ é isotrópico $\Longleftrightarrow(V, B) \simeq \mathbb{H} \oplus^{\perp} \mathbb{H}^{\perp}$;

(ii) $(V, B)$ é isotrópico $\Longrightarrow(V, B)$ é universal;

(iii) $d \in D_{\mathbb{K}}(V, B) \Longleftrightarrow(V, B) \oplus\langle-d\rangle$ é isotrópico;

(iv) $(V, B) \oplus^{\perp}\left(V^{\prime}, B^{\prime}\right)$ é isotrópico $\Longleftrightarrow D_{\mathbb{K}}(V, B) \cap-D_{\mathbb{K}}\left(V^{\prime}, B^{\prime}\right) \neq \emptyset$;

(v) Sejam um corpo fixado $\mathbb{K}$ e $r \in \mathbb{N}$. São equivalentes:

(U) Todo EBS regular de dimensão $r$ é universal;

(I) Todo EBS com dimensão $r+1$ é isotrópico.

Demonstração.

(i) Seja $v \in V-\{0\} \operatorname{com} B(v, v)=0, \operatorname{logo} W=\mathbb{K} v$ é subespaço totalmente isotrópico. Pelo Teorema 7. existe $v^{\prime} \in V$ tal que $\left\{v, v^{\prime}\right\}$ gera um plano hiperbólico $\mathbb{H} \subseteq V$. Em virtude da Proposição 9 , $(V, B) \simeq\left(\mathbb{H} \oplus^{\perp} \mathbb{H}^{\perp}, h\right)$. Inversamente, se $\mathbb{H}$ está imerso em $V$, então $(V, B)$ será isotrópico, pois todo plano hiperbólico é isotrópico.

(ii) Se $(V, B)$ é isotrópico, pelo item (i) temos que $V$ possui um plano hiperbólico como somando direto (ortogonal) e o resultado segue da universalidade dos planos hiperbólicos.

(iii)Se $B(v, v)=d \in \mathbb{K}$ com $v \in V$, então $\left(B \oplus^{\perp}\langle-d\rangle\right)\left(\left(v, 1_{\mathbb{K}}\right),\left(v, 1_{\mathbb{K}}\right)\right)=B(v, v)+-d .1^{2}=0$. Inversamente, seja $(v, a) \neq 0, v \in V, a \in \mathbb{K}$ tal que $\left(B \oplus^{\perp}\langle-d\rangle\right)((v, a),(v, a))=0$, então $B(v, v)=$ $d . a^{2}$. Se $a \neq 0$, então $B\left(\frac{1}{a} v, \frac{1}{a} v\right)=d$; se $a=0$, então $v \neq 0$ e $B(v, v)=0$, logo $(v, B)$ é isotrópico e o resultado segue do item (ii).

(iv)Se $\left(B \oplus^{\perp} B^{\prime}\right)\left(\left(v, v^{\prime}\right),\left(v, v^{\prime}\right)\right)=0 \operatorname{com}\left(v, v^{\prime}\right) \neq 0$, então $B(v, v)=-B^{\prime}\left(v^{\prime}, v^{\prime}\right)=a:$ se $a \in \dot{\mathbb{K}}$ então este é um elemento na interseção; se $a=0$ então $B$ ou $B^{\prime}$ seria isotrópico e portanto universal, garantindo (junto com a regularidade) a não vacuidade da intersecção. Inversamente, se $a \in D(V, B) \cap-D\left(V^{\prime}, B^{\prime}\right)$, então existem $v \in V-\{0\}$ e $v^{\prime} \in V^{\prime}-\{0\}$ tais que $B(v, v)=a$ e $B^{\prime}\left(v^{\prime}, v^{\prime}\right)=-a$. Logo $\left(B \oplus^{\perp} B^{\prime}\right)\left(\left(v, v^{\prime}\right),\left(v, v^{\prime}\right)\right)=0 \mathrm{com}\left(v, v^{\prime}\right) \neq 0$, atestando a isotropia da soma direta.

(v) Suponhamos $(\mathrm{U})(V, B)$ um EBS de dimensão $r+1$. Se $B=0$, então $(V, B)$ é isotrópico já que $\operatorname{dim}(V)>0$; se $B \neq 0$ seja $a \in D\left(q_{B}\right)$ e $\operatorname{assim}(V, B)=\left(V^{\prime}, B^{\prime}\right) \perp\langle a\rangle$, e por (U) $\left(V^{\prime}, B^{\prime}\right)$ é isotrópico, logo universal, assim existe $v^{\prime} \in V^{\prime}$ tal que $-a=B^{\prime}\left(v^{\prime}, v^{\prime}\right)$ para algum $v^{\prime} \in V^{\prime}$, desta forma $B\left(\left(v^{\prime}, 1\right),\left(v^{\prime}, 1\right)\right)=0$. Reversamente, assumindo (I) e tomando $(W, B)$ um EBS regular de dimensão $r$ e $c \in \dot{K}$, então $(W, B) \perp\langle-c\rangle$ é isotrópico por hipótese logo, pelo item (iii), temos $c \in D\left(q_{B}\right)$ atestando a universalidade de $(W, B)$.

Os resultado (auxiliar) a seguir será útil na próxima subseção 
Lema 8. Todo EBS regular $(V, B)$ admite uma decomposição $(V, B) \simeq\left(H,\left.B\right|_{H \times H}\right) \oplus^{\perp}\left(U,\left.B\right|_{U \times U}\right)$, onde $\left(H,\left.B\right|_{H \times H}\right)$ é subespaço hiperbólico e $\left(U,\left.B\right|_{U \times U}\right)$ é subespaço anisotrópico (estes podem ser nulos, eventualmente).

Demonstração. Por indução na dimensão. Se $(V, B)$ é anisotrópico o resultado segue trivialmente. Caso contrário, $(V, B)$ é regular e isotrópico, logo pelo Corolário 6. (i) acima $(V, B) \simeq \mathbb{H}^{\perp} \mathbb{H}^{\perp}$. Como já observamos, as Proposições 8 e 9 garantem que $\mathbb{H}^{\perp}$ é um EBS regular e, como $\operatorname{dim}\left(\mathbb{H}^{\perp}\right)=$ $\operatorname{dim}(V)-2$, o resultado segue por indução.

\subsubsection{Teoremas de Witt}

Nesta subseção apresentamos os teoremas fundamentais da teoria algébrica das formas quadráticas, essencialmente presentes no artigo clássico de E. Witt de 1937 [Witt].

Proposição 13. (Teorema do cancelamento de Witt)Sejam $\phi, \psi, \phi^{\prime}, \psi^{\prime}$ formas quadráticas sobre um corpo $\mathbb{K}$. Se $\phi \oplus^{\perp} \psi \simeq \phi^{\prime} \oplus^{\perp} \psi^{\prime}$ e $\phi \simeq \phi^{\prime}$, então $\psi \simeq \psi^{\prime}$.

Demonstração. Consulte [Lam3, pág 12, teor. 4.2, para a prova desse importante teorema.

Uma forma alternativa de enunciar o teorema do cancelamento de Witt é a seguinte 2

Proposição 14. (Teorema de extensão de Witt) Sejam $(V, B)$ um EBS regular e $W_{1}, W_{2}$ subespaços de $V$. Então toda isometria $\sigma: W_{1} \longrightarrow W_{2}$ admite alguma extensão a uma isometria $\tilde{\sigma}: V \longrightarrow V$.

Proposição 15. (Teorema de decomposição de Witt) Todo EBS $\phi$ admite uma decomposição

$$
\phi_{t} \oplus^{\perp} \phi_{h} \oplus^{\perp} \phi_{a}
$$

onde $\phi_{t}$ é totalmente isotrópico, $\phi_{h}$ é hiperbólico, $\phi_{a}$ é anisotrópico e cada componente é única a menos de isometria.

Demonstração. Existência: Segue diretamente dos Lemas 6 e 8.

Unicidade: Se $\phi \simeq \phi_{t} \oplus^{\perp} \phi_{h} \oplus^{\perp} \phi_{a}, \operatorname{temos} \operatorname{Rad}(\phi) \simeq \operatorname{Rad}\left(\phi_{t}\right) \oplus^{\perp} \operatorname{Rad}\left(\phi_{h} \oplus^{\perp} \phi_{a}\right)=\phi_{t} \oplus^{\perp}\{0\} \simeq \phi_{t}$, pois o primeiro é totalmente isotrópico e o segundo é regular. Assim, se $\phi \simeq \phi_{t}^{\prime} \oplus^{\perp} \phi_{h}^{\prime} \oplus^{\perp} \phi_{a}^{\prime}$, teremos $\phi_{t} \simeq \operatorname{Rad}(\phi) \simeq \phi_{t}^{\prime}$. Logo, pelo Teorema de Cancelamento de Witt, obtemos

$$
(*) \quad \phi_{h} \oplus^{\perp} \phi_{a} \simeq \phi_{h}^{\prime} \oplus^{\perp} \phi_{a}^{\prime} .
$$

Se escrevemos $\phi_{h} \simeq k\langle 1,-1\rangle$ e $\phi_{h}^{\prime} \simeq k^{\prime}\langle 1,-1\rangle$ e supomos, por absurdo, que $k<k^{\prime}$, aplicando o Teorema de Cancelamento de Witt a isometria $(*)$ obtemos $\phi_{a} \simeq\left(k^{\prime}-k\right)\langle 1,-1\rangle \oplus^{\perp} \phi_{a}^{\prime}$, contradizendo a anisotropia de $\phi_{a}$. Logo $k=k^{\prime}$, assim $\phi_{h} \simeq \phi_{h}^{\prime} \mathrm{e}$, aplicando novamente o Teorema de Cancelamento de Witt a isometria $(*)$ acima, obtemos finalmente $\phi_{a} \simeq \phi_{a}^{\prime}$, encerrando a prova.

Corolário 7. Seja $\phi$ um espaço bilinear simétrico regular. Se $\varphi$ for um subespaço totalmente isotrópico maximal de $\phi$, então $\operatorname{dim}(\varphi)=\frac{1}{2} \operatorname{dim}\left(\phi_{h}\right)$.

Demonstração. Pelo teorema 7, consideremos $H \supseteq \varphi$ com $H$ hiperbólico e $\operatorname{dim}(H)=2 \operatorname{dim}(\varphi)$. Pela regularidade de $H$ temos $\phi \simeq H \oplus^{\perp} H^{\perp}$ onde necessariamente $H^{\perp}$ é anisotrópico (hipótese) .

\footnotetext{
${ }^{2}$ Ver [Lam3], pág 23, exercício 11.
} 
Pela unicidade a menos de isometria acima ter-se-á $\phi_{t}=\emptyset, \phi_{h} \simeq H$ e $\phi_{a} \simeq H^{\perp}, \operatorname{logo} \frac{1}{2} \operatorname{dim}\left(\phi_{h}\right)=$ $\frac{1}{2} \operatorname{dim}(H)=\operatorname{dim}(\varphi)$.

Definição 16. A dimensão comum à todos os espaços totalmente isotrópicos maximais de um EBS regular $\phi$ é chamado de índice de Witt do espaço e o denotamos por $\omega(\phi)$.

Observação 14. Para todo espaço regular $\phi$ tem-se $\phi \simeq \omega(\phi) \mathbb{H} \oplus^{\perp} \phi_{a}$.

Definição 17. Sejam $n \geq 1$ e $\left\langle\alpha_{1}, \cdots, \alpha_{n}\right\rangle$ e $\left\langle\beta_{1}, \cdots, \beta_{n}\right\rangle$ formas quadráticas diagonais com dimensão $n$.

(i) Dizemos que $\left\langle\alpha_{1}, \cdots, \alpha_{n}\right\rangle$ e $\left\langle\beta_{1}, \cdots, \beta_{n}\right\rangle$ são simplesmente equivalentes quando existem $i, j$ tais que $\alpha_{l}=\beta_{l}, \forall l \notin\{i, j\}$ e $\left\langle\alpha_{i}, \alpha_{j}\right\rangle \simeq\left\langle\beta_{i}, \beta_{j}\right\rangle$ (caso $i=j$, isto significa que $a_{i}=b_{i} . x^{2}$, para algum $x \neq 0$ ). Usaremos o símbolo $\sim$ para denotar a equivalência simples de duas formas de mesma dimensão.

(ii) Tomando o fecho transitivo $\approx$ de $\sim$ teremos a noção de equivalência de cadeia de formas de mesma dimensão: i.e., $\left\langle\alpha_{1}, \cdots, \alpha_{n}\right\rangle \approx\left\langle\beta_{1}, \cdots, \beta_{n}\right\rangle$ se, e somente se, existir uma cadeia $\phi_{1}, \cdots, \phi_{k}$ de formas diagonais tal que $\phi_{i} \sim \phi_{i+1}, \forall i<k e\left\langle\alpha_{1}, \cdots, \alpha_{n}\right\rangle=\phi_{1}$ e $\left\langle\beta_{1}, \cdots, \beta_{n}\right\rangle=\phi_{k}$

Proposição 16. (Teorema de equivalência de cadeia de Witt) Duas formas diagonais regulares são equivalentes por cadeia se, e somente se são isométricas.

Demonstração. Sej $n \geq 1$ e $\vec{\alpha}=\left\langle\alpha_{1}, \cdots, \alpha_{n}\right\rangle$ e $\vec{\beta}=\left\langle\beta_{1}, \cdots, \beta_{n}\right\rangle$ duas $n$-formas diagonais.

Claramente $\left\langle\alpha_{1}, \cdots, \alpha_{n}\right\rangle \approx\left\langle\beta_{1}, \cdots, \beta_{n}\right\rangle \Longrightarrow\left\langle\alpha_{1}, \cdots, \alpha_{n}\right\rangle \simeq\left\langle\beta_{1}, \cdots, \beta_{n}\right\rangle$.

Suponhamos agora que $\left\langle\alpha_{1}, \cdots, \alpha_{n}\right\rangle \simeq\left\langle\beta_{1}, \cdots, \beta_{n}\right\rangle$ e vamos mostrar que estas n-formas são equivalentes por cadeia. Como o grupo de permutações em $n$-elementos $S_{n}$ é gerado por transposições, então existem permutações $\sigma, \tau \in S_{n}$ tais que $\left\langle\alpha_{1}, \cdots, \alpha_{n}\right\rangle \approx\left\langle\alpha_{\sigma(1)}, \cdots, \alpha_{\sigma(n)}\right\rangle$ e $\left\langle\beta_{1}, \cdots, \beta_{n}\right\rangle \approx$ $\left\langle\beta_{\tau(1)}, \cdots, \beta_{\tau(n)}\right\rangle$ e as entradas nulas nas formas permutadas estão em segmentos finais de comprimentos $k$ e $l$ respectivamente. Como $\vec{\alpha}^{\sigma} \simeq \vec{\beta}^{\tau}$, pela unicidade no Teorema da Decomposição acima temos que $k=l$ e portanto basta provar que:

$\left(^{*}\right)$ Dadas $\left\langle a_{1}, \cdots, a_{r}\right\rangle,\left\langle b_{1}, \cdots, b_{r}\right\rangle$ r-formas diagonais e regulares, se elas são isométricas então são equivalentes por cadeia.

A prova deste enunciado segue por indução em $r$, sendo trivial para $r=1,2$. Para $r \geq 3$ consideremos uma forma diagonal (e regular) $\left\langle c_{1}, \cdots, c_{r}\right\rangle \approx\left\langle a_{1}, \cdots, a_{r}\right\rangle$ e tal que $b_{1} \in D\left(\left\langle c_{1}, \cdots, c_{p}\right\rangle\right)$ com $p$ menor possível.

Afirmação: $p=1$.

Caso contrário, como $b_{1}=c_{1} x_{1}^{2}+c_{2} x_{2}^{2}+\cdots+c_{p} x_{p}^{2}$, tería-se $d:=c_{1} x_{1}^{2}+c_{2} x_{2}^{2} \neq 0$ e assim, pelo Corolário 3. $\left.\left\langle c_{1}, c_{2}\right\rangle \simeq d, d c_{1} c_{2}\right\rangle$, mas nesse caso teríamos $\left\langle d, c_{3}, \cdots, c_{r}, d c_{1} c_{2}\right\rangle \approx\left\langle a_{1}, \cdots, a_{r}\right\rangle \mathrm{e}$ $b_{1} \in D\left(\left\langle d, c_{3}, \cdots, c_{p}\right\rangle\right)$, contradizendo a minimalidade de $p$.

Logo $\left\langle c_{1}\right\rangle \simeq\left\langle b_{1}\right\rangle$ e portanto $\left\langle b_{1}, b_{2} \cdots, b_{r}\right\rangle \simeq\left\langle a_{1}, \cdots, a_{r}\right\rangle \approx\left\langle b_{1}, c_{2}, \cdots, c_{r}\right\rangle$. Pelo Teorema do Cancelamento obtemos $\left\langle b_{2}, \cdots, b_{r}\right\rangle \approx\left\langle c_{2}, \cdots, c_{r}\right\rangle$ e, usando a hipótese de indução $\left\langle b_{2}, \cdots, b_{r}\right\rangle \simeq$ $\left\langle c_{2}, \cdots, c_{r}\right\rangle$. O resultado segue pois:

$$
\left\langle b_{1}, b_{2}, \cdots, b_{r}\right\rangle \approx\left\langle b_{1}, c_{2}, \cdots, c_{r}\right\rangle \approx\left\langle a_{1}, a_{2}, \cdots, a_{r}\right\rangle .
$$

Observação 15. Existem outras descrições da isometria entre formas quadráticas regulares de mesma dimensão. Uma das mais interessantes, que dá sustentação a uma teoria abstrata de formas 
quadráticas denominada Teoria dos Grupos Especiais ([DM1]), tem caráter indutivo ${ }^{3}$ :

Para formas diagonais e regulares $\vec{a}$ e $\vec{b}$ de dimensão $n \geqslant 3$, são equivalentes

(i) $\left\langle a_{1}, a_{2}, \cdots, a_{n}\right\rangle \simeq\left\langle b_{1}, b_{2}, \cdots, b_{n}\right\rangle$;

(ii) existem $a, b, c_{3}, \cdots, c_{n} \in \dot{\mathbb{K}}$ tais que:

- $\left\langle a_{1}, a\right\rangle \simeq\left\langle b_{1}, b\right\rangle$;

- $\left\langle a_{2}, \cdots, a_{n}\right\rangle \simeq\left\langle a, c_{3}, \cdots, c_{n}\right\rangle$;

- $\left\langle b_{2}, \cdots, b_{n}\right\rangle \simeq\left\langle b, c_{3}, \cdots, c_{n}\right\rangle$.

\subsubsection{Formas de Pfister}

Pode-se dizer que uma segunda revolução na teoria das formas quadráticas ocorreu três décadas após o trabalho original de E. Witt: a noção de forma de Pfister ([Pfi3 $)$ mostrou-se central para novos desenvolvimentos da teoria. Nesta subseção nos limitamos a definir este conceito e apresentar uns poucos resultados a respeito deste tema.

Definição 18. Uma $n$ - forma de Pfister é uma forma quadrática regular do tipo $\bigotimes_{i=1}^{n}\left\langle 1, a_{i}\right\rangle$ (i.e., $\left.a_{i} \in \dot{K}, \forall i \leq n\right)$. Nós a simbolizamos simplesmente por $\left\langle\left\langle a_{1}, \ldots, a_{n}\right\rangle\right\rangle$. Convencionamos chamar 0 -forma de Pfister ao espaço quadrático $\langle 1\rangle$. O conjunto das $n$-formas de Pfister sobre um copor $\mathbb{K}$ será denotado por $P_{n}(\mathbb{K})$.

Lema 9. Valem as seguintes propriedades

(1) $\operatorname{Se} \phi \in P_{n}(\mathbb{K})$, então $\operatorname{dim}_{\mathbb{K}}(\phi)=2^{n}$;

(2) $\langle\langle 1, \ldots, 1\rangle\rangle \simeq 2^{n}\langle 1\rangle$ (para $n$ entradas 1$)$;

(3) $\langle\langle 1\rangle\rangle \otimes\left\langle\left\langle a_{1}, \ldots, a_{n}\right\rangle\right\rangle \simeq 2\left\langle\left\langle a_{1}, \ldots, a_{n}\right\rangle\right\rangle$;

(4) $\langle\langle-1\rangle\rangle \otimes\left\langle\left\langle a_{1}, \ldots, a_{n}\right\rangle\right\rangle \simeq 2^{n} \mathbb{H} ;$

(5) $1 \in D_{\mathbb{K}}(\phi), \forall \phi \in P_{n}(\mathbb{K})$

(6) $\phi \simeq\langle 1\rangle \oplus \phi^{\prime}, \forall \varphi \in P_{n}(\mathbb{K})$, onde $\phi^{\prime}$ é uma forma quadrática regular unicamente determinada a menos de isometria, denomida a parte puro de $\phi$;

(7) $\langle\langle a, b\rangle\rangle \simeq\langle\langle a, b y\rangle\rangle, \forall y \in D_{\mathbb{K}}(\langle\langle a\rangle\rangle)$

(8) $\langle\langle a, b\rangle\rangle \simeq\langle\langle x, a b\rangle\rangle, \forall x \in D_{\mathbb{K}}(\langle a, b\rangle)$

Demonstração. Os itens 1,2,3,4,5 e 6 são imediatos. Para uma prova dos itens 7 e 8 basta considerar as isometrias

$$
\begin{gathered}
\langle\langle a, b\rangle\rangle \simeq\langle 1, a\rangle \oplus\langle b\rangle\langle 1, a\rangle \simeq\langle 1, a\rangle \oplus\langle b\rangle\langle y, y a\rangle \simeq\langle\langle a, b y\rangle\rangle \\
\langle\langle a, b\rangle\rangle \simeq\langle 1, a b, a, b\rangle \simeq\langle 1, a b, x, a b x\rangle \simeq\langle\langle x, a b\rangle\rangle
\end{gathered}
$$

Definição 19. O conjunto de fatores de similaridade de uma forma quadrática regular $\phi$ é definido por

$$
G(\phi)=G_{\mathbb{K}}(\phi):=\{a \in \dot{\mathbb{K}}: \phi \simeq a \phi:=\langle a\rangle \otimes \phi\}
$$

\section{Lema 10.}

\footnotetext{
${ }^{3}$ Veja Teorema 1.13 em Mar2] e Teorema 1.32 em [DM1.
} 
1) $\dot{\mathbb{K}}^{2} \subseteq G(\phi) \subseteq \dot{\mathbb{K}}$

2) $G(\phi)$ é um subgrupo de $\dot{\mathbb{K}}$;

3) $G(\mathbb{H})=G(m \mathbb{H})=\dot{K}, \forall m$;

4) $\lambda \in D(\phi) \Longrightarrow \lambda G(\phi) \subseteq D(\phi)$

5) $1 \in D(\phi) \Longleftrightarrow G(\phi) \subseteq D(\phi)$

6) $D(\langle\langle\alpha\rangle\rangle)=G(\langle\langle\alpha\rangle\rangle), \forall \alpha \in \dot{\mathbb{K}}$

Demonstração. (1) e (2) são imediatos (note que, para $a \in \dot{\mathbb{K}}, a^{-1}=a \cdot\left(a^{-1}\right)^{2}$ )

3) Pois $a \bigoplus_{i=1}^{m}\langle 1,-1\rangle \simeq \bigoplus_{i=1}^{m}\langle a,-a\rangle \simeq \bigoplus_{i=1}^{m}\langle 1,-1\rangle, \forall a \in \dot{K}$

4) Pois $\lambda \in D(\phi), a \in G(\phi) \Longrightarrow \exists \psi(\phi \simeq\langle\lambda\rangle \oplus \psi) \Longrightarrow \phi \simeq a \phi \simeq\langle a \lambda\rangle \oplus a \psi$

5) A implicação não trivial segue diretamente de (4).

6) Como $1 \in D(\langle 1, \alpha\rangle)$ a inclusão $G(\langle\langle\alpha\rangle\rangle) \subseteq D(\langle\langle\alpha\rangle\rangle)$ segue de (5). Por outro lado, se $b \in \mathbb{K}$ for tal que $b \in D(\langle 1, \alpha\rangle)$, então, pelo Corolário 3 , temos $\langle 1, \alpha\rangle \simeq\langle b, b \alpha\rangle$, mas $\langle b, b \alpha\rangle=b\langle 1, \alpha\rangle$, logo $b \in G(\langle 1, \alpha\rangle)$.

Um dos resultados mais úteis sobre formas de Pfister é o seguinte:

Proposição 17. (Teorema da subforma pura) Seja $n \geq 1$.

$$
\forall \phi \in P_{n}(\mathbb{K}) \forall b \in D\left(\phi^{\prime}\right) \exists \psi \in P_{n-1}(\mathbb{K})(\phi \simeq\langle\langle b\rangle\rangle \otimes \psi)
$$

Demonstração. Consulte a prova em [Lam3], pág 318, Teor. 1.5.

Corolário 8. Seja $\phi \in P_{n}(\mathbb{K}), n \geq 1$. Então $\phi$ é isotrópica se e só se $\phi$ é hiperbólica.

Demonstração. Como $n \geq 1$, a implicação $(\Leftarrow)$ é clara. Se $\phi$ é isotrópica, temos alguma forma regular $\theta\left(=\mathbb{H}^{\perp}\right)$ tal que

$$
\langle 1,-1\rangle \oplus \theta \simeq \phi \simeq\langle 1\rangle \oplus \phi^{\prime} .
$$

Pela lei de cancelamento, obtemos $-1 \in D\left(\phi^{\prime}\right)$ e, pela Proposição acima, existe $\psi \in P_{n-1}(\mathbb{K})$ tal que

$$
\phi \simeq\langle\langle-1\rangle\rangle \otimes \psi \simeq 2^{n-1} \mathbb{H} \quad(\text { Lema 9.(4)). }
$$

Corolário 9. $\forall \phi \in P_{n}(\mathbb{K}), D(\phi)=G(\phi)$.

Demonstração. Para $n=0$ o resultado é claro. Assumimos $n \geq 1$. Como $1 \in D(\phi)$, a inclusão $G(\phi) \subseteq D(\phi)$ segue do item (5) do Lema anterior. Por outro lado, seja $b \in D(\phi)=D\left(\langle 1\rangle \oplus \phi^{\prime}\right)$, então $b=x^{2}+c$ para algum $c \in D\left(\phi^{\prime}\right) \cup\{0\}$. Se $c=0$, então $x \neq 0$ e $b=x^{2} \in G(\phi)$ (Lema 10.(1)), como queríamos. Se $c \neq 0$, pelo Teorema da subforma pura acima, $\phi \simeq\langle\langle c\rangle\rangle \otimes \psi$ para alguma $\psi \in P_{n-1}(\mathbb{K}) ;$ como $b \in D(\langle\langle c\rangle\rangle)$, pelo Lema 10.(6), temos $b \in G(\langle\langle c\rangle\rangle)$, logo $b \phi \simeq\langle b\rangle \otimes\langle\langle c\rangle\rangle \otimes \psi \simeq$ $\langle\langle c\rangle\rangle \otimes \psi \simeq \phi$, obtendo $b \in G(\phi)$, como queríamos.

O símbolo $\langle\langle\cdot\rangle\rangle$ guarda outras semelhanças formais com o símbolo $\langle\cdot\rangle$ além daquela expressa no Teorema da subforma purat Mais uma evidência é dada a seguir:

\footnotetext{
${ }^{4}$ Se $\phi=\left\langle\left\langle a_{1}, \cdots, a_{n}\right\rangle\right\rangle$, note que $b \in D\left(\phi^{\prime}\right)$ se e só se existem $c_{2}, \cdots, c_{n} \in \mathbb{K}$ tais que $\left\langle\left\langle a_{1}, \cdots, a_{n}\right\rangle\right\rangle \simeq$ $\left\langle\left\langle b, c_{2}, \cdots, c_{n}\right\rangle\right\rangle$.
} 


\section{Definição 20.}

1) Diremos que as formas de Pfister $\left\langle\left\langle\alpha_{1}, \ldots, \alpha_{n}\right\rangle\right\rangle,\left\langle\left\langle\alpha_{1}^{\prime}, \ldots, \alpha_{n}^{\prime}\right\rangle\right\rangle \in P_{n}(\mathbb{K}),(n \geq 1)$ são $P$ simplesmente equivalentes $(\sim)$ quando existirem indices $i, j$ tais que:

$\left.P_{1}\right) \quad\left\langle\left\langle\alpha_{i}, \alpha_{j}\right\rangle\right\rangle \simeq\left\langle\left\langle\alpha_{i}^{\prime}, \alpha_{j}^{\prime}\right\rangle\right\rangle ;$

$\left.P_{2}\right) \quad \alpha_{k}=\beta_{k}, \forall k \neq i, j$

2) Duas formas de Pfister $\phi, \phi^{\prime} \in P_{n}(\mathbb{K})$ são ditas P-equivalentes por cadeia $(\approx)$ quando existir uma sequência $\phi_{1}, \ldots, \phi_{r} \in P_{n}(\mathbb{K})$ tal que :

$\left.P C_{1}\right) \quad \phi=\phi_{1}$ e $\phi^{\prime}=\phi_{r}$;

$\left.P C_{2}\right) \quad \phi_{i} \sim \phi_{i+1},(i<r)$

Proposição 18. (Teorema de P-equivalência por cadeia)

$$
\forall \phi, \varphi \in P_{n}(\mathbb{K})(\phi \simeq \varphi \Longleftrightarrow \phi \approx \varphi)
$$

Demonstração. Consulte a prova em [Lam3], pág 321, Teor. 1.12.

\subsubsection{Formas quadráticas e os Quatérnios}

A estreita conexão entre formas quadráticas binárias e álgebras de quatérnios constitui um dos pontos chave da teoria de formas quadráticas após os anos 1970, sendo uma das componentes essenciais na terceira revolução nesta teoria realizada por J. Milnor ([Mil1] ), que desenvolveremos em capítulos futuros.

Definição 21. Seja $\mathbb{K}$ um corpo com $\operatorname{char}(\mathbb{K}) \neq 2$.

1) Consideremos a $\mathbb{K}$-álgebra associativa (mas não comutativa) $\mathbb{K}\{i, j\}$ que é livre e gerada pelas indeterminadas $i, j$. Para cada $\alpha, \beta \in \mathbb{K}$, denotamos por $(\alpha, \beta)_{\mathbb{K}}$ a seguinte $\mathbb{K}$-álgebra associativa com unidade dada por geradores e relações como segue

$$
(\alpha, \beta)_{\mathbb{K}}:=\frac{\mathbb{K}\{i, j\}}{\left\langle i^{2}-\alpha, j^{2}-\beta, i j+j i\right\rangle} .
$$

2) Dizemos que uma $\mathbb{K}$-álgebra é uma álgebra de quatérnios se ela for isomorfa a $(\alpha, \beta):=(\alpha, \beta)_{\mathbb{K}}$ para alguns $\alpha, \beta \in \mathbb{K}$.

3) Denotamos $k:=i . j . O \mathbb{K}$-subespaço vetorial de $(\alpha, \beta)_{\mathbb{K}}$ gerado pelo conjunto $\{i, j, k\}$ é denotado por $Q_{0}(\alpha, \beta):=Q_{0}=\left\{z \in(\alpha, \beta)_{\mathbb{K}}: z=z_{1} i+z_{2} j+z_{3} k\right\}$ e é denominado o subespaço dos quatérnios puros de $(\alpha, \beta)$.

\section{Fato 3.}

1) $\{1, i, j, k\}$ é uma base de $(\alpha, \beta)$. Assim, $\operatorname{dim}_{\mathbb{K}}(\alpha, \beta)=4$;

2) $i, j, k$ são 2 a 2 anticomutativos;

3) $(-1,-1)_{\mathbb{R}}$ é a álgebra hamiltoniana usual;

4) $(-1,1)_{\mathbb{K}} \cong M_{2 x 2}(\mathbb{K})$, como $\mathbb{K}$-álgebras;

5) Para cada $\alpha, \beta, x, y \in \mathbb{K}$, temos $(\alpha, \beta) \cong\left(\alpha x^{2}, \beta y^{2}\right)$;

6) (Funtorialidade) $\mathbb{L} \otimes_{\mathbb{K}}(\alpha, \beta)_{\mathbb{K}} \cong(\alpha, \beta)_{\mathbb{L}}$ para toda extensão de corpos $\mathbb{K} \subseteq \mathbb{L}$. 
Uma propriedade interessante (e característica) das álgebras de quatérnios é a seguinte:

Proposição 19. Seja A uma $\mathbb{K}$-álgebra de dimensão 4, então são equivalentes:

(i) A é uma $\mathbb{K}$-álgebra de quatérnios;

(ii) A é uma $\mathbb{K}$-álgebra central simples;

Demonstração. (Esboço)

$(i) \Rightarrow(i i)$ : Seja $A \cong(\alpha, \beta)_{\mathbb{K}}$ e consideremos $\mathbb{L} \supseteq \mathbb{K}$ uma extensão quadráticamente fechada de $\mathbb{K}$ (tome, por exemplo, $\mathbb{L}$ um fecho algébrico de $\mathbb{K}$ ). Por "funtorialidade"(item (6) acima) e uma aplicação dos itens (4) e (5), temos

$$
\mathbb{L} \otimes_{\mathbb{K}}(\alpha, \beta)_{\mathbb{K}} \cong(\alpha, \beta)_{\mathbb{L}} \cong(-1,1)_{\mathbb{L}} \cong M_{2 x 2}(\mathbb{L})
$$

Como $M_{2 x 2}(\mathbb{L})$ é uma $\mathbb{L}$-álgebra simples e de centro $\mathbb{L}$, conseguimos garantir que $(\alpha, \beta)_{\mathbb{K}}$ é uma $\mathbb{K}$-álgebra simples e de centro $\mathbb{K}$.

$(i i) \Rightarrow(i)$ : segue da hipótese sobre dimensão, utilizando o Teorema de Artin-Wedderburn (para detalhes, veja [Lam3], pág 74, Teor. 5.1)

Definição 22. Seja $(\alpha, \beta)_{\mathbb{K}}$ uma álgebra de quatérnios.

1) A conjugação em $(\alpha, \beta)_{\mathbb{K}}$ é a função $\overline{(~)}:(\alpha, \beta)_{\mathbb{K}} \longrightarrow(\alpha, \beta)_{\mathbb{K}}$ definida por $\bar{z}=z_{0}-z_{1} i-z_{2} j-z_{3} k$, para cada $z=z_{0}+z_{1} i+z_{2} j+z_{3} k \in(\alpha, \beta)_{\mathbb{K}}$.

2) A forma traço de $(\alpha, \beta)_{\mathbb{K}}$ é a aplicação $\operatorname{Tr}:(\alpha, \beta)_{\mathbb{K}} \longrightarrow \mathbb{K}$ definida por $\operatorname{Tr}(z):=z+\bar{z}=2 z_{0}$.

3) Denominamos de forma norma da álgebra $(\alpha, \beta)_{\mathbb{K}}$ a aplicação $N:(\alpha, \beta)_{\mathbb{K}} \longrightarrow$ definida por $N(z):=z \bar{z}=z_{0}^{2}-\alpha z_{1}^{2}-\beta z_{2}^{2}+\alpha \beta z_{3}^{2}$.

Fato 4. Para cada $x, y \in(\alpha, \beta)$, temos:

1) $\overline{\bar{x}}=x ; \overline{x y}=\bar{y} \bar{x}$;

2) $x \in \mathbb{K} .1$ sse $\bar{x}=x ; x \in Q_{0}$ sse $\bar{x}=-x$;

3) $\overline{N(x)}=N(x) ; N(1)=1 ; N(x y)=N(x) N(y)$;

4) $x$ invertível sse $N(x) \neq 0$; no caso positivo temos: $x^{-1}=x / N(x)$;

5) $\overline{\operatorname{Tr}(x)}=\operatorname{Tr}(x)$;

6) $x$ é raiz do polinômio $\in \mathbb{K}[X]$ dado por $X^{2}-\operatorname{Tr}(x) X+N(x)$.

\section{Proposição 20.}

1) $A$ aplicação $B:(\alpha, \beta)_{\mathbb{K}} \times(\alpha, \beta)_{\mathbb{K}} \longrightarrow \mathbb{K}$ definida por $B(x, y):=\frac{1}{2} \operatorname{Tr}(x \bar{y})=(x \bar{y}+y \bar{x}) / 2$ é um forma bilinear simétrica, cuja forma quadrática associada é a norma : $q_{B}(x)=N(x), \forall x, y \in(\alpha, \beta)$;

2) $A$ base $\{1, i, j, k\}$ de $(\alpha, \beta)$ é ortogonal para $B$, diagonalizando este $E B S$ como $B \simeq\langle 1,-\alpha,-\beta, \alpha \beta\rangle \simeq$ $\langle 1,-\alpha\rangle \otimes\langle 1,-\beta\rangle$;

3) $\left((\alpha, \beta)_{\mathbb{K}}, B\right)$ é um EBS regular;

4) $D\left(q_{B}\right)=D(N)$ é um subgrupo de $\dot{\mathbb{K}}$ que contem $\dot{K}^{2}$;

5) $D(N)=\{\lambda \in \dot{\mathbb{K}}:\langle\lambda\rangle \otimes N \simeq N\}$

Demonstração.

1) Segue por computação direta. 
2) Note que $B(z, \lambda .1)=\lambda \operatorname{Tr}(z) / 2=\lambda z_{0}$, para $\lambda \in \mathbb{K}$, logo $\mathbb{K} \perp Q_{0}$. Se $x, y \in Q_{0}$, então $B(x, y)=$ $(x \bar{y}+y \bar{x}) / 2=-(x y+y x) / 2$ e como $\{i, j, k\}$ anticomutam 2 a 2 , temos que $\{i, j, k\}$ é base ortogonal para $\left.B\right|_{Q_{0} \times Q_{0}}$. Calculando: $q_{B}(1)=N(1)=1, q_{B}(i)=N(i)=-\alpha, q_{B}(j)=N(j)=-\beta ; q_{B}(k)=$ $N(k)=\alpha \beta$.

3) Se $\varepsilon=(1, i, j, k)$, note que $\operatorname{det}\left(B_{\varepsilon}\right)=(1)(-\alpha)(-\beta)(\alpha \beta)=(\alpha \beta)^{2} \in \dot{K}$.

4) Segue por computação direta, utilizando os itens (3) e (4) do fato acima ou, alternativamente, como decorrência do item (3) e de resultados gerais sobre formas de Pfister apresentados na subseção anterior.

5) É uma decorrência do item (3) e de resultados gerais sobre formas de Pfister apresentados na subseção anterior. Alternativamente, se $\langle\lambda\rangle \otimes N \simeq N$, obtemos $\lambda \in D(\langle\lambda\rangle \otimes N)=D(N)$. Por outro lado, se $\lambda \in D(N)$ e $x \in(\alpha, \beta)$ é tal que $N(x)=\lambda \in \dot{K}$, temos pelo item (4) do Fato acima que $x$ é invertível, logo a função $l_{x}:((\alpha, \beta),\langle\lambda\rangle \otimes N) \longrightarrow((\alpha, \beta), N)$ definida por $l_{x}(w)=x w$ é uma isometria entre esses EBSs, pois é um isomorfismo linear tal que

$$
N\left(l_{x}(w)\right)=N(x w)=N(x) N(w)=\lambda N(w)=(\langle\lambda\rangle \otimes N)(w)
$$

\section{Lema 11.}

1) $Q_{0}-\{0\}=\left\{z \in(\alpha, \beta): z^{2} \in \mathbb{K}, z \notin \mathbb{K}\right\}$;

2) Se $\Phi:(\alpha, \beta) \stackrel{\cong}{\longrightarrow}\left(\alpha^{\prime}, \beta^{\prime}\right)$ é um isomorfismo de $\mathbb{K}$-álgebras, então $\Phi_{\mid}: Q_{0} \stackrel{\cong}{\longrightarrow} Q_{0}^{\prime}$ é um isomorfismo de $\mathbb{K}$-espaços vetoriais.

\section{Demonstração.}

1) Seja $z=z_{1} i+z_{2} j+z_{3} k \neq 0$, então $z^{2}=a z_{1}^{2}+b z_{2}^{2}-a b z_{3}^{2} \in \mathbb{K}$ e para algum $l=1,2,3$, tem-se $z_{l} \neq 0$, logo $z \notin \mathbb{K}$. Por outro lado, se $w=w_{0}+w_{1} i+w_{2} j+w_{3} k$ é tal que $w^{2}=$ $\left(w_{0}^{2}+a w_{1}^{2}+b w_{2}^{2}-a b w_{3}^{2}\right)+2 w_{0}\left(w_{1} i+w_{2} j+w_{3} k\right) \in \mathbb{K}$ então $w_{0} w_{1}=w_{0} w_{2}=w_{0} w_{3}=0$ e como $w \notin \mathbb{K}$, para algum $l=1,2,3$, tem-se $w_{l} \neq 0$, logo $w_{0}=0$.

2) Seja $z \in Q_{0}-\{0\}, \operatorname{logo} z \notin \mathbb{K}=\operatorname{Centro}(\alpha, \beta)$. Assim $\Phi(z) \notin \operatorname{Centro}\left(\alpha^{\prime}, \beta^{\prime}\right)=\mathbb{K}$. Por outro lado, como $z^{2} \in \mathbb{K}$, temos $\Phi(z)^{2}=\Phi\left(z^{2}\right) \in \mathbb{K}$. Pelo item (1) obtemos $\Phi\left[Q_{0}\right] \subseteq Q_{0}^{\prime}$. Procedendo de forma análoga para o isomorfismo inverso $\Phi^{-1}:\left(\alpha^{\prime}, \beta^{\prime}\right) \stackrel{\cong}{\longrightarrow}(\alpha, \beta)$, obtemos $\Phi^{-1}\left[Q_{0}^{\prime}\right] \subseteq Q_{0}$, concluindo a prova.

Proposição 21. São equivalentes para todos $a, b, c, d \in \dot{\mathbb{K}}$

1) $(a, b) \cong\left(a^{\prime}, b^{\prime}\right)$, como $\mathbb{K}$-álgebras;

2) $\langle\langle-a,-b\rangle\rangle \simeq\left\langle\left\langle-a^{\prime},-b^{\prime}\right\rangle\right.$, como EBSs;

3) $\langle-a,-b, a b\rangle \simeq\left\langle-a^{\prime},-b^{\prime}, a^{\prime} b^{\prime}\right\rangle$, como EBSs

Demonstração.

$1) \Longrightarrow 2)$ Seja $\Phi:(\alpha, \beta) \stackrel{\cong}{\longrightarrow}\left(\alpha^{\prime}, \beta^{\prime}\right)$ um isomorfismo de $\mathbb{K}$-álgebras então, pelo item (2) do Lema acima, obtemos $\Phi\left[Q_{0}\right]=Q_{0}^{\prime}$ e daí $\overline{\Phi(z)}=\Phi(\bar{z}), \forall z \in(\alpha, \beta)$. Assim

$$
N^{\prime}(\Phi(z))=\Phi(z) \overline{\Phi(z)}=\Phi(z \bar{z})=\Phi(N(z))=N(z) \in \mathbb{K} .
$$

2) $\Longrightarrow 3$ ) Segue do Teorema de Cancelamento de Witt, pois

$$
\langle\langle-a,-b\rangle\rangle=\langle 1,-a\rangle \otimes\langle 1,-b\rangle=\langle 1,-a,-b, a b\rangle
$$


$3) \Longrightarrow 1)$ Se $\tau:\langle-a,-b, a b\rangle \longrightarrow\left\langle-a^{\prime},-b^{\prime}, a^{\prime} b^{\prime}\right\rangle$ for uma isometria, então $\tilde{\tau}:(a, b) \longrightarrow\left(a^{\prime}, b^{\prime}\right)$ dado por $\tilde{\tau}(\lambda+z)=\lambda+\tau(z)$ é um isomorfismo linear. Note agora que $a=-N(i)=-N^{\prime}(\tau(i))=$ $-\tau(i) \overline{\tau(i)}=-\tau(i)(-\tau(i))=\tau(i)^{2}$; analogamente $b=\tau(j)^{2}$. Além disso, como $-(\tau(i) \tau(j)+$ $\tau(j) \tau(i)) / 2=B^{\prime}(\tau(i), \tau(j))=B(i, j)=0$, temos que o par $\tau(i), \tau(j)$ satisfaz as mesmas relações que o par $i, j$, donde $\tilde{\tau}:(a, b) \longrightarrow\left(a^{\prime}, b^{\prime}\right)$ é um isomorfismo de $\mathbb{K}$-álgebras.

Como consequências diretas da caracterização acima, obtemos:

Corolário 10. Sejam $a, b \in \dot{K}$.

$$
\begin{aligned}
& \text { 1) }(a, b) \cong\left(a \alpha^{2}, b \beta^{2}\right) \\
& \text { 2) }(a, b) \cong(b, a) \\
& \text { 3) }(a, a) \cong(a,-1) \\
& \text { 4) }(1,1) \cong(1, a) \cong(b,-b)
\end{aligned}
$$

Demonstração.

1) Pois: $\langle-a,-b, a b\rangle \simeq\left\langle-a \alpha^{2},-b \beta^{2}, a b(\alpha \beta)^{2}\right\rangle$

2) Pois: $\langle-a,-b, a b\rangle \simeq\langle-b,-a, b a\rangle$

3) $\langle 1,-a,-1, a\rangle,\left\langle 1,-b, b,-b^{2}\right\rangle,\langle 1,-c,-(1-c), c(1-c)\rangle$ são isotrópicas.

4) Pois são formas de Pfister hiperbólicas:

$$
\begin{aligned}
& \langle\langle-1,-1\rangle\rangle=\langle 1,-1\rangle \otimes\langle 1,-1\rangle ; \\
& \langle\langle-1,-a\rangle\rangle=\langle 1,-1\rangle \otimes\langle 1,-a\rangle ; \\
& \langle\langle b,-b\rangle\rangle=\langle 1, b\rangle \otimes\langle 1,-b\rangle=\left\langle 1, b,-b,-b^{2}\right\rangle \simeq\left\langle 1,-b^{2}, b,-b\right\rangle \simeq\langle 1,-1,1,-1\rangle .
\end{aligned}
$$

Outra consequência interessante da Proposiçao acima é dada pela seguinte caracterização, semelhante àquela apresentada no Corolário 3.

Corolário 11. Sejam $a, b \in \dot{K}$. São equivalentes:

1) $\langle a, b\rangle \simeq\left\langle a^{\prime}, b^{\prime}\right\rangle$;

2) $a b \dot{\mathbb{K}}^{2}=a^{\prime} b^{\prime} \dot{\mathbb{K}}^{2} e(a, b) \cong\left(a^{\prime}, b^{\prime}\right)$

Demonstração.

$(1) \Longrightarrow(2)$ : Se $\langle a, b\rangle \simeq\left\langle a^{\prime}, b^{\prime}\right\rangle$ temos, pelo Corolário $3 a b \dot{\mathbb{K}}^{2}=a^{\prime} b^{\prime} \dot{\mathbb{K}}^{2}, \operatorname{logo}\langle a b\rangle \simeq\left\langle a^{\prime} b^{\prime}\right\rangle$.Usando novamente a hipótese e somando formas isométricas obtemos a isometria $\langle-a,-b, a b\rangle \simeq\left\langle-a^{\prime},-b^{\prime}, a^{\prime} b^{\prime}\right\rangle$ logo, pela Proposição acima, obtemos $(a, b) \cong\left(a^{\prime}, b^{\prime}\right)$.

$(2) \Longrightarrow(1)$ : Da hipótese $(a, b) \cong\left(a^{\prime}, b^{\prime}\right)$ e da Proposição acima obtemos $\langle-a,-b, a b\rangle \simeq\left\langle-a^{\prime},-b^{\prime}, a^{\prime} b^{\prime}\right\rangle$. Como $a b \mathbb{K}^{2}=a^{\prime} b^{\prime} \dot{\mathbb{K}}^{2}$, obtemos $\langle a b\rangle \simeq\left\langle a^{\prime} b^{\prime}\right\rangle$ e a isometria $\langle a, b\rangle \simeq\left\langle a^{\prime}, b^{\prime}\right\rangle$ segue da Lei de Cancelamento.

Finalizamos a subseção com a seguinte caracterização:

Proposição 22. Dados $a, b \in \dot{\mathbb{K}}$. São equivalentes

1) $(a, b) \cong(1,1)$;

2) $\langle 1,-a,-b, a b\rangle \simeq 2 \mathbb{H}$;

3) $\langle-a,-b, a b\rangle \simeq \mathbb{H} \oplus\langle-1\rangle$;

4) $(a, b)$ possui divisores de zero; 
5) $1 \in D(\langle a, b\rangle)$.

\section{Demonstração.}

1) $\Longrightarrow 2)(a, b) \cong(1,1) \Longrightarrow\langle 1,-a,-b, a b\rangle \simeq\langle 1,-1,-1,1\rangle \simeq 2 \mathbb{H}$.

2) $\Longrightarrow 3)\langle 1\rangle \oplus\langle-a,-b, a b\rangle \simeq\langle 1\rangle \oplus\langle-1,-1,1\rangle \Longrightarrow\langle-a,-b, a b\rangle \simeq\langle-1\rangle \oplus \mathbb{H}$.

$3) \Longrightarrow 4)\langle-a,-b, a b\rangle$ é isotrópica, então existe $x \neq 0$ tal que $0=N(x)=x \cdot \bar{x}, \operatorname{logo} x, \bar{x}$ são um divisores de zero em $(a, b)$.

$4) \Longrightarrow 5)$ Sejam $z, w \in(a, b)-\{0\} \operatorname{com} z w=0$. Assim, $N(z) N(w)=0 \Longrightarrow N(z)=0$ ou $N(w)=0$. Seja $z=z_{0}+z_{1} i+z_{2} j+z_{3} k$ e suponhamos $0=N(z)=z_{0}^{2}-a z_{1}^{2}-b z_{2}^{2}+a b z_{3}^{2}, \operatorname{logo}$

$$
z_{0}^{2}+a b z_{3}^{2}=a z_{1}^{2}+b z_{2}^{2}
$$

Se $\lambda=a z_{1}^{2}+b z_{2}^{2} \neq 0$, teremos $\lambda \in D(\langle 1, a b\rangle) \cap D(\langle a, b\rangle)$ e, como $1 a b \cdot \dot{\mathbb{K}}^{2}=a \cdot b \dot{\mathbb{K}}^{2}$, o Corolário 3 acarreta que $1 \in D(\langle 1, a b\rangle)=D(\langle a, b\rangle)$. Se $\lambda=0$, como $z \neq 0$ e $N(z)=0$, temos duas possibilidades: (i) que $\left(z_{1}, z_{2}\right)$ é vetor isotrópico de $\langle a, b\rangle$ e o resultado segue (pois isotropia acarreta universalidade); ou (ii) $z_{1}=z_{2}=0$, $\log$ o $z_{3} \neq 0$ e $\left(z_{0} / z_{3}\right)^{2}-a b=0$, assim $z_{0} \neq 0$ e a forma de Pfister $\langle 1,-a b\rangle$ é isotrópica e $a \dot{\mathbb{K}}^{2}=b \dot{\mathbb{K}}^{2}$, assim $\langle a, b\rangle \simeq\langle a, a\rangle$. Como $N(z)=0$ e $z \neq 0$, a forma de Pfister $\langle 1,-a,-b, a b\rangle$ é isotrópica, logo é hiperbólica, então

$$
\langle 1,-1,-1,1\rangle \simeq\langle 1,-1,1,-1\rangle \simeq\langle 1,-a,-b, a b\rangle \simeq\left\langle 1,-a,-a, a^{2}\right\rangle \simeq\langle 1,-a,-a, 1\rangle
$$

e, pela Lei do Cancelamento,

$$
\langle-1,-1\rangle \simeq\langle-a,-a\rangle
$$

portanto

$$
\langle 1,1\rangle \simeq\langle a, a\rangle
$$

finalizando esta prova.

$5) \Longrightarrow 1)$ Segue da hipótese e do Corolário 3 que $\langle 1, a b\rangle \simeq\langle a, b\rangle$, logo $\langle 1, a b\rangle \oplus\langle-a,-b\rangle \simeq\langle a, b\rangle \oplus$ $\langle-a,-b\rangle \simeq\langle a,-a, b,-b\rangle \simeq\langle 1,-1,1,-1\rangle \simeq\langle 1,-1,-1,1\rangle$ assim, pela Proposição 21, temos $(a, b) \cong$ $(1,1)$.

subfile 


\section{Capítulo 2}

\section{Anéis de Witt}

Neste Capítulo apresentamos a noção algébrica central que codifica a geometria das formas quadráticas (regulares) sobre um corpo $\mathbb{K}$, que foi desenvolvida no Capítulo anterior: a noção de anel de Witt de $\mathbb{K}$, introduzida em Witt]. Introduziremos aqui os functores de anel de Witt-Grothendieck e de anel de Witt; descreveremos apresentações (canônicas) destes anéis; relacionaremos assinaturas de corpos com suas ordens; descreveremos a propriedade local-global de Pfister (uma generalização do teorema de Sylvester sobre formas quadráticas reais); calcularemos o anel de Witt de alguns corpos e também calcularemos os primeiros termos do anel graduado de Witt. Referências principais: [Lam1], Lam2], Lam3], [Sch2].

Todos corpos neste Capítulo são assumidos terem característica $\neq 2$.

\subsection{Semi-anéis de formas quadráticas}

Na seção 1.5 do Capítulo 1 observamos que, se $\mathbb{K}$ é um corpo com característica $\neq 2$, existem bijeções entre conjuntos de objetos representantes, módulo isomorfismo (ou isometria), das diferentes categorias quadráticas apresentadas $\left(B I L S_{\mathbb{K}}, P L S_{\mathbb{K}}, Q U A D_{\mathbb{K}}, B S_{\mathbb{K}}, M S_{\mathbb{K}}\right)$.

Deste modo, e utilizando que as operações que soma ortogonal e produto tensorial de formas bilineares são compatíveis com isometria, obtemos um semi-anel comutativo e com unidad 1 $(\operatorname{Form}(\mathbb{K}) / \simeq, \bar{\oplus}, \bar{\otimes},[\langle\rangle],[\langle 1\rangle])$, único a menos de isomorfismo. A Lei de Cancelamento de Witt garante que este semi-anel tem cancelamento (uma propriedade válida também no semi-anel dos naturais $(\mathbb{N},+,$.$) .).$

Lembrando que todo EBS se decompõe, de forma única a menos de isometria, como a soma ortogonal de um subespaço totalmente isotrópico (i.e., onde a forma é nula) e de um subespaço regular e que a classe dos espaços regulares é fechada por isometria, soma ortogonal e produto tensorial, obtemos um sub-semianel $M(\mathbb{K})=(\operatorname{Reg}(\mathbb{K}) / \simeq, \bar{\oplus}, \bar{\otimes}) \subseteq(\operatorname{Form}(\mathbb{K}) / \simeq, \bar{\oplus}, \bar{\otimes})(\operatorname{logo}$ também comutativo, com unidade e com cancelamento), obtido considerando-se apenas formas quadráticas regulares.

Em particular, assumimos, de agora em diante, que as formas quadráticas consideradas serão diagonais $\left\langle a_{1}, \cdots, a_{n}\right\rangle$ e regulares, i.e. $a_{i} \in \dot{K}, \forall i \leq n$, para $n \geq 1$. Caso $n=0$, denotamos \langle\rangle a forma diagonal zero-dimensional.

\footnotetext{
${ }^{1}$ Lembramos que uma estrutura de semi-anel com unidade sobre um conjunto $X$ é constituida de um monóide abeliano $(X,+, 0)$ e de um monóide $(X, ., 1)$ tais que vale a lei distributiva. No caso em questão, o elemento neutro do monóide aditivo é a classe de isometria do única forma sobre um $\mathbb{K}$-espaço vetorial $V$ de dimensão zero $(V=\{0\})$, cuja diagonalização- sobre a base vazia- é denotada por \langle\rangle .
} 


\subsection{Anéis de Witt-Grothendieck e de Witt de corpos}

Definição 23. Seja $\mathbb{K}$ um corpo de característica $\neq 2$. O anel de Witt-Grothendieck de $\mathbb{K}$ como "o melhor anel"(comutativo, com unidade) associado ao semi-anel com cancelamento $M(K)$, i.e. $W G(\mathbb{K}):=\mathcal{K} M(\mathbb{K})$ ou, alternativamente, $W G(\mathbb{K}):=\mathcal{G}(M)$, conforme o Apêndice A.

Observação 16. Como $M(\mathbb{K})$ é um monóide com cancelamento, o homomorfismo canônico $\rho_{\mathbb{K}}$ : $M(\mathbb{K}) \longrightarrow W G(\mathbb{K})$ é injetor 2 , logo se $\phi, \phi^{\prime}$ são formas regulares, $\bar{\phi}=\bar{\phi}^{\prime}$ em $W G(\mathbb{K}) \Longleftrightarrow \phi \simeq \phi^{\prime}$.

Lema 12. Seja $\mathbb{Z} \mathbb{H}$ o subgrupo aditivo de $W G(\mathbb{K})$ gerado pela imagem em $W G(\mathbb{K})$ da classe de isometria do plano hiperbólico $\mathbb{H}$. Então, $\mathbb{Z} \mathbb{H} \triangleleft W G(\mathbb{K})$ (é ideal).

Demonstração. Sejam $(\bar{\phi}-\bar{\psi}) \in W G(\mathbb{K})$ e $n \in \mathbb{N}$. Então, pelo Corolário 5 do Capítulo 1 temos:

$$
(\bar{\phi}-\bar{\psi}) \bar{\otimes} n \overline{\mathbb{H}}=n(\overline{\phi \otimes \mathbb{H}}-\overline{\psi \otimes \mathbb{H}})=n(\operatorname{dim}(\phi) \overline{\mathbb{H}}-\operatorname{dim}(\psi) \overline{\mathbb{H}}) \in \mathbb{Z} \overline{\mathbb{H}}
$$

Definição 24. O anel de Witt de um corpo $\mathbb{K}$ é definido como sendo o anel quociente $W(\mathbb{K})=$ $W G(\mathbb{K}) / \mathbb{Z} \mathbb{H}$.

Observação 17. Todo elemento de $W(\mathbb{K})$ pode ser representado por uma forma regular $\phi$, pois:

$$
\begin{gathered}
{[\langle a\rangle]+[\langle-a\rangle]=[\langle a,-a\rangle]=[\langle 1,-1\rangle]=[0]} \\
\operatorname{logo}[\langle-a\rangle]=-[\langle a\rangle] . \text { Portanto, } \\
{\left[\left\langle a_{1}, \cdots, a_{m}\right\rangle\right]-\left[\left\langle b_{1}, \cdots, b_{n}\right\rangle\right]=\left[\left\langle a_{1}, \cdots, a_{m},-b_{1}, \cdots,-b_{n}\right\rangle\right] .}
\end{gathered}
$$

O resultado de classificação a seguir indica que a noção de anel de Witt de um corpo retém a informação mais relevante da geometria das formas quadráticas sobre aquele corpo.

Proposição 23. A aplicação $a: A n i s(\mathbb{K}) / \simeq \longrightarrow W(\mathbb{K}),[\phi] \mapsto \bar{\phi}$ entre o conjunto das classes de isometria das formas anisotrópicas e o anel de Witt é bijetora.

Demonstração. A sobrejetividade da função vem da observação anterior pois, se utilizamos uma forma regular $\phi$ para representar um dado elemento de $W(K)$, a lei de decomposição de Witt (Prop. 15, Cap.1) garante que $\phi \simeq \phi_{a} \oplus^{\perp} \phi_{h}$, com $\phi_{a}$ anisotrópica e $\phi_{h}$ hiperbólica, então $[\phi]=\left[\phi_{a}\right]$.

Por ouro lado, sejam $\phi, \psi$ formas anisotrópicas tais que $[\phi]=[\psi]$ em $W(\mathbb{K})$, então $\bar{\phi}+\mathbb{Z} \mathbb{H}=$ $\bar{\psi}+\mathbb{Z} \mathbb{H}$ em $W G(\mathbb{K}$ ), assim existe $n \in \mathbb{N}$ tal que $\bar{\phi}=\bar{\psi} \bar{\oplus} n \overline{\mathbb{H}}$ (ou equação semelhante, trocando os papéis de $\phi, \psi)$. Assim $\phi \simeq \psi \oplus n \mathbb{H}$ e, como $\phi$ e $\psi$ são anisotrópicas, temos $n=0, \operatorname{logo} \phi \simeq \psi$. Portanto o mapa $a$ é injetor

Corolário 12. Sejam $\phi, \phi^{\prime}$ formas regulares. Então:

(i) $[\phi]=\left[\phi^{\prime}\right]$ em $W(\mathbb{K}) \Longleftrightarrow \bar{\phi}_{a}=\bar{\phi}_{a}^{\prime}$ em $M(\mathbb{K})$.

(ii) $[\phi]=\left[\phi^{\prime}\right]$ em $W(\mathbb{K}) \Longleftrightarrow \bar{\phi}=\bar{\phi}^{\prime}$ em $M(\mathbb{K})$, desde que $\operatorname{dim}(\phi)=\operatorname{dim}\left(\phi^{\prime}\right)$.

\footnotetext{
${ }^{2}$ Da mesma forma que o semi-anel com cancelamento $\mathbb{N}$ injeta-se como subanel de $\mathbb{Z}$.
} 
A aplicação $\operatorname{dim}: M(\mathbb{K}) \longrightarrow \mathbb{Z}$ definida por $\operatorname{dim}([\phi])=\operatorname{dim}(\phi)$ é um homomorfismo de semianéis. Pela propriedade universal que caracteriza o anel $W G(\mathbb{K})$ o morfismo dim estende-se para um único morfismo de anéis $\widetilde{\operatorname{dim}}: W G(\mathbb{K}) \longrightarrow \mathbb{K}, \widetilde{\operatorname{dim}}\left(\phi-\phi^{\prime}\right)=\operatorname{dim}(\phi)-\operatorname{dim}\left(\phi^{\prime}\right)$ de modo que o diagrama abaixo seja comutativo

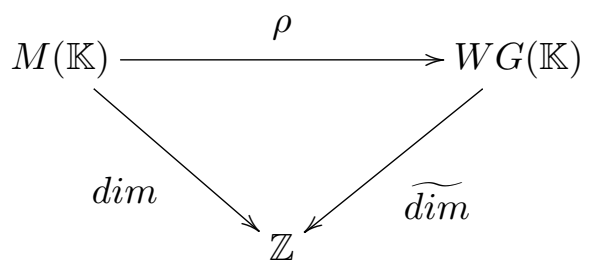

É corriqueiro denotar o morfismo $\widetilde{\text { dim }}$ simplesmente por dim. Chamamos de ideal de aumento de $W G(\mathbb{K})$ ao núcleo $\operatorname{Ker}(\operatorname{dim})$ do homomorfismo $\operatorname{dim}: W G(\mathbb{K}) \longrightarrow \mathbb{Z}$ e o denotamos simplesmente por $\hat{I}(\mathbb{K})$. Desde que $\widetilde{\operatorname{dim}}$ é um epimorfismo, segue do teorema do homomorfismo que $W G(\mathbb{K}) / \hat{I}(\mathbb{K}) \cong \mathbb{Z}$

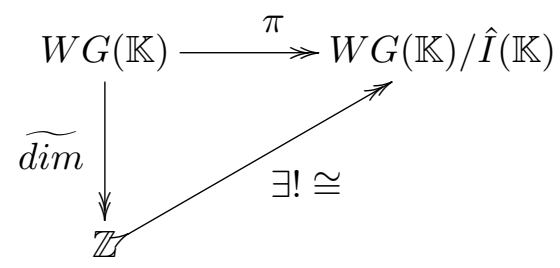

Adotaremos, de agora em diante, a prática usual de omitir a simbologia para classes de equivalência de formas quadráticas sempre que isto não possibilitar interpretação diferente da pretentida.

Proposição 24. Temos o seguinte diagrama comutativo de grupos abelianos:

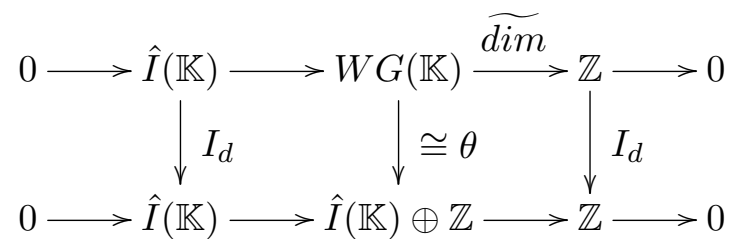

Demonstração. O homomorfismo de semi-aneis $\xi: \mathbb{N} \longrightarrow M(\mathbb{K}), n \mapsto n\langle 1\rangle$ determina um homomorfismo de anéis $\check{\xi}: \mathbb{Z} \longrightarrow W G(\mathbb{K})$ que é uma seção do homomorfismo de grupos $\widetilde{\text { dim }}$ : $W G(\mathbb{K}) \longrightarrow \mathbb{Z}$

Definição 25. Seja $\pi: W G(\mathbb{K}) \longrightarrow W(\mathbb{K})$ a projeção canônica. Chamamos de ideal fundamental de $W(\mathbb{K})$ ao ideal $I(\mathbb{K}) \doteq \pi(\hat{I}) \triangleleft W(\mathbb{K})$.

Observação 18. Observe que

$$
\begin{aligned}
\overline{\phi-\phi^{\prime}}=\overline{\psi-\psi^{\prime}} \in W(\mathbb{K}) & \Longrightarrow n \in \mathbb{N},[\phi]-\left[\phi^{\prime}\right]=[\psi]-\left[\psi^{\prime}\right]+n \mathbb{H} \\
& \Longrightarrow \widetilde{\operatorname{dim}}(\phi)-\widetilde{\operatorname{dim}}\left(\phi^{\prime}\right)=\widetilde{\operatorname{dim}}(\psi)-\operatorname{dim}\left(\psi^{\prime}\right)+2 n \\
& \Longrightarrow \widetilde{\operatorname{dim}}(\phi)-\widetilde{\operatorname{dim}}\left(\phi^{\prime}\right)=\widetilde{\operatorname{dim}}(\psi)-\widetilde{\operatorname{dim}}\left(\psi^{\prime}\right)(\bmod 2 \mathbb{Z})
\end{aligned}
$$

Ou seja, o epimorfismo $\widetilde{\text { dim }}: W G(\mathbb{K}) \longrightarrow \mathbb{Z}$ induz um epimorfismo $\operatorname{dim}_{0}: W(\mathbb{K}) \longrightarrow \mathbb{Z} / 2 \mathbb{Z}$.

\section{Lema 13.}


(i) $\hat{I}(\mathbb{K})=\left\{\phi-\phi^{\prime}: \operatorname{dim}(\phi)=\operatorname{dim}\left(\phi^{\prime}\right)\right\}$

(ii) $\hat{I}(\mathbb{K})$ é gerado aditivamente (i.e., como grupo abeliano) por $\{\langle a\rangle-\langle 1\rangle: a \in \dot{\mathbb{K}}\}$.

(iii) $I(\mathbb{K})$ é gerado por $\{\langle 1,-a\rangle: a \in \mathbb{K}\}$.

(iv) $I(\mathbb{K})=\{\bar{\varphi}: \varphi \in M(\mathbb{K}), \widetilde{\operatorname{dim}}(\varphi)$ é par $\}$

(v) $I(\mathbb{K})=\operatorname{Ker}\left(\operatorname{dim}_{0}\right)$.

(vi) $W(\mathbb{K}) / I(\mathbb{K}) \stackrel{\overline{\operatorname{dim}_{0}}}{\cong} \mathbb{Z} / 2 \mathbb{Z}$.

(vii) $\hat{I}(\mathbb{K}) \cap \mathbb{Z} \mathbb{H}=0$.

(viii) $\hat{I}(\mathbb{K}) \cong I(\mathbb{K})$.

(ix) $\hat{I}(\mathbb{K}) / \hat{I}^{2}(\mathbb{K}) \cong I(\mathbb{K}) / I^{2}(\mathbb{K})$

(xi) $\quad \hat{I}^{n}(\mathbb{K}) / \hat{I}^{n+1}(\mathbb{K}) \cong I^{n}(\mathbb{K}) / I^{n+1}(\mathbb{K})$

Demonstração.

(i) Pois $[\phi]-\left[\phi^{\prime}\right] \in \operatorname{Ker}(\widetilde{\operatorname{dim}}) \Longleftrightarrow \widetilde{\operatorname{dim}}\left([\phi]-\left[\phi^{\prime}\right]\right)=0 \Longleftrightarrow \operatorname{dim}(\phi)=\operatorname{dim}\left(\phi^{\prime}\right)$

(ii) Pois, em $W G(\mathbb{K})$, tem-se $\left\langle a_{1}, \cdots, a_{n}\right\rangle-\left\langle b_{1}, \cdots b_{n}\right\rangle=\sum_{i}^{n}\left\langle a_{i}\right\rangle-\sum_{i}^{n}\left\langle b_{i}\right\rangle=\sum_{i=1}^{n}\left(\left\langle a_{i}\right\rangle-\langle 1\rangle\right)-$ $\sum_{i=1}^{n}\left(\left\langle b_{i}\right\rangle-\langle 1\rangle\right)$

(iii) Como a projeção induzida $\tilde{\pi}: \hat{I}(\mathbb{K}) \longrightarrow I(\mathbb{K})$ é um epimorfismo de grupos, e $\overline{\langle a\rangle-\langle 1\rangle}=$ $-\overline{\langle 1,-a\rangle}$, o resultado segue do item (ii).

(iv) Utilizando que todo elemento de $W(\mathbb{K})$ é representado por alguma forma regular que $\varphi$. Então, se $\widetilde{\operatorname{dim}}(\varphi)=2 m \in 2 \mathbb{N}$, digamos $\varphi=\left\langle a_{1}, \cdots, a_{2 m}\right\rangle$, temos que, em $W(\mathbb{K})$

$$
\varphi=\left\langle a_{1}, \ldots, a_{2 m}\right\rangle=\sum_{i=0}^{m}\left\langle a_{2 i-1}, a_{2 i}\right\rangle=\left(\sum_{i=1}^{m}\left\langle a_{2 m-1}\right\rangle\right)-\left(\sum_{i=1}^{m}\left\langle-a_{2 m}\right\rangle\right) \in \pi(\hat{I}(\mathbb{K}))=I(\mathbb{K})
$$

Por outro lado, se assumimos $\bar{\varphi} \in I(\mathbb{K})$, então pelo item (i), existem $\phi, \psi \operatorname{com} \operatorname{dim}(\phi)=\operatorname{dim}(\psi) \mathrm{e}$ $\bar{\varphi}=\overline{\phi-\psi}$. Logo $\exists n \in \mathbb{Z}$ tal que $[\varphi]=[\varphi]-[\psi]+n \mathbb{H}$, portanto $\widetilde{\operatorname{dim}}(\varphi)=\operatorname{dim}(\phi)-\operatorname{dim}(\psi)+2 n=2 n$.

(v) É uma reformulação do item (iv) provado acima.

(vi) Segue do Teorema do homomorfismo e do item (v) acima.

(vii)

$$
\begin{aligned}
\varphi-\psi \in \hat{I} \cap\langle\mathbb{H}\rangle & \Longrightarrow \quad \exists n \in \mathbb{Z}(\varphi-\psi=n \mathbb{H} \in W G(\mathbb{K})) ; \operatorname{dim} \varphi=\operatorname{dim} \psi \\
& \Longrightarrow \quad 2 n=\widetilde{\operatorname{dim}}(n \mathbb{H})=\widetilde{\operatorname{dim}}(\varphi-\psi)=0 \\
& \Longrightarrow \quad \varphi-\psi=0 \in W G(\mathbb{K}) \Longrightarrow \hat{I} \cap\langle\mathbb{H}\rangle=0 .
\end{aligned}
$$

(viii) Como a projeção induzida $\tilde{\pi}: \hat{I}(\mathbb{K}) \longrightarrow I(\mathbb{K})$ é um epimorfismo (de grupos) e $\operatorname{Ker}(\tilde{\pi})=$ $\operatorname{Ker}(\pi) \cap \hat{I}(\mathbb{K})=\mathbb{Z} \mathbb{H} \cap \hat{I}(\mathbb{K})$,segue do teorema do homomorfismo que $I(\mathbb{K}) \cong \hat{I}(\mathbb{K}) / \hat{I}(\mathbb{K}) \cap \mathbb{Z} \mathbb{H}$, e pelo item $($ vii $), I(\mathbb{K}) \cong \hat{I}(\mathbb{K}) / 0 \cong \hat{I}(\mathbb{K})$.

(ix) $\mathrm{O}$ isomorfismo de grupos abelianos $\hat{I}(\mathbb{K}) \cong I(\mathbb{K})$ induz um isomorfismo $\hat{I}^{2}(\mathbb{K}) \cong I^{2}(\mathbb{K})$ e por conseguinte $\hat{I}(\mathbb{K}) / \hat{I}^{2}(\mathbb{K}) \cong I^{2}(\mathbb{K}) / I(\mathbb{K})$.

(xi) É uma consequência do isomorfismo de grupos abelianos $\hat{I}(\mathbb{K}) \cong I(\mathbb{K})$. 
Observação 19. Registramos que, para todo $a \in \dot{\mathbb{K}}$, tem-se

$$
(\langle a\rangle-\langle 1\rangle)(\langle a\rangle+\langle 1\rangle)=0 \quad \text { em } W(\mathbb{K}) .
$$

Isto segue, observando-se que

$$
\langle a\rangle^{2}=\left\langle a^{2}\right\rangle=\langle 1\rangle=1 \text { em } W(\mathbb{K})
$$

ou, alternativamente, e de forma mais direta, das isometrias

$$
\langle 1, a\rangle \otimes\langle 1,-a\rangle=\left\langle 1, a,-a,-a^{2}\right\rangle \simeq\langle 1, a,-a,-1\rangle \simeq\langle 1,-1, a,-a,\rangle \simeq 2 \mathbb{H}
$$

\section{Proposição 25.}

(i) Se $\mathfrak{J}(\mathbb{K})$ for um ideal primo próprio de $W(\mathbb{K})$, então temos únicos isomorfismos $W(\mathbb{K}) / \mathfrak{J}(\mathbb{K}) \cong$ $\mathbb{Z} / n \mathbb{Z}$, onde $n=0$ ou $n$ é primo.

(ii) $I(\mathbb{K})$ é o único ideal primo próprio em $W(\mathbb{K})$ de característica 2 (i.e. o anel quociente tem característica 2)

Demonstração.

(i) Basta mostrar que temos $\mathfrak{J}(\mathbb{K})=\mathbb{Z}\langle 1\rangle$, pois assim garantimos que o único homomorfismo $\mathbb{Z} \longrightarrow W(\mathbb{K}) / \mathfrak{J}(\mathbb{K})$ é um epimorfismo de anéis e o resultado existencial seguiria do Teorema do homomorfismo (e da classificação de $\operatorname{Spec}(\mathbb{Z})$ ), pois $W(\mathbb{K}) / \mathfrak{J}(\mathbb{K})$ é, por hipótese, um domínio de integridade. Para a unicidade dos isomorfismos, basta notar que todos os anéis quocientes de $\mathbb{Z}$ são rígidos, i.e. possuem apenas o automorfismo identidade.

Segue da observação acima que, para cada $a \in \operatorname{dot} \mathbb{K}$,

$$
\begin{gathered}
(\langle a\rangle-\langle 1\rangle)(\langle a\rangle+\langle 1\rangle)=0 \text { no dom. de integr. } W(\mathbb{K}) / \mathfrak{J}(\mathbb{K}) \\
\Longrightarrow\langle a\rangle+\mathfrak{J}(\mathbb{K}) \in\{1+\mathfrak{J}(\mathbb{K}),-1+\mathfrak{J}(\mathbb{K})\}
\end{gathered}
$$

e, como $\{\langle a\rangle: a \in \dot{\mathbb{K}}\}$ é um conjunto de geradores aditivos para $W(\mathbb{K})$ segue que $W(\mathbb{K}) / \mathfrak{J}(\mathbb{K})=$ $\mathbb{Z}\langle 1\rangle$.

(ii) Pelo item (vi) do Lema 13 acima, $W(\mathbb{K}) / I(K) \cong \mathbb{Z} / 2 \mathbb{Z}$ logo temos que $I(\mathbb{K})$ é um ideal primo próprio de característica 2 . Note que, como $\mathbb{Z} / 2 \mathbb{Z}$ é um corpo, $I(\mathbb{K})$ é um ideal maximal. Se $\mathfrak{J}(\mathbb{K})$ for um ideal primo próprio de $W(\mathbb{K})$ de característica 2 , então pelo item (i) acima temos necessariamente um (único) isomorfismo de anéis $\bar{u}: \mathbb{Z} / 2 \mathbb{Z} \stackrel{\cong}{\longrightarrow} W(\mathbb{K}) / \mathfrak{J}(\mathbb{K})$ obtido, por passagens ao quociente, do único homomorfismo $u: \mathbb{Z} \longrightarrow W(\mathbb{K})$. Então, como $u(2)=\overline{\langle 1,1\rangle}$, temos $0+\mathfrak{J}(\mathbb{K})=$ $\bar{u}(0+2 \mathbb{Z})=\bar{u}(2+2 Z)=\overline{\langle 1,1\rangle}+\mathfrak{J}(\mathbb{K}), \operatorname{logo} \overline{\langle 1,1\rangle} \in \mathfrak{J}(\mathbb{K}) . A \operatorname{ssim}, \overline{\langle a\rangle} \equiv 1(\bmod \mathfrak{J}(\mathbb{K})), a \in \dot{\mathbb{K}}$. Desta forma, se $\phi$ é uma forma que representa algum elemento de $I(K)$, então $\operatorname{dim}(\phi)=2 n$ para algum $n \in \mathbb{N}$ (Lema 13 (iv) $)$ e então $\bar{\phi} \equiv 2 n \overline{\langle 1\rangle} \equiv n \overline{\langle 1,1\rangle} \equiv 0(\bmod \mathfrak{J}(\mathbb{K}))$, provando que $I(\mathbb{K}) \subseteq \mathfrak{J}(\mathbb{K})$. Como $I(\mathbb{K})$ é ideal maximal e $\mathfrak{J}(\mathbb{K})$ é ideal próprio, temos $I(\mathbb{K})=\mathfrak{J}(\mathbb{K})$.

Proposição 26. Temos um diagrama comutativo e exato de grupos abelianos. 


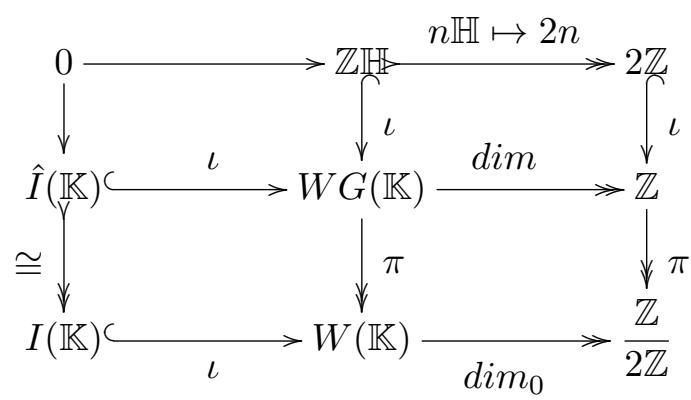

Demonstração. Consequência do Lema 13.

Observação 20. Até aqui temos isomorfismos

(1) $W G(\mathbb{K}) / \hat{I}(\mathbb{K}) \cong \mathbb{Z} ;$

(2) $W(\mathbb{K}) / I(\mathbb{K}) \cong \mathbb{Z} / 2 \mathbb{Z}$;

(3) $\hat{I}(\mathbb{K}) \cong I(\mathbb{K})$;

(4) $\hat{I} / \hat{I}^{2}(\mathbb{K}) \cong I / I^{2}(\mathbb{K})$.

Estes isomorfismos podem ser melhor visualizados através do próximo diagrama

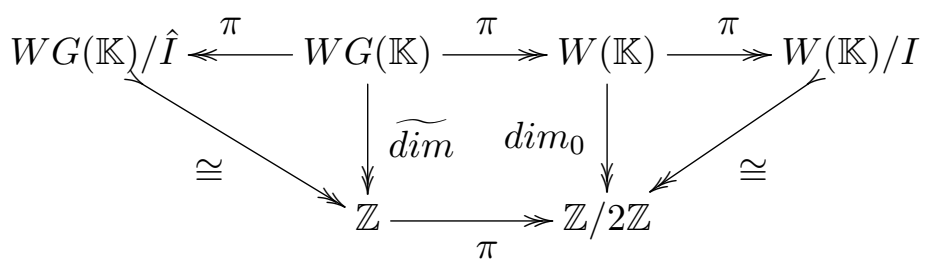

Onde $\pi$ é usado para denotar as projeções canônicas.

\subsection{Extensão de escalares: Funtor Anel de Witt - Grothendieck e Funtor Anel de Witt}

Nesta seção examiraremos o caráter funtorial das construções anteriores, i.e. como elas são coerentes com o processo de extensão de escalares. Denotaremos Field $\frac{1}{2}$ a categoria dos corpos de característica diferente de 2.

Fato 5. Restrição e extensão de escalares na Álgebra Linear:

1) Para cada morfismo de corpos $j: \mathbb{K} \longrightarrow \mathbb{K}^{\prime}$ temos um processo de "restrição de escalares"que a cada $\mathbb{K}^{\prime}$-espaço vetorial $V^{\prime}$ associa o $\mathbb{K}$-espaço vetorial $V_{\mathbb{K}}^{\prime}$ que mantém o mesmo grupo abeliano de vetores mas cuja ação dos escalares de $\mathbb{K}$ é dada por a.v $:=j(a) v, a \in \mathbb{K}, v \in V$. Claramente esta definição transforma uma aplicação $\mathbb{K}^{\prime}$ - linear $T: V^{\prime} \longrightarrow W^{\prime}$ em uma aplicação $\mathbb{K}$-linear $T: V_{\mathbb{K}}^{\prime} \longrightarrow W_{\mathbb{K}}^{\prime}$, associação que preserva composição e identidades, constituindo assim um funtor $j_{*}: V e c_{\mathbb{K}^{\prime}} \longrightarrow V e c_{\mathbb{K}}$. 
2) Para cada morfismo de corpos $j: \mathbb{K} \longrightarrow \mathbb{K}^{\prime}$ temos um processo de "extensão de escalares": note inicialmente que o morfismo de corpo $j$ induz uma estrutura de $\mathbb{K}$-espaço vetorial no grupo aditivo $\mathbb{K}^{\prime}$ por a. $x^{\prime}:=j(a) x^{\prime}, a \in \mathbb{K}, x^{\prime} \in \mathbb{K}^{\prime} . A$ extensão de escalares associa a cada $\mathbb{K}$-espaço vetorial $V$ o $\mathbb{K}^{\prime}$-espaço vetorial $V^{\mathbb{K}^{\prime}}:=\mathbb{K}^{\prime} \otimes_{K} V$ tal que $x^{\prime}\left(\sum_{i=1}^{n} x_{i}^{\prime} \otimes v_{i}\right)=\sum_{i=1}^{n} x^{\prime} x_{i}^{\prime} \otimes v_{i}, \forall x^{\prime} \in \mathbb{K}^{\prime}$.

3) O processo de extensão de escalares associado ao morfismo de corpos $j: \mathbb{K} \longrightarrow \mathbb{K}^{\prime}$ induz um functor $j^{*}: V e c_{\mathbb{K}} \longrightarrow V e c_{\mathbb{K}^{\prime}}$ que é adjunto à esquerda do functor $j_{*}$. Para isto, basta notar que, para cada $\mathbb{K}$-espaço $V$ o mapa canônico $\lambda_{V}: V \longrightarrow\left(V^{\mathbb{K}^{\prime}}\right)_{\mathbb{K}}$, dado por $v \mapsto 1^{\prime} \otimes v$, é $\mathbb{K}$-linear e satisfaz a seguinte propriedade universal: para $\mathbb{K}^{\prime}$-espaço $W^{\prime}$ e cada mapa $\mathbb{K}$-linear $L: V \longrightarrow W_{\mathbb{K}}^{\prime}$ existe um único mapa $\mathbb{K}^{\prime}$-linear $\check{T}: V^{\mathbb{K}^{\prime}} \longrightarrow W^{\prime}$ tal que $\check{L} \circ \lambda_{V}=T$.

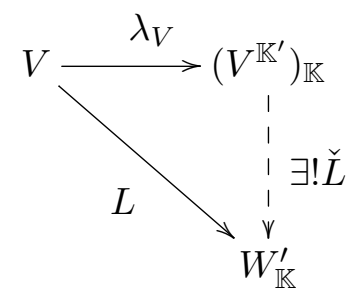

A transformação $\mathbb{K}^{\prime}$-linear associada a uma transformação $\mathbb{K}$-linear $T: V \longrightarrow W$ é $T^{\mathbb{K}^{\prime}}$ : $V^{\mathbb{K}^{\prime}} \longrightarrow W^{K^{\prime}}$, unicamente determinada por $T^{\mathbb{K}^{\prime}}\left(1^{\prime} \otimes v\right)=1^{\prime} \otimes T(v)$.

4) $S e \epsilon=\left(v_{i}\right)_{i \in I}$ é base ordenada do $\mathbb{K}$-espaço $V$, então $\epsilon^{\prime}:=\left(1^{\prime} \otimes v_{i}\right)_{i \in I}$ é base ordenada do $\mathbb{K}^{\prime}$-espaco $V^{\mathbb{K}^{\prime}}$. Em particular $\operatorname{dim}_{\mathbb{K}^{\prime}}\left(V^{\mathbb{K}^{\prime}}\right)=\operatorname{dim}_{\mathbb{K}}(V)$.

5) O processo de extensão de escalares comuta, a menos de isomorfismo, com as construçóes de soma direta e produto tensorial de espaços vetoriais:

a) $\oplus_{i \in I}^{\prime} V_{i}^{\mathbb{K}^{\prime}}=\oplus_{i \in I}^{\prime}\left(\mathbb{K}^{\prime} \otimes_{\mathbb{K}} V_{i}\right) \cong \mathbb{K}^{\prime} \otimes_{\mathbb{K}}\left(\oplus_{i \in I} V_{i}\right) \cong\left(\oplus_{i \in I} V_{i}\right)^{\mathbb{K}^{\prime}}$.

b) $\otimes_{i \in I}^{\prime} V_{i}^{\mathbb{K}^{\prime}}=\otimes_{i \in I}^{\prime}\left(\mathbb{K}^{\prime} \otimes_{\mathbb{K}} V_{i}\right) \cong \mathbb{K}^{\prime} \otimes_{\mathbb{K}}\left(\otimes_{i \in I} V_{i}\right) \cong\left(\otimes_{i \in I} V_{i}\right)^{\mathbb{K}^{\prime}}$.

6) Relativamente a morfismos de corpos, valem as relações funtoriais entre categorias de espaços vetoriais:

a) Para o morfismo id $d_{\mathbb{K}}: \mathbb{K} \longrightarrow \mathbb{K}$, temos $\left(i d_{\mathbb{K}}\right)_{*}=i d_{V e c_{\mathbb{K}}}: V e c_{\mathbb{K}} \longrightarrow V e c_{\mathbb{K}} e\left(i d_{\mathbb{K}}\right)^{*} \cong i d_{V e c_{\mathbb{K}}}$.

b) Para morfismos $\mathbb{K} \stackrel{j}{\longrightarrow} \mathbb{K}^{\prime} \stackrel{j^{\prime}}{\longrightarrow} \mathbb{K}^{\prime \prime}$, tem-se $\left(j^{\prime} \circ j\right)_{*}=j_{*} \circ j_{*}^{\prime} e\left(j^{\prime} \circ j\right)^{*} \cong j^{*} \circ j^{*}$.

Fato 6. Extensão de escalares para categorias quadráticas $\unlhd^{3} \operatorname{Seja} j: \mathbb{K} \longrightarrow \mathbb{K}^{\prime}$ um homomorfismo entre corpos de características $\neq 2$.

1) Seja $(V, B)$ um EBS sobre o corpo $\mathbb{K}$. Uilizando a propriedade universal dos produtos tensoriais, garantimos a existência de uma única forma $\mathbb{K}^{\prime}$-bilinear (e simétrica) $B^{\prime}: V^{\mathbb{K}^{\prime}} \times V^{\mathbb{K}^{\prime}} \longrightarrow \mathbb{K}^{\prime}$ tal que $B^{\prime}\left(x^{\prime} \otimes u, y^{\prime} \otimes v\right)=x^{\prime} y^{\prime} \cdot j(B(u, v))$. De fato, tomando $L_{B}: V \otimes_{K} V \longrightarrow \mathbb{K}$ o mapa $\mathbb{K}$-linear associado ao mapa $\mathbb{K}$-bilinear $B: V \times V \longrightarrow \mathbb{K}$, associamos o mapa $\mathbb{K}^{\prime}$-bilinear associado à aplicação $\mathbb{K}^{\prime}$-linear dada pela composição do mapa $\mathbb{K}^{\prime}$-linear extensão ao corpo $\tilde{L}_{B}: \mathbb{K}^{\prime} \otimes_{\mathbb{K}}\left(V \otimes_{K} V\right) \longrightarrow \mathbb{K}^{\prime} \otimes_{\mathbb{K}} \mathbb{K}$ com os $\mathbb{K}^{\prime}$-isomorfismo linear canônicos $\mathbb{K}^{\prime} \otimes_{\mathbb{K}} \mathbb{K} \cong \mathbb{K}^{\prime} e\left(V^{\mathbb{K}^{\prime}} \otimes_{\mathbb{K}^{\prime}} V^{\mathbb{K}^{\prime}}\right) \cong \mathbb{K}^{\prime} \otimes_{\mathbb{K}}\left(V \otimes_{K} V\right)$.

Se $T:\left(V_{1}, B_{1}\right) \longrightarrow\left(V_{2}, B_{2}\right)$ é um morfismo de EBSs sobre $\mathbb{K}$, então

$$
\begin{gathered}
B_{2}^{\prime}\left(T^{\mathbb{K}^{\prime}}\left(x^{\prime} \otimes v_{1}\right), T^{\mathbb{K}^{\prime}}\left(y^{\prime} \otimes u_{1}\right)\right)=B_{2}^{\prime}\left(x^{\prime} \otimes T\left(v_{1}\right), y^{\prime} \otimes T\left(u_{1}\right)\right)= \\
x^{\prime} y^{\prime} j\left(B_{2}\left(T\left(v_{1}\right), T\left(u_{1}\right)\right)\right)=x^{\prime} y^{\prime} j\left(B_{1}\left(v_{1}, u_{1}\right)\right)=B_{1}^{\prime}\left(x^{\prime} \otimes v_{1}, y^{\prime} \otimes u_{1}\right)
\end{gathered}
$$

logo $T^{\mathbb{K}^{\prime}}:\left(V_{1}^{\mathbb{K}^{\prime}}, B_{1}^{\prime}\right) \longrightarrow\left(V_{2}^{\mathbb{K}^{\prime}}, B_{2}^{\prime}\right)$ é um morfismo de EBSs sobre $\mathbb{K}^{\prime}$. Desta forma obtemos um funtor $j^{*}: E B S_{\mathbb{K}} \longrightarrow E B S_{\mathbb{K}^{\prime}}$.

\footnotetext{
${ }^{3}$ De fato, nos ocuparemos apenas das categorais formadas por EBS, os outros casos podem serobtidos deste pelas equivalêncais categoriais estabelecidas no Capítulo 1.
} 
2) Seja $(V, B)$ um EBS sobre o corpo $\mathbb{K}$ e $\epsilon$ uma $\mathbb{K}$-base ordenada de $V$. Segue diretamente das definições acima que $\left[B^{\prime}\right]_{\epsilon^{\prime}}=j\left([B]_{\epsilon}\right)$. Disto, obtemos que:

a) $S e(V, B)$ é $E B S_{\mathbb{K}}$-regular, então $\left(V^{\mathbb{K}^{\prime}}, B^{\prime}\right)$ é $E B S_{\mathbb{K}^{\prime}}$-regular.

b) $S e(V, B) \simeq_{\mathbb{K}}\left(a_{1}, \cdots a_{n}\right)$, então $\left(V^{\mathbb{K}^{\prime}}, B^{\prime}\right) \simeq_{\mathbb{K}^{\prime}}\left(j\left(a_{1}\right), \cdots, j\left(a_{n}\right)\right)$.

c) $S e(V, B)$ é um $E B S_{\mathbb{K}}$-hiperbólico, então $\left(V^{\mathbb{K}^{\prime}}, B^{\prime}\right)$ é um $E B S_{\mathbb{K}}$-hiperbólico.

3) O processo de extensão de escalares comuta, a menos de isomorfismo, com as construções de soma direta ortogonal e produto tensorial de EBSs.

4) Relativamente a morfismos de corpos, valem as relações funtoriais entre categorias de EBSs:

a) Para o morfismo $i d_{\mathbb{K}}: \mathbb{K} \longrightarrow \mathbb{K}$, temos $\left(i d_{\mathbb{K}}\right)^{*} \cong i d_{E B S_{\mathbb{K}}}: E B S_{\mathbb{K}} \longrightarrow E B S_{\mathbb{K}}$.

b) Para morfismos $\mathbb{K} \stackrel{j}{\longrightarrow} \mathbb{K}^{\prime} \stackrel{j^{\prime}}{\longrightarrow} \mathbb{K}^{\prime \prime}$, tem-se $\left(j^{\prime} \circ j\right)^{*} \cong j^{* *} \circ j^{*}$.

1. Do que foi exposto acima, é fácil ver que para cada extensão de corpos $f: \mathbb{F} \longrightarrow \mathbb{K}$ a aplicação $M(f): M(\mathbb{F}) \longrightarrow M(\mathbb{K})$ dada por $[(V, B)] \mapsto\left[f^{*}(V, B)\right]=\left[\left(V^{\mathbb{K}}, B^{\mathbb{K}}\right)\right]$ esta bem definida e é um homomorfismo de semi-anéis. Além disso, valem as seguintes propriedades:

1) Se gf é uma composição em Field ${ }_{1 / 2}$, então $M(g f)=M(g) M(f)$;

2) $M\left(i d_{\mathbb{F}}\right)=i d_{M(\mathbb{F})}$.

Em outras palavras, $M:$ Field $_{1 / 2} \longrightarrow \mathcal{S} \mathcal{S}$ ing é um funtor covariante da categoria de corpos com característica diferente dois para a categoria dos semi-anéis comutativos, com unidade (e com cancelamento).

2. Seja $f: \mathbb{F} \longrightarrow \mathbb{K}$ uma extensão de corpos. A universalidade de $\rho_{\mathbb{F}}: M(\mathbb{F}) \longrightarrow W G(\mathbb{F})$ fornece um único diagrama comutativo

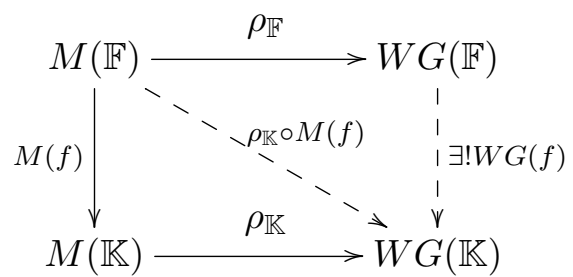

Além disso, se $\mathbb{K} \stackrel{f}{\longrightarrow} \mathbb{F} \stackrel{g}{\longrightarrow} \mathbb{L}$ são extensões de corpos em Field ${ }_{1 / 2}$, então o seguinte diagrama é comutativo.

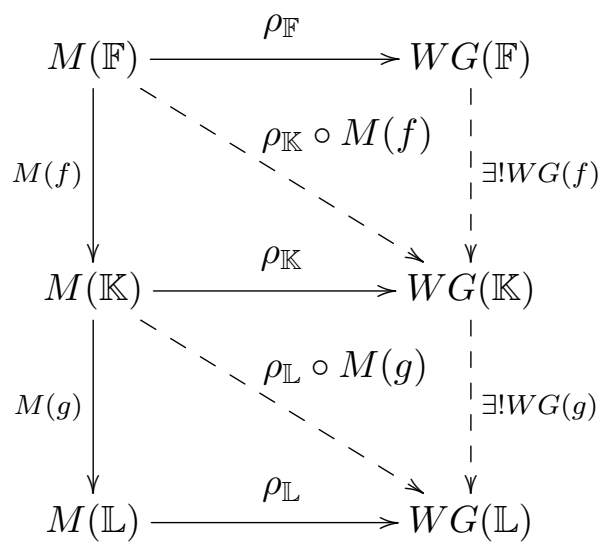


Temos assim:

1) $W G(g f)=W G(g) W G(f)$;

2) $W G\left(i d_{\mathbb{F}}\right)=i d_{W G(\mathbb{F})}$.

Assim, $W G:$ Field $_{1 / 2} \longrightarrow \mathcal{R}$ ing é um funtor covariante.

3. Seja $f: \mathbb{F} \longrightarrow \mathbb{K}$ uma extensão de corpos. Conforme registramos no item (2).(c) do Fato 6. se $(V, B)$ é um $E B S_{\mathbb{F}}$-hiperbólico, então $f^{*}(V, B)$ é um $E B S_{\mathbb{K}}$-hiperbólico. Disto segue que o homomorfismo de anéis induzido por $f, W G(f): W G(\mathbb{F}) \longrightarrow W G(\mathbb{K})$, mapeia o ideal $\mathbb{Z} \mathbb{H} \subseteq$ $W G(\mathbb{F})$ dentro do ideal $\mathbb{Z} \mathbb{H} \subseteq W G(\mathbb{K})$. Assim, o teorema do homomorfismo, fornece uma única fatoração

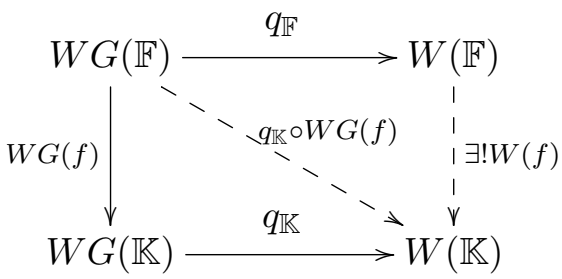

Consequentemente, $W:$ Field $_{1 / 2} \longrightarrow$ Ring é um funtor covariante, i.e.

1) $W(g f)=W(g) W(f)$;

2) $W\left(i d_{\mathbb{F}}\right)=i d_{W(\mathbb{F})}$.

Em suma, temos a seguinte

Proposição 27. As seguintes correspondências

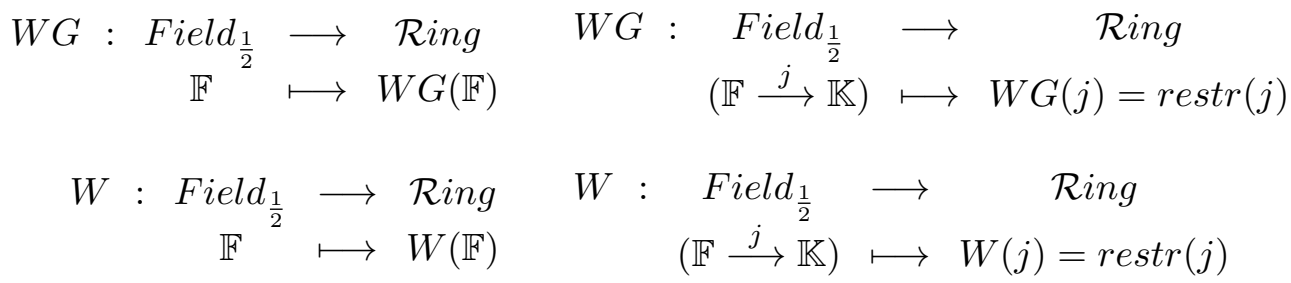

são funtores covariantes da categoria dos corpos de característica $\neq 2$ para a categoria dos anéis comutativos com unidade.

\subsection{Particularizações do anel de Witt}

Esta seção é devotada para o cálculo do anel de Witt e do anel de Witt- Grothendieck de alguns corpos específicos, a saber, corpos quadraticamente fechados, corpos euclidianos, corpos pitagóricos e corpos finitos.

\subsubsection{Corpos quadraticamente fechados}

Definição 26. Dizemos que um corpo $\mathbb{K}$ é quadraticamente fechado quando $\mathbb{K}=\mathbb{K}^{2}$, ou seja, $\forall x \in \mathbb{K}, \exists a \in \mathbb{K}, x=a^{2}$.

\section{Fato 7.}


(1) Todo corpo algebricamente fechado é quadraticamente fechado.

(2) Todo corpo possui um "fecho quadrático", i.e., uma extensão algébrica e quadraticamente fechada.

(3) Fechos quadráticos são únicos a menos de isomorfismo fixando pontualmente o corpo base.

Proposição 28. As seguintes afirmações se equivalem

(i) $\mathbb{K}$ é quadraticamente fechado.

(ii) $W G(\mathbb{K}) \cong \widetilde{\operatorname{dim}} \mathbb{Z}$

(iii) $W(\mathbb{K}) \cong \operatorname{dim}_{0} \mathbb{Z} / 2 \mathbb{Z}$

Demonstração.

$(i) \Longrightarrow($ ii $)$ Segue das isometrias entre formas regulares $\oplus_{i=1}^{n}\left\langle a_{i}\right\rangle \simeq \oplus_{i=1}^{n}\left\langle b_{i}^{2}\right\rangle \simeq n\langle 1\rangle, n \in \mathbb{N}$, que o homomorfismo canônico $u: \mathbb{Z} \longrightarrow W G(\mathbb{K})$ é sobrejetor. Como $\widetilde{\operatorname{dim}}: W G(\mathbb{K}) \longrightarrow \mathbb{Z}$ é uma retração, i.e, $\widetilde{\operatorname{dim}} \circ u=i d$, segue que $u$ e $\widetilde{\operatorname{dim}}$ são isomorfismos inversos.

$($ ii $) \Longrightarrow($ iii $)$ Seja $\phi=\left\langle a_{1}, \cdots, a_{n},-b_{1}, \cdots,-b_{n}\right\rangle$ uma forma que representa um elemento de $I(\mathbb{K})=$ $\operatorname{Ker}\left(\operatorname{dim}_{0}\right)$. Como $\widetilde{\operatorname{dim}}\left(\left[\left\langle a_{1}, \cdots, a_{n}\right\rangle\right]-\left[\left\langle b_{1}, \cdots, b_{n}\right\rangle\right]\right)=0$, a hipótese garante que $\left\langle a_{1}, \cdots, a_{n}\right\rangle \simeq$ $\left\langle b_{1}, \cdots, b_{n}\right\rangle$, logo $\phi$ é forma hiperbólica. Portanto o homomorfismo sobrejetor $\operatorname{dim}_{0}: W(K) \longrightarrow$ $\mathbb{Z} / 2 \mathbb{Z}$ tem núcleo trivial, sendo assim um isomorfismo.

$($ iii $) \Longrightarrow(i)$ Da hipótese obtemos a isometria entre formas anisotrópicas de dimensão 1: $\langle a\rangle \simeq$ $\langle 1\rangle, \forall a \in \dot{\mathbb{K}}$. Assim, para cada $a \neq 0$ existe $x$ tal que $a=x^{2}$.

Exemplo 1. Como o corpo dos números complexos $\mathbb{C}$ é algebricamente fechado, então $W G(\mathbb{C})$ é abeliano cíclico infinito e $W(\mathbb{C})$ é abeliano cícico de ordem 2.

\subsubsection{Corpos ordenados e corpos pitagóricos}

Motivados pelo $17^{\circ}$ problema de Hilbert, a dupla Artin-Schreier desenvolveu, na década de 1920, a interessante teoria dos corpos ordenáveis, através da qual se estabelece uma primeira relação entre os conceitos de formas quadráticas e ordens em corpos.

\section{Definição 27.}

(i) Um cone positivo em um corpo $\mathbb{K}$ é um subconjunto $P \subseteq \mathbb{K}$ tal que

$\left.P_{1}\right) \quad P+P \subseteq P$;

$\left.P_{2}\right)$ P.P $\subseteq P$;

$\left.P_{3}\right) \quad P \neq \mathbb{K}$

$\left.P_{4}\right) \quad P \cup-P=\mathbb{K}$.

(ii) Um corpo ordenado é um par $(\mathbb{K}, P)$, onde $\mathbb{K}$ é um corpo e $P$ é um cone positivo de $\mathbb{K}$.

(iii) Um morfismo de corpo ordenados $j:(\mathbb{K}, P) \longrightarrow\left(\mathbb{K}^{\prime}, P^{\prime}\right)$ é um morfismo de corpos $j: \mathbb{K} \longrightarrow \mathbb{K}^{\prime}$ tal que $j^{-1}\left[P^{\prime}\right]=P$.

(iv) Um corpo é dito formalmente real quando -1 não é somatório de quadrados.

(v) Um corpo $\mathbb{K}$ é dito pitagórico quando $\sum \mathbb{K}^{2}=\mathbb{K}^{2}$.

\section{Fato 8 .}

(1) Os elementos de um cone positivo $P$ são chamados de elementos positivos do corpo, enquanto os elementos de $-P$ são ditos negativos. 
(2) Para corpos de característica $\neq 2$ : na presença de $\left(P_{1}\right)$ e $\left(P_{2}\right)$, a condição $\left(P_{3}\right)$ pode ser substituída por $\left(P_{3}\right)^{\prime}:-1 \notin P$ ou por $\left(P_{3}\right)^{\prime \prime}: P \cap-P=\{0\}$.

(3) Cones positivos em um corpo estão em correspondência bijetiva com ordens lineares no corpo que são compatíveis com soma e produto.

(4) Corpos ordenáveis têm característica zero.

(5) O conjunto dos elementos totalmente positivos em um corpo, i.e., aqueles que são positivos em todas as ordens do corpo, coindide com o conjunto das somatórias de quadrados.

(6) Um corpo admite alguma ordem (linear, compatível com as operações) se e só se for formalmente real.

(7) Um corpo $\mathbb{K}$ é formalmente real se e só se $n\langle 1\rangle$ é forma anisotrópica, para todo $n \in \mathbb{N}$.

(8) Um corpo $\mathbb{K}$ é pitagórico $\Leftrightarrow 1+\mathbb{K}^{2} \subseteq \mathbb{K}^{2} \Leftrightarrow D(\langle 1,1\rangle)=\dot{\mathbb{K}}^{2}$.

(9) Todo corpo possui um "fecho pitagórico", i.e., uma extensão algébrica e pitagórica. Tais fechos são "essencialmente únicos", i.e. são únicos a menos de isomorfismo fixando pontualmente o corpo base.

(10) O fecho pitagórico de um corpo $L$ coincide com o fecho quadrático de $L$ se e só se $L$ não for formalmente real.

Demonstração. Esboçaremos apenas uma construção envolvida na parte existencial no item (9). Dado um corpo $\mathbb{K}$, fixamos $\mathbb{K}^{\text {alg }}$ um fecho algébrico deste e tomamos $\Gamma$ o conjunto de todas as sub-extensões intermediárias $\mathbb{K} \subseteq \mathbb{L} \subseteq \mathbb{K}^{\text {alg }}$ tais que: $[\mathbb{L}: \mathbb{K}]$ é finita e existe cadeia (finita) de subcorpos $\mathbb{K}=\mathbb{M}_{0} \subseteq \mathbb{M}_{1} \subseteq \cdots \subseteq \mathbb{M}_{r}=\mathbb{M}$, onde $\mathbb{M}_{i+1}=\mathbb{M}_{i}\left(\sqrt{1+a_{i}^{2}}\right)$, para algum $a_{i} \in \mathbb{M}_{i}$. Claramente $\Gamma$ é um conjunto dirigido de subcorpos de $\mathbb{K}^{\text {alg }}$ contendo $\mathbb{K}$ e $\cup \Gamma$ é o fecho pitagórico de $\mathbb{K}$ contido em $\mathbb{K}^{\text {alg }}$.

\section{Exemplo 2.}

(i) Todo corpo quadraticamente fechado é pitagórico. Em particular $\mathbb{C}$ é pitagórico.

(ii) $\mathbb{R}$ é um corpo ordenável, com única ordem, e é pitagórico.

(iii) $\mathbb{Q}$ é um corpo ordenável, com única ordem, mas não é pitagórico: como consequência do teorema de Lagrange, os elementos (totalmente) positivos de $\mathbb{Q}$ são somatórios de 4 quadrados.

Proposição 29. Seja $\mathbb{K}$ de característica $\neq 2$.

(1) São equivalentes:

(1a) $\mathbb{K}$ é um corpo pitagórico e $-1 \in \sum \mathbb{K}^{2}$;

(1b) $D(\langle 1,1\rangle)=\dot{\mathbb{K}}^{2}$ e $\langle 1,1\rangle$ é forma universal;

(1c) $\mathbb{K}$ é corpo quadraticamente fechado;

(1d) $W(\mathbb{K}) \cong \mathbb{Z} / 2 \mathbb{Z}$.

(2a) $\mathbb{K}$ é um corpo pitagórico e $-1 \notin \sum \mathbb{K}^{2}$;

(2b) $W(\mathbb{K})$ é grupo abeliano livre de torsão.

Demonstração. Já registramos que: $\mathbb{K}$ é um corpo pitagórico $\Leftrightarrow D(\langle 1,1\rangle)=\dot{\mathbb{K}}^{2}$. 
(1) A equivalência (1c) $\Leftrightarrow(1 d)$ foi estabelecida na Proposição 28. Provamos a equivalência (1a) $\Leftrightarrow$ (1b) desde que estabeleçamos que, para corpos pitagóricos, $-1 \in \mathbb{K}^{2} \Leftrightarrow\langle 1,1\rangle$ é forma universal. Se $-1=a^{2}$ para algum $a \in \dot{\mathbb{K}}$ então $\langle-1\rangle \simeq\langle 1\rangle$ e portanto $\langle 1,1\rangle \simeq\langle 1,-1\rangle$ que é uma forma universal. Por outro lado, se $\langle 1,1\rangle$ for forma universal e $\sum \mathbb{K}^{2}=\mathbb{K}^{2}$, então existem $x, y, z \in \mathbb{K}$ tais que $-1=x^{2}+y^{2}=z^{2}$.

Suponhamos que $\mathbb{K}$ é quadraticamente fechado, então $\mathbb{K}=\mathbb{K}^{2}$, logo $\sum \mathbb{K}^{2} \subseteq \mathbb{K} \subseteq \mathbb{K}^{2}$, i.e. $\mathbb{K}$ é pitagórico, e $-1 \in \mathbb{K} \subseteq \mathbb{K}^{2}$. : desta forma estabecemos (1c) $\Rightarrow$ (1a). Por outro lado, se $\langle 1,1\rangle$ é forma universal e $D(\langle 1,1\rangle)=\dot{K}^{2}$, então para cada $a \in \dot{K}$ existem $x, y, z \in \mathbb{K}$ tais que $a=x^{2}+y^{2}=z^{2}$, $\operatorname{logo} \mathbb{K}=\mathbb{K}^{2}$, estabelecendo $(1 \mathrm{~b}) \Rightarrow(1 \mathrm{c})$.

(2) Se $-1 \notin \sum \mathbb{K}^{2}=\mathbb{K}^{2}$, então para cada forma anisotrópica $\phi=\left\langle a_{1}, \cdots, a_{n}\right\rangle$ e cada $k>0$, se $k \phi$ anular a $n k$-upla $\left(x_{11}, \cdots, x_{1 k}, \cdots, x_{n 1}, \cdots, x_{n k}\right)$, então como para cada $i \leq n$ temos $\sum_{j=1}^{k} x_{i j}^{2}=y_{i}^{2}$ para algum $y_{i} \in \mathbb{K}$, segue que a $n$-upla $\left(y_{1}, \cdots, y_{n}\right)$ é anulada por $\left\langle a_{1}, \cdots, a_{n}\right\rangle$, como $\phi$ é assumida ser anistrópica obtemos $y_{1}=\cdots y_{n}=0$ e com $\mathbb{K}$ é formalmente real, $x_{i 1}=\cdots=x_{i k}=0$ para cada $i \leq n$, ou seja $k \phi$ é anistrópica. Desta forma provamos que $(2 \mathrm{a}) \Rightarrow(2 \mathrm{~b})$.

Seja $c \in D(\langle 1,1\rangle)$, então $\langle c, c\rangle \simeq\langle 1,1\rangle$ e $2\langle 1,-c\rangle \simeq\langle 1,1,-c,-c\rangle \simeq\langle c, c,-c,-c\rangle$ é hiperbólica. Da hipótese $(2 \mathrm{~b})$ concluimos que $\langle 1,-c\rangle$ é isotrópica, e pela lei de cancelamento de Witt $\langle-c\rangle \simeq$ $\langle-1\rangle$, concluindo que $c \in \mathbb{K}^{2}$ e portanto $\mathbb{K}$ é corpo pitagórico. Como $W(\mathbb{K}) ¥ \mathbb{Z} / 2 \mathbb{Z}$, temos pelo item (1) que $-1 \notin \sum \mathbb{K}^{2}=\mathbb{K}^{2}$.

\subsubsection{Corpos euclidianos e corpos reais fechados}

\section{Definição 28.}

(i) Um corpo $\mathbb{E}$ é dito euclidiano quando $\dot{\mathbb{E}} / \dot{\mathbb{E}}^{2}=\{ \pm 1\} \cong \mathbb{Z} / 2 \mathbb{Z}$.

(ii) Um corpo $\mathcal{R}$ é dito (formalmente) real fechado quando for formalmente real e não possuir extensão algébrica própria que é formalmente real.

\section{Fato 9.}

(1) Um corpo $\mathbb{E}$ é euclidiano se e só se for pitagórico e tiver uma única ordenação (cujo cone positivo é $\left.\sum \mathbb{E}^{2}=\mathbb{E}^{2}\right)$.

(2) Um corpo $\mathcal{R}$ é real fechado se e só se for euclidiano e todo polinômio $f(x) \in \mathcal{R}[x]$ de grau impar tem alguma raiz em $\mathcal{R}$.

(3) Seja a $L$ um corpo tal que $-1 \notin L^{2}$. São equivalentes:

(a) L é corpo euclidiano (respectivamente, real fechado);

(b) $L(\sqrt{-1})$ é corpo quadraticamente fechado (respectivamente, algebricamente fechado).

(4) Todo corpo ordenado $(\mathbb{K}, P)$ possui um "fecho euclidiano"(respectivamente, "fecho real"). Fechos euclidianos (respectivamente, fechos reais) são essencialmente únicos.

Demonstração. Esboçaremos apenas parte do item (4), forneçendo uma construção (por baixo) de um fecho euclidiano de um corpo ordenado $(\mathbb{K}, P$ ). Fixamos $A$ um fecho algébrico de $\mathbb{K}$ (poderia ser também tomado um fecho quadrático). Consideramos as afirmação auxiliar, considerando $(F, Q)$ um corpo ordenado, onde $F \subseteq A$ :

(A) Para cada $n, r \in \mathbb{N}$, cada $b_{1}, \cdots, b_{n} \in F$, cada $a_{1}, \cdots, a_{r} \in Q-\{0\}$, a forma $\left\langle b_{1}, \cdots, b_{n}\right\rangle$ é anisotrópica sobre $F\left(\sqrt{a_{1}}, \cdots, \sqrt{a_{r}}\right)$.

Segue de (A), utilizando que $n\langle 1\rangle$ é anisotrópica sobre $F^{\prime}:=F(\{\sqrt{a}: a \in Q\})$ é corpo formalmente real. Da definição de $F^{\prime}$ obtemos:

(B) Toda ordem no corpo $F^{\prime}=F(\{\sqrt{a}: a \in Q\})$ (e existe alguma, pois este é formalmente real) 
estende a ordem associada ao cone positivo $Q$ de $F$. Com base em (B), fazendo escolhas de extensões de corpos ordenados ${ }^{4}(F, Q) \rightsquigarrow\left(F^{\prime}, Q^{\prime}\right)$, construimos recursivamente uma cadeia enumerável de corpos ordenados $\left(F_{0}, Q_{0}\right) \subseteq\left(F_{1}, Q_{1}\right) \subseteq \cdots$, onde $\left(F_{0}, Q_{0}\right)=(\mathbb{K}, P),\left(F_{i+1}, Q_{i+1}=\left(F_{i}^{\prime}, Q_{i}^{\prime}\right)\right.$. Desta forma temos que:

- $E:=\bigcup_{n \in \mathbb{N}} F_{n}$ é corpo tal que $\mathbb{K} \subseteq E \subseteq A$;

- $E^{2}=\bigcup_{n \in \mathbb{N}} Q_{n}$;

- E é corpo euclidiano.

Proposição 30. Seja $\mathbb{K}$ um corpo. As seguintes afirmações são equivalentes

(i) $\mathbb{K}$ é euclidiano.

(ii) $n\langle 1\rangle$ e $n\langle-1\rangle$ são as únicas classes de anisotropia, $\forall n \in \mathbb{N}$.

(iii) $W(\mathbb{K}) \cong \mathbb{Z}$.

(iv) $W G(\mathbb{K}) \cong \mathbb{Z}[\mathbb{Z} / 2 \mathbb{Z}]$.

Demonstração. $(i) \Longrightarrow(i i)$ Seja $\phi=\left\langle a_{1}, \cdots, a_{n}\right\rangle$ uma forma regular, como $\mathbb{K}^{2}$ é (o único) cone positivo de $\mathbb{K}$ temos que exatamente uma das opções ocorre:

$$
a_{i} \in+\mathbb{K}^{2} \text { ou } a_{i} \in-\mathbb{K}^{2}
$$

Definindo $\epsilon_{i}:=1$ caso $(+)$ ocorra e $\epsilon_{i}:=-1$, se (-) for o caso, temos $\left\langle a_{1}, \cdots, a_{n}\right\rangle \simeq\left\langle\epsilon_{1}, \cdots, \epsilon_{n}\right\rangle$. Definimos $\operatorname{Sgn}\left(\left\langle a_{1}, \cdots, a_{n}\right\rangle\right)=\sum_{i=1}^{n} \epsilon_{i} \in \mathbb{Z}$. Notemos que:

- A forma $\left\langle\epsilon_{1}, \cdots, \epsilon_{n}\right\rangle$ é anisotrópica se e só se esta sequência tiver entradas constante;

- $\left\langle a_{1}, \cdots, a_{n}\right\rangle \simeq\left\langle a_{1}^{\prime}, \cdots, a_{n}^{\prime}\right\rangle \Leftrightarrow \sum_{i=1}^{n} \epsilon_{i}=\sum_{i=1}^{n} \epsilon_{i}^{\prime}$ (Teorema de inércia de Jacobi e Sylvester).

(ii) $\Longrightarrow($ iii $)$ O homomorfismo canônico de anéis com unidade $u: \mathbb{Z} \longrightarrow W(\mathbb{K})$ é um isomorfismo devido a bijeção $a: \operatorname{Anis}(\mathbb{K}) / \simeq \longrightarrow W(\mathbb{K})$, estabelecida na Proposição 23 .

(iii) $\Longrightarrow($ i) Como só existe um único homomorfismo de anéis com unidade $u: \mathbb{Z} \longrightarrow W(\mathbb{K})$, este tem que ser o isomorfismo da hipótese. Logo, como $W(\mathbb{K})$ está em bijeção com o conjunto das classes de isometria de formas anisotrópicas, para cada $b \in \dot{K}$, temos que exatamente uma das possibilidades ocorre: $[\langle b\rangle]=u(1)$ ou $[\langle b\rangle]=u(-1)$. Assim $\dot{\mathbb{K}} / \dot{\mathbb{K}}^{2} \cong \mathbb{Z} / 2 \mathbb{Z}$, i.e. $\mathbb{K}$ é euclidiano.

$(i) \Longrightarrow(i v)$ Como $\{\langle 1\rangle,\langle-1\rangle\}$ é um conjunto de classes de formas invertíveis no anel $W G(\mathbb{K})$, obtemos assim um homomorfismo canônico $h: \mathbb{Z}[\mathbb{Z} / 2 \mathbb{Z}] \longrightarrow W G(\mathbb{K})$. Notemos que $\{\langle 1\rangle,\langle-1\rangle\}$ gera aditivamente $W G(\mathbb{K})$ e se $m\langle 1\rangle+n\langle-1\rangle=0$ em $W G(\mathbb{K})$, então, como $\mathbb{K}^{2}$ é cone positivo de $\mathbb{K}$, temos $m=n=0$. Desta forma, temos o homomorfismo canônico $\mathbb{Z}[\mathbb{Z} / 2 \mathbb{Z}] \longrightarrow W G(\mathbb{K})$ é um isomorfismo.

$(i v) \Longrightarrow($ iii $)$ O homomorfismo $h: \mathbb{Z}[\mathbb{Z} / 2 \mathbb{Z}] \longrightarrow W G(\mathbb{K})$ é tal que $J=h^{-1}[\mathbb{Z} \mathbb{H}]=\left\{n \cdot(-1)^{0}+\right.$ $\left.m \cdot(-1)^{1}: n=m\right\}$. Notemos que o homomorfismo canônico $\mathbb{Z} \longrightarrow \mathbb{Z}[\mathbb{Z} / 2 \mathbb{Z}] / J$ tem como inverso o mapa $\mathbb{Z}[\mathbb{Z} / 2 \mathbb{Z}] / J \longrightarrow \mathbb{Z}$ dado por $\left(k \cdot(-1)^{0}+l .(-1)^{1}\right)+J \mapsto(k-l) \in \mathbb{Z}$. Assim: $W(\mathbb{K})=$ $W G(\mathbb{K}) / \mathbb{Z} \mathbb{H} \cong \mathbb{Z}[\mathbb{Z} / 2 \mathbb{Z}] / J \cong \mathbb{Z}$.

$(v) \Longrightarrow(i) n\langle 1\rangle \approx \mathbb{H} \oplus \phi^{\prime} \Longrightarrow D(n\langle 1\rangle)=\mathbb{K} \Longrightarrow \mathbb{K}=\mathbb{K}^{2} \Longrightarrow \# \dot{K} / \mathbb{K} \neq 2$.

Exemplo 3. O corpo dos números reais algébricos $\overline{\mathbb{Q}} \cap \mathbb{R}(\overline{\mathbb{Q}}$ é o fecho algébrico de $\mathbb{Q}$ em $\mathbb{C}$ ) é um corpo real fechado (na verdade, é o menor de todos estes) logo também é euclidiano. Portanto, $W G(\overline{\mathbb{Q}} \cap \mathbb{R})$ é a algebra de grupo $\mathbb{Z}[\mathbb{Z} / 2 \mathbb{Z}]$ e $W(\overline{\mathbb{Q}} \cap \mathbb{R})$ é cíclico infinito.

\footnotetext{
${ }^{4}$ Isto pode ser feito através do axioma das escolhas dependentes, uma versão mais fraca do axioma da escolha.
} 
Exemplo 4. (Números construtíveis) Seja $\widetilde{\mathbb{Q}}$ o fecho quadrático (também chamado de corpo de todos os números construtiveis) do corpo dos números racionais $\mathbb{Q}$. O corpo dos números reais construtiveis é $C_{\mathbb{R}}=\widetilde{\mathbb{Q}} \cap \mathbb{R}$. Como $\sqrt{-1} \notin C_{\mathbb{R}}$ e $C_{\mathbb{R}}(\sqrt{-1})$ é quadraticamente fechado, segue que $C_{\mathbb{R}}$ é um corpo euclidiano (= o fecho euclidiano de $\mathbb{Q}$ ) e, portanto $W G\left(C_{\mathbb{R}}\right) \cong \mathbb{Z}[\mathbb{Z} / 2 \mathbb{Z}]$ e $W\left(C_{\mathbb{R}}\right) \cong \mathbb{Z}$.

Observação 21. Dado $(\mathbb{K}, P)$ um corpo ordenado, para cada $\phi \simeq\left\langle a_{1}, \cdots, a_{n}\right\rangle \in B I L S_{\mathbb{K}}$, denota$\operatorname{mos} \phi^{(+)}=\#\left\{j: a_{j} \in P\right\}$ e $\phi^{(-)}=\#\left\{j: a_{j} \in-P\right\}$. O número $\operatorname{Sgn}_{P}(\phi):=\phi^{(+)}-\phi^{(-)} \in \mathbb{Z} e ́$ invariante por isometria. Desta forma tem-se o mapa de assinatura com relação a ordem $P$.

$$
\operatorname{Sgn}_{P}: M(\mathbb{K}) \longrightarrow \mathbb{Z}
$$

Proposição 31. Seja $(\mathbb{K}, P)$ um corpo ordenado. Então o mapa de assinatura $\operatorname{Sgn}_{P}: M(\mathbb{K}) \longrightarrow \mathbb{Z}$ é um homomorfismo de semianéis que determinada um único morfismo de anéis (também denoto-

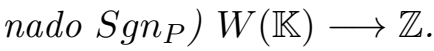

Demonstração. Consideremos o fecho euclidiano $E$ do corpo ordenado $(\mathbb{K}, P)$. Como vimos na seção anterior, o homomorfismo de corpos $j: \mathbb{K} \longmapsto E$ (tal que $j^{-1}\left(E^{2}\right)=P$ ), induz um homomorfismo de anéis $W(j): W(\mathbb{K}) \longrightarrow W(E)$, dado por $\left[\left\langle a_{1}, \cdots, a_{n}\right\rangle\right] \mapsto\left[\left\langle j\left(a_{1}\right), \cdots, j\left(a_{n}\right)\right\rangle\right]$. Pela Proposição 30 acima temos isomorfismo $W\left(E^{(P)}\right) \cong \mathbb{Z}$ dado por $\left[\left\langle x_{1}, \cdots, x_{k}\right\rangle\right] \mapsto \sum_{i=1}^{k} \epsilon_{i}$, onde $\epsilon_{j}= \pm$ dependendo de $x_{j} \in \pm \dot{E}^{2}$. Desta forma, como $j\left(a_{i}\right) \in \pm \dot{E}^{2}$ sse $a_{i} \in \pm \dot{P}$, temos que a composição $W(\mathbb{K}) \stackrel{W(j)}{\longrightarrow}$ $W(E) \stackrel{\cong}{\longrightarrow}$, é tal que $\left[\left\langle a_{1}, \cdots, a_{n}\right\rangle\right] \mapsto S g n_{P}\left(\left[\left\langle a_{1}, \cdots, a_{n}\right\rangle\right]\right)$.

\subsubsection{Corpos finitos}

Lema 14. Seja $\mathbb{F}_{q}$ o corpo finito com $q$ elementos, onde $q=p^{n}$ com $p \neq 2$ primo. Então

(i) $\left|\dot{\mathbb{F}}_{q} / \dot{\mathbb{F}}_{q}^{2}\right|=2$ e denotamos $\dot{\mathbb{F}}_{q} / \dot{\mathbb{F}}_{q}^{2}=\{[1],[\lambda]\} ;$

(ii) $\quad-1 \in \dot{\mathbb{F}}_{q}^{2} \Longleftrightarrow q \equiv 1(\bmod 4) ; \quad-1 \notin \dot{\mathbb{F}}_{q}^{2} \Longleftrightarrow q \equiv 3(\bmod 4)$;

(iii) $\langle\lambda, \lambda\rangle \simeq\langle 1,1\rangle$;

(iv) $D_{\mathbb{F}_{q}}(\langle a, b\rangle)=\dot{\mathbb{F}}_{q}, \forall a, b \in \dot{\mathbb{F}}_{q}$.

(v) Toda forma regular $n$-dimensional sobre $\mathbb{F}_{q}$ é isométrica a exatamente uma das formas: $n\langle 1\rangle$; $(n-1)\langle 1\rangle \oplus\langle\lambda\rangle$.

(vi) Um conjunto completo de representantes de formas regulares anisotrópicas módulo isometria é:

- $\{\langle\rangle,\langle 1\rangle,\langle\lambda\rangle,\langle 1, \lambda\rangle\}$, se $q \equiv 1(\bmod 4)$;

- $\{\langle\rangle,\langle 1\rangle,\langle\lambda\rangle,\langle 1,1\rangle\}$, se $q \equiv 3(\bmod 4)$;

Demonstração.

(i) Como $\varphi: \dot{\mathbb{F}}_{q} \longrightarrow \dot{\mathbb{F}}_{q}^{2}, x \mapsto x^{2}$ é um epimorfismo de grupos com $\operatorname{Ker}(\varphi)=\{ \pm 1\}$, e $-1 \neq 1$ (pois $p \neq 2$ ), temos $\left|\dot{\mathbb{F}}_{q}\right|=2\left|\dot{\mathbb{F}}_{q}^{2}\right|$.

(ii) Como $\left|\dot{\mathbb{F}}_{q}\right|=q-1$ é par temos $q-1=i .2^{m}$ onde $i$ é ímpar e $m>0, \operatorname{logo} q \equiv 1(\bmod 4)$ ou $q \equiv 3(\bmod 4)$. Como $\left(\dot{\mathbb{F}}_{q}, \cdot, 1\right)$ é grupo cíclico, temos $\dot{\mathbb{F}}_{q} \cong \mathbb{Z} / i \mathbb{Z} \times \mathbb{Z} / 2^{m} \mathbb{Z}$. Este isomorfismo restringe-se a $\dot{\mathbb{F}}_{q}^{2} \cong \mathbb{Z} / i \mathbb{Z} \times 2 \mathbb{Z} / 2^{m} \mathbb{Z}$. Como o elemento $-1 \in \dot{\mathbb{F}}_{q}$ é determinado por $(-1)^{2}=1$, $-1 \neq 1$, este deve corresponder ao elemento $\left(0+i \mathbb{Z}, 2^{m-1}+2^{m} \mathbb{Z}\right) \in \mathbb{Z} / i \mathbb{Z} \times \mathbb{Z} / 2^{m} \mathbb{Z}$. Portanto:

$$
-1 \in \dot{\mathbb{F}}_{q}^{2} \Leftrightarrow 2^{m-1} \in 2 \mathbb{Z} \Leftrightarrow m>1 \Leftrightarrow 4 \text { divide }(q-1)
$$


(iii) Como ambas as formas têm mesmo discriminante módulo $\dot{\mathbb{F}}_{q}^{2}$, basta garantir que $D(\langle 1,1\rangle) \cap$ $D(\langle\lambda, \lambda\rangle) \neq \emptyset$. Se $[-1]=[1]$ então $\langle 1,1\rangle \simeq\langle 1,-1\rangle, \operatorname{logo} D(\langle 1,1\rangle)=\dot{\mathbb{F}}_{q}$ e o resultado segue. Por outro lado, assumindo $[-1]=[\lambda]$, como os conjuntos finitos $\dot{\mathbb{F}}_{q}^{2}$ e $\left(1+\dot{\mathbb{F}}_{q}^{2}\right)$ têm mesma cardinalidade (= (q$1) / 2$ ), são distintos (pois $1 \notin 1+\dot{\mathbb{F}}_{q}^{2}$ ) e assim existe $a \in \dot{\mathbb{F}}_{q}$ tal que $1+a^{2} \notin \dot{\mathbb{F}}_{q}^{2}$, temos que $1+a^{2} \neq 0$ (pois senão $[-1]=[1]$, contrariando a hipótese) $\operatorname{logo}[\lambda]=\left[\left(1+a^{2}\right)\right]$ então $\lambda \in D(\langle 1,1\rangle) \cap D(\langle\lambda, \lambda\rangle)$.

(iv) Por $(i)$ tem-se que toda forma regular binária é isométrica a alguma das formas no conjunto $\{\langle 1,1\rangle,\langle\lambda, \lambda\rangle,\langle 1, \lambda\rangle\}$. Segue diretamente de $(i)$ e da definição de representação que $D\left(\langle 1, \lambda)=\dot{\mathbb{F}}_{q}\right.$. Por $(i i i),\{1, \lambda\} \in D(\langle 1,1\rangle) \cap D\left(\langle\lambda, \lambda)\right.$, logo $D\left(\langle 1,1)=D\left(\langle\lambda, \lambda)=\dot{\mathbb{F}}_{q}\right.\right.$

(v) Por $(i)$ tem-se que toda forma regular binária é isométrica a alguma das formas no conjunto $\{\langle 1,1\rangle,\langle\lambda, \lambda\rangle,\langle 1, \lambda\rangle\}$. Por $(i i i),\langle\lambda, \lambda\rangle \simeq\langle 1,1\rangle$. Desta forma, claramente $\{\langle 1,1\rangle,\langle 1, \lambda\rangle\}$ é um conjunto completo de representantes de formas binárias módulo isometria. Como a relação de isometria de formas coincide com a equivalência de cadeias, segue que toda forma quadrática é isometrica n-dimensional é isométrica a $n\langle 1\rangle$ ou (exclusivo) a $(n-1)\langle 1\rangle \oplus\langle\lambda\rangle$.

(vi) Se $q \equiv 1(\bmod 4)$, então pelo item (ii) temos $\langle-1\rangle \simeq\langle 1\rangle$, logo $2 k\langle 1\rangle$ é forma hiperbólica. Utilizando o item (v) do acima temos: para $n$ par, as (classes de isometria de) formas anisotrópicas são \langle\rangle$,\langle 1, \lambda\rangle$; para $n$ ímpar, as formas anisotrópicas são $\langle 1\rangle,\langle\lambda\rangle$. Assim, um conjunto completo de representantes de formas regulares anisotrópicas módulo isometria é $\{\langle\rangle,\langle 1\rangle,\langle\lambda\rangle,\langle 1, \lambda\rangle\}$.

Se $q \equiv 3(\bmod 4)$, então pelo item (ii) temos $\langle-1\rangle \simeq\langle\lambda\rangle$; como $\langle 1,1\rangle \simeq\langle\lambda, \lambda\rangle$ (item (iii)), temos que $4 k\langle 1\rangle$ é forma hiperbólica. Utilizando o item (v) acima temos: para $n$ par, as (classes de isometria de) formas anisotrópicas são \langle\rangle$,\langle 1,1\rangle$; para $n$ ímpar, as formas anisotrópicas são $\langle 1\rangle,\langle\lambda\rangle$ (note que $\langle 1,1,1\rangle \simeq\langle-1\rangle \oplus\langle-1,1\rangle,\langle 1,1,-1\rangle=\langle 1\rangle \oplus\langle 1,-1\rangle$ ). Assim, um conjunto completo de representantes de formas regulares anisotrópicas módulo isometria é $\{\langle\rangle,\langle 1\rangle,\langle\lambda\rangle,\langle 1,1\rangle\}$.

Proposição 32. O anel de Witt de $\mathbb{F}_{q}$ é descrito por

$$
W\left(\mathbb{F}_{q}\right) \cong\left\{\begin{array}{lll}
\mathbb{Z} / 2 \mathbb{Z}\left[\dot{\mathbb{F}}_{q} / \dot{\mathbb{F}}_{q}^{2}\right] & \text { se } & q \equiv 1(\bmod 4) \\
\mathbb{Z} / 4 \mathbb{Z} & \text { se } & q \equiv 3(\bmod 4)
\end{array}\right.
$$

Demonstração. Se $q \equiv 1$ (mod4), então pelo item (vi) do Lema acima, $\{\langle\rangle,\langle 1\rangle,\langle\lambda\rangle,\langle 1, \lambda\rangle\}$ é um conjunto completo de representantes de formas regulares anisotrópicas módulo isometria. Temos portanto bijeção $W\left(\mathbb{F}_{q}\right) \cong\{\langle\rangle,\langle 1\rangle,\langle\lambda\rangle,\langle 1, \lambda\rangle\}$. É fácil ver que $\{\langle 1\rangle,\langle\lambda\rangle\}$ é $\mathbb{Z} / 2 \mathbb{Z}$-base deste constituída de formas invertíveis, então o mapa (bem definido) $\epsilon_{0}(1)+\epsilon_{1}(\lambda) \mapsto \epsilon_{0}\langle 1\rangle+\epsilon_{1}\langle\lambda\rangle, \epsilon_{i} \in \mathbb{Z} / 2 \mathbb{Z}$, estabelece um isomorfismo de anéis $\mathbb{Z} / 2 \mathbb{Z}\left[\dot{\mathbb{F}}_{q} / \dot{\mathbb{F}}_{q}^{2}\right] \stackrel{\cong}{\longrightarrow} W\left(\mathbb{F}_{q}\right)$.

Se $q \equiv 3(\bmod 4)$, então pelo item (vi) do Lema acima, $\{\langle\rangle,\langle 1\rangle,\langle\lambda\rangle,\langle 1,1\rangle\}$ é um conjunto completo de representantes de formas regulares anisotrópicas módulo isometria. Como $[\lambda]=[-1]$ e $\langle 1,1,1\rangle \simeq\langle-1,-1,1\rangle=\langle-1\rangle \oplus\langle-1,1\rangle$, é fácil ver que a bijeção $n \mapsto n\langle 1\rangle, 0 \leq n<4$, estabelece um isomorfismo de anéis $\mathbb{Z} / 4 \mathbb{Z} \stackrel{\cong}{\longrightarrow} W\left(F_{q}\right)$.

\subsection{Apresentações de $W G(\mathbb{K})$ e $W(\mathbb{K})$}

4. Uma descrição por geradores e relações:

(A) Para cada espaço bilinear simétrico regular $\phi$ tem-se $\phi \simeq\left\langle a_{1}, \ldots, a_{n}\right\rangle$, tomando $R \subseteq \mathbb{K}$ um conjunto de representantes de $\dot{\mathbb{K}}$ módulo o subgrupo $\dot{\mathbb{K}}^{2}$, segue que $\hat{S}=\{\langle a\rangle \in W G(\mathbb{K}): a \in R\}$ 
e $S=\{\langle a\rangle \in W(\mathbb{K}): a \in R\}$ são geradores dos grupos abelianos $W G(\mathbb{K})=\langle\hat{S}\rangle$ e $W(\mathbb{K})=\langle S\rangle$. Como cada (classes de equivalência de) forma regular $\langle a\rangle$ é invertível em $W G(\mathbb{K})$ e $W(\mathbb{K})$ (pois $\left.\langle a\rangle \otimes\langle a\rangle=\left\langle a^{2}\right\rangle \simeq\langle 1\rangle\right)$, obtemos os epimorfismos de anéis

$$
\begin{aligned}
& \hat{\varphi}: \mathbb{Z}\left[\dot{\mathbb{K}} / \dot{\mathbb{K}}^{2}\right] \longrightarrow W G(\mathbb{K}) \quad e \quad \varphi: \mathbb{Z}\left[\dot{\mathbb{K}} / \dot{\mathbb{K}}^{2}\right] \longrightarrow W(\mathbb{K}) \\
& \delta_{a} \longmapsto\langle a\rangle \quad \delta_{a} \longmapsto\langle a\rangle
\end{aligned}
$$

(B) Segue do teorema do homomorfismo que existem únicos isomorfismos

$\hat{\Phi}: \mathbb{Z}\left[\dot{\mathbb{K}} / \dot{\mathbb{K}}^{2}\right] / \operatorname{Ker}(\hat{\varphi}) \stackrel{\cong}{\longrightarrow} W G(\mathbb{K})$ e $\Phi: \mathbb{Z}\left[\dot{\mathbb{K}}^{\prime} / \dot{\mathbb{K}}^{2}\right] / \operatorname{Ker}(\varphi) \stackrel{\cong}{\longrightarrow} W(\mathbb{K})$ que fazem, respectivamente, os seguintes diagramas comutarem
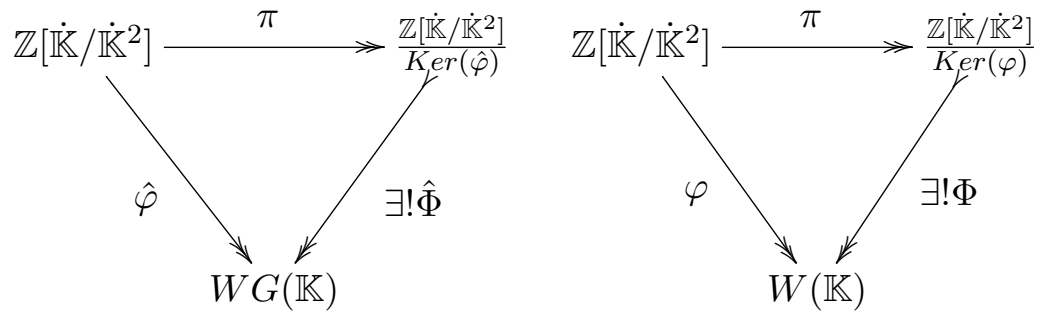

(C) Assim, para dar uma descrição de $W G(\mathbb{K})$ e $W(\mathbb{K})$ por geradores e relações é fundamental que se investigue a estrutura de $\operatorname{Ker}(\hat{\varphi})$ e $\operatorname{Ker}(\varphi)$.

Proposição 33. $G=\left\{\alpha+(-\alpha): \alpha \in \dot{\mathbb{K}} / \dot{\mathbb{K}}^{2}\right\} \cup\{\alpha+\beta-\gamma-\delta:\langle\alpha, \beta\rangle \simeq\langle\gamma, \delta\rangle\}$ é um conjunto de geradores para o subgrupo $\operatorname{Ker}(\varphi)$ de $\mathbb{Z}\left[\mathbb{K} / \dot{\mathbb{K}}^{2}\right]$.

Demonstração. Como formas hiperbólicas têm dimensão par $\left\langle a_{1}, \cdots, a_{n},-a_{1}^{\prime}, \cdots,-a_{n}^{\prime}\right\rangle$, cada $\omega \in \operatorname{Ker}(\varphi)$ possui escrita $\omega=\sum_{i=1}^{n} \alpha_{i}-\sum_{i=1}^{n} \alpha_{i}^{\prime} \operatorname{com} \alpha_{i}, \alpha_{i}^{\prime} \in$ $\dot{\mathbb{K}} / \dot{\mathbb{K}}^{2}, \alpha_{i}=a_{i} \cdot \dot{\mathbb{K}}^{2}$. Seja $(V, q)$ o (único a menos de isometria) espaço quadrático associado a $\left\langle\alpha_{1}, \cdots, \alpha_{n}\right\rangle$.Existem bases ortogonais $\varepsilon=\left\{e_{i}: 1 \leq i \leq n\right\}, \varepsilon^{\prime}=\left\{e_{i}^{\prime}: 1 \leq i \leq n\right\}$ de $\mathrm{V}$ tais que $q\left(e_{i}\right)=\alpha_{i}$ e $q\left(e_{i}^{\prime}\right)=\beta_{i}$. Pelo teorema de equivalência de cadeias de Witt podemos escolher uma cadeia de bases ortogonais $\varepsilon=\varepsilon_{0}, \varepsilon_{1}, \cdots, \varepsilon_{m}=\varepsilon^{\prime}$ tal que $\varepsilon_{i}, \varepsilon_{i+1}$ diferem em no máximo dois vetores. Seja $\left\langle\alpha_{1}^{i}, \cdots, \alpha_{n}^{i}\right\rangle$ a forma diagonal de $(V, q)$ com relação a $\varepsilon_{i}$. Se $\varepsilon_{i}, \varepsilon_{i+1}$ diferem exatamente em um vetor,então $\sum_{i=1}^{n} \alpha_{j}^{i}-\sum_{i=1}^{n} \alpha_{j}^{i+1}=\alpha+(-\alpha)$ para algum $\alpha \in \mathbb{K} / \dot{\mathbb{K}}^{2}$.em caso contrário, $\sum_{i=1}^{n} \alpha_{j}^{i}-\sum_{i=1}^{n} \alpha_{j}^{i+1}=\alpha+\beta-\gamma-\delta$ para alguns $\alpha, \beta, \gamma, \delta \in \dot{\mathbb{K}} / \dot{\mathbb{K}}^{2}$ onde $\langle\alpha, \beta\rangle \approx\langle\gamma, \delta\rangle$. $O$ resultado segue da igualdade

$$
\sum_{i=1}^{n} \alpha_{i}-\sum_{i=1}^{n} \alpha_{i}^{\prime}=\left(\sum_{i=1}^{n} \alpha_{i}^{0}-\sum_{i=1}^{n} \alpha_{i}^{1}\right)+\cdots+\left(\sum_{i=1}^{n} \alpha_{i}^{m-1}-\sum_{i=1}^{n} \alpha_{i}^{m}\right)
$$

Na sequência vamos dar uma descrição de $W G(\mathbb{K})$ e $W(\mathbb{K})$ utilizando $\mathbb{Z}[\dot{\mathbb{K}}]$. Iniciamos com o seguinte lema

Lema 15. As seguintes relações valem no anel $W G(\mathbb{K})$

$\left.\mathcal{R}_{1}\right)\langle 1\rangle=1$;

$\left.\mathcal{R}_{2}\right)\langle\alpha \beta\rangle=\langle\alpha\rangle\langle\beta\rangle, \forall \alpha, \beta \in \dot{\mathbb{K}} ;$

$\left.\mathcal{R}_{3}\right)\langle\alpha\rangle+\langle\beta\rangle=\langle\alpha+\beta\rangle .(1+\langle\alpha \beta\rangle), \alpha, \beta, \alpha+\beta \in \mathbb{K}$. 
Demonstração. $\mathcal{R}_{1}$ e $\mathcal{R}_{2}$ seguem imediatamente do producto de Kronecker. Para $\mathcal{R}_{3}$ seja $\phi=$ $\langle\alpha+\beta\rangle(1+\langle\alpha \beta\rangle)$ e $\phi^{\prime}=\langle\alpha\rangle+\langle\beta\rangle$. É fácil ver que $d(\phi)=d\left(\phi^{\prime}\right)$ e $\alpha+\beta \in D_{\mathbb{K}}(\phi) \cap D_{\mathbb{K}}\left(\phi^{\prime}\right)$.

Corolário 13. Para cada $\alpha \in \mathbb{K}$ vale a seguinte relação

$$
\left\langle\alpha^{2}\right\rangle=\langle 1\rangle
$$

Demonstração. Basta observar que

$$
\langle\alpha\rangle+\langle\alpha\rangle=\langle 2 \alpha\rangle\left(1+\left\langle\alpha^{2}\right\rangle\right)\left(\text { Por } \quad \mathcal{R}_{3}\right)
$$

e

$$
\begin{aligned}
\langle\alpha\rangle+\langle\alpha\rangle & =\langle\alpha\rangle\langle 1\rangle+\langle\alpha\rangle\langle 1\rangle \\
& =\langle\alpha\rangle(\langle 1\rangle+\langle 1\rangle) \\
& =\langle\alpha\rangle[\langle 2\rangle(1+\langle 1\rangle)] \\
& =\langle 2 \alpha\rangle(1+\langle 1\rangle)
\end{aligned}
$$

Usamos $\mathcal{R}_{3}$ na penúltima igualdade e $\mathcal{R}_{2}$ na útilma igualdade.Por fim, $\mathcal{R}_{2}$ permite escrever

$$
\left\langle 2^{-1} \alpha^{-1}\right\rangle\langle 2 \alpha\rangle=\left\langle 2^{-1} \alpha^{-1} 2 \alpha\right\rangle=\langle 1\rangle
$$

Observação 22. Em qualquer anel onde valha formalmente as propriedades do lema 5 pode-se fazer um cálculo similar ao que foi feito na demonstração do corolário anterior.

Proposição 34. Temos um isomorfismo de anéis $W G(\mathbb{K}) \cong \frac{\mathbb{Z}[\mathbb{K}]}{\mathcal{R}}$, onde $\mathcal{R}$ é o ideal gerado pelas relações $\mathcal{R}_{1}=\left\{\delta_{1}-1\right\}, \mathcal{R}_{2}=\left\{\delta_{\alpha \beta}-\delta_{\alpha} \delta_{\beta}: \alpha, \beta \in \dot{\mathbb{K}}\right\}$ e $\mathcal{R}_{3}=\left\{\delta_{\alpha}+\delta_{\beta}-\delta_{(\alpha+\beta)}\left(1+\delta_{\alpha \beta}\right): \alpha, \beta, \alpha+\beta \in\right.$ $\dot{\mathbb{K}}\}$.

Demonstração. Pelo teorema de equivalencia por cadeias de Witt isometria de $n$ - formas é essencialmente isometria de $2-$ formas.Seja $\langle\alpha, \beta\rangle \simeq\langle\gamma, \omega\rangle$ e assim $\alpha \beta=\gamma \omega \varepsilon^{2}$ e $\gamma=\alpha \xi^{2}+\beta \eta^{2}$.Se $\xi=0$,então $\alpha=\beta \eta^{2} \omega \varepsilon^{2} \beta^{-1}$ e ,portanto $\delta_{\alpha}=\delta_{\omega}$. Se $\xi, \eta \neq 0$,então $\delta_{\alpha}+\delta_{\beta}=\delta_{\alpha \xi^{2}}+\delta_{\beta \eta^{2}}=$ $\delta_{\alpha \xi^{2}+\beta \eta^{2}}\left(1+\delta_{\alpha \beta \xi^{2} \eta^{2}}\right)=\delta_{\gamma}\left(1+\delta_{\gamma \omega}\right)=\delta_{\gamma}+\delta_{\omega}$. Assim, temos um homomorfismo de monóides

$$
\psi^{\prime}: \begin{array}{cc}
M(\mathbb{K}) & \longrightarrow \mathbb{Z}[\dot{\mathbb{K}}] / \mathcal{R} \\
\left\langle\alpha_{1}, \ldots, \alpha_{n}\right\rangle & \longmapsto \sum_{i=1}^{n} \delta_{\alpha_{i}}
\end{array}
$$

e pela universalidade de $W G(\mathbb{K})$ existe um homomorfismo de grupos $\psi: W G(\mathbb{K}) \longrightarrow \mathbb{Z}[\dot{\mathbb{K}}] / \mathcal{R}$ tal que $\psi \circ \rho=\psi^{\prime}$.

O morfismo de semianéis $\varphi^{\prime}: \mathbb{Z}[\mathbb{K}] \longrightarrow M(\mathbb{K}), \delta_{\alpha} \mapsto\langle\alpha\rangle$ é tal que $\varphi^{\prime}(\mathcal{R})$ é trivial. Seja $\varphi$ o morfismo de anéis tal que o diagrama

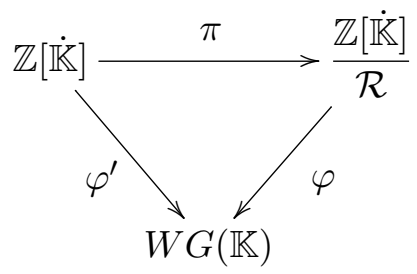


é comutativo.É fácil ver que $\varphi \circ \psi=I_{d}$ e $\psi \circ \varphi=I_{d}$.

Fato 10.

(i) Um argumento similar ao da demonstração da proposição anterior mostra que o grupo WG(KK) é o quociente do livre $\mathbb{Z}[\dot{\mathbb{K}}]$ pelo ideal $\mathcal{R}$ gerado pelas relações

(1) $\delta_{\alpha \beta^{2}}-\delta_{\alpha}$.

(2) $\delta_{\alpha}+\delta_{\beta}-\delta_{\alpha+\beta}-\delta_{\alpha \beta(\alpha+\beta)}$

(ii) $O$ anel de witt $W(\mathbb{K})$ é determinado completamente pelas relações

1) $\mathcal{R}_{1}=\left\{\delta_{1}-1\right\}$,

2) $\mathcal{R}_{2}=\left\{\delta_{\alpha \beta}-\delta_{\alpha} \delta_{\beta}: \alpha, \beta \in \dot{\mathbb{K}}\right\} e$

3) $\mathcal{R}_{3}=\left\{\delta_{\alpha}+\delta_{\beta}-\delta_{(\alpha+\beta)}\left(1+\delta_{\alpha \beta}\right): \alpha, \beta, \alpha+\beta \in \dot{\mathbb{K}}\right\}$

4) $\mathcal{R}_{4}=\delta_{1}+\delta_{-1}$

enquanto as relações de (i) acrescida da relação $\delta_{1}+\delta_{-1}$ determina inteiramente o grupo de Witt $W(\mathbb{K})$

Observação 23. Todas as descrições por geradores e relações nesta seção são canônicas, i.e., elas produzem isomorfismos naturais entre funtores Field ${ }_{1 / 2} \longrightarrow \mathcal{C} \mathcal{R}$ ing dados por $W G$ (respectivamente $W$ ) e por certos funtores Field ${ }_{1 / 2} \longrightarrow \mathcal{C} \mathcal{R} i n g$ que são dados canonicamente por quocientes de anéis de grupos.

\subsection{O espaço de ordens de um corpo e assinaturas}

Seja $\Delta$ o grupo multiplicativo $\{ \pm 1\}$. Conside em $\Delta$ a topologia discreta $\wp(\Delta)$ e tome $\tau_{\pi}$ para ser a topologia inicial das projeções $\pi=\left(\pi_{j}: \Delta^{J} \longrightarrow \Delta\right)_{j \in J}$, para algum conjunto $J$. O espaço produto é $\left(\Delta^{J}, \tau_{\pi}\right)$. Definimos o suporte de $\Omega \in \tau_{\pi}^{J}$ como sendo o conjunto $\operatorname{Supp}(\Omega)=\{j \in$ $\left.J ; \Omega_{j} \neq \Delta\right\}$. Uma base para $\tau_{\pi}$ é dada por $\mathcal{B}_{\pi}=\left\{\Omega \in \tau_{\pi}^{J}: \operatorname{supp}(\Omega)<\aleph_{0}\right\}$,ou ainda, $\mathcal{B}_{\pi}=$ $\left\{\prod_{\in t \notin \operatorname{Supp}(\Omega)} \Omega_{t} \times \Delta^{J \backslash \operatorname{supp}(\Omega)}: \Omega \in \tau_{\pi}^{J} ; \operatorname{supp}(\Omega)<\aleph_{0}\right\}$.Assim, $\Omega \in \tau_{\pi}$ é completamente determinado por $\left\{\omega \in \Delta^{J}: \omega(s)=\varepsilon_{s}, s \in \operatorname{supp}(\Omega), \varepsilon_{s} \in \Delta\right\}$ e como $\Delta^{J} \backslash \Omega=\bigcup\left\{\omega \in \Delta^{J}: \omega(s)=-\varepsilon_{s}\right\}$ é reunião de abertos ,segue que $\Omega$ é clopen. Denotaremos $\left\{\omega \in \Delta^{\mathbb{K}}: \omega\left(x_{1}\right)=\varepsilon_{1}, \cdots, \omega\left(x_{k}\right)=\varepsilon_{k}\right\}$ por $H_{\varepsilon_{1}, \cdots, \varepsilon_{k}}\left(x_{1}, \cdots, x_{k}\right)$.

Seja $X_{\mathbb{K}}=\{P \in \wp(\mathbb{K}): P$ é uma ordem de $\mathbb{K}\}$. Mergulhamos $X_{\mathbb{K}}$ em $\{ \pm 1\}^{\mathbb{K}^{\dot{K}} / \dot{\mathbb{K}}^{2}}$ mediante uma aplicação $\sigma: X_{\mathbb{K}} \longrightarrow\{ \pm 1\}^{\mathbb{K}^{K} / \dot{\mathbb{K}}^{2}}, P \mapsto \sigma_{P}$ definida por $\sigma_{P}\left(x \dot{\mathbb{K}}^{2}\right)=1 \Longleftrightarrow x \in P$. Dessa forma,

$$
\sigma^{-1}\left[H_{\varepsilon_{1}, \cdots, \varepsilon_{k}}\left(\bar{x}_{1}, \cdots, \bar{x}_{k}\right) \cap \sigma\left[X_{\mathbb{K}}\right]\right]=\bigcap\left\{H\left(\varepsilon_{j} x_{j}\right): 1 \leq j \leq k\right\}
$$

onde $H(x)=\left\{P \in X_{\mathbb{K}}: x \in P\right\}, \forall x \in \mathbb{K}$. Observe que $\mathcal{H}=\{H(x): x \in \mathbb{K}\}$ cumpre os pré-requisitos para ser uma subbase de uma topologia sobre $X_{\mathbb{K}}$; de fato, $H(1)=X_{\mathbb{K}}, H(-1)=\emptyset$ e além disso $H(-x)=X_{\mathbb{K}} \backslash H(x)$ e, portanto, cada $H(x)$ é clopen. Seja $\tau_{\mathcal{H}}$ a topologia gerada por $\mathcal{H}$; o espaço de Harrison será $\left(X_{\mathbb{K}}, \tau_{\mathcal{H}}\right)$ (ou espaço de ordens do corpo $\mathbb{K}$ )e denotaremos por $H\left(a_{1}, \cdots, a_{n}\right)$ um aberto básico $H\left(a_{1}\right) \cap \cdots \cap H\left(a_{n}\right)$ de $\tau_{\mathcal{H}}$.

\section{Proposição 35.}

(i) $\{ \pm 1\}^{\mathbb{K}} / \dot{\mathbb{K}}^{2}$ é um espaço booleano.

(ii) $\sigma: X_{\mathbb{K}} \longrightarrow\{ \pm 1\}^{\mathbb{K}^{\dot{K}} / \dot{\mathbb{K}}^{2}}$ é injetora. 
(iii) $\sigma^{\prime}: X_{\mathbb{K}} \longrightarrow \sigma\left[X_{\mathbb{K}}\right], P \mapsto \sigma^{\prime}(P)=\sigma(P)$ é um homeomorfismo.

(iv) $\sigma\left[X_{\mathbb{K}}\right] \subseteq\{ \pm 1\}^{\mathbb{K} / \dot{\mathbb{K}}^{2}}$ é subespaço fechado.

(v) $X_{\mathbb{K}}$ é um espaço booleano.

(vi) $\varphi \in \sigma\left[X_{\mathbb{K}}\right]$ se, e somente se,cumpre as seguintes condições

1) $\varphi$ é um homomorfismo de grupos.

2) $\varphi(-1)=-1$.

3) $\langle\alpha, \beta\rangle \simeq\langle\gamma, \delta\rangle \Longrightarrow \varphi(\alpha)+\varphi(\beta)=\varphi(\gamma)+\varphi(\delta)$ (soma em $\mathbb{Z})$.

Demonstração.

(i) Como $(\{ \pm 1\}, \wp(\{ \pm 1\}))$ é compacto Hausdorff e com base de clopens, então $\{ \pm 1\}^{\dot{\mathbb{K}} / \dot{\mathbb{K}}^{2}}$ é compacto Hausdorff e com base de clopens e assim um espaço booleano.

(ii) Se $P, Q \in X_{\mathbb{K}}$ e $P \neq Q$, então existe $x \in \dot{\mathbb{K}}$ tal que $x \in P$ e $-x \in Q$, logo $\sigma_{P}\left(x \dot{\mathbb{K}}^{2}\right)=1 \neq-1=$ $\sigma_{Q}\left(x \dot{K}^{2}\right)$.

(iii) Imediato a partir de (ii) e (†).

(iv)Se $\varphi \notin \sigma\left[X_{\mathbb{K}}\right]$, então $T=\{0\} \cup\{x \in \mathbb{K}: \varphi \circ \pi(x)=1\}$ é tal que $-1 \in T$ ou $T+T \nsubseteq T$ ou $T . T \nsubseteq$ $T$ ou $\quad \dot{K} \nsubseteq T \cup-T$ o que nos fornece, respectivamente, para certos $x_{1}, x_{2} \in \dot{\mathbb{K}}: \varphi \in H_{-1}$ (1) ou $\varphi \in$ $H_{1,1,-1}\left(x_{1}, x_{2}, x_{1}+x_{2}\right)$ ou $\varphi \in H_{1,1,-1}\left(x_{1}, x_{2}, x_{1} . x_{2}\right)$ ou $\varphi \in H_{-1,-1}\left(x_{1},-x_{2}\right)$ ou $H_{-1,-1}\left(x_{1}, x_{1}\right)$, mas cada um desses abertos básicos é disjunto de $\sigma\left[X_{\mathbb{K}}\right]$.

(v)Todo subespaço fechado de um espaço booleano é também booleano.

(vi) Claramente $\sigma_{P}$ é um homomorfismo tal que $\sigma_{P}(-1)=-1$. Além disso, se $\langle\alpha, \beta\rangle \simeq\langle\gamma, \delta\rangle$,então $\alpha \beta \dot{\mathbb{K}}^{2}=\gamma \delta \dot{\mathbb{K}}^{2}$ e $\alpha \xi^{2}+\beta \eta^{2}=\gamma \xi^{2}+\delta \eta^{2}$ e, portanto $\sigma_{P}(\alpha)+\sigma_{P}(\beta)=\sigma_{P}(\gamma)+\sigma_{P}(\delta)$.

Inversamente, seja $\varphi \in\{ \pm 1\}^{\mathbb{K} / \mathbb{K}^{2}}$ cumprindo 1), 2) e 3) e considere $P:=\{0\} \cup \operatorname{ker}(\varphi \circ \pi)$, onde $\pi: \dot{\mathbb{K}} \longrightarrow \dot{\mathbb{K}} / \dot{\mathbb{K}}^{2}$ é a projeção canônica. Segue de (1) que $P . P \subseteq P$ e de $(2)$ obtemos $-1 \notin P$. Caso $a \in \dot{\mathbb{K}} \backslash P$, então $\varphi(\bar{a})=-1$ e de $(1)$ e $(2)$ obtemos $\varphi\left({ }^{-} a\right)=\varphi\left({ }^{-} 1\right) \varphi(\bar{a})=(-1)(-1)=1$, $\operatorname{logo}-a \in P$, i.e. $P \cup-P=\mathbb{K}$. Verificaremos agora que $P+P \subseteq P$. Sejam $\alpha, \beta \in \dot{K}$ e seja $\gamma=\left(\alpha x^{2}+\beta y^{2}\right) \in D(\langle\alpha, \beta\rangle)$. Assim $\langle\alpha, \beta\rangle \simeq\langle\gamma, \alpha, \beta, \gamma\rangle$, e segue que

$$
\begin{aligned}
\varphi(\alpha)+\varphi(\beta) & =\varphi(\gamma)+\varphi(\alpha \beta \gamma)) \\
& =\varphi(\gamma)+\varphi(\alpha) \varphi(\beta) \varphi(\gamma) \\
& =\varphi(\gamma)(1+\varphi(\alpha)(\varphi(\beta)) .
\end{aligned}
$$

e portanto, quando $\varphi(\alpha)=\varphi(\beta)=1$ ter-se-á $1+\varphi(\alpha) \varphi(\beta)=2$ e assim $\varphi(\gamma)=1$, em particular, $P+P \subseteq P$. Logo, $P \in X_{\mathbb{K}}$ e $\varphi=\sigma_{P}$.

Em DM1 é definida a noção de grupo especial (SG) e de morfismo entre estas estruturas (retomaremos a este tema no último Capítulo). Em particular

Definição 29. Uma aplicação $\varphi: \dot{\mathbb{K}} / \dot{\mathbb{K}}^{2} \longrightarrow\{ \pm 1\}$ é um $S G$-morfismo quando

$\left.S G_{1}\right) \varphi$ é um homomorfismo de grupos

$\left.S G_{2}\right) \varphi(-1)=-1$ 
$\left.S G_{3}\right)\langle\alpha, \beta\rangle \simeq\langle\gamma, \delta\rangle \Longrightarrow \varphi(\alpha)+\varphi(\beta)=\varphi(\gamma)+\varphi(\delta)($ soma em $\mathbb{Z})$

Denotamos por $S G\left(\dot{\mathbb{K}} / \dot{\mathbb{K}}^{2},\{ \pm 1\}\right)$ o conjunto de todos os $S G$-morfismos de $\dot{\mathbb{K}} / \dot{\mathbb{K}}^{2}$ em $\{ \pm 1\}$.

Proposição 36. Existem bijeções canônicas

$$
X_{\mathbb{K} \succ} \gg S G\left(\dot{\mathbb{K}} / \dot{\mathbb{K}}^{2},\{ \pm 1\}\right) \gg \operatorname{Hom}(W(\mathbb{K}), \mathbb{Z})
$$

Demonstração. A primeira bijeção já foi exibida na proposição anterior. Indicaremos agora a segunda bijeção sem apresentar todos os detalhes.

- Se $\varphi \in S G\left(\dot{\mathbb{K}} / \dot{\mathbb{K}}^{2},\{ \pm 1\}\right)$, definimos uma função $h_{\varphi}: M(\mathbb{K}) \longrightarrow \mathbb{Z}$ tomando uma forma regular diagonal $\left\langle a_{1}, \cdots, a_{n}\right\rangle$ e associando

$h_{\varphi}\left(\left[\left\langle a_{1}, \cdots, a_{n}\right\rangle\right]\right)=\sum_{i=1}^{n} \varphi\left(a_{i}\right)$. O axioma $S G_{3}$ e o teorema de equivalencia de cadeias de Witt garante que esta definição não depende da escolha particular do representante módulo isometria. Verifica-se imediatamente que este é um homomorfismo de semi-anéis e que induzirá um homomorfismo de anéis $h_{\varphi}: W(\mathbb{K}) \longrightarrow \mathbb{Z}$.

- É fácil ver que para cada $h \in \operatorname{Hom}(W(\mathbb{K}), \mathbb{Z})$ obtemos, por restrição, homomorfismos de grupos de $h_{1}: I(\mathbb{K}) \longrightarrow 2 \mathbb{Z}$ e $h_{2}: I^{2}(\mathbb{K}) \longrightarrow 4 \mathbb{Z}$, desta forma obtemos homomorfismo de grupo $\bar{h}: I(\mathbb{K}) / I^{2}(\mathbb{K}) \longrightarrow 2 \mathbb{Z} / 4 \mathbb{Z}$. Como $\mathbb{K} / \dot{\mathbb{K}}^{2} \cong I(\mathbb{K}) / I^{2}(\mathbb{K})$ (veja Lema ??) e $\{-1,+1\} \cong$ $2 \mathbb{Z} / 4 \mathbb{Z}$, obtemos homomorfismo de grupos $\varphi_{h}: \dot{\mathbb{K}} / \dot{\mathbb{K}}^{2} \longrightarrow\{-1,+1\}$. Segue da construção que $\varphi_{h} \in S G\left(\dot{\mathbb{K}} / \dot{\mathbb{K}}^{2},\{ \pm 1\}\right)$.

- Verifica-se rapidamente que os mapas indicados acima constituem bijeções inversas.

Observação 24. Para toda extensão de corpos $j: \mathbb{F} \longrightarrow \mathbb{K}$, tem-se um diagrama comutativo que expressa isomorfismos naturais de funtores contravariantes

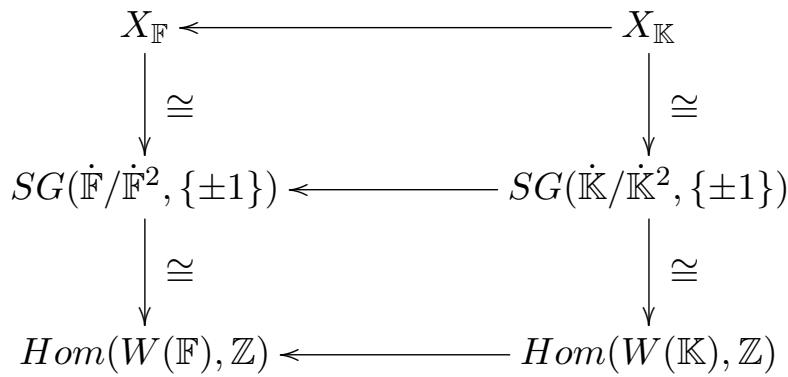

Seja $\mathbb{K}_{P}$ um fecho real para cada corpo ordenado $(\mathbb{K}, P)$. Sendo $r_{P}: \mathbb{K} \longrightarrow \mathbb{K}_{P}$ o homomorfismo inclusão teremos um mapa funtorial $W\left(r_{P}\right): W(\mathbb{K}) \longrightarrow W\left(\mathbb{K}_{P}\right)$. Por outro lado, a Proposição 30 nos fornece um isomorfismo canônico $W\left(\mathbb{K}_{P}\right) \cong \mathbb{Z}$. Como vimos na na Proposição 31, o homomorfismo de assinatura $\operatorname{sgn}_{P}: W(\mathbb{K}) \longrightarrow \mathbb{Z}$ coincide com a composição

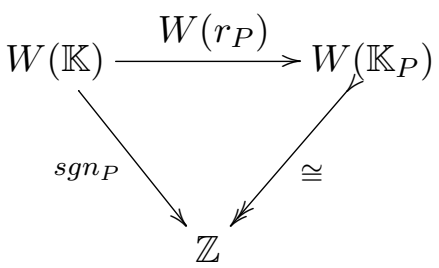


Observe que $\operatorname{sgn}_{P}$ é sobrejetor e além disso não depende da escolha particular de $\mathbb{K}_{P}$, uma vez que, fechos reais são únicos a menos de $\mathbb{K}$ - isomorfismos.

Definição 30. A aplicação

$$
\begin{aligned}
\text { sgn : } W(\mathbb{K}) & \longrightarrow \prod_{P \in X_{\mathbb{K}}} W\left(\mathbb{K}_{P}\right) \cong \prod_{P \in X_{\mathbb{K}}} \mathbb{Z} \\
\phi & \longmapsto
\end{aligned}
$$

é chamada de assinatura total.

Proposição 37. Considere o subespaço do espaço produto $\mathcal{C}\left(X_{\mathbb{K}}, \mathbb{Z}\right) \subseteq \prod_{P \in X_{\mathbb{K}}} \mathbb{Z}$, onde $\mathbb{Z}$ é munido da topologia discreta, o anel das funções contínuas $X_{\mathbb{K}} \longrightarrow \mathbb{Z}$. Então, $\operatorname{Im}(\operatorname{sgn}) \subseteq \mathcal{C}\left(X_{\mathbb{K}}, \mathbb{Z}\right)$.

Demonstração. $\operatorname{sgn}(\phi)(P)=\operatorname{sgn}_{P}(\phi)$ é contínua,pois $(\operatorname{sgn}\langle\alpha\rangle)^{-1}[\{\varepsilon\}]=H(\varepsilon \alpha)$, $\forall \alpha \in \dot{\mathbb{K}}, \varepsilon \in\{ \pm 1\}$ e $(\operatorname{sgn}\langle\alpha\rangle)^{-1}[\{z\}]=\emptyset, \forall z \in \mathbb{Z} \backslash\{ \pm 1\}$. Assim,

$$
\operatorname{sgn}\left\langle\alpha_{1}, \cdots, \alpha_{n}\right\rangle=\sum_{i=1}^{n} \operatorname{sgn}\left(\alpha_{i}\right)
$$

é contínua por ser uma soma de funções contínuas.

Complementando a informação sobre $\operatorname{Spec}(W(\mathbb{K}))$ apresentada na Proposição 25, temos a:

Proposição 38. Se $\mathfrak{J}$ é um ideal primo e próprio de $W(\mathbb{K})$ que não contém 2. Então,

$$
\hat{\mathfrak{J}}:=\{0\} \cup\{a \in \dot{\mathbb{K}}:\langle a\rangle \equiv\langle 1\rangle(\bmod \quad \mathfrak{J})\}
$$

é uma ordem em $\mathbb{K}$

Demonstração. $\quad$ - Claramente $\hat{\mathfrak{J}} \cdot \hat{\mathfrak{J}} \subseteq \hat{\mathfrak{J}}$.

- Como $\langle a\rangle^{2}=1$ em $W(\mathbb{K})$, para cada $a \in \dot{K}$, temos que no domínio $W(\mathbb{K}) / \mathfrak{J}$ ocorre $\overline{\langle a\rangle}=1$ ou $\overline{\langle-a\rangle}=1, \log \mathrm{K}=\hat{\mathfrak{J}} \cup-\hat{\mathfrak{J}}$.

- Como $2 \notin \mathfrak{J}$, temos $\langle-1\rangle \not \equiv 1(\bmod \quad \mathfrak{J}), \log 0-1 \notin \hat{\mathfrak{J}}$.

- Sejam $a, b \in \hat{\mathfrak{J}}$. Da isometria $\langle a, b\rangle \simeq\langle a+b\rangle\langle 1, a b\rangle, \forall a+b \neq 0$, resulta $2 \equiv 2\langle a+b\rangle(\bmod \mathfrak{J})$. Como $2 \notin \mathfrak{J}$, obtemos $\langle a+b\rangle \equiv 1(\bmod \quad \mathfrak{J})$ e, portanto $a+b \in \hat{\mathfrak{J}}$. Assim $\hat{\mathfrak{J}}+\hat{\mathfrak{J}} \subseteq \hat{\mathfrak{J}}$.

\subsection{O princípio local-global de Pfister}

Definição 31. Seja A um anel comutativo. O nilradical $\mathfrak{N}(A)$ de $A$ é o ideal de todos os elementos nilpotentes de A.

Lema 16. Seja $A$ um anel comutativo com unidade. Seja $\mathfrak{P}$ o conjunto de todos os ideais primos de $A$. $O$ nilradical de $A$, denotado por $\mathfrak{N}(A)$, pode ser escrito como

$$
\mathfrak{N}(A)=\bigcap\{\mathfrak{J} \in \wp(A): \mathcal{J} \in \mathfrak{P}\}
$$

Demonstração. Para uma demonstração desse resultado consultar [NJac1]. 
Proposição 39. Se $\mathbb{K}$ é um corpo não formalmente real, então

$$
W(\mathbb{K})=W_{\text {tors }}(\mathbb{K})
$$

Demonstração. Se $\mathbb{K}$ é um corpo não formalmente real, então segue da Proposição 38 que $I(\mathbb{K})$ é o único ideal primo de $W(\mathbb{K})$. Assim, $I(\mathbb{K})=\mathfrak{N}(W(\mathbb{K}))$ pelo Lema 16 .

\section{Lema 17.}

1) Uma forma quadrática anisotrópica $\phi$ sobre um corpo $\mathbb{K}$ é isotrópica sobre $\mathbb{F} \doteq \mathbb{K}(\sqrt{a})$ se $e$ somente se $\phi \simeq(\langle b\rangle \cdot\langle\langle-a\rangle\rangle) \perp \phi^{\prime}$ para algum $b \in \dot{\mathbb{K}}$.

2) Uma forma quadrática $\phi$ sobre $\mathbb{K}$ torna-se hiperbólica sobre $\mathbb{F}$ se e somente se $\phi \simeq\langle\langle-a\rangle\rangle \otimes \psi$ para alguma forma quadrática $\psi$ sobre $\mathbb{F}$.

3) $\operatorname{Ker}(W(\mathbb{K}) \stackrel{W(r)}{\longrightarrow} W(\mathbb{F}))=\langle\langle-a\rangle\rangle W(\mathbb{K})$.

Demonstração.

1) Como $\langle\langle-a\rangle\rangle \simeq \mathbb{H}$ sobre $\mathbb{F}$, a implicação $(\Leftarrow)$ é imediata. Reversamente, suponhamos que $\phi$ torna-se isotrópica sobre $\mathbb{F}$. Tomemos $\left\langle b_{1}, \ldots, b_{n}\right\rangle$ uma diagonalização para $\phi$. Então, existem $x \doteq\left(x_{1}, \ldots, x_{n}\right), y \doteq\left(y_{1}, \ldots, y_{n}\right) \in \mathbb{K}^{n}$ não todos nulos tais que

$$
\sum_{i=1}^{n} b_{i}\left(x_{i}+y_{i} \sqrt{a}\right)^{2}=\sum_{i=1}^{n} b_{i}\left(x_{i}^{2}+a y_{i}^{2}\right)+\sum_{i=1}^{n} b_{i} x_{i} y_{i} \sqrt{a}=0
$$

desta última tiramos $x \perp y$ e $\phi(x)+a \phi(y)=0$ e portanto $\phi$ contém a forma binária

$$
\langle\phi(x), \phi(y)\rangle \simeq\langle-a \phi(y), \phi(y)\rangle \simeq\langle\phi(y)\rangle \cdot\langle\langle-a\rangle\rangle
$$

2) Vamos usar indução em $m \doteq \operatorname{dim}(\phi) / 2$. O caso $m=0$ é trivial. Se $m>0$,então o ítem anterior fornece uma isometria $\phi \simeq\langle\langle-a\rangle\rangle\langle b\rangle \perp \phi^{\prime}$, onde $b \in \dot{\mathbb{K}}$ e $\operatorname{dim}\left(\phi^{\prime}\right) / 2=m-1$.Pelo teorema do cancelamento de Witt $\phi^{\prime}$ torna-se hiperbólica sobre $\mathbb{F}$.Existe ,por hipótese de indução, uma forma $\phi^{\prime \prime}$ tal que $\phi^{\prime} \simeq\langle\langle-a\rangle\rangle \otimes \phi^{\prime \prime}$. Assim, teremos

$$
\phi \simeq\langle b\rangle\langle\langle-a\rangle\rangle \perp \phi^{\prime} \simeq\langle b\rangle\langle\langle-a\rangle\rangle \perp \phi^{\prime \prime} \otimes\langle\langle-a\rangle\rangle \simeq \psi \otimes\langle\langle-a\rangle\rangle
$$

com $\psi=\langle b\rangle \perp \phi^{\prime \prime}$. A recíproca decorre do fato que $\langle\langle-a\rangle\rangle$ torna-se hiperbólica sobre $\mathbb{F}$.

3) Segue imediatamente do item anterior.

Lema 18. Seja $\phi$ uma forma quadrática sobre $\mathbb{K}$ de dimensão $2 m$ que torna-se hiperbólica sobre $\mathbb{K}(\sqrt{a})$, então

1) $-a \cdot \phi \simeq \phi$ sobre $\mathbb{K}$.

2) Se $\phi$ é anisotrópica sobre $\mathbb{K}$, então $d(\phi)=(-a)^{m}$.

3) Se torna-se hiperbólica também sobre $\mathbb{K}(\sqrt{-a})$, então $2 \phi=0 \in W(\mathbb{K})$.

Demonstração.

1) Pelo lema anterior podemos escrever $\phi \simeq r \mathbb{H} \perp\langle\langle-a\rangle\rangle \otimes \psi$ para alguma $\mathbb{K}-$ forma $\psi$. Como $-a \mathbb{H} \simeq \mathbb{H} \mathrm{e}-a \cdot\langle\langle-a\rangle\rangle \simeq\langle\langle-a\rangle\rangle$ ter-se-á $-a \cdot \phi \simeq \phi$.

2) Se $\phi$ for anisotrópica, então $r=0$ e $\phi \simeq \psi \otimes\langle\langle-a\rangle\rangle$. Computando determinantes módulo quadrados teremos $d(\phi)(-a)^{m}=d(\phi) \operatorname{logo} d(\phi)=(-a)^{m}$ (módulo $\dot{\mathbb{K}}^{2}$ ) 
3) Se $\mathbb{K}(\sqrt{-a})=\mathbb{K}$, então $\phi=0 \in W(\mathbb{K})$. Caso contrário teremos, usando (1), $-a \cdot \phi \simeq \phi$ e $a \cdot \phi \simeq \phi$. Adicionando estas últimas ter-se-á $2 \phi=0 \in W(\mathbb{K})$.

\section{Teorema 19. Princípio local-global de Pfister (PLGP)}

A seguinte sequência

$$
0 \longrightarrow W_{\text {tors }}(\mathbb{K}) \longleftrightarrow W(\mathbb{K}) \stackrel{r^{*}}{\longrightarrow} \prod_{P \in X_{\mathbb{K}}} W\left(\mathbb{K}_{P}\right)
$$

é exata, onde $W_{\text {tors }}(\mathbb{K})$ denota o subgrupo de torsão de $W(\mathbb{K})$ e $r^{*} \doteq\left(W\left(r_{P}\right)\right)_{P \in X_{\mathbb{K}}}$. Além disso, todo elemento de $W_{\text {tors }}$ é torsão 2-primário.

Demonstração. Como $\prod_{P \in X_{\mathbb{K}}} W\left(\mathbb{K}_{P}\right) \cong \prod_{P \in X_{\mathbb{K}}} \mathbb{Z}$ é livre de torsão, segue que $W_{\text {tors }}(\mathbb{K}) \subseteq K e r($ sgn $)$. Para terminar, vamos mostrar que se $\phi \in W(\mathbb{K})$ não é de 2-torsão primário, então $\operatorname{sgn}_{P}(\phi) \neq 0$ para alguma ordem $P \in X_{\mathbb{K}}$. Existe, pelo lema de Zorn, um corpo $\mathbb{F} \supseteq \mathbb{K}$ dentro do fecho algébrico de $\mathbb{K}$ que é maximal com relação a propriedade que $\phi_{\mathbb{F}} \in W(\mathbb{F})$ não é de torsão 2-primário. Afirmamos que $\mathbb{F}$ é um corpo euclidiano. Pela Proposição 39, o corpo $\mathbb{F}$ deve ser formalmente real. Suponhamos, por um momento, que $\mathbb{F}$ possui um elemento $a \neq 0$ tal que $a \notin \pm \dot{\mathbb{F}}^{2}$. Assim, pela maximalidade de $\mathbb{F}$ a forma $\phi_{\mathbb{F}}$ deverá ser 2-primária em $\mathbb{F}(\sqrt{a})$ e $\mathbb{F}(\sqrt{-a})$ de modo que para um inteiro $\mathrm{N}$ suficientemente grande ter-se-á $2^{N} \phi_{\mathbb{F}}$ hiperbólica sobre $\mathbb{F}(\sqrt{a})$ e $\mathbb{F}(\sqrt{-a})$. Mas o Lema 18. (3) acima nos fornece $2 \cdot 2^{N} \phi_{\mathbb{F}}=0 \in W(\mathbb{F})$, uma contradição. Isso mostra que $\mathbb{F}$ é euclidiano e além disso $\operatorname{sgn}_{P}(\phi) \neq 0$ para uma ordem $P \in X_{\mathbb{K}}$ induzida em $\mathbb{K}$ pela única ordem em $\mathbb{F}$.

\section{Observação 25.}

(1) Observe que $-1 \in \sum \mathbb{K}^{2} \Longleftrightarrow W_{\text {tors }}(\mathbb{K}) \neq \emptyset$. Se fosse $W_{\text {tors }}(\mathbb{K})=\emptyset$, então pela proposição 29(2) teríamos que $\mathbb{K}$ é pitagórico e $-1 \notin \sum \mathbb{K}^{2}$. Inversamente, se $-1 \in \sum \mathbb{K}^{2}$, então qualquer fecho real $\mathbb{K}_{P}$ seria pitagórico e $-1 \notin \sum \mathbb{K}_{P}^{2}$, donde pela proposição 29 (1) ter-se-ia $W\left(\mathbb{K}_{P}\right) \cong \mathbb{Z} / 2 \mathbb{Z}$ e como pela proposição anterior o núcleo de $r^{*}: W(\mathbb{K}) \longrightarrow W\left(\mathbb{K}_{P}\right)$ é 2-torsão, segue que $W_{\text {tors }} \neq \emptyset$.

(2) Uma caracterização alternativa para um corpo formamalmente real seria a seguinte :

$$
\mathbb{K} \text { é formalmente real se, e somente se, }\langle 1,1\rangle \text { é nilpotente em } \mathcal{W}_{g}(\mathbb{K})
$$

(3) No capítulo 5 daremos uma caracterização de um corpo formalmente real em função de sua K-teoria graduada.

Observação 26. Uma forma de enunciar a primeira parte do teorema anterior é a seguinte : Duas formas quadráticas $\phi$ e $\phi^{\prime}$ sobre $\mathbb{K}$ têm a mesma assinatura relativa a todas as ordens em $\mathbb{K}$ se $e$ somente se $n \phi=n \phi^{\prime}$ para algum inteiro $n>0$. A segunda parte do teorema, nos diz que nessas circunstâncias podemos escolher $n$ da forma $2^{r}$ para algum $r$. Finalmente, uma forma alternativa para o teorema anterior é dizer que se uma forma quadrática $\phi$ é hiperbólica em todos os fechos reais de $\mathbb{K}$, então $2^{r} \cdot \phi$ também o será para todo $r \geq 0$ e isso justifica o nome princípio local - global.

Definição 32. Para cada $S \subseteq X_{\mathbb{K}}$. Definimos a função $\xi_{S}: X_{\mathbb{K}} \longrightarrow \mathbb{Z}$ por $\xi_{S}(P)=1$ se $P \in S$ e $\xi_{S}(P)=0$ caso contrário. Em outros termos, $\xi_{S}$ é a função característica em $S$.

Lema 20. Sejam $\Omega, \Lambda \in \wp\left(X_{\mathbb{K}}\right)$ clopens tais que valham as igualdades $2^{r} \xi_{\Omega}=\operatorname{sgn}(\phi)$ e $2^{s} \xi_{\Lambda}=$ $\operatorname{sgn}(\psi)$, para certos $r, s \in \mathbb{N} e \phi \in I^{r}(\mathbb{K}), \psi \in I^{s}(\mathbb{K})$. Então, existem $t \in \mathbb{N}$ e $\rho \in I^{t}(\mathbb{K})$ tais que $2^{t} \xi_{\Omega \cup \Lambda}=\operatorname{sgn}(\rho)$.

Demonstração. Como, em geral, $2^{n} \operatorname{sgn}(\theta)=\operatorname{sgn}\left(2^{k} \otimes \theta\right)$, podemos supor $r=s$. Agora multiplicamos a equação

$$
\xi_{\Omega \cup \Lambda}=\xi_{\Omega}+\xi_{\Lambda}-\xi_{\Omega} \xi_{\Lambda}
$$


por $2^{2 r}$ e obtemos $2^{t} \xi_{\Omega \cup \Lambda}=\operatorname{sgn}(\rho)$ onde $t=2 r$ e $\rho=2^{r}(\phi+\psi)-\phi \psi \in I^{t}(\mathbb{K})$.

Lema 21. Para todo clopen $\Omega \in \wp\left(X_{\mathbb{K}}\right)$, existe $\phi \in I^{n}(\mathbb{K})$ tal que $\operatorname{sgn}(\phi)=2^{n} \xi_{\Omega}$.

Demonstração. Como $X_{\mathbb{K}}$ é compacto, $\Omega$ admite escrita

$$
\Omega=\bigcup_{1 \leq j \leq n} H\left(\alpha_{1}^{j}, \cdots, \alpha_{k_{j}}^{j}\right)
$$

além disso,

$$
\operatorname{sgn}\left\langle\left\langle\alpha_{1}^{j}, \cdots, \alpha_{k_{j}}^{j}\right\rangle\right\rangle=\prod_{i=1}^{k_{j}}\left(1+\operatorname{sgn}\left(\alpha_{i}^{j}\right)\right)=2^{k_{j}} \xi_{H\left(\alpha_{1}^{j}, \cdots, \alpha_{k_{j}}^{j}\right)}, \forall j \in\{1,2, \cdots, n\}
$$

Assim, o resultado segue do lema anterior.

Proposição 40. Coker(sgn) é um grupo de torsão 2-primário.

Demonstração. Se $\varphi \in \mathcal{C}\left(X_{\mathbb{K}}, \mathbb{Z}\right)$, então $\Omega_{j}=\varphi^{-1}[\{j\}], \forall j \in \mathbb{Z}$ são clopens e como $X_{\mathbb{K}}$ é compacto $S=\left\{j: \Omega_{j} \neq \emptyset\right\}$ é finito e portanto $\varphi=\sum_{j \in S} j \xi_{\Omega_{j}}$. Pelo lema anterior, concluimos que $2^{n} \varphi \in$ $\operatorname{Im}(\operatorname{sgn})$.

Proposição 41. Se $F, G \in \wp\left(X_{\mathbb{K}}\right)$ são fechados disjuntos em $X_{\mathbb{K}}$, então existe $\phi \in I^{n}(\mathbb{K})$ tal que $\operatorname{sgn}(\phi)(F)=0$ e $\operatorname{sgn}(\phi)(G)=2^{n}$.

Demonstração. Como $X_{\mathbb{K}}$ é booleano, existe um clopen $\Omega$ tal que $G \subseteq \Omega$ e $F \subseteq X_{\mathbb{K}} \backslash \Omega$ e o resultado segue do Lema 21

Proposição 42. $\operatorname{sgn}(\phi) \in \mathcal{C}\left(X_{\mathbb{K}}, 2^{n} \mathbb{Z}\right)$ se, e somente se, $2^{t} \phi \in I^{t+n}$ para algum $t \in \mathbb{N}$.

Demonstração. Claramente $\operatorname{sgn}(I(\mathbb{K})) \subseteq \mathcal{C}\left(X_{\mathbb{K}}, 2 \mathbb{Z}\right)$. Como sgn é morfismo de anéis, resulta $\operatorname{sgn}\left(I^{m}(\mathbb{K})\right) \subseteq$ $\mathbb{C}\left(X_{\mathbb{K}}, 2^{m} \mathbb{Z}\right), \forall m$. Assim, $2^{t} \phi \in I^{t+n}(\mathbb{K}) \Longrightarrow 2^{t} \operatorname{sgn}(\phi) \in 2^{t+n} \mathbb{Z}$ e, portanto $\operatorname{sgn}(\phi) \in \mathcal{C}\left(X_{\mathbb{K}}, 2^{n} \mathbb{Z}\right)$. Inversamente, a família $\left(\Omega_{j}:=(\operatorname{sgn}(\phi))^{-1}\left(2^{n} j\right)_{j \in \mathbb{Z}}\right)$ é um cobertura por clopens do compacto $X_{\mathbb{K}}$. Assim, temos uma soma finita $\operatorname{sgn}(\phi)=\sum 2^{n} j \xi_{\Omega_{j}}$. Pelo Lema 21 existem $m_{j}$ e $\phi_{j} \in I^{m_{j}}(\mathbb{K})$ com $2^{m_{j}} \xi_{\Omega_{j}}$. Assumindo $m_{j}=m, \forall j$ teremos $\left.2^{m} \operatorname{sgn}(\phi)=\sum 2^{n} j \operatorname{sgn}\left(\phi_{j}\right)\right)$. Pelo PLGP, resulta $2^{m} \phi \in\left(\sum 2^{n} j \phi_{j}+W_{\text {tors }}(\mathbb{K})\right.$.Lembrando que $W_{\text {tors }}(\mathbb{K})$ é subgrupo de torsão 2-primário, obtemos o resultado.

Observação 27. Uma questão importante, que retornaremos no Capítulo final, é como se relacionam as afirmações

(1) $\operatorname{sgn}(\phi) \in \mathcal{C}\left(X_{\mathbb{K}}, 2^{n} \mathbb{Z}\right)$

(2) $2^{t} \phi \in I^{t+n}$ para algum $t \geq 0$

(3) $\phi \in I^{n}(\mathbb{K})+W_{\text {tors }}(\mathbb{K})$

Vimos acima que (1) $\Longleftrightarrow(2)$. Claramente, $(3) \Longrightarrow(2)$ e (3) $\Longrightarrow(1) ;$ em Lam4 T.Y.Lam perguntou se valem as recíprocas. Essas questôes ficaram conhecidas como conjecturas de Lam. As mesmas questões foram levantadas por M. Marshal para corpos formalmente real e pitagóricos e o mesmo deu uma resposta afirmativa no caso em que o espaço de ordens é finito. A conjectura geral 
foi resolvida por Dickmann-Miraglia ([DM2, [DM3]) e Arason-Elman [AE usando a solução da conjectura de Milnor provada por Voevodsky Voe1 e Orlov-Vishik-Voevodsky OVV].

Proposição 43. $W(\mathbb{K}) \cong W_{\text {tors }}(\mathbb{K}) \oplus G$, onde $G$ é um grupo abeliano livre com rank $(G)=$ $\operatorname{rank}\left(C\left(X_{\mathbb{K}}, \mathbb{Z}\right)\right)$.Se $\left|X_{\mathbb{K}}\right|=r<\infty$, então $\operatorname{rank}(G)=r$.

Demonstração. Usando PLGP e o teorema do homomorfismo teremos

$$
W(\mathbb{K}) / W_{\text {tors }}(\mathbb{K}) \cong \operatorname{Im}(\text { sgn }) \subseteq C\left(X_{\mathbb{K}}, \mathbb{Z}\right)
$$

como $C\left(X_{\mathbb{K}}, \mathbb{Z}\right)$ é um subconjunto do conjunto das funções limitadas $B\left(X_{\mathbb{K}}, \mathbb{Z}\right)$ de $X_{\mathbb{K}}$ em $\mathbb{Z}$ segue que $W(\mathbb{K}) / W_{\text {tors }}(\mathbb{K})$ pode ser visto como um subconjunto de $B\left(X_{\mathbb{K}}, \mathbb{Z}\right)$.Um teorema clássico devido a Specker e Nobeling afirma que $B\left(X_{\mathbb{K}}, \mathbb{Z}\right)$ é um grupo abeliano livre. Desde que, todo subgrupo de um grupo abeliano livre é livre, segue que a sequência exata curta abaixo cinde.

$$
0 \longrightarrow W_{\text {tors }}(\mathbb{K}) \longleftrightarrow W(\mathbb{K}) \stackrel{\text { sgn }}{\longrightarrow} \operatorname{Im}(\text { sgn })
$$

Além disso, $\operatorname{rank}(\operatorname{Im}(\operatorname{sgn}))=\operatorname{rank}\left(C\left(X_{\mathbb{K}}\right), \mathbb{Z}\right)$, pois coker $(\operatorname{sgn})=0$.Finalmente, note que se $\left|X_{\mathbb{K}}\right|=r<\infty$,então

$$
C\left(X_{\mathbb{K}}, \mathbb{Z}\right)=\mathbb{Z}^{X_{\mathbb{K}}} \cong \mathbb{Z}^{r}
$$

onde $\mathbb{Z}^{X_{\mathbb{K}}}$ denota o conjunto das funções de $X_{\mathbb{K}}$ em $\mathbb{Z}$.

\subsection{O grupo de classes de quadrado}

Nestas últimas seções deste capítulo, completaremos o trabalho iniciado na seção 2 sobre informações a respeito de (sub)quocientes dos anéis de Witt e de Witt-Grothendieck.

A aplicação $d: M(\mathbb{K}) \longrightarrow \mathbb{K}^{*} / \mathbb{K}^{* 2}$ é um morfismo de monóides, uma vez que $d(\langle\rangle)=1 \mathrm{e}$ $d\left(\phi \oplus \phi^{\prime}\right)=d(\phi) d\left(\phi^{\prime}\right)$. Nesse caso, temos sua extensão a um homomorfismo de grupos

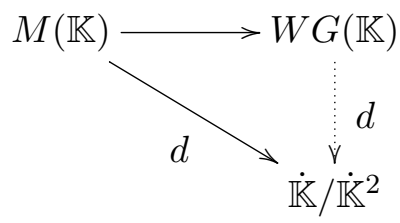

Claramente ambas as funções acima são sobrejetoras. Agora considere o problema de "estender" $d$ para $W(\mathbb{K})$. Inicialmente notamos que não existe morfismo de grupos $\tilde{d}: W(\mathbb{K}) \longrightarrow \mathbb{K}^{*} / \mathbb{K}^{* 2}$ que legitime a comutatividade do diagrama

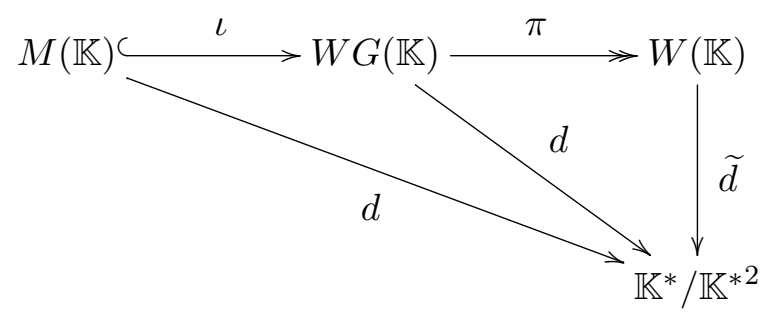

Em outros termos o morfismo $d$ não se fatora através de $W(\mathbb{K})$ e isso se deve ao fato de termos $d(n \mathbb{H})=(-1)^{n} \dot{K}^{2}$. Veremos que uma quebra de complexidade de $W(\mathbb{K})$ será dada por uma de- 
composição do anel quociente $W(\mathbb{K}) / I^{2}(\mathbb{K}) \cong\left(\mathbb{Z} / 2 \mathbb{Z} \oplus \dot{\mathbb{K}} / \dot{\mathbb{K}}^{2}\right)$, onde $\mathbb{Z} / 2 \mathbb{Z} \oplus \dot{\mathbb{K}} / \dot{\mathbb{K}}^{2}$ será munido de uma multiplicação conveniente. Começamos procurando por uma fórmula que altere o sinal de $d$ de modo que espaços triviais (= hiperbólicos) em $W(\mathbb{K})$ tenham "discriminante"trivial.

Definição 33. Definimos o discriminante com sinal de um espaço regular $\phi$ por

$$
d_{ \pm}(\phi)=(-1)^{\frac{n(n-1)}{2}} d(\phi) .
$$

Observação 28. 1. Para cada $n \in \mathbb{N}$,

$$
d_{ \pm}(n \mathbb{H})=(-1)^{\frac{2 n(2 n-1)}{2}} d(n \mathbb{H})=(-1)^{\frac{2 n(2 n-1)}{2}}(-1)^{n}=(-1)^{2 n^{2}}=1 .
$$

2. Se $\phi, \psi$ são formas regulares de dimensões, respectivamente, $k, m$, temos

$$
\begin{aligned}
d_{ \pm}(\phi \oplus \psi) & =(-1)^{\frac{(k+m)(k+m-1)}{2}} d(\phi \oplus \psi) \\
& =\quad(-1)^{\frac{(k+m)(k+m-1)}{2}} d(\phi \oplus \psi) \\
& =\quad(-1)^{\frac{(k+m)(k+m-1)}{2}} d(\phi) \cdot d(\psi) \\
& =(-1)^{\frac{(k+m)(k+m-1)}{2}}(-1)^{\frac{k(k-1)}{2}}(-1)^{\frac{m(m-1)}{2}} d_{ \pm}(\phi) \cdot d_{ \pm}(\psi) \\
& =
\end{aligned}
$$

3. Se $\psi \simeq(n \mathbb{H} \oplus \phi)$, e escrevemos $\operatorname{dim}(\phi)=k$, então

$$
\begin{aligned}
d_{ \pm}(\psi) & =(-1)^{2 n \cdot k} d_{ \pm}(n \mathbb{H}) d_{ \pm}(\phi) \\
& =(-1)^{2 n \cdot k} \cdot 1 \cdot d_{ \pm}(\phi) \\
& =\quad d_{ \pm}(\phi) .
\end{aligned}
$$

Como todo elemento de $W(\mathbb{K})$ é representado por alguma forma anisotrópica, do que notamos acima, podemos concluir que:

- Temos um mapa bem definido

$$
\begin{aligned}
d_{ \pm}: W(\mathbb{K}) & \longrightarrow \dot{\mathbb{K}} / \dot{\mathbb{K}}^{2} \\
\phi & \longmapsto d_{ \pm}(\phi)=(-1)^{\frac{\operatorname{dim}(\phi)(\operatorname{dim}(\phi)-1)}{2}} d(\phi)
\end{aligned}
$$

- Este mapa $d_{ \pm}$é um homomorfismo de grupos se, e somente se $-1 \in \dot{\mathbb{K}}^{2}$ (uma hipótese bastante restritiva).

- Por outro lado, o mapa restrito $d_{ \pm \mid}: I(\mathbb{K}) \longrightarrow \dot{\mathbb{K}} / \dot{\mathbb{K}}^{2}$ é um homomorfismo, pois se $\phi$ e $\psi$ são formas de dimensão par $k, m$ temos

$$
d_{ \pm}(\phi \oplus \psi)=(-1)^{k m} d_{ \pm}(\phi) d_{ \pm}(\psi)=d_{ \pm}(\phi) d_{ \pm}(\psi)
$$


- Notando que $d_{ \pm \mid}(\langle 1, a\rangle)=a \cdot \dot{\mathbb{K}}^{2}$, para cada $a \in \dot{\mathbb{K}}$, então $d_{ \pm \mid}: I(\mathbb{K}) \longrightarrow \dot{\mathbb{K}} / \dot{\mathbb{K}}^{2}$ é um epimorfismo.

- Como $d_{ \pm}(\langle 1,-a\rangle \otimes\langle 1,-b\rangle)=d_{ \pm}(\langle 1, a b\rangle) d_{ \pm}(\langle-a,-b\rangle)=(a b) \cdot(-a)(-b) \cdot \dot{\mathbb{K}}^{2}=1 \cdot \dot{\mathbb{K}}^{2}$ e $I^{2}(\mathbb{K})$ é gerado aditivamente por formas de Pfister de grau 2 , temos o epimorfismo induzido $\bar{d}_{ \pm}$: $I(\mathbb{K}) / I^{2}(\mathbb{K}) \longrightarrow \dot{\mathbb{K}} / \dot{\mathbb{K}}^{2}$.

Completamos apresentando a

Proposição 44. $\bar{d}_{ \pm}: I(\mathbb{K}) / I^{2}(\mathbb{K}) \longrightarrow \dot{\mathbb{K}} / \dot{\mathbb{K}}^{2}$ é um isomorfismo (natural) de grupos.

Demonstração. Consideremos a aplicação $h: \dot{\mathbb{K}} / \dot{\mathbb{K}}^{2} \longrightarrow I(\mathbb{K}) / I(\mathbb{K})^{2}, x \cdot \dot{\mathbb{K}}^{2} \mapsto \overline{(\langle x\rangle-\langle 1\rangle)}$. Esta, além de bem definida, é um homomorfismo de grupos, pois por indução temos

$$
\overline{\sum_{i=1}^{n}\left(\left\langle x_{i}\right\rangle-\langle 1\rangle\right)}=\overline{\left\langle\prod_{i=1}^{n} x_{i}\right\rangle-\langle 1\rangle} .
$$

É imediato que $\bar{d}_{ \pm} \circ h=I_{d}$. A sobrejetividade de $h$ decorre da igualdade

$$
\overline{\sum_{i=1}^{n}\left(\left\langle x_{i}\right\rangle-\langle 1\rangle\right)-\sum_{i=1}^{n}\left(\left\langle y_{i}\right\rangle-\langle 1\rangle\right)}=\overline{\left\langle\prod_{i=1}^{n} x_{i}\right\rangle-\left\langle\prod_{i=1}^{n} y_{i}\right\rangle}=\overline{\left\langle-\prod_{i=1}^{n} x_{i} \prod_{i=1}^{n} y_{i}\right\rangle+\langle 1\rangle}
$$

Assim $h$ e $\bar{d}_{ \pm}$são isomorfismos inversos.

Nossa próxima tarefa será estender o isomorfismo $\bar{d}_{ \pm}: I(\mathbb{K}) / I^{2}(\mathbb{K}) \longrightarrow \dot{K}^{2} / \dot{\mathbb{K}}^{2}$ a um isomorfismo $D: W(\mathbb{K}) / I^{2}(\mathbb{K}) \longrightarrow Q(\mathbb{K})$ para algum grupo $Q(\mathbb{K})$ que estende o grupo $\dot{\mathbb{K}} / \dot{\mathbb{K}}^{2}$.

Recordamos (ver seção 2) que $\operatorname{dim}_{0}: W(\mathbb{K}) \longrightarrow \mathbb{Z} / 2 \mathbb{Z}$ denota o epimorfismo de dimensão, que é naturalmente isomorfo a projeção no quociente $W(\mathbb{K}) \longrightarrow W(\mathbb{K}) / I(\mathbb{K})$. Como o diagrama abaixo é comutativo

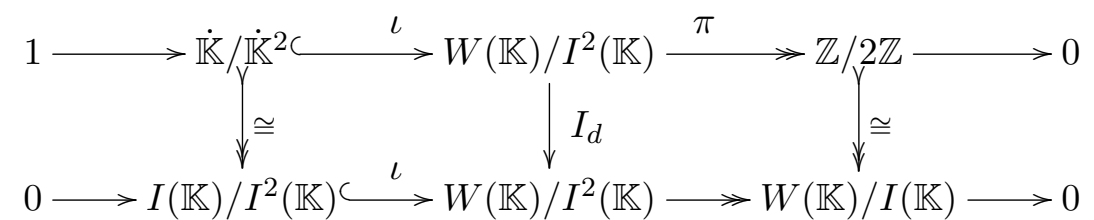

é natural considerar alguma estrutura de grupo sobre o conjunto $\mathbb{Z} / 2 \mathbb{Z} \times \dot{\mathbb{K}} / \dot{\mathbb{K}}^{2}$ que estenda as estruturas de grupo acima, definidas sobre $\mathbb{Z} / 2 \mathbb{Z}$ e sobre $\dot{\mathbb{K}} / \dot{\mathbb{K}}^{2}$.

Assim consideremos $Q(\mathbb{K})=\mathbb{Z} / 2 \mathbb{Z} \times \dot{\mathbb{K}} / \dot{\mathbb{K}}^{2}$ e transformamos $Q(\mathbb{K})$ em um grupo mediante a seguinte operação

$$
\begin{aligned}
\odot: & Q(\mathbb{K}) \times Q(\mathbb{K}) \quad \longrightarrow Q(\mathbb{K}) \\
\left((s, x),\left(s^{\prime}, x^{\prime}\right)\right) & \longmapsto\left(s+s^{\prime},(-1)^{s s^{\prime}} x x^{\prime}\right)
\end{aligned}
$$

Alguns cálculos nos mostram que $(Q(\mathbb{K}), \odot)$ é uma estrutura associativa, comutativa e com elemento neutro $(0,1)$; cada elemento $(s, x) \in Q(\mathbb{K})$ possui inverso $\left(s,(-1)^{s} x\right)$. Além disso, o mapa $x \in \dot{K} / \dot{K}^{2} \mapsto(0, x) \in Q(\mathbb{K})$ é um homomorfismo injetor de grupos que identifica $\dot{\mathbb{K}} / \dot{\mathbb{K}}^{2}$ com um subgrupo de índice 2 de $Q(\mathbb{K})$. Note que $Q(\mathbb{K})$ tem estrutura de produto semidireto se e só se $-1 \in \dot{\mathbb{K}}^{2}$.

Vamos construir o isomorfismo de grupos requerido $D: W(\mathbb{K}) / I^{2}(\mathbb{K}) \longrightarrow Q(\mathbb{K})$ seguindo os passos abaixo. 
- $\left(\operatorname{dim}_{0}, d_{ \pm}\right): M(\mathbb{K}) \longrightarrow Q(\mathbb{K})$ é um homomorfismo de monóides.

De fato, $\left(\operatorname{dim}_{0}, d_{ \pm}\right)(\langle\rangle)=(0,1)$ e se $\operatorname{dim}\left(\phi_{i}\right)=k_{i}$, temos

$$
\begin{gathered}
\left(\operatorname{dim}_{0}, d_{ \pm}\right)\left(\phi_{1} \oplus \phi_{2}\right)=\left(k_{1}+k_{2}+2 \mathbb{Z},(-1)^{\left(k_{1}+k_{2}\right)\left(k_{1}+k_{2}-1\right) / 2} d\left(\phi_{1} \oplus \phi_{2}\right)\right) \\
=\left(k_{1}+k_{2}+2 \mathbb{Z},(-1)^{k_{1} k_{2}} \cdot(-1)^{\left(k_{1}\right)\left(k_{1}-1\right) / 2+\left(k_{2}\right)\left(k_{2}-1\right) / 2} d\left(\phi_{1}\right) d\left(\phi_{2}\right)\right) \\
=\left(\operatorname{dim}_{0}, d_{ \pm}\right)\left(\phi_{1}\right) \cdot\left(\operatorname{dim}_{0}, d_{ \pm}\right)\left(\phi_{2}\right)
\end{gathered}
$$

- Obtemos $\left(\operatorname{dim}_{0}, d_{ \pm}\right): W G(\mathbb{K}) \longrightarrow Q(\mathbb{K})$, um epimorfismo de grupos.

Devido a propriedade universal de $W G(\mathbb{K})$ e porque $\left(\operatorname{dim}_{0}, d_{ \pm}\right): M(\mathbb{K}) \longrightarrow Q(\mathbb{K})$ é um epimorfismo de monóides:

$$
\begin{gathered}
\left(\operatorname{dim}_{0}, d_{ \pm}\right)(\langle a\rangle)=\left(1+2 \mathbb{Z}, a \cdot \dot{\mathbb{K}}^{2}\right) \\
\left(\operatorname{dim}_{0}, d_{ \pm}\right)(\langle 1,-a\rangle)=\left(0+2 \mathbb{Z}, a \cdot \dot{\mathbb{K}}^{2}\right) .
\end{gathered}
$$

- Temos um epimorfismo induzido de grupos $\overline{\left(\operatorname{dim}_{0}, d_{ \pm}\right)}: W(\mathbb{K}) / I^{2}(\mathbb{K}) \longrightarrow Q(\mathbb{K})$.

Como

$$
\left(\operatorname{dim}_{0}, d_{ \pm}\right)(n \mathbb{H})=(2 n+2 \mathbb{Z}, 1)=(0,1),
$$

o epimorfismo $\left(\operatorname{dim}_{0}, d_{ \pm}\right): W G(\mathbb{K}) \longrightarrow Q(\mathbb{K})$, induz um epimorfismo $W(\mathbb{K}) \longrightarrow Q(\mathbb{K})$. Por outro lado, como $I^{2}(\mathbb{K})$ é gerado aditivamente pelo conjunto das formas de Pfister de grau 2 e

$$
\left(\operatorname{dim}_{0}, d_{ \pm}\right)(\langle 1,-a\rangle \otimes\langle 1,-b\rangle)=\left(4+2 \mathbb{Z},(-1)^{4 \cdot 3 / 2} \cdot(1 \cdot(-a),(-b) \cdot(a b))\right)=(0,1),
$$

obtemos o epimorfismo induzido $\overline{\left(\operatorname{dim}_{0}, d_{ \pm}\right)}: W(\mathbb{K}) / I^{2}(\mathbb{K}) \longrightarrow Q(\mathbb{K})$.

- O mapa $h: Q(\mathbb{K}) \longrightarrow W(\mathbb{K}) / I^{2}(\mathbb{K})$ dado por $h\left(0+2 \mathbb{Z}, a \cdot \dot{\mathbb{K}}^{2}\right)=(\langle 1,-a\rangle)+I^{2}(\mathbb{K})$ e $h(1+$ $\left.2 \mathbb{Z}, a \cdot \dot{\mathbb{K}}^{2}\right)=\langle a\rangle+I^{2}(\mathbb{K})$, para cada $a \in \dot{\mathbb{K}}$, é a função inversa do homomorfismo $\overline{\left(\operatorname{dim}_{0}, d_{ \pm}\right)}$: $W(\mathbb{K}) / I^{2}(\mathbb{K}) \longrightarrow Q(\mathbb{K})$.

Uma análise de (três) casos mostra que $h$ é homomorfismo de grupos e, como a imagem de $h$ contem um conjunto gerador do quociente $W(\mathbb{K}) / I^{2}(\mathbb{K})\left(\right.$ pois $h\left(1+2 \mathbb{Z}, a . \dot{\mathbb{K}}^{2}\right)=\langle a\rangle+I^{2}(\mathbb{K})$, $a \in \dot{\mathbb{K}})$, temos que $h$ é epimorfismo. Como $\overline{\left(\operatorname{dim}_{0}, d_{ \pm}\right)} \circ h\left(0+2 \mathbb{Z}, a \cdot \dot{\mathbb{K}}^{2}\right)=\left(0+2 \mathbb{Z}, a \cdot \dot{\mathbb{K}}^{2}\right) \mathrm{e}$ $\overline{\left(\operatorname{dim}_{0}, d_{ \pm}\right)} \circ h\left(1+2 \mathbb{Z}, a . \dot{\mathbb{K}}^{2}\right)=h\left(1+2 \mathbb{Z}, a \cdot \dot{\mathbb{K}}^{2}\right), a \in \dot{\mathbb{K}}$, temos que $h$ é o isomorfismo inverso de $\overline{\left(\operatorname{dim}_{0}, d_{ \pm}\right)}$.

Em resumo, provamos o

Teorema 22. $\overline{\left(\operatorname{dim}_{0}, d_{ \pm}\right)}: W(\mathbb{K}) / I^{2}(\mathbb{K}) \longrightarrow Q(\mathbb{K})$ é um isomorfismo natural de grupos que estende o isomorfismo $\bar{d}_{ \pm}: I(\mathbb{K}) / I^{2}(\mathbb{K}) \longrightarrow \mathbb{K} / \dot{\mathbb{K}}^{2}$.

Procedemos agora uma análise da construção do grupo de classes de quadrado $Q(\mathbb{K})$.

- Verifica-se imediatamente que o diagrama abaixo é comutativo e satisfaz a propriedade universal de soma amalgamada (ou pushout) 


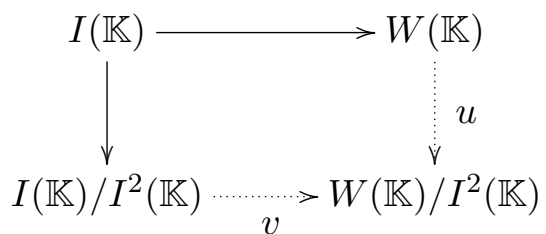

do diagrama $I(\mathbb{K}) / I^{2}(\mathbb{K}) \longleftarrow I(\mathbb{K}) \stackrel{\iota}{\longleftrightarrow} W(\mathbb{K})$.

$I(\mathbb{K}) / I^{2}(\mathbb{K})<I(\mathbb{K}) \stackrel{\iota}{\longrightarrow} W(\mathbb{K})$

- Como $I(\mathbb{K}) / I^{2}(\mathbb{K}) \cong \dot{\mathbb{K}} / \dot{\mathbb{K}}^{2}$ e $W(\mathbb{K}) / I^{2}(\mathbb{K}) \cong Q(\mathbb{K})$, podemos caracterizar (a menos de único isomorfismo) o grupo $Q(\mathbb{K})$ como sendo o pushout

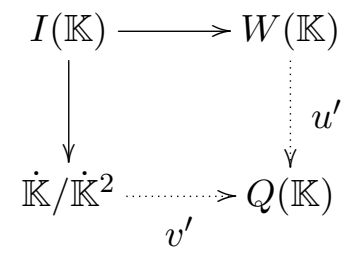

do diagrama $\dot{\mathbb{K}} / \dot{\mathbb{K}}^{2} \longleftarrow I(\mathbb{K}) \stackrel{\iota}{\longleftrightarrow} W(\mathbb{K})$

- Como $\hat{I}(\mathbb{K}) / \hat{I}^{2}(\mathbb{K}) \cong I(\mathbb{K}) / I^{2}(\mathbb{K})$ (seção 2 ), verificamos diretamente que o diagrama abaixo é pushout

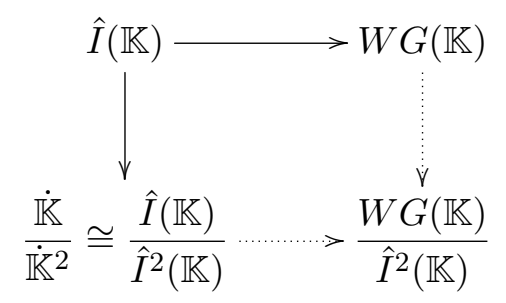

do diagrama $\hat{I}(\mathbb{K}) / \hat{I}^{2}(\mathbb{K}) \stackrel{p}{\longleftarrow} \hat{I}(\mathbb{K}) \stackrel{\iota}{\longleftrightarrow} W G(\mathbb{K})$, onde $\iota$ denota a inclusão e $p$ a projeção canônica. Assim, como $W G(\mathbb{K}) \cong \mathbb{Z}$, teremos um sequência exata cindida

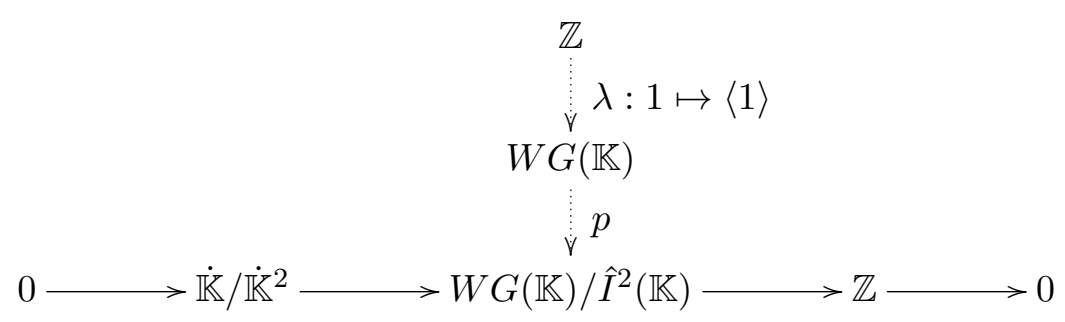

que por sua vez nos fornece uma equivalência de sequências exatas 


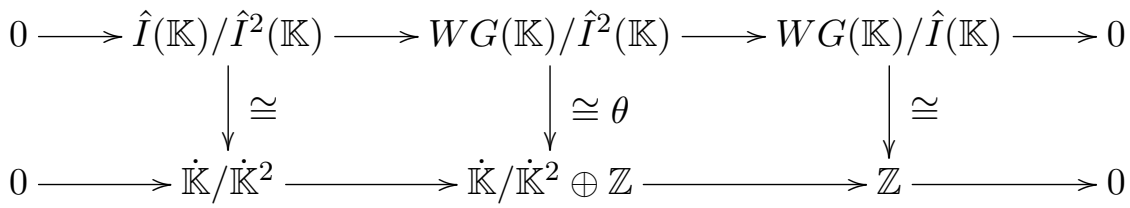

- Agora seja $\gamma: \dot{K} / \dot{\mathbb{K}}^{2} \oplus \mathbb{Z} \longrightarrow Q(\mathbb{K})$ o único epimorfismo de grupos que faz o próximo diagrama comutar

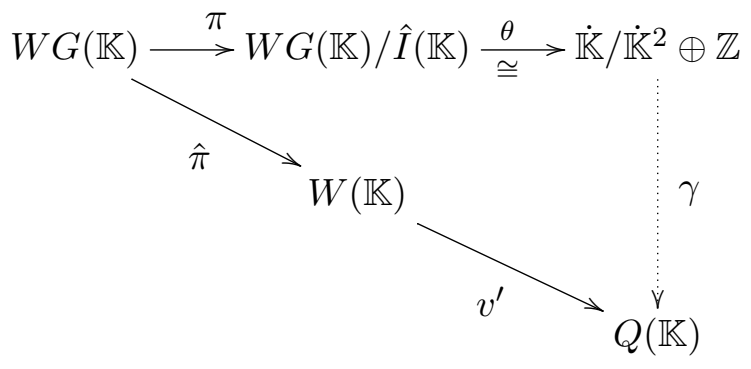

\section{Fato 11.}

(i) $\theta \pi(\phi)=(d(\phi)$, dim $(\phi))$, para cada forma regular $\phi$.

(ii) $\operatorname{Ker}(\gamma)=\left\{\left((-1)^{s}, 2 s\right): s \in \mathbb{Z}\right\}$

(iii) A aplicação $\bar{\gamma}: \frac{\dot{\mathbb{K}} / \dot{\mathbb{K}}^{2} \oplus \mathbb{Z}}{\operatorname{Ker}(\gamma)} \longrightarrow Q(\mathbb{K})$ definida por $\overline{(x, m)} \mapsto\left(m+2 \mathbb{Z},(-1)^{\frac{m(m-1)}{2}} x\right)$ é um isomorfismo de grupos.

\subsection{Invariantes de Hasse e de Witt}

Seja $\mathbb{K}$ um corpo e $(A, \cdot)$ um grupo abeliano. Dizemos que um mapa $\sigma: \dot{\mathbb{K}}^{n} \longrightarrow A$ é $n$-multiplicativo quando

$$
\sigma\left(\alpha_{1}, \ldots, \alpha_{i} \alpha_{i}^{\prime}, \ldots, \alpha_{n}\right)=\sigma\left(\alpha_{1}, \ldots, \alpha_{i}, \ldots, \alpha_{n}\right) \cdot \sigma\left(\alpha_{1}, \ldots, \alpha_{i}^{\prime}, \ldots, \alpha_{n}\right)
$$

Definição 34. (a) Dizemos que um mapa $\sigma: \dot{K}^{n} \longrightarrow A$ é um n-símbolo de Steinberg quando $\sigma$ for um mapa n-multiplicativo e satisfizer $\sigma\left(\alpha_{1}, \ldots, \alpha_{n}\right)=1$ sempre que existir $i<n$ tal que $\alpha_{i}+\alpha_{i+1}=0$.

(b) Dizemos que um n-símbolo de Steinberg $\sigma: \mathbb{K}^{n} \longrightarrow A$ é universal quando para cada $n$-símbolo de Steinberg $\sigma^{\prime}: \dot{\mathbb{K}}^{n} \longrightarrow A^{\prime}$ existe um único homomorfismo de grupos abelianos $\xi: A \longrightarrow A^{\prime}$ que torna o seguinte diagrama comutativo

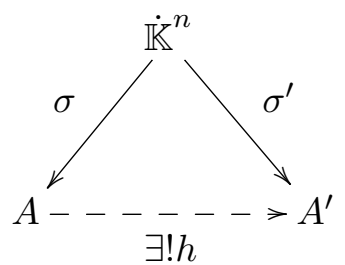

Observação 29. Se $\sigma: \dot{\mathbb{K}}^{n} \longrightarrow A$ é um n-símbolo de Steinberg e $h: A \longrightarrow A^{\prime}$ é um homomorfismo de grupos abelianos, então $h \sigma: \mathbb{K}^{n} \longrightarrow A^{\prime}$ é um n-símbolo de Steinberg. 
Proposição 45. n-símbolos de Steinberg universais são essencialmente únicos.

Demonstração. Se $\sigma$ e $\sigma^{\prime}$ são símbolos universais, então existem únicos morfismos de grupos $h$ : $A \longrightarrow A^{\prime}$ e $h^{\prime}: A^{\prime} \longrightarrow$ tais que $\sigma^{\prime}=h \sigma, \sigma=h^{\prime} \sigma^{\prime}$. Obtemos $i d^{\prime} \sigma^{\prime}=h h^{\prime} \sigma^{\prime}, I d \sigma=h^{\prime} h \sigma$ e assim $h h^{\prime}=i d^{\prime}$ e $h^{\prime} h=i d$ por unicidade.

Na sequência desta seção, assumiremos que todos os grupos abelianos, em contradomínios de símbolos de Steinberg, terão expoente 2.

Lema 23. Se $\sigma$ for um 2-símbolo de Steinberg a valores em um grupo abeliano (de expoente 2), então para todos $\alpha, \beta, \gamma, \delta \in \dot{\mathbb{K}}$

$\left.\sigma_{1}\right) \quad \sigma\left(\alpha \gamma^{2}, \beta \delta^{2}\right)=\sigma(\alpha, \beta)$

$\left.\sigma_{2}\right) \quad \sigma(1, \alpha)=1=\sigma(\alpha, 1)$;

$\left.\sigma_{3}\right) \quad \sigma(\alpha,-\alpha)=1$

$\left.\sigma_{4}\right) \quad \sigma(\alpha, \beta)=\sigma(\beta, \alpha)$;

$\left.\sigma_{5}\right) \quad \sigma(\alpha, \alpha)=\sigma(\alpha,-1)$;

$\left.\sigma_{6}\right) \quad \sigma(\alpha \gamma, \beta \gamma)=\sigma(\alpha, \beta) . \sigma(\gamma, \alpha \beta \gamma)$;

Demonstração.

$\left.\sigma_{1}\right) \sigma\left(\alpha \gamma^{2}, \beta \delta^{2}\right)=\sigma(\alpha, \beta) \sigma(\alpha, \delta)^{2} \sigma(\gamma, \beta)^{2} \sigma(\gamma, \delta)^{4}=\sigma(\alpha, \beta) \cdot 1 \cdot 1 \cdot 1=\sigma(\alpha, \beta)$

$\left.\sigma_{2}\right) \sigma(1, x)=\sigma(1 \cdot 1, x)=\sigma(1, x) \sigma(1, x)$ e portanto $\sigma(1, x)=1$. Analogamanente obtemos $\sigma(x, 1)=$ 1 .

$\left.\sigma_{3}\right)$ Segue de $\left(\sigma_{2}\right)$ que $\sigma(1,-1)=1$. Consideramos agora o caso $\alpha \neq 1$. Como $-\alpha=(1-\alpha) .(1-$ $\left.\alpha^{-1}\right)^{-1}$ temos, aplicando $\left(\sigma_{1}\right)$ e $\left(\sigma_{2}\right)$

$$
\begin{gathered}
\sigma(\alpha,-\alpha)=\sigma(\alpha, 1-\alpha) \sigma\left(\alpha,\left(1-\alpha^{-1}\right)^{-1}\right)=1 . \sigma\left(\alpha,\left(1-\alpha^{-1}\right)^{-1}\right)= \\
\quad=\sigma\left(\alpha \cdot\left(\alpha^{-1}\right)^{2},\left(1-\alpha^{-1}\right)^{-1} \cdot\left(1-\alpha^{-1}\right)^{2}\right)=\sigma\left(\alpha^{-1}, 1-\alpha^{-1}\right)=1 .
\end{gathered}
$$

$\left.\sigma_{4}\right) \sigma(\alpha, \beta) \sigma(\beta, \alpha)=\sigma(\alpha,-\alpha) \sigma(\alpha, \beta) \sigma(\beta,-\beta) \sigma(\beta, \alpha)=\sigma(\alpha \beta,-\alpha \beta)=1$.

$\left.\sigma_{5}\right) \sigma(\alpha, \alpha) \cdot \sigma(\alpha,-1)=\sigma(\alpha,-\alpha)=1$.

$\left.\sigma_{6}\right) \sigma(\alpha \gamma, \beta \gamma)=\sigma(\alpha, \beta \gamma) \cdot \sigma(\gamma, \beta \gamma)=\sigma(\alpha, \beta) \sigma(\gamma, \alpha) \cdot \sigma(\gamma, \beta \gamma)=\sigma(\alpha, \beta) . \sigma(\gamma, \alpha \beta \gamma)$

Lema 24. Seja $\sigma$ um 2-símbolo de Steinberg. $O$ valor $\sigma(\alpha, \beta)$ só depende da classe de isometria do espaço 2-dimensional $\langle\alpha, \beta\rangle$. Além disso, $\sigma(\alpha, \beta)=1$ quando $\langle\alpha, \beta\rangle$ for isotrópica (= hiperbólica).

Demonstração. Se $\langle\alpha, \beta\rangle$ é isotrópica, então $\beta=-\alpha \gamma^{2}$ e $\sigma(\alpha, \beta)=1$ por $\left(\sigma_{1}\right),\left(\sigma_{3}\right)$ do Lema anterior. Se $\langle\alpha, \beta\rangle \simeq\langle\gamma, \delta\rangle$, então existem $\xi, \eta \in \mathbb{K}$ tais que $\alpha \xi^{2}+\beta \eta^{2}=\gamma$. Se $\eta=0$, então $\alpha \equiv \gamma$ $\bmod \dot{\mathbb{K}}^{2}$ e assim, pelo cancelamento de Witt, $\beta \equiv \delta \bmod \dot{\mathbb{K}}^{2}$. Dessa forma, $\sigma(\alpha, \beta)=\sigma(\gamma, \delta)$ por $\left(\sigma_{1}\right)$ do Lema. Usamos um argumento semelhante se $\xi=0$. Se $\xi, \eta \neq 0$, então

$$
\alpha \xi^{2} \gamma^{-1}+\beta \eta^{2} \gamma^{-1}=1
$$

Assim, por $\left(\sigma_{1}\right),\left(\sigma_{6}\right)$,

$$
1=\sigma\left(\alpha \xi^{2} \gamma^{-1}, \beta \eta^{2} \gamma^{-1}\right)=\sigma(\alpha \gamma, \beta \gamma)=\sigma(\alpha, \beta) \sigma(\gamma, \alpha \beta \gamma)
$$

e portanto $\sigma(\alpha, \beta)=\sigma(\gamma, \alpha \beta \gamma)=\sigma(\gamma, \delta)$, pois $\delta \equiv \alpha \beta \gamma \bmod \dot{\mathbb{K}}^{2}$. 
O Teorema de equivalência de cadeia e o Lema anterior grantem a correção da seguinte

Definição 35. O invariante de Hasse de um forma $\phi \simeq\left\langle\alpha_{1}, \ldots, \alpha_{n}\right\rangle$ relativo a um 2-símbolo de Steinberg $\sigma$ é dado pela fórmula

$$
s_{\sigma}(\phi):=\prod_{i<j} \sigma\left(\alpha_{i}, \alpha_{j}\right)
$$

Lema 25. Para quaisquer duas formas quadráticas $\phi \simeq\left\langle\alpha_{1}, \ldots, \alpha_{r}\right\rangle \psi \simeq\left\langle\alpha_{r+1}, \ldots, \alpha_{r+t}\right\rangle$ tem -se

$$
s_{\sigma}(\phi \perp \psi)=s_{\sigma}(\phi) s_{\sigma}(\psi) \sigma(d(\phi), d(\psi))
$$

Demonstração.

$$
\begin{aligned}
s_{\sigma}(\phi \perp \psi) & =\prod_{i=1, i<j}^{r+t} \sigma\left(\alpha_{i}, \alpha_{j}\right) \\
& =\prod_{i=1, i<j}^{r} \sigma\left(\alpha_{i}, \alpha_{j}\right) \prod_{i=r+1, i<j}^{r+t} \sigma\left(\alpha_{i}, \alpha_{j}\right) \prod_{i=1}^{r} \prod_{j=r+1}^{r+t} \sigma\left(\alpha_{i}, \alpha_{j}\right) \\
& =s_{\sigma}(\phi) s_{\sigma}(\psi) \prod_{i=1}^{r} \sigma\left(\alpha_{i}, \prod_{j=r+1}^{r+t} \alpha_{j}\right) \\
& =s_{\sigma}(\phi) s_{\sigma}(\psi) \sigma\left(\prod_{i=1}^{r} \alpha_{i}, d(\psi)\right) \\
& =s_{\sigma}(\phi) s_{\sigma}(\psi) \sigma(d(\phi), d(\psi))
\end{aligned}
$$

Definição 36. Definimos o invariante de Witt de uma forma n-dimensional $\phi$ relativo a um 2símbolo de Steinberg $\sigma$ mediante a seguinte fórmula

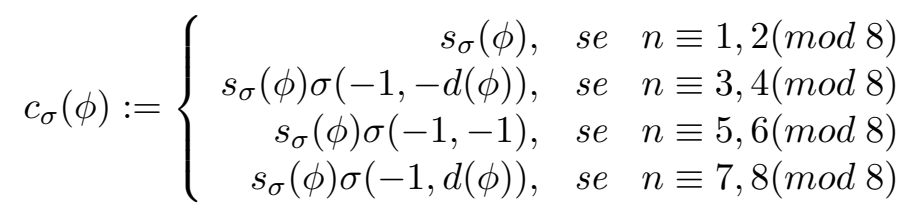

Proposição 46. $c_{\sigma}(\phi)$ só depende de sua classe no anel de Witt.

Demonstração. Nos limitaremos ao caso onde $\operatorname{dim}(\phi) \equiv 3,4(\bmod 8)$, pois os outros casos são análogos

$$
\begin{aligned}
c_{\sigma}(\phi \perp \mathbb{H}) & =s_{\sigma}(\phi \perp \mathbb{H}) \sigma(-1,-1) \\
& =s_{\sigma}(\phi) \sigma(-1, d(\phi)) \sigma(-1,-1) \\
& =s_{\sigma}(\phi) \sigma(-1,-d(\phi)) \\
& =c_{\sigma}(\phi)
\end{aligned}
$$

Assim, por indução em $n \in \mathbb{N}, c_{\sigma}(\phi \perp n \mathbb{H})=c_{\sigma}(\phi)$. 
Lema 26. Se $\sigma$ é um 2-símbolo de Steinberg a valores em um grupo abeliano A,então

1) $c_{\sigma}: I^{2}(\mathbb{K}) \longrightarrow A$ é um homomorfismo de grupos;

2) $c_{\sigma}\left(I^{3}(\mathbb{K})\right)=0$.

Demonstração.

1) Seja $\phi=\langle\langle-\alpha,-\beta\rangle\rangle$ e $\psi=\langle\langle-\gamma,-\xi\rangle\rangle$. Note que $d(\phi)=d(\psi)=1$ e usando que $\sigma(1, x)=1$, obtemos

$$
\begin{array}{r}
c_{\sigma}(\phi \oplus \psi)=\sigma(-\alpha,-\beta) \sigma(-\alpha, \alpha \beta) \sigma(-\alpha, \gamma) \sigma(-\alpha, \xi) \sigma(-\alpha, \gamma \xi) \\
\sigma(-\beta, \alpha \beta) \sigma(-\beta, \gamma) \sigma(-\beta, \xi) \sigma(-\beta, \gamma \xi) \\
\sigma(\alpha \beta, \gamma) \sigma(\alpha \beta, \xi) \sigma(\alpha \beta, \gamma \xi) \\
\sigma(\gamma, \xi) \sigma(\gamma, \gamma \xi) \\
\sigma(\xi, \gamma \xi) \\
=s_{\sigma}(\phi) s_{\sigma}(\psi) \sigma\left(\alpha^{2} \beta^{2}, \gamma\right) \sigma\left(\alpha^{2} \beta^{2}, \xi\right) \sigma\left(\alpha^{2} \beta^{2}, \xi \gamma\right) \\
=s_{\sigma}(\phi) s_{\sigma}(\psi) \\
=s_{\sigma}(\phi) \sigma(-1,-1)^{2} s_{\sigma}(\psi) \\
=c_{\sigma}(\phi) c_{\sigma}(\psi)
\end{array}
$$

e o resultado segue pois $I^{2}(\mathbb{K})$ é gerado aditivamente por formas de Pfister de grau 2.

2) Para cada $\phi=\langle\langle-\alpha,-\beta,-\gamma\rangle\rangle$, temos

$$
\begin{aligned}
c_{\sigma}(\phi) & =s_{\sigma}(\phi) \sigma(-1, d(\phi)) \\
& =\sigma(1,-\alpha) \sigma(-\alpha,-\beta) \sigma(\alpha \beta,-\gamma) \sigma(-\alpha \beta \gamma, \alpha \beta) \sigma(-\gamma, \alpha \gamma) \\
& \cdot \sigma(-\alpha, \beta \gamma) \sigma(-\alpha \beta \gamma,-\alpha \beta \gamma) \sigma(-1,1) \\
& =\sigma(-\alpha,-\gamma) \sigma(\beta \gamma,-\gamma) \sigma(-\alpha \beta \gamma,-\gamma) \\
& =\sigma(-\alpha \beta \gamma,-\gamma) \sigma(-\alpha \beta \gamma,-\gamma) \\
& =\sigma\left(-\alpha \beta \gamma, \gamma^{2}\right) \\
& =\sigma(-\alpha \beta \gamma, 1) \\
& =1
\end{aligned}
$$

Proposição 47. A aplicação $\sigma: \dot{\mathbb{K}} \times \dot{\mathbb{K}} \longrightarrow I^{2}(\mathbb{K}) / I^{3}(\mathbb{K})$ definida por $\sigma(\alpha, \beta) \doteq\langle\langle-\alpha,-\beta\rangle\rangle+I^{3}(\mathbb{K})$ é um 2-símbolo de Steinberg universal.

Demonstração. $\sigma$ é um 2-símbolo de Steinberg, pois $\langle\langle-\alpha,-\beta\rangle\rangle+\langle\langle-\alpha,-\gamma\rangle\rangle \equiv\langle\langle-\alpha, \beta \gamma\rangle\rangle \bmod$ $\left(I^{3}(\mathbb{K})\right)$ e $\langle\langle-\alpha,-1+\alpha\rangle\rangle \in 0+I^{3}(\mathbb{K})$. Se $\sigma^{\prime}: \dot{\mathbb{K}} \times \dot{K} \longrightarrow A^{\prime}$ é um 2-símbolo de Steinberg, combinando o Lema anterior com o cálculo

$$
\begin{aligned}
c_{\sigma}^{\prime}(\sigma(\alpha, \beta)) & =c_{\sigma^{\prime}}\langle\langle-\alpha,-\beta\rangle\rangle=s_{\sigma^{\prime}}\langle\langle-\alpha,-\beta\rangle\rangle \sigma^{\prime}(-1,-1) \\
& =\sigma^{\prime}(\alpha, \beta) \sigma^{\prime}(-1,-1) \sigma^{\prime}(-1,-1)=\sigma^{\prime}(\alpha, \beta)
\end{aligned}
$$


obtemos um diagrama comutativo

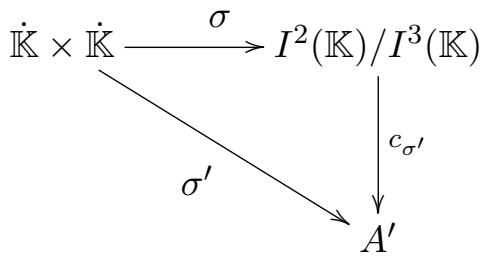

onde $c_{\sigma^{\prime}}$ é um homomorfismo de grupos; este é o único homomorfismo com tal propriedade, pois $I^{2}(\mathbb{K}) / I^{3}(\mathbb{K})$ é gerado aditivamente pela imagem de $\sigma$.

O grupo de Brauer $\operatorname{Br}(\mathbb{K})$ de um corpo $\mathbb{K}$ consiste das classes de isomorfismo de álgebras centrais simples com operação induzida pelo produto tensorial de álgebras. Entraremos em detalhes sobre o tema no Capítulo 3.

Denotaremos por

$$
{ }_{2} \operatorname{Br}(\mathbb{K}):=\left\{x \in \operatorname{Br}(\mathbb{K}): x^{2}=1\right\}
$$

o subgrupo de Brauer dos elementos de ordem $\leq 2$. Cada álgebra de quatérnio $(\alpha, \beta)_{\mathbb{K}}$ (veja última seção do Capítulo 1) é um elemento de ordem (no máximo) 2 do grupo de Brauer de modo que o subgrupo de $B r(\mathbb{K})$ gerado pelas álgebra de quatérnios têm expoente 2 ou é trivial. Uma questão natural é se este grupo contém todos os elementos de ordem 2 em $\operatorname{Br}(\mathbb{K})$ e A. A. Albert tinha conjecturado que este é sempre o caso. No entanto, foi apenas em 1981 que Merkurjev deu uma resposta afirmativa a essa questão utilizando que o mapa $():, \dot{\mathbb{K}} \times \dot{\mathbb{K}} \longrightarrow{ }_{2} B r(\mathbb{K})$ definido por $(\alpha, \beta) \doteq(\alpha, \beta)_{\mathbb{K}}$ é um 2-símbolo de Steinberg.

Proposição 48. (Teorema de Merkurjev)O símbolo de quatérnio

$$
(,,): \dot{\mathbb{K}} \times \dot{\mathbb{K}} \longrightarrow{ }_{2} \operatorname{Br}(\mathbb{K})
$$

é um 2-símbolo de Steinberg universal. Em particular, existe um isomorfismo

$$
I^{2}(\mathbb{K}) / I^{3}(\mathbb{K}) \cong{ }_{2} B r(\mathbb{K})
$$

Demonstração. Consultar [Wad] ou [AKa para demonstrações elementares desse resultado.

\subsection{Os Funtores Anel Filtrado e Anel Graduado de Witt}

Vimos que o ideal fundamental $I(\mathbb{K}) \subseteq W(\mathbb{K})$ é gerado por $P_{1}(\mathbb{K})$, o conjunto das formas de Pfister de grau 1. Assim, a potência n-ésima deste ideal, $I^{n}(\mathbb{K})$, é gerado por $P_{n}(\mathbb{K})$. Note que $\left(I^{n}(\mathbb{K})\right)_{n \in \omega}$ define uma sequência decrescente de ideais em $W(\mathbb{K})=I^{0}(\mathbb{K})$.

Denotaremos por $C$ Ring $_{1}$ a categoria na qual os objetos são anéis comutativos com unidade e os morfismos são morfismos de anéis que preserva unidade.

Definição 37. Diremos que $\mathcal{A}=\left(A,\left(J^{(n)}\right)_{n \in \omega},+, \cdot, 0,1\right)$ é um anel filtrado comutativo com unidade quando

$\left.\mathcal{A}_{1}\right)(A,+, \cdot, 0,1) \in C \operatorname{Ring}_{1}$

$\left.\mathcal{A}_{2}\right) \quad \forall n \in \omega\left(J^{(n)} \subseteq A\right.$ é ideal $)$

$\left.\mathcal{A}_{3}\right) \quad \forall m, n \in \omega\left(m \leq n \Longrightarrow J^{(m)} \supseteq J^{(n)}\right)$ 
$\left.\mathcal{A}_{4}\right) \quad \forall n \in \omega\left(J^{(m)} \cdot J^{(n)} \subseteq J^{(m+n)}\right)$

$\left.\mathcal{A}_{5}\right) \quad J^{(0)}=A$

Quando valerem adicionalmente as condições abaixo, diremos que $\mathcal{A}$ é 2-filtrado.

A $\left.\mathcal{A}_{6}\right) J^{(0)} / J^{(1)} \cong \mathbb{F}_{2}=\mathbb{Z} / 2 \mathbb{Z}$

$\left.\mathcal{A}_{7}\right) \quad \forall n \in \omega\left(\bar{J}^{(n)}:=J^{(n)} / J^{(n+1)}\right.$ é grupo de expoente 2 sob + )

Observação 30. Se $\mathcal{A}$ é 2-filtrado, então:

(i) $2=1+1 \in J^{(1)}$, por $\mathcal{A}_{6}$.

(ii) $2 J^{(n)} \subseteq J^{(n+1)}$ e $2^{n} \in J^{(n)}$, por (i) e $\mathcal{A}_{7}$

Proposição 49. $\mathcal{W}_{f}(\mathbb{K})=\left(W(\mathbb{K}),\left(I^{n}(\mathbb{K})\right)_{n \in \omega}, \bar{\oplus}, \bar{\otimes}, \overline{\langle\rangle}, \overline{\langle 1\rangle}\right)$ é um anel 2-filtrado, comutativo, com unidade.

Demonstração. Já vimos que $W(\mathbb{K}) / I(\mathbb{K}) \cong \mathbb{Z} / 2 \mathbb{Z} \cong \mathbb{K}_{2}$. Além disso, cada $\bar{I}^{n}(\mathbb{K}):=I^{n}(\mathbb{K}) / I^{n+1}(\mathbb{K})$ é de expoente 2 , pois $2 I^{n}(\mathbb{K})=\langle 1,1\rangle I^{n}(\mathbb{K}) \subseteq I^{n+1}(\mathbb{K})$. As outras propriedades que definem um anel filtrado são imediatas para $\mathcal{W}_{f}(\mathbb{K})$.

Lema 27. Para cada anel 2-filtrado, comutativo, com unidade $\mathcal{A}=\left(A,\left(J^{(n)}\right)_{n \in \omega},+, \cdot, 0,1\right)$ anel 2-filtrado, comutativo, com unidade temos que:

i) Temos produtos induzidos nos grupos quocientes de expoente $2, *_{m, n}: \bar{J}^{(m)} \times \bar{J}^{(n)}$, definida por $([\phi],[\psi]) \mapsto[\phi . \psi]$, e estas são aplicações biaditivas.

ii) A aplicação $t_{n}:=2 .\left(\mathrm{)}: \bar{J}^{(n)}(\mathbb{K}) \longrightarrow \bar{J}^{(n+1)}(\mathbb{K}),[\phi] \mapsto t_{n}([\phi])=[2 \cdot \phi]\right.$ é um homomorfismo de $\mathbb{Z} / 2 \mathbb{Z}$-módulos tal que $t_{n}\left(\left[2^{n}\right]\right)=\left[2^{n+1}\right]$.

iii) O grupo abeliano $(\mathcal{A})_{g}:=\bigoplus_{n \in \mathbb{N}} \bar{J}^{(n)}$ tem estrutura de anel (comutativo, com unidade) com multiplicação dada como a única extensão $\mathbb{Z}$-linear (ou $\mathbb{Z} / 2 \mathbb{Z}$-linear) das funçôes $*_{m, n}$, para cada $m, n \in \mathbb{N}$.

Demonstração. Dados $\phi, \phi^{\prime} \in J^{(n)}$ e $\psi, \psi^{\prime} \in J^{(m)}$ tais que $\phi \equiv \phi^{\prime}\left(\bmod J^{(n+1)}\right)$ e $\psi \equiv \psi^{\prime}(\bmod$ $\left.J^{(m+1)}\right)$, então

$$
\phi . \psi \equiv \phi^{\prime} \cdot \psi \equiv \phi^{\prime} \cdot \psi^{\prime}\left(\bmod J^{(m+n+1)}\right) .
$$

Assim o produto induzido nos quocientes de expoente $2, *_{m, n}: \bar{J}^{(m)} \times \bar{J}^{(n)}$, definida por $([\phi],[\psi]) \mapsto$ $[\phi . \psi]$, é uma aplicação biaditiva.

\section{Definição 38.}

a) Um anel graduado e 2-indutivo é uma estrutura $\mathcal{G}=\left(\left(\mathcal{G}_{n}\right)_{n \in \mathbb{N}},\left(*_{n, m}\right)_{n, m \in \mathbb{N}},\left(t_{n}\right)_{n \in \mathbb{N}}\right)$ tal que

G $\left.\mathcal{G}_{1}\right) \mathcal{G}_{n}=\left(G_{n},{ }_{n}, 0_{n}, d_{n}\right)$ é um grupo de expoente 2 com elemento neutro $0_{n}$ e elemento distinguido $d_{n}$

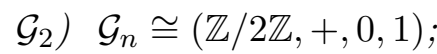

$\left.\mathcal{G}_{3}\right) *_{n, m}: G_{n} \times G_{m} \longrightarrow G_{n+m}$ é aplicação $\mathbb{Z} / 2 \mathbb{Z}$-linear;

G4) $O$ grupo abeliano $\bigoplus_{n \in \mathbb{N}} G_{n}$ tem estrutura de anel comutativo, com unidade $1 \in G_{0}$ e com multiplicação dada como a única extensão $\mathbb{Z} / 2 \mathbb{Z}$-linear das funções $*_{m, n}$, para cada $m, n \in \mathbb{N}$.

$\left.\mathcal{G}_{5}\right) t_{n}: G_{n} \longrightarrow G_{n+1}$ é um homomorfismo de $\mathbb{Z} / 2 \mathbb{Z}$-módulos tal que $t_{n}\left(d_{n}\right)=d_{n+1}$. 
b) $O$ anel graduado de Witt do corpo $\mathbb{K}$ é o anel graduado e 2-indutivo dado por $\mathcal{W}_{g}(\mathbb{K}):=$ $\left(\operatorname{Grad}^{n}(\mathcal{W}(\mathbb{K}))_{n \in \mathbb{N}},\left(*_{n, m}\right)_{n, m \in \mathbb{N}},(2[])_{n \in \mathbb{N}}, *\right)$, onde $\operatorname{Grad}^{n}(\mathcal{W}(\mathbb{K}))=\left(\bar{I}^{n}(\mathbb{K}),+, 0,\left[2^{n}\right]\right)$ é o grupo de expoente 2 com elemento distinguido $\left[2^{n}\right]$.

Lema 28. Dados anéis 2-filtrados, comutativos, com unidades $\mathcal{A}=\left(A,\left(J^{(n)}\right)_{n \in \omega},+, \cdot, 0,1\right)$ e $\mathcal{A}^{\prime}=$ $\left(A^{\prime},\left(J^{\prime(n)}\right)_{n \in \omega},+^{\prime}, .^{\prime}, 0^{\prime}, 1^{\prime}\right)$ e um homomorfismo de anéis com unidade $h: A \longrightarrow A^{\prime}$ tal que $h\left[J^{(n)}\right] \subseteq$ $J^{\prime(n)}$, para cada $n \in \mathbb{N}$, temos:

i) Família de homomorfismos induzidos de grupos de expoente $2, h_{n}: \bar{J}^{(n)} \longrightarrow \bar{J}^{(n)}$ dado por $h_{n}\left(\phi+J^{(n+1)}\right)=h(\phi)+J^{\prime(n+1)}$;

ii) $h_{n} \circ t_{n}=t_{n}^{\prime}, n \in \mathbb{N}$;

iii) Temos único homomorfismo induzido de anéis com unidade $\hat{h}: \bigoplus_{n \in \mathbb{N}} \bar{J}^{(n)} \longrightarrow \bigoplus_{n \in \mathbb{N}}{\overline{J^{\prime}}}^{(n)}$, que estende $\mathbb{Z} / 2 \mathbb{Z}$-linearmente cada homomorfismo de $\mathbb{Z} / 2 \mathbb{Z}$-módulo $h_{n}: \bar{J}^{(n)} \longrightarrow \bar{J}^{(n)}, n \in \mathbb{N}$.

\section{Definição 39.}

a) Um morfismo entre anéis filtrados $\mathcal{A}$ e $\mathcal{A}^{\prime}$ é uma tripla $\left\langle\mathcal{A}, h, \mathcal{A}^{\prime}\right\rangle$,onde $h \in C$ Ring ${ }_{1}$ e $\forall n \in$ $\omega\left(h\left(J^{(n)} \subseteq J^{\prime(n)}\right)\right.$. Se $\left\langle\mathcal{A}, h, \mathcal{A}^{\prime}\right\rangle$ e $\left\langle\mathcal{A}^{\prime}, h^{\prime}, \mathcal{A}^{\prime \prime}\right\rangle$ a composição será dada por $\left\langle\mathcal{A}, h^{\prime} \circ h, \mathcal{A}^{\prime \prime}\right\rangle$. É fácil ver que os anéis filtrados comutativos com unidades com a composição anterior e as identidades

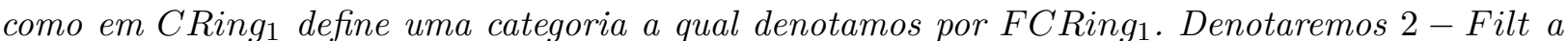
subcategoria dos anéis 2-filtrados, comutativos, com unidade.

b) Um morfismo entre anéis graduados 2-indutivos $\mathcal{G}$ e $\mathcal{G}^{\prime}$ é uma sequência de mapas $\left(G_{n} \stackrel{h_{n}}{\longrightarrow}\right.$ $\left.G_{n}^{\prime}\right)_{n \in \mathbb{N}}$ tal que:

- $h_{n}$ é um homomorfismo de grupos tal que $h_{n}\left(d_{n}\right)=d_{n}^{\prime}$;

- O mapa $\hat{h}: \bigoplus_{n \in \mathbb{N}} G_{n} \longrightarrow \bigoplus_{n \in \mathbb{N}} G_{n}^{\prime}$, que é a única extensão $\mathbb{Z} / 2 \mathbb{Z}$-linear de cada homomorfismo de $\mathbb{Z} / 2 \mathbb{Z}$-módulo $h_{n}$, deve ser um homomorfismo de anéis com unidade.

É fácil ver que os anéis graduados e 2-indutivos, munidos de uma definição natural de composição, fornecem uma categoria que denotaremos 2 - IndGr.

Finalizamos esta seção e capítulo registrando os seguintes

Fato 12. Temos um funtor Grad: $2-$ Filt $\longrightarrow 2-$ IndGr dado por

$\mathcal{A}=\left(A,\left(J^{(n)}\right)_{n \in \omega},+, \cdot, 0,1\right) \mapsto \operatorname{Grad}(\mathcal{A}):=\left(\bigoplus_{n \in \mathbb{N}} \bar{J}^{(n)}, *,\left(2^{n}\right)_{n \in \mathbb{N}},\left(t_{n}\right)_{n \in \mathbb{N}}\right) ;\left(\mathcal{A} \stackrel{h}{\longrightarrow} \mathcal{A}^{\prime}\right) \mapsto$ $\left(\operatorname{Grad}(\mathcal{A}) \stackrel{\hat{h}}{\longrightarrow} \operatorname{Grad}\left(\mathcal{A}^{\prime}\right)\right)$

Fato 13. Temos o diagrama comutativo de categorias e funtores.

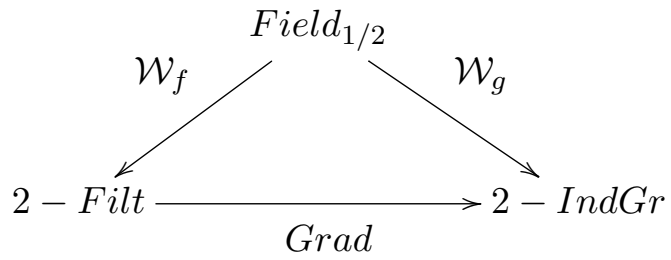

subfile 


\section{Capítulo 3}

\section{Elementos de cohomologia galoisiana}

Neste Capítulo nos dedicamos a apresentar os ingredientes necessários para tratar da cohomologia galoisiana e apresentar algumas conexões com a teoria algébrica das formas quadráticas estabelecidas, em particular, através do conceito de grupo de Brauer de um corpo e de seus elementos de ordem dois 11. Trataremos aqui brevemente dos grupos profinitos e de sua cohomologia; reservamos um apêndice da dissertação para tratar de questões gerais de cohomologia e funtores derivados. As principais referências são: [Rib], [RZ], [SIon2], [Serre2].

\subsection{Grupos topológicos}

Um grupo topológico é um grupo $G$ munido de uma topologia compatível com a operação do grupo, ou seja,

$$
\begin{aligned}
\mu: G \times G & \longrightarrow G \\
(g, h) & \longmapsto g h^{-1}
\end{aligned}
$$

é contínua. Uma aplicação entre grupos topológicos $\varphi: G \longrightarrow G^{\prime}$ é dita um morfismo de grupos topológicos se for homomorfismo de grupos contínuo. Um isomorfismo de grupos topológicos é um homomorfismo de grupos que também é um homeomorfismo. Os grupos topológicos e os seus morfismos definem uma categoria a qual denotamos por $\mathcal{G}_{\text {Top }}$.

Definição 40. Para cada $g \in G$ definimos a aplicação translação à esquerda por $L_{g}: G \longrightarrow G, x \mapsto$ $L_{g}(x)=g x$.

Proposição 50. Seja G um grupo topológico e $g \in G$. A aplicação $L_{g}$ é um homeomorfismo.

Demonstração. $L_{g}$ é a composição das funções contínuas $m: G \times G \longrightarrow G,(g, x) \mapsto g x$ e $(g,-)$ : $G \times G \longrightarrow G, x \mapsto(g, x)$. Por fim, $L_{g}^{-1}=L_{g^{-1}}$.

Definição 41. Seja $(G, \tau)$ um grupo topológico e $g \in G$.

1) Dizer que $V \subseteq G$ é uma vizinhança de $g \in G$ significa

$$
\exists \Omega \in \tau(g \in \Omega \subseteq V)
$$

Denotaremos por $\mathcal{V}(g)$ o conjunto das vizinhanças de $g$.

\footnotetext{
${ }^{1}$ Retornaremos a este tema no Capítulo final desta dissertação.
} 
2)Dizer que $\mathcal{B}(g) \in \wp(\mathcal{V}(g)) \backslash\{\emptyset\}$ é uma base de vizinhanças em g significa

$$
\forall \Omega \in V(g)(V \subseteq \Omega)
$$

Lema 29. Seja $G$ um grupo topológico com elemento neutro 1 e g um elemento de $G$.

1) $\forall V \in \mathcal{V}(g) \exists \Omega \in \mathcal{V}(1)\left(V=L_{g}(\Omega)\right)$.

2) $O$ conjunto

$$
\mathcal{B}(g)=\left\{L_{g}(V): V \in \mathcal{B}(1)\right\}
$$

é uma base de vizinhanças em g,sempre que $\mathcal{B}(1)$ for uma base de vizinhanças em 1 .

Demonstração.

1) Basta tomar $\Omega \doteq L_{g^{-1}}(V)$.

2) Se $\Omega$ é um aberto que contém g,então $\Omega^{\prime} \doteq L_{g^{-1}}(\Omega)$ é um aberto que contém 1.Por hipótese, existe $V \in \mathcal{V}(1)$ tal que $V \subseteq \Omega^{\prime}$. Logo, $L_{g}\left(\Omega^{\prime}\right)$ é um aberto que contém g e está em $\mathcal{B}(g)$.

\subsection{Limites projetivos e Grupos profinitos}

Um sistema dirigido para cima consiste em um conjunto $I \neq \emptyset$ equipado com uma relação binária $\preceq$ cumprindo as seguintes propriedades:

$\left.\preceq_{1}\right) \forall i \in I(i \preceq i)($ Reflexividade $)$;

$\left.\preceq_{2}\right) \forall i, j, k \in I(i \preceq j \wedge j \preceq k \Longrightarrow i \preceq k)$ (Transitividade);

$\left.\preceq_{3}\right) \forall i, j \in I(i \preceq j \wedge j \preceq i \Longrightarrow i=j)($ Anti - simetria $)$;

$\left.\preceq_{4}\right) \forall i, j \in I \exists l \in I(i \preceq l \wedge j \preceq l)($ Limite superior $)$;.

Definição 42. Seja $(I, \preceq)$ um sistema dirigido.

1)Um sistema projetivo(ou inverso) de grupos topológicos consiste em uma família de grupos topológicos $\left\{G_{i}\right\}_{i \in I}$ juntamente com uma familia de homomorfismos contínous de grupos $\left\{\varphi_{j i}: G_{i} \longrightarrow\right.$ $\left.G_{j}\right\}$ tal que o diagrama

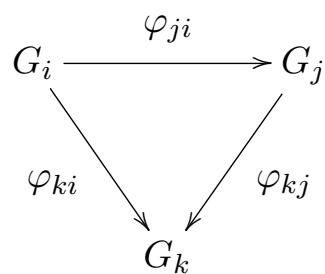

é comutativo para todos $k \preceq j \preceq i$ e $\varphi_{i i}$ é a identidade $\forall i \in I$. Quando os grupos de um sistema projetivo forem finitos e equipados com a topologia discreta, diremos que é um sistema projetivo discreto finito.

2)Uma família de morfismos de grupos topológicos $\left\{\psi_{i}: G \longrightarrow G_{i}\right\}_{i \in I}$ é dita compatível com um sistema projetivo $\left(\varphi_{j i}, G_{i}\right)$ quando o diagrama

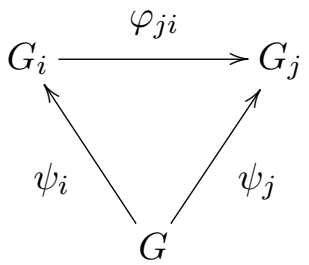


for comutativo para todo $j \preceq i$.

A cada sistema projetivo $\left(\varphi_{j i}, G_{i}\right)$ podemos associar uma categoria $\mathcal{L} P$ definida como segue. Os objetos de $\mathcal{L} P$ são pares $\left(G,\left\{\delta_{i}\right\}_{i \in I}\right)$, onde $\mathrm{G} \in \mathcal{G}_{\text {Top }}$ e $\delta_{i}: G \longrightarrow G_{i}$ é um morfismo na categoria $\mathcal{G}_{\text {Top }}$ tal que $\delta_{j}=\varphi_{j i} \circ \delta_{i}, \forall j \preceq i$.

Um morfismo de $\left(G,\left\{\delta_{i}\right\}_{i \in I}\right)$ para $\left(G^{\prime},\left\{\delta_{i}^{\prime}\right\}_{i \in I}\right)$ é uma tripla $\left(G, h, G^{\prime}\right)$, onde h é um morfismo de grupos topológicos que faz o seguinte diagrama comutar

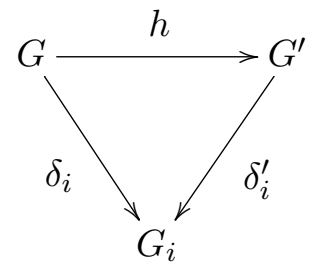

Proposição 51. A categoria $\mathcal{L} P$ (com a topologia produto) admite objeto terminal.

Demonstração. Seja $P \doteq \prod_{i \in I} G_{i}$ com projeções $\left(\pi_{i}\right)_{i \in I}$. Definimos

$$
G=\left\{x \in P: \varphi_{i j} \pi_{j}(x)=\pi_{i}(x), \forall i \leq j\right\}
$$

com a topologia de subespaço. $G \in \mathcal{G}_{T o p}$, pois $1 \in G$ e $\varphi_{i j}, \pi_{i}$ são morfismos contínuos de grupos. Seja $\psi_{i}=\left.\pi_{i}\right|_{G}$. Claramente, $\left(\psi_{i}\right)$ é uma família compatível. Para cada família compatível $\left(\phi_{i}\right.$ : $\left.H \longrightarrow G_{i}\right)_{i \in I}$ definimos $\phi: H \longrightarrow P, h \mapsto\left(\phi_{i}(h)\right.$ ) (note que $\phi$ é homomorfismo contínuo de grupos topológicos e $\operatorname{Im}(\phi) \subseteq G$ ) e seja $\phi^{*}: H \longrightarrow G, h \mapsto \phi(h)$ o único morfismo em $\mathcal{G}_{\text {Top }}$ tal que $\psi_{i} \phi^{*}=\phi_{i}$. Por definição $\phi^{*}$ é única

Definição 43. Seja $\left(G_{i}, \varphi_{i j}\right)_{i \in I}$ um sistema projetivo de grupos topológicos. Um limite projetivo (ou inverso) é um grupo topológico $G$ com uma família compatível $\left(\psi_{i}: G \longrightarrow G_{i}\right)_{i \in I}$ que cumpre a seguinte propriedade universal: Para qualquer familia compatível $\left(G^{\prime}, \phi_{i}: G^{\prime} \longrightarrow G_{i}\right)$ existe um único morfismo de grupos topológicos $\Phi: G^{\prime} \longrightarrow G$ que torna o seguinte diagrama comutativo $\forall j \preceq i$

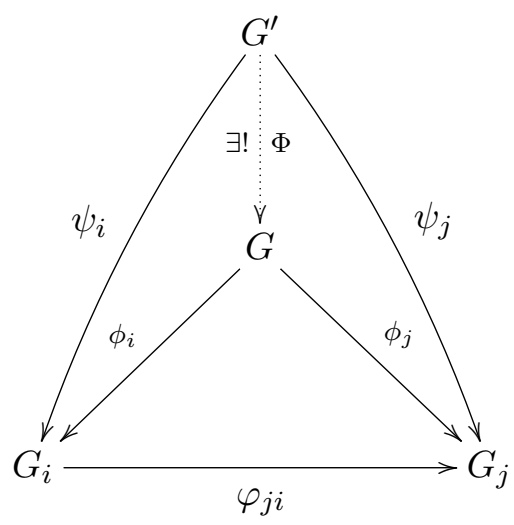

Usamos o símbolo $\varliminf_{i \in I} G_{i}$ para um limite projetivo.

\section{Observação 31.}

1) Os conceitos de sistema projetivo e limite projetivo podem ser dualizados por introduzir a noção de sistema indutivo e limite indutivo. 
2)Um mofismo entre sistemas projetivos $\left(\varphi_{i}, G_{i j}\right)_{i \in I}$ e $\left(\varphi_{i}^{\prime}, G_{i j}^{\prime}\right)_{i \in I}$ consiste em um familia $\left(\rho_{i}\right)_{i \in I}$ de morfismos em $\mathcal{G}_{\text {Top }}\left(G, G^{\prime}\right)$ tal que o seguinte diagrama comuta para todo $j \preceq i$.

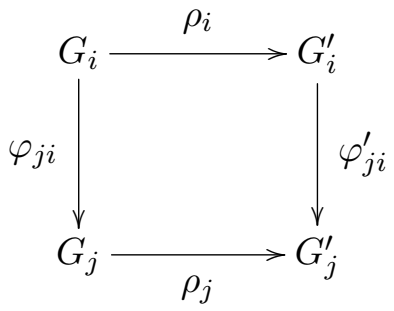

Denotamos por $\mathcal{S}_{\text {Proj }}\left(\left(\varphi_{i}, G_{i j}\right),\left(\varphi_{i}^{\prime}, G_{i j}^{\prime}\right)\right)$ a coleção dos morfismos de $\left(\varphi_{i}, G_{i j}\right)$ em $\left(\varphi_{i}^{\prime}, G_{i j}^{\prime}\right)$.

$\operatorname{Se}\left(\rho_{i}\right) \in \mathcal{S}_{\text {Proj }}\left(\left(\varphi_{i}, G_{i j}\right),\left(\varphi_{i}^{\prime}, G_{i j}^{\prime}\right)\right)$ e $\left(\rho_{i}^{\prime}\right) \in \mathcal{S}_{\text {Proj }}\left(\left(\varphi_{i}^{\prime}, G_{i j}^{\prime}\right),\left(\varphi_{i}^{\prime \prime}, G_{i j}^{\prime \prime}\right)\right)$, então o próximo diagrama comuta $\forall j \preceq i$.

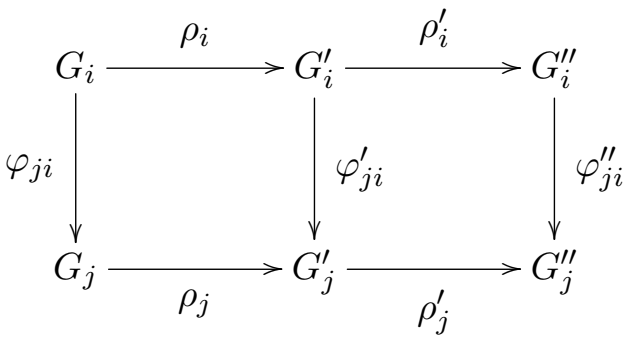

ou seja, $\left(\rho_{i}^{\prime} \circ \rho_{i}\right) \in \mathcal{S}_{\text {Proj }}\left(\left(G_{i}, \varphi_{i j}\right),\left(G_{i}^{\prime \prime}, \varphi_{i j}^{\prime \prime}\right)\right)$. Sistemas projetivos e morfismos de sistemas projetivos definem uma categoria. Usamos $\mathcal{S}_{\text {Proj }}$ para simbolizá-la. Além disso, é fácil ver que lim é um funtor de $\mathcal{S}_{\text {Proj }}$ para $\mathcal{G}_{\text {Top }}$.

Definição 44. Um grupo topológico $G$ é dito profinito quando existe um isomorfismo de grupos topológicos $G \cong \lim _{i \in I} G_{i}$, onde $\left(G_{i}\right)_{i \in I}$ é um sistema projetivo discreto finito.

Proposição 52. Seja G um grupo topológico. As seguintes condições são equivalentes :

(1) G é um grupo profinito;

(2) G é compacto e Hausdorff;

(3) G é compacto, Hausdorff e totalmente desconexo;

(4) $G$ é compacto e o elemento neutro e de $G$ possui um sistema fundamental de vizinhanças consistindo de subgrupos abertos de $G$;

(5) $O$ elemento neutro de $G$ possui um sistema fundamental de vizinhanças $\left\{H_{i}: i \in I\right\}$ consistindo de subgrupos abertos de $G$, e $G=\lim _{i \in I} G / H_{i}$;

(6) Existe um sistema inverso $\left\{G_{i}, \phi_{i j}\right\}$ de grupos finitos, onde cada morfismo $\phi_{i j}$ é um epimorfismo, e $G=\lim _{i \in I} G_{i}$.

Demonstração. Ver [RZ prop.5.1.2, pág.161.

Exemplo 5. (Completamento profinito) Um completamento profinito para um grupo G é um $\operatorname{par}(\hat{G}, \varphi)$, onde $\hat{G}$ é um grupo profinito e $\varphi$ é um morfismo de grupos tal que $\operatorname{Im}(\varphi)$ é denso em $\hat{G}$ e cumpre a seguinte propriedade universal: Se $G^{\prime}$ for profinito e $\varphi^{\prime}: G \longrightarrow G^{\prime}$ for um homomorfismo de grupos, então existe um único homomorfismo contínuo $\hat{\varphi}$ que faz o próximo diagrama comutar 


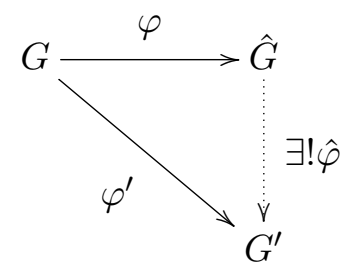

Para cada grupo G considere o conjunto

$$
\Omega \doteq\left\{N \in \wp(G): N \triangleleft G,|G / N|<\aleph_{0}\right\}
$$

A relação binária

$$
\forall N, N^{\prime} \in \Omega\left(N \preceq N^{\prime} \Longleftrightarrow N^{\prime} \subseteq N\right)
$$

faz de $\Omega$ um sistema dirigido. Para cada $N, N^{\prime} \in \Omega$ com $N \preceq N^{\prime}$ nós definimos o homomorfismo de grupos

$$
\varphi_{N N^{\prime}}: G / N^{\prime} \longrightarrow G / N, \quad g N^{\prime} \mapsto g N
$$

$O$ par $\left(G / N, \varphi_{N N^{\prime}}\right)_{N \in \Omega}$ é um sistema projetivo discreto finito. Seja $\varphi: G \longrightarrow \lim _{N \in \Omega} G / N o$ homomorfismo natural induzido pela propriedade universal de $\lim _{N \in \Omega} G / N$. Pode-se mostrar que $\left(\lim _{N \in \Omega} G / N, \varphi\right)$ é um completamento profinito para $G$. Além disso, a universalidade de $\hat{G}$ garante a unicidade.

Exemplo 6. (O grupo aditivo do anel de Prüfer) Em $\omega$ consideramos a ordem parcial

$$
\forall i, j \in \omega(i \preceq j \Longleftrightarrow i \mid j)
$$

Quando $j \preceq i$ existe um único homomorfismo de grupos que faz o seguinte diagrama comutar

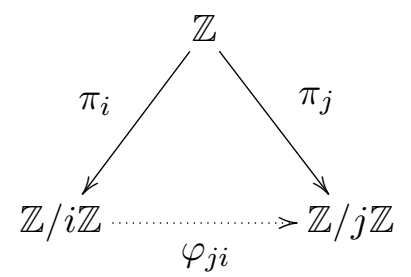

Facilmente observa-se que $\left(\mathbb{Z} / i \mathbb{Z}, \varphi_{i j}\right)$ constitui um sistema projetivo discreto finito. A compatibilidade da família $\left(\pi_{i}\right)_{i \in \omega}$ e a universalidade de $\hat{\mathbb{Z}} \doteq \lim _{i \in \omega} \mathbb{Z} / i \mathbb{Z}$ fornecem um único homomorfismo $\varphi: \mathbb{Z} \longrightarrow \hat{\mathbb{Z}}$. Pode-se mostrar que $\mathbb{Z}$ é denso em $\hat{\mathbb{Z}}$ e que $(\hat{\mathbb{Z}}, \varphi)$ é universal, ou seja, $(\hat{\mathbb{Z}}, \varphi)$ é o completamento profinito de $\mathbb{Z}$.

\subsection{Grupos de Galois}

\subsubsection{Grupo de automorfismos de extensões de corpos}

Seja $\mathbb{K}$ um corpo. Dizemos que um corpo $(\mathbb{E}, \cdot)$ é um extensão de $\mathbb{K}$ quando este for um subcorpo daquele. Denotamos por $\mathbb{E} \mid \mathbb{K}$ quando este for o caso. Temos, naturalmente, a seguinte ação 


$$
\begin{aligned}
\star: & \mathbb{K} \times \mathbb{E} \\
(k, e) & \longmapsto \mathbb{E} \\
& \longmapsto k \cdot e
\end{aligned}
$$

esta ação faz de $\mathbb{E}$ um $\mathbb{K}$-espaço vetorial. A sua dimensão é, corriqueiramente, anotada por $[\mathbb{E}: \mathbb{K}]$ e é chamado o grau de $\mathbb{E} \mid \mathbb{K}$. A extensão $\mathbb{E} \mid \mathbb{K}$ é dita finita se o seu grau for finito. Caso contrário, a extensão será dita infinita.

Seja $\mathbb{E} \mid \mathbb{K}$ uma extensão não nula de corpos. Um elemento $a \in \mathbb{E}$ é dito algébrico sobre $\mathbb{K}$ quando existir um polinômio $p(x) \in \mathbb{K}[x]$ tal que $p(a)=0$, ou seja, quando $a$ for raiz de algum polinômio em $\mathbb{K}[x]$. Uma extensão de corpos $\mathbb{E} \mid \mathbb{K}$ será dita algébrica se todo elemento de $\mathbb{E}$ for algébrico sobre $\mathbb{K}$. Para cada extensão de corpos $\mathbb{E} \mid \mathbb{K}$ denotaremos por $A_{\mathbb{E}}(\mathbb{K})$ o conjunto dos elementos algébricos de $\mathbb{E}$ que são algébricos sobre $\mathbb{K}$.

Para todo $a \in A_{\mathbb{E}}(\mathbb{K})$ considere o homomorfismo avaliação em $a$ definido por

$$
\begin{aligned}
\varphi_{a}: \mathbb{K}[x] & \longrightarrow \mathbb{K} \\
p(x) & \longmapsto p(a)
\end{aligned}
$$

Desde que, $\mathbb{K}[x]$ é um domínio de ideais principais e $\operatorname{Ker}\left(\varphi_{a}\right)$ é um de seus ideais,e $\operatorname{Ker}\left(\varphi_{a}\right) \neq 0$ (pois $a \in A_{\mathbb{E}}(\mathbb{K})$ ), segue que existe um único polinômio mônico $p_{a, \mathbb{K}}$ que o gera. Tal polinômio é chamado de polinômio minimal de $a$ sobre $\mathbb{K}$.

Para extensões algébricas valem os seguintes fatos 2 :

Fato 14. Seja $\mathfrak{M}$ um subconjunto de uma extensão de corpos $\mathbb{E} \mid \mathbb{K}$ tal que $\mathfrak{M} \subseteq A_{\mathbb{E}}(\mathbb{K})$. Então, a extensão $\mathbb{K}(\mathfrak{M}) \mid \mathbb{K}$ será algébrica.

Fato 15. Sejam $\mathbb{L} \mid \mathbb{E}$ e $\mathbb{E} \mid \mathbb{K}$ extensões de corpos. Vale a seguinte propriedade de transitividade algébrica :

$$
\mathbb{L} \mid \mathbb{E} \text { e } \mathbb{E} \mid \mathbb{K} \text { são algébricas } \Longleftrightarrow \mathbb{L} \mid \mathbb{K} \text { é algébrica. }
$$

Seja $\mathcal{K}_{\mathbb{E}}$ o conjunto dos subcorpos de $\mathbb{E}$. A propriedade de transitividade algébrica expressa no fato 2 permite definir a seguinte operação de fecho em $\mathcal{K}_{\mathbb{E}}$

$$
\begin{aligned}
A_{\mathbb{E}}: \mathcal{K}_{\mathbb{E}} & \longrightarrow \mathcal{K}_{\mathbb{E}} \\
\mathbb{K} & \longmapsto A_{\mathbb{E}}(\mathbb{K})
\end{aligned}
$$

- Seja $\mathbb{K} \in \mathcal{K}_{\mathbb{E}}$. Pelo fato $1, \mathbb{K}\left(A_{\mathbb{E}}(\mathbb{K})\right) \mid \mathbb{K}$ é algébrica e, portanto $\mathbb{K}\left(A_{\mathbb{E}}(\mathbb{K})\right) \subseteq A_{\mathbb{E}}(\mathbb{K})$ e como $A_{\mathbb{E}}(\mathbb{K}) \subseteq \mathbb{K}\left(A_{\mathbb{E}}(\mathbb{K})\right)$ temos a igualdade

$$
\mathbb{K}\left(A_{\mathbb{E}}(\mathbb{K})\right)=A_{\mathbb{E}}(\mathbb{K})
$$

concluímos que $\left(A_{\mathbb{E}}\left(\mathcal{K}_{\mathbb{E}}\right)\right) \subseteq \mathcal{K}_{\mathbb{E}}$.

- Vale, trivialmente, a seguinte implicação

$$
\mathbb{K} \subseteq \mathbb{F} \Longrightarrow A_{\mathbb{E}}(\mathbb{K}) \subseteq A_{\mathbb{E}}(\mathbb{F}), \forall \mathbb{K}, \mathbb{F} \in \mathcal{K}_{\mathbb{E}}
$$

- Para cada $a \in A_{\mathbb{E}}\left(A_{\mathbb{E}}(\mathbb{K})\right)$ a extensão $A_{\mathbb{E}}(\mathbb{K})(a) \mid A_{\mathbb{E}}(\mathbb{K})$ é algébrica, e decorre do fato 2 que $A_{\mathbb{E}}(\mathbb{K})(a) \mid \mathbb{K}$ também é algébrica; portanto $a \in A_{\mathbb{E}}(\mathbb{K})$. Desde que, as inclusões $\mathbb{K} \subseteq A_{\mathbb{E}}(\mathbb{K}) \subseteq$ $A_{\mathbb{E}}\left(A_{\mathbb{E}}(\mathbb{K})\right)$ são imediatas ter-se-á

\footnotetext{
${ }^{2}$ Ver o excelente livro de teoria de corpos de Otto Endler End para uma demonstração dessas propriedades.
} 


$$
\mathbb{K} \subseteq A_{\mathbb{E}}(\mathbb{K})=A_{\mathbb{E}}\left(A_{\mathbb{E}}(\mathbb{K})\right)
$$

- Obviamente, $A_{\mathbb{E}}(\mathbb{K})=\mathbb{E}$ se, e somente se, $\mathbb{E} \mid \mathbb{K}$ for uma extensão algébrica. Por exemplo, $A_{\mathbb{C}}(\mathbb{Q})$ é o corpo de todos os números complexos que são algébricos sobre $\mathbb{Q}$.

- $\Omega \doteq A_{\mathbb{E}}(\mathbb{K})$ é o único corpo entre $\mathbb{K}$ e $\mathbb{E}$ com a seguinte propriedade ${ }^{3}$ :

$\Omega \mid \mathbb{K}$ é uma extensão algébrica tal que $A_{\mathbb{E}}(\Omega)=\Omega$.

- $\mathrm{O}$ corpo $A_{\mathbb{E}}(\mathbb{K})$ é chamado o fecho algébrico de $\mathbb{K}$ em $\mathbb{E}$.

Definição 45. Seja $\mathbb{E} \mid \mathbb{K}$ uma extensão de corpos. Diremos que $\mathbb{K}$ é algebricamente fechado em $\mathbb{E}$ quando $A_{\mathbb{E}}(\mathbb{K})=\mathbb{K}$.

Observação 32. Para toda extensão de corpos $\mathbb{E} \mid \mathbb{K}$ o corpo $A_{\mathbb{E}}(\mathbb{K})$ é algebricamente fechado em $\mathbb{E}$

Noções absolutas de fecho algébrico e algebricamente fechado serão introduzidas na sequência.

\section{Definição 46.}

1) Diremos que um corpo $\mathbb{F}$ é algebricamente fechado, quando for algebricamente fechado em toda extensão $\mathbb{E} \mid \mathbb{F}$.

2) Seja $\mathbb{F} \mid \mathbb{K}$ um extensão de corpos. Dizemos que $\mathbb{F}$ é um fecho algébrico de $\mathbb{K}$, quando for algebricamente fechado e $\mathbb{F} \mid \mathbb{K}$ for algébrica.

Proposição 53. Seja $\Omega \mid \mathbb{K}$ uma extensão de corpos algebricamente fechado. Então, $A_{\Omega}(\mathbb{K})$ é o único fecho algébrico de $\mathbb{K}$ contido em $\Omega$.

Demonstração. Ver End pág. 43 e prop. 2.15.

\section{Proposição 54.}

1) Todo corpo $\mathbb{K}$ está contido em um corpo algebricamente fechado $\Omega$.

2) Todo corpo possui um fecho algébrico.

3) Todos os fechos algébricos de um corpo $\mathbb{K}$ são $\mathbb{K}$-isomorfos.

Demonstração. Consulte [End] pág. 44 ,prop. 2.16. e pág 47 ,cor. 2.21.

Observação 33. Decorre do item 3 da proposição anterior que se $\mathbb{K}$ está contido em corpos algebricamente distintos $\Omega$ e $\Omega^{\prime}$, então existe um $\mathbb{K}$ - isomorfismo de corpos $A_{\Omega}(\mathbb{K}) \cong A_{\Omega^{\prime}}(\mathbb{K})$. Doravante, usaremos $\mathbb{K}^{\text {alg }}$ para um fecho algébrico de $\mathbb{K}$ (salvo exceções).

Seja $\mathbb{K}$ um corpo e $p(x) \in \mathbb{K}[x]$. Seja $a \in \mathbb{K}$ uma raiz desse polinômio. Definimos a sua multiplicidade como sendo o inteiro não-negativo

$$
\operatorname{mult}(a, p) \doteq \max \left\{m \in \omega: \exists p^{\prime}(x) \in \mathbb{K}[x] ; p(x)=(x-a)^{m} \cdot p^{\prime}(x)\right\}
$$

\footnotetext{
${ }^{3}$ Confira End página 37 e exercício 2.8.
} 
Definição 47. Seja $\mathbb{K}$ um corpo e $p(x) \in \mathbb{K}[x]$. Dizemos que uma raiz a $\in \mathbb{K}$ de $p(x)$ é simples quando mult $(a, p)=1$. Caso contrário, a é dita uma raiz múltipla de $p(x)$.

Um critério, bastante conhecido, da teoria de corpos para decidir quando uma raíz é ou não simples, faz uso do operador derivação $D: \mathbb{K}[x] \longrightarrow \mathbb{K}[x]$ que definiremos na sequência ${ }^{4}$

Definição 48. Seja $R$ um anel. Definimos a derivada no anel de polinômios $R[x]$ como segue

$$
D: \begin{gathered}
R[x] \\
\sum_{j=0}^{m} a_{j} x^{j}
\end{gathered} \stackrel{\sum_{j=1}^{m} j \cdot a_{j} x^{j-1}}{\longrightarrow}
$$

Um polinômio não - nulo $p(x) \in \mathbb{K}[x]$ é denominado separável, sempre que $m d c(p(x), D p(x))=$ 1. Em outras palavras, um polinômio será separável quando ele e a sua derivada forem primos entre si. Note que essa definição de separabilidade independe ${ }^{5}$ de $\mathbb{K}$, pois a noção de primos entre si independe de $\mathbb{K}$.

Seja $\mathbb{E} \mid \mathbb{K}$ uma extensão de corpos. Dizemos que $a \in \mathbb{E}$ é separável sobre $\mathbb{K}$ quando for raiz de algum polinômio separável de $\mathbb{K}[x]$.

Definição 49. Dizemos que uma extensão algébrica de corpos $\mathbb{E} \mid \mathbb{K} e ́$

1) Separável, quando todo a $\in \mathbb{K}$ for separável sobre $\mathbb{K}$.

2) Normal, quando para todo $a \in \mathbb{K}$, o polinômio minimal $p_{a, \mathbb{K}}$ se decompor em fatores lineares em $\mathbb{E}[x]$.

3) Galoisiana(ou de Galois), quando for normal e separável.

Definição 50. Seja $\mathbb{K}$ um corpo e $\Omega$ um corpo algebricamente fechado que contém $\mathbb{K}$. Seja $\mathbb{E} \mid \mathbb{K}$ uma extensão de $\mathbb{K}$ tal que $\mathbb{K} \subseteq \mathbb{E} \subseteq \Omega$. Dizemos que um homomorfismo de corpos $\sigma: \mathbb{E} \longrightarrow \Omega$ é um $\mathbb{K}$-homomorfismo de $\mathbb{E}$ em $\Omega$, quando $\left.\sigma\right|_{\mathbb{K}}=i d_{\mathbb{K}}$. Simbolizamos por $H_{o m}(\mathbb{E}, \Omega)$ o conjunto dos $\mathbb{K}$-homomorfismos de $\mathbb{E}$ em $\Omega$.

Seja $\mathbb{K}$ um corpo e $\Omega$ um corpo algebricamente fechado que o contém. Para cada $\mathbb{E}$ entre $\mathbb{K}$ e $\Omega$. Dizemos que um endomorfismo de corpos $\sigma: \mathbb{E} \longrightarrow \mathbb{E}$ é um $\mathbb{K}$-automorfismo, quando $\sigma(\mathbb{K})=\mathbb{K}$, ou seja, quando $\sigma$ fixar o corpo $\mathbb{K}$. Denotaremos por $\operatorname{Gal}(\mathbb{E} \mid \mathbb{K})$ o conjunto de todos os $\mathbb{K}$-automorfismos de $\mathbb{E}$ que fixa $\mathbb{K}$. É fácil ver que $\operatorname{Gal}(\mathbb{E} \mid \mathbb{K})$ com a composição de funções o é um grupo, com unidade $i_{d_{\mathbb{E}}}$.

Definição 51. O grupo Gal $(\mathbb{E} \mid \mathbb{K})$ é chamado de grupo de Galois da extensão $\mathbb{E} \mid \mathbb{K}$.

Com as condições acima o seguinte fato é central na teoria de Galois finita

Fato 16. Seja $\mathbb{E} \mid \mathbb{K}$ uma extensão finita. Então:

1) $|\operatorname{Gal}(\mathbb{E} \mid \mathbb{K})| \leq\left|\operatorname{Hom}_{\mathbb{K}}(\mathbb{E}, \Omega)\right| \leq[\mathbb{E}: \mathbb{K}]$.

2) $|\operatorname{Gal}(\mathbb{E} \mid \mathbb{K})|=\left|\operatorname{Hom}_{\mathbb{K}}(\mathbb{E}, \Omega)\right| \Longleftrightarrow \mathbb{E} \mid \mathbb{K}$ for normal.

3) $|\operatorname{Gal}(\mathbb{E} \mid \mathbb{K})|=[\mathbb{E}: \mathbb{K}] \Longleftrightarrow \mathbb{E} \mid \mathbb{K}$ for galoisiana.

Seja $\mathbb{E} \mid \mathbb{K}$ uma extensão de corpos. Denotaremos por

$$
\mathcal{J}_{\mathbb{E}} \doteq\left\{\mathbb{I} \in \mathcal{K}_{\mathbb{E}}:[\mathbb{I}: \mathbb{K}]<\aleph_{0}, \mathbb{I} \mid \mathbb{K} \text { galoisiana }\right\}
$$

\footnotetext{
${ }^{4}$ Uma raiz $a \in \mathbb{K}$ de $p(x) \in \mathbb{K}[x]$ é simples se, e somente se, $D p(a) \neq 0$. Consulte, por exemplo, End pág. $52 \mathrm{e}$ prop. 3.1.

${ }^{5}$ Veja o exer. 2.7,pág.48 em End].
} 
o conjunto de todas as sub-extensões galoisianas intermediárias e finita da extensão $\mathbb{E} \mid \mathbb{K}$. Convém destacar, também, o conjunto

$$
\mathcal{G}_{\mathbb{E}} \doteq\left\{\operatorname{Gal}(\mathbb{E} \mid \mathbb{I}): \mathbb{I} \in \mathcal{J}_{\mathbb{E}}\right\}
$$

Lema 30. Para toda extensão galoisiana arbitrária $\mathbb{E} \mid \mathbb{K}$ e para todo $\mathbb{I} \in \mathcal{J}_{\mathbb{E}}$ valem as seguintes asserções:

1) $\operatorname{Gal}(\mathbb{E} \mid \mathbb{I}) \triangleleft \operatorname{Gal}(\mathbb{E} \mid \mathbb{K})$;

2) $\operatorname{Gal}(\mathbb{E} \mid \mathbb{K}) / \operatorname{Gal}(\mathbb{E} \mid \mathbb{I}) \cong \operatorname{Gal}(\mathbb{I} \mid \mathbb{K})$;

3) $|\operatorname{Gal}(\mathbb{E} \mid \mathbb{K}) / \operatorname{Gal}(\mathbb{E} \mid \mathbb{I})|<\aleph_{0}$

Demonstração. Consulte a prova em [Mor] lema 17.2 ,seção 17 e cap. IV.

Definição 52. Definimos uma relação binária em $\mathcal{J}_{\mathbb{E}}$ da seguinte maneira:

$$
\mathbb{I} \preceq \mathbb{I}^{\prime} \doteq \mathbb{I} \subseteq \mathbb{I}^{\prime}
$$

\section{Lema 31.}

1) $(\mathcal{I}, \preceq)$ é um sistema dirigido.

2) Se $\mathbb{I} \preceq \mathbb{I}^{\prime}$, então todo automorfismo $\sigma \in G a l(\mathbb{I} \mid \mathbb{K})$ induz um homomorfismo

$$
\varphi_{\mathbb{I}^{\prime}, \mathbb{I}}: \operatorname{Gal}\left(\mathbb{I}^{\prime} \mid \mathbb{K}\right) \longrightarrow \operatorname{Gal}(\mathbb{I} \mid \mathbb{K}),\left.\sigma \mapsto \sigma\right|_{I}
$$

2) $\left(\operatorname{Gal}(\mathbb{I}, \mathbb{K}), \varphi_{\mathbb{I}^{\prime}, \mathbb{I}}\right)_{\mathbb{I}, \mathbb{I}^{\prime} \in \mathcal{J}_{\mathbb{E}}}$ é um sistema projetivo.

Demonstração.

1)

$\left(\preceq_{1}\right) \mathbb{I} \preceq \mathbb{I} \Longleftrightarrow \mathbb{I} \subseteq \mathbb{I}($ Reflexiva $) ;$

$\left(\preceq_{2}\right) \mathbb{I} \preceq \mathbb{I}^{\prime} \wedge \mathbb{I}^{\prime} \preceq \mathbb{I} \Longleftrightarrow \mathbb{I} \subseteq \mathbb{I}^{\prime} \wedge \mathbb{I}^{\prime} \subseteq \mathbb{I} \Longrightarrow \mathbb{I}=\mathbb{I}^{\prime}($ Anti - simetria $) ;$

$\left(\preceq_{3}\right) \quad \mathbb{I} \preceq \mathbb{I}^{\prime} \wedge \mathbb{I}^{\prime} \preceq \mathbb{I}^{\prime \prime} \Longrightarrow \mathbb{I} \subseteq \mathbb{I}^{\prime} \wedge \mathbb{I}^{\prime} \subseteq \mathbb{I}^{\prime \prime} \Longrightarrow \mathbb{I} \subseteq \mathbb{I}^{\prime \prime}($ Transitiva $) ;$

$\left(\preceq_{4}\right) \quad \forall \mathbb{I}, \mathbb{I}^{\prime} \in \mathcal{J}_{\mathbb{E}}\left(\mathbb{I} \preceq \mathbb{I} \cdot \mathbb{I}^{\prime} \wedge \mathbb{I}^{\prime} \preceq \mathbb{I} \cdot \mathbb{I}^{\prime}\right)$ (Limite superior ${ }^{6}$ )

2)Basta notar que a aplicação restrição

$$
\varphi_{\mathbb{I}^{\prime}, \mathbb{I}}: \operatorname{Gal}\left(\mathbb{I}^{\prime} \mid \mathbb{K}\right) \longrightarrow \operatorname{Gal}(\mathbb{I} \mid \mathbb{K})
$$

é bem definida. 7

3) Claramente se $\mathbb{I} \subseteq \mathbb{I}^{\prime} \subseteq \mathbb{I}^{\prime \prime}$, então $\varphi_{\mathbb{I}^{\prime}, \mathbb{I}} \circ \varphi_{\mathbb{I}^{\prime \prime}, \mathbb{I}^{\prime}}=\varphi_{\mathbb{I}^{\prime \prime}, \mathbb{I}}$.

Podemos introduzir de maneira semelhante uma ordem $\preceq$ em $\mathcal{G}_{\mathbb{E}}$ considerando inclusões. Definimos aplicações $\varphi_{\mathbb{I}^{\prime}, \mathbb{I}}^{*}$ mediante a composição

\footnotetext{
${ }^{6} \mathrm{O}$ compósito de extensões finitas galoisianas também é finita galoisiana.

${ }^{7}$ Veja o teorema 3.3 na página 237 de [SLan ou [End prop.5.13, pág.81.
} 


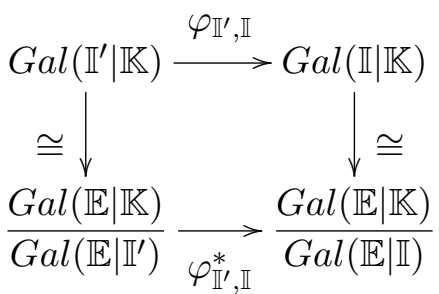

Não é difícil ver que o par

$$
\left(\frac{\operatorname{Gal}(\mathbb{E} \mid \mathbb{K})}{\operatorname{Gal}(\mathbb{I} \mid \mathbb{K})}, \varphi_{\mathbb{I}^{\prime}, \mathbb{I}}^{*}\right)_{\mathbb{I}, \mathbb{I}^{\prime} \in \mathcal{J}_{\mathbb{E}}}
$$

constitui um sistema projetivo de grupos finitos. Os dois sistemas projetivos anteriores se equivalem no sentido que existe um diagrama comutativo

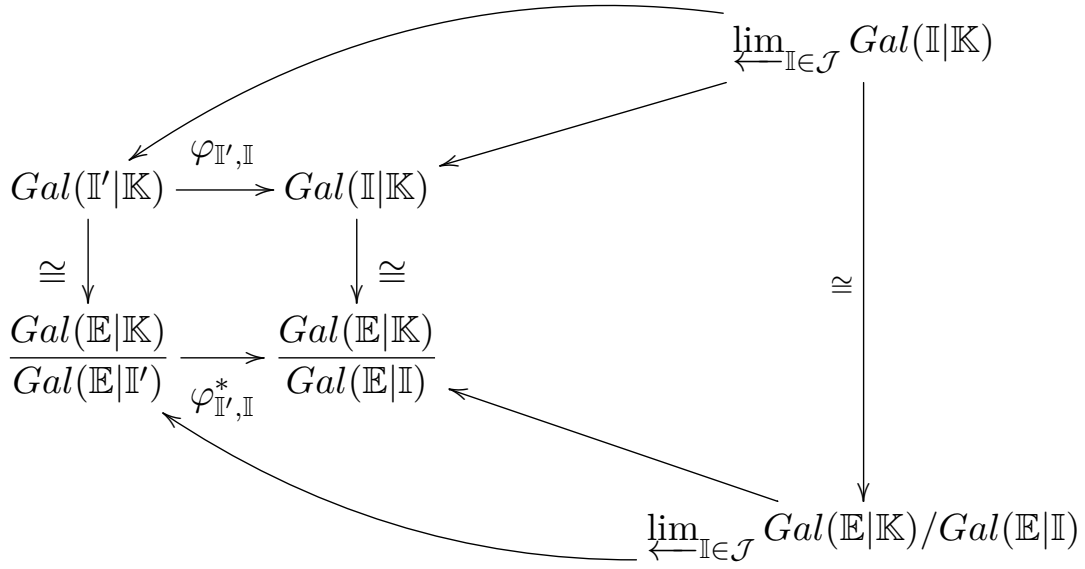

Proposição 55. Existem isomorfismos naturais de grupos

$$
\operatorname{Gal}(\mathbb{E} \mid \mathbb{K}) \cong \lim _{\mathbb{I} \in \mathcal{J}} \operatorname{Gal}(\mathbb{I} \mid \mathbb{K}) \cong \lim _{\mathbb{I} \in \mathcal{J}} \operatorname{Gal}(\mathbb{E} \mid \mathbb{K}) / \operatorname{Gal}(\mathbb{E} \mid \mathbb{I})
$$

Demonstração. Consulte [RZ] pág. 68 , teor. 2.11.1.

Para uma extensão galoisiana arbitrária $\mathbb{E} \mid \mathbb{K}$, segue da proposição anterior que $\operatorname{Gal}(\mathbb{E} \mid \mathbb{K})$ é um grupo profinito. Nesse caso, a proposição 52 garante que $\mathbb{E} \mid \mathbb{K}$ é um grupo topológico, Hausdorff, compacto e totalmente desconexo.

Vimos acima que todo grupo de galois é profinito. Na realidade, esses são, essencialmente, os únicos grupos profinitos. Em outras palavras, todo grupo profinito $\mathcal{P}$ é isomorfo a um grupo de galois de alguma extensão de corpos ${ }^{8}$

Seja $\mathbb{E} \mid \mathbb{K}$ uma extensão galoisiana. É possível mostrar que existe uma única topologia em $\operatorname{Gal}(\mathbb{E} \mid \mathbb{K})$ compatível com a estrutura de grupo de $G a l(\mathbb{E} \mid \mathbb{K})$ tal que o conjunto

$$
\mathcal{G}_{\mathbb{E}}=\left\{\operatorname{Gal}(\mathbb{E} \mid \mathbb{I}): \mathbb{I} \in \mathcal{J}_{\mathbb{E}}\right\}
$$

\footnotetext{
${ }^{8}$ Para uma prova dessa afirmação e mais detalhes sobre grupo de galois como grupo profinito consulte, por exemplo, a seção 2.11 na página $68 \mathrm{em}[\mathbf{R Z}$.
} 
é um sistema fundamental de vizinhanças do automorfismo identidade de $G a l(\mathbb{E} \mid \mathbb{K})$ (ver [Bou2]). Essa topologia é conhecida como topologia de Krull. Pode-se mostrar que o isomorfismo

$$
\operatorname{Gal}(\mathbb{E} \mid \mathbb{K}) \cong \lim _{\mathbb{I} \in \mathcal{J}} \operatorname{Gal}(\mathbb{I} \mid \mathbb{K})
$$

da proposição anterior é um homeomorfismo de grupos topológicos, onde $G a l(\mathbb{E} \mid \mathbb{K})$ está munido com a topologia de Krull.

\subsubsection{O grupo de Galois absoluto}

Para cada extensão algébrica $\mathbb{E} \mid \mathbb{K}$ convém destacar o conjunto

$$
S_{\mathbb{E}}(\mathbb{K}) \doteq\{a \in \mathbb{E}: a \text { é separável sobre } \mathbb{K}\}
$$

Lema 32. $S_{\mathbb{E}}(\mathbb{K})$ é uma sub-extensão de $\mathbb{E} \mid \mathbb{K}$.

Demonstração. Se $a, b \in S_{\mathbb{E}}(\mathbb{K})$, então $\mathbb{K}(a, b) \mid \mathbb{K}(a)$ e $\mathbb{K}(a) \mid \mathbb{K}$ são extensões separáveis ${ }^{9}$ e, portanto $\mathbb{K}(a, b) / \mathbb{K}$ também o é10

O lema anterior nos habilita a definir uma aplicação

$$
\begin{aligned}
S_{\mathbb{E}}: \mathcal{K}_{\mathbb{E}} & \longrightarrow \mathcal{K}_{\mathbb{E}} \\
\mathbb{K} & \longmapsto S_{\mathbb{E}}(\mathbb{K})
\end{aligned}
$$

Essa aplicação possui as seguintes propriedades:

- $S_{\mathbb{E}}\left(S_{\mathbb{E}}(\mathbb{K})\right)=S_{\mathbb{E}}(\mathbb{K})$;

- $\forall \mathbb{K}, \mathbb{K}^{\prime} \in \mathcal{K}_{\mathbb{E}}\left(\mathbb{K} \subseteq \mathbb{K}^{\prime} \Longrightarrow S_{\mathbb{E}}(\mathbb{K}) \subseteq S_{\mathbb{E}}\left(\mathbb{K}^{\prime}\right)\right) ;$

- $S_{\mathbb{E}}(\mathbb{K}) \subseteq A_{\mathbb{E}}(\mathbb{K})$

- $S_{\mathbb{E}}(\mathbb{K})=A_{\mathbb{E}}(\mathbb{K})$, sempre que $\mathbb{K}$ for perfeito.

- Se $\mathbb{F} \mid S_{\mathbb{E}}(\mathbb{K})$ for uma extensão algébrica e separável de $S_{\mathbb{E}}(\mathbb{K})$,então claramente $S_{\mathbb{E}}(\mathbb{K})=\mathbb{F}$. Em outras palavras, $S_{\mathbb{E}}(\mathbb{K})$ não admite extensões algébricas separáveis não triviais.

\section{Definição 53.}

1) Seja $\mathbb{E} \mid \mathbb{K}$ uma extensão de corpos. O corpo $\mathbb{K}$ é dito separavelmente fechado em $\mathbb{E}$, quando $S_{\mathbb{E}}(\mathbb{K})=\mathbb{K}$.

2) $S_{\mathbb{E}}(\mathbb{K})$ é chamado de o fecho separável de $\mathbb{K}$ em $\mathbb{E}$.

3) Um corpo $\mathbb{F}$ é dito separavelmente fechado, quando não admitir extensão algébrica separável não trivial.

4) Seja $\mathbb{K}$ um corpo. Por um fecho separável de $\mathbb{K}$ entendemos uma extensão algébrica e separável $\mathbb{F} \mid \mathbb{K}$ tal que $\mathbb{F}$ é separavelmente fechado.

\section{Observação 34 .}

1) $S_{\mathbb{E}}(\mathbb{K})$ é separavelmente fechado em $\mathbb{E}$.

\footnotetext{
${ }^{9}$ Seja $\mathbb{E} \mid \mathbb{K}$ uma extensão e seja $\mathfrak{M}$ um subconjunto de $\mathbb{E}$. Se todo $a \in \mathfrak{M}$ for separável sobre $\mathbb{K}$, então $\mathbb{K}(\mathfrak{M})$ será separável.([End], prop.3.12, pág. 59)

${ }^{10}$ Ver End prop.3.13, pág.60
} 
2) Se $\mathbb{K}$ for algebricamente fechado em $\mathbb{E}$, então será separavelmente fechado em $\mathbb{E}$;

3) Todo corpo separavelmente fechado $\mathbb{F} \supset \mathbb{K}$ contém um único fecho separável de $\mathbb{K}$, a saber $S_{\mathbb{E}}(\mathbb{K})$.

4) Todo fecho algébrico $\mathbb{F} \mid \mathbb{K}$ contém um único fecho separável de $\mathbb{K}$.

5) Doravante, denotaremos por $\mathbb{K}^{\text {sep }}$ um fecho separável de $\mathbb{K}$ dentro de $\mathbb{K}^{\text {alg }}$.

Definição 54. Chamamos de grupo de Galois absoluto de um corpo $\mathbb{K}$ ao grupo de Galois $\operatorname{Gal}\left(\mathbb{K}^{s e p} \mid \mathbb{K}\right)$.

Exemplo 7. (O grupo de Galois absoluto de um corpo finito) Para cada primo p sabemos que $\mathbb{F}_{p}$ é um corpo perfeito e, portanto uma extensão galoisiana. A família $\left(\mathbb{F}_{p^{n}}\right)$ fornece uma classificação das extensões galoisianas de grau n para o corpo $\mathbb{F}_{p}$. Além disso, um fecho separável para $\mathbb{F}_{p}$ é dado por $\mathbb{F}_{p}^{a l g}=\bigcup_{n \in \omega} \mathbb{F}_{p^{n}}$. Nesse caso, o grupo de Galois absoluto de $\mathbb{F}_{p}$ é dado por

$$
\operatorname{Gal}\left(\mathbb{F}_{p}^{a l g} / \mathbb{F}_{p}\right) \cong \hat{\mathbb{Z}}
$$

onde $\hat{\mathbb{Z}}:=\left\lfloor\lim \mathbb{Z} / n \mathbb{Z}=\left\{\left(\bar{x}_{n}\right) \in \prod \mathbb{Z} / n \mathbb{Z}: x_{k} \equiv x_{j}(\bmod k), \forall k \mid j\right\}\right.$ com as operações de soma (e produto) componente a componente é o chamado grupo (anel) de Prüfer (Ver o exemplo 6 da secção 3.3).

\subsection{Cohomologia profinita}

\subsubsection{G-módulos profinitos}

Seja $(G, \cdot)$ um grupo profinito. Um $G$-módulo é um par $(A, \star)$ consistindo de um grupo topológico abeliano e uma aplicação contínua $(G \times A$ munido com a topologia produto )

$$
\begin{aligned}
\star: G \times A & \longrightarrow A \\
(g, a) & \longmapsto g \star a
\end{aligned}
$$

Satisfazendo

(1) $\forall g, g^{\prime} \in G \quad \forall a \in A\left(\left(g g^{\prime}\right) \star a=g \star\left(g^{\prime} \star a\right)\right)$;

(2) $\forall g \in G \quad \forall a, a^{\prime} \in A\left(g \star\left(a+a^{\prime}\right)=g \star a+g \star a^{\prime}\right)$;

(3) $\forall a \in A(1 \star a=a)$.

Definição 55. Um G-módulo $(A, \star)$ é dito discreto quando for um grupo topológico discreto. E será chamado profinito quando for profinito.

Proposição 56. Seja A um G-módulo e $\mathcal{T}$ a topologia de $G$. As afirmações a seguir são equivalentes

(1) A é um G-módulo discreto.

(2) $\operatorname{Stab}_{G}(a) \doteq\{g \in G: g a=a\}$ é aberto em $G, \forall a \in A$.

(3) $A=\bigcup\left\{A^{\Omega}: \Omega\right.$ é um subgrupo aberto de $\left.G\right\}$, onde $A^{\Omega} \doteq\{a \in A: g \star a=a, \forall g \in \Omega\}$.

Demonstração. 
$(1) \Longrightarrow(2) \operatorname{Stab}_{G}(a)=\mu^{-1}(\{a\}) \in \mathcal{T}$, onde $\mu$ é aplicação contínua $g \mapsto g a$.

$(2) \Longrightarrow(3) a \in A \Longrightarrow a \in A^{\operatorname{Stab}_{G}(a)} \Longrightarrow A \subseteq \bigcup\left\{A^{\Omega}: \Omega\right.$ é vizinhança aberta de $\left.1 \mathrm{em} G\right\}$.

$(3) \Longrightarrow(1)$. Basta observar que $\{1\} \times\{a\} \subseteq \Omega \times\{a\} \subseteq \star^{-1}(\{a\})$ para algum aberto $\Omega$ (que é vizinhança de 1) de G.

Definição 56. Um $G$-homomorfismo entre $G$-módulos $A, B$ é um homomorfismo de grupos abelianos $\varphi: A \longrightarrow B$ compativel com a ação, ou seja,

$$
\varphi(g a)=g \varphi(a) \quad \forall g \in G, \forall a \in A
$$

Dizemos que $\varphi: A \longrightarrow B$ é um $G$-morfismo de $G$-módulos se for um $G$-homomorfismo contínuo.

Verifica-se facilmente que a classe de $G$-módulos juntamente com a classe de $G$ - homomorfismos cumprem os axiomas de uma categoria. Tal categoria é conhecida como a categoria de G-módulos e a denotamos por $\mathcal{M}_{G}$. Destacamos duas subcategorias abelianas daquela categoria, a saber, a categoria de $G$-módulos discretos $\mathcal{D}_{G}$ e a categoria de $G$-módulos profinitos $\mathcal{P}_{G}$.

\subsubsection{Cadeias não-homogêneas}

Seja $G$ um grupo profinito. Munimos o produto $G^{n}$ com a topologia produto. Para cada $A \in \mathcal{D}_{G}$ seja $\mathcal{C}\left(G^{n}, A\right)$ o anel das funções contínuas de $G^{n}$ em A. Colocamos

$$
C_{\text {cont }}^{n}(G, A) \doteq\left\{\varphi: G^{n} \longrightarrow A: \varphi \in \mathcal{C}\left(G^{n}, A\right)\right\}
$$

Em $C_{\text {cont }}^{n}(G, A)$ definimos a operação pontual

$$
\begin{aligned}
\oplus: C_{\text {cont }}^{n}(G, A) \times C_{\text {cont }}^{n}(G, A) \longrightarrow C_{\text {cont }}^{n}(G, A) \\
\left(\varphi, \varphi^{\prime}\right) \longmapsto \varphi \oplus \varphi^{\prime}
\end{aligned}
$$

onde $\varphi \oplus \varphi^{\prime}$ está definida como segue

$$
\begin{aligned}
\varphi \oplus \varphi^{\prime}: G^{n} & \longrightarrow A \\
x & \longrightarrow \varphi(x)+\varphi^{\prime}(x)
\end{aligned}
$$

é fácil ver que $\varphi \oplus \varphi^{\prime}$ é contínua e que o par $\left(C_{c o n t}^{n}(G, A), \oplus\right)$ é um grupo abeliano. Agora, cada morfismo $h: A \longrightarrow A^{\prime}$ em $\mathcal{D}_{G}$ determina por composição um homomorfismo de grupos abelianos

$$
\begin{aligned}
& C_{\text {cont }}^{n}(G, h): C_{\text {cont }}^{n}(G, A) \longrightarrow C_{\text {cont }}^{n}\left(G, A^{\prime}\right) \\
& \varphi \longmapsto \\
& h \circ \varphi
\end{aligned}
$$

Facilmente observa-se que $C_{\text {cont }}^{n}(G,-)$ é um funtor que sai da categoria $\mathcal{D}_{G}$ e chega na categoria $\mathcal{A} b$ de grupos abelianos.

Proposição 57. O funtor $C^{n}(G,-): \mathcal{D}_{G} \longrightarrow \mathcal{A} b$ é exato. 
Demonstração. Considere a sequência exata de G-módulos

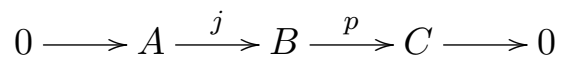

Mostraremos que a sequência

$$
0 \longrightarrow C_{\text {cont }}^{n}(G, A) \stackrel{j^{*}}{\longrightarrow} C_{\text {cont }}^{n}(G, B) \stackrel{p^{*}}{\longrightarrow} C_{\text {cont }}^{n}(G, C) \longrightarrow 0
$$

é exata, onde $j^{*} \doteq C_{\text {cont }}^{n}(G, j)$ e $p^{*} \doteq C_{\text {cont }}^{n}(G, p)$.

Injetividade de $j^{*}$

$$
j^{*}(\varphi)=j\left(\varphi^{\prime}\right) \Longleftrightarrow j \circ \varphi=j \circ \varphi^{\prime} \Longleftrightarrow \varphi=\varphi^{\prime}
$$

Sobrejetividade de $p^{*}$ Seja $\varphi \in C_{\text {cont }}^{n}(G, C)$. Por hipótese, temos

$$
\forall x \in G^{n} \exists b \in B(\varphi(b)=p(x))
$$

Fazendo escolha $b_{x} \in B$ para cada $x \in G^{n}$ como acima definimos a seguinte função

$$
\tilde{\varphi}: G^{n} \longrightarrow B, x \mapsto b_{x}
$$

Uma vez que,

$$
\tilde{\varphi}\left(\left\{b_{x}\right\}\right)=\emptyset \text { se } b \notin \operatorname{Im}(\varphi)
$$

$\mathrm{e}$

$$
\tilde{\varphi}^{-1}(\{\tilde{\varphi}(x)\})=\tilde{\varphi}^{-1}\left(\left\{b_{x}\right\}\right)=\varphi^{-1}(\{p(b x)\})
$$

segue que $\tilde{\varphi} \in C_{\text {cont }}^{n}(G, B)$.

Finalmente, mostremos que $\operatorname{Ker}\left(p^{*}\right)=\operatorname{Im}\left(j^{*}\right)$. Note que $p^{*} \circ j^{*}=0$, pois $p^{*} \circ j^{*}(h)=p^{*}(j \circ$ $h)=p \circ(j \circ h)=j \circ h=0$. Logo, $\operatorname{Im}\left(j^{*}\right) \subseteq \operatorname{Ker}\left(p^{*}\right)$. Por outro lado, se $\varphi \in \operatorname{Ker}\left(p^{*}\right)$, então $\varphi(x) \in \operatorname{Ker}(p)=\operatorname{Im}(j), \forall x \in G^{n}$. Assim, $\varphi(x)=j\left(a_{x}\right)$ para um único $a_{x} \in A$ (pois j é injetora). Segue que $\operatorname{Ker}\left(p^{*}\right) \subseteq \operatorname{Im}\left(j^{*}\right)$.

Definição 57. Definimos a aplicação cobordo para $n>0$

$$
\partial^{n}: C_{\text {cont }}^{n}(G, A) \longrightarrow C_{\text {cont }}^{n+1}(G, A)
$$

mediante a seguinte fórmula

$$
\begin{aligned}
\partial^{n} \varphi\left(g_{1}, g_{2}, \ldots, g_{n}, g_{n+1}\right) & =g_{1} \varphi\left(g_{2}, \ldots, g_{n}, g_{n+1}\right) \\
& +\sum_{k=1}^{n}(-1)^{k} \varphi\left(g_{1}, \ldots, g_{k} g_{k+1}, g_{k+2}, \ldots, g_{n+1}\right) \\
& +(-1)^{n+1} \varphi\left(g_{1}, \ldots, g_{n}\right)
\end{aligned}
$$

e para para $n=0$ definimos $\left(\partial^{0} \varphi\right)\left(g_{1}\right)=g_{1} \varphi-\varphi$.

Lema 33. A aplicação cobordo $\partial^{n}: C_{\text {cont }}^{n}(G, A) \longrightarrow C_{\text {cont }}^{n+1}(G, A)$ é um homomorfismo de grupos. 
Demonstração.

$$
\begin{aligned}
& \partial^{n}(\varphi \oplus \psi)\left(g_{1}, \ldots, g_{n+1}\right)=\quad g_{1}(\varphi \oplus \psi)\left(g_{2}, \ldots, g_{n+1}\right) \\
& +\sum_{k=1}^{n}(-1)^{k}(\varphi \oplus \psi)\left(g_{1}, \ldots, g_{k} g_{k+1}, \ldots, g_{n+1}\right) \\
& +\quad(-1)^{n+1}(\varphi \oplus \psi)\left(g_{1}, \ldots, g_{n}\right) \\
& =g_{1} \varphi\left(g_{2}, \ldots, g_{n+1}\right)+g_{1} \psi\left(g_{2}, \ldots, g_{n+1}\right) \\
& +\quad \sum_{k=1}^{n}(-1)^{k} \varphi\left(g_{1}, \ldots, g_{k} g_{k+1}, \ldots, g_{n+1}\right) \\
& +\quad \sum_{k=1}^{n}(-1)^{k} \psi\left(g_{1}, \ldots, g_{k} g_{k+1} \ldots g_{n+1}\right) \\
& +(-1)^{n+1} \varphi\left(g_{1}, \ldots, g_{n}\right)+(-1)^{n+1} \psi\left(g_{1}, \ldots, g_{n}\right) \\
& =\quad\left(\partial^{n}(\varphi) \oplus \partial^{n}(\psi)\right)\left(g_{1}, \cdots, g_{n+1}\right) \text {. }
\end{aligned}
$$

Proposição 58. A sequência de homomorfismos de grupos

$$
\begin{gathered}
C_{\text {cont }}^{0}(G, A) \stackrel{\partial^{0}}{\longrightarrow} C_{\text {cont }}^{1}(G, A) \stackrel{\partial^{1}}{\longrightarrow} C_{\text {cont }}^{2}(G, A) \longrightarrow \cdots \\
\longrightarrow C_{\text {cont }}^{n-1}(G, A) \stackrel{\partial^{n-1}}{\longrightarrow} C_{\text {cont }}^{n}(G, A) \stackrel{\partial^{n}}{\longrightarrow} C_{\text {cont }}^{n+1}(G, A) \longrightarrow \cdots
\end{gathered}
$$

é um complexo de cadeias, i.e, $\partial^{n} \partial^{n-1}=0, \forall n \geq 1$.

Demonstração. Para $\mathrm{n}=1$, temos

$$
\begin{aligned}
\left(\partial^{1} \partial^{0} \varphi\right)(u, v) & =u\left(\left(\partial^{0} \varphi\right)(v)\right)-\left(\partial^{0} \varphi\right)(u v)+\left(\partial^{0} \varphi\right)(u) \\
& =u(v \varphi-\varphi)-(u v \varphi-\varphi)+(u \varphi-\varphi) \\
& =0
\end{aligned}
$$

E para $n>1$, iniciamos com

$$
\begin{aligned}
\left(\partial^{n} \partial^{n-1} \varphi\right)\left(g_{1}, \ldots, g_{n+1}\right) & =g_{1}\left(\left(\partial^{n-1} \varphi\right)\left(g_{2}, \ldots, g_{n+1}\right)\right) \\
& +\sum_{k=1}^{n}\left(\partial^{n-1} \varphi\right)(-1)^{k}\left(g_{1}, \ldots, g_{k} g_{k+1}, \ldots, g_{n+1}\right) \\
& +(-1)^{n+1}\left(\partial^{n-1} \varphi\right)\left(g_{1}, \ldots, g_{n}\right) \\
& =I+I I+I I I
\end{aligned}
$$

Onde 


$$
\begin{aligned}
& I=g_{1} g_{2}\left(\varphi\left(g_{3}, \ldots, g_{n+1}\right)\right) \\
& +\sum_{k=2}^{n}(-1)^{k-1} g_{1}\left(\varphi\left(g_{2}, \ldots, g_{k} g_{k+1}, \ldots, g_{n+1}\right)\right) \\
& +(-1)^{n} g_{1}\left(\varphi\left(g_{2}, \ldots, g_{n}\right)\right) \\
& =I A+I B+I C \\
& I I=-\left(\partial^{n-1} \varphi\right)\left(g_{1} g_{2}, g_{3}, \ldots, g_{n}\right) \\
& +\sum_{k=2}^{n}(-1)^{k}\left(\partial^{n-1} \varphi\right)\left(g_{1}, \ldots, g_{k} g_{k+1}, \ldots, g_{n+1}\right) \\
& =I I A+I I B \\
& I I I=(-1)^{n+1} g_{1}\left(\varphi\left(g_{2}, \ldots, g_{n}\right)\right) \\
& +(-1)^{n+1}(-1) \varphi\left(g_{1} g_{2}, g_{3}, \ldots, g_{n}\right) \\
& +(-1)^{n+1} \sum_{k=2}^{n-1}(-1)^{k} \varphi\left(g_{1}, \ldots, g_{k} g_{k+1}, \ldots, g_{n}\right) \\
& +(-1)^{n+1}(-1)^{n} \varphi\left(g_{1}, \ldots, g_{n-1}\right) \\
& =I I I A+I I I B+I I I C+I I I D
\end{aligned}
$$

Os termos $I I A$ e $I I B$, ainda se decompõem, como

$$
\begin{aligned}
I I A & =-g_{1} g_{2}\left(\varphi\left(g_{3}, \ldots, g_{n+1}\right)\right) \\
& +\varphi\left(g_{1} g_{2} g_{3}, g_{4}, \ldots, g_{n+1}\right) \\
& -\sum_{k=3}^{n}(-1)^{k+1} \varphi\left(g_{1} g_{2}, \ldots, g_{k} g_{k+1}, \ldots, g_{n+1}\right) \\
& -(-1)^{n} \varphi\left(g_{1} g_{2}, g_{3}, \ldots, g_{n+1}\right) \\
& =I I A a+I I A b+I I A c+I I A d
\end{aligned}
$$




$$
\begin{aligned}
I I B & =\sum_{k=2}^{n}(-1)^{k} g_{1}\left(\varphi\left(g_{2}, \ldots, g_{k} g_{k+1}, \ldots, g_{n+1}\right)\right) \\
& +(-1)^{2}(-1) \varphi\left(g_{1} g_{2} g_{3}, g_{4}, \ldots, g_{n+1}\right) \\
& +\sum_{k=3}^{n}(-1)^{k}(-1) \varphi\left(g_{1} g_{2}, \ldots, g_{k} g_{k+1}, \ldots, g_{n+1}\right) \\
& +\sum_{k=2}^{n}(-1)^{k} \sum_{l=2}^{k-2}(-1)^{l} \varphi\left(g_{1}, \ldots, g_{l} g_{l+1}, \ldots, g_{k} g_{k+1}, \ldots, g_{n+1}\right) \\
& +\sum_{k=3}^{n}(-1)^{k}(-1)^{k-1} \varphi\left(g_{1}, \ldots, g_{k-1} g_{k} g_{k+1}, \ldots, g_{n+1}\right) \\
& +\sum_{k=2}^{n-1}(-1)^{k}(-1)^{k} \varphi\left(g_{1}, \ldots, g_{k} g_{k+1} g_{k+2}, \ldots, g_{n+1}\right) \\
& +\sum_{k=2}^{n-2}(-1)^{k} \sum_{l=k+1}^{n}(-1)^{l-1} \varphi\left(g_{1}, \ldots, g_{k} g_{k+1}, \ldots, g_{l} g_{l+1}, \ldots, g_{n+1}\right) \\
& +\sum_{k=2}^{n-1}(-1)^{k}(-1)^{n} \varphi\left(g_{1}, \ldots, g_{k} g_{k+1}, \ldots, g_{n}\right) \\
& +(-1)^{n}(-1)^{n} \varphi\left(g_{1}, \ldots, g_{n-1}\right) \\
& =I I B a+I I B b+I I B c+I I B d+I I B e+I I B f+I I B g+I I B h+I I B i .
\end{aligned}
$$

Inspeções mostram-nos que temos cancelamentos entre o termo IA e o termo IIAa, termo IB e o termo IIBa, termo IC e o termo IIIA, termo IIAb e o termo IIBb, termo IIAc e o termo IIBc, termo IIAd e o termo IIIB, termo IIBd e o termo IIB e o termo IIBg, termo IIBe e o termo IIBf, termo IIBh e o termo IIIC, e o termo IIBi e o termo IIID. Todos os termos cancelam e concluímos que $\partial^{n} \partial^{n-1} \varphi=0$.

Corolário 14. Para todo $n \geq 1$ tem-se $\operatorname{Im}\left(\partial^{n-1}\right) \subseteq \operatorname{Ker}\left(\partial^{n}\right)$.

Demonstração. Seja $\partial^{n-1}(\varphi) \in \operatorname{Im}\left(\partial^{n-1}\right)$.Temos,

$$
\partial^{n}\left(\partial^{n-1}(\varphi)\right)=\partial^{n} \partial^{n-1}(\varphi)=0(\varphi)=0
$$

Definição 58. Para cada $n \in \boldsymbol{\omega}$ definimos o grupo de n-cociclos por

$$
Z^{n}(G, A) \doteq \operatorname{Ker}\left(\partial^{n}\right)
$$

e o grupo de $\boldsymbol{n}$-cobordos por

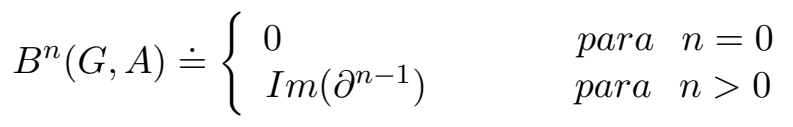

Finalmente, definimos o $n$-ésimo grupo de cohomologia continud ${ }^{11}$ com coeficientes em A

\footnotetext{
${ }^{11}$ A cohomologia de grupos contínua é uma variação da cohomologia ordinária de grupos. A sua origem remonta
} 
como sendo o grupo quociente

$$
H_{\text {cont }}^{n}(G, A) \doteq \frac{Z^{n}(G, A)}{B^{n}(G, A)}
$$

Observação 35. Uma forma alternativ ${ }^{12}$ de descrever $H_{\text {cont }}^{n}(G, A)$ é através das cadeias homogêneas. Para cada grupo profinito $G$ e cada $G$-módulo discreto $A$, definimos $\tilde{C}_{\text {cont }}(G, A)$ como sendo o conjunto das funções contínuas $\varphi: G^{n+1} \longrightarrow A$ tais que

$$
\varphi\left(g g_{0}, \ldots, g g_{n}\right)=g \varphi\left(g_{0}, \ldots, g_{n}\right), \forall g, g_{0}, \ldots, g_{n} \in G
$$

Definimos operadores cobordos $\partial^{n+1}: \tilde{C}_{\text {cont }}^{n}(G, A) \longrightarrow \tilde{C}_{\text {cont }}^{n+1}(G, A)$ através da seguinte fórmula

$$
\partial^{n+1} \varphi\left(g_{0}, \ldots, g_{n+1}\right)=\sum_{i=0}^{n+1}(-1)^{i} \varphi\left(g_{0}, \ldots, \hat{g}_{i}, \ldots g_{n+1}\right)
$$

onde $g_{0}, \ldots, g_{n+1} \in G$. É possível mostrar que a sequência de homomorfismos de grupos

$$
\begin{gathered}
\tilde{C}_{\text {cont }}^{0}(G, A) \stackrel{\partial^{0}}{\longrightarrow} \tilde{C}_{\text {cont }}^{1}(G, A) \stackrel{\partial^{1}}{\longrightarrow} \tilde{C}_{\text {cont }}^{2}(G, A) \longrightarrow \cdots \\
\longrightarrow \tilde{C}_{\text {cont }}^{n-1}(G, A) \stackrel{\partial^{n-1}}{\longrightarrow} \tilde{C}_{\text {cont }}^{n}(G, A) \stackrel{\partial^{n}}{\longrightarrow} \tilde{C}_{\text {cont }}^{n+1}(G, A) \longrightarrow \cdots
\end{gathered}
$$

é um complexo de cadeias que é isomorfo ao complexo $\left(C_{\text {cont }}^{n}\left(G^{n}, A\right)\right)$. Logo existe um isomorfismo natural $\tilde{H}_{\text {cont }}^{n}(G, A) \cong H_{\text {cont }}(G, A)$.

Definição 59. Seja $(A, *) \in \mathcal{D}_{G},\left(A^{\prime}, \star\right) \in \mathcal{D}_{G^{\prime}}$. Seja $\psi: A^{\prime} \longrightarrow A$ um homomorfismo de grupos e $\varphi: G \longrightarrow G^{\prime}$ um homomorfismo contínuo de grupos profinitos. Dizemos que há compatilidade entre os mapas $\varphi$ e $\psi$ (ou que são compatíveis)quando o diagrama abaixo for comutativo

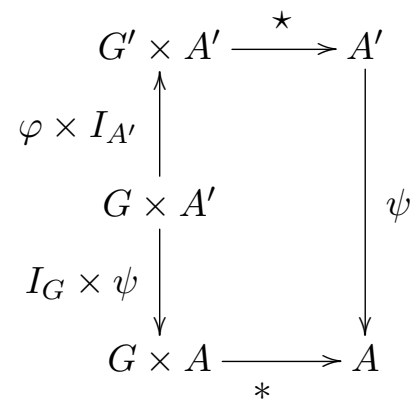

Ou seja, se $\psi$ for um $G$ - homomorfismo quando consideramos $A^{\prime}$ com a $G$-ação

$$
g a^{\prime} \doteq \varphi(g) a^{\prime}, g \in G, a^{\prime} \in A^{\prime}
$$

Lema 34. Um par compatível $\langle\varphi, \psi\rangle$ origina uma sequência de homomorfismos de grupos

$$
\begin{gathered}
(\varphi, \psi)_{n}: C_{\text {cont }}^{n}\left(G^{\prime}, A^{\prime}\right) \\
\sigma \quad C_{\text {cont }}^{n}(G, A) \\
\sigma \quad \longmapsto \circ \sigma \circ \varphi^{n}
\end{gathered}
$$

ao final dos anos 1950 nos trabalhos de John Tate motivado por considerações da teoria dos feixes introduzida por Alexander Grothendieck. O objetivo original de Tate era encontrar um formalismo adequado para a teoria de corpos de classe.

${ }^{12}$ Consulte $[\mathbf{R Z}$ seção 6.4, página 213 e o exercício 6.4 .2 na mesma página. 
para cada $n \in \omega$. Além disso, o seguinte diagrama é comutativo

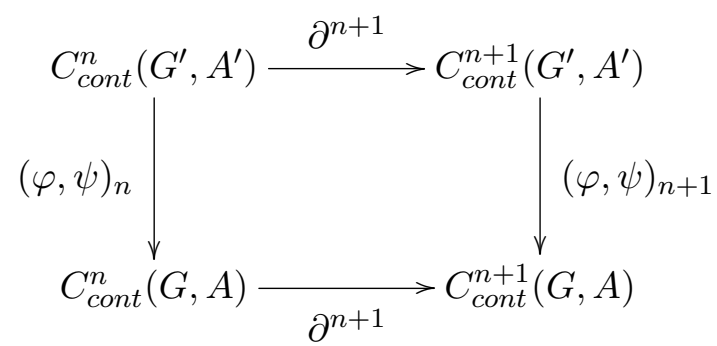

ou seja,$(\varphi, \psi)$ é um mapa de complexo de cadeias.

Demonstração. Claramente, $(\varphi, \psi)_{j}$ é um homomorfismo, para cada $j \in \omega$. Finalmente, para cada $\sigma \in C_{\text {cont }}^{n}\left(G^{\prime}, A^{\prime}\right)$ e $\left(x_{1}, \cdots, x_{n+1}\right) \in G^{n+1}$

$$
\begin{array}{rlc}
\partial^{n+1}\left((\varphi, \psi)_{n} \sigma\right)\left(x_{1}, \ldots, x_{n+1}\right) & = & x_{1} \psi \sigma\left(\varphi x_{2}, \ldots, \varphi x_{n+1}\right) \\
& + & \sum_{i=1}^{n}(-1)^{i} \psi \sigma\left(\varphi x_{1}, \ldots, \varphi x_{i} x_{i+1}, \ldots, \varphi x_{n+1}\right) \\
& + & (-1)^{n+1} \psi \sigma\left(\varphi x_{1}, \ldots, \varphi x_{n}\right) \\
& = & x_{1} \psi \sigma\left(\varphi x_{2}, \ldots, \varphi x_{n+1}\right) \\
& + & \sum_{i=1}^{n}(-1)^{i} \psi \sigma\left(\varphi x_{1}, \ldots, \varphi x_{i} \varphi x_{i+1}, \ldots, \varphi x_{n+1}\right) \\
+ & & (-1)^{n+1} \psi \sigma\left(\varphi x_{1}, \ldots, \varphi x_{n}\right) \\
& = & (\varphi, \psi)_{n+1} \partial^{n+1} \sigma\left(x_{1}, \ldots, x_{n+1}\right)
\end{array}
$$

Corolário 15. $(\varphi, \psi)_{n}$ induz um homomorfismo nos grupos de cohomologia

$$
(\varphi, \psi)^{n}: H_{\text {cont }}^{n}\left(G^{\prime}, A^{\prime}\right) \longrightarrow H_{\text {cont }}^{n}(G, A)
$$

Demonstração. De acordo com o resultado anterior há sentido na seguinte composição

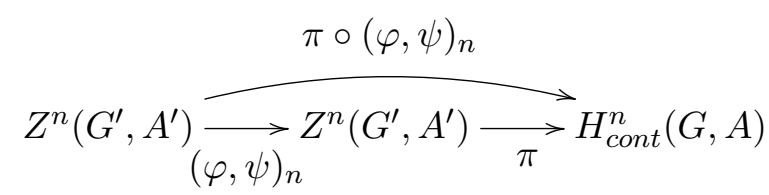

Finalmente, se $\sigma \in B^{n}\left(G^{\prime}, A^{\prime}\right)$,então existe $\theta \in Z^{n-1}\left(G^{\prime}, A^{\prime}\right)$ tal que

$$
\begin{aligned}
(\varphi, \psi)_{n} \sigma & =\quad(\varphi, \psi) \partial^{n} \theta \\
& =\partial^{n}(\varphi, \psi)_{n} \theta \in B^{n}(G, A)
\end{aligned}
$$

Proposição 59. Seja $G_{1} \stackrel{\varphi_{1}}{\longrightarrow} G_{2} \stackrel{\varphi_{2}}{\longrightarrow} G_{3}$ uma sequência de homomorfismos contínuos entre grupos profinitos e $A_{1} \lessdot{ }_{1} A_{2} \longleftarrow{ }_{2} A_{3}$ uma sequência de homomorfismos de grupos abelianos. Se 
$\left(\varphi_{1}, \psi_{1}\right)$ e $\left(\varphi_{2}, \psi_{2}\right)$ são pares compatíveis, então $\left(\varphi_{2} \circ \varphi_{1}, \psi_{1} \circ \psi_{2}\right)$ será compatível e $\left(\varphi_{1}, \psi_{1}\right)^{n} \circ\left(\varphi_{2}, \psi_{2}\right)^{n}$ é dado por $\left(\varphi_{2} \varphi_{1}, \psi_{1} \psi_{2}\right)^{n}$. Além disso, se $\varphi_{1}$ e $\psi_{1}$ são as identidades também o é $\left(\varphi_{1}, \psi_{1}\right)^{n}$.

Demonstração. Para ver que $\left(\varphi_{2} \varphi_{1}, \psi_{1} \psi_{2}\right)$ é um par compatível, basta observar que para cada $a_{3} \in A_{3}$ e cada $g_{1} \in G_{1}$ tem-se

$$
\begin{aligned}
\psi_{1} \psi_{2}\left(\varphi_{2} \varphi_{1}\left(g_{1}\right) a_{3}\right) & =\psi_{1}\left(\psi_{2}\left(\varphi_{2}\left(\varphi_{1} g_{1}\right) a_{3}\right)\right) \\
& =\psi_{1}\left(\varphi_{1}\left(g_{1}\right) \psi_{2}\left(a_{3}\right)\right) \\
& =g_{1} \psi_{1}\left(\psi_{2}\left(a_{3}\right)\right) \\
& =g_{1} \psi_{1} \psi_{2}\left(a_{3}\right)
\end{aligned}
$$

Finalmente, como temos igualdade dos morfismos de cadeia

$$
\begin{aligned}
\left(\varphi_{1}, \psi_{1}\right)_{n}\left(\varphi_{2}, \psi_{2}\right)_{n} \gamma\left(g_{1}, \ldots, g_{n}\right) & =\psi_{1}\left(\varphi_{2}, \psi_{2}\right)_{n} \gamma\left(\varphi_{1} g_{1}, \ldots, \varphi_{1} g_{n}\right) \\
& =\psi_{1} \psi_{2} \gamma\left(\varphi_{2} \varphi_{1} g, \ldots, \varphi_{2} \varphi_{1} g_{n}\right) \\
& =\left(\varphi_{2} \varphi_{1}, \psi_{1} \psi_{2}\right)-n \gamma\left(g_{1}, \ldots, g_{n}\right) .
\end{aligned}
$$

Temos

$$
\left(\varphi_{1}, \psi_{1}\right)^{n} \circ\left(\varphi_{2}, \psi_{2}\right)^{n}=\left(\varphi_{2} \varphi_{1}, \psi_{1} \psi_{2}\right): H_{\text {cont }}^{n}\left(G_{3}, A_{3}\right) \longrightarrow H_{\text {cont }}^{n+1}\left(G_{1}, A_{1}\right)
$$

Corolário 16. $H_{\text {cont }}^{n}(G,-)$ é um funtor de $\mathcal{D}_{G}$ para a categoria de grupos abelianos $\mathcal{A} b$.

Demonstração. É uma consequência direta da proposição anterior.

Proposição 60. Seja $\left(G_{i}, \varphi_{i j}\right)_{i \in I}$ um sistema projetivo de grupos profinitos e $\left(A_{i}, \psi_{i j}\right)_{i \in I}$ um sistema indutivo dirigido para cima de $G_{i}$-módulos discretos com $\varphi_{i j}$ e $\psi_{i j}$ compatíveis para $i \leq j$. Então, para cada $n \geq 0$

$$
H_{\text {cont }}^{n}\left(\lim _{\longleftarrow} G_{i}, \lim _{\longrightarrow} A_{i}\right) \cong \lim _{\longrightarrow} H_{\text {cont }}^{n}\left(G_{i}, A_{i}\right)
$$

Demonstração. Consulte $\mathbf{R Z}$ pág 218, prop.6.5.3.

\subsubsection{A sequência exata longa de cohomologia}

Lema 35. Seja $0 \longrightarrow A_{1} \stackrel{\varphi_{1}}{\longrightarrow} A_{2} \stackrel{\varphi_{2}}{\longrightarrow} A_{3} \longrightarrow 0$ uma sequência exata de G-módulos discretos e G-homomorfismos. Existem homorfismos canônicos $\left\{\delta^{n}: H_{\text {cont }}^{n}\left(G, A_{3}\right) \rightarrow H_{\text {cont }}^{n+1}\left(G, A_{1}\right)\right\}_{n \in \omega}$ tal que a sequência exata longa de cohomologia

$$
\begin{aligned}
& H_{\text {cont }}^{0}\left(G, A_{1}\right) \stackrel{\varphi_{1}^{0}}{\longrightarrow} H_{\text {cont }}^{0}\left(G, A_{2}\right) \stackrel{\varphi_{2}^{0}}{\longrightarrow} H_{\text {cont }}^{0}\left(G, A_{3}\right) \stackrel{\delta^{0}}{\longrightarrow} \\
& \rightarrow H_{\text {cont }}^{1}\left(G, A_{1}\right) \stackrel{\varphi_{1}^{1}}{\longrightarrow} H_{\text {cont }}^{1}\left(G, A_{2}\right) \stackrel{\varphi_{2}^{1}}{\longrightarrow} H_{\text {cont }}^{1}\left(G, A_{3}\right) \stackrel{\delta^{1}}{\longrightarrow} \\
& \rightarrow H_{\text {cont }}^{n}\left(G, A_{1}\right) \stackrel{\varphi_{1}^{n}}{\longrightarrow} H_{\text {cont }}^{n}\left(G, A_{2}\right) \stackrel{\varphi_{2}^{n}}{\longrightarrow} H_{\text {cont }}^{n}\left(G, A_{3}\right) \stackrel{\delta^{n}}{\longrightarrow}
\end{aligned}
$$


é exata, com os mapas $\varphi_{1}$ e $\varphi_{2}$ induzindo, respectivamente, $\varphi_{1}^{i} e \varphi_{2}^{i}$.

Demonstração. Considere a sequência exata curta de complexos de cocadeia induzido pelos mapas $\varphi_{1}$ e $\varphi_{2}$.

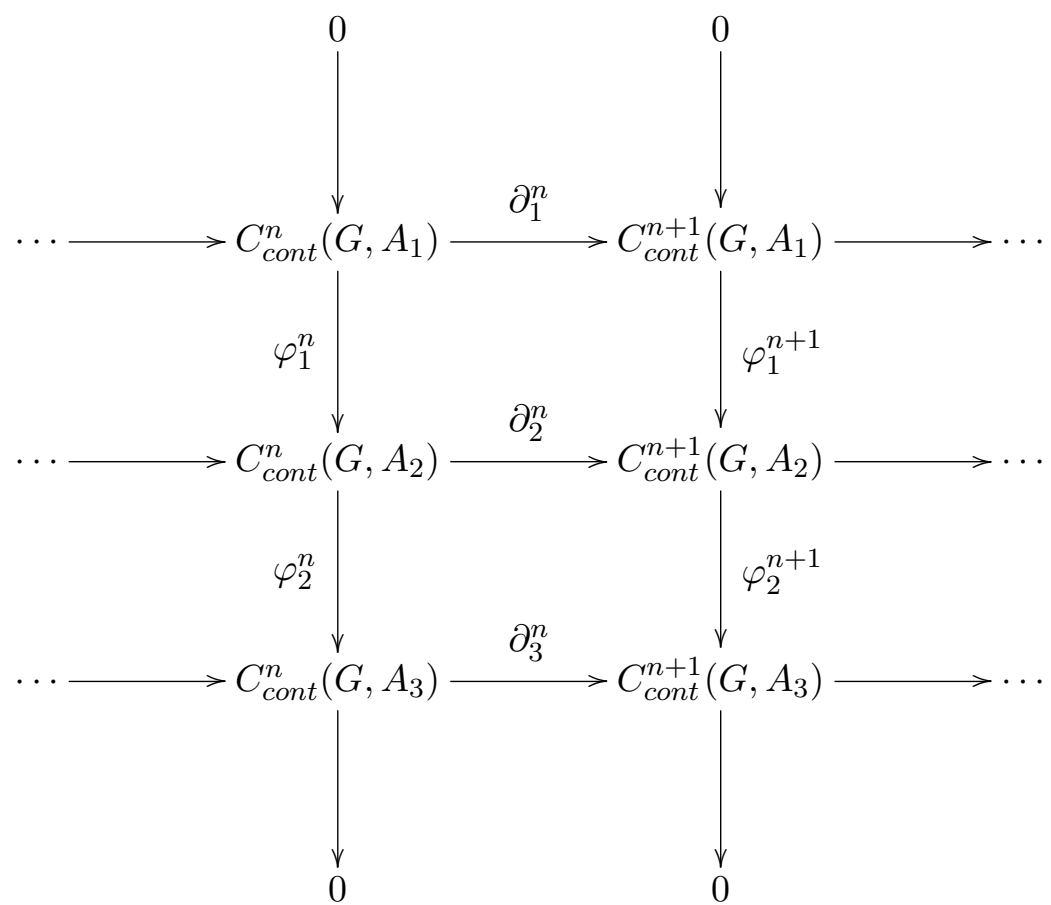

Pela proposição 7 cada linha vertical do diagrama é uma sequência exata de grupos abelianos. Ao longo da prova devemos representar um elemento de $\mu \in H_{\text {cont }}\left(G, A_{i}\right)$ por $[\mu]$, onde $\mu \in C_{\text {cont }}^{n}\left(G, A_{i}\right)$ é um cociclo, i.e, $\partial_{i}^{n}(\mu)=0$.

Definição de $\delta^{n}$ : Seja $\left[\sigma_{n}\right] \in H_{\text {cont }}^{n}\left(G, A_{3}\right)$, então $\partial_{3}^{n}\left(\sigma_{n}\right)=0$. Seja $\rho_{n} \in C_{\text {cont }}^{n}\left(G, A_{2}\right)$ com $\varphi_{2}^{n}\left(\overline{\left.\rho_{n}\right)=\sigma_{n} \text {. Então, }}\right.$

$$
0=\partial_{3}^{n} \varphi_{2}^{n}\left(\rho_{n}\right)=\varphi_{2}^{n+1} \partial_{2}^{n}\left(\rho_{n}\right)
$$

Assim, existe $\nu_{n+1} \in C_{\text {cont }}^{n+1}\left(G, A_{1}\right)$ com

$$
\varphi_{1}^{n+1}\left(\nu_{n+1}\right)=\partial_{2}^{n}\left(\rho_{n}\right)
$$

Claramente, $\partial_{1}^{n+1}\left(\nu_{n+1}\right)=0$. Definimos

$$
\delta^{n}\left(\left[\sigma_{n}\right]\right)=\left[\nu_{n+1}\right] \in H_{\text {cont }}^{n+1}\left(G, A_{1}\right)
$$

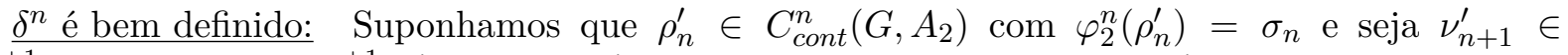
$C_{\text {cont }}^{n+1}\left(G, A_{1}\right)$ tal que $\varphi_{1}^{n+1}\left(\nu_{n+1}^{\prime}\right)=\partial_{2}^{n}\left(\rho_{n}^{\prime}\right)$. Vamos mostrar que $\left[\nu_{n+1}\right]=\left[\nu_{n+1}^{\prime}\right]$. Com efeito, desde que, $\varphi_{2}^{n}\left(\rho_{n}^{\prime}-\rho_{n}\right)=0$ deverá existir $\nu_{n} \in C_{\text {cont }}^{n}\left(G, A_{1}\right) \operatorname{com} \varphi_{1}^{n}\left(\nu_{n}\right)=\rho_{n}^{\prime}-\rho_{n}$ e, então

$$
\varphi_{1}^{n+1} \partial_{1}^{n}\left(\nu_{n}\right)=\partial_{2}^{n} \varphi_{1}^{n}\left(\nu_{n}\right)=\partial_{2}^{n}\left(\rho_{n}^{\prime}-\rho_{n}\right)=\varphi_{1}^{n+1}\left(\nu_{n+1}^{\prime}-\nu_{n+1}\right)
$$

Assim,

$$
\partial_{1}^{n}\left(\nu_{n}\right)=\nu_{n+1}^{\prime}-\nu_{n+1}
$$


pois, $\varphi_{1}^{n+1}$ é injetora. Em outras palavras, $\nu_{n+1}^{\prime}-\nu_{n+1}$ é um cobordo, ou seja, $\left[\nu_{n+1}^{\prime}\right]=\left[\nu_{n+1}\right]$.

Exatidão em $H_{c o n t}^{n+1}\left(G, A_{1}\right)$ : Vamos mostrar que a sequência

$$
H_{\text {cont }}^{n}\left(G, A_{3}\right) \stackrel{\delta^{n}}{\longrightarrow} H_{\text {cont }}^{n+1}\left(G, A_{1}\right) \stackrel{\varphi_{1}^{n+1}}{\longrightarrow} H_{\text {cont }}^{n+1}\left(G, A_{2}\right)
$$

é exata, ou seja,

$$
\operatorname{Im}\left(\delta^{n}\right)=\operatorname{Ker}\left(\varphi_{1}^{n+1}\right)
$$

Por construção de $\delta^{n}$, segue que $\varphi_{1}^{n+1} \delta^{n}=0$ e, portanto

$$
\operatorname{Im}\left(\delta^{n}\right) \leq \operatorname{Ker}\left(\varphi_{1}^{n+1}\right)
$$

Inversamente, seja $\left[\nu_{n+1}\right] \in H_{\text {cont }}^{n+1}$ tal que $\varphi_{1}^{n+1}\left(\left[\nu_{n+1}\right]=0\right)$,isto é, $\varphi_{1}^{n+1}\left(\nu_{n+1}\right)=\partial_{2}^{n}\left(\nu_{n}\right)$ para algum $\nu_{n} \in C_{\text {cont }}^{n}\left(G, A_{2}\right)$. Resulta da construção de $\delta^{n}$

$$
\delta^{n}\left(\left[\varphi_{2}^{n}\left(\nu_{n}\right)\right]\right)=\left[\nu_{n+1}\right]
$$

e assim

$$
\operatorname{Ker}\left(\varphi_{1}^{n+1}\right) \leq \operatorname{Im}\left(\delta^{n}\right)
$$

A exatidão geral da sequência do enunciado do lema é uma simples caça ao diagrama (como foi feito,por exemplo, acima)e deixamos aos cuidados do leitor. Além disso, é fácil ver que os $\left\{\delta^{n}\right\}$ são homomorfismos.

Definição 60. Os homomorfismos $\left\{\delta^{n}\right\}_{n \in \omega}$ da proposição anterior são chamados de homomorfismos de conexão. Indicamos, $\delta=\delta^{n}$ simplesmente.

Fato 17. Segue direto da definição dos morfismos de conexão que cada diagrama comutativo

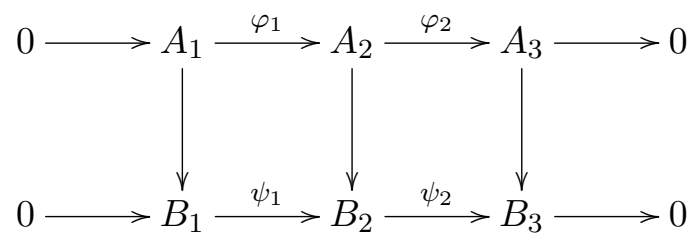

de G-módulos e G-homomorfismos com linhas exatas induz um diagrama comutativo

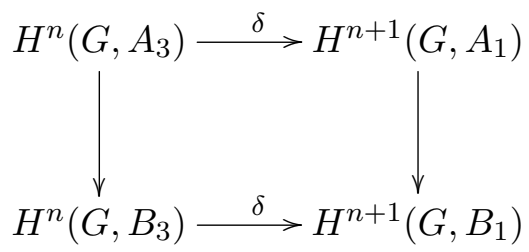

\subsubsection{Funtores cohomológicos}

Sejam $\mathcal{A}$ e $\mathcal{A}^{\prime}$ categorias abelianas. Um funtor cohomológico covariante de $\mathcal{A}$ em $\mathcal{A}^{\prime}$ é uma sequência de funtores covariantes aditivos $\mathbf{H}^{*} \doteq\left(H^{n}\right)_{n \in \omega}$ de $\mathcal{A}$ em $\mathcal{A}^{\prime}$ tal que para cada sequência 
exata

$$
0 \longrightarrow A_{1} \longrightarrow A_{2} \longrightarrow A_{3} \longrightarrow 0
$$

em $\mathcal{A}$ e cada $n \in \omega$, existe um morfismo conexão

$$
\delta=\delta^{n}: H^{n}\left(A_{3}\right) \longrightarrow H^{n+1}\left(A_{1}\right)
$$

cumprindo as seguintes propriedades :

$\mathbf{H}_{1}^{*}$ : Para cada diagrama comutativo com linhas exatas em $\mathcal{A}$

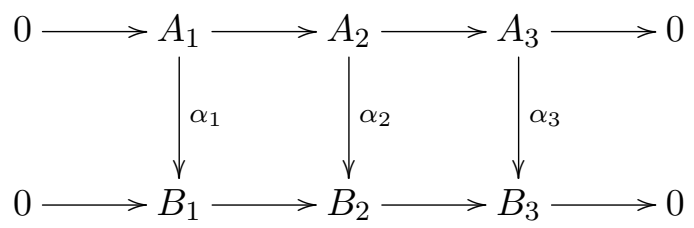

o próximo diagrama

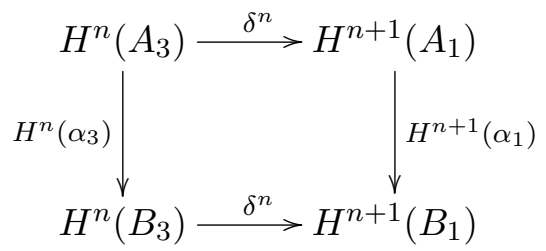

é comutativo para todo $n \in \omega$.

$\mathbf{H}_{2}^{*}$ : A sequência exata longa

$$
\begin{aligned}
& H^{0}\left(A_{1}\right) \longrightarrow H^{0}\left(A_{2}\right) \longrightarrow H^{0}\left(A_{3}\right) \stackrel{\delta^{0}}{\longrightarrow} \\
& \rightarrow H^{1}\left(A_{1}\right) \longrightarrow H^{1}\left(A_{2}\right) \longrightarrow H^{1}\left(A_{3}\right) \stackrel{\delta^{1}}{\longrightarrow} \\
& \rightarrow H^{n}\left(A_{1}\right) \longrightarrow H^{n}\left(A_{2}\right) \longrightarrow H^{n}\left(A_{3}\right) \stackrel{\delta^{n}}{\longrightarrow}
\end{aligned}
$$

é exata.

Um morfismo entre funtores cohomológicos covariantes $\mathbf{H}_{1}^{*}$ e $\mathbf{H}_{2}^{*}$ consiste em uma família de transformações naturais de funtores $\left(\varphi^{n}: H_{1}^{n} \rightarrow H_{2}^{n}\right)_{n \in \omega}$ tal que para cada sequência exata $0 \longrightarrow$ $A_{1} \longrightarrow A_{2} \longrightarrow A_{3} \longrightarrow 0$ em $\mathcal{A}$ o seguinte diagrama comuta para cada $n \in \omega$

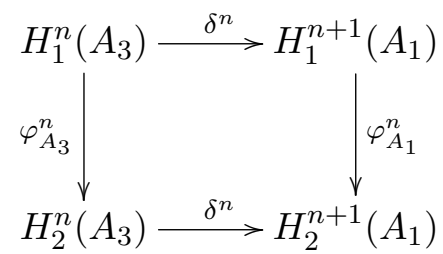

Definição 61. Um funtor cohomológico $\boldsymbol{H}_{1}^{*}: \mathcal{A} \longrightarrow \mathcal{A}^{\prime}$ é dito universal quando satifaz a seguinte propriedade: Se $\boldsymbol{H}_{2}^{*}: \mathcal{A} \longrightarrow \mathcal{A}^{\prime}$ for outro funtor cohomológico, então para cada morfismo $\varphi^{\prime}: H_{1}^{0} \longrightarrow$ $H_{2}^{0}$ existe um único morfismo $\varphi: H_{1}^{*} \longrightarrow H_{2}^{*}$ tal que $\varphi^{0}=\varphi^{\prime}$. Tal morfismo é dito uma extensão 
de $\varphi^{\prime}$.

Lema 36. Sejam $\boldsymbol{H}_{1}^{*}, \boldsymbol{H}_{2}^{*}: \mathcal{A} \longrightarrow \mathcal{A}^{\prime}$ funtores cohomológicos universais. Seja

$$
\varphi^{0}: H_{1}^{0} \longrightarrow H_{2}^{0}
$$

um morfismo de funtores e seja

$$
\varphi: \boldsymbol{H}_{1}^{*} \longrightarrow \boldsymbol{H}_{2}^{*}
$$

sua extensão correspondente. Então, $\varphi$ é um isomorfismo se, e somente se $\varphi^{0}$ for um isomorfismo.

Demonstração. Segue da definição da universalidade dos funtores.

A sequência de funtores $\mathbf{H}_{\text {cont }}^{*} \doteq\left\{H_{\text {cont }}^{n}(G,-): \mathcal{D}_{G} \longrightarrow \mathcal{A} b\right\}_{n \in \omega}$ pode ser caracterizada globalmente como sendo um funtor cohomológico covariante universal ${ }^{13}$

\subsubsection{Inflação, Restrição e corestrição}

Seja K um subgrupo normal e fechado de um grupo profinito G. A ação canônica que faz de $A^{K}$ um $G / K$-módulo é definida da seguinte maneira

$$
\begin{aligned}
*: G / K \times A^{K} & \longrightarrow A^{K} \\
(x K, a) & \longmapsto x a,
\end{aligned}
$$

note que a projeção $\pi: G \longrightarrow G / K$ e a inclusão $\iota: A^{K} \longrightarrow A$ são mapas compatíveis. Assim, eles induzem homomorfismos

$$
i n f_{G}^{G / K}: H_{\text {cont }}^{n}\left(G / K, A^{K}\right) \longrightarrow H^{n}(G, A)
$$

que são chamados de inflações. Explicitamente,

$$
\text { inf : } H_{\text {cont }}^{0}\left(G / K, A^{K}\right)=\left(A^{K}\right)^{G / K} \longrightarrow H^{0}(G, A)=A^{G}
$$

é o mapa identidade.

Seja $G$ um grupo profinito e $N$ um subgrupo fechado de G. É possível mostrar que $N$ é ainda profinito. Nesse caso, se $A \in \mathcal{D}_{G}$, então $A \in \mathcal{D}_{N}$. O mapa inclusão $\iota: N C G$ juntamente com o mapa identidade $i_{d}: A \longrightarrow A$ é um par compatível de modo que temos homomorfismos restrições

$$
r e s=\operatorname{res}_{G, N}: H^{n}(G, A) \longrightarrow H^{n}(N, A)
$$

Para $\varphi \in H^{n}(G, A)$ tem-se $\operatorname{res}(\varphi)(x)=\varphi(x)$ para todo $x \in N^{n}$. A comutatividade do próximo diagrama é imediata

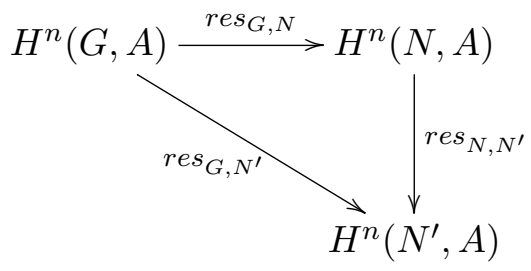

\footnotetext{
${ }^{13}$ Para mais detalhes consulte $[\mathbf{R Z}$ págs. 222-223, prop 6.6.2 e prop 6.6.3.
} 
Seja G um grupo profinito e $N$ um subgrupo aberto de G. Nesse caso,

$$
G=\bigcup_{t \in T} t N
$$

,onde $T$ é um conjunto completo de classes de representantes à esquerda de N em G. Como G é compacto, então T é finito. Observe que existe um homomorfismo

$$
\begin{aligned}
& \text { cor : } H_{\text {cont }}^{0}(N, A) \longrightarrow H_{\text {cont }}^{0}(G, A) \\
& a \longmapsto \sum_{t \in T} t a
\end{aligned}
$$

A universalidade de $\mathbf{H}_{\text {cont }}^{*}$ permite estender este homomorfismo de grupos para um morfismo de funtores cohomológicos

$$
\operatorname{cor}: H^{*}(N,-) \longrightarrow H^{*}(G,-)
$$

Por conseguinte teremos uma sequência de transformações naturais chamadas de corestrições

$$
\text { cor } \doteq \operatorname{cor}_{N, G}: H_{\text {cont }}^{n}(N, A) \longrightarrow H_{\text {cont }}^{n}(G, A)
$$

Proposição 61. Seja $G$ um grupo profinito e $N^{\prime} \subseteq N$ subgrupos abertos de G. Então, o próximo diagrama é comutativo

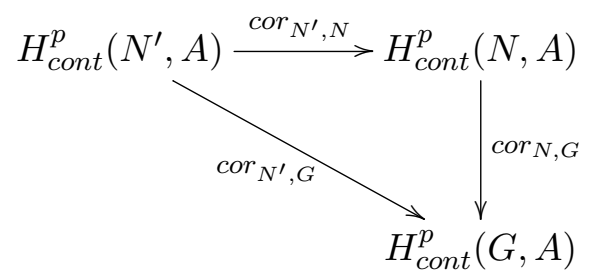

ou seja, $\operatorname{cor}_{N, G} \operatorname{cor}_{N^{\prime}, N}=\operatorname{cor}_{N^{\prime}, G}$.

Demonstração. Pelo lema 11 é suficiente mostrar o resultado em dimensão zero. para tanto, basta observar que se $\left\{h_{j}\right\}$ e $\left\{g_{i}\right\}$ são tranversais a esquerda ,respectivamente, de $N^{\prime}$ em $N$ e $N$ em $G$,então $\left\{h_{j} g_{i}\right\}$ será uma transversal a esquerda de $N^{\prime}$ em G.

Proposição 62. Para todo subgrupo aberto de um grupo profinito $G$ vale a seguinte fórmula

$$
\operatorname{cor}_{N, G} r e s_{G, N}=[G: N] i_{d}
$$

onde $[G: N]$ é o índice de $N$ em $G$ e $i_{d}$ é a transformação natural identidade de $H^{n}(G,-), \forall n \in \omega$. Demonstração. Pelo lema 11 é suficiente mostrar o resultado em dimensão zero. Temos

$$
\operatorname{cor}_{N, G} \operatorname{res}_{G, N}=\sum_{t \in T} t a=[G: N] a
$$

como queríamos.

\subsubsection{O produto cup}

Sejam $\mathrm{G}$ um grupo profinito, e $A, A^{\prime}, T \in \mathcal{D}_{G}$. Considere um mapa biaditivo

$$
*: A \times A^{\prime} \longrightarrow T
$$


tal que

$$
g a * g a^{\prime}=g\left(a * a^{\prime}\right)
$$
fórmula

Para $\varphi \in C_{\text {cont }}^{p}(G, A)$ e $\varphi^{\prime} \in C_{\text {cont }}^{q}\left(G, A^{\prime}\right)$ definimos $\varphi \cup \varphi^{\prime} \in C_{\text {cont }}^{p+q}(G, T)$ por intermédio da

$$
\varphi \cup \varphi^{\prime}\left(g_{1}, \ldots, g_{p+q}\right) \doteq \varphi\left(g_{1}, \ldots, g_{p}\right) * \prod_{i=1}^{p} g_{i} \varphi^{\prime}\left(g_{p+1}, \ldots, g_{p+q}\right)
$$

\section{Lema 37.}

$$
\forall \varphi \in C_{\text {cont }}^{p}(G, A) \forall \varphi^{\prime} \in C_{\text {cont }}^{q}\left(G, A^{\prime}\right)\left(\partial \varphi \cup \varphi^{\prime}=\partial \varphi \cup \varphi^{\prime}+(-1)^{p} \varphi \cup \partial \varphi^{\prime}\right)
$$

Demonstração. Faremos um esboço para o caso $p+q$ ímpar e $q$ par. Para tanto, note que por um lado

$$
\begin{aligned}
\partial \varphi \cup \varphi^{\prime}\left(g_{1}, \ldots, g_{p+q+1}\right) & =\partial \varphi\left(g_{1}, \ldots, g_{p+1}\right) * \prod_{i=1}^{p+1} g_{i} \varphi^{\prime}\left(g_{p+2}, \ldots, g_{p+q+1}\right) \\
& =g_{1} \varphi\left(g_{2}, \ldots, g_{p+1}\right) * \prod_{i=1}^{p+1} g_{i} \varphi^{\prime}\left(g_{p+2}, \ldots, g_{p+q+1}\right) \\
& +\sum_{i=1}^{p}(-1)^{i} \varphi\left(g_{1}, \ldots, g_{i} g_{i+1}, \ldots g_{p+1}\right) \\
& * \prod_{i=1}^{p+1} g_{i} \varphi^{\prime}\left(g_{p+2}, \ldots, g_{p+q+1}\right) \\
& +(-1)^{p+1} \varphi\left(g_{1}, \ldots, g_{p}\right) * \prod_{i=1}^{p+1} g_{i} \varphi^{\prime}\left(g_{p+2}, \ldots, g_{p+q+1}\right)
\end{aligned}
$$

Por outro lado,

$$
\begin{aligned}
\varphi \cup \partial \varphi^{\prime}\left(g_{1}, \ldots, \ldots, g_{p+q+1}\right)= & \varphi\left(g_{1}, \ldots, g_{p}\right) \\
& * \prod_{i=1}^{p} g_{i}\left[g_{p+1} \varphi^{\prime}\left(g_{p+2}, \ldots g_{p+q+1}\right)+\xi\right] \\
& =\varphi\left(g_{1}, \ldots, g_{p}\right) * \prod_{i=1}^{p} g_{i} g_{p+1} \varphi^{\prime}\left(g_{p+2}, \ldots\right) \\
& +\varphi\left(g_{1}, \ldots, g_{p}\right) * \xi
\end{aligned}
$$

Onde $\xi=\partial \varphi^{\prime}\left(g_{1}, \ldots, g_{p+q+1}\right)-g_{p+1} \varphi\left(g_{p+2}, \ldots g_{p+q+1}\right)$. Observe que por hipótese podemos cancelar os termos $(-1)^{p} \varphi\left(g_{1}, \ldots, g_{p}\right) * \prod_{i=1}^{p} g_{i} g_{p+1} \varphi^{\prime}\left(g_{p+2}, \ldots\right)$ e $\varphi\left(g_{1}, \ldots, g_{p}\right) * \prod_{i=1}^{p+1} g_{i} \varphi^{\prime}\left(g_{p+2}, \ldots, g_{p+q+1}\right)$ restando em $\partial \varphi \cup \varphi^{\prime}+(-1)^{p} \varphi \cup \partial \varphi^{\prime}$ exatamente $\partial\left(\varphi \cup \varphi^{\prime}\right)$.

\section{Fato 18.}

1) $\forall \varphi \forall \varphi^{\prime}\left(\partial \varphi=\partial \varphi^{\prime}=0 \Longrightarrow \partial\left(\varphi \cup \varphi^{\prime}\right)=0\right)$;

2) $\forall \varphi, \varphi^{\prime}, \psi, \psi^{\prime}\left([\varphi]=\left[\varphi^{\prime}\right] \wedge[\psi]=\left[\psi^{\prime}\right] \Longrightarrow\left[\varphi \cup \psi^{\prime}\right]=\left[\varphi^{\prime} \cup \psi^{\prime}\right]\right)$; 
Seja $\mathrm{G}$ um grupo profinito e $A, B$ G-módulos. Seja $A \otimes_{\mathbb{Z}} B$ o produto tensorial de $\mathrm{A}$ e $\mathrm{B}$ sobre $\mathbb{Z}$ (onde A e B são vistos como grupos abelianos). Pode-se mostrar que existe uma G-ação em $A \otimes_{\mathbb{Z}} B$ tal que

$$
g \cdot(a \otimes b)=g \cdot a \otimes g \cdot b
$$

$\forall g \in G, \forall a \in A, \forall b \in B$. Além disso,

$$
A \otimes_{\mathbb{Z}} B=\bigcup_{\Omega \in \mathcal{T}}\left(A \otimes_{\mathbb{Z}} B\right)^{\Omega}
$$

onde $\mathcal{T}$ é conjunto de vizinhanças abertas de 1 ou subgrupos normais abertos contendo 1 . Assim, $A \otimes_{\mathbb{Z}} B$ é um G-módulo discreto (ver a proposição 56 ).

Definição 62. O mapa biaditivo

$$
\cup: H_{\text {cont }}^{p}(G, A) \times H_{\text {cont }}^{q}\left(G, A^{\prime}\right) \longrightarrow H_{\text {cont }}^{p+q}\left(G, A \otimes_{\mathbb{Z}} A^{\prime}\right)
$$

definido por

$$
\varphi \cup \varphi^{\prime}\left(g_{1}, \ldots, g_{p+q}\right)=\varphi\left(g_{1}, \ldots, g_{p}\right) \otimes \prod_{i=1}^{p} g_{i} \varphi^{\prime}\left(g_{p+1}, \ldots, g_{p+q}\right)
$$

é chamado de produto cup.

Na sequência enunciamos vários fatos envolvendo o produto cup 14

Fato 19. A família de aplicações

$$
\left\{\cup: H_{\text {cont }}^{p}(G, A) \otimes H_{\text {cont }}^{p}(G, B) \longrightarrow H_{\text {cont }}^{p+q}(G, A \otimes B)\right\}_{p, q \in \omega}
$$

é a única família de homomorfismos de grupos abelianos satisfazendo

$\left.\cup_{1}\right) \cup$ é natural em $A$ e $B$;

$\cup_{2}$ )Para $p=q=0$ a identidade em $A \otimes_{\mathbb{Z}} B$ induz o produto cup

$$
A^{G} \otimes_{\mathbb{Z}} B^{G} \longrightarrow(A \otimes B)^{G}
$$

$\left.\cup_{3}\right)$ Seja $0 \longrightarrow A \longrightarrow A^{\prime} \longrightarrow A^{\prime \prime} \longrightarrow 0$ uma sequência exata em $\mathcal{D}_{G}$. Se a sequência

$0 \longrightarrow A \otimes B \longrightarrow A^{\prime} \otimes B \longrightarrow A^{\prime \prime} \otimes B \longrightarrow 0$ for exata para algum $B \in \mathcal{D}_{G}$, então o diagrama

$$
\begin{aligned}
H_{\text {cont }}^{p}\left(G, A^{\prime \prime}\right) & \otimes_{\mathbb{Z}} H_{\text {cont }}^{q}(G, B) \stackrel{\cup}{\longrightarrow} H_{\text {cont }}^{p+q}\left(G, A^{\prime \prime} \otimes_{\mathbb{Z}} B\right) \\
& \downarrow \delta \\
H_{\text {cont }}^{p+1}(G, A) & \otimes H_{\text {cont }}^{q}(G, B) \stackrel{\cup}{\longrightarrow} H_{\text {cont }}^{p+q+1}\left(G, A \otimes_{\mathbb{Z}} B\right)
\end{aligned}
$$

comuta.

$\left.\cup_{4}\right)$ Seja $0 \longrightarrow B \longrightarrow B^{\prime} \longrightarrow B^{\prime \prime} \longrightarrow 0$ uma sequência exata em $\mathcal{D}_{G}$. Se a sequência $0 \longrightarrow A \otimes B \longrightarrow A \otimes B^{\prime} \longrightarrow A \otimes B^{\prime \prime} \longrightarrow 0$ for exata para algum $A \in \mathcal{D}_{G}$, então o diagrama

\footnotetext{
${ }^{14}$ Para uma descrição detalhada via cadeias homogêneas ver [RZ],seção 7.9.
} 


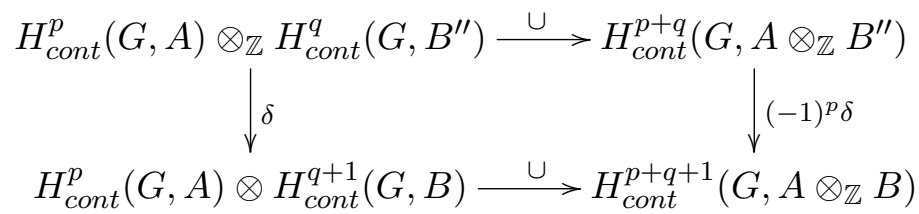

comuta.

Fato 20.

i) $\varphi \cup \varphi^{\prime}=(-1)^{p q} \varphi^{\prime} \cup \varphi, \varphi \in H_{\text {cont }}^{p}(G, A), \varphi^{\prime} \in H_{\text {cont }}^{q}\left(G, A^{\prime}\right)$;

ii) $\left(\varphi \cup \varphi^{\prime}\right) \cup \varphi^{\prime \prime}=\varphi \cup\left(\varphi^{\prime} \cup \varphi^{\prime \prime}\right) e m \in H_{\text {cont }}^{p+q+r}\left(G, A \otimes A^{\prime} \otimes A^{\prime \prime}\right)$

Fato 21. Seja $G$ um grupo profinito e $A, B \in \mathcal{D}_{G}$.Para cada subgrupo fechado $H$ de $G$ existem diagramas comutativos

i)

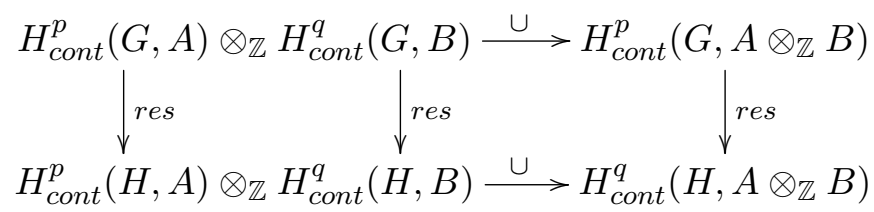

ii)

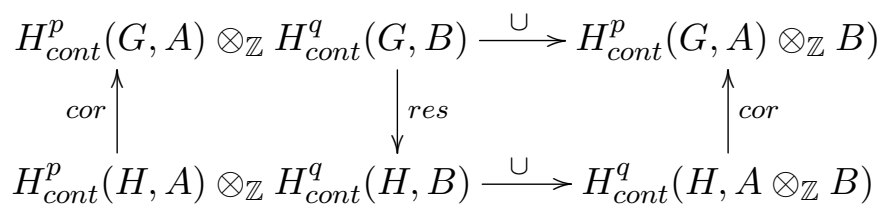

\subsection{Cohomologia em dimensões 0, 1 e 2}

\subsubsection{Descrição de $H_{\text {cont }}^{0}(G, A)$}

Lembremos que $H_{\text {cont }}^{0}(G, A)=Z^{0}(G, A) / B^{0}(G, A)$, onde $B^{0}(G, A)$ é trivial por definição. Denotamos por $A^{G}$ o módulo de invariantes pela ação de G. Em símbolos, temos $A^{G} \doteq\{a \in A: g a=$ $a, \forall g \in G\}$.

Proposição 63. $H_{\text {cont }}^{0}(G, A) \cong A^{G}$

Demonstração. $H_{\text {cont }}^{0}(G, A)=Z^{0}(G, A) / B^{0}(G, A)=K e r \partial^{0} /\{0\} \cong K e r \partial^{0}$, onde $K e r \partial^{0}=\{\varphi \in$ $\left.C_{\text {cont }}^{0}(G, A): \partial^{0}(\varphi)(g)=g a-a=0, \forall g \in G\right\}$. Como $C_{\text {cont }}^{0}(G, A) \cong A$, segue que $K e r \partial^{0} \cong A^{G}$.

\subsubsection{Descrição de $H_{\text {cont }}^{1}(G, A)$}

Dizemos que uma aplicação contínua $\varphi: G \longrightarrow A$ é um homorfismo cruzado (hc) ou uma derivação de $\mathrm{G}$ para A, quando $\varphi\left(g g^{\prime}\right)=\varphi(g)+g \varphi\left(g^{\prime}\right)$. Uma aplicação contínua $\varphi: G \longrightarrow A$ é chamada de homomorfismo cruzado principal (hcp) ou derivação interna, quando para cada $g \in G$ existe $a \in A$ tal que $\varphi(g)=g a-a$. Denotamos por $\operatorname{Der}(G, A)$ as derivações de $\mathrm{G}$ em A e por $\operatorname{Der}^{\circ}(G, A)$ as derivações internas.

\section{Proposição 64.}


1) $\varphi \in Z^{1}(G, A) \Longleftrightarrow \varphi$ for homomorfismo cruzado;

2) $\varphi \in B^{1}(G, A) \Longleftrightarrow \varphi$ for homomorfismo cruzado principal;

3) $H_{\text {cont }}^{1}(G, A)=\operatorname{Der}(G, A) / \operatorname{Der}^{\circ}(G, A)$.

Demonstração.

1) $\varphi \in Z^{1}(G, A) \Longleftrightarrow \forall g, g^{\prime} \in G\left(g \varphi\left(g^{\prime}\right)-\varphi\left(g g^{\prime}\right)+\varphi(g)=0\right)$.

2) $\varphi \in B^{1}(G, A) \Longleftrightarrow \exists a \in A\left(\partial^{0}(a)=\varphi\right) \Longleftrightarrow \forall g \in G(\varphi(g)=g a-a)$;

3) É uma consequência imediata de 1) e 2).

Existe uma correspondência bijetora entre o conjunto de derivações

$$
d: G \longrightarrow A
$$

e o conjunto de homomorfismos contínuos de grupos

$$
\varphi: G \longrightarrow A \rtimes G
$$

tal que a composição $G \stackrel{\varphi}{\longrightarrow} A \rtimes G \stackrel{f}{\longrightarrow} G$ é o homomorfismo identidade 15

Quando A é um G-módulo. O grupo de cohomologia ordinária $H^{1}(G, A)$ está diretamente ligado as classes de conjugações dos complementos de $\mathrm{A}$ em $A \rtimes G^{16}$.

\subsubsection{Descrição de $H_{\text {cont }}^{2}(G, A)$}

Considere a sequência exata de grupos profinitos e homomorfismos contínuos

$$
0 \longrightarrow K \stackrel{\iota}{\longrightarrow} E \stackrel{\pi}{\longrightarrow} Q \longrightarrow 1
$$

onde $\mathrm{K}$ é um grupo finito abeliano.

Uma aplicação contínua $t: Q \longrightarrow E$ tal que $\pi \circ t=i d_{Q}$ e $t(0)=1$ é chamada de aplicação transversal. Aplicações tranversais sempre existem. Uma seção para $\pi$ é uma aplicação transversal que é um homomorfismo de grupos profinitos. Aqui sempre vamos supor que $K$ e um Q-módulo com ação

$$
q \cdot x \doteq t(q) x t(q)^{-1}
$$

Dado um grupo profinito Q e um Q-módulo finito K, uma extensão de K por Q é uma sequência exata

$$
0 \longrightarrow K \stackrel{\iota}{\longrightarrow} E \stackrel{\pi}{\longrightarrow} Q \longrightarrow 1
$$

com homomorfismos contínuos onde E é um grupo profinito.

Dizemos que duas extensões de grupos

$$
0 \longrightarrow K \stackrel{\iota}{\longrightarrow} E \stackrel{\pi}{\longrightarrow} Q \longrightarrow 1
$$

$\mathrm{e}$

$$
0 \longrightarrow K \stackrel{\iota}{\longrightarrow} E^{\prime} \stackrel{\pi}{\longrightarrow} Q \longrightarrow 1
$$

\footnotetext{
${ }^{15}$ Ver o exercício 6.8 .2 ,pág. 32 de $\mathbf{R Z}$

${ }^{16} \mathrm{O}$ primeiro grupo de cohomologia ordinária $H^{1}(G, A)$ está em bijeção com as classes de conjugação dos complementos de A no produto semidireto $A \rtimes G$. Em particular, $H^{1}(G, A)$ será trivial se, e somente se, os complementos de A em $A \rtimes G$ forem conjugados entre si.
} 
são equivalentes (escrevemos $E \simeq E^{\prime}$ ) quando existir um isomorfismo contínuo de grupos $\varphi: E \longrightarrow$ $E^{\prime}$

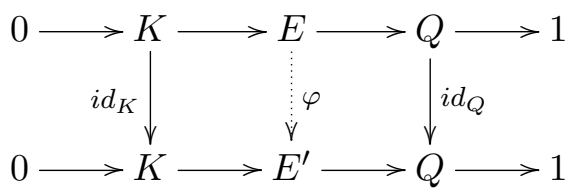

Observe que nesse caso o homomorfismo $\varphi$ é necessariamente um isomorfismo de grupos topológicos profinitos. Se $E \simeq E^{\prime}$ e $E^{\prime} \simeq E^{\prime \prime}$, então o próximo diagrama é comutativo

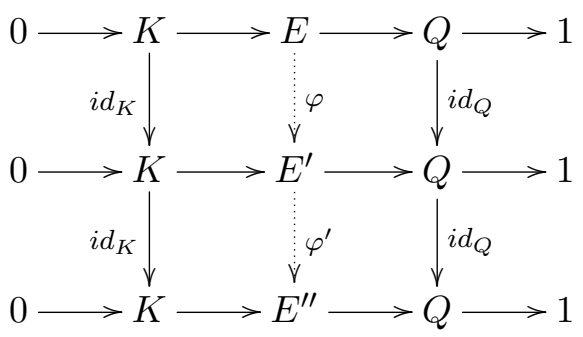

ou seja, $E \simeq E^{\prime \prime}$ e, portanto $\simeq$ é uma relação de equivalência. Doravante, usaremos $E x t(Q, K)$ para representar a totalidade dos pares $([\varepsilon], t)$,onde $[\varepsilon]$ é a classe de equivalência de uma extensão $0 \longrightarrow K \stackrel{\iota}{\longrightarrow} E \stackrel{\pi}{\longrightarrow} Q \longrightarrow 1$ e $t: Q \longrightarrow K$ é um mapa transversal.

Para todos $x, y \in Q$, temos $\pi\left(t(x) t(y) t(x y)^{-1}\right)=x y(x y)^{-1}=1$, ou seja, $t(x) t(y) t(x y)^{-1} \in K$. Assim, temos a seguinte

Definição 63. Seja $0 \longrightarrow K \stackrel{\iota}{\longrightarrow} E \stackrel{\pi}{\longrightarrow} Q \longrightarrow 1$ uma extensão de grupo profinitos e $t$ : $Q \longrightarrow E$ um mapa transversal. Um 2-cociclo normalizado é uma aplicação $f: Q \times Q \longrightarrow E$ tal que $t(x) t(y)=f(x, y) t(x y)$.

Observação 36. Note que um 2-cociclo normalizado é uma aplicação contínua, uma vez que, a aplicação transversal associada a ele é contínua.

Lema 38. Um 2-cociclo normalizado $f: Q \times Q \longrightarrow E$ satisfaz as seguintes identidades

$\left.f_{1}\right) \quad \forall x, y, z \in Q(x f(y, z) f(x, y z)=f(x, y) f(x y, z))$;

$\left.f_{2}\right) \quad \forall x, y \in Q(f(x, 1)=f(1, y)=1)$.

Demonstração.

$f_{1}$ )Temos por um lado

$$
\begin{aligned}
x f(y, z) f(x, y z) & =x\left(t(y) t(z) t(y z)^{-1}\right)+ \\
& +t(x) t(y z) t(x y z)^{-1} \\
& =t(x) t(y) t(z) t(x y z)^{-1}
\end{aligned}
$$

E por outro lado

$$
\begin{aligned}
f(x, y) f(x y, z) & =\left(t(x) t(y) t(x y)^{-1}\right) \cdot\left(t(x y) t(z) t(x y z)^{-1}\right) \\
& =t(x) t(y) t(z) t(x y z)^{-1}
\end{aligned}
$$

$f_{2}$ ) Resulta imediato de $t(0)=1$. 
Fato 22. A toda função $f$ que satisfaça $f_{1}$ e $f_{2}$ pode-se associar uma extensão $E(Q, K, f)$ e uma transversalt $: Q \longrightarrow E(Q, K, f)$ tal que $f(x, y)=t(x) t(y) t(x y)^{-1}$. Alem disso, se $\varepsilon$ for um extensão de $K$ por $Q$, então $\varepsilon \cong E(Q, K, f)$.

Lema 39. Dois 2-cociclos normalizados associados a uma mesma extensão diferem por um cobordo.

Demonstração. Sejam $f, f^{\prime}$ cociclos normalizados associados, respectivamente, às tranversais $t$ e $t^{\prime}$. Note que $h(x) \doteq t^{\prime}(x) \cdot t(x) \in K, \forall x \in Q$. De fato, $\pi(h(x))=\pi\left(t^{\prime}(x) \cdot t(x)^{-1}\right)=x \cdot x^{-1}=1$. Definindo uma aplicação $h: Q \longrightarrow K$ por $h(x) \doteq t^{\prime}(x) t(x)^{-1}$ teremos

$$
\begin{aligned}
f^{\prime}(x, y) & =t^{\prime}(x) t^{\prime}(y) t^{\prime}(x y)^{-1} \\
& =\left[t^{\prime}(x) t^{\prime}(y) t(y)^{-1} t(x)^{-1}\right]+\left[t(x) t(y) t(x y)^{-1}\right]+\left[t(x y) t^{\prime}(x y)^{-1}\right] \\
& =h(x) x h(y) f(x, y) h(x y)^{-1} \\
& =f(x, y) \partial^{2} h(x, y)
\end{aligned}
$$

Proposição 65. Existe uma bijeção canônica $\operatorname{Ext}(Q, K) \cong H^{2}(Q, K)$.

Demonstração. O lema anterior nos habilita a definir uma aplicação

$$
\begin{gathered}
\Upsilon: \operatorname{Ext}(Q, K) \longrightarrow H^{2}(Q, K) \\
([\varepsilon], t) \longmapsto f_{t}
\end{gathered}
$$

Se $\Upsilon([\varepsilon], t)=\Upsilon\left(\left[\varepsilon^{\prime}\right], t^{\prime}\right)$, então $f_{t}=f_{t^{\prime}}$ e,portanto $E\left(Q, K, f_{t}\right) \cong \varepsilon$ e $E\left(Q, K, f_{t^{\prime}}\right) \cong \varepsilon^{\prime}$. Finalmente, a cada $[f] \in H^{2}(Q, K)$ associamos $E(Q, K, f)$.

Observação 37. Usando pull-back e pushout podemos introduzir uma operação de grupo em $\operatorname{Ext}(Q, K)$. Assim, teremos um isomorfismo de grupos abelianos natural $\operatorname{Ext}(Q, K) \cong H^{2}(Q, K)$.

\subsection{O grupo de Brauer}

\subsubsection{R-álgebras}

Nesta seção R será sempre um anel comutativo com unidade. Uma $R^{\bullet}$ - álgebra é um par $\langle A, \mu\rangle$, onde $A=\langle A, *\rangle$ é um anel com unidade e $\langle A, \mu\rangle$ é um $R$-módulo tais que existe compatibilidade entre operações ação e produto.

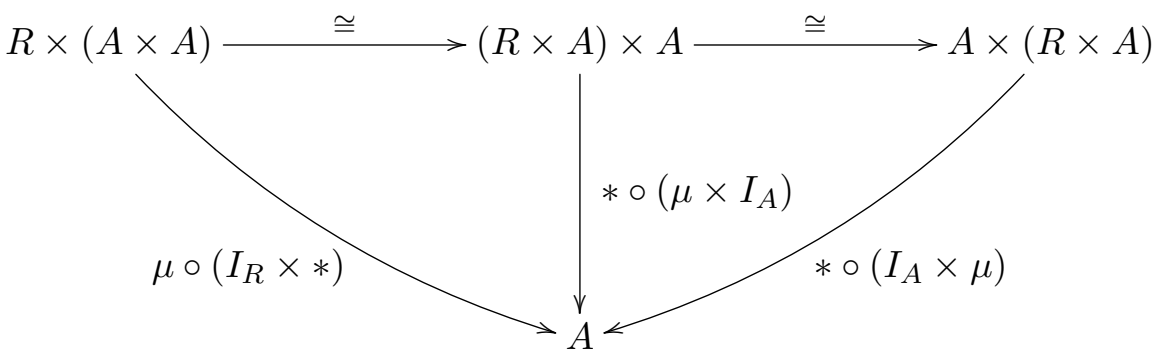

Um morfismo entre $R^{\bullet}$ - álgebras $\langle A, \mu\rangle$ e $\langle B, \xi\rangle$ é uma tripla $\langle A, \varphi, B\rangle$ que induz morfismos nas categorias $\boldsymbol{R i n g}$ e $\boldsymbol{R}$-mod. É fácil ver que isso define uma categoria de $R^{\bullet}$ - álgebras a qual denotamos por $\boldsymbol{A} \boldsymbol{L} \boldsymbol{G}_{R}^{\bullet}$. 
Alternativamente uma $R$-álgebra (ou uma álgebra sobre $\mathrm{R}$ )é um par $\langle A, \varphi\rangle$, onde $\mathrm{A}$ é um anel e $\varphi: R \longrightarrow A$ é um morfismo de anéis tal que $\operatorname{Im}(\varphi) \subseteq Z(A)$,onde $Z(A)$ é definido por

$$
Z(A) \doteq\{x \in A: x a=a x, \forall a \in A\}
$$

Um morfismo $f$ entre $R$-álgebras $\langle A, \varphi\rangle$ e $\langle B, \psi\rangle$ é definido por um morfismo entre anéis e por um diagrama comutativo

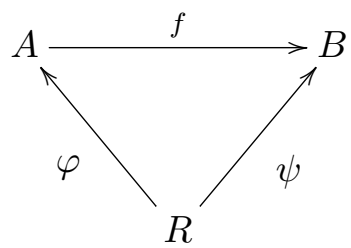

Isso define a categoria das $R$-algebras simbolizada por $\boldsymbol{A} \boldsymbol{L} \boldsymbol{G}_{R}$. As categorias $A L G_{R}^{\bullet}$ e $A L G_{R}$ são essencialmente as mesmas,ou seja, existe um isomorfismo de categorias $A L G_{R} \cong A L G_{R}^{\bullet}$. Dizemos que um subconjunto $S \subseteq A$ é uma $R$-subalgebra de A quando for uma $R$-álgebra com as operações induzidas. Para cada $R$-álgebra $A$ nós associamos a álgebra oposta $A^{o p}=\langle A, \tau \circ \mu\rangle$, com $\tau(a, b)=$ $(b, a)$. Observe que essa asociação é funtorial. Uma R-álgebra A tal que A é um anel com divisão é chamada de R-álgebra de divisão ou simplesmente de álgebra de divisão.

Lema 40. Para cada R-álgebra $\langle A, \varphi\rangle$; o anel de R-endomofismos $\operatorname{End}_{R}(A)$ admite uma estrutura de R-álgebra.

Demonstração.

1) Note que a seguinte aplicação é um morfismo de anéis

$$
\begin{aligned}
& \mu: R \longrightarrow \quad \operatorname{End}_{R}(A) \\
& r \longmapsto \begin{aligned}
\mu_{r}: A & \longrightarrow A \\
a & \longmapsto \varphi(r) a
\end{aligned}
\end{aligned}
$$

que dá ao anel $\operatorname{End}_{R}(A)$ uma estrutura de $R$-álgebra.

Definição 64. (Produto tensorial de R-álgebras) $O$ produto tensorial de duas R-álgebras $A$ e $B$ é uma tripla $\left\langle A \otimes_{R} B, \eta_{A}, \eta_{B}\right\rangle$, onde $A \otimes_{R} B$ é uma $R$-álgebra e $\eta_{A}: A \longrightarrow A \otimes_{R} B, \eta_{B}: B \longrightarrow A \otimes_{R} B$ são morfismos de R-álgebras satisfazendo as seguintes condições:

$\left.\otimes_{1}\right) \forall a \in A, \forall b \in B\left(\eta_{A}(a) \eta_{B}(b)=\eta_{B}(b) \eta_{A}(a)\right)$.

$\left.\otimes_{2}\right)$ Se $\alpha: A \longrightarrow C$ e $\beta: B \longrightarrow C$ são homomorfismos de $R$-álgebras tais que

$$
\alpha(a) \beta(b)=\beta(b) \alpha(a), \forall a \in A, \forall b \in B
$$

então existe um único homomorfismo de $R$-álgebras $\varphi: A \otimes_{R} B \longrightarrow C$ que torna o seguinte diagrama comutativo 


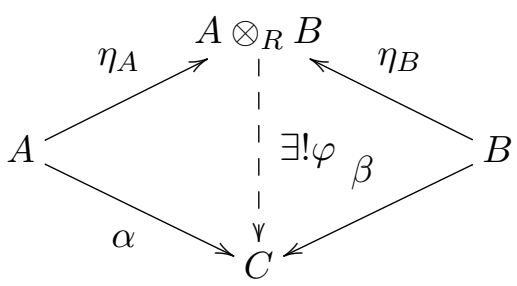

Proposição 66. (Unicidade do produto tensorial)Sejam $A$ e $B$ duas R-álgebras. Se $\left\langle A \otimes_{R} B, \eta_{A}, \eta_{B}\right\rangle$ $e\left\langle A \hat{\otimes}_{R} B, \hat{\eta}_{A}, \hat{\eta}_{B}\right\rangle$ são dois produtos tensoriais para essas álgebras, então $A \otimes_{R} B \cong A \hat{\otimes}_{R} B$.

Demonstração. Por hipótese existem únicos homomorfismos de R-álgebras $\varphi$ e $\hat{\varphi}$ que fornecem diagramas comutativos
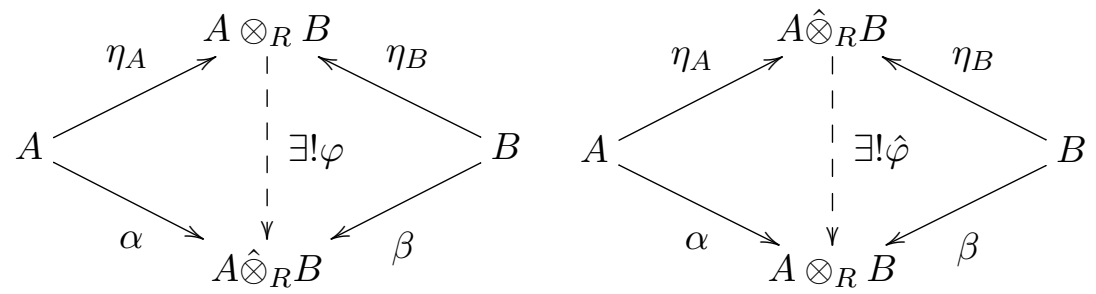

e, portanto a unicidade nos dá $\hat{\varphi} \circ \varphi=I d_{A \otimes_{R} B}$ e de maneira similar teremos $\varphi \circ \hat{\varphi}=I d_{A \hat{\otimes}_{R} B}$. Isso prova a proposição.

Definição 65. Seja $A \otimes_{R} B$ um produto tensorial para as $R$-álgebras $A$ e B. Dizemos que um mapa $\eta: A \times B \longrightarrow A \otimes_{R} B$ é multiplicativo quando para todos $a, a^{\prime} \in A$ e $b, b^{\prime} \in B$ tivermos

$$
\eta\left(a a^{\prime}, b b^{\prime}\right)=\eta(a, b) \eta\left(a^{\prime}, b^{\prime}\right)
$$

Proposição 67. (Caracterização alternativa do produto tensorial) Sejam $A, B$ duas $R$-álgebras. Suponha que existe um produto tensorial $\left\langle A \otimes_{R} B, \eta_{A}, \eta_{B}\right\rangle$, então existe uma aplicação bilinear multiplicativa

$$
\eta: A \times B \longrightarrow A \otimes_{R} B
$$

que cumpre a seguinte propriedade universal: Se $C$ é uma $R$-álgebra e $\hat{\eta}: A \times B \longrightarrow C$ for uma aplicação bilinear multiplicativa, então existe um único homomorfismo de R-álgebras $\varphi: A \otimes_{R} B \longrightarrow$ $C$ que faz o seguinte diagrama comutar

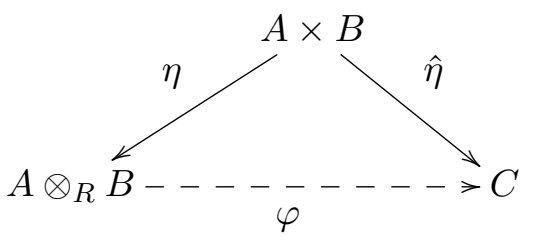

Inversamente, se existe uma bilinear multiplicativa $\eta: A \times B \longrightarrow A \otimes_{R} B$ satifazendo a propriedade universal obtemos um produto tensorial de acordo com a definição 64 .

Demonstração. Defina $\eta(a, b) \doteq \eta_{A}(a) \eta_{B}(b)$. Ela é multiplicativa, pois 


$$
\begin{aligned}
\eta\left(a a^{\prime}, b b^{\prime}\right) & =\eta_{A}\left(a a^{\prime}\right) \eta_{B}\left(b b^{\prime}\right) \\
& =\eta_{A}(a) \eta_{A}\left(a^{\prime}\right) \eta_{B}(b) \eta_{B}\left(b^{\prime}\right) \\
& =\eta_{A}(a) \eta_{B}(b) \eta_{A}\left(a^{\prime}\right) \eta_{B}\left(b^{\prime}\right) \\
& =\eta\left(a, a^{\prime}\right) \eta\left(b, b^{\prime}\right)
\end{aligned}
$$

Claramente os mapas

$$
\begin{aligned}
& \hat{\eta}_{A}: A \cong A \times\{1\} \longrightarrow A \otimes_{R} B \quad \eta_{B}: B \cong\{1\} \times B \longrightarrow A \otimes_{R} B \\
& a \mapsto(a, 1) \quad \longmapsto \hat{\eta}(a, 1) \quad b \mapsto(1, b) \quad \longmapsto \hat{\eta}(1, b)
\end{aligned}
$$

são morfismos de álgebras tais que

$$
\hat{\eta}_{A}(a) \hat{\eta}_{B}(b)=\hat{\eta}_{B}(b) \hat{\eta}_{A}(a)
$$

Dessa forma, a universalidade do produto tensorial nos dá o mapa requerido.

Proposição 68. (Existência do produto tensorial )Sejam $A$ e $B$ duas R-álgebras. Existem um produto tensorial para o par $(A, B)$.

Demonstração. Daremos a seguir um esboço dessa construção e deixamos os detalhes para o leitor. Denotemos por $A \tilde{\otimes}_{R} B$ o produto tensorial de R-módulos. Inicialmente, considere a aplicação

$$
\begin{aligned}
& g: A \times B \longrightarrow \quad\left(A \tilde{\otimes}_{R} B\right)^{A \times B}
\end{aligned}
$$

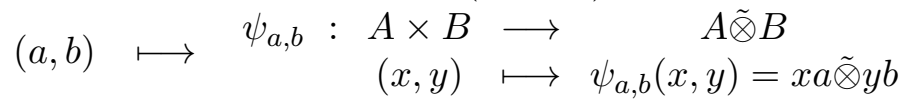

que associa a cada par $(a, b)$ um aplicação R-bilinear $\psi_{a, b}$. A universalidade do produto tensorial $A \tilde{\otimes}_{R} B$ nos fornece um único homomorfismo de R-módulos que faz o seguinte diagrama comutar

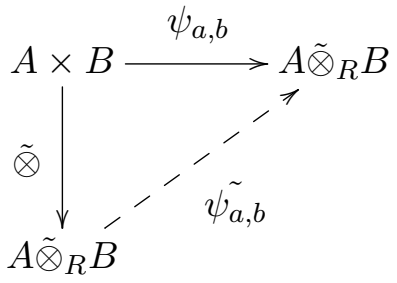

Verifique que a seguinte aplicação é R-bilinear

$$
\begin{aligned}
& \beta: A \times B \longrightarrow \operatorname{End}_{R}\left(A \tilde{\otimes}_{R} B\right) \\
& (a, b) \longmapsto \quad \tilde{\psi_{a}, b}
\end{aligned}
$$

Mais uma vez, a universalidade do produto tensorial nos dar um diagrama comutativo 


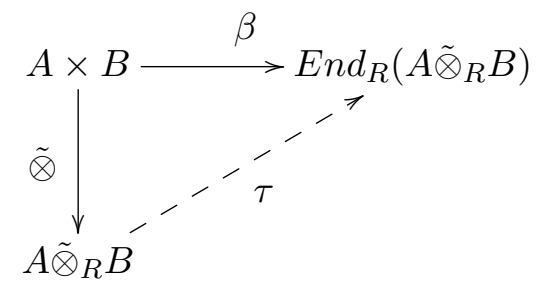

onde $\tau$ é homomorfismo de R-módulos tal que $\tau(a \tilde{\otimes} b)=\tilde{\psi_{a}, b}$. A multiplicação

$$
\begin{gathered}
\odot:\left(A \tilde{\otimes}_{R} B\right) \times\left(A \tilde{\otimes}_{R} B\right) \\
(u, v) \\
\longmapsto \tau(u)(v)
\end{gathered}
$$

dá a $A \tilde{\otimes}_{R} B$ uma estrutura de R-álgebra. Além disso, $\odot$ satisfaz

$$
(a \tilde{\otimes} b) \odot\left(a^{\prime} \tilde{\otimes} b^{\prime}\right)=\left(a a^{\prime}\right) \tilde{\otimes}\left(b b^{\prime}\right)
$$

Deixamos ao leitor a verificação de que a R-álgebra $A \tilde{\otimes}_{R} B$ juntamente com os mapas naturais

$$
\begin{aligned}
& \eta_{A}: A \longrightarrow A \tilde{\otimes}_{R} B \quad \eta_{B}: B \longrightarrow A \tilde{\otimes}_{R} B \\
& a \longmapsto a \tilde{\otimes} 1 \quad b \longmapsto 1 \tilde{\otimes} b
\end{aligned}
$$

constitui um produto tensorial para as álgebras A e B.

Definição 66. Sejam $\varphi: A \longrightarrow B$ e $\varphi^{\prime}: A^{\prime} \longrightarrow B$ morfismos de R-álgebras. Dizemos que $\varphi$ centra $\varphi^{\prime}\left(\varphi \rightleftharpoons \varphi^{\prime}\right)$ quando $\operatorname{Im}\left(\varphi^{\prime}\right) \subseteq Z(\operatorname{Im}(\varphi))$, ou seja, $\varphi^{\prime}\left(a^{\prime}\right) \varphi(a)=\varphi(a) \varphi^{\prime}\left(a^{\prime}\right), \forall a \in A, \forall a^{\prime} \in A^{\prime}$.

Observação 38. A relação

$$
\varphi \rightleftharpoons \varphi^{\prime}, \forall\left(\varphi, \varphi^{\prime}\right) \in A L G_{R}(A, B) \times A L G_{R}\left(A^{\prime}, B\right)
$$

é simétrica, mas não é reflexiva e nem transitiva em geral. Definimos o conjunto

$$
A L G_{R}\left(A, A^{\prime}, B\right) \doteq\left\{\left(\varphi, \varphi^{\prime}\right) \in A L G_{R}(A, B) \times A L G_{R}\left(A^{\prime}, B\right): \varphi \rightleftharpoons \varphi^{\prime}\right\}
$$

Proposição 69. Existe uma bijeção canônica

$$
A L G_{R}\left(A, A^{\prime}, B\right) \longleftrightarrow A L G_{R}\left(A \otimes_{R} A^{\prime}, B\right)
$$

Demonstração. Considere os morfismos naturais de álgebras

$$
\begin{aligned}
& \eta_{A}: A \longrightarrow A \otimes_{R} A^{\prime} \quad \eta_{A^{\prime}}: A^{\prime} \longrightarrow A \otimes_{R} A^{\prime} \\
& a \longmapsto a \otimes 1 \quad a^{\prime} \longmapsto 1 \otimes a^{\prime}
\end{aligned}
$$

claramente, $\eta_{A} \rightleftharpoons \eta_{A^{\prime}}$. Para $f \in A L G_{R}\left(A \otimes_{R} A^{\prime}, B\right)$ não é difícil ver que $f \circ \eta_{A} \rightleftharpoons f \circ \eta_{A^{\prime}}$. Dessa forma, temos uma aplicação

$$
\begin{aligned}
\Phi: A L G_{R}\left(A \otimes_{R} A^{\prime}, B\right) & \longrightarrow A L G_{R}\left(A, A^{\prime}, B\right) \\
f & \longmapsto\left(f \circ \eta_{A}, f \circ \eta_{A^{\prime}}\right)
\end{aligned}
$$

Mostremos que essa aplicação a bijetora.

Injetividade: $\Phi(f)=\Phi\left(f^{\prime}\right)$ se e só se $\left(f \circ \eta_{A}, f \circ \eta_{A^{\prime}}\right)=\left(f^{\prime} \circ \eta_{A}, f^{\prime} \circ \eta_{A^{\prime}}\right)$. Assim, 


$$
f\left(a \otimes a^{\prime}\right)=f^{\prime}\left(a \otimes a^{\prime}\right), \forall a \in A, \forall a^{\prime} \in A^{\prime}
$$

e poranto $\Phi$ é injetora.

Sobrejetividade:Seja $\left(\varphi, \varphi^{\prime}\right) \in A L G_{R}\left(A, A^{\prime}, B\right)$. A aplicação R-bilinear

$$
\beta: A \times A^{\prime} \longrightarrow B,\left(a, a^{\prime}\right) \mapsto \varphi(a) \varphi^{\prime}\left(a^{\prime}\right)
$$

induz um único morfismo $f: A \otimes_{R} A^{\prime} \longrightarrow B$ tal que $f\left(a \otimes a^{\prime}\right)=\varphi(a) \varphi^{\prime}\left(a^{\prime}\right), \forall a \in A, \forall a \in A^{\prime}$. Além disso, $f \circ \eta_{A}=\varphi$ e $f \circ \eta_{A^{\prime}}=\varphi^{\prime}$.

Proposição 70. Sejam A,B, $C$ três $R$-álgebras com $R$ comutativo. Existem isomorfismos canônicos

1) $A \otimes_{R} B \cong B \otimes_{R} A$.

2) $A \otimes_{R}\left(B \otimes_{R} C\right) \cong\left(A \otimes_{R} B\right) \otimes_{R} C$

Demonstração. Decorrem sa propriedade universal. Para (1) ver [Row prop. 1.7.13,pág 105 e para (2) consulte [Row cor.1.7.13,pág 105.

Proposição 71. Sejam A e B duas R-álgebras com $R$ um anel comutativo. As seguintes afirmações são válidas

1) $\forall m, n \in \omega\left(M_{m}\left(M_{n}(A)\right) \cong M_{m n}(A)\right)$.

2) $\forall n \in \omega\left(A \otimes_{R} M_{n}(R) \cong M_{n}(A)\right)$

3) $\forall m, n \in \omega\left(M_{m}(A) \otimes_{R} M_{n}(B) \cong M_{m n}\left(A \otimes_{R} B\right)\right)$

Demonstração.

1)Seja $B \doteq A^{o p}$. Basta observar que

$$
M_{m n}(A) \cong \operatorname{End}_{B}\left(B^{m n}\right) \cong M_{m}\left(\operatorname{End}_{B}\left(B^{n}\right)\right) \cong M_{m}\left(M_{n}\left(\operatorname{End}_{B}(B)\right)\right) \cong M_{m}\left(M_{n}(A)\right)
$$

2)Seja I a identidade da álgebra de matrizes $M_{n}(R)$. Note que

$$
\begin{aligned}
f: A & \longrightarrow M_{n}(A) \\
a & \longmapsto a I
\end{aligned}
$$

é um morfismo de R-álgebras. Também é um homomorfismo de R-álgebras o mapa

$$
\begin{aligned}
g: M_{n}(R) & \longrightarrow M_{n}(A) \\
\left(a_{i j}\right) & \longmapsto\left(\varphi\left(a_{i j}\right)\right)
\end{aligned}
$$

Claramente $f \rightleftharpoons g$ em $M_{n}(A)$ e, assim, a proposição 69 garante a existência de um único morfismo de R-álgebras

$$
\begin{aligned}
h: A \otimes_{R} M_{n}(R) & \longrightarrow M_{n}(A) \\
a \otimes M & \longmapsto a g(M)
\end{aligned}
$$

Seja $S \doteq\left\{e_{i j}: i, j \in\{1, \ldots, n\}\right\}$ a base standard de $M_{n}(R)$. Observe que o conjunto $\{a g(e): a \in$ $A, e \in S\}$ é uma R-base de $M_{n}(A)$. Segue que $f$ é um isomorfismo de R-álgebras.

3) Pelo item anterior e pela comutatividade do produto tensorial temos

$$
M_{m}(A) \otimes_{R} M_{n}(B) \cong\left(A \otimes_{R} B\right) \otimes_{R}\left(M_{m}(R) \otimes_{R} M_{n}(R)\right)
$$

agora combinando (2) e (1) obtemos 


$$
M_{m}(R) \otimes_{R} M_{n}(R) \cong M_{m}\left(M_{n}(R)\right) \cong M_{m n}(R)
$$

Assim,

$$
M_{m}(A) \otimes_{R} M_{n}(B) \cong\left(A \otimes_{R} B\right) \otimes_{R} M_{m n}(R) \cong M_{m n}\left(A \otimes_{R} B\right)
$$

\subsection{2 Álgebras simples e centrais simples}

Seja $\mathbb{K}$ um corpo. Dizemos que uma $\mathbb{K}$ - álgebra A é simples quando não admite ideais bilaterais não triviais. Para cada $n \in \omega$, o anel de matrizes $M_{n}(\mathbb{K})$ é um exemplo clássico de uma $\mathbb{K}$-álgebra simples(ver, por exemplo, Mil1] pág. 119 , exemplo 1.7 ).

Definição 67. Seja $A$ uma $R$-álgebra e $S \subseteq A$. O conjunto definido por

$$
C_{S}(A) \doteq\{x \in A: x s=s x, \forall s \in S\}
$$

é chamado de centro de $A$ em $S$. Em particular, $C_{A}(A)=Z(A)$ é chamado de centro da álgebra $A$.

Definição 68. Dizemos que uma $\mathbb{K}$-álgebra $A$ é central se $Z(A) \cong \mathbb{K}$ e central simples se for central e simples.

Proposição 72. (Artin - Wedderburn) Toda $\mathbb{K}$-álgebra simples e de dimensão finita A é isomorfa a $M_{n}(D)$ para algum inteiro positivo n e alguma $\mathbb{K}$ - álgebra de divisão $D$, também de dimensão finita. Além disso, $n$ é unicamente determinado por A e álgebra de divisão $D$ é unicamente determinada a menos de isomorfismo.

Demonstração. Para ver uma demonstração da primeira parte do teorema, consulte [Mil1]pág 122, Teorema 1.15, enquanto a segunda parte pode ser consultada, também, em [Mil1] pág 123, corolário 1.20 .

Lema 41. Se A é uma álgebra de divisão de dimensão finita sobre um corpo algebricamente fechado $\mathbb{K}$, então $A \cong \mathbb{K}$. Em particular, $A$ é central $(Z(A) \cong A \cong \mathbb{K})$.

Demonstração. Seja $D \cong A$ uma álgebra de divisão de dimensão finita sobre o corpo algebricamente fechado $\mathbb{K}$ tal que $\mathbb{K} \subseteq D$ e tomemos $a \in D$. Observe que $\mathbb{K}(a)$ é uma extensão comutativa finita de $\mathbb{K}$, uma vez que, D tem dimensão finita sobre $\mathbb{K}$. Como toda extensão de corpo de grau finito é algébrica e $\mathbb{K}$ é algebricamente fechado por hipótese, segue que $\mathbb{K}(a)=\mathbb{K}$. Portanto, $D \cong \mathbb{K}$.

Lema 42. Seja $\mathbb{K}$ um corpo algebricamente fechado. Seja A uma $\mathbb{K}$-álgebra central simples de dimensão finita. Existe um inteiro positivo $n$ tal que

$$
A \cong M_{n}(\mathbb{K})
$$

Demonstração. Pela proposição 72 existe um isomorfismo $A \cong M_{n}(D)$, onde n é um inteiro positivo e D é uma álgebra de divisão. O resultado segue agora do lema anterior.

Lema 43. Seja $\mathbb{K}$ um corpo. Para toda $\mathbb{K}$-álgebra central simples valem:

1) $\operatorname{dim}_{\mathbb{K}}(A)=n^{2}$ para algum $n \in \omega$.

2) $A \otimes_{\mathbb{K}} A^{o p} \cong M_{n}(\mathbb{K})$ como $\mathbb{K}$-álgebras . 
Demonstração.

1) Seja $\mathbb{K}^{\text {alg }}$ um fecho algébrico de $\mathbb{K}$. Ponhamos $A \mathbb{K}^{\text {alg }} \doteq A \otimes_{\mathbb{K}} \mathbb{K}^{\text {alg }}$ para ser a álgebra estendida por escalares. Pelo lema 42 existe um inteiro positivo n tal que $A \mathbb{K}^{\text {alg }} \cong M_{n}\left(\mathbb{K}^{\text {alg }}\right)$. Dessa forma,

$$
[A: \mathbb{K}]=\left[A \mathbb{K}^{\text {alg }}: \mathbb{K}^{\text {alg }}\right]=\left[M_{n}\left(\mathbb{K}^{\text {alg }}\right): \mathbb{K}^{\text {alg }}\right]=n^{2}
$$

2)Considere os seguintes homomorfismos de $\mathbb{K}$-álgebras

$$
\begin{aligned}
& f: A \longrightarrow \quad \operatorname{End}_{\mathbb{K}}(A) \\
& \begin{aligned}
a \longmapsto f(a): A & \longrightarrow c \\
x & \longmapsto f(a)(x)=a x
\end{aligned} \\
& g: A \longrightarrow \quad \operatorname{End}_{\mathbb{K}}(A)
\end{aligned}
$$

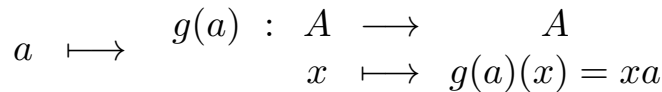

Observe que $f \rightleftharpoons g$ em $\operatorname{End}_{\mathbb{K}}(A)$. Assim, existe um único homomorfismo de $\mathbb{K}$-álgebras

$$
\begin{aligned}
h: A \otimes_{\mathbb{K}} A^{o p} & \longrightarrow \operatorname{End}_{\mathbb{K}}(A) \\
a \otimes b & \longmapsto f(a) g(b)
\end{aligned}
$$

h é injetivo, pois $A \otimes_{\mathbb{K}} A^{o p}$ é simples e, também, sobrejetivo, uma vez que, $\left[A \otimes_{\mathbb{K}} A^{o p}: \mathbb{K}\right]=n^{2}$ e $\left[\operatorname{End}_{\mathbb{K}}(A): \mathbb{K}\right]=n^{2}$. Finalmente, temos $\operatorname{End}_{\mathbb{K}}(A) \cong M_{n}(\mathbb{K})$ como $\mathbb{K}$-álgebras.

Lema 44. Sejam $A$ e $A^{\prime}$ álgebras sobre um corpo $\mathbb{K}$ com sub-álgebras $B$ e $B^{\prime}$, respectivmente. Então,

$$
C_{A \otimes_{\mathbb{K}} A^{\prime}}\left(B \otimes_{\mathbb{K}} B^{\prime}\right)=C_{A}(B) \otimes_{\mathbb{K}} C_{A^{\prime}}\left(B^{\prime}\right)
$$

Demonstração. É fácil ver $C\left(B \otimes_{\mathbb{K}} B^{\prime}\right) \supset C(B) \otimes_{\mathbb{K}} C\left(B^{\prime}\right)$. Seja $\left\{f_{i}\right\}_{i \in I}$ uma base para $A^{\prime}$ (como $\mathbb{K}$ - espaço vetorial). Nesse caso, $\left\{1 \otimes f_{i}\right\}_{i \in I}$ é uma base para $A \otimes_{\mathbb{K}} A^{\prime}$ como um A-módulo, e então cada $\alpha \in A \otimes_{\mathbb{K}} A^{\prime}$ escreve-se como

$$
\alpha=\sum_{i \in I} \alpha_{i} \otimes f_{i}, \quad \alpha_{i} \in A
$$

Seja $\beta \in B$. Então, $\alpha$ comuta com $\beta \otimes 1$ se, e somente se, $\beta \alpha_{i}=\alpha_{i} \beta, \forall i$. Portanto, o centralizador de $B \otimes 1$ em $A \otimes A^{\prime}$ é $C_{A}(B) \otimes A^{\prime}$. Analogamente, o centralizador de $1 \otimes B^{\prime}$ em $C_{A}(B) \otimes A^{\prime}$ é $C_{A}(B) \otimes C_{A^{\prime}}\left(B^{\prime}\right)$. Obviamente, $C\left(B \otimes B^{\prime}\right) \subset C(B \otimes 1)$, e assim $C\left(B \otimes B^{\prime}\right) \subset C(B) \otimes A^{\prime}$ e, de fato está contido no centralizador de $1 \otimes B^{\prime}$ em $C(B) \otimes A^{\prime}$, que é $C(B) \otimes C\left(B^{\prime}\right)$. Isto completa a prova.

Corolário 17. Sejam $A$ e $B$ duas $\mathbb{K}$-álgebras. Temos o seguinte isomorfismo

$$
Z\left(A \otimes_{\mathbb{K}} B\right) \cong Z(A) \otimes_{\mathbb{K}} Z(B)
$$

Corolário 18. Se A é uma $\mathbb{K}$-álgebra simples de dimensão finita, então $Z(A) \cong \mathbb{K}^{\prime}$, onde $\mathbb{K}^{\prime} \mid \mathbb{K}$ é uma extensão finita de corpos. Em outros termos, o centro de toda $\mathbb{K}$-álgebra simples é um corpo.

Demonstração. Pelo proposição 72 temos um isomorfismo $A \cong M_{n}(D)$ para alguma álgebra de divisão D de dimensão finita sibre $\mathbb{K}$. Assim, $A \cong M_{n}(D) \cong M_{n}(\mathbb{K}) \otimes_{\mathbb{K}} D$ de modo que $Z(A) \cong$ $Z\left(M_{n}(\mathbb{K})\right) \otimes_{\mathbb{K}} Z(D) \cong \mathbb{K} \otimes_{\mathbb{K}} Z(D) \cong Z(D)$. Como $Z(D)$ é um corpo, temos o resultado. 
Corolário 19. Se $A$ e $A^{\prime}$ são $\mathbb{K}$-álgebras centrais, então $A \otimes_{\mathbb{K}} A^{\prime}$ também o é.

Demonstração.

$$
Z\left(A \otimes_{\mathbb{K}} B\right) \cong Z(A) \otimes_{\mathbb{K}} Z(B) \cong \mathbb{K} \otimes \mathbb{K} \cong \mathbb{K}
$$

Proposição 73. Sejam $A$ e $A^{\prime}$ duas $\mathbb{K}$-álgebras simples de dimensão finita. Se $A$ ou $A^{\prime}$ for central, então $A \otimes_{\mathbb{K}} A^{\prime}$ será simples.

Demonstração. Ver [Mil1] prop 2.6,pág.125 ou [Ker2] sec. 2.6, pág. 27 para uma demonstração desse resultado.

Fato 23. Seja $\mathbb{K} \stackrel{\phi}{\longrightarrow} \mathbb{F}$ uma extensão de corpos. Seja $A$ uma $\mathbb{K}$-álgebra de divisão de dimensão finita. Qualquer ideal bilateral em $A \otimes_{\mathbb{K}} \mathbb{F}$ é gerado como $A$-módulo por sua intersecção com $\mathbb{K} e$, portanto é 0 ou $A \otimes_{\mathbb{K}} \mathbb{F}$.

Proposição 74. Seja $\mathbb{K} \stackrel{\phi}{\longrightarrow} \mathbb{F}$ uma extensão de corpos. Uma $\mathbb{K}$-álgebra $A$ de dimensão finita é central simples se, e somente se, a álgebra estendida por escalares $A \otimes_{\mathbb{K}} \mathbb{F}$ for central simples de dimensão finita sobre $\mathbb{F}$.

Demonstração. Suponhamos que A é uma $\mathbb{K}$-álgebra central simples. $A \otimes_{\mathbb{K}} \mathbb{F}$ é central, pois

$$
Z\left(A \otimes_{\mathbb{K}} \mathbb{F}\right) \cong Z(A) \otimes_{\mathbb{K}} Z(\mathbb{F}) \cong \mathbb{K} \otimes_{\mathbb{K}} \mathbb{F} \cong \mathbb{F}
$$

pela proposição 72 existe um isomorfismo $A \cong M_{n}(D)$ para alguma álgebra de divisão D. Assim,

$$
\begin{aligned}
A \otimes_{\mathbb{K}} \mathbb{F} & \cong M_{n}(D) \otimes_{\mathbb{K}} \mathbb{F} \\
& \cong\left(M_{n}(\mathbb{K}) \otimes_{\mathbb{K}} D\right) \otimes_{\mathbb{K}} \mathbb{F} \\
& \cong M_{n}(\mathbb{K}) \otimes_{\mathbb{K}}\left(D \otimes_{\mathbb{K}} \mathbb{F}\right) \\
& \cong M_{n}\left(D \otimes_{\mathbb{K}} \mathbb{F}\right) \\
& \cong M_{n}(\mathbb{F}) \otimes_{\mathbb{F}}\left(D \otimes_{\mathbb{K}} \mathbb{F}\right)
\end{aligned}
$$

que é simples. Inversamente, se $A \otimes_{\mathbb{K}} \mathbb{F}$ for uma álgebra central simples sobre $\mathbb{F}$, então

$$
\mathbb{K} \otimes_{\mathbb{F}} \mathbb{F} \cong \mathbb{F} \cong Z\left(A \otimes_{\mathbb{K}} \mathbb{F}\right) \cong Z(A) \otimes_{\mathbb{K}} Z(\mathbb{F}) \cong Z(A) \otimes_{\mathbb{K}} \mathbb{F}
$$

e, então, $Z(A) \cong \mathbb{K}$. Finalmente, se A admite um ideal bilateral não trivial $\mathcal{I}$, então $\mathcal{I} \otimes_{\mathbb{K}} \mathbb{F}$ seria um ideal não trivial de $A \otimes_{\mathbb{K}} \mathbb{F}$. Uma contradição.

\subsubsection{Definição do grupo de Brauer}

Definição 69. Dizemos que duas $\mathbb{K}$-álgebras centrais simples $A$ e B sobre um corpo $\mathbb{K}$ são similares, $A \approx B$, se existe uma álgebra de divisão $D$ e inteiros positivos $r$,s tais que $A \cong M_{r}(D)$ e $B \cong$ $M_{s}(D)$.

Proposição 75. Sejam $A, A^{\prime}, B, B^{\prime} \mathbb{K}$-álgebras centrais simples de dimensão finita. Se $A \approx A^{\prime} e$ $B \approx B^{\prime}$, então $A \otimes_{\mathbb{K}} B \approx A^{\prime} \otimes_{\mathbb{K}} B^{\prime}$. 
Demonstração. Com efeito, A (resp. $\left.A^{\prime}\right)$ é isomorfa a uma álgebra da forma $M_{p}(D)\left(\operatorname{resp} . M_{r}(D)\right)$, B (resp. $\left.B^{\prime}\right)$ é isomorfa a uma álgebra da forma $\left.M_{q}(E)\right)\left(\right.$ resp. $M_{s}(E)$ ), onde $\mathrm{D}$ e E são $\mathbb{K}$ - álgebras de divisão. A álgebra $D \otimes_{\mathbb{K}} E$ é central simples e, também, é isomorfa a uma $\mathbb{K}$-álgebra da forma $M_{h}(L)$, onde L é uma $\mathbb{K}$-álgebra de divisão. Segue da proposição 71 que $A \otimes_{\mathbb{K}} B \cong M_{h p q}(L)$ e $A^{\prime} \otimes_{\mathbb{K}^{\prime}} B^{\prime} \cong M_{h r s}(L)$.

Definição 70. Denotaremos por $S m_{\mathbb{K}}(A, B)$ a seguinte relação : $\boldsymbol{A}$ e $\boldsymbol{B}$ são duas $\mathbb{K}$ - álgebras centrais simples de dimensão finita que são similares.

Observação 39. Pelo teorema de Artin - Wedderburn a relação de similaridade é uma relação de equivalência. Denotamos por $[A]$ a classe de equivalência de cada $\mathbb{K}$-álgebra central simples A. Seja $\mathcal{A}_{n} \doteq\left\{S: S\right.$ é $\mathbb{K}$ - álgebra central simples com $\mathbb{K}$ - espaço vetorial subjacente $\left.\mathbb{K}^{n}\right\}$ para cada $n \in \omega$. Seja $\mathcal{C} \subseteq \bigcup_{n \in \omega} \mathcal{A}_{n}$ o conjunto de todas $\mathbb{K}$-álgebras centrais simples. Definimos $[A] \uparrow_{\mathcal{C}} \doteq$ $\{B \in \mathcal{C}: A \approx B\}$. Observe que $B r(\mathbb{K}) \doteq\{[A] \uparrow \mathcal{C}: A \in \mathcal{C}\}$ é um conjunto.

Proposição 76. O conjunto $\operatorname{Br}(\mathbb{K})$ juntamente com a operação

$$
\begin{aligned}
\odot: \operatorname{Br}(\mathbb{K}) \times \operatorname{Br}(\mathbb{K}) & \longrightarrow \quad \operatorname{Br}(\mathbb{K}) \\
([A],[B]) & \longmapsto\left[A \otimes_{\mathbb{K}} B\right]
\end{aligned}
$$

é um grupo abeliano.

Demonstração. A associatividade e a comutatividade seguem da proposição 70 . Claramente, $\left[M_{n}(\mathbb{K})\right]=$ $[\mathbb{K}]$ é o elemento neutro. Finalmente, $\left[A^{o p}\right]$ é o inverso de $[A]$ pelo lema 43 .

Definição 71. O grupo $(\operatorname{Br}(\mathbb{K}), \odot)$ é conhecido como grupo de Brauer ${ }^{17}$

Proposição 77. Cada morfismo de corpos $\varphi: \mathbb{K} \longrightarrow \mathbb{F}$ determina um morfismo de grupos abelianos $\operatorname{Br}(\varphi): \operatorname{Br}(\mathbb{K}) \longrightarrow \operatorname{Br}(\mathbb{F})$.

Demonstração. Seja $\varphi: \mathbb{K} \longrightarrow \mathbb{F}$ um homomorfismo de corpos. Definimos $\operatorname{Br}(\varphi): \operatorname{Br}(\mathbb{K}) \longrightarrow$ $\operatorname{Br}(\mathbb{F})$ por $\operatorname{Br}(\varphi)([A]) \doteq\left[A \otimes_{\mathbb{K}} \mathbb{F}\right]$. Pelas proposições 74 e 75 essa definição é legítima. Vamos mostrar que $\operatorname{Br}(\varphi)$ é um homorfismo de grupos abelianos. De fato

$$
\begin{aligned}
\operatorname{Br}(\varphi)([A][B]) & =\operatorname{Br}(\varphi)\left(\left[A \otimes_{\mathbb{K}} B\right]\right) \\
& =\left[\left(A \otimes_{\mathbb{K}} B\right) \otimes_{\mathbb{K}} \mathbb{F}\right] \\
& =\left[\left(A \otimes_{\mathbb{K}} B\right) \otimes_{\mathbb{K}}\left(\mathbb{F} \otimes_{\mathbb{F}} \mathbb{F}\right)\right] \\
& =\left[A \otimes_{\mathbb{K}} \mathbb{F} \otimes_{\mathbb{F}} \mathbb{F} \otimes_{\mathbb{K}} B\right] \\
& =\left[\left(A \otimes_{\mathbb{K}} \mathbb{F}\right) \otimes_{\mathbb{F}}\left(B \otimes_{\mathbb{K}} \mathbb{F}\right)\right] \\
& =\left[A \otimes_{\mathbb{K}} \mathbb{F}\right]\left[B \otimes_{\mathbb{K}} \mathbb{F}\right] \\
& =\operatorname{Br}(\varphi)([A]) \operatorname{Br}(\varphi)([B])
\end{aligned}
$$

\footnotetext{
${ }^{17}$ Grupos de Brauer foram definidos e exaustivamente estudados em vários artigos de R. Brauer, E. Noether, A. Albert, H. Hasse. Este grupo mantém estreitas conexões com a teoria dos números.
} 
Proposição 78. Seja Field a categoria de corpos e Ab a categoria de grupos abelianos. As aplicaçôes

$$
\begin{gathered}
\text { Br : Field } \longrightarrow A b \\
\mathbb{K} \in \text { Ob(Field }) \longmapsto \operatorname{Br}(\mathbb{K}) \in \operatorname{Ob}(A b) \\
\varphi \in \operatorname{Hom}(\mathbb{K}, \mathbb{F}) \longmapsto \operatorname{Br}(\varphi) \in \operatorname{Hom}(\operatorname{Br}(\mathbb{K}), \operatorname{Br}(\mathbb{F}))
\end{gathered}
$$

Definem um funtor covariante da categoria de corpos para a categoria de grupos abelianos.

Demonstração. Claramente $\operatorname{Br}\left(1_{\mathbb{K}}\right)=1_{B r(\mathbb{K})}$ para todo corpo $\mathbb{K}$. Finalmente, para cada composição $\mathbb{K} \stackrel{\varphi}{\longrightarrow} \mathbb{F} \stackrel{\phi}{\longrightarrow} \mathbb{L}$ de morfismos de corpos teremos $\operatorname{Br}(\phi) \circ \operatorname{Br}(\varphi)([A])=\left[\left(A \otimes_{\mathbb{K}} \mathbb{F}\right) \otimes_{\mathbb{F}} \mathbb{L}\right]=\left[A \otimes_{\mathbb{K}}\right.$ $\left.\left(\mathbb{F} \otimes_{\mathbb{F}} \mathbb{L}\right)\right]=\left[A \otimes_{\mathbb{K}} \mathbb{L}\right]=\operatorname{Br}(\phi \circ \varphi)([A])$.

Proposição 79. Seja $\mathbb{K}$ um corpo. Os elementos de Br( $\mathbb{K})$ estão em correspondência bijetora com as álgebras de divisão centrais sobre $\mathbb{K}$.

Demonstração. Pela proposição 72 toda $\mathbb{K}$-algebra central simples é da forma $M_{n}(D)$ para algum inteiro positivo n e alguma álgebra de divisão D. Desde que , $A \cong M_{n}(D) \cong D \otimes M_{n}(\mathbb{K})$ teremos $[A]=[D]$ em $\operatorname{Br}(\mathbb{K})$.A proposição 72 também afirma que $\mathrm{D}$ é determinada unicamente a menos de isomorfismo. Assim, $[D]=\left[D^{\prime}\right]$ implica que existe uma álgebra de divisão $D^{\prime \prime}$ e inteiros positivos $r, s \in \omega$ tal que $D \cong M_{r}\left(D^{\prime \prime}\right)$ e $D^{\prime} \cong M_{s}\left(D^{\prime \prime}\right)$. Resulta da unicidade em 72 que $D \cong D^{\prime \prime} \cong D^{\prime}$ e $r=s=1$.

Colocamos agora mais alguns resultados úteis sobre os elementos de $\operatorname{Br}(\mathbb{K})$ determinados por álgebras de quatérnios.

Proposição 80. (Linearidade)Para $a, b, c \in \mathbb{K}$, temos

$$
(a, b)_{\mathbb{K}} \otimes(a, c)_{\mathbb{K}} \cong(a, b c)_{\mathbb{K}} \otimes\left(a,-a^{2} c\right)_{\mathbb{K}} \cong(a, b c)_{\mathbb{K}} \otimes M_{2}(\mathbb{K})
$$

Demonstração. Ver [Lam1] teor.2.11 e pág. 60.

Lema 45. Se uma álgebra de quatérnios $(a, b)_{\mathbb{K}}$ não é uma álgebra de divisão,então $(a, b)_{\mathbb{K}} \cong$ $M_{2}(\mathbb{K})$.

Demonstração. Seja $(a, b)_{\mathbb{K}}$ uma álgebra de quatérnios. Pela proposição $22(1),(a, b)_{\mathbb{K}} \cong(1,1)_{\mathbb{K}}$.Agora note que a base $\{1, i, j, k\}$ de $(1,1)_{\mathbb{K}}$ satisfaz as mesmas relações como as matrizes

$$
\left(\begin{array}{ll}
1 & 0 \\
0 & 1
\end{array}\right),\left(\begin{array}{cc}
1 & 0 \\
0 & -1
\end{array}\right),\left(\begin{array}{ll}
0 & 1 \\
1 & 0
\end{array}\right),\left(\begin{array}{cc}
0 & 1 \\
-1 & 0
\end{array}\right)
$$

Proposição 81. Toda álgebra de quatérnios $(a, b)_{\mathbb{K}}$ tem ordem dois como um elemento de $\operatorname{Br}(\mathbb{K})$.

Demonstração. Usando o corolário 10 e a proposição 80 deste capítulo, teremos: 


$$
\begin{aligned}
{\left[(a, b)_{\mathbb{K}}\right]^{2} } & =\left[(a, b)_{\mathbb{K}}\right] \odot\left[(a, b)_{\mathbb{K}}\right] \\
& =\left[(a, b)_{\mathbb{K}} \otimes(a, b)_{\mathbb{K}}\right] \\
& =\left[\left(a, b^{2}\right) \otimes M_{2}(\mathbb{K})\right] \\
& =\left[(a, 1)_{\mathbb{K}}\right] \otimes\left[M_{2}(\mathbb{K})\right]
\end{aligned}
$$

pelo corolário $10(a, 1)_{\mathbb{K}} \cong(1,1)_{\mathbb{K}}$ e como pela proposição 22 a álgebra $(1,1)_{\mathbb{K}}$ não é uma álgebra de divisão, segue que $(a, 1)_{\mathbb{K}}$ não é uma álgebra de divisão. Em virtude do lema 45 teremos

$$
\begin{aligned}
{\left[(a, b)_{\mathbb{K}}\right]^{2} } & =\left[(a, 1)_{\mathbb{K}} \otimes M_{2}(\mathbb{K})\right] \\
& =\left[M_{2}(\mathbb{K}) \otimes M_{2}(\mathbb{K})\right] \\
& =\left[M_{4}(\mathbb{K})\right] \\
& =[\mathbb{K}] \\
& =1
\end{aligned}
$$

\subsection{Cohomologia galoisiana}

Seja $\mathbb{E} \mid \mathbb{K}$ uma extensão galoisiana. Seja $(\dot{\mathbb{E}}, \cdot)$ o grupo abeliano multiplicativo do corpo $\mathbb{E}$ com a topologia discreta. Fazemos de $\dot{\mathbb{E}}$ um $\operatorname{Gal}(\mathbb{E} \mid \mathbb{K})$ - módulo mediante a aplicação

$$
\begin{aligned}
\cdot \operatorname{Gal}(\mathbb{E} \mid \mathbb{K}) \times \dot{\mathbb{E}} & \longrightarrow \dot{\mathbb{E}} \\
(\sigma, e) & \longmapsto \sigma(e)
\end{aligned}
$$

De fato,

$$
\begin{gathered}
i d \cdot e=e \\
\forall \sigma, \tau \in G a l(\mathbb{E} \mid \mathbb{K}) \forall e \in \dot{\mathbb{E}}((\sigma \tau) \cdot e=\sigma(\tau(e))=\sigma \cdot(\tau \cdot e))
\end{gathered}
$$

e,

$$
\forall \sigma \in G a l(\mathbb{E} \mid \mathbb{K}) \forall e, e^{\prime} \in \dot{\mathbb{E}}\left(\sigma \cdot\left(e e^{\prime}\right)=\sigma(e) \sigma\left(e^{\prime}\right)=\sigma \cdot e \cdot \sigma \cdot e^{\prime}\right)
$$

Com essa mesma ação o grupo aditivo $\mathbb{E}_{+}$do corpo $\mathbb{E}$, também é um $G a l(\mathbb{E} \mid \mathbb{K})$-módulo.

Definição 72. Seja $G$ um grupo e $\mathbb{K}$ um corpo. Um carácter 18 de $G$ em $\mathbb{K}$ é um homomorfismo de grupos $\chi: G \longrightarrow \dot{\mathbb{K}}$.

\footnotetext{
${ }^{18}$ A origem de teoria de caracteres e teoria de representação remonta aos anos de 1896 com correspondências entre Dedekind e Georg Frobenius. Um problema primordial que Dedekind propôs a Frobenius tinha a ver com a fatoração de certos polinômios homogêneos oriundos de um determinante associado a um grupo finito. Frobenius inventou a teoria de caracteres e resolveu completamente o problema proposto por Dedekind. A sua primeira definição de caracter foi $a d$ hoc. Mas, em 1897 ele dá uma definição moderna de uma representação de um grupo G como sendo um homomorfismo de grupos $D: G \longrightarrow G L_{n}(\mathbb{C})$. Na sequência, define a função $\chi_{D}: G \longrightarrow \mathbb{C}$ por $\chi_{D}(g)=$ $\operatorname{Tr}(D(g)), \forall g \in G$,onde $\operatorname{Tr}(D(g))$ é a soma de suas entradas diagonais. Frobenius chamará essa função de carácter
} 
Definição 73. Um conjunto de caracteres $\left\{\chi_{1} \ldots \chi_{n}\right\}$ de $G$ em $\mathbb{K}$ é dito independente, quando

$$
\sum_{i=1}^{n} a_{i} \chi_{i}(x)=0, \forall a_{1}, \ldots, a_{n} \in \mathbb{K}, \forall x \in G
$$

implicar

$$
a_{1}=a_{2}=\cdots=a_{n}=0
$$

Proposição 82. (Dedekind)Toda lista $\chi_{1}, \ldots, \chi_{n}$ de caracteres distintos de um grupo $G$ em um corpo $\mathbb{K}$ é independente.

Demonstração. Vamos usar indução finita para demonstrar esse resultado.

Base de indução $(n=1)$ : Seja $\chi: G \longrightarrow \dot{\mathbb{K}}$ um carácter de $G$ em $\mathbb{K}$. Suponhamos que $a \chi(x)=$

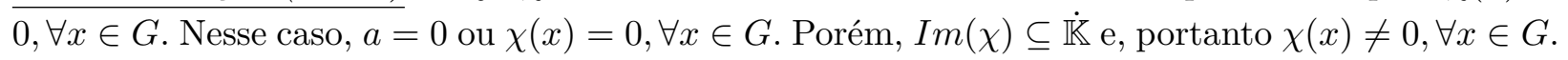
Logo, $a=0$.

Hipótese de indução: Nossa hipótese de indução consiste em supor que o resultado vale para $n-1, n>0$.

Tese de indução: $\mathrm{O}$ resultado vale para $\mathrm{n}$.

Sejam $\chi_{1}, \cdots, \chi_{n}: G \longrightarrow \dot{K}$ caracteres distintos de $G$ em $\mathbb{K}$. Suponhamos que eles não sejam independentes. Então, existem $a_{1}, \cdots, a_{n} \in \mathbb{K}$ não todos nulos tais que :

$$
\sum_{i=1}^{n} a_{i} \chi(x)=0, \forall x \in G
$$

se algum $a_{i}=0$, então teremos uma contradição com a hipótese de indução. Assim, podemos supor todos os $a_{i} \neq 0$. Podemos supor, também, que $a_{n}=1$, pois caso contrário multiplicaríamos $(*)$ por $a_{n}^{-1}$. Desde que, $\chi_{n} \neq \chi_{1}$, existe $y \in G$ tal que $\chi_{n}(y) \neq \chi_{1}(y)$. Trocando $x$ por $y x$ em $(*)$, obtemos

$$
\left(\sum_{i=1}^{n_{1}} a_{i} \chi_{i}(y) \chi_{i}(x)\right)+\chi_{n}(y) \chi_{n}(x)=0
$$

pois, $\chi_{i}(y x)=\chi_{i}(y) \chi_{i}(x), \forall i=1, \cdots, n$. Agora multiplicamos $(* *)$ por $\chi_{n}(y)^{-1}$ e obtemos a equação

$$
\left(\sum_{i=1}^{n_{1}} a_{i} \chi_{n}(y)^{-1} \chi_{i}(y) \chi_{i}(x)\right)+\chi_{n}(x)=0 \quad(* * *)
$$

subtraindo a equação $(* * *)$ de $(*)$ teremos a soma de $n-1$ termos:

$$
\sum_{i=1}^{n-1} a_{i}\left[1-\chi_{n}(y)^{-1} \chi_{i}(y)\right] \chi_{i}(x)=0
$$

por hipótese de indução

$$
a_{i}\left[1-\chi_{n}(y)^{-1} \chi_{i}(y)\right]=0
$$

da representação D. Finalmente, A definição geral de carácter e representação(que são atualmente utilizadas) foram introduzidas por Dedekind. O Leitor interessado em mais detalhes pode consultar [Cur1]. 
para todo $i=1, \cdots, n-1$. Como $a_{i} \neq 0$, então $\chi_{n}(y)^{-1} \chi_{i}(y)=1$ para todo $i<n-1$. Em particular,

$$
\chi_{n}(y)=\chi_{1}(y)
$$

contradizendo a escolha de $\mathrm{y}$.

Proposição 83. (Hilbert 90 multiplicativo) Para toda extensão galoisiana $\mathbb{E} \mid \mathbb{K}$ tem-se

$$
H_{\text {cont }}^{1}(G a l(\mathbb{E} \mid \mathbb{K}), \dot{\mathbb{E}})=1
$$

Demonstração. Suponhamos, inicialmente, que $[\mathbb{E}: \mathbb{K}]<\aleph_{0}$. Seja $\varphi \in Z^{1}(\operatorname{Gal}(\mathbb{E} \mid \mathbb{K}), \dot{\mathbb{E}})$. Como $\mathbb{E} \mid \mathbb{K}$ é uma extensão finita, segue do fato 16 que o grupo $\operatorname{Gal}(\mathbb{E} \mid \mathbb{K})$ é finito. Assim, a proposição 82 garante que $\{\varphi(\sigma): \sigma \in G a l(\mathbb{E} \mid \mathbb{K})\}$ é um conjunto independente. Em particular, o endomorfismo de grupos abelianos

$$
\begin{aligned}
& \hat{\varphi}: \mathbb{E}_{+} \longrightarrow \quad \mathbb{E}_{+} \\
& e \longmapsto \sum_{\sigma \in \operatorname{Gal}(\mathbb{E} \mid \mathbb{K})} \varphi(\sigma) \sigma(e)
\end{aligned}
$$

é não nulo. Seja $e \in \mathbb{E}$ tal que $\varepsilon \doteq \hat{\varphi}(e) \neq 0$. Usando a igualdade

$$
\varphi(\sigma \tau) \sigma(\varphi(\tau)), \quad \forall \sigma, \tau \in \operatorname{Gal}(\mathbb{E} \mid \mathbb{K})
$$

Teremos

$$
\begin{aligned}
\tau(\varepsilon) & =\sum_{\sigma \in \operatorname{Gal}(\mathbb{E} \mid \mathbb{K})} \tau(\varphi(\sigma) \sigma(e)) \\
& =\sum_{\sigma \in \operatorname{Gal}(\mathbb{E} \mid \mathbb{K})} \tau(\varphi(\sigma)) \tau(\sigma(e)) \\
& =\sum_{\sigma \in \operatorname{Gal}(\mathbb{E} \mid \mathbb{K})} \varphi(\tau)^{-1} \varphi(\tau \sigma) \tau(\sigma(e)) \\
& =\varphi(\tau)^{-1} \sum_{\sigma \in \operatorname{Gal}(\mathbb{E} \mid \mathbb{K})} \varphi(\tau \sigma) \tau(\sigma(e)) \\
& =\varphi(\tau)^{-1} \sum_{\sigma \in \operatorname{Gal}(\mathbb{E} \mid \mathbb{K})} \varphi(\tau \sigma)(\tau \sigma)(e) \\
& =\varphi(\tau)^{-1} \sum_{\sigma^{\prime} \in \operatorname{Gal}(\mathbb{E} \mid \mathbb{K})} \varphi\left(\sigma^{\prime}\right) \sigma^{\prime}(e) \\
& =\varphi(\tau)^{-1} \varepsilon
\end{aligned}
$$

Por conseguinte,

$$
\tau(\varepsilon)=\varphi(\tau)^{-1} \varepsilon \Longrightarrow \varphi(\tau)=\frac{\tau\left(\varepsilon^{-1}\right)}{\varepsilon^{-1}}=\frac{\varepsilon}{\tau(\varepsilon)}=\varepsilon \tau(\varepsilon)^{-1} \Longrightarrow \varphi \in B^{1}
$$

portanto, 


$$
H_{\text {cont }}^{1}(\operatorname{Gal}(\mathbb{E} \mid \mathbb{K}), \dot{\mathbb{K}})=\frac{Z^{1}(\operatorname{Gal}(\mathbb{E} \mid \mathbb{K}), \dot{\mathbb{K}})}{B^{1}(\operatorname{Gal}(\mathbb{E} \mid \mathbb{K}), \dot{\mathbb{K}})}=\frac{B^{1}(\operatorname{Gal}(\mathbb{E} \mid \mathbb{K}), \dot{\mathbb{K}})}{B^{1}(\operatorname{Gal}(\mathbb{E} \mid \mathbb{K}), \dot{\mathbb{K}})}=\{1\}
$$

Finalmente, combinando as proposições 55 e 60 , teremos

$$
\begin{aligned}
& H_{\text {cont }}^{1}(\operatorname{Gal}(\mathbb{E} \mid \mathbb{K}), \dot{\mathbb{K}})=H_{\text {cont }}^{1}\left(\lim _{\mathbb{I} \in \mathcal{J}} \operatorname{Gal}(\mathbb{E} \mid \mathbb{I}), \underset{\underset{\mathbb{I} \in \mathcal{J}}{\lim } \dot{\mathbb{I}}}{ }\right) \\
& =\lim _{\mathbb{I} \in \mathcal{J}} H_{\text {cont }}^{1}(\operatorname{Gal}(\mathbb{E} \mid \mathbb{I}), \dot{\mathbb{I}}) \\
& =\underset{\mathbb{I} \in \mathcal{J}}{\lim }\{1\} \\
& =\{1\}
\end{aligned}
$$

Proposição 84. (Hilbert 90 aditivo) Para toda extensão galoisiana $\mathbb{E} \mid \mathbb{K}$ e todo $n>0$ tem-se

$$
H_{\text {cont }}^{n}\left(\operatorname{Gal}(\mathbb{E} \mid \mathbb{K}), \mathbb{E}_{+}\right)=0
$$

Demonstração. De acordo com as proposições 55 e 60 é suficiente mostrar o caso em que a extensão tem grau finito. Suponhamos que $[\mathbb{E}: \mathbb{K}]<\aleph_{0}$ e denotemos $G \doteq \operatorname{Gal}\left(\mathbb{K}^{s e p} \mid \mathbb{K}\right)$ e $A \doteq \mathbb{E}_{+}$. Agora o teorema da base normal 19 permite escrever

$$
\mathbb{E} \cong \bigoplus_{\sigma \in G} \mathbb{K} \sigma \alpha \cong \mathbb{K}[G]
$$

onde $\{\sigma \alpha\}_{\sigma \in G}$ é uma base para o $\mathbb{K}$-espaço $\mathbb{E}$. Desde que, $A_{1}^{G} \cong \mathbb{K}[G]$, segue da proposição 132 do apêndice $\mathrm{B}$ que

$$
H^{n}\left(G, \mathbb{E}_{+}\right) \cong H^{n}\left(G, A_{1}^{G}\right) \cong H^{n}(1, A)=0, \forall n>0
$$

\section{Proposição 85.}

$$
H^{2}\left(\operatorname{Gal}\left(\mathbb{K}^{s e p} \mid \mathbb{K}\right), \dot{\mathbb{K}}^{s e p}\right) \cong \operatorname{Br}(\mathbb{K})
$$

Demonstração. Ver [BLA] cap. IV.

Seja $\mathbb{K}$ um corpo e $m>0$ um inteiro positivo.Convém considerar o grupo

$$
\mu_{m} \doteq\left\{\zeta \in \mathbb{K}^{s e p}: \zeta^{m}=1\right\}
$$

tal grupo é chamado de grupo de m-raízes da unidade em $\mathbb{K}^{\text {sep }}$.Observe que $\mu_{m}$ é um $\operatorname{Gal}\left(\mathbb{K}^{\text {sep }} \mid \mathbb{K}\right)$-módulo com ação induzida pela ação em $\mathbb{K}^{s e p}$.

A aplicação potência definida por

\footnotetext{
${ }^{19} \mathrm{O}$ teorema da base normal afirma que se $\mathbb{E} \mid \mathbb{K}$ é Galois finita, então existe $\alpha \in \mathbb{E}$ tal que o conjunto $\{\sigma \alpha: \sigma \in$ $\operatorname{Gal}(\mathbb{E} \mid \mathbb{K})\}$ é uma $\mathbb{K}$-base de $\mathbb{E}$ (consulte, por exemplo, SLan] Teor. 13.1 da secção 13).
} 


$$
\begin{aligned}
m: \dot{\mathbb{K}}^{s e p} & \longrightarrow \dot{\mathbb{K}}^{s e p} \\
x & \longmapsto x^{m}
\end{aligned}
$$

é um epimorfismo,pois o polinômio $x^{m}-a$ é sempre separável para todo $a \in \mathbb{K}^{s e p}$. Assim, teremos a sequência exata de $\operatorname{Gal}\left(\mathbb{K}^{\operatorname{sep}} \mid \mathbb{K}\right)$-módulos

$$
1 \longrightarrow \mu_{m} \longrightarrow \dot{\mathbb{K}}^{s e p} \stackrel{m}{\longrightarrow} \dot{\mathbb{K}}^{s e p} \longrightarrow 1
$$

Proposição 86. (Teoria de Kummer)Seja $\mathbb{K}$ um corpo e $m>0$ tal que char $(\mathbb{K}) \neq 0$. Existem isomorfismos canônicos

$$
H_{\text {cont }}^{n}\left(\operatorname{Gal}\left(\mathbb{K}^{s e p} \mid \mathbb{K}\right), \mu_{m}\right) \cong\left\{\begin{array}{cl}
\mu_{m}, & \text { se } n=0 \\
\dot{K}_{\mathbb{K}} \dot{\mathbb{K}}^{m}, & \text { se } n=1 \\
\left.{ }_{m} B r \mathbb{K}\right), & \text { se } n=2
\end{array}\right.
$$

Demonstração. Seja $G \doteq \operatorname{Gal}\left(\mathbb{K}^{\text {sep }} \mid \mathbb{K}\right)$. A sequência exata de G-módulos (†) origina uma sequência exata longa em grupos de cohomologia (ver 35)

$$
\begin{aligned}
1 \longrightarrow H_{\text {cont }}^{0}\left(G, \mu_{m}\right) \longrightarrow H_{\text {cont }}^{0}\left(G, \dot{\mathbb{K}}^{\text {sep }}\right) \longrightarrow H_{\text {cont }}^{0}\left(G, \dot{\mathbb{K}}^{\text {sep }}\right) \longrightarrow \\
\longrightarrow H^{1}\left(G, \mu_{m}\right) \longrightarrow H_{\text {cont }}^{1}\left(G, \dot{\mathbb{K}}^{\text {sep }}\right) \longrightarrow H_{\text {cont }}^{1}\left(G, \dot{\mathbb{K}}^{\text {sep }}\right) \longrightarrow \\
\longrightarrow H_{\text {cont }}^{2}\left(G, \mu_{m}\right) \longrightarrow H_{\text {cont }}^{2}\left(G, \dot{\mathbb{K}}^{\text {sep }}\right) \longrightarrow H_{\text {cont }}^{2}\left(G, \dot{\mathbb{K}}^{\text {sep }}\right) \longrightarrow \cdots
\end{aligned}
$$

Existem os seguintes isomorfismos canônicos

- $H^{0}\left(G, \dot{\mathbb{K}}^{\text {sep }}\right) \cong \dot{\mathbb{K}}$ (Por teoria de Galois)

- $H^{1}\left(G, \dot{K}^{s e p}\right) \cong 1$ (ver proposição 83

- $H^{2}\left(G, \dot{\mathbb{K}}^{s e p}\right) \cong B r(\mathbb{K})$ (ver proposição 85 .

Assim, a sequência exata longa anterior converte-se em

$$
\begin{aligned}
& 1 \longrightarrow \mu_{m} \longrightarrow \dot{\mathbb{K}} \stackrel{m}{\longrightarrow} \dot{\mathbb{K}} \longrightarrow \\
& \longrightarrow H_{\text {cont }}^{1}\left(G, \mu_{m}\right) \longrightarrow 1 \longrightarrow \\
& \longrightarrow H_{\text {cont }}^{2}\left(G, \mu_{m}\right) \longrightarrow \operatorname{Br}(\mathbb{K}) \stackrel{\beta}{\longrightarrow} \operatorname{Br}(\mathbb{K}) \longrightarrow \cdots
\end{aligned}
$$

Da sequência anterior obtemos isomorfismos

- $H_{\text {cont }}^{1}\left(G, \mu_{m}\right) \cong \dot{\mathbb{K}} / \dot{\mathbb{K}}^{m}$

- $H_{\text {cont }}^{2}\left(G, \mu_{m}\right) \cong \operatorname{Ker}(\beta)={ }_{m} \operatorname{Br}(\mathbb{K})$

Observação 40. Desde que $\mu_{2}$ é um G-módulo trivial podemos utilizar a identificação canônica $\mu_{2} \cong \mathbb{Z} / 2 \mathbb{Z}$. Nesse caso, $H_{\text {cont }}^{1}\left(G a l\left(\mathbb{K}^{\text {sep }} \mid \mathbb{K}\right), \mu_{2}\right)$ é essencialmente $H_{\text {cont }}^{1}\left(G a l\left(\mathbb{K}^{\text {sep }} \mid \mathbb{K}\right), \mathbb{Z} / 2 \mathbb{Z}\right)$ e $H_{\text {cont }}^{2}\left(G a l\left(\mathbb{K}^{s e p} \mid \mathbb{K}\right), \mu_{2}\right)$ é essencialmente $H_{\text {cont }}^{1}\left(G a l\left(\mathbb{K}^{\text {sep }} \mid \mathbb{K}\right), \mathbb{Z} / 2 \mathbb{Z}\right)$. Registramos para uso futuro a seguinte relação 


$$
2 \cdot H^{n}\left(\operatorname{Gal}\left(\mathbb{K}^{s e p} \mid \mathbb{K}\right), \mathbb{Z} / 2 \mathbb{Z}\right)=\{0\}
$$

Seja $\mathbb{K}$ um corpo com $C h a r(\mathbb{K})=p>0$. O endomorfismo aditivo

$$
\begin{aligned}
\wp: \mathbb{K} & \longrightarrow \mathbb{K} \\
x & \longmapsto x^{p}-x
\end{aligned}
$$

é conhecido como o mapa de Artin-Schreier. Na sequência usaremos este mapa para complementar a teoria de Kummer em característica positiva.

Proposição 87. (Teoria de Artin-Schreier)Seja $\mathbb{K}$ um corpo com char $(\mathbb{K})=p>0$. Existem isomorfismos canônicos

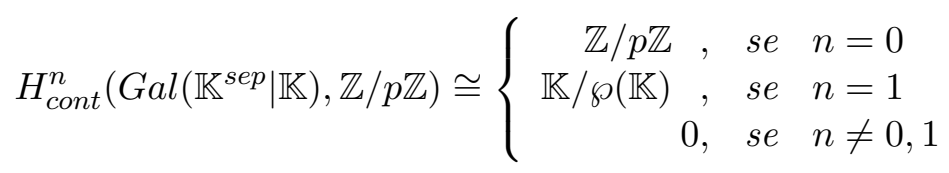

Demonstração. Seja $\wp^{*}: \mathbb{K}^{\text {sep }} \longrightarrow \mathbb{K}^{\text {sep }}$ definido por $\wp^{*}(x)=x^{p}-x, \forall x \in \mathbb{K}^{\text {sep }}$. Observe que $\operatorname{Ker}\left(\wp^{*}\right) \cong \mathbb{F}_{p} \cong \mathbb{Z} / p \mathbb{Z}$ (Isomorfismo de G-módulos). Como o polinômio $p(x)=x^{p}-x-a$ é separável para cada $a \in \mathbb{K}^{s e p}$, segue que a sequência de $\operatorname{Gal}\left(\mathbb{K}^{s e p} \mid \mathbb{K}\right)$-módulos

$$
0 \longrightarrow \mathbb{Z} / p \mathbb{Z} \longrightarrow \mathbb{K}_{+}^{\text {sep }} \stackrel{\wp^{*}}{\longrightarrow} \mathbb{K}_{+}^{\text {sep }} \longrightarrow 0
$$

é exata. Essa sequência origina uma sequência exata longa em grupos de cohomologia (ver o lema 35 )

$$
\begin{aligned}
1 \longrightarrow H_{\text {cont }}^{0}(G, \mathbb{Z} / p \mathbb{Z}) \longrightarrow H_{\text {cont }}^{0}\left(G, \mathbb{K}_{+}^{\text {sep }}\right) \longrightarrow H_{\text {cont }}^{0}\left(G, \mathbb{K}_{+}^{\text {sep }}\right) \longrightarrow \\
\longrightarrow H^{1}(G, \mathbb{Z} / p \mathbb{Z}) \longrightarrow H_{\text {cont }}^{1}\left(G, \mathbb{K}_{+}^{\text {sep }}\right) \longrightarrow H_{\text {cont }}^{1}\left(G, \mathbb{K}_{+}^{\text {sep }}\right) \longrightarrow \\
\longrightarrow H_{\text {cont }}^{2}(G, \mathbb{Z} / p \mathbb{Z}) \longrightarrow H_{\text {cont }}^{2}\left(G, \mathbb{K}_{+}^{\text {sep }}\right) \longrightarrow H_{\text {cont }}^{2}\left(G, \mathbb{K}_{+}^{\text {sep }}\right) \longrightarrow \cdots
\end{aligned}
$$

combinando $H_{\text {cont }}^{0}\left(G, \mathbb{K}_{+}^{\text {sep }}\right) \cong \mathbb{K}$ e a proposição 84 obtemos

$$
\begin{array}{r}
1 \longrightarrow \mathbb{Z} / p \mathbb{Z} \longrightarrow \mathbb{K} \longrightarrow \mathbb{K} \longrightarrow \\
\longrightarrow H^{1}(G, \mathbb{Z} / p \mathbb{Z}) \longrightarrow 1 \longrightarrow 1 \longrightarrow \\
\longrightarrow H_{\text {cont }}^{2}(G, \mathbb{Z} / p \mathbb{Z}) \longrightarrow 1 \longrightarrow 1 \longrightarrow \cdots
\end{array}
$$

o que conclui a prova.

\subsection{Classes de Stiefel-Whitney}

Nesta seção $\mathbb{K}$ é um corpo com característica $\neq 2$. Seja $G=\operatorname{Gal}\left(\mathbb{K}^{s e p} \mid \mathbb{K}\right)$. O produto cup induz uma estrutura de anel graduado em $H_{*}(\mathbb{K}) \doteq \prod_{i \in \omega} H^{i}(G, \mathbb{Z} / 2 \mathbb{Z})$. Denotaremos por

$$
H_{*}(\mathbb{K})[[x]] \doteq\left\{\sum_{i \in \omega} \alpha_{i} x^{i}: \alpha_{i} \in H^{i}(G, \mathbb{Z} / 2 \mathbb{Z})\right\}
$$


o anel de séries de potências em $x$ com coeficientes homogêneos. A aplicação

$$
\begin{aligned}
w: \mathbb{Z}[\dot{\mathbb{K}}] & \longrightarrow H_{*}(\mathbb{K})[[x]] \\
\langle\alpha\rangle & \longmapsto 1+(\alpha) x
\end{aligned}
$$

induz um homomorfismo de anéis

$$
w: W G(\mathbb{K}) \longrightarrow H_{*}(\mathbb{K})[[x]]
$$

Tal homomomofismo é conhecido como o invariante total de Stiefel-Whitney. Observe que para cada $\phi \in W G(\mathbb{K})$ o mapa $w$ admite escrita

$$
w(\phi)=\sum_{i \in \omega} w_{i}(\phi)
$$

onde cada componente

$$
w_{i}: W G(\mathbb{K}) \longrightarrow H_{*}(\mathbb{K})[[x]]
$$

de $w$ é chamado de i-ésimo invariante de Stiefel-Whitney 20 de $\phi$.

Quando $\phi=\left\langle a_{1}, \ldots, a_{n}\right\rangle$, então

$$
w_{k}\left(\left\langle a_{1}, \ldots, a_{n}\right\rangle\right)=\xi_{k}\left(\left(a_{1}\right), \ldots,\left(a_{n}\right)\right)
$$

onde $\xi_{k}$ é o $k$-ésimo polinômio simétrico elementar em n-indeterminadas, ou seja,

$$
\xi_{k}\left(x_{1}, \ldots, x_{n}\right) \doteq \sum_{i_{1}<i_{2}<\cdots<i_{k}} \prod_{j=1}^{k} x_{i_{j}}
$$

Note que $w_{1}$ é essencialmente o determinante de $\phi$; enquanto $w_{2}$ é essencialmente o invariante de Hasse, considerado no Capítulo 2. Não obstante, a família $\left(w_{i}\right)_{i \in \omega}$ não é um sistema completo de invariantes de formas quadráticas(ver [Sch2], pag. 84, teor. 12.12 e a pag. 88).

subfile

\footnotetext{
${ }^{20}$ Milnor considerou esses invariantes no contexto da K-teoria algébrica: detalharemos este ponto no Capítulo 5 desta dissertação.
} 


\section{Capítulo 4}

\section{Os funtores $\mathbf{K}_{0}, \mathbf{K}_{1}$ e $\mathbf{K}_{2}$ da $\mathbf{K}$-teoria algébrica clássica}

A K-teoria algébrica clássica é, em algum sentido, uma generalização da álgebra linear clássica, de fato, a K-teoria algébrica procura generalizar a forma como contabilizamos soluções de sistemas lineares. A K-teoria fornece um correlato matemático para a idéia de mensurar obstruções algébricas e topológicas. Por exemplo, quando tentamos reduzir uma matriz para uma matriz identidade via o método gaussiano, então K-teoria mensura a obstrução de uma tal operação.

Informalmente podemos dizer que a K-teoria algébrica clássica estuda funtores $K_{n}: \operatorname{Ring} \longrightarrow$ $A b, \forall n \in \omega$ com fonte na categoria de anéis e valores na categoria dos grupos abelianos. Neste capítulo exibiremos construções razoáveis de tais funtores em níveis 0,1,2. Além disso, mostraremos a estreita conexão entre K-teoria e (co)homologia de grupos lineares clássicos e, portanto ela pode ser vista como uma (co)homologia simplicadora para anéis.

A principal referência para este capítulo é o livro de John Milnor :Introduction to algebraic K-theory ([Miln4]).

\subsection{O Funtor $K_{0}$}

Seja $\operatorname{Ring}_{1}$ a categoria de anéis com unidade e $R \in O b\left(\operatorname{Ring}_{1}\right)$. Dizemos que $P \in R-$ Mod é projetivo quando todo diagrama de morfismos de R-módulos com linha inferior exata

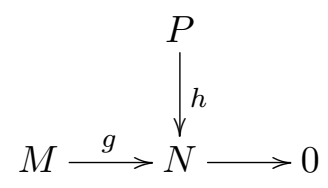

pode ser completado para um diagrama comutativo

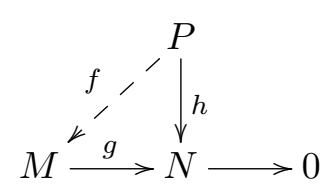

Os principais objetos da K-teoria algébrica clássica são os módulos finitamente gerados. Denotaremos por $\operatorname{Proj}_{R}$ a subcategoria de $R-M o d$, formada pelos R-módulos projetivos finitamente gerado, e por $[P]$ a classe de isomorfismos de um objeto $P \in \operatorname{Proj}_{R}$.

Lema 46. $\operatorname{Proj}_{R}$ é fechado sob a operação de soma direta $\oplus$. 
Demonstração. Se $P, P^{\prime} \in \mathcal{P}(R)$,então existem $Q, Q^{\prime}, n, n^{\prime}$ tais que $P \oplus Q \cong R^{n}$ e $P \oplus Q^{\prime} \cong R^{n^{\prime}}$.Logo,

$$
\left(P \oplus P^{\prime}\right) \oplus\left(Q \oplus Q^{\prime}\right) \cong(P \oplus Q) \oplus\left(P^{\prime} \oplus Q^{\prime}\right) \cong R^{n} \oplus R^{n^{\prime}} \cong R^{n+n^{\prime}}
$$

Fato 24. Sejam $P, P^{\prime}, P^{\prime \prime} \in \operatorname{Proj}_{R}$.Valem as seguintes propriedades

1) $\left[\left(P \oplus P^{\prime}\right) \oplus P^{\prime \prime}\right]=\left[P \oplus\left(P^{\prime} \oplus P^{\prime \prime}\right)\right]$;

2) $[P \oplus 0]=[P]$;

3) $\left[P \oplus P^{\prime}\right]=\left[P^{\prime} \oplus P\right]$.

Denotando por $\mathcal{P}_{R}$ a coleção das classes de isomorfismos de R-módulos projetivos finitamente gerados, podemos definir uma operação $\bar{\oplus}$ em $\mathcal{P}_{R}$. De acordo com o fato 24 o par $(\mathcal{P}(R), \bar{\oplus})$ é comutativo, associativo e admite elemento neutro.Não obstante, não está legitimado que o par $\left(\mathcal{P}_{R}, \bar{\oplus}\right)$ é um monóide, pois $\mathcal{P}(R)$ não é um conjunto. Sanamos essa obstrução conjuntista por considerar o seguinte fato, bastante conhecido, na teoria de R-módulos

Fato 25. As seguintes afirmações são equivalentes

1) $P$ é um R-módulo projetivo finitamente gerado.

2) $P \oplus Q \cong R^{n}$ para algum $R$-módulo $Q$.

3) Existe um isomorfismo de R-módulos

$$
P \cong\left\langle\left\{\left(e_{i 1}, \cdots, e_{i n}\right), \cdots,\left(e_{n 1}, \cdots, e_{n n}\right)\right\}\right\rangle
$$

onde $\left(e_{i j}\right) \in M_{n}(R)$ é uma matriz idempotente 1 .

O fato 25 nos permite fabricar um conjunto $\operatorname{Proj}_{R}^{*}$ tal que para cada $P \in \operatorname{Proj}_{R}$, existe um único objeto $\langle P\rangle \in \mathcal{P} \operatorname{Proj}_{R}$, onde $P \cong\langle P\rangle$. Em outros termos, a categoria $\operatorname{Proj}_{R}$ é esqueleticamente pequena. Definimos para cada $P \in \operatorname{Proj}_{R}$ a classe relativa de $\mathrm{P}$ em $\operatorname{Proj}_{R}^{*}$ por

$$
[P]_{P r o j}^{*} \doteq \operatorname{Proj}_{R}^{*} \cap[P]
$$

é fácil ver que existe uma bijeção entre $\mathcal{P}(R)$ e o conjunto $\mathcal{M}_{\operatorname{Proj}_{R}^{*}}(R)$ definido por

$$
\mathcal{M}_{\operatorname{Proj}_{R}^{*}}(R) \doteq\left\{[P]_{\operatorname{Proj}_{R}^{*}}: P \in \operatorname{Proj}_{R}\right\}
$$

A operação $+: \mathcal{M}_{\operatorname{Proj}_{R}^{*}}(R) \times \mathcal{M}_{\operatorname{Proj}_{R}^{*}}(R) \longrightarrow \mathcal{M}_{\operatorname{Proj}_{R}^{*}}(R)$ definida por

$$
[P]_{\operatorname{Proj}_{R}^{*}}+\left[P^{\prime}\right]_{\operatorname{Proj}_{R}^{*}} \doteq\left[P \oplus P^{\prime}\right]_{\operatorname{Proj}_{R}^{*}}
$$

faz de $\mathcal{M}_{\operatorname{Proj}_{R}^{*}}(R)$ um monóide comutativo. Além disso, se $\operatorname{Proj}_{R}^{\prime *}$ for um outro conjunto de representantes dos objetos de $\operatorname{Proj}_{R}$, a menos de isomorfismos, então existe um isomorfismo de monóides

$$
\begin{aligned}
\eta: \mathcal{M}_{\text {Proj }_{R}^{*}}(R) & \longrightarrow \mathcal{M}_{\text {Proj }_{R}{ }_{R}}(R) \\
{[P]_{\text {Proj }_{R}^{*}} } & \longmapsto[P]_{\text {Proj }_{R}^{\prime *}}
\end{aligned}
$$

Definição 74. Definimos o primeiro grupo da K-teoria algébrica clássica de um anel $R$ como sendo $K_{0}(R)=\operatorname{Groth}\left(\mathcal{M}_{\operatorname{Proj}_{R}^{*}}(R)\right)$ (cf. Apêndice 1).

\footnotetext{
${ }^{1}$ Consulte NJac1
} 
Observe que $K_{0}$ é um funtor covariante da categoria de anéis para a categoria de grupos abelianos. Em outras palavras, se $\varphi: R \longrightarrow R^{\prime}$ for um homomorfismo de anéis, então existe um homomorfismo de grupos abelianos

$$
K_{0}(\varphi): K_{0}(R) \longrightarrow K_{0}\left(R^{\prime}\right)
$$

satisfazendo

$$
K_{0}(\varphi \circ \psi)=K_{0}(\varphi) \circ K_{0}(\psi) \quad \text { e } \quad K_{0}\left(i d_{R}\right)=i d_{K_{0}(R)}
$$

Para ver isso, note que $\varphi$ dá a $R^{\prime}$ uma estrutura de R-módulo. Se $\mathrm{P}$ é um R-módulo finitamente gerado e projetivo, então $R^{\prime} \otimes_{\varphi} P$ é finitamente gerado e projetivo sobre $R^{\prime}$, pois

$$
\left(R^{\prime} \otimes_{\varphi} P\right) \oplus\left(R^{\prime} \otimes_{\varphi} Q\right) \cong R^{\prime} \otimes_{\varphi}(P \oplus Q) \cong\left(R^{\prime} \otimes_{\varphi} R^{n}\right) \cong R^{\prime n}
$$

Assim, $\varphi$ induz um homomorfismo

$$
\begin{aligned}
\mathcal{M}_{\operatorname{Proj}_{R}^{*}}(\varphi): \mathcal{M}_{\operatorname{Proj}_{R}^{*}}(R) & \longrightarrow \mathcal{M}_{\operatorname{Proj}_{R}^{*}}\left(R^{\prime}\right) \\
{[P] } & \longmapsto\left[R^{\prime} \otimes_{\varphi} P\right]
\end{aligned}
$$

Além disso, se $i d: R \longrightarrow R$ for o homomorfismo identidade e $\psi: R^{\prime} \longrightarrow R^{\prime \prime}$ for um homomorfismo de anéis, então

$$
\mathcal{M}_{\operatorname{Proj}_{R}^{*}}\left(i d_{R}\right)=i d_{\mathcal{M}_{P j_{R}^{*}}(R)} \quad \text { e } \mathcal{M}_{\operatorname{Proj}_{R}^{*}}(\psi \circ \varphi)=\mathcal{M}_{\operatorname{Proj}_{R}^{*}}(\psi) \circ \mathcal{M}_{\operatorname{Proj}_{R}^{*}}(\varphi)
$$

em outras palavras, $\mathcal{M}_{\operatorname{Proj}_{R}^{*}}(\mathrm{)}): \operatorname{Ring} \longrightarrow$ Mon é um funtor covariante. Usando a funtorialidade da construção de Grothendieck é fácil ver que

$$
K_{0}()=\text { Groth } \circ \mathcal{M}_{\operatorname{Proj}_{R}^{*}}(\text { ) }
$$

O funtor $K_{0}$ satisfaz as seguintes propriedades(para mais detalhes e, também as demonstrações dessas propriedades, consulte [Ros], capítulo 1, seção 2)

\section{Proposição 88.}

1) Para todo anel $R$ e todo inteiro positivo n, existe um isomorfismo natural

$$
K_{0}(R) \cong K_{0}\left(M_{n}(R)\right)
$$

2) Existe um isomorfismo natural

$$
K_{0}\left(R \times R^{\prime}\right) \cong K_{0}(R) \oplus K_{0}\left(R^{\prime}\right)
$$

para todos anéis $R$ e $R^{\prime}$.

3) Todo sistema dirigido de anéis

$$
\left\langle\left(R_{\lambda}\right)_{\lambda \in \Lambda},\left(\phi_{\lambda \mu}: R_{\lambda} \longrightarrow R_{\mu}\right)_{\lambda \leq \mu}\right\rangle
$$

induz um sistema dirigido

$$
\left\langle\left(K_{0}\left(R_{\lambda}\right)\right)_{\lambda \in \Lambda},\left(\left(K_{0}\left(R_{\lambda}\right) \longrightarrow K_{0}\left(R_{\mu}\right)\right)_{\lambda \leq \mu}\right\rangle\right.
$$

Além disso, 


$$
\underset{\lambda \in\left(\lim _{(\Lambda, \leq)}\right.}{\longrightarrow} K_{0}\left(R_{\lambda}\right) \stackrel{\cong}{\longrightarrow} K_{0}\left(\underset{\lambda \in(\Lambda, \leq)}{\lim _{\lambda}} R_{\lambda}\right)
$$

\section{Exemplo 8.}

1) Se $R$ é um corpo ou um anel de divisão, então $K_{0}(R) \cong \mathbb{Z}$.

2) Mais geralmente, se $R$ é um anel local comutativo, então $K_{0}(R) \cong \mathbb{Z}$.

3) Se $R$ é um domínio de ideais principais comutativo, então $K_{0}(R) \cong \mathbb{Z}$.

Exemplo 9. Dizemos que um R-módulo é estavelmente livre de rank $n-m$, quando

$$
P \oplus R^{m} \cong R^{n}
$$

Se $R$ satisfaz a a propriedade da base invariante ${ }^{2}$

\section{Exemplo 10.}

1) Se $R$ é um anel tal que

$$
R \cong M_{n_{1}}\left(D_{1}\right) \times \cdots \times M_{n_{r}}\left(D_{r}\right)
$$

onde cada $D_{i}$ é um anel de divisão, então

$$
K_{0}(R) \cong \prod K_{0}\left(M_{n_{i}}\left(D_{i}\right)\right) \cong \mathbb{Z}^{r}
$$

2) Seja $n \neq 1$ um inteiro positivo. Por álgebra elementar temos um isomorfismo de anéis

$$
\mathbb{Z} / n \mathbb{Z} \cong \prod_{i=1}^{r} \mathbb{Z} / p_{i}^{n_{i}} \mathbb{Z}
$$

onde $\left\{p_{1}^{n_{1}}, \cdots, p_{r}^{n_{r}}\right\}$ é uma decomposição em primos para $n$. Desde que, cada $\mathbb{Z} / p_{i}^{n_{i}} \mathbb{Z}$ é um anel comutativo local (de fato, $\left\langle p_{i}\right\rangle /\left\langle p_{i}^{n_{i}}\right\rangle$ é seu único ideal maximal), resulta da proposição 88 (2) e do exemplo 8 (2) que

$$
K_{0}(\mathbb{Z} / n \mathbb{Z}) \cong K_{0}\left(\prod_{i=1}^{r} \mathbb{Z} / p_{i}^{n_{i}} \mathbb{Z}\right) \cong \prod_{i=1}^{r} \mathbb{Z}=\mathbb{Z}^{r}
$$

Exemplo 11. A idéia de medir a obstrução para que um dominio de Dedekind seja um domínio de ideais principais tem o seu correlato matemático em $K_{0}(R)$. De forma precisa, Um domínido de Dedekind é um domínio de ideais principais se, e somente se existe um isomorfismo de grupos $K_{0}(R) \cong \mathbb{Z}$.

\subsection{O Funtor $K_{1}$}

Daremos nesta seção uma construção para o segundo funtor da K-teoria algébrica. A posteriori mostrou-se que o tal era um excelente candidato para um possível segundo funtor que sai da categoria de anéis e chega na categoria dos grupos abelianos.

Existe uma sequência de funtores matriciais $\left(M_{n}: \operatorname{Ring} \longrightarrow \text { Ring }\right)_{n \in \omega}$ de ordem $n$, onde $M_{n}(R)$ é o anel de matrizes sobre $\mathrm{R}$, e quando $\varphi: R \longrightarrow R^{\prime}$ for um morfismo de anéis ter-se-á

\footnotetext{
${ }^{2}$ Dizemos que um anel $\mathrm{R}$ satisfaz propriedade da base invariante, quando $R^{m}$ e $R^{n}$ não são isomorfos para $m \neq n$. Neste caso, o rank do R-módulo livre M é invariante, independente da escolha da base de M.
} 
$M_{n}(\varphi): M_{n}(R) \longrightarrow M_{n}\left(R^{\prime}\right)$ definido por $M_{n}(\varphi)\left(a_{i j}\right)=\left(\varphi\left(a_{i j}\right)\right)$. Além disso, existe o funtor das unidades $U N:$ Ring $\longrightarrow$ Grp; que trabalha em objetos como $U N(R)=\dot{R}$, e em morfismos como $U N(\varphi: R \longrightarrow S): \dot{R} \longrightarrow \dot{S}, u \mapsto U N(\varphi)(u)=\varphi(u)$.

Definição 75. Definimos o funtor linear de ordem $n$ através da composição

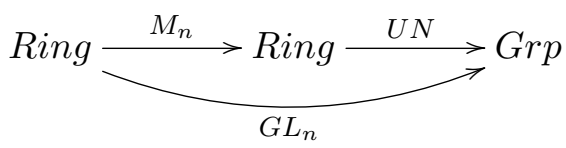

Fato 26. A família $\left(\operatorname{det}_{n}(R): G L_{n}(R) \longrightarrow U N(R)\right)_{n \in \omega}$ é uma transformação natural entre os funtores $M_{n}$ e UN. Em outras palavras, para cada morfismo de anéis $\varphi: R \longrightarrow R^{\prime}$ existe um diagrama comutativo

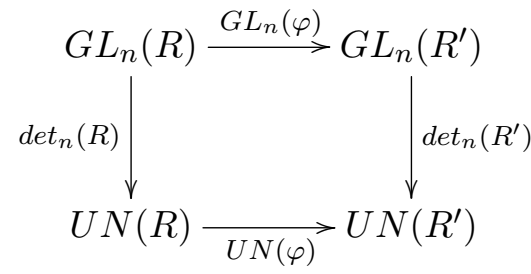

Associamos de forma óbvia uma categoria para a estrutura bem ordenada $(\omega, \leq)$ e a denotamos por $\operatorname{Poset}(\omega)$. Claramente temos um funtor $G L_{-}(R): \operatorname{Poset}(\omega) \longrightarrow G r p$ definido por $G L_{-}(R)(m)=G L_{m}(R)$ nos objetos e por $\delta_{m, n} \doteq G L_{-}(R)(m \longrightarrow n): G L_{m}(R) \longrightarrow G L_{n}(R), A \mapsto$ $\Delta\left(A, I_{n-m}\right)$, onde $\Delta\left(A, I_{n-m}\right)$ é matriz diagonal em A e na matriz identidade $I_{n-m}$ de ordem $n-m$. Note que para cada morfismo de anéis $\varphi: R \longrightarrow R^{\prime}$, existe um diagrama comutativo

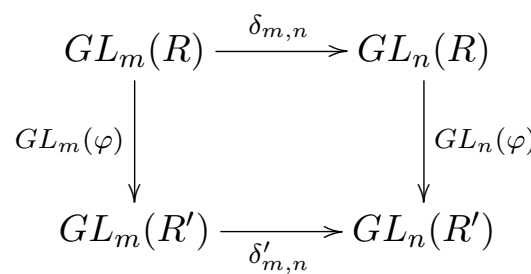

Proposição 89. A família $\left(\delta_{m, n}\right)$ constitui um sistema inverso, ou seja, o seguinte diagrama comuta

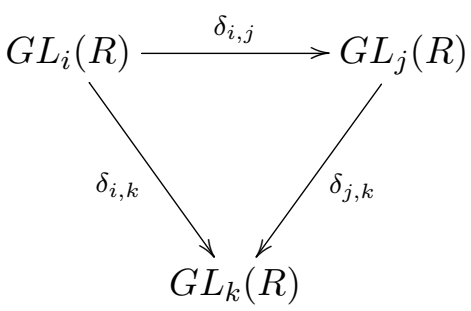

Para todo $i \leq j \leq k$ e $\delta_{l, l}=I d, \forall l \in \omega$.

Seja $\lambda_{i}: G L_{i}(R) \longrightarrow \bigcup_{n \in \omega} G L_{n}(R)$ a inclusão canônica para cada $i \in \omega$. Observe que o par $\left(\bigcup_{n \in \omega} G L_{n}(R),\left(\lambda_{n}\right)_{n \in \omega}\right)$ é tal que $\lambda_{i}=\lambda_{j} \circ \delta_{i, j}$ e a para cada par $\left(G,\left(\phi_{i}\right)_{i \in \omega}\right)$, onde $\mathrm{G}$ é um grupo 
e $\phi_{i}=\phi_{j} \circ \delta_{i, j}$, existe um único morfismo de grupos $u$ que faz o próximo diagrama comutar

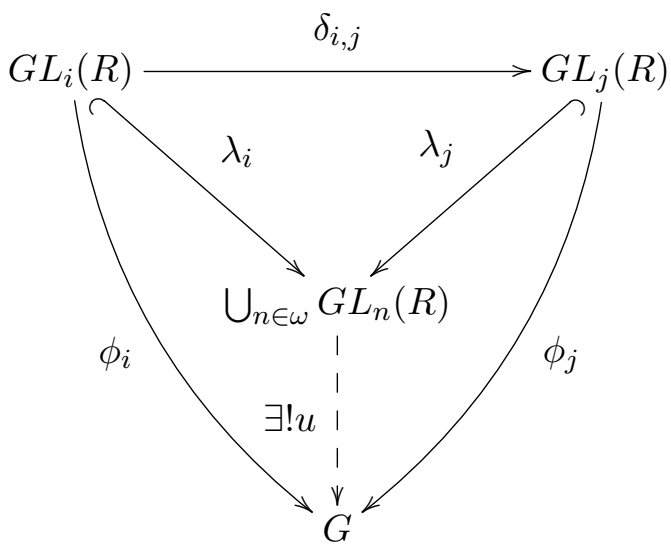

Dessa forma, $\left(\bigcup_{n \in \omega} G L_{n}(R),\left(\lambda_{n}\right)_{n \in \omega}\right)$ é um (co)limite sobre o sistema dirigido $\left(\delta_{m, n}\right)$.

Definição 76. Definimos o grupo linear geral estável sobre $R$ como sendo o limite direto $G L(R) \doteq$ $\lim _{\longrightarrow} G L_{n}(R)$.

Observação 41. Seja $\varphi: R \longrightarrow R^{\prime}$ um homomorfismo de anéis. A universalidade de $G L(R)$ permite construir o seguinte diagrama comutativo de grupos

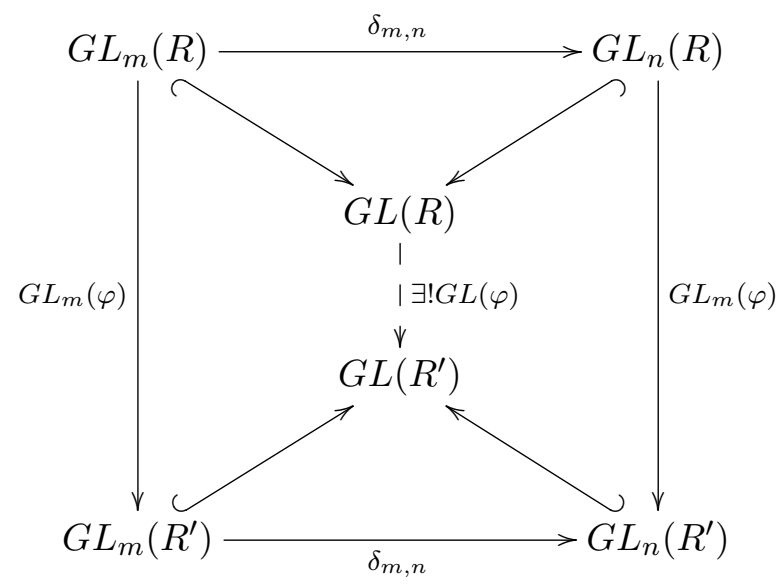

Portanto, temos um funtor $\boldsymbol{G L}:$ Ring $\longrightarrow$ Grp. Este funtor é chamado de funtor linear geral estável.

Proposição 90. Seja $R$ um anel comutativo. Existe um único homomorfismo de grupos

$$
\operatorname{det}: G L(R) \longrightarrow \dot{R}
$$

compatível com a familia de homomorfismos $\left(\operatorname{det}_{n}(R)\right)_{n \in \omega}$.

Demonstração. Basta observar que $\operatorname{det}_{n}(R) \circ \delta_{m, n}=\operatorname{det}_{m}(R)$ e usar a propriedade universal que caracteriza $G L(R)$.

Para cada $n \in \omega$ e positivo considere $n \bullet\{1, \cdots, n\}$. Seja

$$
\left\{\xi_{i j}:(i, j) \in n^{\bullet} \times n^{\bullet}\right\}
$$


a base canônica de $M_{n}(R)$. Lembre-se que cada função

$$
\xi_{i j}: n^{\bullet} \times n^{\bullet} \longrightarrow R
$$

é tal que $\xi_{i j}(k, l)=0, \forall(k, l) \neq(i, j)$ e $\xi_{i j}(i, j)=1$

Chamaremos uma matriz $\left(a_{i j}\right)_{n \times n} \in G L_{n}(R)$ de matriz elementar em $R$, quando $a_{i i}=1 \mathrm{e}$ $\left\{a_{i j}: i \neq j ; a_{i j} \neq 0\right\}$ for um conjunto unitário. Usaremos $e_{i j}^{\lambda}$ para simbolizar a matriz elementar com $\lambda \neq 0$ na posição $(i, j)$ com $i \neq j$. O subgrupo de $G L_{n}(R)$ gerado pelas matrizes elementares será indicado por $E_{n}(R)$. Definimos $E(R)$ como sendo $\underset{\longrightarrow}{\longrightarrow} E_{n}(R)$.

Lema 47. Para inteiros positivos $i, j, k, l$ valem

1) $\xi_{i j} \xi_{k l}=\delta_{j k} \xi_{i l}$

2) $e_{i j}^{\lambda}=I_{n}+\lambda \xi_{i j}$.

3) $e_{i j}^{\lambda} \cdot e_{i j}^{\mu}=e_{i j}^{\lambda+\mu}$

4) $e_{i j}^{\lambda}+e_{i j}^{\mu}=I_{n}+e_{i j}^{\lambda} e_{i j}^{\mu}$

5) $\left(e_{i j}^{\lambda}\right)^{-1}=e_{i j}^{-\lambda}$

6) $e_{i j}^{\lambda} e_{k l}^{\mu} e_{i j}^{-\lambda} e_{k l}^{-\mu}=I_{d}, \forall j \neq k, \forall i \neq l$

7) $e_{i j}^{\lambda} e_{k l}^{\mu} e_{i j}^{-\lambda} e_{k l}^{-\mu}=e_{i j}^{\lambda \mu}, \forall j=k, \forall i \neq l$

8) $e_{i j}^{\lambda} e_{k l}^{\mu} e_{i j}^{-\lambda} e_{k l}^{-\mu}=e_{i j}^{-\lambda \mu}, \forall j \neq k, \forall i=l$

Demonstração.

1)Seja $\xi_{i j}=\left(a_{r s}\right)$ e $\xi_{k l}=\left(b_{p q}\right)$. Um elemento genérico de $\xi_{r s} \cdot \xi_{p q}$ é

$$
a_{r 1} b_{1 q}+a_{r 2} b_{2 q}+\ldots+a_{r n} b_{n q}
$$

Desde que, $j \neq k$, então $a_{i j} \cdot b_{k l} \neq a_{r s} \cdot b_{s q}$. Nesse caso, $\xi_{i j} \cdot \xi_{k l}=0$. No caso, $j \neq k$ basta usar $(*)$ para ver que $\xi_{i l} \cdot \xi_{k l}=\xi_{i l}$.

2) Seja $I_{n}=\left(a_{r s}\right)$ e $\xi_{i, j}=\left(b_{p q}\right)$ se $r=s$, então $a_{r s}+\lambda b_{r s}=1++\lambda 0=1$. Caso contrário, $\left(a_{r s}+\lambda b_{r s}\right)=0+\lambda b_{r s}=\lambda b_{r s}$. Se $(r, s) \neq(i, j)$, então $\lambda b_{r s}=0$. Caso contrário, $\lambda b_{r s}=\lambda b_{i j}=\lambda$.

$3)$

$$
\begin{aligned}
e_{i j}^{\lambda} \cdot e_{i j}^{\mu} & =I_{n}+(\lambda+\mu) \xi_{i j}+\lambda \mu \xi_{i j}^{2} \\
& =I_{n}+(\lambda+\mu) \xi_{i j} \\
& =e_{i j}^{\lambda+\mu}
\end{aligned}
$$

4)

$$
\begin{aligned}
I_{n}+e_{i j}^{\lambda} e_{i j}^{\mu} & =I_{n}+e_{i j}^{(\lambda+\mu)} \\
& =I_{n}+I_{n}+(\lambda+\mu) \xi_{i j} \\
& =\left(I_{n}+\lambda \xi_{i j}\right)+\left(I_{n}+\mu \xi_{i j}\right) \\
& =e_{i j}^{\lambda}+e_{i j}^{\mu}
\end{aligned}
$$


5) $e_{i j}^{\lambda} \cdot e_{i j}^{-\lambda}=e_{i j}^{\lambda-\lambda}=e_{i j}^{0}=I_{n}$

(6),(7) e (8) Inicialmente observe que

- $\left(I_{n}+\lambda \xi_{i j}\right) \cdot\left(I_{n}+\mu \xi_{k l}\right)=I_{n}+\mu \xi_{k l}+\lambda \xi_{i j}+\lambda \mu \xi_{i j} \xi_{k l}$

- $\left(I_{n}-\lambda \xi_{i j}\right) \cdot\left(I_{n}-\mu \xi_{k l}\right)=I_{n}-\mu \xi_{k l}-\lambda \xi_{i j}+\lambda \mu \xi_{i j} \xi_{k l}$

$$
\begin{aligned}
e_{i j}^{\lambda} e_{k l}^{\mu} e_{i j}^{-\lambda} e_{i j}^{-\mu} & =\left(I_{n}+\mu \xi_{k l}+\lambda \xi_{i j}+\lambda \mu \xi_{i j} \xi_{k l}\right)\left(I_{n}-\mu \xi_{k l}-\lambda \xi_{i j}+\lambda \mu \xi_{i j} \xi_{k l}\right) \\
& =I_{n}-\mu \xi_{k l}-\lambda \xi_{i j}+\lambda \mu \xi_{i j} \xi_{k l} \\
& +\mu \xi_{k l}-\mu^{2} \xi_{k l}^{2}-\lambda \mu \xi_{k l} \xi_{i j}+\lambda \mu^{2} \xi_{k l} \xi_{i j} \xi_{k l} \\
& +\lambda \xi_{i j}-\lambda \mu \xi_{i j} \xi_{k l}-\lambda^{2} \xi_{i j}^{2}+\lambda^{2} \mu \xi_{i j}^{2} \xi_{k l} \\
& +\lambda \mu \xi_{i j} \xi_{k l}-\lambda \mu^{2} \xi_{i j} \xi_{k l}^{2}-\lambda^{2} \mu \xi_{i j} \xi_{k l} \xi_{i j}+\lambda^{2} \mu^{2}\left(\xi_{i j} \xi_{k l}\right)^{2}
\end{aligned}
$$

Usando (1) obtemos

$$
\xi_{i j}^{2}=\xi_{k l}^{2}=0
$$

e cancelando $-\mu \xi_{k l}$ com $\mu \xi_{k l},-\lambda \xi_{i j}$ com $\lambda \xi_{i j}$ e $-\lambda \mu \xi_{i j} \xi_{k l}$ com $\lambda \mu \xi_{i j} \xi_{k l}$, teremos

$$
\begin{aligned}
e_{i j}^{\lambda} e_{k l}^{\mu} e_{i j}^{-\lambda} e_{k l}^{-\mu} & =I_{n}+\lambda \mu \xi_{i j} \xi_{k l}-\lambda \mu \xi_{k l} \xi_{i j}+\lambda \mu^{2} \xi_{k l} \xi_{i j} \xi_{k l} \\
& -\lambda^{2} \mu \xi_{i j} \xi_{k l} \xi_{i j}+\lambda^{2} \mu^{2}\left(\xi_{i j} \xi_{k l}\right)^{2}
\end{aligned}
$$

Desta última equação, derivamos facilmente, (6), (7) e (8).

Lembremos que o comutador de elementos $a$ e $b$ de um grupo G é definido por $[a, b] \doteq a b a^{-1} b^{-1}$. Denotamos por $[G, G]$ o subgrupo (normal) de $G$ formado pelos símbolos $[a, b]$. Existe uma construção canônica que associa a cada grupo $\mathrm{G}$ o melhor subgrupo abeliano possível. Explicitamente, a cada grupo $G \in G r p$ associamos o grupo abeliano $G^{a b} \doteq G /[G, G]$. Além disso, se $h: G \longrightarrow G^{\prime}$ for um homomorfismo de grupos, então existe um único homomorfismo $\varphi^{a b}: G^{a b} \longrightarrow G^{a b}$ fazendo o seguinte diagrama comutativo

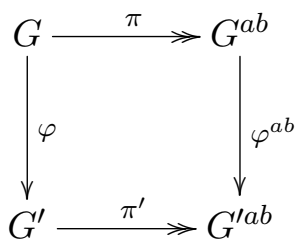

Assim, temos um funtor ( $)^{a b}: G r p \longrightarrow A b$ adjunto à esquerda para o funtor esquecimento $\mathcal{U}: A b \longrightarrow G r p$.

\section{Observação 42 .}


(1) De acordo com os ítens (6), (7) e (8) do lema 47 temos

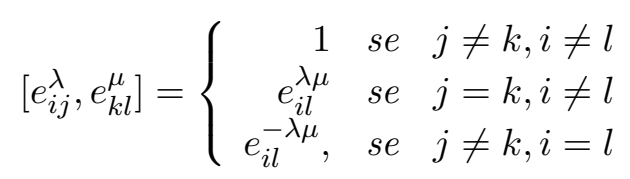

Vimos na demonstração do lema 47 que vale a igualdade

$$
\begin{aligned}
e_{i j}^{\lambda} e_{k l}^{\mu} e_{i j}^{-\lambda} e_{i j}^{-\mu} & =I_{n}+\lambda \mu \xi_{i j} \xi_{k l}-\lambda \mu \xi_{k l} \xi_{i j}+\lambda \mu^{2} \xi_{k l} \xi_{i j} \xi_{k l} \\
& -\lambda^{2} \mu \xi_{i j} \xi_{k l} \xi_{i j}+\lambda^{2} \mu^{2}\left(\xi_{i j} \xi_{k l}\right)^{2}
\end{aligned}
$$

Em particular,

$$
\left[e_{i j}^{\lambda}, e_{j i}^{\mu}\right]=1+\lambda \mu \xi_{i i}-\lambda \mu \xi_{j j}+\lambda \mu^{2} \xi_{j i}-\lambda^{2} \mu \xi_{i j}
$$

Ou seja, $\left[e_{i j}^{\lambda}, e_{j i}^{\mu}\right]$ não é expressável em termos de $e_{i j}^{\lambda}$ e $e_{j i}^{\mu}$.

(2) Vale a seguinte igualdade

$$
[E(R), E(R)]=E(R)
$$

De fato, temos

$$
e_{i l}^{\lambda}=\left[e_{i j}^{\lambda}, e_{j l}^{1}\right]
$$

para $i, j, l$ distintos.

(3) Cada inclusão

$$
\begin{gathered}
E_{n}(R) \longleftrightarrow E_{n+1}(R) \\
e_{i j}^{\lambda} \longmapsto e_{i j}^{\lambda}
\end{gathered}
$$

Permite obter matrizes $e_{i j}^{\lambda} \in E(R)$ como a imagem comum de todas as matrizes $e_{i j}^{\lambda}$ (Cada $e_{i j}^{\lambda}$ em algum $E_{n}(R)$ ) para $i, j \leq n$. É possivel mostrar que $E(R)$ é o subgrupo gerado de $G L(R)$ gerado pelos elementos $e_{i j}^{\lambda}$ satisfazendo as seguintes identidades

$\left.\boldsymbol{E}_{1}\right) e_{i j}^{\lambda} e_{i j}^{\mu}=e_{i j}^{\lambda+\mu}, \forall \lambda, \mu \in R$

$\left.\boldsymbol{E}_{2}\right) e_{i j}^{\lambda} e_{k l}^{\mu}=1$, se $j \neq k, i \neq l$.

$\left.\boldsymbol{E}_{3}\right) e_{i j}^{\lambda} e_{k l}^{\mu}=e_{i k}^{\lambda \mu}$, se $i \neq k, \forall \lambda, \mu \in R$

Lema 48. Se A, B são matrizes de ordem n, então

(1) $\left(\begin{array}{cc}I_{n} & A \\ 0 & I_{n}\end{array}\right),\left(\begin{array}{cc}I_{n} & 0 \\ A & I_{n}\end{array}\right) \in E_{2 n}(R)$

(2) $\left(\begin{array}{cc}B & 0 \\ 0 & B^{-1}\end{array}\right) \in E_{2 n}(R)$

Demonstração.

(1) Para $(i, j) \in\{(i, n+1): 1 \leq i \leq n-1\}$ defina

$$
P_{i j}=\left(\prod_{l=0}^{n-i} e_{i+l j+l}^{a_{i+l j+l}}\right)
$$


e para $(i, j) \in\{(1, j): n+1<j \leq 2 n\}$ defina

$$
P_{1 j} \doteq\left(\prod_{l=0}^{2 n-j} e_{1+l j+l}^{a_{1+l j+1}}\right)
$$

agora observe que a matriz

$$
\left(\begin{array}{cc}
I_{n} & A \\
0 & I_{n}
\end{array}\right)
$$

pode ser escrita como

$$
\left(\prod_{i=1}^{n-1} P_{i, n+1}\right)\left(\prod_{j=n+2}^{2 n} P_{1, j}\right)\left(e_{1,2 n}^{a_{1,2 n}} e_{n n+1}^{a_{n n+1}}\right)
$$

Uma decomposição semelhante existe para a matriz

$$
\left(\begin{array}{cc}
I_{n} & 0 \\
A & I_{n}
\end{array}\right)
$$

(2) Pelo item anterior, o resultado é uma consequência das identidades

$$
\begin{gathered}
\left(\begin{array}{cc}
B & 0 \\
0 & B^{-1}
\end{array}\right)=\left(\begin{array}{cc}
I_{n} & B \\
0 & I_{n}
\end{array}\right)\left(\begin{array}{cc}
I_{n} & 0 \\
-B^{-1} & I_{n}
\end{array}\right)\left(\begin{array}{cc}
I_{n} & B \\
0 & I_{n}
\end{array}\right)\left(\begin{array}{cc}
0 & -I_{n} \\
I_{n} & 0
\end{array}\right) \\
\left(\begin{array}{cc}
0 & -I_{n} \\
I_{n} & 0
\end{array}\right)=\left(\begin{array}{cc}
I_{n} & -I_{n} \\
0 & I_{n}
\end{array}\right)\left(\begin{array}{cc}
I_{n} & 0 \\
I_{n} & I_{n}
\end{array}\right)\left(\begin{array}{cc}
I_{n} & -I_{n} \\
0 & I_{n}
\end{array}\right)
\end{gathered}
$$

Lema 49. (Whitehead)

$$
E(R)=[G L(R), G L(R)]
$$

Demonstração. Usando a observação 42 (ii), temos

$$
E(R) \subseteq G L(R) \Longrightarrow E(R)=[E(R), E(R)] \subseteq[G L(R), G L(R)]
$$

Por fim, seja $A, B \in G L_{n}(R)$. Mergulhando $G L_{n}(R)$ em $G L_{2 n}(R)$ ter-se-á

$$
\left(\begin{array}{cc}
A B A^{-1} B^{-1} & 0 \\
0 & I_{n}
\end{array}\right)=\left(\begin{array}{cc}
A B & 0 \\
0 & B^{-1} A^{-1}
\end{array}\right)\left(\begin{array}{cc}
A^{-1} & 0 \\
0 & A
\end{array}\right)\left(\begin{array}{cc}
B^{-1} & 0 \\
0 & B
\end{array}\right)
$$

agora pelo lema 48 (1) cada menbro do lado direito da equação anterior está em $E_{2 n}$ e, portanto

$$
[G L(R), G L(R)] \subseteq E(R)
$$

Definição 77. Definimos o segundo funtor da K-teoria algébrica através da composição de funtores

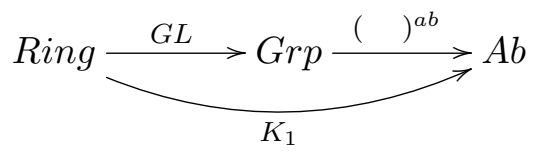


Observe que $K_{1}$ trabalha em objetos como

$$
K_{1}(R)=\frac{G L(R)}{[G L(R), G L(R)]}=\frac{G L(R)}{E(R)}
$$

O funtor $K_{1}$ possui propriedades semelhantes àquelas satisfeitas pelo funtor $K_{0}$ na proposição 88

Proposição 91. Sejam $R$ e $R^{\prime}$ anéis, então valem:

1) $K_{1}\left(R^{o p}\right)=K_{1}(R)($ Simetria);

2) $K_{1}(R)=K_{1}\left(M_{n}(R)\right)$ (Invariância de Morita);

3) $K_{1}\left(R \times R^{\prime}\right)=K_{1}(R) \times K_{1}\left(R^{\prime}\right)($ Preservação de produto);

4) Todo sistema dirigido de anéis $\left(\varphi_{i j}: R_{i} \longrightarrow R_{j}\right)$ induz um sistema dirigido $\left(K_{1}\left(\varphi_{i j}\right): K_{1}\left(R_{i}\right) \longrightarrow\right.$ $\left.K_{1}\left(R_{j}\right)\right)$ na categoria $A b$.

5) O funtor $K_{1}$ preserva limites diretos de sistemas dirigidos, ou seja

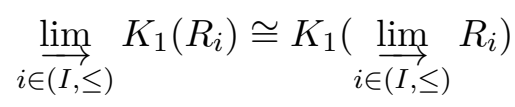

Seja $R$ um anel comutativo com unidade e $S L_{n}(R) \doteq\left\{A \in M_{n \times n}(R): \operatorname{det}_{n}(A)=1\right\}$. A sequência exata cindida

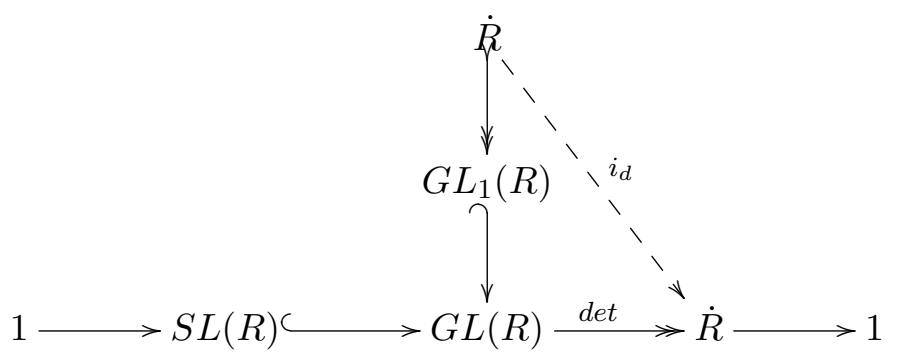

Permite construir um diagrama comutativo

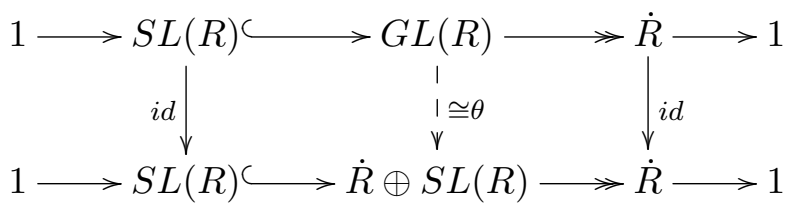

onde $S L(R)=\lim _{i \in \omega} S L_{i}(R)$. De acordo com o lema dos cinco ${ }^{3}$ o homomorfismo $\theta$ é um isomorfismo.

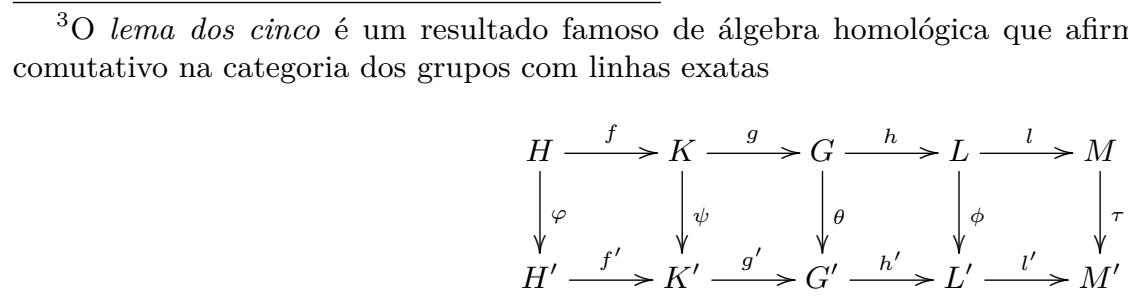

Se $\varphi, \psi, \phi, \tau$ são isomorfismos, então $\theta$ também o será. 
Como $E(R) \triangleleft S L(R)$ o quociente $S K_{1}(R) \doteq S L(R) / E(R)$ é um grupo .Considere o epimorfismo

$$
\Psi: G L(R) \longrightarrow \dot{R} \oplus S K_{1}(R)
$$

definido pela composição

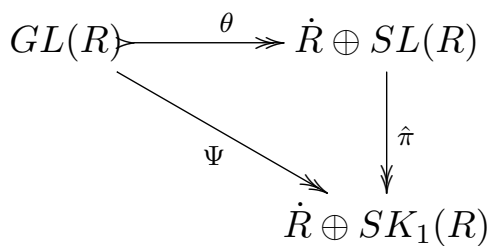

onde $\hat{\pi}(r, X)=(r,[X])$. Desde que, $\operatorname{Ker}(\Psi)=E(R)=[G L(R), G L(R)]$ existe um único isomorfismo

$$
\Phi: K_{1}(R) \longrightarrow \dot{R} \oplus S K_{1}(R)
$$

que torna o seguinte diagrama comutativo

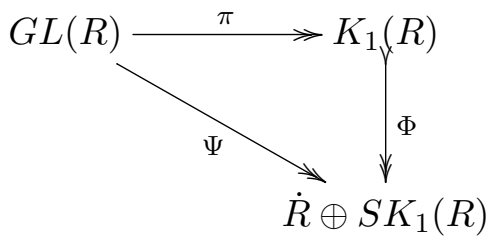

Assim, temos a seguinte

Proposição 92. Para todo anel comutativo $R$, temos

$$
K_{1}(R)=\dot{R} \oplus S K_{1}(R)
$$

A proposição anterior mostra-nos que conhecido $\dot{R}$, a informação de $K_{1}(R)$ está completamente codificada em $S K_{1}(R)$.

Proposição 93. Se $\mathbb{K}$ é um corpo, então existe um isomorfismo

$$
K_{1}(\mathbb{K}) \cong \dot{\mathbb{K}}
$$

Demonstração. Seja $A \in G L_{n}(\mathbb{K})$. Como $\operatorname{det}(A) \neq 0$, existe $a_{i 1} \neq 0$. Podemos supor que $i=1$, pois caso contrário tomaríamos $\tilde{A} \doteq e_{1 i}^{1} e_{i 1}^{-1} e_{1 i}^{1} A$. Aplicando o método de eliminação gaussiana em A, obtemos

$$
\prod_{i=2}^{n} e_{i 1}^{\varepsilon_{i 1}} A=\left(\begin{array}{cc}
a_{11} & * \\
0 & A^{\prime}
\end{array}\right)
$$

onde $\varepsilon_{i 1} \doteq-a_{i 1} a_{11}^{-1}$ e $A^{\prime} \in G L_{n-1}(\mathbb{K})$. Repetindo o procedimento, chegaremos na matriz

$$
A=\left(\begin{array}{ccc}
\alpha_{11} & * & * \\
0 & \alpha_{22} & * \\
0 & 0 & A^{\prime \prime}
\end{array}\right)
$$

com $A^{\prime \prime} \in G L_{n-2}(\mathbb{K})$. Por indução, vamos obter uma matriz triangular superior 


$$
S=\left(\begin{array}{cccc}
\alpha_{11} & * & \cdots & * \\
0 & \alpha_{12} & \cdots & * \\
\vdots & \vdots & \ddots & \vdots \\
0 & 0 & \cdots & \alpha_{n n}
\end{array}\right)
$$

Aplicando o método gaussiano em $\mathrm{S}$, obtemos uma matriz diagonal

$$
D=\left\langle d_{11}, \cdots, d_{n n}\right\rangle
$$

Sabemos pelo lema 48 (2) que

$$
\left\langle 1, \cdots, 1, a, a^{-1}, 1, \cdots, 1\right\rangle \in E(\mathbb{K})
$$

multiplicando D por matrizes do tipo anterior conseguiremos uma matriz diagonal

$$
\langle\operatorname{det}(D), 1, \ldots, 1\rangle
$$

Finalmente, se $\operatorname{det}(A)=1$ (i.e, $A \in S L_{n}(\mathbb{K})$ ), concluiremos imediatamente que $A \in E(\mathbb{K})$. Logo, $S K_{1}(\mathbb{K})=\{1\}$ e $K_{1}(\mathbb{K}) \cong \dot{K} \oplus\{1\} \cong \dot{K}$.

Proposição 94. Se $R$ é um anel de divisão, então a inclusão natural

$$
\dot{R} \cong G L_{1}(R) \longleftrightarrow G L_{n}(R)
$$

induz um epimorfismo

$$
\dot{R} /[\dot{R}, \dot{R}] \longrightarrow K_{1}(R)
$$

Demonstração. A demonstração da proposição anterior mostra que toda matriz $A \in G L_{n}(R)$ é equivalente a uma matriz diagonal $\langle a, 1, \cdots, 1\rangle$, ou seja, a aplicação $\varphi: \dot{R} \longrightarrow G L_{n}(R)$ definida por $a \mapsto\langle a, 1, \cdots, 1\rangle$ é um epimorfismo. Desde que, $K_{1}(R)$ é abeliano, o epimorfismo anterior determina um diagrama comutativo

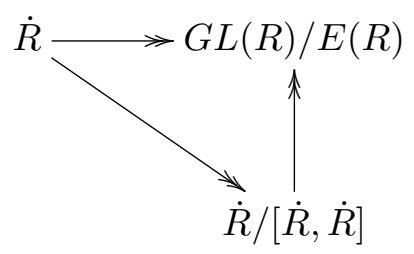

Observação 43. Seja $R$ um anel uma anel local ${ }^{4}$ não necessariamente comutativo. Existe uma

\footnotetext{
${ }^{4}$ Diremos que um anel é um Anel local quando satisfizer qualquer uma (e, portanto todas) das seguintes condições equivalentes :

- $\mathrm{R}$ possui um único ideal maximal à esquerda.

- $\mathrm{R}$ possui um único ideal maximal à direita.

- $R / J(R)$ é um anel de divisão, onde $J(R)$ é o radical de Jacobson.

- $R \backslash \dot{R}$ é um ideal de $\mathrm{R}$.

- $R \backslash \dot{R}$ é um grupo com relação a adição.

- $\forall n \forall a_{1}, \cdots, a_{n} \in R \exists a_{i}\left(a_{1}+a_{2}+\cdots+a_{n} \in \dot{R} \Longrightarrow a_{i} \in \dot{R}\right)$.
} 
família compatível de aplicaçôes

$$
\operatorname{det}_{n}: G L_{n}(R) \longrightarrow \dot{R} /[\dot{R}, \dot{R}]
$$

caracterizada pelas seguintes propriedades

$\left.\boldsymbol{d e t}_{n}^{1}\right)$ Se $A \in G L_{n}(R)$, então

$$
\operatorname{det}_{n}\left(e_{i j}^{\lambda} \cdot A\right)=\operatorname{det}_{n}(A)
$$

para todo $\lambda \in \dot{R}$ e $1 \leq i, j \leq n, i \neq j$.

$\left.\boldsymbol{d e t}_{n}^{2}\right)$ Se $A \in G L_{n}(R)$, então para todo $\lambda \in \dot{R}$ tem-se

$$
\operatorname{det}_{n}\left(\left(\lambda \xi_{i i}+I_{n}\right) A\right)=\bar{\lambda} \operatorname{det}_{n}(A)
$$

onde $\bar{\lambda}$ representa a classe de $\lambda$ em $\dot{R} /[\dot{R}, \dot{R}]$.

$\left.\operatorname{det}_{n}^{1}\right) \operatorname{det}_{n}\left(I_{n}\right)=\overline{1}$

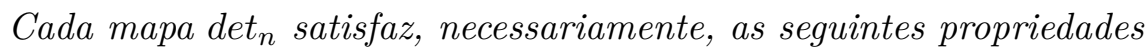

- $\left.\operatorname{det}_{n}\right|_{G L_{n-1}}=\operatorname{det}_{n-1}$

- $\operatorname{det}_{n}(A B)=\operatorname{det}_{n}(A) \operatorname{det}_{n}(B)$

- $\operatorname{det}_{n}(P A)=\overline{(-1)} \operatorname{det}_{n}(A)$, onde $P$ é uma matriz que permuta exatamente duas linhas de $A$.

- $\operatorname{det}_{n}\left(A^{t}\right)=\operatorname{det}_{n}(A)$

A família $\left\{\right.$ det $\left._{n}\right\}$ induz uma única aplicação (chamada de determinante ${ }^{5}$ )

$$
\operatorname{det}: G L(R) \longrightarrow \dot{R} /[\dot{R}, \dot{R}]
$$

com as seguintes propriedades

$\left.\operatorname{det}_{1}\right) \operatorname{det}(A B)=\operatorname{det}(A) \operatorname{det}(B)$

$\left.\operatorname{det}_{2}\right) \operatorname{det}(A)=1$ para todo $A \in E(R)$

$\left.\operatorname{det}_{3}\right)$ A composição

$$
\dot{R} \cong G L_{1}(R) \longleftrightarrow G L(R) \stackrel{\operatorname{det}}{\longrightarrow} \dot{R} /[\dot{R}, \dot{R}]
$$

coincide com a projeção natural

$$
\pi: \dot{R} \longrightarrow \dot{R} /[\dot{R}, \dot{R}]
$$

Proposição 95. Se $R$ é um anel local, não necessariamente comutativo, então o determinante induz um isomorfismo

$$
K_{1}(R) \cong \dot{R} /[\dot{R}, \dot{R}]
$$

- $a+b \in \dot{R} \Longrightarrow a \in \dot{R} \quad$ ou $\quad b \in \dot{R}$.

${ }^{5} \mathrm{O}$ leitor interessado em mais detalhes poderá consultar o clássico livro de Artin Geometric Algebra Art2] capítulo IV ou Ros o teorema 2.2.5 na pág. 64 ou Srin a página 5. 
Demonstração. Em um anel local o radical de Jacobson $J(R)$ é conjunto das não-unidades de R. ${ }^{6}$ Se a $i$-ésima linha de A tivesse todas as entradas em $J(R)$, então aconteceria o mesmo para a matriz $A B$, para toda matriz $\mathrm{B}$ e, então $\mathrm{A}$ não teria inversa à direita. Semelhantemente, se $\mathrm{A}$ tivesse a $j$-ésima coluna com entradas em $J(R)$,então o mesmo seria válido para $B A$, qualquer que fosse $\mathrm{B}$ e, então A não teria inversa à esquerda. Portanto, existe um epimorfismo

$$
\pi: \dot{R} \longrightarrow \dot{R} /[\dot{R}, \dot{R}]
$$

desde que, este epimorfismo coincide com a composição

$$
\dot{R} \cong G L_{1}(R) \longleftrightarrow G L(R) \stackrel{\operatorname{det}}{\longrightarrow} \dot{R} /[\dot{R}, \dot{R}]
$$

temos o resultado.

Corolário 20. (Dieudonné) Se $R$ é um anel de divisão,então o determninate de Dieudonné 7

$$
\text { det }: G L(R) \longrightarrow \dot{R} /[\dot{R}, \dot{R}]
$$

induz um isomorfismo

$$
K_{1}(R) \cong \dot{R} /[\dot{R}, \dot{R}]
$$

Exemplo 12. (Anel de séries formais) Sejam $\mathbb{K}$ um corpo e $\mathbb{K}[[T]]$ o anel de séries formais em T. O isomorfismo

$$
\frac{\mathbb{K}[[T]]}{\langle T\rangle} \cong \mathbb{K}
$$

permite concluir que $\langle T\rangle$ é um ideal maximal de T. Além disso,

$$
\mathbb{K}[[T]] \backslash\langle T\rangle=\dot{\mathbb{K}}
$$

Portanto, o anel $\mathbb{K}[[T]]$ é local. Em virtude da proposição 95 temos um isomorfismo

$$
K_{1}(\mathbb{K}[[T]]) \cong \mathbb{K}^{\bullet}[[T]] /\left[\mathbb{K}^{\bullet}[[T]], \mathbb{K}^{\bullet}[[T]]\right]
$$

onde $\mathbb{K}^{\bullet}[[T]]$ é o grupo de unidades de $\mathbb{K}[[T]]$.

Exemplo 13. (O anel das séries de potência torcida)Seja $R$ um anel de divisão (não necessariamente comutativo) e $\sigma \in \operatorname{End}(R)$ não trivial. Definimos $R[x, \sigma]$ para ser

$$
R[x, \sigma] \doteq\left\{\sum_{i=n}^{\infty} r_{i} x^{i}: r_{i} \in R, n \in \omega\right\}
$$

$O$ produto em $R[x, \sigma]$ é definido da segunte maneira

$$
\left(\sum a_{i} x^{i}\right) \cdot\left(\sum a_{j} x^{j}\right)=\left(\sum a_{i} \sigma^{i}\left(b_{j}\right) x^{i+j}\right)
$$

\footnotetext{
${ }^{6}$ Por definição de anel local, temos que o conjunto de não unidades $R \backslash \dot{R}$ de $\mathrm{R}$ é um ideal próprio e, portanto está contido em $J(R)$. Inversamente, se $a \notin J(R)$, então existe um ideal maximal $\mathcal{M}$ de $\mathrm{R}$ tal que $a \notin \mathcal{M}$. Assim, existiria uma decomposição $b a+c=1$ com $a b, c \in \mathcal{M} \subseteq R \backslash \dot{R}$ : uma contradição. Logo, $J(R) \subseteq R \backslash \dot{R}$.

${ }^{7} \mathrm{O}$ determinante de matrizes sobre anéis de divisão foi definido por Jean Dieudonné em [Die. O leitor poderá encontrar as aplicações desse determinante em [Bre1]. Veja, também, as correções desse último artigo em Bre2].
} 
O conjunto $R[x, \sigma]$ com produto anterior e a soma usual é um anel não comutativo local. De acordo com a proposição 95 tem-se

$$
K_{1}(R[x, \sigma]) \cong R^{\bullet}[x, \sigma] /\left[R^{\bullet}[x, \sigma], R^{\bullet}[x, \sigma]\right]
$$

\section{Exemplo 14. (Anéis euclidianos)}

(1) Se $R$ é um anel euclidiano, então $K_{1}(R) \cong \dot{R}$.

(2) $K_{1}(\mathbb{Z}) \cong\{ \pm 1\}$.

(3) $K_{1}(\mathbb{Z}[i])=\{ \pm 1, \pm i\}$

(4) $\left.K_{1}(\mathbb{Z}[(-1+i \sqrt{3}) / 2]) \cong\left\{\zeta \in \mathbb{C}: \zeta^{6}=1\right\}\right)$

(5) $K_{1}(\mathbb{K}[t])=\dot{\mathbb{K}}$

Nos exemplos (2), (3), (4) e (5) as normas de $\mathbb{Z}, \mathbb{Z}[i], \mathbb{Z}[1+\sqrt{3} / 2]$ e $\mathbb{K}[t]$ são dadas, respectivamente por

- $|a|$ (valor absoluto ou módulo).

- $|a+b i|=a^{2}+b^{2}($ Norma complexa)

- $|a+b(i \sqrt{3})-1 / 2|=a^{2}-a b+b^{2}$.

- $|f(t)|=2^{\operatorname{grau}(f)}($ com a convenção $\operatorname{grau}(0)=-\infty)$

Exemplo 15. (Símbolos de Mennicke ) Seja $R$ um anel comutativo e $\mathfrak{J}$ um ideal de $R$. Definimos o conjunto $W_{\mathfrak{J}}$ de elementos $\mathfrak{J}$-unimodulares em $R^{2}$ como sendo o conjunto

$$
W_{\mathfrak{J}} \doteq\left\{(a, b) \in R^{2}:(a, b) \equiv(1,0)(\bmod \quad \mathfrak{J}) ; a R+b R=R\right\}
$$

Seja $G$ um grupo abeliano. Um símbolo de Mennicke 8 é um mapa

$$
\begin{aligned}
& \text { [ ] : } W_{\mathfrak{J}} \longrightarrow G \\
& (a, b) \longmapsto\left[\begin{array}{l}
a \\
b
\end{array}\right]
\end{aligned}
$$

satisfazendo os seguintes axiomas

$$
\begin{aligned}
& \boldsymbol{M S}_{1}:\left[\begin{array}{c}
b+t a \\
a
\end{array}\right]=\left[\begin{array}{l}
b \\
a
\end{array}\right] \text { se }(a, b) \in W_{\mathfrak{J}} \text { et } \in \mathfrak{J} \\
& \boldsymbol{M S}_{2}:\left[\begin{array}{c}
b \\
a+t b
\end{array}\right]=\left[\begin{array}{l}
b \\
a
\end{array}\right] \text { se }(a, b) \in W_{\mathfrak{J}} \text { et } \in \mathfrak{J} \\
& \boldsymbol{M S}_{3}:\left[\begin{array}{c}
b_{1} \\
a
\end{array}\right]\left[\begin{array}{c}
b_{2} \\
a
\end{array}\right]=\left[\begin{array}{c}
b_{1} b_{2} \\
a
\end{array}\right] \text { se }\left(a, b_{1}\right),\left(a, b_{2}\right) \in W_{\mathfrak{J}} \\
& \boldsymbol{M S}_{4}:\left[\begin{array}{c}
b \\
a_{1}
\end{array}\right]\left[\begin{array}{c}
b \\
a_{2}
\end{array}\right]=\left[\begin{array}{c}
b \\
a_{1} a_{2}
\end{array}\right] \text { se }\left(a_{1}, b\right),\left(a_{2}, b\right) \in W_{\mathfrak{J}}
\end{aligned}
$$

Os axiomas anteriores não são independentes e uma definição mais enxuta pode ser encontrada em [BMS na página 6\% Um símbolo de Mennicke [ ] : $W_{\mathfrak{J}} \longrightarrow G$ é dito universal se para cada

\footnotetext{
65.

${ }^{8}$ Símbolos de Mennicke foram observados inicialmente em [Men] e generalizados em [BMS], a saber, na página
} 
símbolo de Mennicke ( ) : $W_{\mathfrak{J}} \longrightarrow H$ existe um único homomorfismo de grupos $\varphi: G \longrightarrow H$ que torna o seguinte diagrama comutativo

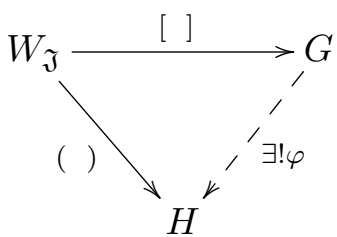

É claro da definição que deve existir um símbolo de Mennicke universal. Ele pode ser construído, por exemplo, como o grupo com geradores $W_{\mathfrak{J}}$ módulo o subgrupo gerado pelas relações $\boldsymbol{M} \boldsymbol{S}_{1}, \boldsymbol{M} \boldsymbol{S}_{2}$, $\boldsymbol{M S}_{3}$ e $\boldsymbol{M} \boldsymbol{S}_{4}$.

Tomando $\mathfrak{J}=R$ e escolhendo $c, d \in R$ tais que ad $-c d=1$, então a aplicação

$$
\text { [ ] : } \begin{array}{cc}
W_{R} & \longrightarrow \\
(a, b) & \longmapsto[a, b]
\end{array}
$$

onde $[a, b]$ é a classe de $\left[\begin{array}{ll}a & b \\ c & d\end{array}\right]$ em $S K_{1}(R)$ é um símbolo de Mennicke. Se $R$ é um anel Noetheriano com dimensão de Krull $\leq 1$, então $S K_{1}(R)$ é completamente determinado pelo símbolo de Mennicke anterior (veja o capitulo VI de [Bass2] )

Exemplo 16. (Anéis de tipo aritmético) Um corpo valorado é um par $(\mathbb{K},||)$, onde $\mathbb{K}$ é um corpo $e||$ é uma função ||$: \mathbb{K} \longrightarrow \mathbb{R}$ cumprindo as seguintes propriedades

||$_{1}:|a| \geq 0$ e $|a|=0 \Longleftrightarrow a=0$

||$_{2}:|a b|=|a||b|$

||$_{3}:|a+b| \leq \max (|a|,|b|)$

Se em lugar de ||$_{3}$, a aplicação | | satisfaz a inequação usual

$$
||_{3^{\prime}}:|a+b| \leq|a|+|b|
$$

então | | é arquimediana, caso contrário,| | é não-arquimediana. Obviamente, o par $(\mathbb{K}, \mid$ |) determina, do modo usual, um espaço ultramétrico.

Dizemos que dois corpos valorados $\left(\mathbb{K},||^{1}\right)$ e $\left(\mathbb{K},||^{2}\right)$ são equivalentes quando suas valorações induzem a mesma topologia em $\mathbb{K}$.

Dizemos que um corpo $\mathbb{K}$ é global quando ele é um corpo numérico 9 ou um corpo de funções 10. Seja $\Omega$ um sistema completo de representantes de valorações não-triviais em $\mathbb{K}$ e seja $S_{\infty}$ um subconjunto de valorações arquimedianas. Temos

- $S_{\infty}=\emptyset$ se $\mathbb{K}$ for um corpo de funções.

- $S_{\infty}$ é finito se $\mathbb{K}$ for um corpo numérico.

Seja $S \subseteq \Omega$ contendo $S_{\infty} . O$ conjunto

$$
\mathcal{O}_{\mathbb{K}}(S) \doteq\{x \in \mathbb{K}:|x| \leq 1,|| \in \Omega-S\}
$$

\footnotetext{
${ }^{9}$ Dizemos que um corpo $\mathbb{K}$ é um corpo numérico se ele é uma extensão finita do corpo dos números racionais $\mathbb{Q}$.

${ }^{10}$ Dizemos que um corpo $\mathbb{K}$ é um corpo de funções quando ele é uma extensão finita do corpo quociente do anel de polinômios em uma variável sobre um corpo finito com $d$ elementos, a saber, $\mathbb{F}_{d}[X]$
} 
é um subanel de $\mathbb{K}$ chamado $\boldsymbol{O}$ anel de $\boldsymbol{S}$-inteiros de $\mathbb{K}$. Se $S$ e $\Omega$ forem substituídos por conjuntos equivalentes de valorações, então $\mathcal{O}_{\mathbb{K}}(S)$ permanece inalterado. Observe que $\mathcal{O}_{\mathbb{K}}(S)$ é um dominio de Dedekind. Se $S$ é finito,então $S$ é um conjunto de valorações de Hasse em $\mathbb{K}$ e $\mathcal{O}_{\mathbb{K}}(S)$ é dito um dominio de Hasse ou um dominio de Dedekind de tipo aritmético.Se $\mathbb{K}$ é um corpo numérico e $S=S_{\infty}$, então $\mathcal{O}_{\mathbb{K}}$ é o anel de inteiros de $\mathbb{K}$. Nesse caso, foi demonstrado em um excelente artigo devido a H.Bass, J. Milnor e J.P.Serre a seguinte igualdade

$$
S L_{n}\left(\mathcal{O}_{\mathbb{K}}\right)=E_{n}\left(\mathcal{O}_{\mathbb{K}}\right)
$$

Ou seja, $S K_{1}\left(\mathcal{O}_{\mathbb{K}}\right)=0$ (ver $[\boldsymbol{B M S}$ o corolário 4.3 da página 95). A computação completa de $K_{1}\left(\mathcal{O}_{\mathbb{K}}\right)$ é, agora, uma consequência do Teorema da unidade de Dirichilet.

Vamos terminar essa seção com um exemplo histórico que impulsionou muitos dos desenvolvimentos da K-teoria algébrica e, também, da teoria de homotopia.

Exemplo 17. (O grupo de Whitehead)Seja $\Pi$ um grupo multiplicativo e $\mathbb{Z} \Pi$ o anel de grupo correspondente com coeficientes inteiros. Seja $[-1] \in K_{1}(\mathbb{Z} \Pi)$ denotando o elemento de ordem 2 correspondente para unidade

$$
-1 \in G L_{1}(\mathbb{Z} \Pi) \longleftrightarrow G L(\mathbb{Z} \Pi)
$$

O grupo de Whitehead reduzido de $\mathbb{Z} \Pi$ é definido pelo quociente

$$
\overline{K_{1}}(\mathbb{Z} \Pi) \doteq \frac{K_{1}(\mathbb{Z} \Pi)}{\langle 0,[-1]\rangle}
$$

onde $\langle 0,[-1]\rangle$ é o subgrupo gerado pelo conjunto $\{0,[-1]\}$.

A sequência de grupos

$$
\Pi \hookrightarrow(\mathbb{Z} \Pi)^{\bullet} \stackrel{\cong}{\longrightarrow} G L_{1}(\mathbb{Z} \Pi) \longleftrightarrow G L(\mathbb{Z} \Pi)
$$

permite construir os homomorfismos naturais

$$
\Pi \longrightarrow K_{1}(\mathbb{Z} \Pi) \longrightarrow \overline{K_{1}}(\mathbb{Z} \Pi)
$$

Chamamos de grupo de Whitehead ${ }^{11}$ ao cokernel

\footnotetext{
${ }^{11}$ O grupo $W h(G)$ tem origem nos anos de 1950 no trabalho de J.H.C Whitehead sobre tipos de homotopias simples (Whi2 $)$. Seja X um CW-complexo conexo e Y um subcomplexo de X que é uma retrato por deformação de X. Nesse caso, a inclusão $Y C X$ é uma equivalência de homotopia que induz um isomorfismo nos grupos fundamentais pontuados $\Pi_{1}(Y, y) \longrightarrow \Pi \doteq \Pi_{2}(X, y)$, onde $y \in Y$. Se $P: \widetilde{X} \longrightarrow X$ é um recobrimento universal, então $\widetilde{Y} \doteq p^{-1}(Y)$ é um recobrimento universal de Y e retrato por deformação de $\widetilde{X}$. Portanto, o complexo de cadeia $C(\widetilde{X}, \widetilde{Y})$ é acíclico. Assim, se $i d: \mathbb{Z} \longrightarrow \mathbb{Z} \Pi$ é o homomorfismo identidade, nós temos a torção

$$
\tau(X, Y)=\tau_{i d}(X, Y)=\tau(C(\widetilde{X}, \widetilde{Y})) \in W h(\Pi)
$$

essa torção é conhecida como torção de Whitehead e o seu conteúdo geométrico foi dado em Whi1. Explicitamente, seja $X, Y \mathrm{CW}$-espaços conexos e seja $f: X \longrightarrow Y$ uma equivalência de homotopia celular. A torção de Whitehead de $f$ é definida por

$$
\tau(f) \doteq p_{*}\left(\tau\left(M_{f}, X\right)\right) \in W h(\Pi)
$$

onde $p_{*}: W h\left(\Pi_{1}(X)\right) \longrightarrow W h\left(\Pi_{1}(Y)\right)$ é o isomorfismo induzido por determinado isomorfismo $p_{\#}: \Pi_{1}\left(M_{f}\right) \longrightarrow$ $\Pi_{1}(Y)$ (aqui $M_{f}$ é o mapa cilindro associado a f) e $\Pi=\Pi_{1} X=\Pi_{1} Y=\Pi_{1} M_{f}$. Whitehead mostrou o seguinte :

Uma equivalência $f$ entre $C W$-complexos conexos finitos é simples $(\tau(f)=1)$ se, e somente se puder ser de-
} 


$$
W h(\Pi) \doteq \frac{\overline{K_{1}}(\mathbb{Z} \Pi)}{\operatorname{Im}(\Pi)}
$$

Essa construção pode ser descrita pela sequência exata

$$
0 \longrightarrow \Pi /[\Pi, \Pi] \longrightarrow \overline{K_{1}}(\mathbb{Z} \Pi) \longrightarrow W h(\Pi) \longrightarrow 0
$$

Note que qualquer homomorfismo $f: \Pi \longrightarrow \Pi^{\prime}$ induz de forma coerente um homomorfismo Wh $(f)$ : $W h(\Pi) \longrightarrow W h\left(\Pi^{\prime}\right)$. Em outras palavras, Wh é um funtor covariante.

- (Higman 1940) Se $\Pi \cong \mathbb{Z}$ ou $\Pi$ é isomorfo á $\mathbb{Z}_{2}, \mathbb{Z}_{3}$ ou $\mathbb{Z}_{4}$, então Higman mostrou em Hig que Wh(П) é trivial.

- (Bass -Heller -Swan 1964)Hyman Bass, Alex Heller e Richard G. Swan mostraram em [BHS que o grupo de Whitehead de qualquer grupo abeliano livre é trivial.

- Stephen Gersten ([Gers, Teorema 1$)$ e Jonh Stallings ([Sta Teorema 6.4) mostraram que o grupo de Whitehead de qualquer grupo livre é zero. Explicitamente, este último mostrou que

$$
W h\left(\mathbb{Z} \Pi_{1} * \mathbb{Z} \Pi_{2}\right) \cong W h\left(\mathbb{Z} \Pi_{1}\right) \oplus W h\left(\mathbb{Z} \Pi_{2}\right)
$$

onde $\mathbb{Z} \Pi_{1} * \mathbb{Z} \Pi_{2}$ é o produto livre como anéis.

\subsection{O Funtor $K_{2}$}

Um problema importante em K-teoria algébrica consiste em mostrar a existência de um possível funtor $K_{2}$ que satisfaça certos axiomas de exatidão e de excisão. Uma definição aproximada foi dada por Milnor em [Miln4] usando geradores e relações de Steinberg.

Definição 78. Para $n \geq 3$ o n-ésimo grupo de Steinberg é definido pelo quociente

$$
S t_{n}(R)=F_{n}\left(S_{n}\right) / R_{n}\left(S_{n}\right)
$$

onde $F_{n}\left(S_{n}\right)$ é gerado pelos símbolos

$$
S_{n} \doteq\left\{x_{i j}^{\lambda} \mid 1 \leq i, j \leq n, i \neq j, \lambda \in \dot{R}\right\}
$$

e $R_{n}\left(S_{n}\right)$ é o menor subgrupo normal onde valem as relações

St1) $x_{i j}^{\lambda} \cdot x_{i j}^{\mu}=x_{i j}^{\lambda+\mu}$;

St2) $x_{i j}^{\lambda} x_{k l}^{\mu} x_{i j}^{-\lambda} x_{k l}^{-\mu}=i_{d}, \forall j \neq k, \forall i \neq l$

St3) $x_{i j}^{\lambda} x_{k l}^{\mu} x_{i j}^{-\lambda} x_{k l}^{-\mu}=x_{i j}^{\lambda \mu}, \forall j=k, \forall i \neq l$

St4 $x_{i j}^{\lambda} x_{k l}^{\mu} x_{i j}^{-\lambda} x_{k l}^{-\mu}=x_{i j}^{-\lambda \mu}, \forall j \neq k, \forall i=l$

composta em uma sequência finita de expansões e colapsos elementares.

O leitor pode consultar Coh para mais detalhes sobre esse resultado. Finalmente, nos anos de 1960 a torsão de Whitehead revela, ainda mais, o seu poder geométrico ao mostrar-se crucial em uma formulação mais geral do Teorema de s-cobordismos culminando em 1966 com o teorema de classificação de h-cobordismos (consulte Miln2 A classificação de h-cobordismos na página 398 e seção 11). 
Lema 50. As aplicações, para $m \leq n$

$$
\begin{aligned}
\xi_{m, n}: \begin{array}{l}
S_{m} \\
x_{i j}^{\lambda}
\end{array} & \longmapsto x_{i j}^{\lambda}
\end{aligned}
$$

podem ser estendidas a homomorfismos de grupos

$$
\begin{aligned}
\xi_{m, n}: S t_{m} & \longrightarrow S t_{n} \\
x_{i j}^{\lambda} & \longmapsto x_{i j}^{\lambda}
\end{aligned}
$$

para todos $m \leq n$.

Demonstração. Para ver a existência basta observar que a propriedade universal do grupo livre fornece um diagrama comutativo

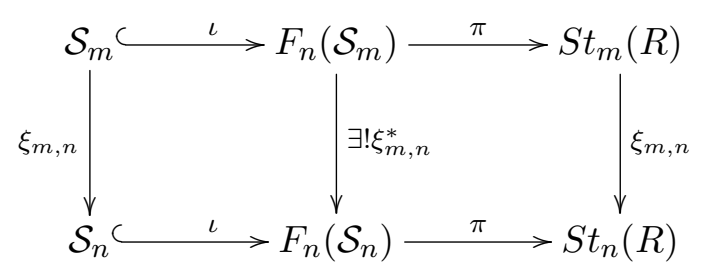

\section{Fato 27.}

1) Se $\phi: R \longrightarrow R^{\prime}$ for um homomorfismo de anéis, então temos um diagrama comutativo
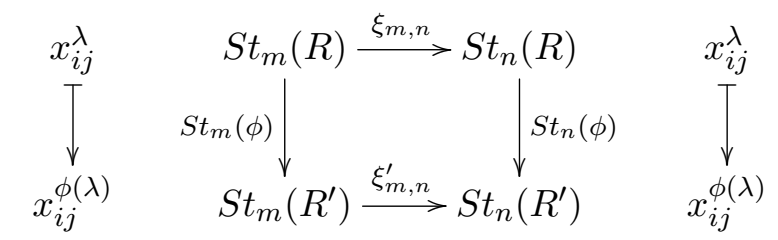

2) A família de morfismos $\left(\xi_{m, n}\right)$ é um sistema inverso, ou seja, o seguinte diagrama comuta

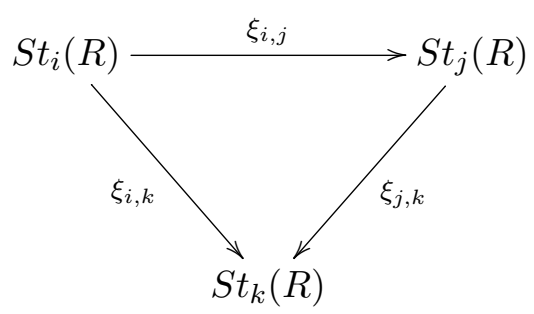

Para todo $i \leq j \leq k$ e $\xi_{l, l}=I d, \forall l \in \omega \backslash\{1,2\}$.

3) Existem funtores de Steinberg $S t_{n}:$ Ring $\longrightarrow$ Grp de ordem n que sai da categoria dos anéis e chega na categoria dos grupos.

Definição 79. O grupo infinito de Steinberg é definido como sendo o limite

$$
S t(R) \doteq \lim _{\longrightarrow} S t_{n}(R)
$$

Observe que as matrizes elementares $e_{i j}^{\lambda}$ que geram o grupo elementar $E_{n}(R)$ de ordem n também satisfazem as relações de Steinberg. A aplicação 


$$
\begin{aligned}
\theta_{n}: S t_{n}(R) & \longrightarrow G L_{n}(R) \\
x_{i j}^{\lambda} & \longmapsto e_{i j}^{\lambda}
\end{aligned}
$$

é um homomorfismo de grupos. Além disso, $\theta_{n}\left(S t_{n}(R)\right)=E_{n}(R)$. A partir daí, podemos usar a universalidade de $S t(R)$ para definir um epimorfismo de grupos $\theta: S t(R) \longrightarrow E(R)$. Além disso, não é difícil ver que existe um funtor covariante $S t: \operatorname{Ring} \longrightarrow G r p$ da categoria de anéis para a categoria de grupos.

Fato 28. $S t(R)$ pode ser visto como o grupo gerado por símbolos abstratos $x_{i j}^{\lambda}, \lambda \in R$, módulo o menor subgrupo normal cumprindo relações semelhantes as relações $\left(E_{1}\right),\left(E_{2}\right),\left(E_{3}\right)$ da observação 42 (3) $\operatorname{com} x_{i j}^{\lambda}$ no lugar de $e_{i j}^{\lambda}$.

Definição 80. Para cada anel $R$ definimos o terceiro grupo da K-teoria algébrica como sendo o grupo

$$
K_{2}(R) \doteq \operatorname{Ker}(\theta: S t(R) \longrightarrow E(R))
$$

Para cada $n \geq 3$ definimos $P_{n}$ como sendo o subgrupo de $S t(R)$ gerado pelo conjunto

$$
\left\{x_{i n}^{\mu}: 1 \leq i \leq n-1, \mu \in R\right\}
$$

Note que $P_{n}(R)$ é comutativo pela condição $\mathbf{S t}_{2}$.

\section{Lema 51.}

1) $A \cdot e_{k l}^{\lambda}=e_{k l}^{\lambda} \cdot A, \forall e_{k l}^{\lambda} \in E_{n}(R) \Longleftrightarrow A=\langle a, \cdots, a\rangle, \forall A \in M_{n}(R)$.

2) $O$ centro de $E(R)$ é trivial.

Demonstração.

1) Seja $A=\left(a_{i j}\right)$. Por um lado, temos

$$
\begin{aligned}
\left(\sum_{i=1}^{n} \sum_{j=1}^{n} a_{i j} \xi_{i j}\right) \cdot e_{k l}^{\lambda} & =\left(\sum_{i=1}^{n} \sum_{j=1}^{n} a_{i j} \xi_{i j}\right) \cdot\left(1+\lambda \xi_{k l}\right) \\
& =\sum_{i=1}^{n} \sum_{j=1}^{n} a_{i j} \xi_{i j}+\sum_{i=1}^{n} \sum_{j=1}^{n} \lambda a_{i j} \xi_{i j} \xi_{k l} \\
& =\sum_{i=1}^{n} \sum_{j=1}^{n} a_{i j} \xi_{i j}+\sum_{i=1}^{n} \sum_{j=1}^{n} \lambda a_{i j} \delta_{j k} \xi_{i l} \\
& =\sum_{i, j} a_{i j} \xi_{i j}+\sum_{i} \lambda a_{i k} \xi_{i l} \\
& =\sum_{i, j \neq l} a_{i j} \xi_{i j}+\sum_{i}\left(a_{i l}+\lambda a_{i k}\right) \xi_{i l}
\end{aligned}
$$

e por outro lado 


$$
\begin{aligned}
e_{k l}^{\lambda} \cdot\left(\sum_{i=1}^{n} \sum_{j=1}^{n} a_{i j} \xi_{i j}\right) & =\left(1+\lambda \xi_{k l}\right) \cdot\left(\sum_{i=1}^{n} \sum_{j=1}^{n} a_{i j} \xi_{i j}\right) \\
& =\sum_{i=1}^{n} \sum_{j=1}^{n} a_{i j} \xi_{i j}+\sum_{i=1}^{n} \sum_{j=1}^{n} \lambda a_{i j} \xi_{k l} \xi_{i j} \\
& =\sum_{i=1}^{n} \sum_{j=1}^{n} a_{i j} \xi_{i j}+\sum_{i=1}^{n} \sum_{j=1}^{n} \lambda a_{i j} \delta_{l i} \xi_{k j} \\
& =\sum_{i, j} a_{i j} \xi_{i j}+\sum_{j} \lambda a_{l j} \xi_{k j} \\
& =\sum_{i, j \neq k} a_{i j} \xi_{i j}+\sum_{j}\left(a_{k j}+\lambda a_{l j}\right) \xi_{k j}
\end{aligned}
$$

Agora é fácil derivar o resultado.

2) Decorre do item anterior que $E_{n-1}(R) \cap E_{n}(R)=\{1\}$. Portanto, no limite temos $E(R)=\{1\}$.

\section{Lema 52.}

1) Cada elemento de $\gamma \in P_{n}$ admite uma escrita

$$
\gamma=\prod_{i=1}^{n-1} x_{i n}^{\mu_{i}}
$$

2) A restrição $\left.\theta\right|_{P_{n}}$ é injetora.

3) Para cada $n$ temos a seguinte inclusão

$$
x_{i j}^{\lambda} P_{n} x_{i j}^{-\lambda} \subseteq P_{n}
$$

para todos $i, j<n$.

Demonstração.

1) Por definição cada $\gamma \in P_{n}(R)$ pode ser escrito como

$$
\gamma=x_{i_{1} n}^{\lambda_{1}} \cdots x_{i_{k} n}^{\lambda_{k}}
$$

Agora para cada $1 \leq t \leq n-1$ definimos o conjunto

$$
S_{t} \doteq\left\{j: i_{j}=t\right\}
$$

Como $P_{n}(R)$ é comutativo, podemos escrever

$$
\left(\prod_{j \in S_{1}} x_{1 n}^{\lambda_{j}}\right) \cdots\left(\prod_{j \in S_{n-1}} x_{n-1 n}^{\lambda_{j}}\right)
$$

o resultado segue de $\mathbf{S t}_{1}$.

2) É uma consequência de 


$$
\theta\left(x_{1 n}^{\mu_{1}} \cdots x_{n-1 n}^{\mu_{n-1}}\right)=\left[\begin{array}{cccc}
1 & 0 & \cdots & \mu_{1} \\
0 & 1 & \cdots & \mu_{2} \\
\vdots & \vdots & \ddots & \vdots \\
0 & 0 & \cdots & \mu_{n-1} \\
0 & 0 & \cdots & 1
\end{array}\right]
$$

3) Seja $x_{k n}^{\mu}$ um gerador qualquer de $P_{n}$. O resultado é uma consequência da igualdade

$$
x_{i j}^{\lambda} x_{k n}^{\mu} x_{i j}^{-\lambda}=\left\{\begin{array}{ccc}
x_{i n}^{\lambda \mu} x_{k n}^{\mu}, & \text { se } & j=k \\
x_{k n}^{\mu}, & \text { se } & j \neq k
\end{array}\right.
$$

\section{Proposição 96.}

1) A seguinte sequência de grupos

$$
1 \longrightarrow K_{2}(R) \stackrel{\iota}{\longrightarrow} S t(R) \stackrel{\theta_{R}}{\longrightarrow} G L(R) \stackrel{\pi}{\longrightarrow} K_{1}(R) \longrightarrow 1
$$

é exata.

2) $K_{2}(R)$ é o centro de $\operatorname{St}(R)$.

3) $K_{2}(R)$ é um grupo abeliano.

Demonstração.

1) Decorre imediatamente da definição de $K_{2}(R)$ e da sobrejetividade de $\theta: S t(R) \longrightarrow G L(R)$.

2)Se $z$ pertence ao centro de $S t(R)$, então $\theta(z)$ pertence ao centro de $E(R)$. Assim, pelo lema 51 (2) temos que $\theta(z)=1$. Inversamente, seja $\theta(z)=1$. Escolhemos n suficientemente grande de modo que $z$ seja escrito como uma palavra nos geradores $x_{i j}^{\lambda}$ com $i, j<n$. Assim, resulta do lema 52 (3) que $z P_{n} z^{-1} \subseteq P_{n}$, donde

$$
\theta\left(z p z^{-1}\right)=\theta(z) \theta(p) \theta\left(z^{-1}\right)=\theta(p), \forall p \in P_{n}
$$

Usando novamente o lema 51 (2) ter-se-á $z p z^{-1}=p$. Portanto, $z$ comuta com todo $p \in P_{n}$. Em particular, $z$ comuta com todo gerador $x_{k n}^{\lambda}, k<n$ de $P_{n}$. Semelhantemente, $z$ comuta com todo $x_{n l}^{\mu}, l<n$. Assim, $z$ comuta com o comutador

$$
\left[x_{k n}^{\mu}, x_{n l}^{1}\right]=x_{k l}^{\mu}
$$

para todos $\forall k \neq l, l<n$. Desde que, podemos escolher $n$ arbitrariamente grande, isto completa a prova.

Lema 53. Cada homomorfismo de anéis $\varphi: R \longrightarrow R^{\prime}$ determina um homomorfismo $K_{2}(\varphi)$ : $K_{2}(R) \longrightarrow K_{2}\left(R^{\prime}\right)$.

Demonstração. Basta observar que a igualdade

$$
E(\varphi) \circ \theta_{R}=\theta_{R^{\prime}} \circ S t(\varphi)
$$

define um diagrama comutativo 


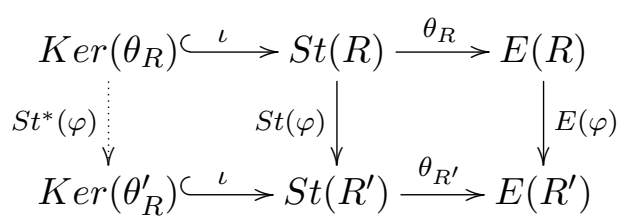

onde

$$
\begin{aligned}
S t^{*}(R): \operatorname{Ker}\left(\theta_{R}\right) & \longrightarrow \operatorname{Ker}\left(\theta_{R^{\prime}}\right) \\
x & \longrightarrow S t^{*}(x) \doteq S t(x)
\end{aligned}
$$

Proposição 97. $K_{2}$ é um funtor covariante da categoria de anéis para a categoria de grupos abelianos.

Demonstração. Se $\varphi: R \longrightarrow R^{\prime}$ e $\psi: R^{\prime} \longrightarrow R^{\prime \prime}$ homomorfismos de anéis, então

$$
\begin{aligned}
S t(\psi \circ \varphi)\left(x_{i j}^{\lambda}\right) & =x_{i j}^{\psi(\varphi(\lambda))} \\
& =\quad \operatorname{St}(\psi)\left(x_{i j}^{\varphi(\lambda)}\right) \\
& =\operatorname{St}(\psi)\left(\operatorname{St}(\varphi)\left(x_{i j}^{\lambda}\right)\right) \\
& =\operatorname{St}(\psi) \circ \operatorname{St}(\varphi)\left(x_{i j}^{\lambda}\right) .
\end{aligned}
$$

E, portanto $S t^{*}(\psi \circ \varphi)=S t^{*}(\psi) \circ S t^{*}(\varphi)$. Obviamente, se $I_{d}: R \longrightarrow R$ for o morfismo identidade de anéis, então $S t\left(I_{d}\right): S t(R) \longrightarrow S t(R)$ também o será e por conseguinte $S t^{*}\left(I_{d}\right): K_{2}(R) \longrightarrow K_{2}(R)$ será identidade.

Observação 44. Os funtores $K_{1}, K_{2}, S t$ e $G L$ fazem parte de um diagrama comutativo

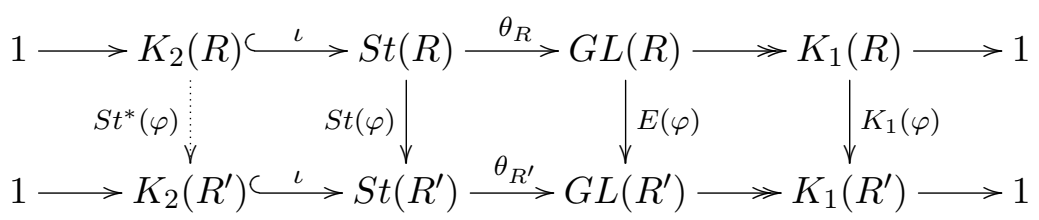

em particular, esse diagrama comutativo mostra que existe uma transformação natural entre os funtores $K_{2}$ e St.

Terminamos esta seção, enuncinado sem demonstração, a seguinte proposição versando sobre algumas propriedades do funtor $K_{2}$

Proposição 98. Valem as seguintes propriedades envolvendo o funtor $K_{2}$ :

1) $K_{2}\left(R^{o p}\right)=K_{2}(R)$;

2) $K_{2}\left(R \times R^{\prime}\right) \cong K_{2}(R) \times K_{2}\left(R^{\prime}\right)$.

3) $K_{2}(R) \cong K_{2}\left(R^{\prime}\right)$ para $R$ e $R^{\prime}$ anéis Morita equivalente.

4) $K_{2}(R) \cong K_{2}\left(M_{n}(R)\right)$.

5) $K_{2}\left(\lim _{i \in I} R_{i}\right) \cong \underline{\lim }_{i \in I} K_{2}\left(R_{i}\right)$, para cada sistema dirigido para cima. 


\subsubsection{Extensões centrais}

\section{Definição 81.}

1) Seja E um grupo e $K$ um grupo abeliano. Uma Extensão central de E por $K$ é um par $(S, \theta)$ constando de um grupo $S$ tal que $K \subseteq Z(S)$ e um epimorfismo de grupos $\theta: S \longrightarrow E$ tal que $K=K e r(\theta)$. Aternativamenmte, uma extensão central de $E$ por $K$ é uma sequência exata curta

$$
1 \longrightarrow K \longrightarrow S \stackrel{\theta}{\longrightarrow} E \longrightarrow 1
$$

onde $K$ é um subgrupo central de $S$.

2) Um morfismo entre extensões centrais $(S, \theta)$ e $\left(S^{\prime}, \theta^{\prime}\right)$ é um diagrama comutativo

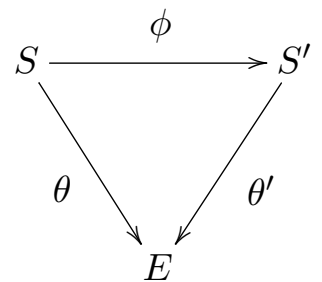

Logo $\operatorname{Ker}(\theta)=\operatorname{Ker}\left(\theta^{\prime} \circ \phi\right)$ e $\phi \mid: \operatorname{Ker}(\theta) \longrightarrow \operatorname{Ker}\left(\theta^{\prime}\right)$ é bem definido.

3) Uma extensão central $(S, \theta)$ é dita uma extensão central universal de $E$ quando para qualquer extensão central $(C, \pi)$ de $E$ existe um único homomorfismo $\phi: S \longrightarrow C$ tal que o diagrama

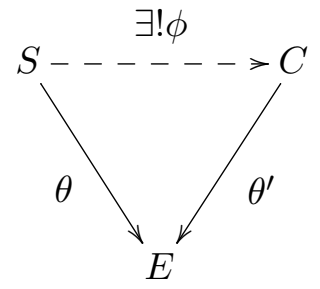

comuta.

4) Dizemos que uma extensão central $(S, \theta)$ cinde, quando existe um homomorfismo $\xi: E \longrightarrow S$ tal que $\theta \circ \xi=i d$. Nesse caso, $\xi$ é chamada uma seção para a extensão central $(S, \theta)$.

\section{Fato 29.}

1) Extensões centrais juntamente com morfismos de extensões centrais definem uma categoria.

2) Qualquer extensão central universal é unicamente determinada a menos de isomorfismo.

3) Se $(S, \theta)$ for uma extensão central que admite seção, então $S \cong E \times K$.

Proposição 99. Se $(S, \theta)$ for uma extensão central universal de $E$, então $[S, S]=S e[E, E]=E$.

Demonstração. Seja $(C, \pi)$ a extensão central de E onde $C \doteq S \times(S /[S, S])$ e $\pi(s, t)=\theta(s), \forall s \in$ $S, \forall t \in S /[S, S]$ (note que $\operatorname{Ker}(\theta) \times S /[S, S]$ é abeliano e $\operatorname{Ker}(\pi) \subseteq Z(C)$ ). Para $i=1,2$, definimos

$$
\phi_{i}: S \longrightarrow S \times S /[S, S]
$$

por $\phi_{1}(s) \doteq(s, 1)$ e $\phi_{2}(s) \doteq(s, \bar{s})$, onde $\bar{s}$ é a classe de s em $S /[S, S]$. Então, $\pi \circ \phi_{i}=\theta$ para $i=1,2$. Assim, $\phi_{1}=\phi_{2}$ e, portanto $S /[S, S]=1$. Finalmente, como $S \cong \operatorname{Im}(\theta)=E$, segue que $[E, E]=E$. 
Definição 82. Dizemos que um grupo $G$ é perfeito, quando $G$ coincide com o seu comutador, ou seja, $G=[G, G]$.

Proposição 100. Seja $(S, \theta)$ e $\left(S^{\prime}, \theta^{\prime}\right)$ extensões centrais de E. Se $S$ for um grupo perfeito, então existe no máximo um homomorfismo $\phi: S \longrightarrow S^{\prime}$ tal que

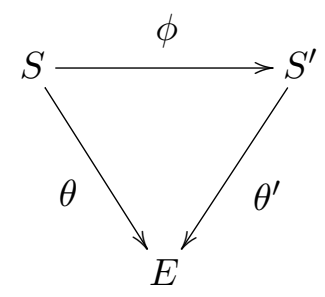

comuta.

Demonstração. Suponhamos que $\phi$ e $\phi^{\prime}$ são dois mapas nas condições desejadas. Sejam $s, s^{\prime} \in S$ elementos quaisquer. Então,

$$
\phi^{\prime}(s)=k \phi(s) \quad e \quad \phi^{\prime}\left(s^{\prime}\right)=k^{\prime} \phi\left(s^{\prime}\right)
$$

para algum $k$ e $k^{\prime}$ em $\operatorname{Ker}\left(\theta^{\prime}\right)$ e, portanto, $k, k^{\prime} \in Z\left(S^{\prime}\right)$. Assim,

$$
\phi^{\prime}\left(\left[s, s^{\prime}\right]\right)=\phi\left(\left[s, s^{\prime}\right]\right)
$$

Desde que, S é gerado por comutadores, resulta que $\phi^{\prime}=\phi$.

Proposição 101. Seja E um grupo perfeito. Então

1) $S e(S, \theta)$ é uma extensão central de $E$, então $[S, S]$ é um grupo perfeito e $\theta([S, S])=E$.

2) O par $\left([S, S],\left.\theta\right|_{[S, S]}\right)$ é uma extensão central de $E$.

Demonstração.

1) De fato,

$$
\theta([S, S])=[\theta(S), \theta(S)]=[E, E]=E
$$

e

$$
S=\operatorname{Ker}(\theta)[S, S] \Longrightarrow[S, S]=[D S, D S]
$$

onde $D S \doteq[S, S]$.

2) É uma consequência de (1).

Definição 83. Dizemos que um grupo E é centralmente fechado se $\left(E, i d_{E}\right)$ for uma extensão central universal de E.

\section{Observação 45.}

1) Se E for centralmente fechado, então $[E, E]=E$ pela proposição 99 . 
2) Seja $\phi: C \longrightarrow E$ um isomorfismo de grupos. Para qualquer grupo abeliano $K$ a aplicação

$$
\begin{aligned}
\phi^{*}: S \times K & \longrightarrow E \\
(c, k) & \longmapsto \phi(c)
\end{aligned}
$$

é claramente um epimorfismo de grupos. Além disso,

$$
\left((c, k) \in \operatorname{Ker}\left(\phi^{*}\right) \Longleftrightarrow c=1\right)
$$

Logo, $\operatorname{Ker}\left(\phi^{\prime}\right) \subseteq Z(S \times K)$, ou seja, o par $\left(C \times K, \phi^{*}\right)$ é uma extensão central de E. Se E for centralmente fechado, então todas as extensões centrais de $G$ possuem, essencialmente, esta forma. De fato, se E é centralmente fechado e $(S, \theta)$ é um extensão central de E, então existe um único homomorfismo $\xi: E \longrightarrow S$ tal que o diagrama

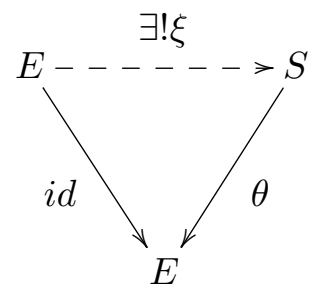

comuta. Dessa forma, a sequência exata curta

$$
1 \longrightarrow \operatorname{Ker}(\theta) \longrightarrow S \stackrel{\theta}{\longrightarrow} E \longrightarrow 1
$$

cinde. De acordo com o fato 29 (3) ter-se-á $S \cong E \times K \operatorname{Ker}(\theta)$.

Proposição 102. Uma extensão central $(S, \theta)$ de E é uma extensão central universal de $E$ se, $e$ somente se $S$ for centralmente fechado.

Demonstração. $(\Longleftarrow)$ Suponhamos que S é centralmente fechado. Seja $(C, \pi)$ uma extensão central qualquer de E. Observe que o seguinte subgrupo de $S \times C$

$$
S \times{ }_{E} C \doteq\{(s, c) \in S \times C: \theta(s)=\pi(c)\}
$$

juntamente com o homomorfismo

$$
\begin{aligned}
\pi^{*}: S \times_{E} C & \longrightarrow S \\
(s, c) & \longmapsto s
\end{aligned}
$$

é uma extensão central de S. Assim, existe um homomorfismo

$$
\psi: S \longrightarrow S \times{ }_{E} C
$$

tal que $\pi^{*} \psi=i d_{S}$. Definimos $\phi: S \longrightarrow C$ pela equação $\psi(s) \doteq(s, \phi(s))$. Note que

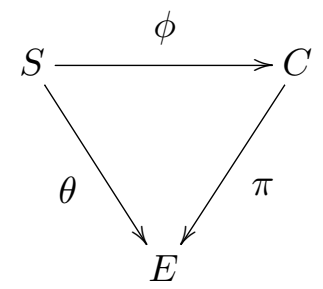


comuta. O homomorfismo $\phi$ é único pela proposição 100

$(\Longrightarrow)$ Suponhamos que $(S, \theta)$ é uma extensão central universal de E. Vamos mostrar que $\left(S, i d_{S}\right)$ é uma extensão central universal de S. Seja $(C, \pi)$ uma extensão central universal qualquer de S. Afirmamos que a composição

$$
C \stackrel{\pi}{\longrightarrow} S \stackrel{\theta}{\longrightarrow} E
$$

é uma extensão central de E. Se $\theta(\pi(c))=1$, então $\pi(c) \in Z(S)$ e o diagrama

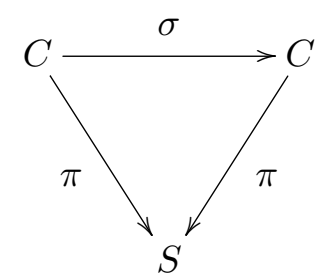

com $\sigma(x) \doteq c x c^{-1}$ comuta. Pelas proposições 101 e 100 a restrição

$$
\begin{aligned}
\sigma^{*}:[C, C] & \longrightarrow[C, C] \\
x & \longmapsto \sigma(x)
\end{aligned}
$$

é o mapa identidade. Assim,

$$
c x=x c, \forall x \in[C, C]
$$

Pela prova da proposição 101, C é gerado por $[C, C]$ juntamente com o subgrupo central $\operatorname{Ker}(\pi)$. De modo que, $c \in Z(C) \mathrm{e}$, de fato

$$
C \stackrel{\pi}{\longrightarrow} S \stackrel{\theta}{\longrightarrow} E
$$

é um extensão central de E. Desde que, $(S, \theta)$ é universal de E; existe um homomorfismo $\phi: S \longrightarrow C$ tal que

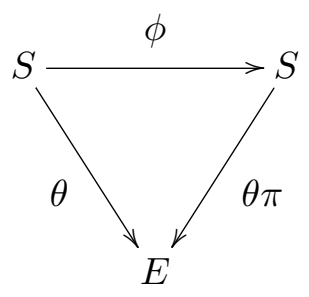

comuta. A composição $\pi \phi$ faz o diagrama

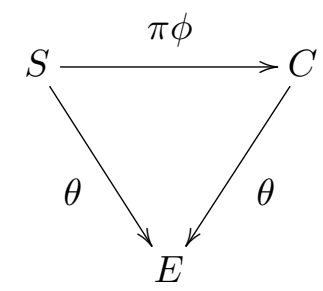

comutativo. Logo, pelas proposições 99 e $100, \pi \phi=i d_{S}$. Finalmente, verifique que a extensão $I d: S \longrightarrow S$ é universal para $\mathrm{S}$. 
Proposição 103. Um grupo admite uma extensão central universal se, e somente se for perfeito.

Demonstração. Se E possui uma extensão central universal,então segue da proposição 99 que $[E, E]=E$. Inversamente, suponhamos que E é um grupo perfeito, vamos construir uma extensão central universal para E.Para tanto, seja $F \doteq F(E)$ o grupo livre no conjunto E. A universalidade de $\mathrm{F}$ e o teorema do homomorfismo fornecem um diagrama comutativo de grupos

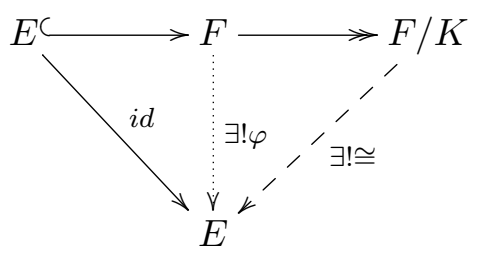

onde $K \doteq \operatorname{Ker}(\varphi)$. Observe que

$$
[K, F] \triangleleft F \quad e \quad[K, F] \subseteq K
$$

é fácil ver que a composição

$$
F /[K, F] \longrightarrow F / K \stackrel{\cong}{\longrightarrow} E
$$

é uma extensão central de $\mathrm{E}$ (note que a sobrejetividade de $\Theta$ decorre de $E=[E, E]$ ). Desde que,

$$
[F /[K, F], F /[K, F]]=[F, F] /[K, F]
$$

segue da proposição 101 que

$$
\Theta:[F, F] /[K, F] \longrightarrow E
$$

é uma extensão central de E. Provaremos que ela é universal. Seja $(C, \pi)$ uma extensão central qualquer de E. Note que existe um homomorfismo $f: F \longrightarrow C$ tal que

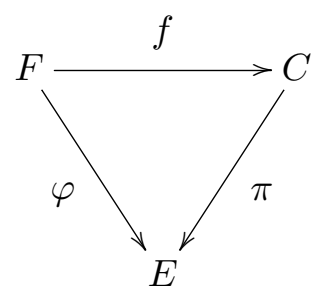

comuta. Agora,

$$
\operatorname{Ker}(\pi) \subseteq Z(C) \Longrightarrow f([K, F])=1
$$

Assim, f induz um homomorfismo

$$
\tilde{f}: F /[K, F] \longrightarrow C
$$

tal que 


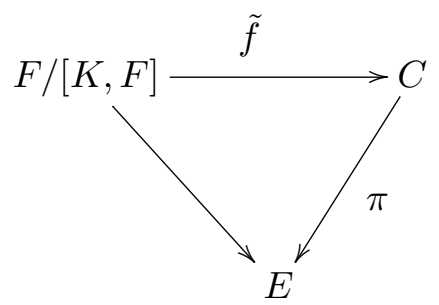

comuta. Aplicando a proposição 100 a restrição

$$
\tilde{f}^{*}:[F, F] /[K, F] \longrightarrow E
$$

conseguimos um único homomorfismo

$$
\gamma:[F, F] /[K, F] \longrightarrow C
$$

tal que

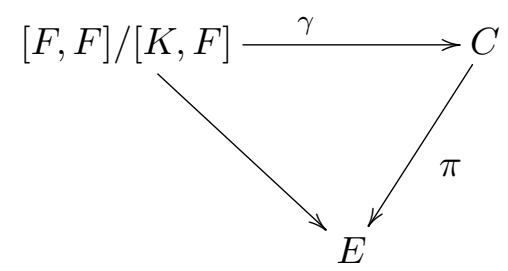

comuta (note que $\gamma$ é único, pois $[E, F] /[K, F]=[F /[K, F], F /[K, F]]$ e este último é perfeito pela proposição 101$)$.

\subsection{2 $\mathrm{St}(\mathrm{R})$ como uma extensão central universal de $\mathrm{E}(\mathrm{R})$}

O principal objetivo desta seção é mostrar que $S t(R)$ é uma extensão central universal de $E(R)$. Já sabemos da proposição 96 (2) que a sequência

$$
1 \longrightarrow K_{2}(R) \longrightarrow S t(R) \stackrel{\theta}{\longrightarrow} E(R) \longrightarrow 1
$$

é uma extensão central de $E(R)$. Para provar que a extensão central $\theta: S t(R) \longrightarrow E(R)$ é universal devemos fabricar para uma extensão central arbitrária $\psi: S \longrightarrow E(R)$ um único homomorfismo $\phi: S t(R) \longrightarrow S$ tal que o diagrama

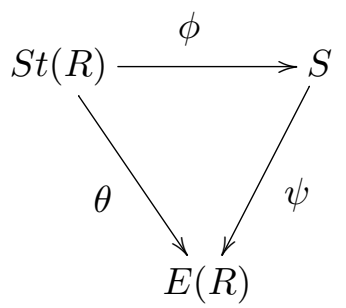

comuta. A estratégia da prova é simples. Nós fabricamos em S um conjunto de elementos $y_{i j}^{\lambda}$ que satisfaçam as relações $\left(\mathbf{E}_{1}\right),\left(\mathbf{E}_{2}\right)$ e $\left(\mathbf{E}_{3}\right)$ (veja a observação $\left.42(3)\right)$ e $\psi\left(y_{i j}^{\lambda}\right)=\psi\left(e_{i j}^{\lambda}\right)$. Por uma combinação de resultados de álgebra, a regra $x_{i j}^{\lambda} \mapsto y_{i j}^{\lambda}$ define o homomorfismo desejado $\phi$. Iniciamos provando algumas identidades em comutadores. 
Lema 54. Seja $G$ um grupo. Para $a, b, c \in G$ valem as seguintes identidades em comutadores

1) $[a b, c][c, a][c, b]=[a,[b, c]]$

2) $[a, c]^{-1}=[c, a]$

3) $[a b, c]=[a,[b, c]][b, c][a, c]$

\section{Demonstração.}

1)

$$
\begin{aligned}
{[a b, c][c, a][c, b] } & =\left(a b c(a b)^{-1} c^{-1}\right)\left(c a c^{-1} a^{-1}\right)\left(c b c^{-1} b^{-1}\right) \\
& =a b c b^{-1} a^{-1} c^{-1} c a c^{-1} a^{-1} c b c^{-1} b^{-1} \\
& =a b c b^{-1} a^{-1} a c^{-1} a^{-1} c b c^{-1} b^{-1} \\
& =a b c b^{-1} c^{-1} a^{-1} c b c^{-1} b^{-1} \\
& =a\left(b c b^{-1} c^{-1}\right) a^{-1}\left(b c b^{-1} c^{-1}\right)^{-1} \\
& =a[b, c] a^{-1}[b, c]^{-1} \\
& =[a,[b, c]]
\end{aligned}
$$

2) Usando o item anterior

$$
\begin{aligned}
{[a \cdot 1, c][c, a][c, 1] } & =[a,[1, c]] \\
{[a, c][c, a] } & =[a, 1] \\
{[a, c][c, a] } & =1
\end{aligned}
$$

2) Combinando 1) e 2) teremos

$$
\begin{aligned}
{[a b, c] } & =[a,[b, c]][c, b]^{-1}[c, a]^{-1} \\
& =[a,[b, c]][b, c][a, c]
\end{aligned}
$$

Lema 55. Se $\phi: G \longrightarrow E$ é uma extensão central, então para a, $b, c, d \in G$ valem

1) $\phi(a)=\phi(b) \quad$ e $\phi(c)=\phi(d) \Longrightarrow[a, c]=[b, d]$

2) $[\phi(a), \phi(b)]=[\phi(a), \phi(c)]=1 \Longrightarrow[a,[b, c]]=1$

Demonstração.

1) Inicialmente observe que

$$
[a, c]=[b, d] \Longleftrightarrow a c a^{-1} c^{-1} d b d^{-1} b^{-1}=1
$$

Por hipótese temos que $a^{-1} b, c^{-1} d \in Z(G)$. Por conseguinte, 


$$
\begin{aligned}
a c a^{-1}\left(c^{-1} d\right) b d^{-1} b^{-1} & =a c a^{-1} b\left(c^{-1} d\right) d^{-1} b^{-1} \\
& =a c\left(a^{-1} b\right) c^{-1}\left(d d^{-1}\right) b^{-1} \\
& =a c\left(a^{-1} b\right) c^{-1} \cdot 1 \cdot b^{-1} \\
& =a\left(a^{-1} b\right)\left(c c^{-1}\right) \cdot 1 \cdot b^{-1} \\
& =\left(a a^{-1}\right) b \cdot 1 \cdot 1 \cdot b^{-1} \\
& =1 \cdot b \cdot 1 \cdot 1 \cdot b^{-1} \\
& =1
\end{aligned}
$$

2) Como $\phi([a, b])=\phi([a, c])=1$, segue que $x \doteq[a, b] \in Z(G)$ e $y \doteq[a, c] \in Z(G)$. Assim,

$$
\phi\left(a b a^{-1}\right)=\phi(x b)=\phi(x) \phi(b)=\phi(b)
$$

e

$$
\phi\left(a c a^{-1}\right)=\phi(y c)=\phi(y) \phi(c)=\phi(c)
$$

E,então pelo item 1) temos

$$
\left[a b a^{-1}, a c a^{-1}\right]=[b, c]
$$

Assim,

$$
\begin{aligned}
{[a,[b, c]] } & =a\left(b c b^{-1} c^{-1}\right) a^{-1}\left(c b c^{-1} b^{-1}\right) \\
& =a b\left(a^{-1} a\right) c\left(a^{-1} a\right) b^{-1}\left(a^{-1} a\right) c^{-1} a^{-1} c b c^{-1} b^{-1} \\
& =\left(a b a^{-1}\right)\left(a c a^{-1}\right)\left(a b^{-1} a^{-1}\right)\left(a c^{-1} a^{-1}\right) c b c^{-1} b^{-1} \\
& =\left(a b a^{-1}\right)\left(a c a^{-1}\right)\left(a b a^{-1}\right)^{-1}\left(a c a^{-1}\right)^{-1} c b c^{-1} b^{-1} \\
& =\left[a b a^{-1}, a c a^{-1}\right][c, b] \\
& =[b, c][c, b] \\
& =1
\end{aligned}
$$

Proposição 104. O homomorfismo $\theta: S t(R) \longrightarrow E(R)$ é uma extensão central universal. Em particular, $S t(R)$ é centralmente fechado.

Demonstração. Seja $\psi: S \longrightarrow E(R)$ uma extensão central qualquer. Para qualquer par $(i, j)$ de inteiros com $i \neq j$ e qualquer $\lambda \in R$ definimos o seguinte subconjunto de $\mathrm{S}$

$$
Y_{i j}^{\lambda} \doteq \psi^{-1}\left(e_{i j}^{\lambda}\right)
$$

Pelo lema 55 (1) temos que o conjunto

$$
\left[Y_{i j}^{\lambda}, Y_{k l}^{\mu}\right] \doteq\left\{[a, b]: a \in Y_{i j}^{\lambda}, b \in Y_{k l}^{\mu} \forall \lambda, \mu \in R\right\}
$$


é unitário. Se $j=k$ e $i \neq l$, então usando $\left(E_{3}\right)$ em $E(R)$, vemos que $\left[Y_{i j}^{\lambda}, Y_{k l}^{\mu}\right]$ está em $Y_{i l}^{\lambda \mu}$.

Afirmação $I:\left[Y_{i j}^{\lambda}, Y_{k l}^{\mu}\right]=1$, se $i \neq l$ e $j \neq k$.

Para ver isto, seja $a \in Y_{i j}^{\lambda}$ e escolhamos $m>i, j, k, l$. Sejam $c \in Y_{k m}^{\mu}, d \in Y_{m l}^{1}$ e ponhamos $b \doteq[c, d]$. Note que $b \in Y_{k l}^{\mu}$. Usando $\left(E_{2}\right)$ em $E(R)$ e o lema 55 (1), vem

$$
a b a^{-1}=a[c, d] a^{-1}=\left[a c a^{-1}, a d a^{-1}\right]=[c, d]=b
$$

e a afirmação está provada.

$\underline{\text { Afirmação } I I:}\left[Y_{i k}^{\lambda}, Y_{k j}^{\mu}\right]=\left[Y_{i m}^{\lambda \mu}, Y_{m j}^{\lambda \mu}\right]$, para $m \neq i, j, k$ e $i \neq j$ e $j \neq k$.

Seja $a \in Y_{i k}^{\lambda}, c \in Y_{k m}^{\mu}$ e $d \in Y_{m j}^{1}$. Seja $b \doteq[c, d]$. Note que $b \in Y_{k j}^{\mu}$. Pela afirmação $I, a d=d a$ e por, simples computação,

$$
a b a^{-1}=a[c, d] a^{-1}=[[a, c] c, d]=[[a, c], b] b[[a, c], d]
$$

Observe que $[a, c] \in Y_{i m}^{\lambda \mu}$, de modo que pela afirmação $I,[[a, c], b]=1$. Assim, $[[a, c], d] \in Y_{i j}^{\lambda \mu}$ comuta com b. Portanto

$$
a b a^{-1}=[[a, c], d] b
$$

e, então

$$
\left[Y_{i k}^{\lambda}, Y_{k j}^{\mu}\right]=\left[Y_{i m}^{\lambda \mu}, Y_{m j}^{1}\right]
$$

Logo, a afirmação está provada.

Tomando $\mu=1$, temos que o único elemento em $\left[Y_{i k}^{\lambda}, Y_{m j}^{1}\right]$ é independente da escolha de $\mathrm{k}$ (distinto de i e j). Definimos

$$
\left\{y_{i j}^{\lambda}\right\} \doteq\left[Y_{i k}^{\lambda}, Y_{k j}^{1}\right]
$$

Note que $y_{i j}^{\lambda} \in Y_{i j}^{\lambda}$. As afirmações I e II nos mostram que $y_{i j}^{\lambda}$ satisfaz as relações $\left(E_{2}\right)$ e $\left(E_{3}\right)$. Resta mostrar que $y_{i j}^{\lambda}$ também satisfaz $\left(E_{1}\right)$. Mas, isto é uma consequência do lema 55 (1) e do lema 54 (3)

$$
\begin{aligned}
y_{i j}^{\lambda+\mu} & =\left[y_{i m}^{\lambda+\mu}, y_{m j}^{1}\right] \\
& =\left[y_{i m}^{\mu} y_{i m}^{\lambda}, y_{m j}^{1}\right] \\
& =\left[y_{i m}^{\mu}, y_{i j}^{\lambda}\right]\left[y_{i m}^{\lambda}, y_{m j}^{1}\right]\left[y_{i m}^{\mu}, y_{m j}^{1}\right] \\
& =y_{i j}^{\lambda} y_{i j}^{\mu}
\end{aligned}
$$

E a existência do homomorfismo $\phi$ está estabelecida. A unicidade segue facilmente : Sejam $\phi^{\prime}$ : $S t(R) \longrightarrow S$ outro homomorfismo tal que $\psi \phi^{\prime}=\theta$. Desde que, $\psi \phi^{\prime}\left(x_{i j}^{\lambda}\right)=e_{i j}^{\lambda}=\psi\left(y_{i j}^{\lambda}\right)$, uma aplicação do lema 55 (1) para $k \neq i, j$ nos fornece 


$$
\phi^{\prime}\left(x_{i j}^{\lambda}\right)=\left[\phi^{\prime}\left(x_{i k}^{\lambda}\right), \phi^{\prime}\left(x_{k j}^{1}\right)\right]=\left[y_{i k}^{\lambda}, x_{k j}^{1}\right]=y_{i j}^{\lambda}
$$

Como $S t(R)$ é gerado pelos símbolos $x_{i j}^{\lambda}$, resulta

$$
\phi^{\prime}=\phi
$$

e o teorema está demonstrado.

A demonstração da proposição anterior pode ser facilmente adaptada para provar a seguinte

Proposição 105. Seja $n \geq 5$. Se $\theta: S t_{n}(R) \longrightarrow E_{n}(R)$ é uma extensão central, então ela é uma extensão central universal.

\subsubsection{O teorema de Matsumoto}

Sejam $\alpha, \beta \in E(R)$ duas matrizes que comutam. Escolhemos $a, b \in S t(R)$ tais que

$$
\theta(a)=\alpha \quad e \quad \theta(b)=\beta
$$

Note que $[a, b]=a b a^{-1} b^{-1} \in K_{2}(R)$. Definimos um produto $\star: E(R) \times E(R) \longrightarrow K_{2}(R)$ da seguinte maneira

$$
\alpha \star \beta \doteq[a, b]
$$

Observe que $\alpha \star \beta$ não depende do par $(a, b)$.Se, por exemplo, $\theta(\bar{a})=\alpha$, então $\bar{a}=a c$ para algum elemento c no centro de $S t(R)$ e, assim

$$
\bar{a} b \bar{a}^{-1} b^{-1}=a\left(c b c^{-1}\right) a^{-1} b^{-1}=a b a^{-1} b^{-1}
$$

Esse produto possui as seguintes propriedades

- (Bimultiplicatividade) Se $\alpha_{1}$ e $\alpha_{2}$ ambos comutam com $\beta$, então

$$
\left(\alpha_{1} \alpha_{2}\right) \star \beta=\left(\alpha_{1} \star \beta\right)\left(\alpha_{2} \star \beta\right)
$$

- (Invariância sob automorfismo interno) Para qualquer $\nu \in E(R)$, temos

$$
\left(\nu \alpha \nu^{-1}\right) \star\left(\nu \beta \nu^{-1}\right)=\alpha \star \beta
$$

- $($ Anti-simetria $) \beta \star \alpha=(\alpha \star \beta)^{-1}$

Agora, seja $A \in G L_{n}(R), B \in G L_{m}(R)$, e seja $I_{n} \in G L_{n}(R), I_{m} \in G L_{m}(R)$, respectivamente, as matrizes identidades. Note que as matrizes $A \otimes I_{m}$ e $I_{n} \otimes B$ de $G L_{m n}(R)$ comutam. Assim, as matrizes diagonais por blocos

$$
\alpha \doteq\left\langle A \otimes I_{m}, A^{-1} \otimes I_{m}, I_{n} \otimes I_{m}\right\rangle
$$

e

$$
\beta \doteq\left\langle I_{n} \otimes B, I_{n} \otimes I_{m}, I_{n} \otimes B^{-1}\right\rangle
$$


de $E_{3 m n}(R)$, também comutam. Definimos

$$
\{A, B\} \doteq \alpha \star \beta \in K_{2}(R)
$$

Isto define uma aplicação anti-simétrica

$$
K_{1}(R) \otimes K_{1}(R) \longrightarrow K_{2}(R)
$$

que é $K_{0}(R)$ - bilinear 12

Em particular, se $m=n=1$, nós temos um produto

$$
\dot{R} \otimes_{\mathbb{Z}} \dot{R} \longrightarrow K_{2}(R)
$$

definido por

$$
(a \otimes b) \mapsto\left(\begin{array}{ccc}
a & 0 & 0 \\
0 & a^{-1} & 0 \\
0 & 0 & 1
\end{array}\right) \star\left(\begin{array}{ccc}
b & 0 & 0 \\
0 & 1 & 0 \\
0 & 0 & b^{-1}
\end{array}\right)
$$

Ele possui as propriedades de Steinberg (ver [Miln4], página 74, lema 9.8)

- $\{a, 1-a\}=1$, desde que $a, 1-a \in \dot{R}$.

- $\{a,-a\}=1, \forall a \in \dot{R}$

Dessa forma, \{\}$: \dot{R} \otimes \dot{R} \longrightarrow K_{2}(R)$ é um 2-símbolo de Steinberg.

Proposição 106. (Matsumoto) Se $\mathbb{K}$ é um corpo, então $K_{2}(\mathbb{K})$ possui uma apresentação como um grupo livre abeliano em símbolos $\{a, b\}$ com $a, b \in \mathbb{K}$ sujeito as seguintes relações

$\left.\boldsymbol{M}_{1}\right)\{a, 1-a\}=1$, para $a \neq 0,1$

$\left.\boldsymbol{M}_{2}\right)\{a b, c\}=\{a, c\}\{b, c\}$.

$\left.M_{3}\right)\{a, b c\}=\{a, b\}\{a, c\}$

Demonstração. Uma prova deste resultado pode ser encontrada em [Miln4] na página 93, capítulo 11.

De acordo com o lema 23 do cap. 2, temos só para grupos de expoente 2 o seguinte

\section{Corolário 21.}

1) $\{a,-a\}=1$

2) $\{a, b\}^{-1}=\{b, a\}$

Observação 46. Observe que em termos de produto tensorial, $K_{2}(\mathbb{K})$ possui a seguinte apresentação

$$
K_{2}(\mathbb{K}) \doteq \frac{\dot{\mathbb{K}} \otimes \dot{\mathbb{K}}}{\langle\{a \otimes(1-a): a \in \dot{K}, a \neq 1\}\rangle}
$$

\footnotetext{
${ }^{12} \mathrm{O}$ leitor interessado em mais detalhes pode consultar Miln4 capítulo 8 na página 63.
} 
onde $\langle\{a \otimes(1-a): a \in \dot{K}, a \neq 1\}\rangle$ é o subgrupo gerado por

$$
\{a \otimes(1-a): a \in \dot{K}, a \neq 1\}
$$

Será essa expressão que Milnor irá se basear em [Miln3] para definir de forma ad hoc a K-teoria algébrica de um corpo, a qual descrevemos no Capítulo 5 desta dissertação.

Seja $\mathbb{K}$ um corpo. Lembremos que um símbolo em $\mathbb{K}$ com valores em um grupo abeliano G é um mapa

$$
(,): \dot{\mathbb{K}} \times \dot{\mathbb{K}} \longrightarrow G
$$

cumprindo os seguintes axiomas

$(,)_{1}:(a b, c)=(a, c)(b, c), \forall a, b, c \in \dot{\mathbb{K}}$.

$(,)_{2}:(a, b)^{-1}=(b, a), \forall a, b \in \mathbb{K}$

$(,)_{3}:(a, 1-a)=1, \forall a \in \mathbb{K} \backslash\{1\}$

Reformulação do teorema de Matsumoto.Existe uma bijeção natural entre símbolos em $\mathbb{K}$ com valores em $G$ e homomorfismos $K_{2}(\mathbb{K}) \longrightarrow G$.

Damos na sequência alguns exemplos de símbolos que surgem, naturalmente, em matemática (para mais detalhes consulte [Serre1], [Tate1] ou [Srin] na página 15).

Exemplo 18. (Símbolo real)O símbolo real é a aplicação

$$
(,)_{\infty}: \mathbb{R} \times \mathbb{R} \longrightarrow\{ \pm 1\}
$$

tal que $(a, b)_{\infty}=-1$, se $a, b<0$ e $(a, b)_{\infty}=1$, caso contrário.

Exemplo 19. (Símbolos de Hilbert) Seja $\mathbb{K}$ um corpo de característica $\neq 2$. Definimos o símbolo quadrático resíduos de Hilbert no corpo $\mathbb{K}$ da seguinte forma

$$
(a, b) \doteq\left\{\begin{array}{rll}
1, & \text { se } & a x^{2}+b y^{2}=1 \text { possui solução em } \mathbb{K} \\
-1, & \text { se } & \text { caso contrário }
\end{array}\right.
$$

Quando $\mathbb{K}$ for um corpo local o símbolo de Hilbert será um símbolo de Steinberg. Em particular, se $\mathbb{K}=\mathbb{R}$, então ele coincide com o símbolo real.

Exemplo 20. (Norma - resíduo)Seja $\mathbb{L}$ um corpo local. As raízes da unidade em $\mathbb{L}$ formam um grupo cíclico finito $\mu$ que coincide com o grupo $\mu_{m}$ das $m$-ésimas raízes da unidade para algum inteiro $m$, com $1 / m \in \mathbb{L}$.

Desde que, $\dot{\mathbb{L}}^{m}$ possui índice finito em $\mathbb{L} ;$ então existe uma extensão de Kummer finita $\mathbb{K} \mid \mathbb{L}$ contendo todas as m-ésimas raízes de todos os elementos de $\mathbb{L}$.

A aplicação

$$
\begin{aligned}
& \Psi: \operatorname{Gal}(\mathbb{K} \mid \mathbb{L}) \quad \longrightarrow \quad \operatorname{Hom}\left(\dot{\mathbb{L}}, \mu_{m}\right) \\
& \phi \quad \begin{aligned}
\quad: \dot{\mathbb{L}} & \longrightarrow \mu_{m} \\
a & \longmapsto \phi(x) / x
\end{aligned}
\end{aligned}
$$

onde $x^{m}=a$, é um isomorfismo.

Seja $N_{\mathbb{K} \mid \mathbb{L}}$ o mapa norma. Por teoria de corpos de classes, temos os seguintes isomorfismos canônicos 


$$
\operatorname{Gal}(\mathbb{K} \mid \mathbb{L}) \cong \operatorname{Hom}\left(\dot{\mathbb{L}}, \mu_{m}\right) \cong N_{\mathbb{K} \mid \mathbb{L}} \dot{\mathbb{K}}
$$

escrito como $x \mapsto(x,-)_{\mathbb{L}}$. O seu adjunto é um mapa bilinear não-degenerado

$$
(,)_{\mathbb{L}}: \dot{\mathbb{L}} \otimes \dot{\mathbb{L}} \longrightarrow \mu_{m}
$$

que é um símbolo de Steinberg.

Exemplo 21. (Símbolo de Tame) Seja $\mathbb{K}$ um corpo com uma valoração discreta ${ }^{13} v$ com corpo residual $\mathbb{K}_{v}$. Seja $K \doteq \operatorname{Ker}(v: \dot{\mathbb{K}} \longrightarrow \mathbb{Z})$ e considere o mapa quociente $\varphi: \mathbb{K} \longrightarrow \mathbb{K}_{v}^{\bullet}$, onde $\mathbb{K}_{v}^{\bullet}$ é o grupo das unidades de $\mathbb{K}_{v}$. Definimos o símbolo de Tame em $v$ como sendo a aplicação

$$
(,)_{v}: \dot{\mathbb{K}} \times \dot{\mathbb{K}} \longrightarrow \mathbb{K}_{v}
$$

definida pela fórmula

$$
(a, b)_{v} \doteq \varphi\left((-1)^{v(a) v(b)} \frac{a^{v(b)}}{b^{v(a)}}\right)
$$

Note que do lado direito da expressão anterior temos exatamente a classe residual do elemento

$$
(-1)^{v(a) v(b)} \frac{a^{v(b)}}{b^{v(a)}}
$$

Ao homomorfismo $K_{2}(\mathbb{K}) \longrightarrow \mathbb{K}_{v}^{\bullet}$ correspondente a (, ), também o chamaremos de símbolo de tame.

Exemplo 22. (Símbolo de Galois) Seja $\mathbb{K}$ um corpo e $n \neq$ char $(\mathbb{K})$ um inteiro positivo.Suponhamos que $\mathbb{K}$ contém as n-ésimas raízes da unidade. Seja $\zeta \in \mathbb{K}$ um n-ésima raiz da unidade primitiva. Chamamos de símbolo de Galois de ordem $n$ ao mapa

$$
\begin{aligned}
(,): \dot{\mathbb{K}} \times \dot{\mathbb{K}} & \longrightarrow{ }_{n} B r(\mathbb{K}) \\
(a, b) & \longmapsto\left[D_{\zeta}(a, b)\right]
\end{aligned}
$$

onde ${ }_{n} \operatorname{Br}(\mathbb{K})$ é o $n$ - subgrupo de torsão do grupo de Brauer $\operatorname{Br}(\mathbb{K})$ e $D_{\zeta}(a, b)$ é a álgebra cíclica de dimensão $n^{2}$ sobre $\mathbb{K}$ com geradores $X, Y$ satisfazendo as relações

$$
X^{n}=a, Y^{n}=b, X Y=\zeta Y X
$$

Exemplo 23. (Símbolo diferencial) Seja $\mathbb{K}$ um corpo. $O \mathbb{K}$ - espaço vetorial de diferenciais absolutos de Kähler (denotado por $\Omega_{\mathbb{K}}^{1} \doteq \Omega_{\mathbb{K} / \mathbb{Z}}^{1}$ )é o $\mathbb{K}$-espaço vetorial gerado por símbolos $\{d x$ : $x \in \mathbb{K}\}$ sujeito as relações

$$
d(x y)=x d y+y d x, \forall x, y \in \mathbb{K}
$$

Seja $\Omega_{\mathbb{K}}^{2} \doteq \bigwedge_{\mathbb{K}}^{2} \Omega_{\mathbb{K}}^{1}$ a segunda potência exterior de $\Omega_{\mathbb{K}}^{1}$. O símbolo diferencial é o mapa

$$
\dot{\mathbb{K}} \times \dot{\mathbb{K}} \longrightarrow \Omega_{\mathbb{K}}^{2}
$$

definido por

$$
(x, y) \mapsto \frac{d x}{x} \wedge \frac{d y}{y}
$$

\footnotetext{
${ }^{13}$ Consulte o capítulo V para mais informações sobre valorações discretas e corpos residuais.
} 
para todos $x, y \in \mathbb{K}$. O homomorfismo $\phi: K_{2}(\mathbb{K}) \longrightarrow \Omega_{\mathbb{K}}^{2}$ associado a este símbolo é tal que

$$
\operatorname{Im}(\phi) \subseteq \operatorname{Ker}\left(d: \Omega_{\mathbb{K}}^{2} \longrightarrow \Omega_{\mathbb{K}}^{3}\right)
$$

onde d é o diferencial de De Rham.

Terminamos esta seção com a computação do $K_{2}$ de um corpo finito.

Exemplo 24. Se $\mathbb{K}_{q}$ é o corpo finito com q elementos, então $K_{2}\left(\mathbb{K}_{q}\right)=1$

Prova. Vamos provar isso usando a proposição 106 . Inicialmente,observe que $K_{2}\left(\mathbb{K}_{q}\right)$ é um grupo cíclico. De fato, se $\{\alpha, \beta\} \in K_{2}\left(\mathbb{K}_{q}\right)$, então como $\mathbb{K}_{q}$ é cíclico, digamos, gerado por $\xi$; existe $r, s \in \omega$ tais que

$$
\{\alpha, \beta\}=\left\{\xi^{r}, \xi^{s}\right\}=\{\xi, \xi\}^{r s}
$$

e, portanto $\{\xi, \xi\}$ é um gerador de $K_{2}\left(\mathbb{K}_{q}\right)$.Agora, temos dois casos, a saber $\operatorname{char}\left(\mathbb{K}_{q}\right)=2$ ou $\operatorname{char}\left(\mathbb{K}_{q} \neq 2\right)$.

- Se $\operatorname{char}\left(\mathbb{K}_{q}\right)=2$, então $\{-\xi, \xi\}=\{\xi, \xi\}=\{\xi,-\xi\}=1$.

- Se $\operatorname{char}\left(\mathbb{K}_{q}\right) \neq 2$, então

$$
\xi^{q-1 / 2}=-1 \Longrightarrow\{\xi, \xi\}^{q+1 / 2}=\left\{\xi, \xi^{q+1 / 2}\right\}=\{\xi,-\xi\}=1
$$

de modo que $\{\xi, \xi\}$ possui ordem par. Agora, note que $\dot{K}_{q} \backslash\{1\}$ é invariante sob a bijeção

$$
\begin{aligned}
\Psi: \dot{\mathbb{K}}_{q} \backslash\{1\} & \longrightarrow \dot{\mathbb{K}}_{q} \backslash\{1\} \\
x & \longmapsto 1-x
\end{aligned}
$$

Desde que, existem $(q-1) / 2$ não-quadrados e $(q-3) / 2$ quadrados em $\mathbb{K}$; deve existir um não-quadrado $x$ tal que $1-x$ é o não-quadrado. Então, existem inteiros $i, j$ ímpares tais que $x=\xi^{i}$ e $\xi^{j}=1-x$. Por conseguinte,

$$
1=\{x, 1-x\}=\left\{\xi^{i}, \xi^{j}\right\}=\{\xi, \xi\}^{i j}
$$

com ij ímpar. Assim, $\{\xi, \xi\}$ é trivial.

\section{4 $\quad \mathrm{K}_{1}(\mathrm{R})$ e $\mathrm{K}_{2}(\mathrm{R})$ como grupos de (co)homologia}

Como havíamos dito na introdução deste capítulo. K-teoria algébrica mantém conexões estreitas com a (co)homologia de grupos clássicos. Nesta seção, daremos indicações dessa relação nos níveis 1 e 2 .

Definição 84. Seja $G$ um grupo e $\mathbb{Z} G$ o anel de grupos com coeficientes inteiros ${ }^{14}$. Chamamos de homomorfismo aumento ao homomorfismo de anéis

$$
\begin{aligned}
& \varepsilon: \mathbb{Z} G \longrightarrow \mathbb{Z} \\
& \sum_{g \in G} n_{g} g \longmapsto \sum_{g \in G} n_{g}
\end{aligned}
$$

\footnotetext{
${ }^{14}$ Consulte o apêndice B desta dissertação para mais informações sobre o anel de grupos de um anel.
} 
O núcleo desse homomorfismo é chamado de ideal aumento, e corriqueiramente denotado por $I G$.

Seja A um G-módulo. Seja $A_{G}$ o maior quociente de A, no qual G age trivialmente. Assim,

$$
A_{G} \doteq \frac{A}{\langle\{g a-a: g \in G, a \in A\}\rangle}
$$

\section{Lema 56.}

1) O grupo aditivo do ideal aumento IG é um grupo livre abeliano com base

$$
\{g-1: g \in \dot{G}\}
$$

onde $\dot{G}=\{g \in G: g \neq 1\}$.

2) $I^{2} G \doteq I G \cdot I G$ é um subgrupo abeliano de $\mathbb{Z} G$ gerado pelo conjunto

$$
\{(g-1)(h-1): g, h \in G\}
$$

3) $A_{G}=A / I(G A) \cong H_{0}(G, A)$

Demonstração.

1) Seja $x=\sum_{n_{g}} g \in \mathbb{Z} G$. Note que

$$
x \in I G \Longleftrightarrow \sum_{g \in G} n_{g}=0
$$

assim,

$$
x=x-\left(\sum_{g \in G} n_{g}\right) \cdot 1=\sum_{g \in \dot{G}} n_{g}(g-1)
$$

ou seja, IG é gerado aditivamente pelo conjunto $\{g-1: g \in \dot{G}\}$. Finalmente, suponhamos que

$$
\sum_{g \in \dot{G}} n_{g}(g-1)=0
$$

então,

$$
\sum_{g \in \dot{G}} n_{g} g-\left(\sum_{g \in \dot{G}} n_{g}\right) 1=0
$$

mas, desde que $\mathbb{Z} G$ é um grupo abeliano livre, com base $\{g \in G\}$ (como grupo abeliano), segue que $n_{g}=0, \forall g \in \dot{G}$.

Os itens 2) e 3) seguem imediatamente de 1).

A sequência exata

$$
0 \longrightarrow I G \longrightarrow \mathbb{Z} G \longrightarrow \mathbb{Z} \longrightarrow 0
$$

origina a seguinte sequência 


$$
H_{1}(G, \mathbb{Z} G) \longrightarrow H_{1}(G, \mathbb{Z}) \longrightarrow H_{0}(G, I G) \stackrel{\lambda}{\longrightarrow} H_{0}(G, \mathbb{Z} G) \longrightarrow H_{0}(G, \mathbb{Z})
$$

O G-módulo $\mathbb{Z} G$ é projetivo, pois é um $\mathbb{Z} G$-módulo livre. Dessa forma,

$$
H_{1}(G, \mathbb{Z} G)=0
$$

Além disso, existem isomorfismos

1. $H_{0}(G, I G) \cong I G / I^{2} G$;

2. $H_{0}(G, \mathbb{Z} G) \cong \mathbb{Z} G / I G$;

3. $H_{0}(G, \mathbb{Z}) \cong \mathbb{Z}$

Portanto, a sequência exata $(\dagger)$ converte-se-á na sequência exata

$$
0 \longrightarrow H_{1}(G, \mathbb{Z}) \longrightarrow I G / I^{2} G \stackrel{\lambda}{\longrightarrow} \mathbb{Z} G / I G \longrightarrow \mathbb{Z} \longrightarrow 0
$$

onde $\lambda$ é induzido pelo mapa inclusão $I G \longleftrightarrow \mathbb{Z} G$. Assim, a sequência exata (††) nos fornece a seguinte

Proposição 107. Existem isomorfismos naturais

$$
H_{1}(G, \mathbb{Z}) \cong I G / I^{2} G, \quad(\mathbb{Z} G)_{G} \cong \mathbb{Z}
$$

Proposição 108. A aplicação

$$
\begin{aligned}
\phi: G & \longrightarrow \\
g & \longmapsto(g-1)+I^{2} G \\
&
\end{aligned}
$$

induz um isomorfismo

$$
\Phi: \begin{array}{ccc}
G^{a b} & \longrightarrow & I G / I^{2} G \\
g+[G, G] & \longmapsto & (g-1)+I^{2} G
\end{array}
$$

Demonstração. A aplicação $\phi$ é um homomorfismo, pois

$$
\begin{aligned}
g h-1 & =(g-1)(h-1)+(g-1)+(h-1) \\
& \equiv(g-1)+(h-1)\left(\bmod I^{2} G\right)
\end{aligned}
$$

para todos $g, h \in G$. Desde que, $I G / I^{2} G$ é abeliano, existe um único homomorfismo $\Phi$ tal que

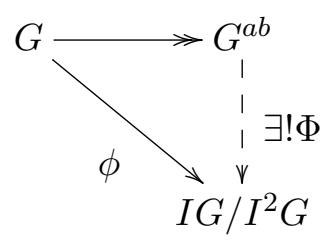

comuta. Para mostrar que $\Phi$ é um isomorfismo, exibimos o seu inverso. Considere o único homomorfismo 


$$
\psi: I G \longrightarrow G^{a b}
$$

tal que $g-1 \mapsto g+[G, G]$ (pois IG é abeliano livre). Segue da identidade

$$
(g-1)(h-1)=(g h-1)-(g-1)-(h-1)
$$

que $\psi((g-1)(h-1))=1$. Portanto, existe um único homomorfismo $\Psi$ tal que o diagrama

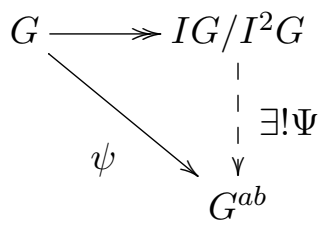

comuta . Os homomorfismos $\Phi$ e $\Psi$ são inversos um do outro.

combinando as proposições 107 e 108 ,obtemos

Proposição 109. $H_{1}(G, \mathbb{Z}) \cong G^{a b}$

Corolário 22. $H_{1}(G L(R), \mathbb{Z}) \cong K_{1}(R)$

Terminamos esta seção esboçando uma demonstração da fórmula de Hopf, e por conseguinte fornecendo um descrição homológica do terceiro grupo da K-teoria algébrica clássica.

Começamos com o seguinte fato (muito conhecido em álgebra homólógica)

Fato 30. (Sequência exata de 5 termos)Seja A um G-módulo. Cada sequência exata de grupos

$$
1 \longrightarrow K>F \longrightarrow G \longrightarrow 1
$$

determina uma sequência exata

$$
H_{2}(F, A) \rightarrow H_{2}(G, A) \rightarrow H_{0}\left(G, H_{1}(K, A)\right) \rightarrow H_{1}(F, A) \rightarrow H_{1}(G, A) \rightarrow 0
$$

Alternativamente,

$$
H_{2}(F, A) \rightarrow H_{2}(G, A) \rightarrow A \otimes_{G} K^{a b} \rightarrow A \otimes_{F} I F \rightarrow A \otimes_{G} I G \rightarrow 0
$$

No caso específico $A=\mathbb{Z}$ (com $\mathrm{G}$ agindo trivialmente em $\mathbb{Z}$ ), os isomorfismos

1. $H_{1}(F, \mathbb{Z}) \cong F^{a b}$

2. $H_{1}(G, \mathbb{Z}) \cong G^{a b}$

3. $\mathbb{Z} \otimes_{G} K^{a b} \cong K /[F, K]$

fornecem-nos a seguinte sequência exata

$$
H_{2}(F, \mathbb{Z}) \rightarrow H_{2}(G, \mathbb{Z}) \rightarrow K /[F, K] \rightarrow F^{a b} \rightarrow G^{a b} \rightarrow 0
$$

se, em adição $\mathrm{F}$ for um grupo livre, então a sequência exata anterior converte-se em 


$$
H_{2}(G, \mathbb{Z}) \rightarrow K /[F, K] \rightarrow F /[F, F] \rightarrow G /[G, G] \rightarrow 0
$$

e, portanto temos a fórmula de Hopf

$$
\left.H_{2}(G, \mathbb{Z}) \cong \operatorname{Ker}(K /[F, K]) \longrightarrow F /[F, F]\right) \cong K \cap[F, F] /[K, F]
$$

Seja $\Theta:[F, F] /[K, F] \longrightarrow E$ a extensão central que aparece na demonstração da proposição 103 Note que

$$
\operatorname{Ker}(\Theta) \cong(K \cap[F, F] /[K, F])
$$

Portanto, existe um isomorfismo canônico

$$
H_{2}(E, \mathbb{Z}) \cong \operatorname{Ker}(\Theta)
$$

em particular, se $E=E(R)$ é o grupo das matrizes elementares sobre um anel R, então

$$
H_{2}(E(R), \mathbb{Z}) \cong \operatorname{Ker}(\theta: S t(R) \longrightarrow E(R))=K_{2}(R)
$$

subfile 


\section{Capítulo 5}

\section{A K-teoria de Milnor e a Conjectura de Marshall}

Neste capítulo final, fechamos esta dissertação através de duas seções que correlacionam definições, resultados e questões postos nos outros capítulos. Na primeira seção, apresentaremos as definições e resultados principais a respeito da construção de John Milnor, realizada na década de 1970, sobre uma K-teoria ad hoc adequada para atacar problemas postos em teoria algébrica de formas quadráticas. Na seção final, apresentamos a conjectura de Murray Marshall sobre assinaturas e esboçamos a solução desta apresentada em [DM2], através da introdução do conceito de grupo especial ([DM1 $)$, e de interligações entre os anéis graduados: de Witt, de k-teoria reduzida de Milnor e de cohomologia.

\subsection{A K-teoria de Milnor}

As principais referências para esta seção são [Miln3] e [Miln4].

\subsubsection{A K-teoria de Milnor de um corpo}

Olhando $K_{1}(\mathbb{K})$ como um $\mathbb{Z}$ - módulo podemos considerar a álgebra tensorial graduada

$$
\text { Tens }_{\mathbb{Z}}\left(K_{1}(F)\right)=\left\langle\mathbb{Z}, K_{1}(F), K_{1}(F) \otimes K_{1}(F), \cdots, K_{1}(F)^{\otimes^{n}}, \cdots\right\rangle
$$

Transformamos corretamente $K_{1}(\mathbb{K})$ em um grupo aditivo mediante o isomorfismo canônico $l: \dot{\mathbb{K}} \longrightarrow K_{1}(\mathbb{K}), a \mapsto l(a)$, onde $l(a b)=l(a)+l(b)$. Na sequência representamos por $\mathfrak{M}$ o ideal homogêneo de $\operatorname{Tens}_{\mathbb{Z}}\left(K_{1}(\mathbb{K})\right)$ gerado por $l(a) l(1-a)$, para todo $a \neq 0,1$.

Definição 85. Seja $\mathbb{K}$ um corpo. A K-teoria de Milnor do corpo $\mathbb{K}$ é definida por

$$
K_{\star}^{M}(\mathbb{K}) \doteq \frac{T e n_{\mathbb{Z}}\left(K_{1}(\mathbb{K})\right.}{\mathfrak{M}}
$$

$A$ classe de $l\left(a_{1}\right) \otimes \ldots \otimes l\left(a_{n}\right)$ em $K_{\star}(\mathbb{K})$ será representada por $l\left(a_{1}\right) \ldots l\left(a_{n}\right)$.

Fato 31 .

1) O anel $K_{\star}^{M}(\mathbb{K})$ é dado por geradores $\{l(a): a \in \dot{K}\}$ e relações

$\mathcal{R}_{1}^{\star}: l(a b)=l(a)+l(b), \forall a, b \in \dot{K}$

$\mathcal{R}_{2}^{\star}: l(a) l(1-a), \forall a \in \dot{K}, a \neq 1$ 
2)Os níveis do anel de Milnor são dados pelos $K_{n}(\mathbb{K})$. Cada $K_{n}(\mathbb{K})$ é gerado pelos símbolos $l\left(a_{1}\right) l\left(a_{2}\right) \ldots l\left(a_{n}\right)$ módulo as seguintes relações

$\mathcal{R}_{1}: l\left(a_{1}\right) l\left(a_{2}\right) \ldots l\left(a_{n}\right)$,onde $a_{i}+a_{i+1}=1$ para algum $1 \leq i \leq n-1$

$\mathcal{R}_{2}: l\left(a_{1}\right) \ldots l\left(a_{i} b_{i}\right) \ldots l\left(a_{n}\right)-l\left(a_{1}\right) \ldots l\left(a_{i}\right) \ldots l\left(a_{n}\right)-l\left(a_{1}\right) \ldots l\left(b_{i}\right) \ldots l\left(a_{n}\right)$

Em outras palavras, $K_{\star}^{M}(\mathbb{K})$ é o anel graduado

$$
K_{\star}^{M}(\mathbb{K})=\left(K_{1}(\mathbb{K}), K_{2}(\mathbb{K}), \ldots, K_{n}(\mathbb{K}), \ldots\right)
$$

3) $\mathfrak{M}=\left(\mathfrak{M} 1, \mathfrak{M}_{2}, \cdots, \mathfrak{M}, \cdots\right)$ é um ideal graduado de $\operatorname{Tens}_{\mathbb{Z}}\left(K_{1}(\mathbb{K})\right)$.

\section{Observação 47.}

1) Temos os seguintes isomorfismos

- $K_{0}^{M}(F) \cong \mathbb{Z} \cong K_{0}(F)$.

- $K_{1}^{M}(F) \cong \dot{\mathbb{F}} \cong K_{1}(F)$.

- $K_{2}^{M}(F) \cong K_{2}(F)$ (Teorema de Matsumoto).

2) $K_{n}^{M}(F)$ é $n$ - Steinberg universal.

\section{Lema 57.}

(1) $\forall a, b \in \dot{\mathbb{K}}(a+b \in\{0,1\} \Longrightarrow l(a) l(b)=0)$;

(2) $\forall a_{1}, \ldots, a_{n} \in \dot{\mathbb{K}} \in\{0,1\} \Longrightarrow l\left(a_{1}\right) \ldots l\left(a_{n}\right)=0$;

(3) $\forall a \in \dot{\mathbb{K}}\left(l(a)^{2}=l(a) l(-1)=l(-1) l(a)\right)$;

(4) $\forall a, b \in \dot{\mathbb{K}}(l(a) l(b)=-l(b) l(a))$;

(5) $\forall a, b \in \mathbb{K}\left(c \doteq a+b \neq 0 \Longrightarrow l(c) l\left(-b a^{-1}\right)=l\left(-b a^{-1}\right) l(c)\right)=l(a) l(b)$;

(6) $\forall a_{1}, \ldots, a_{n} \in \dot{\mathbb{K}} \forall \sigma \in \mathfrak{S}_{n}\left(l\left(a_{\sigma(1)}\right) \ldots l\left(a_{\sigma(n)}\right)=(-1)^{t} l\left(a_{1}\right) \ldots l\left(a_{n}\right)\right)$, onde $\sigma=\prod_{j=1}^{t} \tau_{j}$ e cada $\tau_{j}$ é uma transposiçẫo de $\mathfrak{S}_{n}$.

Demonstração.

$(1) \forall a \neq 1\left(l(a) l(1-a)=l\left(a^{-1}\right) l\left(1-a^{-1}\right)=0\right)$. Assim,

$$
\begin{aligned}
l(a) l(-a) & =l(a) l(-a)+l(a) l\left(1-a^{-1}\right) \\
& =l(a)\left[l(-a)+l\left(1-a^{-1}\right)\right] \\
& =l(a) l(1-a) \\
& =0
\end{aligned}
$$

(2)Se $n=1$, a afirmação é trivial. Se $n=2$ é o ítem anterior. Suponhamos $3 \leq n$. Se $b \doteq a_{1}+a_{2}=0$ terminamos; caso contrário

$$
l\left(a_{1} b^{-1}\right) l\left(a_{2} b^{-1}\right)=0 \Longrightarrow l\left(a_{1}\right) l\left(a_{2}\right)=l\left(a_{1}\right) l(b)+l(b) l\left(a_{2}\right)-l^{2}(b)
$$

Assim,

$$
\begin{aligned}
l\left(a_{1}\right) \ldots l\left(a_{n}\right) & =l\left(a_{1}\right) l\left(a_{2}\right)\left[l\left(a_{3}\right) \ldots l\left(a_{n}\right)\right] \\
& =l\left(a_{1}\right) l(b) \ldots l\left(a_{n}\right)-l\left(a_{2}\right) l(b) \ldots l\left(a_{n}\right)-l(b) l(b) l\left(a_{3}\right) \ldots l\left(a_{n}\right) \\
& =0
\end{aligned}
$$


(3) Decorre de (2). De fato, basta observar que

$$
l((-1)(-a)) l(a)=[l(-1)+l(-a)] l(a)=l(-1) l(a)+l(-a) l(a)=l(-1) l(a)
$$

e,

$$
l(a) l((-1)(-a))=l(a)[l(-1)+l(-a)]=l(a) l(-1)+l(a) l(-a)=l(a) l(-1)
$$

(4)Decorre de (2).

$$
\begin{aligned}
l(a) l(b)+l(b) l(a) & =l(a) l(b)+l(a) l(-a)+l(b) l(a)+l(b) l(-b) \\
& =l(a)[l(b)+l(-a)]+l(b)[l(a)+l(-b)] \\
& =l(a) l(-a b)+l(b) l(-a b) \\
& =l(a b) l(-a b) \\
& =0
\end{aligned}
$$

(5)

$$
\begin{aligned}
l(a) l(b)-l(c) l\left(-b a^{-1}\right) & =l(a) l(b)-l(c)\left[l(-b)+l\left(a^{-1}\right)\right] \\
& =l(a) l(b)-l(c)[l(-1)+l(b)-l(a)] \\
& =l(a) l(b)-l(c) l(-1)-l(c) l(b)+l(c) l(a) \\
& =l(a) l(b)-l(c) l(b)+l(c) l(a)-l(c) l(-1) \\
& =[l(a)-l(c)] l(b)+l(c) l(a)-l(c) l(c) \\
& =l\left(a c^{-1}\right) l(b)+l(c)[l(a)-l(c)] \\
& =l\left(a c^{-1}\right) l(b)+l(c) l\left(a c^{-1}\right) \\
& =l\left(a c^{-1}\right) l(b)-l\left(a c^{-1}\right) l(c) \\
& \left.=l\left(a c^{-1}\right)[l(b)]-l(c)\right] \\
& =l\left(a c^{-1}\right) l\left(b c^{-1}\right) \\
& =0
\end{aligned}
$$

A outra igualdade é provada de maneira similar.

(6) Aplique o ítem (4).

Proposição 110. $\forall \alpha \in K_{m}(\mathbb{K}) \forall \beta \in K_{n}(\mathbb{K})\left(\alpha \beta=(-1)^{m n} \beta \alpha\right)$

Demonstração. Basta usar indução em n e depois em m. Faremos somente em n e nos geradores. Inicialmente, segue por indução a fórmula:

$$
\forall a_{1}, \ldots a_{m}, b \in \mathbb{K} \forall m \in \omega\left(l\left(a_{1}\right) \ldots l\left(a_{m}\right) l(b)=(-1)^{m} l(b) l\left(a_{1}\right) \ldots l\left(a_{m}\right)\right.
$$

De fato,

$$
\begin{aligned}
l\left(a_{1}\right) \ldots l\left(a_{m}\right) l\left(a_{m+1}\right) l(b) & =l\left(a_{1}\right) \ldots l\left(a_{m}\right)(-1) l(b) l\left(a_{m+1}\right) \\
& =(-1) l\left(a_{1}\right) \ldots l\left(a_{m}\right) l(b) l\left(a_{m+1}\right) \\
& =(-1)(-1)^{m} l(b) l\left(a_{1}\right) \ldots l\left(a_{m}\right) l\left(a_{m+1}\right) \\
& =(-1)^{m+1} l(b) l\left(a_{1}\right) \ldots l\left(a_{m}\right) l\left(a_{m+1}\right)
\end{aligned}
$$

Finalmente, 


$$
\begin{aligned}
l\left(a_{1}\right) \ldots l\left(a_{m}\right) l\left(b_{1}\right) \ldots l\left(b_{n}\right) l\left(b_{n+1}\right) & =(-1)^{m n} l\left(b_{1}\right) \ldots l\left(b_{n}\right) l\left(a_{1}\right) \ldots l\left(a_{m}\right) l\left(b_{n+1}\right) \\
& =(-1)^{m n} l\left(b_{1}\right) \ldots l\left(b_{n}\right)(-1)^{m} l\left(b_{n+1}\right) \prod_{i=1}^{m} l\left(a_{i}\right) \\
& =(-1)^{m(n+1)} l\left(b_{1}\right) \ldots l\left(b_{n+1}\right) l\left(a_{1}\right) \ldots l\left(a_{n}\right)
\end{aligned}
$$

Apresentamos agora uma série de exemplos ilustrativos:

Exemplo 25. (n-ésimo grupo de K-teoria de um corpo finito)Se $\mathbb{K}$ for um corpo finito, então $K_{n} \mathbb{K}=0$, para todo $n \geq 2$. De fato, vimos no exemplo 24 do capitulo 4 que $K_{2} \mathbb{K}=0$. Por outro lado, $K_{n} \mathbb{K}$ é gerado por $K_{2} \mathbb{K} \cdot K_{n-2} \mathbb{K}$ e, portanto, $K_{n} \mathbb{K}=0$, para todo $n \geq 2$.

Exemplo 26. (extensão do símbolo real)A aplicação

$$
\begin{aligned}
{[]_{\infty}: \dot{\mathbb{R}} \times \dot{\mathbb{R}} } & \longrightarrow \mathbb{Z} / 2 \mathbb{Z} \\
(x, y) & \longmapsto[x, y]_{\infty}
\end{aligned}
$$

definida por $[x, y]_{\infty} \doteq \frac{1}{2}(1-\sigma(x))(1-\sigma(y))$ é chamada de simbolo real, onde $\sigma(z)=0$ ou $\sigma(z)=1$, conforme z seja positivo ou negativo. É fácil ver que tal aplicação é um 2-símbolo de Steinberg tal que $[x, y]_{\infty}=0$ sempre que $x+y=1$. Assim, existe um único homomorfismo (pela proposição 106 e observação 46$)[]_{\infty}^{*}: K_{2}(\mathbb{R}) \longrightarrow \mathbb{Z} / 2 \mathbb{Z}$ que torna o seguinte diagrama comutativo

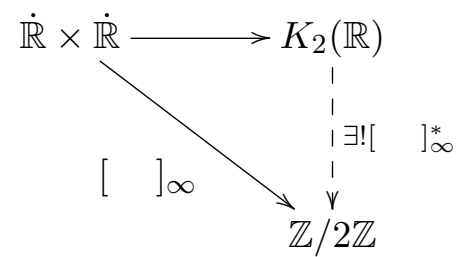

ou seja, $[l(x), l(y)]_{\infty}^{*}=[x, y]_{\infty}$. Usando a extensão do símbolo real, é possível "quebrar a complexidade de $K_{2}(\mathbb{R})$ ". Explicitamente,

$$
K_{2}(\mathbb{R}) \cong \mathbb{Z} / 2 \mathbb{Z} \oplus G
$$

onde $G$ é um grupo divisivel.

Exemplo 27. (Bass - Tate)Seja $m \in \mathbb{Z}$ e $m \geq 1$. Suponhamos que para cada $p(x) \in \mathbb{K}[x]$ tal que $p(x)=x^{m}-a$ valha:

$$
x^{m}-a=\prod_{i=1}^{m}\left(x-\xi_{i}\right)
$$

com $a=\xi_{i}^{m}$. Então, $K_{n} \mathbb{K}$ é um grupo unicamente divísivel por $m$, para $m \geq 2$.

Prova. Inicialmente considere a seguinte:

Afirmação: $S e A$ e $B$ são grupos abelianos unicamente divisíveis por $m$, então $A \otimes B$ é unicamente divisivel por $m$. 
Prova. Para cada $r \geq 1$, seja $m^{r}: A \longrightarrow A$ a multiplicação por $m^{r}$. Considere a parte m-primária de A

$$
A_{m} \doteq \bigcup_{r \geq 1} \operatorname{Ker}\left(m^{r}\right)
$$

Desde que, A é unicamente divisível por $\mathrm{m}$, segue que $A_{m} \otimes B=0$. Tensorando a sequência exata

$$
0 \longrightarrow A \stackrel{m}{\longrightarrow} A \stackrel{\pi}{\longrightarrow} A / A_{m} \longrightarrow 0
$$

obtemos uma sequência exata

$$
0 \longrightarrow A \otimes B \stackrel{m \otimes B}{\longrightarrow} A \otimes B \stackrel{\pi \otimes B}{\longrightarrow} A / A_{m} \otimes B \longrightarrow 0
$$

e como $\operatorname{ker}(\pi \otimes B)=A_{m} \otimes B=0$, segue que $A \otimes B \cong A / A_{m} \otimes B$. Mas, a multiplicação por m é um isomorfismo em $A / A_{m}$ e, assim em $A / A_{m} \otimes B$.

Considere o diagrama

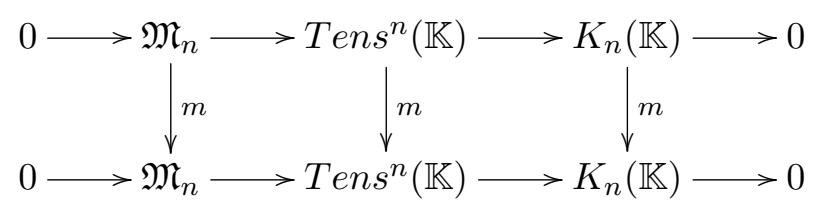

onde $\mathfrak{M}=\left(\mathfrak{M}_{1}, \mathfrak{M}_{2}, \ldots, \mathfrak{M}_{n}, \ldots\right)$. Se mostrarmos que

(i) $m:$ Tens $^{n}(\dot{\mathbb{K}}) \longrightarrow$ Tens $^{n}(\dot{\mathbb{K}})$ é bijetivo, para $n \geq 2$.

(ii) $m: \mathfrak{M}_{n} \longrightarrow \mathfrak{M}_{n}$ é sobrejetivo.

então, concluiremos usando o Lema dos cinco que $m: K_{n} \mathbb{K} \longrightarrow K_{n} \mathbb{K}$ é bijetivo. O item (i) resulta da afirmação anterior, uma vez que $\dot{K}$ é divisível por $\mathrm{m}$. Finalmente, para o ítem (ii) é suficiente mostrar que $l(a) l(1-a) \in m \mathfrak{M}_{2}, \forall a \neq 0,1$. Por hipótese,

$$
x^{m}-a=\prod_{i=1}^{m}\left(x-\xi_{i}\right)
$$

onde cada $\xi_{i} \in \mathbb{K}$ e $a=\xi_{i}^{n}$. Assim,

$$
\begin{aligned}
l(a) l(1-a) & =l(a) l\left(\prod_{i=1}^{n} 1-\xi_{i}\right) \\
& =l(a)\left(\sum_{i=1}^{n} l\left(1-\xi_{i}\right)\right) \\
& =\sum l(a) l\left(1-\xi_{i}\right) \\
& =\sum l\left(\xi_{i}^{m}\right) l\left(1-\xi_{i}\right) \\
& =m \sum l\left(\xi_{i}\right) l\left(1-\xi_{i}\right)
\end{aligned}
$$


Exemplo 28. Se $\mathbb{K}$ é algebricamente fechado, então $K_{n} \mathbb{K}$ é livre de torsão e divisível p, para todo $n \geq 2$.

Exemplo 29. Se $\mathbb{K}$ é um corpo perfeito de característica $p>0$, então $K_{n} \mathbb{K}$ é unicamente divisível por $p$, para $n \geq 2$.

Lema 58. As seguintes afirmações valem:

(1) $\forall \alpha \in K_{n} \exists \varepsilon \in\{ \pm 1\}(\mathbb{K}), \beta \in K_{m}(\mathbb{K})\left((\alpha \cdot \beta)^{s}=\varepsilon \beta^{s} \cdot \alpha^{s}\right)$

(2) $\forall a_{1}, \ldots, a_{n} \in \mathbb{K} \forall n, s \in \omega \exists \varepsilon \in\{ \pm 1\}\left(\prod_{i=1}^{n} l\left(a_{i}\right)\right)^{s}=\varepsilon \prod_{i=1}^{n} l^{s}\left(a_{i}\right)$.

(3) $\forall a \in \mathbb{K} \forall s \in \omega\left(l^{s}(a)=l^{s-1}(-1) l(a)=l(a) l^{s-1}(-1)\right)$.

(4) $\forall a_{1}, \ldots, a_{n} \in \mathbb{K} \forall n, s \in \omega \exists \varepsilon \in\{ \pm 1\}\left(\left(\prod_{i=1}^{n} l\left(a_{n}\right)\right)^{s}=\varepsilon l(-1)^{n(s-1)} \prod_{i=1}^{n} l\left(a_{i}\right)\right.$

Demonstração.

1)Indução em $s \geq 1$. O caso $n=1$ é claro.

$$
\begin{aligned}
(\alpha \cdot \beta)^{s+1} & =(\alpha \cdot \beta)^{s}(\alpha \cdot \beta) \\
& =\varepsilon \beta^{s} \alpha^{s} \alpha \beta \text { (Hipótese de indução) } \\
& =\varepsilon \beta^{s} \alpha^{s+1} \beta \\
& =\varepsilon^{\prime} \beta^{s+1} \alpha^{s+1}
\end{aligned}
$$

(2)Indução em $n \geq 1$. O caso $n=1$ é claro.

$$
\begin{aligned}
\left(\prod_{i=1}^{n+1} l\left(a_{i}\right)\right)^{s} & =\varepsilon^{\prime}\left(\prod_{i=1}^{n} l\left(a_{i}\right)\right)^{s} l^{s}\left(a_{n+1}\right), \varepsilon^{\prime} \in\{ \pm 1\}(\text { por }(1)) \\
& =\varepsilon^{\prime} \varepsilon \prod_{i=1}^{n} l^{s}\left(a_{i}\right) l^{s}\left(a_{n+1}\right) \text { (pela hipótese de indução) } \\
& =\varepsilon^{\prime \prime} \prod_{i=1}^{n+1} l^{s}\left(a_{i}\right)
\end{aligned}
$$

(3)Indução em $s \geq 2$. O caso $n=2$ corresponde ao lema 57 (3).

$$
\begin{aligned}
l(a)^{s+1} & =l^{s}(a) l(a) \\
& =l^{s-1}(-1) l(a) l(a)(\text { pela hipótese de indução) } \\
& =l^{s-1}(-1) l^{2}(a) \\
& =l^{s-1}(-1) l(1) l(a) \\
& =l^{s}(-1) l(a)
\end{aligned}
$$


(4)

$$
\begin{aligned}
\left(\prod_{i=1}^{n} l(a)\right)^{s} & =\varepsilon \prod_{i=1}^{n} l^{s}\left(a_{i}\right)(\text { por }(2)) \\
& =\varepsilon \prod_{i=1}^{n} l^{s-1}(-1) l\left(a_{i}\right)(\operatorname{por}(3)) \\
& =\varepsilon l^{n(s-1)}(-1) \prod_{i=1}^{n} l\left(a_{i}\right)
\end{aligned}
$$

Observação 48. Sejam $\gamma_{1}, \gamma_{2}, \ldots, \gamma_{k}$ geradores de $K_{\star}^{M} \mathbb{K}$. Pode-se mostrar que

$$
\left(\sum_{i=1}^{k} \gamma_{i}\right)^{s}=\sum_{\sum_{l=1}^{k} i_{l}=s} \varepsilon_{i_{1}, \ldots, i_{k}} \prod_{1 \leq t \leq k} \gamma_{i}^{i_{t}}
$$

onde

$$
\left|\varepsilon_{i_{1}, \ldots, i_{k}}\right| \leq \frac{s !}{i_{1} ! \ldots i_{k} !}
$$

e se $\gamma_{i}=l\left(a_{1}^{i}\right) \ldots l\left(a_{n_{i}}^{i}\right)$, então $\gamma^{i_{t}}=\varepsilon_{i_{t}} l(-1)^{d_{i_{t}}} \eta_{i_{t}}$ com $d_{i_{t}} \doteq n_{i}\left(i_{t}-1\right), \varepsilon_{i_{t}} \in\{ \pm 1\}$ e $\eta_{i} \in K_{\star} \mathbb{K}$. Dessa forma podemos escrever

$$
\left(\sum_{i=1}^{k} \gamma_{i}\right)^{s}=\sum_{\sum_{l=1}^{k} i_{l}=s} \varepsilon_{i_{1}, \ldots, i_{k}} \prod_{1 \leq t \leq k} \varepsilon_{i_{t}} l(-1)^{d_{i_{t}}} \eta_{i_{t}}
$$

Assim, se existir $m>0$ tal que $l(-1)^{m}=0$, então fazendo $n \doteq \min \left\{n_{1}, \ldots, n_{k}\right\}$ e tomando $s$ tal que $n(s-k)>m$, teremos

$$
\left(\sum_{i=1}^{k} \gamma_{i}\right)^{s}=0
$$

Proposição 111. Para um corpo $\mathbb{K}$ as seguinte afirmações são equivalentes

(1) $l(-1)$ é nilpotente.

(2) $\mathbb{K}$ não é formalmente real;

(3) Qualquer elemento de $\bigoplus_{n>0} K_{n} \mathbb{K}$ é nilpotente;

Demonstração. (1) $\Longrightarrow(2)$ Se $-1 \notin \sum \dot{K}^{2}$, então $\mathbb{K}$ pode ser imerso e um corpo real fechado e, portanto, pode ser ordenado. Fixamos uma ordem em $\mathbb{K}$ e assinatura correspondente $\sigma$, e definimos uma aplicação $n$-linear $\psi: \dot{\mathbb{K}}^{n} \longrightarrow \mathbb{Z} / 2 \mathbb{Z}$ que transforma $\prod_{i=1}^{n} l\left(a_{i}\right)$ em $\prod_{i=1}^{n} \frac{\left(1-\sigma\left(a_{i}\right)\right)}{2}$, onde $\sigma\left(a_{i}\right)=1$ ou $\sigma\left(a_{i}\right)=-1$ conforme $a_{i}$ seja positivo ou negativo. É fácil ver que $\psi$ é um $n$-símbolo de Steinberg e, portanto, estende-se a um homomorfismo $\varphi: K_{n} \mathbb{K} \longrightarrow \mathbb{Z} / 2 \mathbb{Z}$ que leva $l(-1)^{n}$ em 1. Isso prova que $l(-1)$ não é nilpotente.

$(1) \Longrightarrow(3)$ Segue da observação anterior e $(3) \Longrightarrow(1)$ é imediato. 


\subsubsection{O cálculo de $K_{\star}$ para corpos residuais por valorações}

O principal objetivo desta subseção é mostrar que podemos levantar o símbolo de Tame (ver o exemplo 21 do capítulo 4) para a K-teoria.

$$
(a, b)_{v}=(-1)^{v(a) v(b)} \frac{\bar{a}^{v(b)}}{\bar{b}^{v(a)}}
$$

para uma aplicação

$$
\partial_{v}: K_{\star}^{M}(\mathbb{K}) \longrightarrow K_{\star}^{M}\left(\mathbb{K}_{v}\right)
$$

As principal referência para esta seção é [BT].

\subsubsection{1 $\Lambda$-álgebras}

Seja $\Lambda$ o anel graduado definido por

$$
\Lambda \doteq\left(\mathbb{Z}, \mathbb{Z}_{2} \lambda, \mathbb{Z}_{2} \lambda^{2}, \ldots, \mathbb{Z}_{2} \lambda^{n}, \ldots\right)
$$

ou seja, $\Lambda$ é a álgebra de polinômios em uma indeterminada $\lambda$, com termos constantes em $\mathbb{Z}$, e termos de graus superiores em $\mathbb{Z}_{2}=\mathbb{Z} / 2 \mathbb{Z}$.

Definição 86. Uma $\Lambda$-álgebra graduada é um anel graduado $A=\bigoplus_{n \in \omega} A_{n}$, juntamente com um homomorfismo $\Lambda \longrightarrow A$, definido por $\lambda \mapsto \lambda_{A}$, onde $\lambda_{A}$ é um elemento de grau 1 , no centro de $A$. Se, em adição, $A$ for gerada pelos elementos de grau 1 e,também, $a^{2}=\lambda_{A} a, \forall a \in A_{1}$; diremos que A é uma $\Lambda$-álgebra.

Exemplo 30. Seja $\mathbb{K}$ um corpo. O anel $K_{\star}^{M}(\mathbb{K})$ é uma $\Lambda$-álgebra.

Prova. $l(-1)$ é central, uma vez que, $K_{\star}^{M}(\mathbb{K})$ é anticomutativo e $2 l(-1)=0$. Portanto, $\lambda \mapsto l(-1)$ dá a $K_{\star}^{M}(\mathbb{K})$ uma estrutura de $\Lambda$-álgebra. Finalmente, o lema 57 (3), permite concluir que $K_{\star}^{M}(\mathbb{K})$ é uma $\Lambda$-álgebra.

Coproduto de $\Lambda$ - álgebras. Sejam A e B duas $\Lambda$-álgebras. Denotamos por $A \otimes_{\mathbb{Z}} B$ o anel graduado $\left(\bigoplus_{p+q=n} A_{p} \otimes_{\mathbb{Z}} B_{q}\right)_{n \in \omega}$. O produto em $A \otimes_{\mathbb{Z}} B$ é definido, em componentes homogêneas $a, a^{\prime} \in A$ e $b, b^{\prime} \in B$, da seguinte maneira:

$$
(a \otimes b)\left(a^{\prime} \otimes b^{\prime}\right)=(-1)^{\operatorname{grau}(b) \operatorname{grau}(a)} a a^{\prime} \otimes b b^{\prime}
$$

Fato 32. $O$ conjunto $\left\{a \lambda_{A} \otimes b-a \otimes \lambda_{B} b: a \in A, b \in B\right.$ homogêneos $\}$, gera um ideal graduado $\Delta$ de $A \otimes_{\mathbb{Z}} B$.

Fato 33. $A \otimes_{\Lambda} B$ definido por

$$
A \otimes_{\Lambda} B \doteq \frac{A \otimes_{\mathbb{Z}} B}{\Delta}
$$

é um anel anticomutativo com graduação

$$
\left(A \otimes_{\Lambda} B\right)_{n}=\sum_{p+q=n} A_{p} \otimes B_{q}
$$


Observação 49. A soma anterior não é uma soma direta, pois

$$
A_{p} \lambda_{A} \otimes B_{q}=A_{p} \otimes \lambda_{B} B_{q} \subset\left(A_{p+1} \otimes B_{q}\right) \cap\left(A_{p} \otimes B_{q+1}\right)
$$

Lema 59. $A \otimes_{\Lambda} B$ é uma $\Lambda$-álgebra.

Demonstração. Seja $c=a \otimes 1+1 \otimes b \in\left(A \otimes_{\Lambda} B\right)_{1}$. Então,

$$
c^{2}=a^{2} \otimes 1+a \otimes b-a \otimes b+1 \otimes b^{2}=a \lambda_{A} \otimes 1+1 \otimes \lambda_{B} b=\lambda c
$$

onde, $\lambda=\lambda_{A} \otimes 1=1 \otimes \lambda_{B}$. Portanto, pondo $\lambda_{A \otimes_{\Lambda} B} \doteq \lambda$, vemos que $A \otimes_{\Lambda} B$ possui uma estrutura de $\Lambda$-álgebra.

Definição 87. $A \otimes_{\Lambda} B$ é chamado de produto tensorial das $\Lambda$-álgebras $A$ e $B$.

A $\Lambda$-álgebra livre em um gerador $\eta$ é a $\Lambda$-álgebra

$$
\Lambda\langle\eta\rangle \doteq \frac{\Lambda[X]}{X^{2}-\lambda X}
$$

onde $X=\eta\left(\bmod X^{2}-\lambda X\right)$. Observe que $\Lambda\langle\eta\rangle$ é um $\Lambda$-módulo livre com base $\{1, \eta\}$.

Para qualquer $\Lambda$-álgebra $\mathrm{A}$, colocamos

$$
A\langle\eta\rangle=A \otimes_{\Lambda} \Lambda\langle\eta\rangle=A \oplus A \eta
$$

este é um $\Lambda$-módulo livre com base $\{1, \eta\}$. Além disso,

$$
A\langle\eta\rangle_{p}=A_{p} \oplus A_{p-1} \eta
$$

Se $a+b \eta \in A\langle\eta\rangle_{p}$ e $c+d \eta \in A\langle\eta\rangle_{q}$, então

$$
\begin{aligned}
(a+b \eta)(c+d \eta) & =a c+a d \eta+b \eta c+b \eta d \eta \\
& =a c+a d \eta+(-1)^{q} b c+(-1)^{q-1} b d \eta^{2} \\
& =a c+a d \eta+(-1)^{q} b c+(-1)^{q-1} b d \lambda \eta \\
& =a c+\left(a d+(-1)^{q} b c+(-1)^{q-1} b d \lambda\right) \eta
\end{aligned}
$$

\subsubsection{Valorações discretas}

Definição 88. Dizemos que uma aplicação $v: \dot{K} \longrightarrow \mathbb{Z}$ é uma valoração discreta em $\mathbb{K}$ quando ela cumpre as seguintes propriedades:

$\left.v_{1}\right) v$ é sobrejetora;

$\left.v_{2}\right) \quad \forall a, b(v(a \cdot b)=v(a)+v(b))$;

$\left.v_{3}\right) \forall a, b(a \neq-b \Longrightarrow v(a+b) \geq \min \{v(a), v(b)\})$

ou seja, $v: \dot{\mathbb{K}} \longrightarrow \mathbb{Z}$ é um epimorfismo tal que $v(a+b) \geq \min \{v(a), v(b)\}$, se $a+b \neq 0$.

Lema 60. Para uma valoração discreta $v$ sobre $\mathbb{K}$ valem :

1) $v(1)=v(-1)=0$ 
2) $\forall x(v(x)=v(-x))$

3) $\forall x\left(v\left(x^{-1}\right)=-v(x)\right)$

4) $\forall x \forall n \in \mathbb{Z}\left(v\left(x^{n}\right)=n v(x)\right)$

5) $\forall x, y(v(x) \neq v(y) \Longrightarrow v(x+y)=\min \{v(x), v(y)\})$

6) Se $x_{1}, \ldots, x_{n} \in \dot{K}$ e $v\left(x_{1}\right), \ldots, v\left(x_{n}\right)$ são doi a dois distintos e $\sum_{i=1}^{n} x_{i} \neq 0$, então

$$
v\left(\sum_{i=1}^{n} x_{i}\right)=\min \left\{v\left(x_{1}\right), \ldots, v\left(x_{n}\right)\right\}
$$

Demonstração.

1) $v(1)=v(1 \cdot 1)=v(1)+v(1) \Longrightarrow v(1)=0$. Assim, $0=v(1)=v((-1) \cdot(-1))=v(-1)+v(-1)=$ $2 v(-1) \Longrightarrow v(-1)=0$.

2) $v(-x)=v((-1) \cdot x)=v(-1)+v(x)=0+v(x)=v(x)$.

3) $0=v(1)=v\left(x x^{-1}\right)=v(x)+v\left(x^{-1}\right)$

4)Por indução. Se $n \geq 0$, então $v\left(x^{n+1}\right)=v\left(x^{n}\right)+v(x)=n v(x)+v(x)=(n+1) v(x)$. Se $n<0$, então $v\left(x^{n}\right)=v\left(\left(x^{-n}\right)^{-1}\right)=-v\left(x^{-n}\right)=-(-n) v(x)=n v(x)$

5)Suponhamos $v(x)<v(y)$. Assim, $v(x)=v(x+y+(-y)) \geq \min \{v(x+y), v(-y)\}=\min \{v(x+$ $y), v(y)\}=v(x+y) \geq \min \{v(x), v(y)\}=v(x)$.

6) Por indução usando 5).

\section{Lema 61.}

1) $\mathcal{O}_{v} \doteq\{0\} \cup\{a \in \dot{\mathbb{K}}: v(a) \geq 0\}$ é um subanel de $\mathbb{K}$.

2) $\forall x \in \dot{\mathbb{K}}\left(x \in \mathcal{O}_{v} \vee x^{-1} \in \mathcal{O}_{v}\right)$.

3) $\mathbb{K}$ é o corpo quociente do domínio $\mathcal{O}_{v}$.

4) $\dot{\mathcal{O}}_{v}=\{x \in \dot{\mathbb{F}}: v(x)=0\}$.

5) $\mathcal{O}_{v^{+}} \doteq \mathcal{O}_{v} \backslash \dot{\mathcal{O}}_{v}$ é o único ideal maximal de $\mathcal{O}_{v}$.

6) $\mathcal{O}_{v}$ é um dominio de ideais principais.

7) Seja $\pi$ tal que $v(\pi)=1$. Todo elemento $x \in \mathbb{K}$ admite uma única escrita $x=u \pi^{k}$, com $u \in \dot{\mathcal{O}}_{v} e$ $k \in \mathbb{Z}$. Além disso, $k=v(x)$

\section{Demonstração.}

1) $1 \in \mathcal{O}_{v}$, pois $v(1)=0$. Se $a, b \in \mathcal{O}_{v}$, então $v(a b)=v(a)+v(b) \geq 0$ e, assim, $a b \in \mathcal{O}$. Finalmente, para $a, b \in \dot{\mathcal{O}}_{v}$ tem-se $v(a-b) \geq \min \{v(a), v(b)\} \geq 0$ e, portanto $a-b \in \mathcal{O}_{v}$.

2) $v(x)<0 \Longrightarrow v\left(x^{-1}\right)=-v(x)>0 \Longrightarrow x^{-1} \in \mathcal{O}_{v}$

3) Consequência imediata do item anterior.

4) Se $x \in \mathcal{O}_{v}$ admite $x^{-1} \in \mathcal{O}_{v}$, então $v\left(x^{-1}\right) \geq 0$. Por outro lado, $v\left(x^{-1}\right)=-v(x) \leq 0$. Assim, $v\left(x^{-1}\right)=-v(x)=0$ e,portanto $v(x)=0$.

5) Basta notar que $\mathcal{O}_{v^{+}}=\{0\} \cup\{x \in \dot{K}: v(x) \geq 0\}$ é um ideal de $\mathcal{O}_{v}$ e que $\mathcal{O}_{v^{+}}=\mathcal{O}_{v} \backslash \dot{\mathcal{O}}_{v}$.

6)Seja I um ideal de $\mathcal{O}_{v}$ e defina $\eta \doteq \min v(I)>0$. Se $\lambda \in \dot{\mathbb{K}}$ é tal que $v(\lambda)=\eta$, então $I=\lambda \mathcal{O}_{v}$. 
7)Como $\mathcal{O}_{v}$ é um domínio de ideais principais, em particular, $\mathcal{O}_{v^{+}}=\pi \mathcal{O}_{v}$, ou equivalentemente, $v(\dot{\mathbb{K}})=v(\pi) \mathbb{Z}$, com $v(\pi) \geq 0$. Logo, $v(\pi)= \pm 1$ e $v(\pi) \geq 0$ e, então $v(\pi)=1$. Nesse caso, para cada $x \in \dot{\mathbb{K}}$ existe $k \in \mathbb{Z}$ tal que $v(x)=v(\pi) k$, ou seja, $v\left(\frac{x}{\pi^{k}}\right)=0$ e, portanto, $u \doteq \frac{x}{\pi^{k}}$ é uma unidade. Logo, $x=u \pi^{k}$ e $v(x)=k v(\pi)=k$. Se $x=u^{\prime} \pi^{k^{\prime}}$ para alguma unidade $u^{\prime}$ e algum inteiro $k^{\prime}$, ter-se-á

$$
\begin{aligned}
u \pi^{k}=u^{\prime} \pi^{k^{\prime}} & \Longrightarrow v\left(u \pi^{k}\right)=v\left(u^{\prime} \pi^{k^{\prime}}\right) \\
& \Longrightarrow v(u)+v\left(\pi^{k}\right)=v\left(u^{\prime}\right)+v\left(\pi^{k^{\prime}}\right) \\
& \Longrightarrow 0+k v(\pi)=0+k^{\prime} v(\pi) \\
& \Longrightarrow k \cdot 1=k^{\prime} \cdot 1 \\
& \Longrightarrow k=k^{\prime} \\
& \Longrightarrow \pi^{k}=\pi^{k^{\prime}} .
\end{aligned}
$$

e consequentemente, $u=u^{\prime}$.

Definição 89. $O$ anel $\mathcal{O}_{v}$ é chamado de anel de valoração relacionado a $v$. O corpo quociente $\mathbb{K}_{v} \doteq \frac{\mathcal{O}_{v}}{\mathcal{O}_{v^{+}}}$é denominado de corpo de resíduos (ou corpo residual) de $\mathbb{K}$ com relação a valoração $v$.

\subsubsection{Extensão do símbolo de Tame}

Com as notações anteriores, definimos

$$
\begin{aligned}
d_{\pi}: \dot{\mathbb{K}} & \longrightarrow K_{\star}^{M}\left(\mathbb{K}_{v}\right)\langle\eta\rangle \\
u \pi^{i} & \longmapsto l(\bar{u})+i \eta
\end{aligned}
$$

para $u \in \dot{\mathcal{O}}_{v}, i \in \mathbb{Z}$.

Lema 62. $\forall \alpha, \beta \in \dot{\mathbb{K}}\left(d_{\pi}(\alpha \beta)=d_{\pi}(\alpha)+d_{\pi}(\beta)\right)$

Demonstração. De fato, para $u_{1}, u_{2} \in \dot{\mathcal{O}}_{v}$ e $i_{1}, i_{2} \in \mathbb{Z}$, temos

$$
\begin{aligned}
d_{\pi}\left(u_{1} \pi^{i_{1}} \cdot u_{2} \pi^{i_{2}}\right) & =d_{\pi}\left(u_{1} u_{2} \pi^{i_{1}+i_{2}}\right) \\
& =l\left(\overline{u_{1} u_{2}}\right)+\left(i_{1}+i_{2}\right) \eta \\
& =l\left(\overline{u_{1}}\right)+l\left(\overline{u_{2}}\right)+i_{1} \eta+i_{2} \eta \\
& =\left(l\left(\overline{u_{1}}\right)+i_{1} \eta\right)+\left(l\left(\overline{u_{2}}\right)+i_{2} \eta\right) \\
& =d_{\pi}\left(u_{1} \pi^{i_{1}}\right)+d_{\pi}\left(u_{2} \pi^{i_{2}}\right)
\end{aligned}
$$


Lema 63. Existe um diagrama comutativo

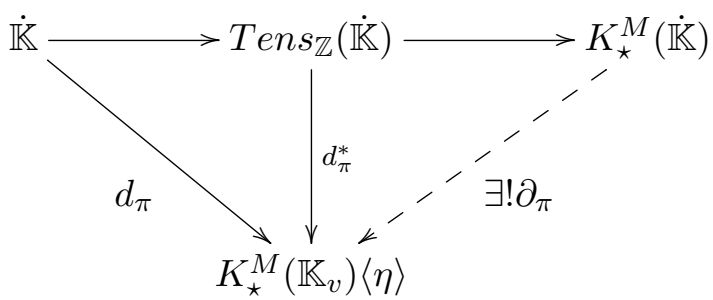

onde

$$
\partial_{\pi}\left(l\left(u_{1} \pi^{i_{1}}\right) \ldots l\left(u_{n} \pi^{i_{n}}\right)\right)=\left(l\left(\overline{u_{1}}\right)+i_{1} \eta\right) \ldots\left(l\left(\overline{u_{n}}\right)+i_{n} \eta\right)
$$

Demonstração. Pelo lema anterior e pela propriedade universal da álgebra tensorial, existe uma extensão $d_{\pi}^{*}:$ Tens $s_{\mathbb{Z}}(\dot{\mathbb{K}}) \longrightarrow K_{\star}^{M}\left(\mathbb{K}_{v}\right)\langle\eta\rangle$ que torna o seguinte diagrama comutativo

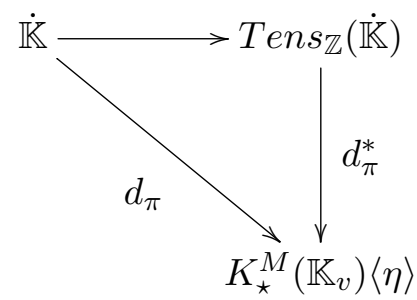

Finalmente, para cada $\alpha \in \dot{\mathbb{K}}$ escreva $\alpha=\pi^{i} u \operatorname{com} i=v(d)$ e $u \in \dot{\mathcal{O}}_{v}$. Se $i>0$, então $\alpha \neq 1,(1-\alpha) \neq$ $0 \Longrightarrow 0=v(1)=\min \{v(\alpha), v(1-\alpha)\} \Longrightarrow v(1-\alpha)=0$ e, assim $1-\alpha$ é uma unidade com classe residual $\overline{1-\alpha}=\overline{1}$. Nesse caso, $d_{\pi}(1-\alpha)=l(\overline{1-\alpha})=l(\overline{1})=0$ e então $d_{\pi}(\alpha) d_{\pi}(1-\alpha)=0$. Suponhamos que $i<0$. Temos $1-\alpha=\pi^{i}\left(\pi^{-i}-u\right)$. Note que $0=v(u)=\min \left\{v\left(\pi^{-i}\right), v\left(\pi^{-i}-u\right)\right\}$, ou seja, $\pi^{-i}-u$ é uma unidade pertencente a $\dot{\mathcal{O}}_{v}$. Nesse caso, teremos

$$
\begin{aligned}
d_{\pi}(\alpha) d_{\pi}(1-\alpha) & =(l(\bar{u})+i \eta)(l(-\bar{u})+i \eta) \\
& =l(\bar{u}) l(-\bar{u})+l(\bar{u}) i \eta+i \eta l(-\bar{u})+i^{2} \eta^{2} \\
& =0+l(\bar{u}) \eta i+\eta i(l(\bar{u})+l(-1)) \\
& =l(\bar{u}) \eta i+\eta i l(\bar{u})+i \eta l(-1)-i^{2} \eta l(-1) \\
& =(l(\bar{u}) \eta+\eta l(\bar{u})) i+\left(i-i^{2}\right) \eta l(-1) \\
& =0 \cdot i+\left(i-i^{2}\right) \eta l(-1) \\
& =\left(i-i^{2}\right) \eta l(-1) \\
& =-(2 l(-1) \eta+\ldots+2 l(-1) \eta) \quad\left(\frac{i^{2}-i}{2} \text { parcelas }\right) \\
& =-(0 \eta+\ldots+0 \eta) \\
& =0 .
\end{aligned}
$$

Resta o caso $i=0$, ou seja, $\alpha=u$ é uma unidade. Se $v(1-\alpha)>0$ o argumento é similar ao primeiro caso trocando $\alpha$ por $1-\alpha$. Finalmente, se $\alpha$ e $1-\alpha$ são unidades, teremos

$$
d_{\pi}(\alpha) d_{\pi}(1-\alpha)=l(\bar{u}) l(\overline{1-u})=0
$$


Considere o mapa $\partial_{v}$ definido através da composição

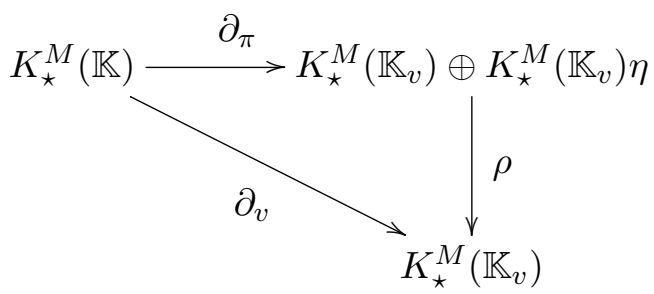

onde $\rho$ é a projeção $\rho(a+b \eta)=b$. Desde que, $d_{\pi}\left(u \pi^{i}\right)=l(\bar{u})+i \eta$ e $d_{\pi}\left(u_{j}\right)=l\left(\overline{u_{j}}\right)$ para todos $u_{1}, \ldots, u_{n} \in \dot{\mathcal{O}}_{v}$, uma fácil computação mostra que

$$
\partial_{v}\left(l\left(u_{1}\right) \ldots l\left(u_{n-1}\right) l(a)\right)=l\left(\overline{u_{1}}\right) \ldots l\left(\overline{u_{n-1}}\right) v(a)
$$

para todo $a \in \dot{K}$. Em particular, segue que $\partial_{v}$ depende somente de $v$ e não do parâmetro $\pi$ Proposição 112. Os seguintes diagramas são comutativos

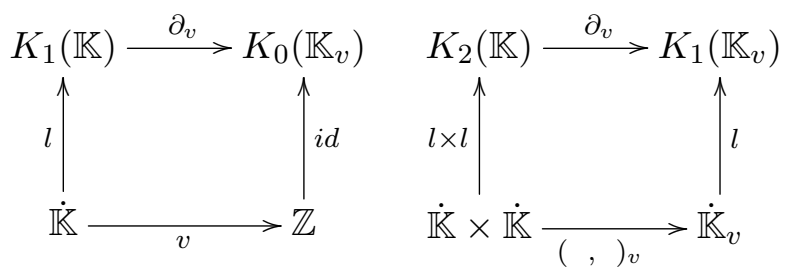

onde (, ) v é o símbolo de Tame.

Demonstração. Seja $a=a_{0} \pi^{\alpha}$ e $b=b_{0} \pi^{\beta}$ em $\dot{\mathbb{K}}$, onde $a_{0}, b_{0} \in \dot{\mathcal{O}_{\sqsubseteq}}$. Temos

$$
\partial_{\pi}(l(a))=l\left(\overline{a_{0}}\right)+\alpha \eta \Longrightarrow \partial_{v}(l(a))=\alpha=v(a)
$$

e, portanto $\partial_{v} \circ l=v$. Para comutatividade do segundo diagrama; note que, por um lado

$$
\begin{aligned}
\partial_{\pi}(l(a) l(b)) & =\left(l\left(\overline{a_{0}}\right)+\alpha \eta\right)\left(l\left(\overline{b_{0}}\right)+\beta \eta\right) \\
& =l\left(\overline{a_{0}}\right) l\left(\overline{b_{0}}\right)+\left(l\left(\overline{a_{0}}\right) \beta-\alpha l\left(\overline{b_{0}}\right)+\alpha \beta l(-1)\right) \eta
\end{aligned}
$$

e por outro lado

$$
l(\bar{c})=l\left(a_{0}\right) \beta-\alpha l\left(\overline{b_{0}}\right)+\alpha \beta l(-1)
$$

com

$$
c=(-1)^{\alpha \beta} \frac{a_{0}^{\beta}}{b_{0}^{\alpha}}=(-1)^{v(a) v(b)} \frac{a_{0}^{v(a)}}{b_{0}^{v(b)}}
$$

o que conclui a prova.

\subsubsection{Stiefel-Whitney de um espaço quadrático}

Seja $\mathbb{K}$ um corpo com $\operatorname{char}(\mathbb{K}) \neq 2$. Para cada $n$, colocamos 


$$
k_{n}(\mathbb{K})=\frac{K_{n}(\mathbb{K})}{2 K_{n}(\mathbb{K})}
$$

A K-teoria de Milnor $\bmod 2$ é definida por

$$
k_{\star}^{M}(\mathbb{K})=\left(\mathbb{Z} / 2 \mathbb{Z}, k_{1} \mathbb{K}, \ldots, k_{n} \mathbb{K}, \ldots\right)
$$

ou seja, é o quociente da K-teoria de Milnor

$$
K_{\star}^{M}\left(\mathbb{Z}, K_{1} \mathbb{K}, K_{2} \mathbb{K}, \ldots, K_{n} \mathbb{K}, \ldots\right)
$$

pelo ideal homogêneo

$$
2 K_{\star}^{M} \mathbb{K} \doteq\left(2 \mathbb{Z}, 2 K_{1} \mathbb{K}, \ldots, 2 K_{n} \mathbb{K}, \ldots\right)
$$

observe que $k_{\star}^{M} \mathbb{K}$ é uma $\mathbb{Z} / 2 \mathbb{Z}$-álgebra graduada, com

- $k_{0} \mathbb{K}=K_{0} \mathbb{K} / 2 K_{0} \mathbb{K} \cong \mathbb{Z} / 2 \mathbb{Z}$

- $k_{1} \mathbb{K}=K_{1} \mathbb{K} / 2 K_{1} \mathbb{K} \cong \dot{\mathbb{K}} / \dot{\mathbb{K}}^{2}$

Lema 64. Valem em $k_{2} \mathbb{K}$ as seguintes identidades

1) $l(\alpha) l\left(x^{2}-\alpha y^{2}\right)=0, \forall \alpha \in \mathbb{K} ; x, y \in \mathbb{K}$

2) $l(a) l(b)=l(a+b) l(a b(a+b)), \forall a+b \in \dot{\mathbb{K}}, a+b \neq 0$

Demonstração.

1)

$$
\begin{aligned}
l(\alpha) l\left(x^{2}-\alpha y^{2}\right) & =l\left(\alpha y^{2} x^{-2}\right) l\left(x^{-2}\left(x^{2}-\alpha y^{2}\right)\right) \\
& =l\left(\alpha\left(y x^{-1}\right)^{2}\right) l\left(1-\alpha\left(y x^{-1}\right)^{2}\right) \\
& =0
\end{aligned}
$$

2) Fazendo $y=1, x=a+b$ e $\alpha=a(a+b)$ em (1), obtemos

$$
\begin{aligned}
0 & =l(\alpha) l\left(x^{2}-\alpha y^{2}\right) \\
& =l(a(a+b)) l\left((a+b)^{2}-a(a+b)\right) \\
& =l(a(a+b)) l(b(a+b)) \\
& =[l(a)+l(a+b)][l(b)+l(a+b)] \\
& =l(a) l(b)+l(a) l(a+b)+l(a+b) l(b)+l(a+b) l(a+b) \\
& =l(a) l(b)+l(a+b) l(a)+l(a+b) l(b)+l(a+b) l(a+b) \\
& =l(a) l(b)+l(a+b) l(a b)+l(a+b) l(a+b) \\
& =l(a) l(b)+l(a+b) l(a b(a+b))
\end{aligned}
$$

Lema 65. Seja $\mathcal{F}(\mathbb{K})$ o grupo abeliano livre no conjunto $\{\langle a\rangle: a \in \mathbb{K}\}$ e $\left(k_{*} \mathbb{K}[[x]]\right)^{\bullet}$ o grupo de unidades da álgebra das séries formais em $x$. A aplicação 


$$
\begin{aligned}
w: \mathcal{F}(\mathbb{K}) & \longrightarrow\left(k_{*} \mathbb{K}[[x]]\right)^{\bullet} \\
\langle a\rangle & \longmapsto 1+l(a) x
\end{aligned}
$$

induz um homomorfismo de grupos $w: W G(\mathbb{K}) \longrightarrow\left(k_{*} \mathbb{K}[[x]]\right)^{\bullet}$.

Demonstração. Sabemos do capítulo 2 que $W G(\mathbb{K}) \cong \frac{\mathbb{Z}[\dot{\mathbb{K}}]}{\mathcal{R}}$, onde $\mathcal{R}$ consiste de certas relações (ver a proposiçãd 34 do cap. 2 ). Assim, basta observar que se $a, b \in \dot{K}$ satisfaz $a+b \neq 0$, então pelo lema anterior teremos

$$
\begin{aligned}
w(\langle a+b\rangle+\langle a b(a+b)\rangle) & =1+l\left(a b(a+b)^{2}\right) x+l(a+b) l(a b(a+b)) x^{2} \\
& =(1+l(a+b) x) \cdot(1+l(a b(a+b)) x) \\
& =1+l(a b) x+l(a) l(b) x^{2} \\
& =1+(l(a)+l(b)) x+l(a) l(b) x^{2} \\
& =(1+l(a) x)(1+l(b) x) \\
& =w(\langle a\rangle+\langle b\rangle) \\
& =w(\langle a\rangle) \cdot w(\langle b\rangle)
\end{aligned}
$$

Definição 90. O mapa $w: W G(\mathbb{K}) \longrightarrow\left(k_{*} \mathbb{K}[[x]]\right)^{\bullet}$ do lema anterior é chamado de mapa total de Stiefel-Whitney.

\section{Observação 50.}

1) Considere um polinômio $p\left(x_{1}, \ldots, x_{n}\right)=\frac{r\left(x_{1}, \ldots, x_{n}\right)}{s\left(x_{1}, \ldots, x_{n}\right)} \in \mathbb{K}\left(x_{1}, \ldots, x_{n}\right)$ tal que $p(\cdots, 0, \cdots)=0$, para cada $x_{i}=0$. Uma vez que, $p\left(0, x_{2}, \ldots, x_{n}\right)=0$, resulta $r\left(x_{1}, \ldots, x_{n}\right)=x_{1} g_{1}\left(x_{2}, \ldots, x_{n}\right)$ em $\mathbb{K}\left(x_{2}, \ldots, x_{n}\right)\left[x_{1}\right]$ e, portanto $p\left(x_{1}, \ldots, x_{n}\right)=x_{1} g_{1}\left(x_{2}, \ldots, x_{n}\right)$ para algum $g_{1}\left(x_{2}, \ldots, x_{n}\right)$. De forma semelhante, existe $f_{2}\left(x_{1}, \ldots, x_{n}\right)$ tal que $p\left(x_{1}, \ldots, x_{n}\right)=x_{1} x_{2} f_{2}\left(x_{1}, \ldots, x_{n}\right)$. Finalmente, concluiremos usando indução que

$$
p\left(x_{1}, \ldots, x_{n}\right)=x_{1} x_{2} \ldots x_{n} f_{n}\left(x_{1}, \ldots, x_{n}\right)
$$

para algum $f_{n}\left(x_{1}, \ldots, x_{n}\right)$ em $\mathbb{K}\left(x_{1}, \ldots, x_{n}\right)$.

2) Seja $\xi\left(x_{1}, \ldots, x_{n}\right)=\prod_{\varepsilon=\left(\varepsilon_{1}, \ldots, \varepsilon_{n}\right) \in\{0,1\}^{n}}\left(1+\sum_{i=1}^{n} \varepsilon_{i} x_{i} x\right)^{\delta_{\varepsilon, n}}$ um elemento de $(\mathbb{Z} / 2 \mathbb{Z}[[x]])\left[\left[x_{1}, \ldots, x_{n}\right]\right]$, onde $\delta_{\varepsilon, n}=(-1)^{\sigma_{\varepsilon}+n}$ e $\sigma_{\varepsilon}=\sum_{i=1}^{n} \varepsilon_{i}$. Avaliando $\xi\left(x_{1}, \ldots, x_{n}\right)$ em $(a, \ldots, a)$, obtemos $\prod_{\varepsilon \in\{0,1\}^{n}}(1+$ $\left.\sigma_{\varepsilon} a x\right)^{\delta_{\varepsilon, n}}$. Se $n=2 r$, então

$$
\xi(a, \ldots, a)=\prod_{\varepsilon \in\{0,1\}^{n}}\left(1+\sigma_{\varepsilon} a x\right)^{\delta \varepsilon}
$$

onde $\delta_{\varepsilon}=(-1)^{\sigma}$. Além disso, $\xi$ contém, exatamente, $\sum_{i \in\{0,2, \ldots, r\}}\left(\begin{array}{c}n \\ i\end{array}\right)=2^{n-1}$ termos da forma $(1+\text { eax })^{e}$ com e par, ou seja, $2^{n-1}$ termos iguais a 1 . Assim, há $2^{n-1}$ termos da forma $(1+a x)^{-1}$. Logo,

$$
\xi(a, \ldots, a)=(1+a x)^{-2^{n-1}}
$$

Um argumento semelhante mostra que, se $n$ for impar, então 


$$
\xi(a, \ldots, a)=(1+a x)^{2^{n-1}}
$$

Lema 66. $O$ valor de $w$ em um gerador

$$
\eta=\left(\left\langle a_{1}\right\rangle-\langle 1\rangle\right)\left(\left\langle a_{2}\right\rangle-\langle 1\rangle\right) \ldots\left(\left\langle a_{n}\right\rangle-\langle 1\rangle\right)
$$

da n-ésima potência do ideal $\hat{I}(\mathbb{K})$ é dado pela fórmula

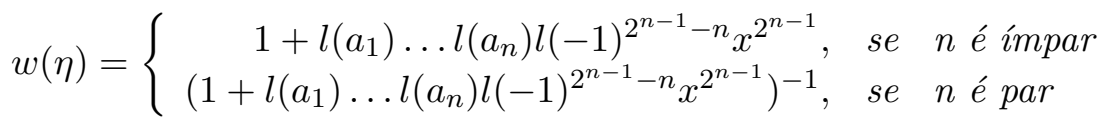

Demonstração. Como $\eta=\sum_{\varepsilon=\left(\varepsilon_{1}, \ldots, \varepsilon_{n}\right) \in\{0,1\}^{n}} \delta_{\varepsilon, n}\left\langle a_{1}^{\varepsilon_{1}} \ldots a_{n}^{\varepsilon_{n}}\right\rangle$, temos

$$
w(\eta)=\prod_{\varepsilon=\left(\varepsilon_{1}, \ldots, \varepsilon_{n}\right) \in\{0,1\}^{n}}\left(1+\sum_{i=1}^{n} \varepsilon_{i} l\left(a_{i}\right) x\right)^{\delta_{\varepsilon, n}}
$$

Assim, pelo item (1) da observação anterior ter-se-á

$$
w(\eta)=1+l\left(a_{1}\right) \ldots l\left(a_{n}\right) g\left(l\left(a_{1}\right) \ldots l\left(a_{n}\right)\right)
$$

e como $l^{2}(a)=l(a) l(-1)$, teremos

$$
w(\eta)=1+l\left(a_{1}\right) \ldots l\left(a_{n}\right) g(l(-1), \ldots, l(-1))
$$

E se $n$ for ímpar, teremos pelo item (2) da observação anterior

$$
1+l(-1)^{n} g(l(-1), \ldots, l(-1)) x^{n}=(1+l(-1) x)^{2^{n-1}}=1+l(-1)^{2^{n-1}} x^{2^{n-1}}
$$

Assim,

$$
g(l(-1), \ldots, l(-1))=l(-1)^{2^{n-1}} x^{2^{n-1}-n}
$$

Portanto,

$$
w(\eta)=1+l\left(a_{1}\right) \ldots l\left(a_{n}\right) l(-1)^{2^{n-1}-n} x^{2^{n-1}}
$$

Um cálculo semelhante pode ser feito no caso $n$ par.

Fato 34. Para cada $\eta \in W G(\mathbb{K})$, $w(\eta)$ admite uma escrita

$$
w(\eta)=\sum_{i \geq 0} w_{i}(\eta) x^{i}
$$

com $w_{i}: W G(\mathbb{K}) \longrightarrow k_{i}(\mathbb{K})$. Além disso,

$$
w\left(\phi+\phi^{\prime}\right)=w(\phi) w\left(\phi^{\prime}\right), \forall \phi, \phi^{\prime} \in W G(\mathbb{K})
$$

e, portanro temos a fórmula de Whitney

$$
w_{n}\left(\phi+\phi^{\prime}\right)=\sum_{i+j=n} w_{i}(\phi) w_{j}\left(\phi^{\prime}\right)
$$


Definição 91. A aplicação $w_{i}: W G(\mathbb{K}) \longrightarrow k_{i} \mathbb{K}$ é chamado de i-ésimo invariante de StiefelWhitney.

Corolário 23. Se $t=2^{n-1}$, então os invariantes de Stiefel-Whitney anulam $\hat{I}^{n}(\mathbb{K})$, ou seja,

$$
w_{j}(\hat{I}(\mathbb{K}))=0
$$

para todos $j=1,2, \ldots, t-1$. Além disso, a aplicação

$$
w_{t}: \hat{I}(\mathbb{K}) \longrightarrow k_{t} \mathbb{K}
$$

induz um homomorfismo 1

$$
w_{t}: \frac{\hat{I}^{n} \mathbb{K}}{\hat{I}^{n+1} \mathbb{K}} \cong \frac{I^{n} \mathbb{K}}{I^{n+1} \mathbb{K}} \longrightarrow k_{t} \mathbb{K}
$$

que leva $\left(\left\langle a_{1}\right\rangle-\langle 1\rangle\right) \cdots\left\langle\left(a_{n}\right\rangle-\langle 1\rangle\right)$ para $l\left(a_{1}\right) \cdots l\left(a_{n}\right) l(-1)^{t-n}$

Demonstração. Seja $\phi=\left(\left\langle a_{1}\right\rangle-\langle 1\rangle\right) \ldots\left(\left\langle a_{n}\right\rangle-\langle 1\rangle\right)$. Pelo lema anterior, $w_{j}(\phi)=0$ para $j=$ $1, \ldots, t-1$. O resultado segue da fórmula de Whitney.

\subsubsection{O triângulo incompleto de Milnor}

Lema 67. A aplicação

$$
\varsigma: \begin{array}{ccc}
\dot{\mathbb{K}}^{n} & \longrightarrow & \overline{I^{n} \mathbb{K}} \\
\left(a_{1}, \ldots, a_{n}\right) & \longmapsto & \left\langle\left\langle-a_{1}, \ldots,-a_{n}\right\rangle\right\rangle
\end{array}
$$

é um n-símbolo de Steinberg

Demonstração. Inicialmente, observe que

$$
\begin{aligned}
\langle\langle-a b\rangle\rangle+\langle\langle-a,-b\rangle\rangle & =\langle 1,-a b\rangle+\langle 1,-a\rangle\langle 1,-b\rangle \\
& =\langle 1,-a b\rangle+\langle 1,-b,-a, a b\rangle \\
& =\langle 1,-a b, 1,-b,-a, a b\rangle \\
& =\langle 1,-a\rangle+\langle 1,-b\rangle+\langle-a b, a b\rangle \\
& =\langle 1,-a\rangle+\langle 1,-b\rangle \\
& =\langle\langle-a\rangle\rangle+\langle\langle-b\rangle\rangle
\end{aligned}
$$

Consequentemente,

$$
\left\langle\left\langle-a_{1}, \ldots,-a_{n} b_{n}\right\rangle\right\rangle+\left\langle\left\langle-a_{1}, \ldots,-a_{n},-b_{n}\right\rangle\right\rangle=\left\langle\left\langle-a_{1}, \ldots,-a_{n}\right\rangle\right\rangle+\left\langle\left\langle-a_{1}, \ldots,-b_{n}\right\rangle\right\rangle
$$

e, portanto, ऽ é multiplicativa. Finalmente, se $a+b=1$, então

\footnotetext{
${ }^{1} \mathrm{O}$ isomorfismo $\hat{I}^{n} / \hat{I}^{n+1} \cong I^{n} / I^{n+1}$ foi demonstrado no capíulo 2 , a saber, prop. 13 (xi).
} 


$$
\begin{aligned}
(\langle a\rangle-\langle 1\rangle)(\langle b\rangle-\langle 1\rangle) & =\langle a\rangle\langle b\rangle-\langle a\rangle-\langle b\rangle+\langle 1\rangle \\
& =\langle a\rangle\langle b\rangle-(\langle a\rangle+\langle b\rangle)+\langle 1\rangle \\
& =\langle a b\rangle-(\langle a+b\rangle(\langle a b\rangle+\langle 1\rangle))+\langle 1\rangle \\
& =\langle a b\rangle-(\langle 1\rangle(\langle a b\rangle+\langle 1\rangle))+\langle 1\rangle \\
& =\langle a b\rangle-\langle a b\rangle-\langle 1\rangle+\langle 1\rangle \\
& =0
\end{aligned}
$$

Lema 68. A aplicação

$$
\hbar: \begin{array}{ccc}
\dot{\mathbb{K}}^{n} & \longrightarrow & H^{n}(\mathbb{K}) \\
\left(a_{1}, \ldots, a_{n}\right) & \longmapsto\left(a_{1}\right) \ldots\left(a_{n}\right)
\end{array}
$$

é um n-símbolo de Steinberg

Demonstração. Segue imediatamente do fato que o símbolo de quatérnio

$$
(,)_{\mathbb{K}}: \dot{\mathbb{K}} \times \dot{\mathbb{K}} \longrightarrow{ }_{2} B(\mathbb{K}) \cong H^{2}(\mathbb{K}),(a, b) \mapsto(a, b)_{\mathbb{K}}=(a) \cup(b)
$$

é um 2- símbolo de Steinberg (confira o Teorema de Merkurjev mencionado no final da seção 2.9 do capítulo 2).

Proposição 113. Para cada $n \geq 0$, existe um triângulo incompleto de homomorfismos de grupos e estes que constituem um triângulo incompleto de morfismos de anéis graduados

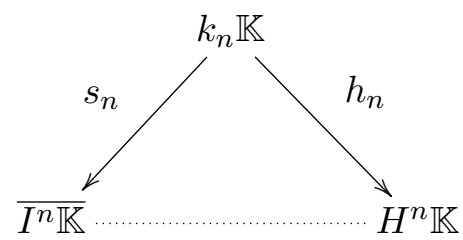

Demonstração. Vamos nos concentrar na construção das famílias de homomorfismos de grupos. Seja $\varsigma$ como no lema 67. A universalidade de $K_{n} \mathbb{K}$ fornece um único homomorfismo de grupos que torna o seguinte diagrama comutativo

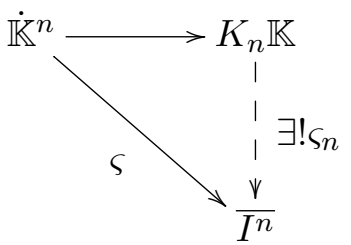

Observe que $\left.\varsigma_{n} l\left(a_{1}\right) \ldots l\left(a_{n}\right)\right)=\left\langle\left\langle-a_{1}, \ldots,-a_{n}\right\rangle\right\rangle+I^{n+1}$. Desde que, $2 \overline{I^{n}}=0$, existe um único homomorfismo de grupos $s_{n}: k_{n} \mathbb{K} \longrightarrow \overline{I^{n}}$ tal que o diagrama abaixo é comutativo: 


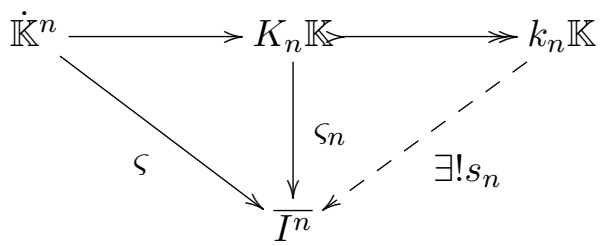

Finalmente, seja $\hbar$ como no lema 68 acima. Pela propriedade universal de $K_{n} \mathbb{K}$, existe um único homomorfismo $\hbar_{n}: K_{n} \mathbb{K} \longrightarrow H^{n} \mathbb{K}$ tal que $\hbar\left(l\left(a_{1}\right) \ldots l\left(a_{n}\right)\right)=\left(a_{1}\right) \ldots\left(a_{n}\right)$ e como $2 H^{n} \mathbb{K}=0$ (ver observação 40 do capítulo 3 ), segue que $\hbar_{n}$ induz um único homomorfismo $h_{n}: k_{n} \mathbb{K} \longrightarrow H^{n} \mathbb{K}$.

Observação 51. Para $t=2^{n-1}$ a composição $w_{t} \circ s_{n}$ é a multiplicação por $l(-1)$, ou seja, $w_{t} \circ s_{n}=$ $l(-1)^{t-n} \cdot(\quad)$.

\section{Proposição 114.}

1) Se $n=1,2$, temos isomorfismos

- $k_{1}(\mathbb{K}) \cong I(\mathbb{K}) / I^{2}(\mathbb{K})$

- $k_{2}(\mathbb{K}) \cong I^{2}(\mathbb{K}) / I^{3}(\mathbb{K})$

Demonstração. Se $n=1$ ou $n=2$, então $2^{n-1}=n$ e, portanto $t=n$. Assim, $w_{n} \circ s_{n}$ é a identidade. Logo, $s_{1}$ e $s_{2}$ são bijetivos (já que $s_{n}$ é sempre sobrejetivo).

Observação 52. Sejam $n \geq 2$ e $t=2^{n-1}$. Se $w_{t} \circ s_{n}=l(-1)^{t-n} \cdot(-): k_{n} \mathbb{K} \longrightarrow k_{t} \mathbb{K}$ é injetora, então a aplicação $s_{n}: k_{n} \mathbb{K} \longrightarrow I^{n}(\mathbb{K}) / I^{n+1}(\mathbb{K})$ é um isomorfismo de grupos.

Combinando a proposição 114 a proposição 44 o lema 13 (vi), a proposição 48 , e a proposição 86. obtemos os seguintes isomorfismos (dados por $s_{n}$ e $h_{n}, n=0,1,2$ )

- $\overline{I^{0}(\mathbb{K})} \cong k_{0}(\mathbb{K}) \cong H^{0}(\mathbb{K}) \cong \mathbb{Z} / 2 \mathbb{Z}$

- $\overline{I^{1}(\mathbb{K})} \cong k_{1}(\mathbb{K}) \cong H^{1}(\mathbb{K}) \cong \dot{K} / \dot{\mathbb{K}}^{2}$

- $\overline{I^{2}(\mathbb{K})} \cong k_{2}(\mathbb{K}) \cong H^{2}(\mathbb{K}) \cong{ }_{2} B r(\mathbb{K})$

Os isomorfismos anteriores levaram o medalhista Fields John Milnor a seguinte questão (central na teoria algébrica de formas quadráticas)

Problema I: É o homomorfismo $s_{n}: k_{n} \mathbb{K} \longrightarrow \overline{I^{n} \mathbb{K}}$ bijetivo para todo valor de $\mathrm{n}$ ?

Essa questão apareceu em Miln3 (Questão 4.3 , página 332), e ficou conhecida na literatura como a Conjectura de Milnor sobre o anel graduado de Witt. Levando em consideração a parte cohomológica, a conjectura de Milnor pode ser colocada da seguinte forma :

Conjectura de Milnor: Existe um diagrama completo comutativo

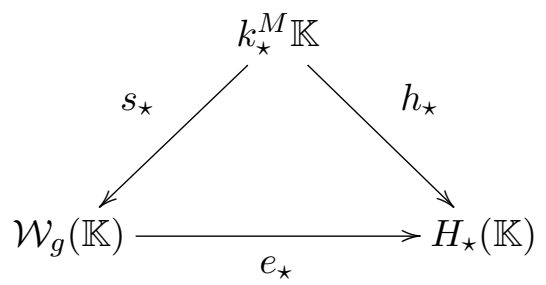


de isomorfismos de anéis graduados ? Ou seja, existe um diagrama completo de isomorfismos em nível $n$

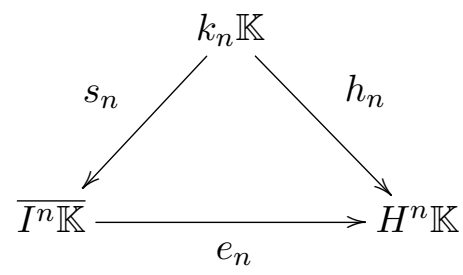

para todo $n$ ?

Um outro problema fundamental em teoria algébrica de formas quadráticas, era saber se o anel de Witt é Hausdorff pela topologia I-ádica. Equivalentemente,

Problema II: É a intersecção dos ideais $I^{n}(\mathbb{K})$ igual a zero ?

Essa questão apareceu primeiro, até onde eu saiba, em [Pfi3] na página 124 e depois em [Sch1] na página 263. John Milnor recoloca a questão em [Miln3], a saber, Questão 4.4 da página 332.

Jonh Milnor forneceu em [Miln3] algumas evidências para a sua primeira conjectura :

- Se $\mathbb{K}$ é um corpo global ou um limite de corpos globais, então $s_{n}$ é bijetivo.

- Se $\mathbb{K}$ é um corpo tal que $k_{2}(\mathbb{K})$ possui no máximo dois elementos distintos, então $s_{n}$ é bijetivo.

- Seja $(\mathbb{K}, v)$ um corpo completo sob a valoração discreta $v$ com característica $\neq 2$. Usando o Teorema de Springer 2 , Milnor mostrou que se

$$
s_{n}^{\mathbb{K}_{v}}: k_{n} \mathbb{K}_{v} \longrightarrow \overline{I^{n} \mathbb{K}_{v}}
$$

for um isomorfismo, então

$$
s_{n}^{\mathbb{K}}: k_{n} \mathbb{K} \longrightarrow \overline{I^{n} \mathbb{K}}
$$

também o será (ver [Miln3], cor 5.2, pág. 334).

- Se $\mathbb{K}$ é um corpo finito, ou local, ou global, ou real fechado; então Milnor mostrou que o homomorfismo induzido pelo homomorfismo de Bass-Tate 3

\footnotetext{
${ }^{2}$ Seja $(\mathbb{K}, v)$ completo sob uma valoração discreta $v$ e seja $\pi \in \dot{K}$ um uniformizador, i.e, $v(\pi)=1$. O Teorema de Springer afirma o seguinte : O anel de Witt $W(\mathbb{K})$ contém um subanel $W_{0}$ canonicamente isomorfo a $W\left(\mathbb{K}_{v}\right)$. Além disso, $W(\mathbb{K}) \cong W_{0} \oplus\langle\pi\rangle W_{0}$.

${ }^{3}$ Tate mostrou em (Tate1], teor.2, pág. 207) que se $m$ é um número natural primo com a característica do corpo $\mathbb{K}$, então o homomorfismo

$$
h_{m}: K_{2}(\mathbb{K}) \longrightarrow H^{2}\left(\mathbb{K}, \mu_{m} \otimes \mu_{m}\right)
$$

induz um isomorfismo

$$
K_{2}(\mathbb{K}) \cong H^{2}\left(\mathbb{K}, \mu_{m} \otimes \mu_{m}\right)
$$

Os argumentos usados por Bass e Tate permitem mostrar que se $\mathbb{K}$ é um corpo de característica $\neq 2$, então o isomorfismo $h^{1}: k_{1} \mathbb{K} \longrightarrow H^{1}(\mathbb{K})$ estende-se de maneira única para um homomorfismo de anéis graduados (ver Miln3, Lema 6.2, pág. 340)

$$
h_{\star}: k_{\star}^{M}(\mathbb{K}) \longrightarrow H_{\star}(\mathbb{K})
$$
}




$$
h_{\mathbb{K}}: k_{\star}(\mathbb{K}) \longrightarrow H_{\star}\left(\operatorname{Gal}\left(\mathbb{K}^{s e p} \mid \mathbb{K}\right), \mathbb{Z} / 2 \mathbb{Z}\right)
$$

é bijetivo. Além disso, se $\mathbb{K}=\lim _{\longrightarrow} \mathbb{K}_{\alpha}$ e cada $h_{\mathbb{K}_{\alpha}}$ é bijetivo, então $h_{\mathbb{K}}$ é bijetivo([Miln3], Lema 6.2, pág. 340).

- Seja $\mathbb{K}((t))$ o corpo de frações do anel de séries formais $\mathbb{K}[[t]]$. Se $h_{\mathbb{K}}$ é bijetivo, então $h_{\mathbb{K}((t))}$ é bijetivo (ver [Miln3], teor.6.3, pág. 340).

John Milnor também forneceu evidências positivas sobre o problema II

- Se $\mathbb{K}$ é um corpo global ou um limite de corpos globais, então $\cap I^{n}(\mathbb{K})$ pode ser mapeado bijetivamente para $\bigcap 2^{n} \mathbb{Z}$. Portanto,

$$
\bigcap_{n \in \omega} I^{n}(\mathbb{K})=0
$$

consulte ([Miln3], Lema 4.5, pág. 332).

- Se $\mathbb{K}$ é um corpo tal que $k_{2}(\mathbb{K})$ possui, no máximo, dois elementos distintos, então

$$
\bigcap_{n \in \omega} I^{n}(\mathbb{K})=0
$$

consulte ([Miln3], Lema 4.6, pág. 333).

- Se $(\mathbb{K}, v)$ um corpo completo sob a valoração discreta $v$ com característica $\neq 2$, então

$$
\bigcap_{n \in \omega} I^{n}(\mathbb{K})=0
$$

consulte ([Miln3], Lema 5.2, pág. 334).

Aqui é o ponto inicial da prova de Voevodsky para a conjectura de Milnor, que é por indução.

Por teoria de Kummer, temos um mapa sobrejetivo

$$
\partial: \dot{\mathbb{K}} \longrightarrow H^{1}\left(\mathbb{K}, \mu_{m}\right)
$$

Considere o produto cup

$$
H^{1}\left(\mathbb{K}, \mu_{m}\right) \otimes \ldots \otimes H^{1}\left(\mathbb{K}, \mu_{m}\right) \longrightarrow H^{n}\left(\mathbb{K}, \mu_{m}^{\otimes_{n}}\right)
$$

onde $\mu_{m}^{\otimes_{n}}$ é o produto tensorial sobre $\mathbb{Z}$ de $\mathrm{m}$ - cópias de $\mu_{m}$ equipado com a ação de Galois

$$
\sigma\left(\omega_{1} \otimes \ldots \otimes \omega_{n}\right)=\sigma(\omega) \otimes \ldots \sigma\left(\omega_{n}\right)
$$

Portanto, temos um homomorfismo

$$
\partial^{n}: \dot{\mathbb{K}} \otimes_{\mathbb{Z}} \ldots \dot{\mathbb{K}} \otimes_{\mathbb{Z}} \dot{\mathbb{K}} \longrightarrow H^{n}\left(\mathbb{K}, \mu_{m}^{\otimes_{n}}\right)
$$

onde $a_{1}, \ldots, a_{n} \in \mathbb{K}$ é uma sequência de elementos tais que $a_{i}+a_{j}=1$ para algum símbolo $1 \leq i<j \leq n$. Um resultado, devido a Jonh Tate, afirma que

$$
\partial^{n}\left(a_{1} \otimes \ldots \otimes a_{n}\right)=0
$$

Assim, $\partial^{n}$ fatora-se através de $K_{n}(\mathbb{K})$ e produz um mapa

$$
h_{\mathbb{K}, m}^{n}: K_{n}(\mathbb{K}) \longrightarrow H^{n}\left(\mathbb{K}, \mu_{m}^{\otimes_{m}^{\otimes n}}\right)
$$

conhecido como símbolo de Galois ou mapa norma residual. 
No ano seguinte ao da publicação do artigo Miln3 de Milnor, surge em [AP uma resposta positiva ao problema II, como consequência de um resultado que tornou-se célebre, o Hauptsatz de Arason-Pfister:

"Se $q$ é uma forma sobre um corpo $\mathbb{K}$ que é: não trivial, anisotrópica e tal que $q \in I^{n}(\mathbb{K})$, então $\operatorname{dim}(q) \geq 2^{n}$."

Por outro lado, passaram-se quase três decádas sem que houvesse uma afirmação positiva ou negativa para a conjectura de Milnor, até que em 1994, o brilhante matemático russo - e também medalhista Fields - Vladimir Voevodsky ${ }^{4}$ provou em [Voe1 o celabradíssimo resultado

Teorema 69. (Vladimir Voevodsky) Para qualquer corpo $\mathbb{K}$ de característica $\neq 2$ e qualquer $n \geq 0$, o homomorfismo norma residual

$$
\bar{h}_{n}: K_{n}(\mathbb{K}) / 2 K_{n}(\mathbb{K}) \longrightarrow H^{n}(\mathbb{K}, \mathbb{Z} / 2 \mathbb{Z})
$$

é um isomorfismo.

Para provar este teorema, Voevodsky usou, fundamentalmente, técnicas da teoria de cohomologia motívica, desenvolvida por ele em colaboração com A. Suslin e E. Friedlander (ver [FV],[SV1], [SV2, [SV3, [Voe1], [Voe2], [Voe3, ,Voe4]).

A prova usa, também, teoria de homotopia estável para esquemas, introduzidas por F. Morel em Morel1 e V. Voevodsky em MV].

Anos mais tarde, em um trabalho conjunto de Orlov, Vishik e Voevodsky ([OVV]), foi solucionado positivamente o problema I: para cada $n \geq 0$, o homomorfismo

$$
s_{n}: K_{n}(\mathbb{K}) / 2 K_{n}(\mathbb{K}) \longrightarrow I^{n}(\mathbb{K}) / I^{n+1}(\mathbb{K})
$$

é um isomorfismo.

Vladmir Voevodsky trabalhou, mais geralmente, com a conjectura de Block- Kato ${ }^{5}$, reduzindoa a questões concretas sobre variedades algébricas. Há diversas exposições na literatura a cerca da prova de Voevodsky da conjectura de Milnor, sendo que, a melhor delas (em minha opinião) é a exposição de Bruno de Kahn ([Kanh2]). Existem, ainda, outras exposições como [Fried] e Morel2.

\footnotetext{
${ }^{4} \mathrm{Um}$ dos problemas centrais na Matemática do século XX, era desenvolver em analogia com K-teoria topológica - iniciada por Alexander Grothendieck (Medalha Fields em 1966 )- uma K-teoria algébrica para anéis. Com essa filosofia, Karoubi e Villamayor dão em [KV] uma definição da K-teoria algébrica para um anel. Daniel Quillen, também, dá uma definição de K-teoria algébrica (considerada,atualmente, como uma definição correta), usando a construção-plus(ver Qui1, Qui3). Se X é um esquema regular, então A K-teoria de Karoubi -Villa Mayor concorda com a K-teoria de Quillen, porém discorda para esquemas singulares. Por outro lado, a versão algébrica de cohomologia singular não estava completamente definida. Um importante avanço veio, quando Voevodsky - baseado numa proposta de Andrei Suslin - introduziu a cohomologia motívica. Essa conquista tem suas raízes na obra de Alexandre Grothendieck. Grothendieck sugeriu que deveriam haver objetos, que ele chamou de motivos, que estão na base da unidade entre teoria dos números e geometria. Os trabalhos de Voevodsky trouxeram uma nova perspectiva para as teorias de cohomologia de variedades algébricas. Uma consequência do trabalho de Voevodsky, e uma de suas realizações mais celebradas, é a solução da conjectura de Milnor.

${ }^{5} \mathrm{O}$ matemático Kazuya Kato colocou em Kato a seguinte questão :
}

Conjectura Kato: O símbolo de Galois produz um isomorfismo

$$
K_{n}(\mathbb{K}) / m \stackrel{\cong}{\longrightarrow} H^{n}\left(\mathbb{K}, \mu_{m}^{\otimes_{n}}\right)
$$

para todo $n \geq 0$, todos corpos $\mathbb{K}$ e todo $m$ primo com a característica de $\mathbb{K}$.

Uma conjectura semelhante a essa (porém, em um versão mais fraca) foi proposta por Spencer Block em Block1]. 


\subsection{Anéis graduados, Teoria algébrica de formas quadráticas e Grupos especiais}

Terminamos este capítulo e, também, a dissertação com uma breve exposição da Conjectura de Marshall e a sua resolução. Seguiremos, quase fielmente, [DM1] e [DM2].

\subsubsection{A conjectura Lam}

Os resultados dessa subseção podem ser concultados em detalhes em Mon. T.Y. Lam colocou em ([Lam4 , Problema aberto B, pág. 49) a seguinte conjectura

Conjectura[LC]: Seja $\mathbb{K}$ um corpo formalmente real, $n \geq 1$ um inteiro e $\phi$ uma forma quadrática sobre $\mathbb{K}$. Se $\operatorname{sgn}_{P}(\phi) \equiv 0\left(\bmod 2^{n}\right), \forall P \in X_{\mathbb{K}}$, então $\phi \in I^{n}(\mathbb{K})+W_{\text {tors }}(\mathbb{K})$.

Essa conjectura ficou conhecida na literatura como Conjectura Lam. Veremos na sequência que esta conjectura é uma versão reduzida da conjectura de Milnor. Ou seja, é a conjectura de Milnor para a parte de torsão do anel de Witt. Doravante, salvo menção, $\mathbb{K}$ sempre denotará um corpo formalmente real.

Seja $\mathcal{C}\left(X_{\mathbb{K}}, \mathbb{Z}\right)$ o anel de funções contínuas de todas as aplicações contínuas

$$
\theta: X_{\mathbb{K}} \longrightarrow \mathbb{Z}
$$

onde $\mathbb{Z}$ é considerado com a topologia discreta. Lembremos do capítulo 2 que a assinatura de uma forma quadrática induz um homomorfismo

$$
\begin{aligned}
& \text { sgn }: W(\mathbb{K}) \longrightarrow \quad \mathcal{C}\left(X_{\mathbb{K}}, \mathbb{Z}\right) \\
& \phi \quad \longmapsto \quad \begin{array}{clc}
\operatorname{sgn}(\phi): X_{\mathbb{K}} & \longrightarrow & \mathbb{Z} \\
P & \longmapsto & \operatorname{sgn}(\phi)(P)=\operatorname{sgn}_{P}(\phi)
\end{array}
\end{aligned}
$$

com núcleo $W_{\text {tors }}(\mathbb{K})$. Observe que $\operatorname{sgn}\left(I^{n}(\mathbb{K})\right) \subseteq \mathcal{C}\left(X_{\mathbb{K}}, 2^{n} \mathbb{Z}\right)$. A composição

$$
I^{n}(\mathbb{K}) \longrightarrow \mathcal{C}\left(X_{\mathbb{K}}, 2^{n} \mathbb{Z}\right) \longrightarrow \mathcal{C}\left(X_{\mathbb{K}}, 2^{n} \mathbb{Z} / 2^{n+1} \mathbb{Z}\right) \longrightarrow \mathcal{C}\left(X_{\mathbb{K}}, \mathbb{Z} / 2 \mathbb{Z}\right)
$$

origina um homomorfismo

$$
\operatorname{sgn}_{n}: I^{n}(\mathbb{K}) / I^{n+1}(\mathbb{K}) \longrightarrow \mathcal{C}\left(X_{\mathbb{K}}, \mathbb{Z} / 2 \mathbb{Z}\right)
$$

Convém considerar agora o seguinte mapa

$$
\begin{aligned}
& h g_{n}: H^{n}(\mathbb{K}) \longrightarrow \quad \mathcal{C}\left(X_{\mathbb{K}}, \mathbb{Z} / 2 \mathbb{Z}\right)
\end{aligned}
$$

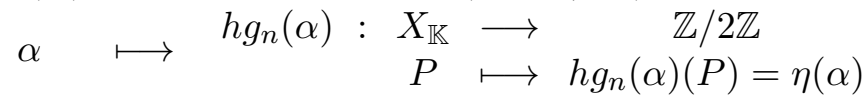

onde $\eta$ é isomorfo ao mapa natural

$$
\eta \cong H^{n}(i): H^{n}(\mathbb{K}) \longrightarrow H^{n}\left(\mathbb{K}_{P}\right) \cong \mathbb{Z} / 2 \mathbb{Z}
$$

induzido pela inclusão $i: \mathbb{K} \hookrightarrow \mathbb{K}_{P}$ em um fecho real de $\mathbb{K}$ para $\mathrm{P}$.

\section{Definição 92.}

1) A n-ésima parte de torsão do ideal fundamental do anel de Witt é definida por 


$$
I_{\text {tors }}^{n}(\mathbb{K}) \doteq W_{\text {tors }}(\mathbb{K}) \cap I^{n}(\mathbb{K})
$$

2) O subgrupo de 1-torsão de $H^{n}(\mathbb{K})$ é definido por

$$
H_{\text {tors }}^{n}(\mathbb{K}) \doteq\left\{\alpha \in H^{n}(\mathbb{K}): \alpha \cup(-1)^{k}=0 \text {, para algum } k\right\}
$$

Fato 35. $\operatorname{Ker}\left(h g_{n}\right)=H_{\text {tors }}^{n}(\mathbb{K})$.

Fato 36. Seja $\mathbb{K}$ um corpo formamente real. Então, a seguinte sequência é exata

$$
0 \longrightarrow I_{\text {tors }}^{2}(\mathbb{K}) \longrightarrow I_{\text {tors }}(\mathbb{K}) \stackrel{e_{1}^{*}}{\longrightarrow} H_{\text {tors }}^{1}(\mathbb{K})
$$

onde $e_{1}^{*}$ é a restrição de e e para a parte de torsão do ideal fundamental.

Conjectura [MT]: Seja $\mathbb{K}$ um corpo formalmente real e $n \geq 1$ um inteiro. Restringindo

$$
e_{n}: \bar{I}^{n}(\mathbb{K}) \longrightarrow H^{n}(\mathbb{K})
$$

para a parte de torsão do grupo de Witt, obtemos uma sequência exata

$$
0 \longrightarrow I_{\text {tors }}^{n+1}(\mathbb{K}) \longrightarrow I_{\text {tors }}^{n}(\mathbb{K}) \stackrel{e_{n}^{*}}{\longrightarrow} H_{\text {tors }}^{n}(\mathbb{K}) \longrightarrow 0
$$

Proposição 115. A conjectura $[\boldsymbol{L C}]$ é equivalente a conjectura $[\boldsymbol{M T}]$

Demonstração. Suponhamos que $[\mathbf{M T}]$ seja válida. Seja $\phi \in W(\mathbb{K}) \backslash\{0\}$ tal que $\operatorname{sgn}_{P}(\phi) \equiv$ $0\left(\bmod 2^{n}\right), \forall p \in X_{\mathbb{K}}$. Como $\bigcap_{r \in \omega} I^{r}(\mathbb{K})=\{0\}$, definimos

$$
m \doteq \max \left(\left\{r \in \omega: \phi \in I^{r}(\mathbb{K})\right\}\right)
$$

supondo $m<n$, temos também o seguinte diagrama comutativo

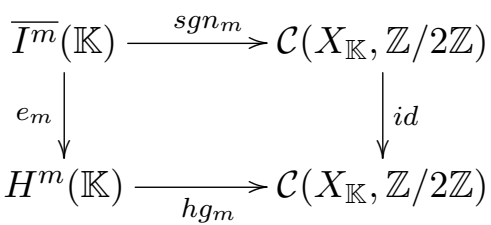

Agora,

$$
\operatorname{sgn}_{m}(\phi / m+1)=0 \Longrightarrow \alpha \doteq e_{m}(\phi / m+1)=e_{m}^{*}(\phi) \in \operatorname{ker}\left(h g_{m}\right)=H_{\text {tors }}^{m}(\mathbb{K})
$$

pela exatidão da sequência

$$
0 \longrightarrow I_{\text {tors }}^{m+1}(\mathbb{K}) \longrightarrow I_{\text {tors }}^{m}(\mathbb{K}) \stackrel{e_{m}^{*}}{\longrightarrow} H_{\text {tors }}^{m}(\mathbb{K}) \longrightarrow 0
$$

existe $\phi^{\prime} \in I_{\text {tors }}^{m}(\mathbb{K})$ tal que $e_{m}^{*}\left(\phi^{\prime}\right)=\alpha$. Assim, $e_{m}\left(\phi^{\prime} / m+1\right)=\alpha$. Como $e_{m}$ é injetivo, segue que $\phi / m+1=\phi^{\prime} / m+1$. Assim, $\psi=\phi-\phi^{\prime} \in I^{m+1}(\mathbb{K})$. Desde que, $\phi^{\prime} \in \operatorname{Ker}(\operatorname{sgn})$, vem $\operatorname{sgn}(\psi)=\operatorname{sgn}(\phi)$. Repetindo o processo, obtemos $\psi \in I^{n}(\mathbb{K})$. Assumindo $[\mathbf{L C}]$, temos que provar que

$$
e_{m}^{*}: I_{\text {tors }}^{n}(\mathbb{K}) \longrightarrow H_{\text {tors }}(\mathbb{K})
$$

é sobrejetivo. Seja $\alpha \in H_{\text {tors }}(\mathbb{K})$. Uma vez que, 


$$
0 \longrightarrow I^{n+1}(\mathbb{K}) \longrightarrow I^{n}(\mathbb{K}) \stackrel{e_{m}}{\longrightarrow} H^{n}(\mathbb{K}) \longrightarrow 0
$$

é exata $\left[6\right.$, existe $\phi \in I^{n}(\mathbb{K})$ tal que $e_{n}(\phi)=\alpha$. A comutatividade do diagrama

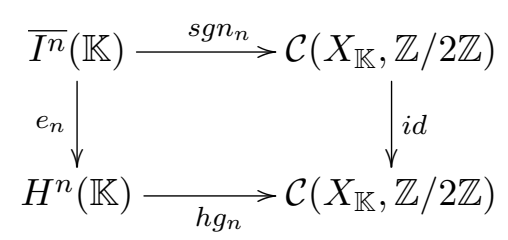

implica que $\operatorname{sgn}_{n}(\phi / n+1)=0$, assim

$$
\operatorname{sgn}(\phi) \equiv 0\left(\bmod 2^{n+1}\right)
$$

por $[\mathbf{L C}]$, podemos escrever $\phi=\phi^{\prime}+\phi^{\prime \prime}, \operatorname{com} \phi^{\prime} \in I^{n+1}(\mathbb{K})$ e $\phi^{\prime \prime} \in W_{\text {tors }}(\mathbb{K})$. Então, $\phi^{\prime \prime}=\phi-\phi^{\prime} \in$ $W_{\text {tors }}(\mathbb{K}) \cap I^{n}(\mathbb{K})=I_{\text {tors }}^{n}(\mathbb{K})$. Claramente $\phi /(n+1)=\phi^{\prime \prime} /(n+1)$ e $e_{n}(\phi)=e_{n}\left(\phi^{\prime}\right)=\alpha$ o que conclui a prova.

\subsubsection{A conjectura Marshall}

Para um corpo formalmente real pitagórico temos $W_{\text {tors }}(\mathbb{K})=0$. Nesse caso, a conjectura de Lam ganha a seguinte versão

Conjectura [MC]: $\operatorname{Se} \operatorname{sgn}_{P}(\phi) \equiv 0\left(\bmod 2^{n}\right), \forall P \in X_{\mathbb{K}}$, então $\phi \in I^{n}(\mathbb{K})$.

Essa questão apareceu em ([Mar1], questão aberta 2, pág. 575), mais geralmente, no contexto de espaços de ordens abstratos e ficou conhecida na literatura como a conjectura de Marshall sobre assinaturas.

A conjectura de Marshal foi resolvida por Dickmann e Miraglia em [DM2]. Finalmente, os mesmos resolveram a conjectura de Lam em [DM3.

Para uma breve exposição desse resultado iniciamos por estabelecer um vocabulário básico da Teoria de Grupos Especiais, desenvolvida em detalhes em [DM1].

Vimos, no capítulo 1, que o teorema de equivalência de cadeias de Witt expressa a isometria entre formas quadráticas de mesma dimensão através de uma sequência de isometrias entre formas de dimensão $\leq 2$. Isso motiva a seguinte

Definição 93. Um grupo pré-especial é uma tripla $\left\langle G, \equiv_{G},-1\right\rangle$ constando de grupo $(G, ., 1)$ de expoente 2, um elemento distinguido -1, e uma relação binária $\equiv_{G}$ em $G^{2}$ satisfazendo, para todos $a, b, c, d \in G$, os seguintes axiomas:

$S_{0}: \equiv_{G}$ é uma relação de equivalência.

$S_{1}:\langle a, b\rangle \equiv_{G}\langle b, a\rangle$.

$S_{2}:\langle a,-a\rangle \equiv_{G}\langle 1,-1\rangle$.

$S_{3}:\langle a, b\rangle \cong\langle c, d\rangle \Longrightarrow a b=c d$.

$S_{4}:\langle a, b\rangle \Longrightarrow\langle a,-c\rangle \equiv_{G}\langle-b, d\rangle$.

\footnotetext{
${ }^{6}$ Pois o triângulo de Milnor foi completado!
} 
$S_{5}:\langle a, b\rangle \equiv\langle c, d\rangle \Longrightarrow\langle x a, x b\rangle \equiv_{G}\langle x c, x d\rangle, \forall x \in G$.

Se $1 \neq-1$ e, em adição, $\left\langle G, \equiv_{G},-1\right\rangle$ satisfaz o axioma de redução

$$
\forall a \in G,\langle a, a\rangle \equiv_{G}\langle 1,1\rangle \Longleftrightarrow a=1
$$

diremos que ele é um grupo pré-especial reduzido.

Fato 37. Seja $X$ um conjunto $e \equiv$ uma relação binária em $X \times X$. Essa relação estende-se indutivamente para uma relação $n$-ária $\equiv_{n}$ em $X^{n}$ tal que:

$(0) \equiv_{1}$ é a relação de igualdade;

$(1) \equiv_{2}$ coincide com $\equiv$;

(2) $\left\langle a_{1}, \ldots, a_{n}\right\rangle \equiv_{n}\left\langle b_{1}, \ldots, b_{n}\right\rangle \Longleftrightarrow \exists x, y, z_{3}, \ldots, z_{n} \in X,\left\langle a_{1}, x\right\rangle \equiv\left\langle b_{1}, y\right\rangle,\left\langle a_{2} \ldots, a_{n}\right\rangle \equiv_{n-1}\left\langle x, z_{3}, \ldots, z_{n}\right\rangle$ $e\left\langle b_{2}, \ldots, b_{n}\right\rangle \equiv_{n-1}\left\langle y, z_{3}, \ldots, z_{n}\right\rangle$

Definição 94. Um grupo especial é um grupo pré - especial $\left\langle G, \equiv_{G},-1\right\rangle$ tal que a extensão de $\equiv_{G}$ para $G^{3}$ é uma relação transitiva.

O grupo especial de um corpo $\mathcal{G}(\mathbb{K})$ :

A principal classe de exemplos de grupos especiais provém (funtorialmente) da classe dos corpos $\mathbb{K}$ de característica $\neq 2$. Neste caso define-se $\mathcal{G}(\mathbb{K}):=\left(\dot{\mathbb{K}}_{\mathbb{K}} / \dot{\mathbb{K}}^{2}, ., \overline{1},-\overline{1}, \equiv\right)$ onde $\langle\bar{a}, \bar{b}\rangle \equiv\langle\bar{c}, \bar{d}\rangle$ sse $\langle a, b\rangle \simeq\langle c, d\rangle$, para $a, b, c, d \in \mathbb{K}$. Esta estrutura de primeira ordem codifica completamente a teoria algébrica de forma quadráticas sobre o corpo $\mathbb{K}$. Em particular, verifica-se facilmente que $\mathbb{K}$ é formalmente real e pitágorico se, e só se, $\mathcal{G}(\mathbb{K})$ é um grupo especial reduzido.

O anel de Witt de um grupo especial $W(G)$ :

Seja $G$ um grupo especial. A partir deste pode-se definir o semi-anel das formas diagonais ("regulares") módulo isometria; pela construção de Grothendieck (ver apêndice 1) obtem-se o anel de Witt-Grothendieck associado e, tomando o quociente pelo ideal das formas hiperbólicas, obtemos $W(G)$, o anel de Witt do grupo especial $G$; seja $I(G) \subseteq W(G)$ o ideal formado por (classes de equivalência) de formas de dimensão par.

$\mathrm{O}$ anel graduado 2-indutivo $\mathcal{K}(\mathbb{K})$ :

Seja $\mathbb{K}$ um corpo com característica $\neq 2$. Para cada $n \geq 1$, existe pelo teorema do homomorfismo, um homomorfismo de grupos

$$
\omega_{n}: k_{n}(\mathbb{K}) \longrightarrow k_{n+1}(\mathbb{K})
$$

definido em geradores como sendo a multiplicação por $l(-1)$. Assim, temos o seguinte diagrama comutativo infinito

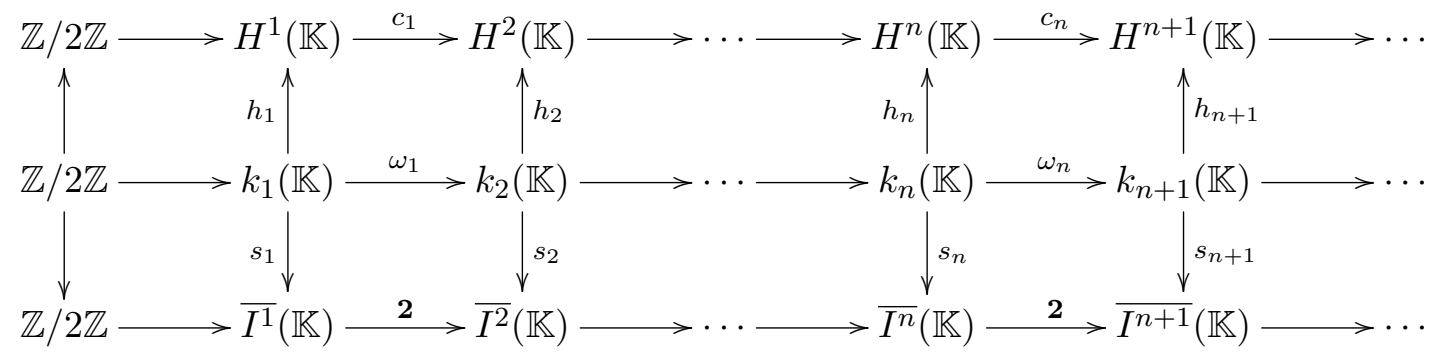

onde $c_{n} \doteq(-1) \cup-$ e $\mathbf{2} \doteq\langle 1,1\rangle \cdot-$ é a multiplicação por $2=\langle 1,1\rangle$. Este arranjo diagramático mostrou-se fundamental na solução da conjectura Marshall realizada em [DM2]. 
- Para cada $n \geq 1$, definimos $\top_{n} \doteq l(-1)^{n}$ como um elemento distinguido de $k_{n}(\mathbb{K})$.

- O homomorfismo $\omega_{n, m}: k_{n}(\mathbb{K}) \longrightarrow k_{m}(\mathbb{K})$ é a multiplicação por $l(-1)^{m-n}$, para $n \leq m$.

- É fácil ver que o anel graduado

$$
\mathcal{K}_{*}(\mathbb{K}) \doteq\left(k_{1}(\mathbb{K}), k_{2}(\mathbb{K}), \ldots, k_{n}(\mathbb{K}), \ldots\right)
$$

é um anel graduado 2-indutivo comutativo (veja a seção final do capítulo 2).

O anel graduado 2-indutivo $\mathcal{W}_{g}(G)$ :

Definimos o anel graduado de Witt do grupo especial $G, \mathcal{W}_{g}(G)$, através seguinte sequência

$$
\mathcal{W}_{g}(G) \doteq\left(\bar{I}(G), \overline{I^{2}}(G), \ldots, \overline{I^{n}}(G), \ldots\right)
$$

onde $\overline{I^{n}}(G) \doteq I^{n}(G) / I^{n+1}(G)$.

- Cada $\overline{I^{n}}(G)$ é um grupo de expoente 2 , pois

$$
\phi \oplus \phi=2 \otimes \phi \in I^{n+1}(G)
$$

qualquer que seja $\phi \in I^{n}(G)$.

- $\mathcal{W}_{g}(G)$ herda uma multiplicação graduada do produto tensorial de formas. Escrevendo $x / n$, com $x \in I^{n}(G)$, para um elemento de $\overline{I^{n}}(G)$, tem-se uma operação bem definida

$$
x / n * y / m \doteq(x \otimes y) / m+n
$$

é uma consequência direta das propriedades de soma e produto de formas que $\mathcal{W}_{g}(G)$ é um anel graduado 2 - indutivo comutativo.

- Para cada $n \geq 1$, podemos levantar a multiplicação por 2

$$
2 \otimes-\doteq\langle 1,1\rangle \otimes-: I^{n}(G) \longrightarrow I^{n+1}(G)
$$

Para homomorfismos de grupos (chamados de transições)

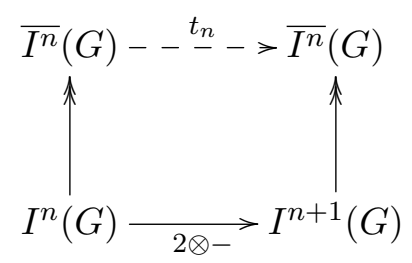

definido por

$$
t_{n}(x / n) \doteq(2 x) / n+1=(\langle 1,1\rangle \otimes x) / n+1
$$

Observe que

$$
t_{n, m}: \overline{I^{n}}(G) \longrightarrow \overline{I^{m}}(G)
$$


é a multiplicação por $2^{m-n}$, para todos $1 \leq n \leq m$. Definindo

$$
\top_{n} \doteq 2^{n} / n \in \overline{I^{n}}(G)
$$

é fácil ver que $\mathcal{W}_{g}(G)$ é um anel graduado 2 -indutivo comutativo.

Na sequência usaremos a abreviação DM para nos referirmos a Dickmann e Miraglia. A prova de $[\mathbf{M C}]$ foi dada em duas partes:

Primeira parte: DM desenvolveram várias técnicas, correlacionando grupos especiais reduzidos, álgebras booleanas e os três anéis graduados 2-indutivos $\left(\mathcal{W}_{g}, \mathcal{K}_{*}, \mathcal{H}^{*}\right)$ para mostrar que a conjectura $[\mathbf{M C}]$ é equivalente à seguinte conjectura fraca de Marshall

Conjectura [WMC]: Se $2 \phi \in I^{n+1}(\mathbb{K})$, então $\phi \in I^{n}(\mathbb{K}), \forall n \geq 1$.

As conjecturas [MC] e [WMC] fazem sentido no contexto de grupos especiais ditos formalmente reais. Por exemplo,

Conjectura [MC]:Se $\operatorname{sgn}_{\sigma}(\phi) \equiv 0\left(\bmod 2^{n}\right), \forall \sigma \in X_{G}$, então $\phi \in I^{n}(G)$.

onde $X_{G}$ denota o espaço de ordens de $G$, ou seja, o conjunto de homomorfismos de grupos especiais de $G$ para o grupo especial $\mathbb{Z}_{2}$ (topologizado adequadamente, como subespaço fechado de $\left.\left(\mathbb{Z}_{2}\right)^{G}\right)$.

A redução de $[\mathbf{M C}]$ para $[\mathbf{W M C}]$ se dá mediante a seguinte sequência :

1. Os limites indutivos

$$
\left\langle\mathcal{W}(G),\left\{\alpha_{n}: n \geq 1\right\}\right\rangle \doteq \lim _{n \in \omega} \mathcal{W}_{g}(G)
$$

e

$$
\left\langle\mathcal{K}(\mathbb{K}),\left\{\beta_{n}: n \geq 1\right\}\right\rangle \doteq \lim _{n \in \omega} \mathcal{K}_{*}(\mathbb{K})
$$

são anéis booleanos. Se, em adição, $\mathbb{K}$ for formalmente real e $G$ satisfaz a propriedade $\mathcal{A P}$ (de Arason-Pfister), então eles são anéis booleanos não-triviais, ou seja, $\perp \neq T$. Como álgebras booleanas, estes limites indutivos possuem uma estrutura natural de grupos especiais reduzidos.

2. Existem homomorfismos naturais de grupos (que são, também, homomorfismos de grupos especiais)

$$
\rho: G \longrightarrow \mathcal{W}(G) \quad \text { e } \quad \kappa: \mathcal{G}(\mathbb{K}) \longrightarrow \mathcal{K}(\mathbb{K})
$$

onde $\mathcal{G}(\mathbb{K})$ é o grupo especial associado a um corpo. Estes são injetivos se, e somente se, $G$ é reduzido e $\mathbb{K}$ é pitagórico. Em virtude da propriedade universal da envoltória booleana $B_{G}$, estes mapas podem ser levantados para homomorfismos de álgebras booleanas

$$
B(\rho): B(G) \longrightarrow \mathcal{W}(G) \quad \text { e } \quad B(\kappa): B(\mathbb{K}) \longrightarrow \mathcal{K}(\mathbb{K})
$$

3. DM mostraram que esses mapas são isomorfismos. De fato, eles possuem inversos 


$$
\mu: \mathcal{W}(G) \longrightarrow B(G) \quad \text { e } \varepsilon: \mathcal{K}(\mathbb{K}) \longrightarrow B(\mathbb{K})
$$

O homomorfismo $\mu$ é definido na imagem de $\varphi / n \in \overline{I^{n}}(G)$ em $\mathcal{W}(G)$ (pelos homomorfismos $\alpha_{n}$ dados pela construção do limite indutivo) como sendo o clopen

$$
\left\{\sigma \in X_{G}: \operatorname{sgn}_{\sigma}(\varphi) \not \equiv 0\left(\bmod 2^{n+1}\right)\right\}
$$

em $X_{G}$. O mapa $\mu$ é o análogo de um mapa considerado por Milnor em [Miln3]. O mapa $\varepsilon$, definido na imagem de $k_{n}(\mathbb{K})$ em $\mathcal{K}(\mathbb{K})$ envia (a imagem de) um gerador $l\left(a_{1}\right) l\left(a_{2}\right) \ldots l\left(a_{n}\right)$ para o elemento $\epsilon\left(a_{1}\right) \wedge \epsilon\left(a_{2}\right) \wedge \ldots \wedge \epsilon\left(a_{n}\right)$ em $B(\mathbb{K})$, onde $\epsilon(a):=\left\{\sigma \in X_{\mathbb{K}}: \sigma(a)=-1\right\}$.

4. Segue da definição do mapa $\mu$ que [MC] vale para um dado inteiro $n \geq 1$ se, e somente se o mapa $\mu \circ \alpha_{n}: \bar{I}^{n}(G) \longrightarrow B(G)$ é injetivo. E este é o caso se, e somente se [WMC] vale em nível $n$, ou equivalentemente, os mapas transições $\overline{I^{n}}(G) \stackrel{2}{\longrightarrow} \overline{I^{n+1}}(G)$ são injetivos.

Segunda parte Na segunda parte da prova, DM trabalharam com corpos formalmente reais e pitagóricos. Eles estabeleceram a injetividade do mapa $\overline{I^{n}}(\mathcal{G}(\mathbb{K})) \stackrel{2}{\longrightarrow} \overline{I^{n+1}}(\mathcal{G}(\mathbb{K}))$ com os seguintes passos:

1. Usando a sequência exata longa em cohomologia associada a extensões quadráticas de $\mathbb{K}$,

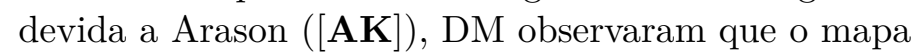

$$
(-1) \cup-: H^{n}(\mathbb{K}) \longrightarrow H^{n+1}(\mathbb{K})
$$

é injetivo, para todo $n \geq 1$.

2. Segue do teorema de Voevodsky (teorema 69) e da comutatividade do diagrama

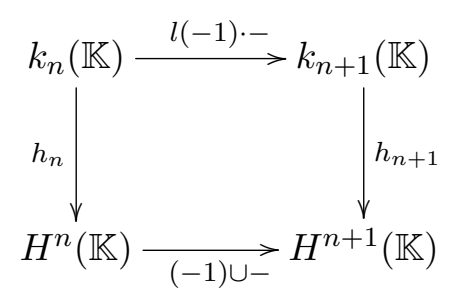

que o mapa da primeira linha do diagrama também é injetivo.

3. Finalmente, a observação 52 e a comutatividade do diagrama

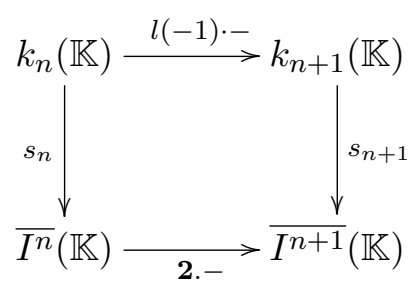

provam $[\mathbf{W M C}]$ e, portanto, [MC].

\subsubsection{Alguns detalhes sobre a resolução da conjectura de Marshall}

Nesta subsessão daremos mais detalhes de como procederam DM para resolver a conjectura Marshall. 
Proposição 116. Se $G$ é um grupo especial $\mathcal{A P}$, então

$$
G \quad \text { satisfaz } \quad[\boldsymbol{M C}] \Longleftrightarrow G \quad \text { satisfaz } \quad[\boldsymbol{W M C}]
$$

Corolário 24. Um corpo formalmente real satisfaz $[\boldsymbol{M C}]$ se, e somente se satisfaz $[\boldsymbol{W M C}]$.

Basta ver que $(-1) \cup-: H^{n}(\mathbb{K}) \longrightarrow H^{n+1}(\mathbb{K})$ é injetora, o que faremos a seguir.

Proposição 117. Seja $\mathbb{K}$ um corpo de característica $\neq 2$. Então,

a) $H_{\star}(\mathbb{K})$ é gerado, como anel graduado, por $H^{1}(\mathbb{K})$.

b) Seja $\mathbb{E}=\mathbb{K}\left(d^{1 / 2}\right)$. Para cada $\beta \in H^{n}(\mathbb{E})$ e $n \geq 1$, existe um conjunto finito de índices juntamente com $a_{1}^{i}, \ldots, a_{n-1}^{i} \in \dot{\mathbb{K}} e b^{i} \in \dot{\mathbb{E}}, i \in I$ tal que

$$
\beta=\sum_{i \in I}\left(a_{1}^{i}, \ldots, a_{n-1}^{i}, b^{i}\right)
$$

Demonstração. Ver ([DM2],corolário 6.3, pág.274).

Proposição 118. Se $\mathbb{K}$ é um corpo formalmente real e pitagórico, então o mapa

$$
(-1) \cup-: H^{n}(\mathbb{K}) \longrightarrow H^{n+1}(\mathbb{K})
$$

é injetivo, para todo $n \geq 1$.

Demonstração. Seja $\mathbb{E}=\mathbb{K}\left(d^{1 / 2}\right)$ a extensão quadrática de $\mathbb{K}$. Pelo colorário 4.6 em $[\mathbf{A K}$, existe uma sequência exata longa de cohomologia

$$
\cdots H^{n}(\mathbb{K}) \stackrel{\text { res }}{\longrightarrow} H^{n}(\mathbb{E}) \stackrel{\text { cor }}{\longrightarrow} H^{n}(\mathbb{K}) \stackrel{-\cup(d)}{\longrightarrow} H^{n+1}(\mathbb{K}) \cdots
$$

onde res e cor são, respectivamente, os homomorfismos restrição e corestrição definidos na seção 3.4.5 do capítulo 3. Estes mapas possuem as seguintes propriedades:

1. res $: H^{1}(\mathbb{K}) \longrightarrow H^{1}(\mathbb{E})$ é dado por $(a) \mapsto(a), \forall a \in \dot{\mathbb{K}}$.

2. cor $: H^{1}(\mathbb{E}) \longrightarrow H^{1}(\mathbb{K})$ é dado por $(b) \mapsto N_{\mathbb{E} \mid \mathbb{K}}(b)$, onde $N_{\mathbb{E} \mid \mathbb{K}}$ é a norma do corpo, ou seja, se $b=x+y \sqrt{d}$, então $N_{\mathbb{E} \mid \mathbb{K}}(b)=x^{2}-y^{2} d \in \dot{\mathbb{K}}$.

De acordo com o Fato 21 e a Proposição 63 da seção 3.4 do capítulo 3, temos:

3. $\operatorname{res}(\eta \cup \zeta)=\operatorname{res}(\eta) \cup \operatorname{res}(\zeta), \forall \eta \in H^{n}(\mathbb{K}), \forall \zeta \in H^{n}(\mathbb{K})$

4. $\operatorname{cor}(\operatorname{res}(\eta) \cup \zeta)=\eta \cup \operatorname{cor}(\zeta), \forall \eta \in H^{p}(\mathbb{K}), \forall \zeta \in H^{q}(\mathbb{E})$.

Em virtude da Proposição 117. (2) , cada $\beta \in H^{n}(\mathbb{E})$ pode ser escrito como:

$$
\beta=\sum_{i \in I}\left(a_{1}^{i}, \cdots, a_{n-1}^{i}, b^{i}\right)
$$

$\operatorname{com} a_{k}^{i} \in \dot{\mathbb{K}}$, para $1 \leq k \leq n-1$, e todo $i \in I$. Escrevendo $\eta_{i}=\left(a_{1}^{i}, \cdots, a_{n-1}^{i}\right)$, então 


$$
\begin{aligned}
\operatorname{cor}(\beta) & =\operatorname{cor}\left(\sum_{i \in I} \operatorname{res}\left(\eta_{i}\right) \cup b^{i}\right) \\
& =\sum_{i \in I} \operatorname{cor}\left(\operatorname{res}\left(\eta_{i}\right) \cup b^{i}\right) \\
& =\sum_{i \in I} \eta_{i} \cup \operatorname{cor}\left(b^{i}\right) \\
& =\sum_{i \in I} \eta_{i} \cup N_{\mathbb{E} \mid \mathbb{K}}\left(b^{i}\right)
\end{aligned}
$$

Observe que se $d=-1$, então, para cada $i \in I, N_{\mathbb{E} \mid \mathbb{K}}\left(b^{i}\right)$ é uma soma de quadrados não-nula em $\mathbb{K}$. Desde que, $\mathbb{K}$ é pitagórico, existe $x_{i} \in \dot{\mathbb{K}}$ tal que $N_{\mathbb{E} \mid \mathbb{K}}\left(b^{i}\right)=x_{i}^{2}, \forall i \in I$. Mas, para cada $i \in I$, temos $\left(x_{i}^{2}\right)=(1)$ (o zero de $H^{1}(\mathbb{K})$ ). Segue que cor é o mapa nulo de $H^{n}(\mathbb{E})$ para $H^{n}(\mathbb{K})$. Agora, a exatidão da sequência $(*)$, produz que o produto cup com $(-1)$ é uma injeção de $H^{n}(\mathbb{K})$ para $H^{n+1}(\mathbb{K})$, como afirmado.

Observe que o seguinte diagrama

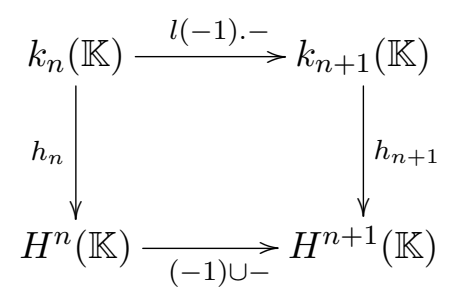

comuta. Combinando o teorema 69 e a proposição 118 , obtemos

Corolário 25. Para todo corpo formalmente real e pitagórico

$$
\omega_{n}=l(-1) .-: k_{n}(\mathbb{K}) \longrightarrow k_{n+1}(\mathbb{K})
$$

é injetivo.

Teorema 70. (Dickamnn - Miraglia)Todo corpo formalmente real e pitagórico satisfaz [MC].

Demonstração. Combinando o corolário 25 e a observação 52 resulta que $s_{n}$ é um isomorfismo, para todo $n \geq 1$. Agora a comutatividade do diagrama

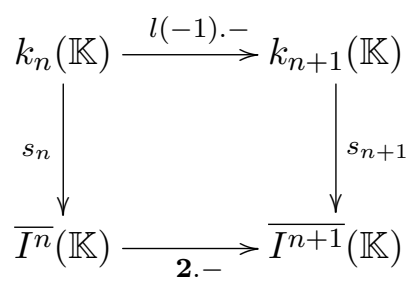

juntamente com o corolário 25 e a bijetividade de $s_{n}$ implica a injetividade de $t_{n}=2 .-$, ou seja, $\mathbb{K}$ satisfaz [WMC] e, portanto, pelo Corolário 24, $\mathbb{K}$ satisfaz [MC]. 


\section{Apêndice A}

\section{O Grupo de Grothendieck}

Neste apêndice vamos apresentar uma construção canônica relacionada ao problema de simetrização de um monóide. Em outras palavras, vamos transformar corretamente um monóide em um grupo. Um instância desse fato genérico é a famosa construção do anel dos números inteiros $\mathbb{Z}$ a partir do conjunto dos números naturais $\mathbb{N}$ (que é um monóide). Um tal procedimento é conhecido como construção de Grothendieck. Iniciamos com a seguinte

Proposição 119. Seja $\mathcal{U}: A b \longrightarrow$ Mon o funtor de esqueciment $\left.\right|^{1}$ da categoria dos grupos abelianos para a categoria dos monóides comutativos. Então $\mathcal{U}$ admite um adjunto à esquerda $\mathcal{K}:$ Mon $\longrightarrow A b$.

Demonstração. Para cada $M \in O b(M o n)$, seja $\mathcal{K}(M):=F(M) / R(M)$, onde $F(M)$ é o grupo abeliano livre com base $\{\langle a\rangle: a \in M\}$ e $R(M)$ é o subgrupo de $F(M)$ gerado pelo conjunto

$$
r(M):=\{\langle a\rangle+\langle b\rangle-\langle a+b\rangle: a, b \in M\} .
$$

Denotamos $\rho_{M}: M \longrightarrow \mathcal{K}(M)$ o mapa dado por $a \mapsto[a]:=\langle a\rangle+R(M)$. Uma vez que $\langle a+$ $b\rangle+R(M)=\langle a\rangle+\langle b\rangle+R(M)$, e que $\langle 0\rangle \in r(M) \subseteq R(M)$, segue que $\rho_{M}: M \longrightarrow \mathcal{K}(M)$ é um homomorfismo de monóides.

Afirmação: $\mathrm{O}$ par $\left(\mathcal{K}(M), \rho_{M}\right)$ satisfaz a propriedade universal abaixo:

Para cada grupo abeliano $A$ e cada homomorfismo de monóides $h: M \longrightarrow A$, existe um único homomorfismo de grupos abelianos $\tilde{h}: \mathcal{K}(M) \longrightarrow A$ que torna comutativo o seguinte diagrama de monóides abelianos e homomorfismos:

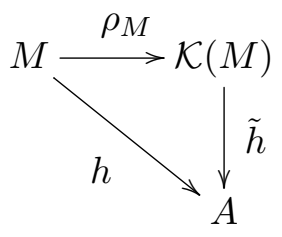

Verificaremos na sequência a afirmação.

Pela propriedade universal do grupo livre $F(M)$, existe um único homomorfismo de grupos $h^{+}: F(M) \longrightarrow A$ que torna comutativo o diagrama abaixo de conjuntos e funções:

\footnotetext{
${ }^{1}$ Como se faz correntemente, sempre que não existir risco de confusão, omitiremos menções explícitas aos funtores de esquecimento ou de inclusão de categorias.
} 


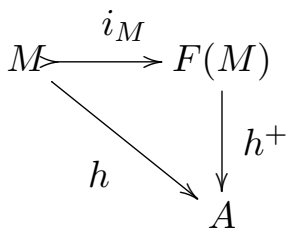

Como para cada $a, b \in M$ tem-se que $h(a+b)=h(a)+h(b)$, temos $h^{+}(\langle a+b\rangle)=h^{+}(\langle a\rangle)+$ $h^{+}(\langle b\rangle)$, assim $\langle a\rangle+\langle b\rangle-\langle a+b\rangle \in \operatorname{ker}\left(h^{+}\right)$. Portanto $R(M) \subseteq \operatorname{ker}\left(h^{+}\right)$e, pelo teorema do homomorfismo, existe um único homomorfismo de grupos $\tilde{h}: \mathcal{K}(M) \longrightarrow A$ tal que o diagrama abaixo de grupos e homomorfismos comuta:

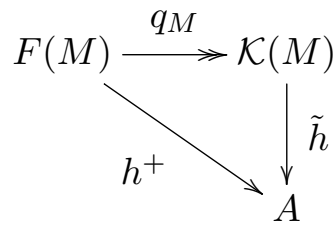

Combinando as informações e notando que o mapa $q_{M} \circ i_{M}=\rho_{M}: M \longrightarrow \mathcal{K}(M)$ é um homomorfismo de monóides a valor em um grupo abeliano, fica estalecida a propriedade universal.

Por um resultado bem conhecido sobre adjunções (veja por exemplo a referência clássica sobre categorias [MLan1], teorema 2, pág. 81), isto basta. Mais precisamente, existe uma única maneira de estender o mapa $M \mapsto \mathcal{K}(M)$, a um funtor $\mathcal{K}: M o n \longrightarrow A b$, de forma a $\rho=\left(\rho_{M}\right)_{M \in O b(M o n)}$ tornar-se uma transformação natural, i.e.: para cada homomorfismo de monóides $f: M \longrightarrow M^{\prime}$, a propriedade universal acima nos fornece um diagrama comutativo

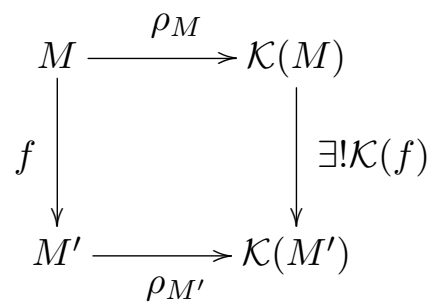

onde $\mathcal{K}(f):=\widetilde{\rho_{M^{\prime}} \circ f}$.

Observação 53. $\mathcal{K}:$ Mon $\longrightarrow A b$ é denominado o funtor de Grothendieck. Para cada monóide $M$, o grupo abeliano associado $\mathcal{K}(M)$, é nomeado o Grupo de Grothendieck de $M$.

Fato 38. $\mathcal{K}(M)=\{[a]-[b]: a, b \in M\}$. Em particular, $\mathcal{K}(M)$ é gerado, como grupo abeliano, pela imagem de $\rho_{M}$.

Demonstração. Basta observar que todo $x \in \mathcal{K}(M)$ admite alguma escrita

$$
x=\sum_{\alpha \in \mathbb{Z}_{0}^{+}} \alpha\left[x_{\alpha}\right]+\sum_{\beta \in \mathbb{Z}_{0}^{-}} \beta\left[x_{\beta}\right]
$$

Observação 54. É corriqueiro usar simplesmente $a-b$ no lugar da notação mais precisa $([a]+$ $R(M))-([b]+R(M))$ 
Na sequência vamos apresentar uma outra forma construir um adjunto à esquerda para o funtor esquecimento $\mathcal{U}: A b \longrightarrow M o n$.

Fato 39. Seja $M$ um monóide comutativo. Considere a relação binária em $M \times M$ definida por

$$
(a, b) \sim\left(a^{\prime}, b^{\prime}\right) \Longleftrightarrow \exists m \in M\left(a+b^{\prime}+m=a^{\prime}+b+m\right)
$$

Então:

(a) A relação $\sim$ é uma relação de equivalência.

(b) Quando $M$ satisfaz a propriedade do cancelamento, a relação acima pode ser descrita pela expressão alternativa e mais simples

$$
(a, b) \sim\left(a^{\prime}, b^{\prime}\right) \Longleftrightarrow a+b^{\prime}=a^{\prime}+b
$$

A classe de equivalência de um elemento $(a, b) \in M \times M$ será denotada simplesmente por $[a, b]$ . É de fácil verificação que a relação definida por

$$
[a, b]+[c, d]:=[a+c, b+d]
$$

é uma operação binária no conjunto quociente $(M \times M) / \sim$. Do mesmo modo, vemos que a relação

$$
-[a, b]:=[b, a]
$$

determina uma operação unária em $(M \times M) / \sim$.

Se denotarmos $0:=[0,0]$, segue rapidamente que:

- $\mathcal{G}(M):=((M \times M) / \sim,+,-, 0)$ é um grupo abeliano;

- O mapa $\gamma_{M}: M \longrightarrow \mathcal{G}(M)$, dado por $a \mapsto[a, 0]$ é um homomorfismo de monóides.

Além disso, verifica-se a validade do seguinte:

Fato 40 (Propriedade universal). Para cada grupo abeliano $G$ e cada homorfismo de monóides $h$ : $M \longrightarrow G$, existe um único homorfismo de grupos $\hat{h}: \mathcal{G}(M) \longrightarrow G$, dado por $\hat{h}([a, b])=h(a)-h(b)$, que torna o seguinte diagrama comutativo:

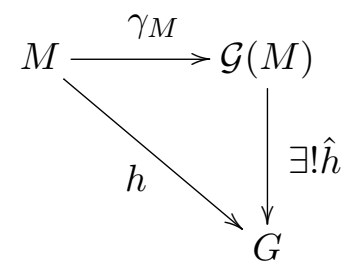

Observação 55. Já mencionamos anteriormente que uma propriedade universail como acima garante a existência de uma única maneira de estender o mapa $M \mapsto \mathcal{G}(M)$, a um funtor $\mathcal{G}$ : Mon $\longrightarrow A b$, de forma a $\gamma=\left(\gamma_{M}\right)_{M \in O b(M o n)}$ tornar-se uma transformação natural, i.e.: para cada homomorfismo de monóides $f: M \longrightarrow M^{\prime}$, a propriedade universal acima nos fornece um diagrama comutativo 


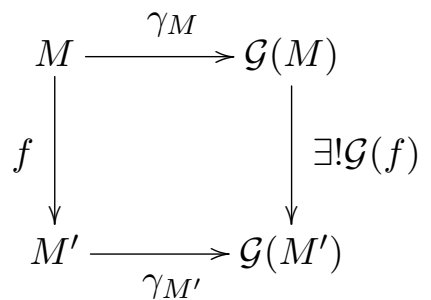

onde $\mathcal{G}(f):=\widehat{\gamma_{M^{\prime}} \circ f}$.

Outra consequência bem conhecida das propriedades universais é que adjuntos de um funtor são únicos a menos de isomorfismo natural (veja por exemplo [MLan1, corolário 1, pág. 83). Disponiblizamos abaixo uma prova, para o caso que temos em vista:

Proposição 120. Existe uma única transformação natural $\eta: \mathcal{K} \longrightarrow \mathcal{G}$ tal que o diagrama de monóides e homomorfismos abaixo comuta, para cada $M \in O b(M o n)$. Além disso, $\eta$ é um isomorfismo natural de funtores.

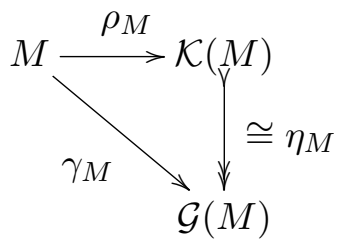

Demonstração. A existência de uma família de isomorfismos de grupos abelianos $\left(\eta_{M}: \mathcal{K}(M) \longrightarrow\right.$ $\mathcal{G}(M))_{M \in O b(M o n)}$ é uma consequência imediata da propriedade universal de cada flecha $\rho_{M}, \gamma_{M}$.

Para terminar vamos mostrar que para cada morfismo de monóides $f: M \longrightarrow M^{\prime}$ o próximo diagrama comuta

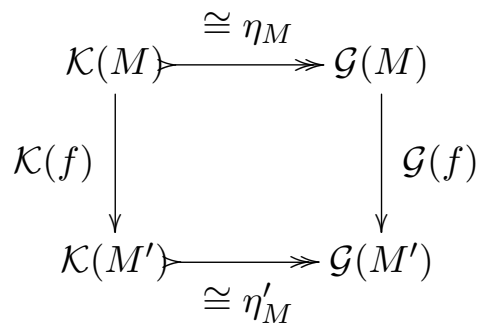

Mas este é o caso, uma vez que,

$$
\begin{array}{rcc}
\eta_{M^{\prime}} \circ \mathcal{K}(f) \circ \rho_{M} & =\eta_{M^{\prime}} \circ \rho_{M^{\prime}} \circ f \\
& =\quad \gamma_{M^{\prime}} \circ f \\
& =\quad \mathcal{G}(f) \circ \gamma_{M} \\
& =\mathcal{G}(f) \circ \eta_{M} \circ \rho_{M} .
\end{array}
$$

e, novamente pela propriedade universal de $\rho_{M}$, obtemos

$$
\eta_{M^{\prime}} \circ \mathcal{K}(f)=\mathcal{G}(f) \circ \eta_{M}
$$


A Proposição acima nos diz que os homomorfismos $\rho_{M}$ e $\gamma_{M}$ compartilham as mesmas propriedades. Em particular pelo Fato 38, o grupo abeliano $\mathcal{G}(M)$ é gerado pela imagem de $\gamma_{M}$. Analisaremos abaixo outra propriedade dos homomorfismos universais isomorfos $\rho_{M}$ e $\gamma_{M}$.

Proposição 121. Seja $M$ um monóide comutativo. São equivalentes:

1) $M$ satisfaz a propriedade do cancelamento.

2) $\gamma_{M}: M \longrightarrow \mathcal{G}(M)$ é um homomorfismo injetor de monóides.

3) $\rho_{M}: M \longrightarrow \mathcal{K}(M)$ é um homomorfismo injetor de monóides.

4) Existe um grupo $G$ e um homomorfismo de monóides $h: M \longrightarrow G$ que é injetivo.

Demonstração. (1) $\Rightarrow(2)$ : Sejam $a, a^{\prime}$ em $M$ tais que $\gamma_{M}(a)=\gamma_{M}\left(a^{\prime}\right)$, então $(a, 0) \sim\left(a^{\prime}, 0\right)$. Como assumimos que $M$ satisfaz a propriedade do cancelamento, o fato 39. (b) nos garante que $a+0=a^{\prime}+0, \operatorname{logo} a=a^{\prime}$ e concluimos que $\gamma_{M}$ é injetivo.

$(2) \Rightarrow(3)$ : Segue diretamente da Proposição 120.

$(3) \Rightarrow(4)$ : Imediato.

$(4) \Rightarrow(1):$ Sejam $a, a^{\prime}, m \in M$ tais que $a+m=a^{\prime}+m$, então $h(a)+h(m)=h(a+m)=$ $h\left(a^{\prime}+m\right)=h\left(a^{\prime}\right)+h(m)$. Como $G$ é um grupo, este satisfaz a propriedade do cancelamento, logo $h(a)=h\left(a^{\prime}\right)$ e como $h$ é injetor, concluimos que $a=a^{\prime}$.

Dispondo dos preparativos acima, podemos considerar agora a situação que mais nos interessa nesta dissertação.

Proposição 122. Sejam CSRing 1 a categoria dos semianéis comutativos com 1 e CRing I $_{1}$ categoria dos anéis comutativos com 1. Então o funtor esquecimento $\mathcal{U}:$ CRing $_{1} \longrightarrow S C R i n g_{1}$ admite um adjunto a esquerda $\mathcal{G}:$ SCRing $_{1} \longrightarrow$ CRing $_{1}$.

Demonstração. (Esboço)

- Verifica-se inicialmente que a relação

$$
\begin{aligned}
& \text { • : } \mathcal{G}(M) \times \mathcal{G}(M) \longrightarrow \quad \mathcal{G}(M) \\
& \left([a, b],\left[a^{\prime}, b^{\prime}\right]\right) \longmapsto\left[a a^{\prime}+b b^{\prime}, a b^{\prime}+b a^{\prime}\right]
\end{aligned}
$$

é uma operação binária.

- Através de longa lista de verificações, garantimos que o grupo abeliano aditivo $(\mathcal{G}(M),+,-, 0)$, munido da operação de multiplicação acima, e da constante $1:=[1,0]$, torna-se um anel comutativo com unidade.

- Segue rapidamente das definições que o homomorfismo de monóides aditivos $\gamma_{M}: M \longrightarrow$ $\mathcal{G}(M)$, preserva produto e 1 .

- Finalmente, prova-se (também rapidamente), que o homomorfimo de semi-anéis comutativos com unidade $\gamma_{M}: M \longrightarrow \mathcal{G}(M)$ satisfaz a propriedade universal: para cada anel comutativo com unidade $A$ e para cada homomorfismo semianéis com unidade $h: M \longrightarrow A$ existe um único homomorfismo de anéis com unidade $\hat{h}: \mathcal{G}(M) \longrightarrow A$ que torna comutativo o seguinte diagrama de semi-aneis comutativos com 1 : 


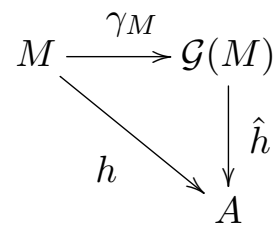

\section{Observação 56.}

(1) Analogamente podemos transformar o grupo abeliano $\mathcal{K}(M)$ em um anel universal mediante a operação

$$
(a-b)\left(a^{\prime}-b^{\prime}\right)=\left(a a^{\prime}+b b^{\prime}\right)-\left(a b^{\prime}+b a^{\prime}\right)
$$

$E$ assim $\mathcal{K}$ é um funtor adjunto para o funtor esquecimento da categoria dos anéis comutativos com unidade para a categoria dos semianéis comutativos com unidade.

(2) De forma análoga a Proposição 120, tem-se um isomorfismo natural $\eta: \mathcal{K} \cong \mathcal{G}$.

(3) De forma análoga a Proposição 121, verifica-se que um semi-anel comutativo com 1 satisfaz a propriedade do cancelamento (aditivo) se, e somente se, $\gamma_{M}: M \longrightarrow \mathcal{G}(M)$ é injetivo.

Observação 57. No início do Capítulo 2, observamos que a construção $\mathbb{K} \mapsto M(\mathbb{K})=\operatorname{Reg}(\mathbb{K}) / \simeq$ se estende a um funtor $\mathcal{M}:$ Field $_{1 / 2} \longrightarrow$ CSRing ${ }_{1}$. Assim obtemos o funtor "anel de WittGrothendieck"WG : Fields $s_{1 / 2} \longrightarrow$ CRing $1_{1}$ - único a menos de isomorfismo natural - através da composição $W G=\mathcal{G} \circ \mathcal{M}$. Devido a lei de cancelamento de Witt, cada semi-anel $M(\mathbb{K})$ satifaz a propriedade de cancelamento (aditivo), portanto o homomorfismo universal $\rho_{\mathbb{K}}: M(\mathbb{K}) \longrightarrow$ $W G(\mathbb{K}), \bar{\phi} \mapsto[\bar{\phi}, \overline{\langle\rangle}]$, é injetivo. 


\section{Apêndice B}

\section{Elementos de Cohomologia}

Reservamos este apêndice para tratar de definições e resultados gerais sobre cohomologia e cohomologia de grupos, sendo assim material complementar ao desenvolvido no Capítulo 3, onde centramos o foco sobre cohomologia profinita e galoisiana. As principais referências para este apêndice são: [BD], [MLan1], [HS], We2], [Mil1], [CE], [Ber] e [Rot2].

\section{B.1 Preliminares}

\section{B.1.1 A categoria de complexos}

Seja $\mathcal{A}$ uma categoria abeliana. Um complexo de cocadeias em $\mathcal{A}$ é uma sequência de morfismos $\left\{\partial^{n}: A^{n} \longrightarrow A^{n+1}\right\}_{n \in \mathbb{Z}}$ de $\mathcal{A}$ tal que $\partial^{n} \circ \partial^{n-1}=0$. É habitual denotar um complexo por $\left(C^{\bullet}, \partial^{\bullet}\right)$. Um complexo é representado diagramaticamente da seguinte forma

$$
\cdots \longrightarrow A_{n-1} \stackrel{\partial^{n-1}}{\longrightarrow} A_{n} \stackrel{\partial^{n}}{\longrightarrow} A_{n+1} \longrightarrow \cdots
$$

A noção dual do conceito de complexos de cocadeias é a noção de complexos de cadeias que nada mais é do que uma sequência morfismos

$$
\cdots \longrightarrow A_{n+1} \stackrel{\partial_{n+1}}{\longrightarrow} A_{n} \stackrel{\partial_{n}}{\longrightarrow} A_{n-1} \longrightarrow \cdots
$$

tais que $\partial_{n} \circ \partial_{n+1}=0$. Denotamos, simplesmente, por $\left(C_{\bullet}, \partial_{\bullet}\right)$. Existem formas de converter um complexo de cocadeias em um complexo de cadeias, sendo que, a mais simples consiste em colocar $A_{n} \doteq A^{-n}$ e $\partial^{n} \doteq \partial_{-n}$. O processo reverso também é possível.

Um morfismo entre complexos de cocadeias $\left(C_{A}^{\bullet}, \partial_{A}^{\bullet}\right)$ e $\left(C_{B}^{\bullet}, \partial_{B}^{\bullet}\right)$ (ou mapa de cocadeias) é uma família de morfismos $\left\{\varphi^{n}: A^{n} \longrightarrow B^{n}\right\}_{n \in \mathbb{Z}}$ com a seguinte propriedade

$$
\partial_{B}^{n} \circ \varphi^{n}=\varphi^{n+1} \circ \partial_{A}^{n}
$$

Ou seja, o diagrama abaixo é comutativo 


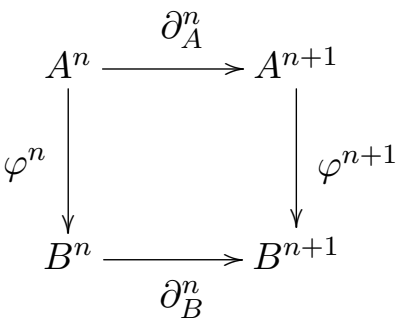

Simbolizamos por $\varphi^{\bullet}:\left(C_{A}^{\bullet}, \partial_{A}^{\bullet}\right) \longrightarrow\left(C_{B}^{\bullet}, \partial_{B}^{\bullet}\right)$ um morfismo de cocadeias. É fácil ver que se $\varphi^{\bullet}$ : $\left(C_{A}^{\bullet}, \partial_{A}^{\bullet}\right) \longrightarrow\left(C_{B}^{\bullet}, \partial_{B}^{\bullet}\right)$ e $\psi^{\bullet}:\left(C_{B}^{\bullet}, \partial_{B}^{\bullet}\right) \longrightarrow\left(C_{C}^{\bullet}, \partial_{C}^{\bullet}\right)$ são morfismos de cocadeias, então a família $\left\{\psi^{n} \circ \varphi^{n}\right\}_{n \in \mathbb{Z}}$ é um morfismo entre os complexos $\left(C_{A}^{\bullet}, \partial_{A}^{\bullet}\right)$ e $\left(C_{C}^{\bullet}, \partial_{C}^{\bullet}\right)$ e o denotamos por $\psi^{\bullet} \circ \varphi^{\bullet}$. Os complexos de cocadeias juntamente com os morfismos de cocadeias definem uma categoria chamada de categoria de complexos a qual denotamos por $C h^{\bullet}(\mathcal{A})$.

Definição 95. O n-ésimo objeto de cohomologia associado ao complexo $\left(C^{\bullet}, \partial^{\bullet}\right)$ é definido por

$$
H^{n}\left(C^{\bullet}, \partial^{\bullet}\right) \doteq \frac{\operatorname{Ker}\left(\partial^{n}\right)}{\operatorname{Im}\left(\partial^{n-1}\right)}
$$

onde o segundo membro significa Coker $\left(\operatorname{Im}\left(\partial^{n-1}\right) \longrightarrow \operatorname{Ker}\left(\partial^{n}\right)\right)$.

Seja $\varphi^{\bullet}:\left(C_{A}^{\bullet}, \partial_{A}^{\bullet}\right) \longrightarrow\left(C_{B}^{\bullet}, \partial_{B}^{\bullet}\right)$ um morfismo de complexo de cocadeias. Cada morfismo $\varphi^{n}$ : $A^{n} \longrightarrow B^{n}$ induz morfismos

$$
\operatorname{Ker}\left(\partial_{A}^{n}\right) \longrightarrow \operatorname{Ker}\left(\partial_{B}^{n}\right), \quad \operatorname{Im}\left(\partial_{A}^{n-1}\right) \longrightarrow \operatorname{Im}\left(\partial_{B}^{n-1}\right)
$$

e, portanto $\varphi^{n}$ define um homomorfismo

$$
H^{n}\left(\varphi^{n}\right): H^{n}\left(C_{A}^{\bullet}\right) \longrightarrow H\left(C_{B}^{\bullet}\right)
$$

As seguintes propriedades

- $H^{n}\left(\varphi^{n} \circ \psi^{n}\right)=H^{n}\left(\varphi^{n}\right) \circ H^{n}\left(\psi^{n}\right)$.

- $H^{n}\left(i_{d_{A^{n}}}\right)=i_{d_{H^{n}(C)}}$.

- $H^{n}\left(\psi^{n}+\varphi^{n}\right)=H^{n}\left(\varphi^{n}\right)+H^{n}\left(\psi^{n}\right)$

Fazem de $H^{n}$ um funtor aditivo.

Observação 58. Procedendo de maneira dual pode-se definir morfismos entre complexos de cadeias e, portanto é possível definir a categoria $C h(\mathcal{A})$ de complexos de cadeias.

\section{B.1.2 Objetos injetivos e projetivos}

Seja $\mathcal{C}$ uma categoria e $\mathcal{M} \subseteq M o r(\mathcal{C})$. Dizemos que $I \in O b(\mathcal{C})$ é $\mathcal{M}$ - injetivo, quando para cada diagrama

$$
A<\stackrel{\varphi}{\longleftarrow} \stackrel{\psi}{\longrightarrow} I
$$

onde $\varphi: B \longrightarrow A$ está em $\mathcal{M}$ e $\psi: B \longrightarrow I \in \operatorname{Mor}(\mathcal{C})$, existe um diagrama comutativo 


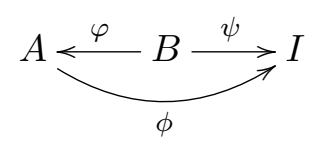

para algum morfismo $\phi: A \longrightarrow I \in \operatorname{Mor}(\mathcal{C})$. Quando todo $\mathcal{M}$ for a classe de todos os monomorfismo s diremos, simplesmente, que I é um objeto injetivo.

A noção dual de objetos $\mathcal{M}$-injetivos é a noção de objetos $\mathcal{M}$-projetivos. Dizemos que um objeto $P \in O b(\mathcal{C})$ é $\mathcal{M}$-projetivo, quando para todo $\varphi: A \longrightarrow B \in \mathcal{M}$ e todo $\psi: P \longrightarrow B$, existe $\phi: P \longrightarrow A \in \operatorname{Mor}(\mathcal{C})$ tal que o seguinte diagrama comuta

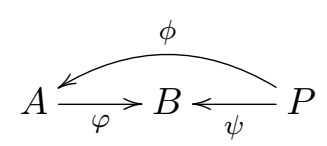

quando $\mathcal{M}$ consistir de todos epimorfismos diremos apenas que $P$ é um objeto projetivo.

\section{Definição 96.}

1) Diremos que uma categoria $\mathcal{C}$ admite suficientes objetos injetivos, quando cada objeto $A \in O b(\mathcal{C})$ é um sub-objeto de algum objeto injetivo I. Em outras palavras, quando existe um monomorfismo $A \longrightarrow I$

2) Diremos que uma categoria $\mathcal{C}$ admite suficientes objetos projetivos se, para cada $A \in \mathcal{C}$, existe um epimorfismo $P \longrightarrow A$ em algum objeto projetivo $P$.

Fato 41. Seja $\mathcal{A}$ uma categoria abeliana. Um objeto I de $\mathcal{A}$ é injetivo se, e somente se, $\operatorname{Hom}_{\mathcal{A}}(-, I)$ for um funtor exato, ou seja, se a sequência

$$
0 \longrightarrow A \stackrel{f}{\longrightarrow} B \stackrel{g}{\longrightarrow} C \longrightarrow 0
$$

for exata em $\mathcal{A}$, então a sequência

$$
0 \longrightarrow \operatorname{Hom}_{\mathcal{A}}(C, I) \stackrel{g^{*}}{\longrightarrow} \operatorname{Hom}(B, I) \longrightarrow \operatorname{Hom}_{\mathcal{A}}(A, I)^{f^{*}} \longrightarrow 0
$$

é exata.

\section{B.1.3 Resoluções injetivas e projetivas}

Seja $\mathcal{A}$ uma categoria abeliana. Dizemos que um complexo $\left(C^{\bullet}, \partial^{\bullet}\right)$ é acíclico (ou exato) quando $\operatorname{Im}\left(\partial^{n-1}\right)=\operatorname{Ker}\left(\partial^{n}\right)$. Dizemos que um complexo de cocadeias é positivo exato quando for exato e $A^{n}=0, \forall n<0$. Dizemos que um complexo positivo exato $I^{\bullet}$ é injetivo se cada objeto de $I^{\bullet}$ for injetivo. De forma semelhante podemos definir um complexo de cadeias negativo exato projetivo.

Definição 97. Seja $\mathcal{A}$ uma categoria abeliana.Uma resolução injetiva de um objeto $A \in O b(\mathcal{A})$ é um complexo de cocadeias positivo exato injetivo

$$
0 \longrightarrow I^{0} \stackrel{\partial^{0}}{\longrightarrow} I^{1} \longrightarrow \cdots \longrightarrow I^{n} \stackrel{\partial^{n}}{\longrightarrow} I^{n+1} \longrightarrow \cdots
$$

equipado com um morfismo $\varepsilon: A \longrightarrow I^{0}$ tal que a sequência

$$
0 \longrightarrow A \stackrel{\varepsilon}{\longrightarrow} I^{0} \stackrel{\partial^{0}}{\longrightarrow} I^{1} \longrightarrow \cdots \longrightarrow I^{n} \stackrel{\partial^{n}}{\longrightarrow} I^{n+1} \longrightarrow \cdots
$$


é exata. Enquanto, uma resolução projetiva de $A \in O b(\mathcal{A})$ é um complexo de cadeias negativo exato projetivo

$$
\cdots \longrightarrow P_{n} \stackrel{\partial_{n}}{\longrightarrow} P_{n-1} \longrightarrow \cdots \longrightarrow P_{1} \stackrel{\partial_{1}}{\longrightarrow} P_{0} \longrightarrow 0
$$

equipado com um morfismo $\varepsilon: P^{0} \longrightarrow$ A tal que sequência

$$
\cdots \longrightarrow P_{n} \stackrel{\partial_{n}}{\longrightarrow} P_{n-1} \longrightarrow \cdots \longrightarrow P_{1} \stackrel{\partial_{1}}{\longrightarrow} P_{0} \stackrel{\varepsilon}{\longrightarrow} A \longrightarrow 0
$$

é exata. Usaremos $A \longrightarrow I^{\bullet}$ para uma resolução injetiva de $A$ e $A \longrightarrow P \bullet$ para um resolução projetiva de $A$.

Lema 71. Seja A um objeto de $\mathcal{A}$, uma categoria abeliana com suficientes injetivos.

1) Existe um resolução injetiva de $A$.

2) $S e A \longrightarrow I^{\bullet}$ e $A \longrightarrow J^{\bullet}$ são duas resoluções injetivas de A, então existe um diagrama comutativo

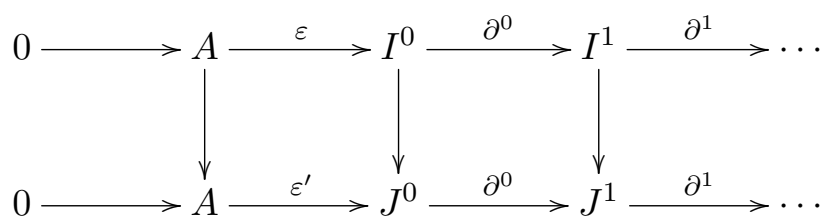

Demonstração.

1) Por hipótese existe um monomorfismo $\varepsilon: A \longrightarrow I^{0}$ onde $I^{0}$ é um objeto injetivd ${ }^{1}$. Definindo $B \doteq I^{0} / \operatorname{Im}(\varepsilon)$ teremos, novamente, por hipótese um monomorfismo $\partial: B \longrightarrow I^{1} \operatorname{com} I^{1}$ objeto injetivo. Assim, teremos um diagrama comutativo

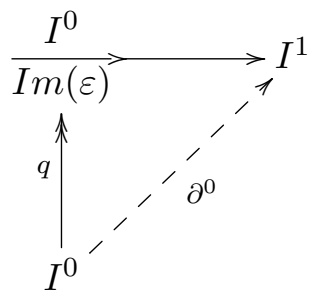

Claramente, a sequência

$$
0 \longrightarrow A \stackrel{\varepsilon}{\longrightarrow} I^{0} \stackrel{\partial^{0}}{\longrightarrow} I^{1}
$$

é exata. Por indução, suponhamos que temos uma sequência exata

$$
0 \longrightarrow A \stackrel{\varepsilon}{\longrightarrow} I^{0} \stackrel{\partial^{0}}{\longrightarrow} I^{1} \longrightarrow \cdots \longrightarrow I^{n-1} \stackrel{\partial^{n-1}}{\longrightarrow} I^{n}
$$

Seja $B^{\prime} \doteq \operatorname{Coker}\left(\partial^{n-1}\right)$. Por hipótese existe um monomorfismo $\partial^{\prime}: B^{\prime} \longrightarrow I^{n+1}$ com $I^{n+1}$ injetivo. Por conseguinte, temos um diagrama comutativo

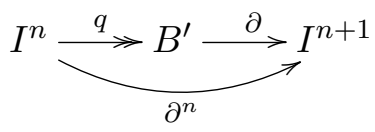

Assim, a seguinte sequência é exata

\footnotetext{
${ }^{1}$ Aqui faz se uso do princípio das escolhas dependentes.
} 


$$
0 \longrightarrow A \stackrel{\varepsilon}{\longrightarrow} I^{0} \stackrel{\partial^{0}}{\longrightarrow} I^{1} \longrightarrow \cdots \longrightarrow I^{n-1} \stackrel{\partial^{n-1}}{\longrightarrow} I^{n} \stackrel{\partial^{n}}{\longrightarrow} I^{n+1}
$$

2) De maneira semelhante podemos usar a definição de objeto injetivo para construir por indução o morfismo de resoluções injetivas.

\section{B.2 Funtores derivados à direita}

Seja $F: \mathcal{A} \longrightarrow \mathcal{B}$ um funtor exato à esquerda em categorias abelianas. Para cada resolução injetiva $A \longrightarrow I^{\bullet}$

$$
0 \longrightarrow A \stackrel{\varepsilon}{\longrightarrow} I^{0} \stackrel{\partial^{0}}{\longrightarrow} I^{1} \stackrel{\partial^{1}}{\longrightarrow} \cdots \longrightarrow I^{n} \stackrel{\partial^{n}}{\longrightarrow} I^{n+1} \longrightarrow \cdots
$$

obtemos o complexo de cocadeias $F\left(I^{\bullet}\right)$

$$
F\left(I^{0}\right) \stackrel{F\left(\partial^{0}\right)}{\longrightarrow} F\left(I^{1}\right) \stackrel{F\left(\partial^{1}\right)}{\longrightarrow} \cdots \longrightarrow I^{n} \stackrel{F\left(\partial^{n}\right)}{\longrightarrow} F\left(I^{n+1}\right) \longrightarrow \cdots
$$

Definição 98. Definimos o n-ésimo objeto de cohomologia associado ao complexo $F\left(I^{\bullet}\right)$ por $H^{n}\left(F\left(I^{\bullet}\right)\right)$.

Observação 59. Aplicando o funtor $F$ exato à esquerda na sequência exata

$$
0 \longrightarrow A \longrightarrow I^{0} \longrightarrow I^{1}
$$

obtemos a sequência exata

$$
0 \longrightarrow F(A) \longrightarrow F\left(I^{0}\right) \longrightarrow F\left(I^{1}\right)
$$

e, portanto

$$
\left.H^{0}\left(F\left(I^{\bullet}\right)\right)\right) \cong \operatorname{Ker}\left(\partial^{0}\right) \cong F(A)
$$

Fato 42. Seja $A \longrightarrow I^{\bullet}$ e $B \longrightarrow J^{\bullet}$ resoluções injetivas, respectivamente, dos objetos $A$ e $B$ de $\mathcal{A}$.

(i) Para cada morfismo $\alpha: A \longrightarrow B$ existe um diagrama comutativo

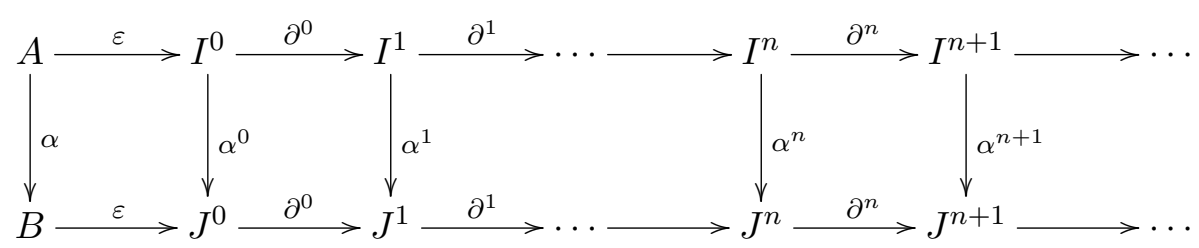

(ii) O diagrama do item (i) induz um morfismo de complexos de cocadeias

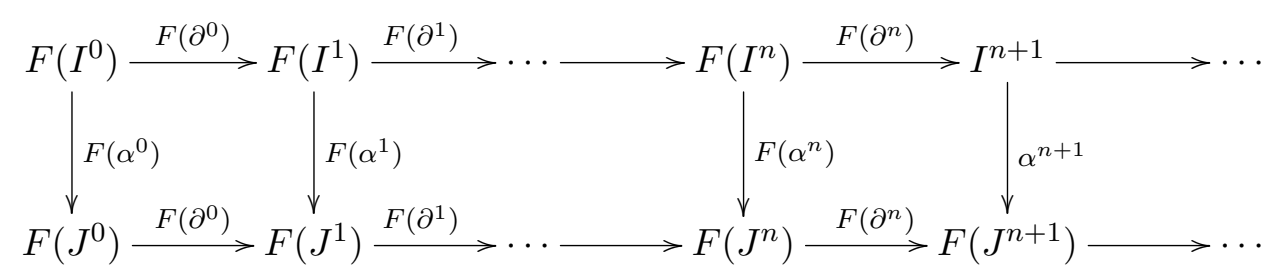


(iii) $O$ morfismo $H^{n}\left(F\left(\alpha^{\bullet}\right)\right): H^{n}\left(F\left(I^{\bullet}\right)\right) \longrightarrow H^{n}\left(F\left(J^{\bullet}\right)\right)$ não depende da escolha de $\alpha^{\bullet}=$ $\left(\alpha^{0}, \alpha^{1}, \cdots\right)$ e $\left.H^{(} F\left(\alpha^{0}\right)\right) \cong F(\alpha): F(A) \longrightarrow F(B)$.

Considerando o morfismo identidade $i_{d}: A \longrightarrow A$ teremos um diagrama comutativo

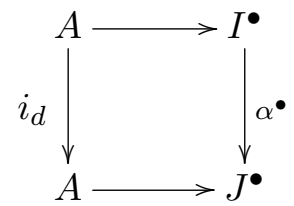

Nesse caso, os mapas induzidos $H^{n}\left(F\left(I^{\bullet}\right)\right) \longrightarrow H^{n}\left(F\left(I^{\bullet}\right)\right)$ são isomorfismos que independem da escolha de $\alpha^{\bullet}$. Assim, os objetos $H^{n}\left(F\left(I^{\bullet}\right)\right)$ são determinados por A e $\mathrm{F}$ a menos de isomorfismo. Para cada objeto A em $\mathcal{A}$ definimos

$$
\left(R^{n} F\right)(A) \doteq H^{n}\left(F\left(I^{\bullet}\right)\right)
$$

onde $A \longrightarrow I^{\bullet}$ é alguma resolução injetiva de A. Um morfismo $\alpha: A \longrightarrow B$ induz um morfismo bem definido (a menos de isomorfismo) $\left(R^{n} F\right)(A) \longrightarrow\left(R^{n} F\right)(B)$ e esses mapas fazem de $R^{n} F$ um funtor ${ }^{2}$ É possível mostrar que $\left\{R^{n} F\right\}_{n \in \omega}$ é um funtor covariante cohomológico universal ${ }^{3}$. Essa sequência de funtores é conhecida como a sequência de funtores derivados a direita de F.

Proposição 123. Toda sequência exata

$$
0 \longrightarrow A \longrightarrow B \longrightarrow C \longrightarrow 0
$$

em $\mathcal{A}$, origina uma sequência exata longa

$$
\begin{aligned}
& 0 \longrightarrow F(A) \longrightarrow F(B) \longrightarrow R^{\longrightarrow} \longrightarrow R^{1} F(A) \longrightarrow \cdots \\
& \cdots \longrightarrow R^{n} F(A) \longrightarrow R^{n} F(B) \longrightarrow R^{n} F(C) \longrightarrow R^{n+1} F(A) \longrightarrow \cdots
\end{aligned}
$$

Além disso, a associação da sequência exata curta com a sequência exata longa é funtorial.

Demonstração.

A segunda condição da proposição anterior significa que todo diagrama comutativo

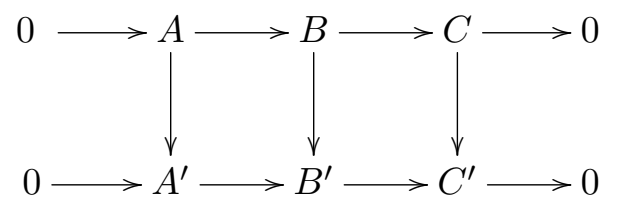

induz um diagrama comutativo e esta associação preserva identidade e composição

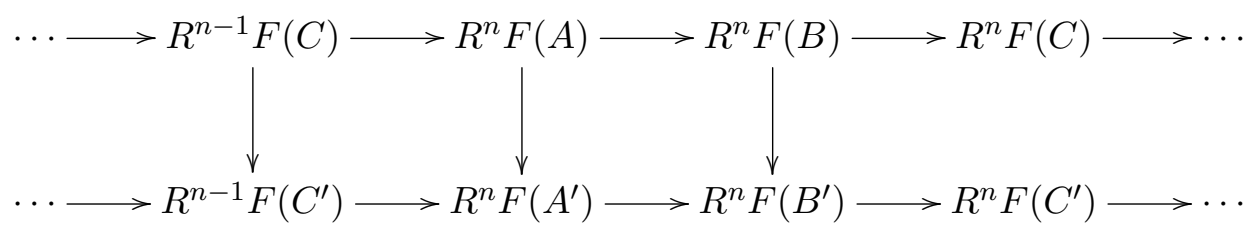

\footnotetext{
${ }^{2}$ Por isto há uma maneira de tomar $H^{n}$ funtores, isto é, preservando composições e identidade.

${ }^{3}$ Para mais detalhes veja as referências MLan1, CE] e Grot
} 
Observação 60. Os funtores derivados à direita de $F$ são unicamente caracterizados(a menos de um único isomofismo de funtores) pelas seguintes propriedades

$(R F)^{1}: R^{0} F=F$.

$(R F)^{2}: R^{n} F(I)=0, \forall n>0$, quando I é injetivo.

$(R F)^{3}:$ A propriedade expressa na proposição 123

\section{B.2.1 Funtores Ext}

Seja $\mathcal{A}$ uma categoria abeliana com suficientes objetos injetivos. Para cada $A \in O b(\mathcal{A})$ denotamos por $\operatorname{Ext}^{n}(A,-)$ o $n$-ésimo funtor derivado à direita do funtor exato à esquerda $\operatorname{Hom}(A,-)$. Como anteriormente, colocamos $\operatorname{Ext}^{n}(A, B)$ como sendo

$$
\operatorname{Ext}^{n}(A, B) \doteq H^{n}\left(\operatorname{Hom}\left(A, I^{\bullet}\right)\right)
$$

para alguma resolução injetiva $B \longrightarrow I^{\bullet}$.

Seja $\mathcal{A}$ uma categoria abeliana com suficientes objetos projetivos. Podemos definir os funtores derivados à direita do funtor contravariante exato à esquerda $\operatorname{Hom}(-, B)$. Simbolizamos por $\operatorname{Ext}^{n}(-, B)$ o $n$-ésimo funtor derivado a direita de $\operatorname{Hom}(-, B)$ e colocamos

$$
\operatorname{Ext}^{n}(A, B) \doteq H^{n}\left(H o m\left(P_{\bullet}, B\right)\right)
$$

para alguma resolução projetiva $A \longrightarrow P_{\bullet}$.

Proposição 124. Seja $\mathcal{A}$ uma categoria abeliana com suficientes objetos injetivos e suficientes objetos projetivos. Então, as definições de $\operatorname{Ext}^{n}(A, B)$ coincidem.

Demonstração. Definimos $E x t^{n}$ via resoluções projetivas e provamos que ele possui as propriedades que caracterizam os funtores derivados a direita de $\operatorname{Hom}(A,-)$.

- Claramente, $\operatorname{Ext}^{0}(A, B)=\operatorname{Hom}(A, B)$

- I é objeto injetivo $\Longleftrightarrow \operatorname{Hom}(-, I)$ é exatd 4 . Assim, $\operatorname{Hom}\left(P_{\bullet}, I\right)$ é exato e, portanto

$$
\operatorname{Ext}^{n}(A, I) \doteq H^{n}\left(H o m\left(P_{\bullet}, I\right)\right)=0
$$

- Finalmente, se

$$
0 \longrightarrow A \longrightarrow B \longrightarrow C \longrightarrow 0
$$

é exata, então

$$
0 \longrightarrow \operatorname{Hom}\left(P_{\bullet}, A\right) \longrightarrow \operatorname{Hom}\left(P_{\bullet}, B\right) \longrightarrow \operatorname{Hom}\left(P_{\bullet}, C\right) \longrightarrow 0
$$

é exata, pois $A \longrightarrow P_{\bullet}$ é uma resolução projetiva de A. Por um processo standard, obtemos de $(*)$ a sequência exata longa

$$
\cdots \rightarrow H^{n}\left(\operatorname{Hom}\left(P_{\bullet}, A\right)\right) \rightarrow H^{n}\left(H o m\left(P_{\bullet}, B\right)\right) \rightarrow H^{n}\left(H o m\left(P_{\bullet}, C\right)\right) \rightarrow \cdots
$$

\footnotetext{
${ }^{4}$ Ver a observação 41
} 


\section{B.3 G-módulos}

Seja $\mathrm{G}$ um grupo. Um G-módulo é um par $(A, \phi)$, onde A é um grupo abeliano e $\phi: G \longrightarrow$ $\operatorname{Aut}(A)$ é um homomorfismo de grupos. Observe que valem as seguintes propriedades

- $g \cdot\left(a+a^{\prime}\right)=g \cdot a+g \cdot a^{\prime}, \quad \forall g \in G, \forall a, a^{\prime} \in A$

- $\left(g g^{\prime}\right) \cdot a=g \cdot\left(g^{\prime} \cdot a\right), \quad \forall g, g^{\prime} \in G, \forall a \in A$

- $1 \cdot a=a, \quad \forall a \in A$

Onde $g \cdot a \doteq \phi(g)(a)$. Nesse caso, fica, automaticamente definida, uma G-ação

$$
\begin{aligned}
\cdot: G \times A & \longrightarrow A \\
(g, a) & \longmapsto \phi(g)(a)
\end{aligned}
$$

Inversamente, toda G-ação $: G \times A \longrightarrow A$ determina um homomorfismo de grupos

$$
\begin{aligned}
\phi: G & \longrightarrow \operatorname{Aut}(A) \\
g & \longmapsto g \cdot a
\end{aligned}
$$

Definição 99. Dizemos que um grupo $G$ age em um grupo abeliano $A$, quando A for um G-módulo. Dizemos que $G$ age trivialmente em $A$ quando $g \cdot a=a$ para todo $g \in G$ e todo $a \in A$.

Um G-homomorfismo de G-módulos é um homomorfismo de grupos abelianos $\varphi: A \longrightarrow B$ compatível com a G-ação, ou seja, tal que

$$
\varphi(g \cdot a)=g \cdot \varphi(a), \quad \forall a \in A, g \in G
$$

Denotamos por $\operatorname{Hom}_{G}(A, B)$ o conjunto de G-homomorfismos de A em B.

Observe que se $\varphi \in \operatorname{Hom}_{G}(A, B)$ e $\psi \in \operatorname{Hom}_{G}(B, C)$, então $\psi \circ \varphi \in \operatorname{Hom}_{G}(A, C)$. Os Ghomomorfismos de G-módulos juntamente com os G-módulos definem uma categoria a qual denotamos por $\operatorname{Mod}_{G}$.

Seja G um grupo e $\mathbb{Z}^{(G)}$ o grupo abeliano livre em G. Para cada $\alpha \in \mathbb{Z}^{(G)}$ definimos o suporte de $\alpha$ por

$$
\operatorname{supp}(\alpha) \doteq\{g \in G: \alpha(g) \neq 0\}
$$

Observe que cada $\alpha \in \mathbb{Z}^{(G)}$ pode ser escrito como

$$
\alpha=\sum_{g \in \operatorname{supp}(\alpha)} n_{g} \cdot \delta_{g}
$$

onde cada $n_{g} \in \mathbb{Z}$ e $\delta_{g}$ é a função característica em $\{g\}$. Na literatura é corriqueiro escrever $\left(^{*}\right)$ simplesmente por

$$
\alpha=\sum_{g \in \operatorname{supp}(\alpha)} n_{g} \cdot g
$$

um procedimento desses significa que estamos identificando o conjunto $\mathrm{G}$ com o conjunto $\Delta(G) \doteq$ $\left\{\delta_{g}: g \in G\right\}$. A multiplicação de elementos em $\mathbb{Z}^{(G)}$ apoia-se na multiplicação do grupo $\mathrm{G}$, ou seja,

$$
\left(\sum_{g \in \text { supp }(\alpha)} n_{g} \cdot g\right)\left(\sum_{n \in \operatorname{supp}(\alpha)} m_{h} \cdot h\right)=\sum_{g \in \operatorname{supp}(\alpha)} \sum_{h \in \operatorname{supp}(\beta)}\left(n_{g} m_{h}\right) g \cdot h
$$


o grupo $\mathbb{Z}^{(G)}$ com a multiplicação definida acima é um anel conhecido como anel do grupo G e é , habitualmente, denotado por $\mathbb{Z}[G]$.

Denotamos por $\mathbb{Z}[G]-\operatorname{Mod}$ a categoria de $\mathbb{Z}[G]$-módulos. Para cada $(A, \phi) \in \operatorname{Mod}_{G}$ existe um único homomorfismo de anéis com unidade $\phi^{*}: \mathbb{Z}[G] \longrightarrow E n d_{+}(A)$ (Pela universalidade de $\mathbb{Z}[G]$ ) que torna o seguinte diagrama comutativo

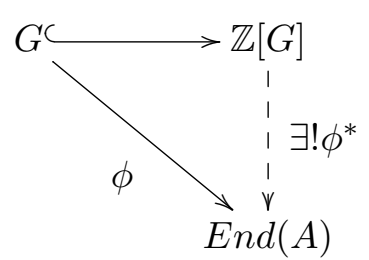

Verifique que $\phi^{*}$ dá a $A$ um estrutura de $\mathbb{Z}$-módulo. Reciprocamente, todo $\mathbb{Z}[G]$-módulo determina, trivialmente, um G-módulo. Além disso, um homomorfismo de grupos abelianos é um morfismo de G-módulos se, e somente se, for um homomorfismo de $\mathbb{Z}[G]$-módulos. Essa correspondência fornece um isomorfismo de categorias

$$
\operatorname{Mod}_{G} \cong \mathbb{Z}[G]-M o d
$$

\section{B.3.1 Módulos induzidos}

Seja G um grupo e H um subgrupo de G. Seja A um H-módulo. Definimos $A_{H}^{G}$ como sendo o conjunto das aplicações $\varphi: G \longrightarrow A$ que são H-lineares. De forma precisa, temos

$$
A_{H}^{G} \doteq\{\varphi: G \longrightarrow A: \varphi(h g)=h \varphi(g)\}
$$

Sejam A e B dois G-módulos. O conjunto $\operatorname{Hom}(A, B)$ de homomorfismos (aqui A e B são considerados com a estrutura de grupos abelianos)com a operação pontual é um grupo abeliano. A estrutura standard de G-módulo em $A_{H}^{G}$ é dada por

$$
\begin{aligned}
& \text { • : } G \times A_{H}^{G} \longrightarrow \quad A_{H}^{G} \\
& \begin{aligned}
(g, \varphi) & \longmapsto g \cdot \varphi: G \\
& \longrightarrow x \\
x & \longmapsto \varphi(x g)
\end{aligned}
\end{aligned}
$$

Cada morfismo de H-módulos $\psi: A \longrightarrow B$ induz um morfismo de G-módulos da seguinte forma

$$
\begin{aligned}
\psi_{H}^{G}: A_{H}^{G} & \longrightarrow B_{H}^{G} \\
\varphi & \longmapsto \psi \circ \varphi
\end{aligned}
$$

É fácil ver que as correspondências

$$
\begin{gathered}
()_{H}^{G}: \operatorname{Mod}_{H} \longrightarrow \operatorname{Mod}_{G} \\
A \in O b\left(\operatorname{Mod}_{H}\right) \longmapsto A_{H}^{G} \in \operatorname{Ob}_{\left(M_{G}\right)} \\
\psi \in \operatorname{Hom}_{H}(A, B) \longmapsto \psi_{H}^{G} \in \operatorname{Hom}_{G}\left(A_{H}^{G}, B_{H}^{G}\right)
\end{gathered}
$$

definem um funtor da categoria dos H-módulos para a categoria dos G-módulos.

Lema 72. Seja $A$ um G-módulo e $B$ um H-módulo. Temos

i) $\operatorname{Hom}_{G}\left(A, B_{H}^{G}\right) \cong \operatorname{Hom}_{H}(A, B)$

ii) O funtor ( $)_{H}^{G}$ é exato. 
Demonstração.

i)Para cada $\varphi \in \operatorname{Hom}_{G}\left(A, B_{H}^{G}\right)$ definimos $\hat{\varphi}: A \longrightarrow B$ por $\hat{\varphi}(a) \doteq \varphi(a)(1)$,onde 1 é o elemento neutro de G. Vamos mostrar que $\hat{\varphi}$ é um morfismo de H-módulos. Por um lado, para todo $g \in G$ temos

$$
\hat{\varphi}(g a)=\varphi(g a)(1)=(g \varphi(a))(1)=\varphi(a)(g)
$$

onde 1 é o elemento neutro de G. Por outro lado, temos para cada $g \in H$

$$
\varphi(a)(g)=\varphi(a)(g \cdot 1)=g(\varphi(a)(1))=g \hat{\varphi}(a)
$$

o que mostra que $\hat{\varphi} \in \operatorname{Hom}_{H}(A, B)$. Assim, fica definida uma aplicação

$$
\begin{aligned}
& \text { ^: } \operatorname{Hom}_{G}(A, B) \longrightarrow \operatorname{Hom}_{H}(A, B) \\
& \varphi \quad \longmapsto \quad \hat{\varphi}
\end{aligned}
$$

que é claramente um homomorfismo de grupos abelianos. Reciprocamente, para cada $\psi \in \operatorname{Hom}_{H}(A, B)$ definimos $\check{\psi}: A \longrightarrow B_{H}^{G}$ por $\check{\psi}(a)(g) \doteq \psi(g a)$. É fácil ver que $\check{\psi} \in \operatorname{Hom}_{G}\left(A, B_{H}^{G}\right)$. Assim, fica definida um homomorfismo

$$
\begin{array}{ccc}
\because \operatorname{Hom}_{H}(A, B) & \longrightarrow \operatorname{Hom}_{G}\left(\underset{\breve{\psi}}{A}, B_{H}^{G}\right) \\
\psi & \longmapsto & \check{\psi}
\end{array}
$$

Claramente^ $\mathrm{e}^{`}$ são inversos um do outro.

ii)Dada uma sequência exata de H-módulos

$$
0 \longrightarrow A \stackrel{\varphi}{\longrightarrow} B \stackrel{\psi}{\longrightarrow} C \longrightarrow 0
$$

vamos mostrar que a sequência de G-módulos

$$
0 \longrightarrow A_{H}^{G} \stackrel{\varphi_{H}^{G}}{\longrightarrow} B_{H}^{G} \stackrel{\psi_{H}^{G}}{\longrightarrow} C_{H}^{G} \longrightarrow 0
$$

é exata.

$\operatorname{Ker}\left(\psi_{H}^{G}\right)=\operatorname{Im}\left(\varphi_{H}^{G}\right):$ Seja $\beta \in \operatorname{Im}\left(\varphi_{H}^{G}\right)$. Nesse caso, existe $\alpha \in A_{H}^{G}$ tal que $\beta=\varphi_{H}^{G}(\alpha)$, ou seja, $\beta(g)=\varphi(\alpha(g)), \forall g \in G$. Como $\left(^{*}\right)$ é exata ter-se-á

$$
0=\psi(\beta(g))=\psi \circ \beta(g)=\psi_{H}^{G}(\beta)(g), \forall g \in G
$$

e, portanto $\beta \in \operatorname{Ker}\left(\psi_{H}^{G}\right)$.

Inversamente, seja $\beta \in \operatorname{Ker}\left(\psi_{H}^{G}\right)$. Então,

$$
\psi_{H}^{G}(\beta)=0 \Longleftrightarrow \psi \circ \beta(g)=0, \forall g \in G \quad(* *)
$$

Seja $\mathcal{R} \subseteq G$ um conjunto de classes de representantes à direita de $\mathrm{H}$ em G. Assim,

$$
G=\bigcup_{r \in \mathcal{R}} H r
$$

A expressão (**) transforma-se em

$$
\psi(\beta(h r))=0, \quad \forall h \in H, \forall r \in \mathcal{R}
$$


escolha $\alpha(r) \in A(\alpha(r)$ é único, pois $\varphi$ é injetor $)$ tal que $\varphi(\alpha(r))=\beta(r)$ e defina a aplicação

$$
\begin{aligned}
\alpha^{*}: G & \longrightarrow A \\
h r & \longmapsto h \alpha(r)
\end{aligned}
$$

Note que por construção $\alpha^{*} \in A_{H}^{G}$. Além disso, temos

$$
\begin{aligned}
\varphi_{H}^{G}\left(\alpha^{*}\right)(h r) & =\varphi \circ \alpha^{*}(h r) \\
& =\varphi(h(\alpha(r))) \\
& =h \varphi(\alpha(r)) \\
& =h \beta(r) \\
& =\beta(h r)
\end{aligned}
$$

e, portanto $\beta=\varphi_{H}^{G}\left(\alpha^{*}\right)$, ou seja, $\beta \in \operatorname{Im}\left(\varphi_{H}^{G}\right)$.

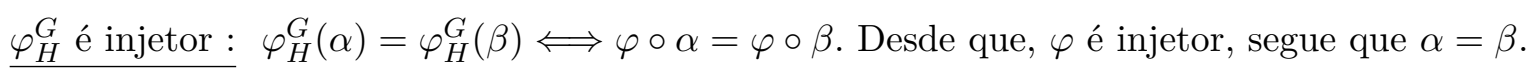

$\psi_{H}^{G}$ é sobrejetor: Seja $\gamma \in C_{H}^{G}$. Para cada $r \in \mathcal{R}$ seja $\beta(r) \in B$ tal que $\psi(\beta(r))=\gamma(r)$. Defina $\beta^{*}: G \longrightarrow B$ por $\beta^{*}(h r) \doteq h \beta(r)$. Note que $\beta^{*}$ é H-linear e $\psi_{H}^{G}\left(\beta^{*}\right)=\gamma$.

Corolário 26. Para cada H-módulo A existe um H-homomorfismo $\phi: A_{H}^{G} \longrightarrow A$ tal que o par $\left(A_{H}^{G}, \phi\right)$ cumpre a seguinte propriedade universal: Para cada H-homomorfismo $\alpha: B \longrightarrow A$, existe um único $G$-homomorfismo $\alpha^{*}: B \longrightarrow A_{H}^{G}$ que torna o seguinte diagrama comutativo, onde $B$ é um G-módulo

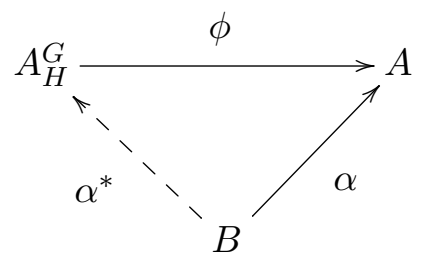

Proposição 125. A categoria Mod $_{G}$ possui suficientes objetos injetivos.

Demonstração. $\quad$ - Se $G=\{1\}$,então $\operatorname{Mod}_{G} \cong A b$.

- Seja A um G-módulo. Existe um mergulho

$$
A \hookrightarrow I
$$

de A (considerado como grupo abeliano) em um grupo abeliano injetivo I. Aplicando o funtor ()$_{1}^{G}$ em (*), obtemos um mergulho de G-módulos

$$
A_{1}^{G C} \longrightarrow I_{1}^{G} \quad(* *)
$$

Agora considere o mergulho de G-módulos 


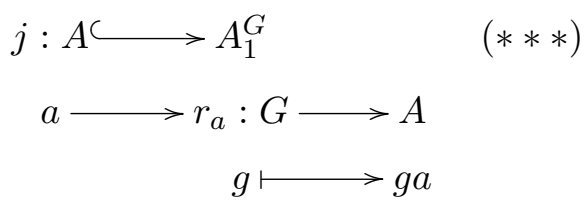

Compondo $(* *)$ e $(* * *)$

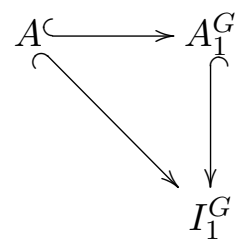

teremos um mergulho $A \hookrightarrow I_{1}^{G}$. Resta mostrar que $I_{1}^{G}$ é um G-módulo injetivo. Mas, o isomorfismo natural

$$
\operatorname{Hom}_{M_{G}}\left(-, I_{1}^{G}\right) \cong \operatorname{Hom}_{A b}(\mathcal{U}(-), I)
$$

(onde $\mathcal{U}(-)$ é um funtor esquecimento da categoria de monóides para a categoria de grupos abelianos)comfirma que $\operatorname{Hom}_{M o d}\left(-, I_{1}^{G}\right)$ é exato.

\section{Observação 61.}

(i) Analizando a prova da proposição anterior, vemos que o funtor ()$_{H}^{G}$ preserva objetos injetivos.

(ii) Pode-se mostrar que a categoria $\operatorname{Mod}_{G}$ é uma categoria abeliana com suficientes objetos projetivos.

\section{B.4 Cohomologia ordinária de grupos}

Seja A um G-módulo. Definimos $A^{G}$ como sendo

$$
A^{G} \doteq\{a \in A: g a=a, \forall g \in G\}
$$

Note que $A^{G}$ é um G-submódulo. Quando $a \in A^{G}$ dizemos que $\mathrm{G}$ fixa a ou que a é fixado por G. Se

$$
f: A \longrightarrow B
$$

for um G-homomorfismo, então a aplicação

$$
\begin{aligned}
f^{G}: A^{G} & \longrightarrow B^{G} \\
a & \longmapsto f(a)
\end{aligned}
$$

está bem definida e é um homomorfismo de grupos abelianos. É fácil ver que

$$
\begin{gathered}
{[]^{G}: \operatorname{Mod}_{G} \longrightarrow A b} \\
A \in O b\left(\operatorname{Mod}_{G}\right) \longmapsto A^{G} \in O b(A b) \\
f \in \operatorname{Hom}_{H}(A, B) \longmapsto f^{G} \in \operatorname{Hom}\left(A^{G}, B^{G}\right)
\end{gathered}
$$


é um funtor chamado de funtor submódulo fixado. Para cada $A \in O b\left(\operatorname{Mod}_{G}\right)$ é homomorfismo de grupos abelianos

$$
\begin{aligned}
& \eta_{A}: A^{G} \longrightarrow \operatorname{Hom}_{G}(\mathbb{Z}, A) \\
& \begin{aligned}
a \longmapsto \eta_{A}: \mathbb{Z} & \longrightarrow A \\
n & \longmapsto n a
\end{aligned}
\end{aligned}
$$

e é bijetora( $\mathbb{Z}$ como G-módulo com ação trivial). De fato,

$$
\eta_{A}(a)=\eta_{A}\left(a^{\prime}\right) \Longleftrightarrow n a=n a^{\prime}, \forall n \in \mathbb{Z} \Longleftrightarrow a=a^{\prime}
$$

e se $\phi \in \operatorname{Hom}_{G}(\mathbb{Z}, A)$, então $a \doteq \phi(1)$ é tal que

$$
g a=g \phi(1)=\phi(g \cdot 1)=\phi(1)=a
$$

e além disso $\eta_{A}(a)=\phi$. Para cada morfismo de G-módulos $f \in \operatorname{Hom}_{G}(A, B)$ temos um diagrama comutativo

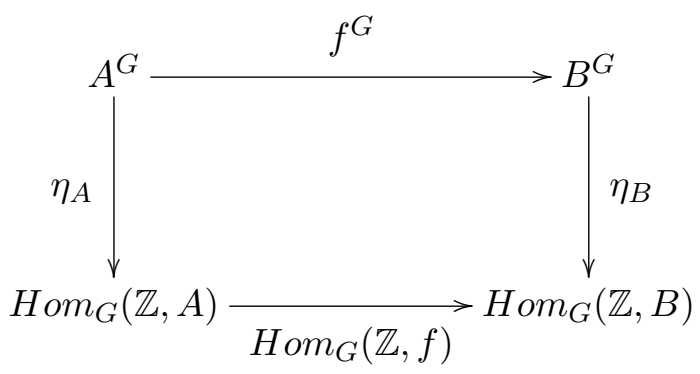

Isso significa que existe um isomorfismo natural

$$
[]^{G} \cong \operatorname{Hom}_{G}(\mathbb{Z},-)
$$

Como $\operatorname{Hom}_{G}(\mathbb{Z},-)$ é um funtor exato a esquerda, segue que o funtor ponto fixado [ $]^{G}$ também o é. Em outros termos, se

$$
0 \longrightarrow A \stackrel{\varphi}{\longrightarrow} B \stackrel{\psi}{\longrightarrow} C
$$

for uma sequência exata de G-módulos, então A sequência

$$
0 \longrightarrow A^{G} \stackrel{\varphi^{G}}{\longrightarrow} B^{G} \stackrel{\psi^{G}}{\longrightarrow} C^{G}
$$

também o será.

Como a categoria $\operatorname{Mod}_{G}$ possui suficientes objetos injetivos, podemos aplicar a teoria de funtores derivados nesse caso específico (ver seção B.2).

Seja $A$ um G-módulo e $A \longrightarrow I^{\bullet}$ uma resolução injetiva de A

$$
A \stackrel{\varepsilon}{\longrightarrow} I^{0} \stackrel{\partial^{0}}{\longrightarrow} I^{1} \stackrel{\partial^{1}}{\longrightarrow} \cdots \longrightarrow I^{n} \stackrel{\partial^{n}}{\longrightarrow} I^{n+1} \longrightarrow \cdots
$$

Aplicando o funtor fixado [ $]^{G}$ em $A \longrightarrow I^{\bullet}$ obtemos o complexo

$$
\left[I^{0}\right]^{G} \stackrel{\left[\partial^{0}\right]^{G}}{\longrightarrow}\left[I^{1}\right]^{G} \stackrel{\left[\partial^{1}\right]^{G}}{\longrightarrow} \cdots \longrightarrow\left[I^{n}\right]^{G} \stackrel{\left[\partial^{n}\right]^{G}}{\longrightarrow}\left[I^{n+1}\right]^{G} \longrightarrow \cdots
$$


Definição 100. Definimos o n-ésimo grupo de cohomologia ordinária de $G$ com coeficientes em A como sendo o grupo quociente

$$
H^{n}(G, A) \doteq \frac{\operatorname{Ker}\left(\left[\partial^{n}\right]^{G}\right)}{\operatorname{Im}\left(\left[\partial^{n-1}\right]^{G}\right)}
$$

Na sequência elencamos algumas propriedades importantes dos grupos de cohomomologia

Proposição 126. $H^{0}(G, A) \cong A^{G}$, pois a sequência

$$
0 \longrightarrow A^{G} \longrightarrow\left[I^{0}\right]^{G} \stackrel{\left[\partial^{0}\right]}{\longrightarrow}\left[I^{1}\right]^{G}
$$

é exata e além disso

$$
H^{0}(G, A) \doteq \frac{\operatorname{Ker}\left(\left[\partial^{0}\right]^{G}\right)}{\operatorname{Im}\left(\left[\partial^{-1}\right]^{G}\right)} \cong \operatorname{Ker}\left(\left[\partial^{0}\right]^{G}\right) \cong A^{G}
$$

Proposição 127. Se $I \in O b\left(\operatorname{Mod}_{G}\right)$ é um objeto injetivo, então para todo $n>0$

$$
H^{n}(G, I)=0
$$

pois a sequência

$$
0 \longrightarrow I \stackrel{i d}{\longrightarrow} I \longrightarrow 0 \longrightarrow \cdots
$$

é uma resolução injetiva.

Proposição 128. Se $A \longrightarrow I^{\bullet} e A \longrightarrow J^{\bullet}$ são resoluções injetivas de A, então todo G-homomorfismo $\alpha: A \longrightarrow B$ estende-se para um morfismo de complexos

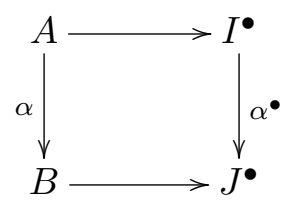

e os homomorfismos

$$
H^{n}\left(\alpha^{\bullet}\right): H^{n}\left(I^{\bullet}\right) \longrightarrow H^{n}\left(J^{\bullet}\right)
$$

independem da escolha de $\vec{\alpha}$.

Proposição 129. Aplicando a propriedade anterior para o G-homomorfismo identidade $i_{d}: A \longrightarrow$ A ter-se-á que os grupos $H^{n}(G, A)$ são bem definidos a menos de um isomorfismo bem definido.

Proposição 130. $A \mapsto H^{n}(G, A)$ é um funtor da categoria de G-módulos para a categoria de grupos abelianos.

Proposição 131. Toda sequência exata curta de G-módulos

$$
0 \longrightarrow A \longrightarrow B \longrightarrow C \longrightarrow 0
$$

dá origem a uma sequência exata longa de grupos abelianos (ver 123) 


$$
\begin{gathered}
0 \longrightarrow H^{0}(G, A) \longrightarrow H^{0}(G, B) \longrightarrow H^{0}(G, C) \stackrel{\delta^{0}}{\longrightarrow} H^{1}(G, A) \longrightarrow \\
\longrightarrow H^{1}(G, A) \longrightarrow H^{1}(G, B) \longrightarrow H^{1}(G, C) \stackrel{\delta^{1}}{\longrightarrow} H^{2}(G, A) \longrightarrow \cdots \\
H^{n}(G, A) \longrightarrow H^{n}(G, B) \longrightarrow H^{n}(G, C) \stackrel{\delta^{n}}{\longrightarrow} H^{n+1}(G, A) \longrightarrow \cdots
\end{gathered}
$$

Observação 62. Vimos que existe um isomorfismo natural

$$
\operatorname{Hom}_{G}(\mathbb{Z},-) \cong[\quad]^{G}
$$

Nesse caso, temos isomorfismos para seus funtores derivados

$$
\operatorname{Ext}_{G}^{n}(\mathbb{Z}, A) \cong H^{n}(G, A)
$$

Usando a proposição 124 deste apêndice, podemos escolher uma resolução projetiva $P_{\bullet} \longrightarrow \mathbb{Z}$ de $\mathbb{Z}$ e colocar

$$
H^{n}(G, A)=H^{n}\left(\operatorname{Hom}_{G}\left(P_{\bullet}, A\right)\right)
$$

Proposição 132. (Lema de Shapiro)Seja $G$ um grupo e $H$ um subgrupo de G. Para cada $n$ existe um isomorfismo natural

$$
H^{n}\left(G, A_{H}^{G}\right) \cong H^{n}(H, A)
$$

para todo H-módulo A.

Demonstração. Analisamos o primeiro caso. Seja $n=0$. Combinando o lema 72 e o isomorfismo natural $\operatorname{Hom}_{G}(\mathbb{Z},-) \cong[]^{G}$ obtemos

$$
H^{0}(H, A) \cong A^{H} \cong \operatorname{Hom}_{H}(\mathbb{Z}, A) \cong \operatorname{Hom}_{G}\left(\mathbb{Z}, A_{H}^{G}\right) \cong\left[A_{H}^{G}\right]^{G} \cong H^{0}\left(G, A_{H}^{G}\right)
$$

Para $n \geq 0$ geral seja $A \longrightarrow I^{\bullet}$ uma resolução injetiva de A. Desde que, ()$_{H}^{G}$ é exato (ver o lema 72 e preserva injetivos (ver a observação 61), obtemos a resolução injetiva $A_{H}^{G} \longrightarrow\left(I^{\bullet}\right)_{H}^{G}$ do G-módulo $A_{H}^{G}$. Assim,

$$
H^{n}\left(G, A_{H}^{G}\right) \cong H^{n}\left(\left[\left(I^{\bullet}\right)_{H}^{G}\right]^{G}\right) \cong H^{n}\left(\left[I^{\bullet}\right]^{H}\right) \cong H^{n}(H, A)
$$

Corolário 27. Se A for um G-módulo induzido, então para todo $n>0$

$$
H^{n}(G, A)=0
$$

Demonstração. Por hipótese $A \cong B_{1}^{G}$ para algum grupo abeliano B. Pela proposição anterior temos

$$
H^{n}(G, A) \cong H^{n}\left(G, B_{1}^{G}\right) \cong H^{n}(1, B)=0, \quad \forall n>0
$$




\section{B.4.1 Grupos de cohomologia via cocadeias}

Seja G um grupo. Denotamos por $G^{n+1}$ o conjunto das $n+1$-uplas de G. Precisamente, temos

$$
G^{n+1}=\left\{\left(g_{0}, g_{1}, \ldots, g_{n}\right): g_{i} \in G\right\}
$$

Para cada $n \in \omega$ definimos $P_{n} \doteq \mathbb{Z}^{\left(G^{n+1}\right)}$ como sendo o $\mathbb{Z}$ - módulo livre com base $G^{n+1}$. Fazemos deste um G-módulo mediante a ação diagonal

$$
g \cdot\left(g_{0}, g_{1}, \ldots, g_{n}\right) \doteq\left(g g_{0}, g g_{1}, \ldots, g g_{n}\right)
$$

Observe que $P_{n}$ é um $\mathbb{Z}[G]$ - módulo livre com base

$$
\left\{\left(1, g_{1}, \ldots, g_{n}\right): g_{i} \in G\right\}
$$

Definimos um homomorfismo $\partial_{n}: P_{n} \longrightarrow P_{n-1}$ em cada gerador pela regra

$$
\partial_{n}\left(1, g_{1}, \ldots, g_{n}\right) \doteq \sum_{i=0}^{n}(-1)^{i}\left(g_{0}, \ldots, \hat{g}_{i}, \ldots, g_{n}\right)
$$

onde o símbolo $\hat{x}$ significa que o símbolo $x$ foi omitido na expressão. Seja

$$
P_{\bullet}: \cdots \longrightarrow P_{n} \longrightarrow P_{n-1} \longrightarrow \cdots \longrightarrow P_{0}
$$

pode-se checar que $\partial_{n-1} \circ \partial_{n}=0$, ou seja, que $P_{\bullet}$ é um complexo. Seja $\varepsilon: P_{0} \longrightarrow \mathbb{Z}$ definida por $\varepsilon\left(g_{i}\right)=1, \forall i$.

Lema 73. O complexo $P_{\bullet} \longrightarrow \mathbb{Z} \longrightarrow 0$ é exato.

Demonstração. Escolhemos um elemento $\theta \in G$ e definimos $\kappa_{n}: P_{n} \longrightarrow P_{n+1}$ por

$$
\kappa_{n}\left(g_{0}, g_{1}, \ldots, g_{n}\right) \doteq\left(\theta, g_{0}, \ldots, g_{n}\right)
$$

Verifica-se facilmente que

$$
\partial_{n+1} \circ \kappa_{n}+\kappa_{n-1} \circ \partial_{n}=i d
$$

e, portanto

$$
\partial_{n}(x)=0 \Longrightarrow x=\partial_{n+1}\left(\kappa_{n}(x)\right)
$$

Proposição 133. Para qualquer G-módulo A vale o seguinte isomorfismo

$$
H^{n}(G, A) \cong H^{n}\left(\operatorname{Hom}_{G}\left(P_{\bullet}, A\right)\right)
$$

Demonstração. Segue do fato que $P_{\bullet} \longrightarrow \mathbb{Z}$ é uma resolução projetiva de $\mathbb{Z}$ (veja a observação 62 ).

Definição 101. Seja Func $\left(G^{n+1}, A\right)$ o conjunto das funções de $G^{n+1}$ em A. Dizemos que $\varphi \in$ Func $\left(G^{n+1}, A\right)$ é uma n-cocadeia homogênea de $G$ com valores em $A$, quando cumpre a seguinte condição

$$
\varphi\left(g g_{0}, \ldots, g g_{n}\right)=g\left(\varphi\left(g_{0}, \ldots, g_{n}\right)\right)
$$


Denotaremos por $\tilde{C}^{n}(G, A)$ o conjunto das n-cocadeias homogêneas.

A propriedade universal de $\mathbb{Z}^{\left(G^{n+1}\right)}$ nos dá para cada função $\varphi: G^{n+1} \longrightarrow A$ um diagrama comutativo

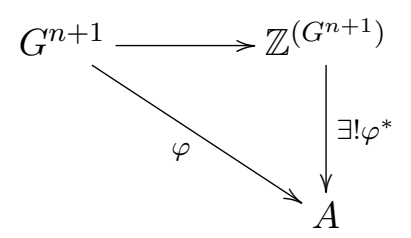

onde $\varphi^{*}$ é um morfismo em $A b$. Assim, temos uma bijeção natural

$$
\operatorname{Hom}\left(\mathbb{Z}^{\left(G^{n+1}\right)}, A\right) \cong \operatorname{Func}\left(G^{n+1}, A\right)
$$

Além disso, $\varphi \in\left[F u n c\left(G^{n+1}, A\right)\right]^{G} \Longleftrightarrow \varphi \in \tilde{C}^{n}(G, A)$. Dessa forma, existe uma bijeção natural

$$
\operatorname{Hom}_{G}\left(P_{n}, A\right) \cong \tilde{C}^{n}(G, A)
$$

Para cada $n \in \omega$ o homomorfismo n-bordo é o mapa

$$
\tilde{\partial}^{n}: \tilde{C}^{n}(G, A) \longrightarrow \tilde{C}^{n+1}(G, A)
$$

induzido por $\partial_{n}$.De acordo com a proposição 133 temos um isomorfismo

$$
H^{n}(G, A) \cong \frac{\operatorname{Ker}\left(\tilde{\partial}^{n}\right)}{\operatorname{Im}\left(\tilde{\partial}^{n-1}\right)}
$$

Na sequência apresentamos uma outra maneira de definir grupos de cohomologia via cocadeias. Veremos que ela permite olhar em $n$ coordenadas do grupo $\mathrm{G}$ ao invés de $n+1$ coordenadas. Em outros termos ela será mais efetiva.

Definição 102. Seja A um G-módulo. Definimos $C^{n}(G, A)$ para ser o conjunto de todos os mapas $\varphi: G^{n} \longrightarrow A$. Cada elemento de $C^{n}(G, A)$ será chamado de $\boldsymbol{n}$-cocadeia não homogênea de $\boldsymbol{G}$ com valores em $\boldsymbol{A}$. Definimos operadores cobordos

$$
\partial^{n}: C^{n}(G, A) \longrightarrow C^{n+1}(G, A)
$$

da seguinte maneira

$$
\begin{aligned}
\partial^{n} \varphi\left(g_{1}, \ldots, g_{n+1}\right) & =g_{1} \varphi\left(g_{2}, \ldots, g_{n}, g_{n+1}\right) \\
& +\sum_{k=1}^{n}(-1)^{k} \varphi\left(g_{1}, \ldots, g_{k} g_{k+1}, g_{k+2}, \ldots, g_{n+1}\right) \\
& +(-1)^{n+1} \varphi\left(g_{1}, \ldots, g_{n}\right)
\end{aligned}
$$

Definimos o grupo de $\boldsymbol{n}$-cociclos por

$$
Z^{n}(G, A)=\operatorname{Ker}\left(\partial^{n}\right)
$$

e o grupo de $\boldsymbol{n}$-cobordos por 


$$
B^{n}(G, A):=\operatorname{Im}\left(\partial^{n-1}\right)
$$

Fato 43. As seguintes aplicações

$$
\begin{aligned}
& \eta_{A}: \tilde{C}^{n}(G, A) \longrightarrow \quad C^{n}(G, A)
\end{aligned}
$$

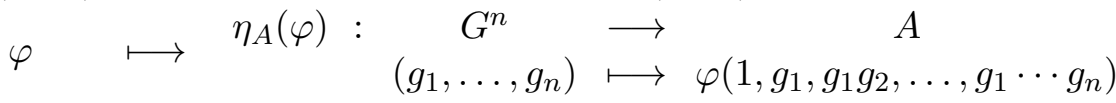

$$
\begin{aligned}
& \xi_{A}: C^{n}(G, A) \longrightarrow \quad \tilde{C}^{n}(G, A)
\end{aligned}
$$

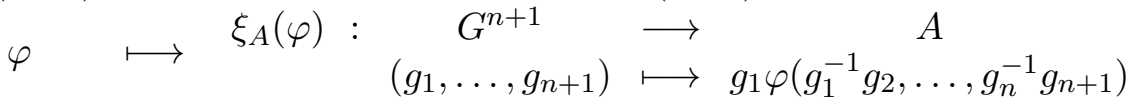

$e$

São homomorfismos inversos de G-módulos.

$\mathrm{O}$ fato anterior nos permite concluir a seguinte

\section{Proposição 134.}

(i)A sequência

$$
C^{0}(G, A) \stackrel{\partial^{0}}{\longrightarrow} C^{1}(G, A) \stackrel{\partial^{1}}{\longrightarrow} \cdots \longrightarrow C^{n}(G, A) \stackrel{\partial^{n}}{\longrightarrow} C^{n+1}(G, A) \longrightarrow \cdots
$$

é um complexo ,ou seja,

$$
\partial^{n} \circ \partial^{n-1}=0
$$

Para todo $n \geq 1$.

(ii)Existe um isomorfismo canônico

$$
H^{n}(G, A) \cong Z^{n}(G, A) / B^{n}(G, A)
$$




\section{Referências Bibliográficas}

[AE] J.K. Arason, R.Elman, Powers of the fundamental ideal in the Witt rings, Journal of Algebra 239 (2001), 150- 160.

[AK] J. K. Arason, M. Knebusch, Über die Grade quadratischer Formen, Math. Ann. 234 (1978), 167-192.

[AKa] Y. André, B. Kahn, A proof of Merkurjev's theorem, Quadratic and Hermitian forms(Hamilton, Ont., 1983), CMS Conf. Proc., vol. 4, Amer. Math. Soc., 1984, 121-130.

[AKM] A. Adem, D. B. Karagueuzian, J. Minác, On the Cohomology of Galois Groups Determined by Witt Rings, Advances in Mathematics 148 (1999), 105-160.

[AP] J. K. Arason, A. Pfister, Beweis des Krullschen Durchschnittsatzes für den Wittring, Invent. Math. 12 (1971), 173-176.

[Aras] J. K. Arason, Cohomologische Invarianten quadratischer Formen, Journal of Algebra 36 (1975), 448-491.

[Art] E. Artin, Galois Theory, University of Notre Dame Press, South Bend, 1942.

[Art2] E. Artin, Geometric Algebra, Interscience, New York, 1957.

[AW] M.F. Atiyah, C.T.C. Wall, Cohomology of Groups, in Cassels and Fröhlich, Academy Press, 1967.

[BD] I.Bucur, A.Deleanu, Introduction to the theory of categories and functors. With the collaboration of Peter J. Hilton and Nicolae Popescu. Pure and Applied Mathematics, Vol. XIX. Interscience Publication John Wiley \& Sons, Ltd., London-New YorkSydney, 1968.

[Bass1] H. Bass, Lectures on topics in Algebraic K-Theory, Tata- Institute of Fundamental Research, Bombay, 1967.

[Bass2] H. Bass, Algebraic K-Theory, Benjamin, New York, 1968.

[Bass3] H. Bass, Unitary algebraic K-theory, in Lecture Notes in Math. 342, SpringerVerlag, 1973.

[BHS] H. Bass, A. Heller, R. Swan, The Whitehead group of a polynomial extension, Publ. Math. IHES 22 (1964), 61-79.

[BLA] A. Blanchard, Les corps non commutatifs, P.U.F., 1972. 
[BMS] H.Bass, J.Milnor, J.P. Serre, Solution of the congruence subgroup problem for $S L_{n}(n \leq$ 3) and $S p_{2 n}(n \leq 2)$, Publ. Math. Inst. Hautes Études Sci. 33(1967), 59 -137.

[Ber] G . Berhuy, An Introduction to Galois Cohomology and its Applications, Cambridge University Press, 2010.

[Block1] S. Block, Lectures on Algebraic Cycles, Duke Univ. Lectures series, 1982.

[Block2] S. Block, Algebraic K-Theory, Motives, and Algebraic Cycles, course notes, University of Chicago.

[Bou1] N.Bourbaki, Algèbre, Chapitres 1-3, Éléments de Mathématiques. Hermann, 1970

[Bou2] N.Bourbaki, Topologie générale. Chapitres 1 - 4, Éléments de mathématique, Hermann, Paris, 1971

[Bou3] N.Bourbaki,Algèbre, Chapitre 10, Éléments de Mathématiques. Masson, 1980.

[Bou4] N.Bourbaki, Algèbre, Chapitre 4 - 7, Éléments de Mathématiques. Masson, 1981.

[Bou5] N.Bourbaki, Algèbre Commutative, Chapitres 8-9, Éléments de Mathématiques. Masson, 1983.

[Bre1] J.L. Bremer, Applications of the Dieudonné determinant, Linear Algebra Appl.1 (1968), 511-536.

[Bre2] J.L. Bremer, Corrections to Applications of the Dieudonné Determinant, Linear Algebra Appl. 13 (1976), 289.

[Brow] K. S. Brown, Cohomology of groups, Springer Verlag 1982.

[BT] H.Bass, J.Tate, The Milnor ring of a global field, in Algebraic K-Theory II, Lectures Notes in Math. 342, 349-446, Springer Verlag, 1973.

[Coh] M. M. Cohen, A course in simple homotopy theory, Graduate Texts in Mathematics, Vol.10, Springer-Verlag, New-York - Berlin, 1973.

[Cur1] C. W. Curtis, Pionners of Representation theory: Frobenius, Burside, Schur and Brauer. American Mathematical Society, Providence, 2000.

[Cur2] C. W. Curtis, Representation Theory of Finite Groups: from Frobenius to Brauer, Math. Intelligencer 14 (1992), 48-57.

[CE $]$ H. Cartan, S.Eilenberg, Homological Algebra, Princeton Univ. Press, Princeton, 1956.

[Del] A. Delzant, Définition des classes de Stiefel-Whitney d'un module quadratique sur un corps de charactéristique différent de 2, Academy Science Paris 255 (1962), 1366-1368.

[Die] J. Dieudonné, Les determinants sur un corps non commutatif, Bull. Soc. Math. France 71 (1943), 27-45.

[DM1] M. Dickmann, F. Miraglia, Special Groups: Boolean-Theoretic Methods in the Theory of Quadratic Forms, Memoirs of the AMS 689, American Mathematical Society, Providence, USA, 2000. 
[DM2] M. Dickmann, F. Miraglia, On Quadratic Forms whose total signature is zero mod $2^{n}$. Solution to a problem of M. Marshall, Inventiones Mathematicae 133 (1998), 243-278.

[DM3] M. Dickmann, F. Miraglia, Lam's Conjecture, Algebra Colloquium 10 (2003), 149-176.

[DM4] M. Dickmann, F. Miraglia, Algebraic K-theory of Special Groups, Journal of Pure and Applied Algebra 204 (2006), 195-234.

[Efr1] I. Efrat, Finitely generated pro-p Galois groups of p-henselian fields, J. Pure Appl. Algebra 138 (1999), 215-228.

[Efr2] I. Efrat, Construction of valuations from K-theory, Math. Research Letters 6 (1999), $335-344$.

[Efr3] I. Efrat, Valuations, orderings and Milnor K-theory, Mathematical Surveys and Monographs 124, (2006).

[EKM] R.Elman, N.Karpenko, A.Merkurjev, The Algebraic and Geometric Theory of Quadratic Forms, course notes.

[EL1] R.Elman, T. Y. Lam, Pfister forms and K-theory of fields, Journal of Algebra 23 (1972), $181-213$.

[EL2] R.Elamn, T. Y. Lam, Quadratic forms over formally real and pythagorean fields, Amer. J. Math. 94 (1972), 1155 - 1194.

[End] O. Endler, Teoria dos Corpos, Monografias de Matemática 44, IMPA, Brasil, 1987.

[ES] S. Eilenberg, N. Steenrod, Foundations of Algebraic Topology, Princeton Univ.Press, Princeton, 1952.

[Fried] E. Friedlander, Motivic complexes of Suslin and Voevodsky, Astérisque, 245, (1997), Exp. No. 833, 355-378.

[FV] E. Friedlander, V. Voevodsky,Bivariant cycle cohomology, preprint (1995).

[Gers] S. Gersten, Whitehead groups of free associative algebras, Amer. Math. Soc. 71 (1965), 157-159.

[Grot] A. Grothendieck, Sur quelques points d'algèbre homologique, Tôhoku Math. J. 9, 119-221 (1957).

[Gru] K. Gruenberg, Profinite Groups in Cassels and Fröhlich, Academy Press, 1967.

[GS] P. Gille, T. Szamuely, Central simple algebras and Galois cohomology, Cambridge Studies in Advanced Mathematics, 2006.

[Has1] H. Hasse, Über die Darstellbarkeit von Zahlen durch quadratische Formen im Körper der rationalen Zahlen, J. Reine Angew. Math.152 (1923), 129-148.

[Has2] H. Hasse, Über die Äquivalenz quadratischer Formen im Körper der rationalen Zahlen, J. Reine Angew. Math. 152 (1923), 205-224

[Has3] H. Hasse, K. Hensel, Über die Normenreste eines relativ zyklischen Körpers von Primzahlgrad nach einem Primteiler l von, Math. Annalen 90 (1923), 262-278. 
[Has4] H. Hasse, Zur Theorie des quadratischen Hilbertschen Normenrestsymbols in algebraischen Körpern, J. Reine Angew. Math. 153 (1923), 76-93.

[Has5] H. Hasse, Symmetrische Matrizen im Körper der rationalen Zahlen, J. Reine Angew. Math. 153 (1924) 113-130.

[Has6] H. Hasse, Darstellbarkeit von Zahlen durch quadratische Formen in einem beliebigen algebraischen Zahlkörper, J. Reine Angew. Math. 153 (1924), 113-130.

[Has7] H. Hasse, Äquivalenz quadratischer Formen in einem beliebigen algebraischen Zahlkörper, J. Reine Angew. Math. 153 (1924), 158-162.

[Hig] G. Higman, The units of group rings, Proc. London Math. Soc. 46, 236 - 248.

[Hilb1] D. Hilbert, Die Theorie der algebraischen Zahlkörper, Jahresberich der DMV 4 (1897), $175-546$.

[Hilb2 $]$ D. Hilbert, The Theory of Algebraic Number Fields, Springer-Verlag, Berlin, 1998.

[HM] A. J. Hanh, O. T. O'Meara, The Classical Groups and K-Theory, Springer-Verlag, New York, 1989.

[HS] P. Hilton, U. Stammbach, A Course in Homological Algebra, Springer-Verlag, 1970.

[JR] B. Jacob, M. Rost, Degree four cohomological invariants for quadratic forms, Invent. Math. 96 (1989), 551-570.

[Kna] A. W. Knapp, Advanced Algebra, Cornerstones, Boston, Birkhäuser Boston, 2007.

[Kanh2] B. Kahn, La conjecture de Milnor d'aprés V. Voevodsky, Astérisque, 245, (1997), Exp. No. 834, 5, 379-418, Séminaire Bourbaki, Vol. 1996/97.

[Kato] K. Kato, A generalization class field theory by using K-groups I, J. Fac. Sci. Univ. Tokyo 27 (1979), 303 -376.

[KSS] M. Kula, L. Szczepanik, K. Szymiczek, Quadratic form schemes and quaternionic schemes, Fund.Math, 130 (1988), 181 - 190.

[KV] M. Karoubi, O. Villamayor, K-théorie algébrique et K-théorie topologique, Math. Scand. 28(1971), 265-307.

[Ker1] I. Kersten, Brauergruppen von Körpern, Friedr. Vieweg, 1990.

[Ker2] I. Kersten, Brauergruppen, Universitätsverlag Göttingen, 2007.

[Lam1] T. Y. Lam, The algebraic theory of quadratic forms, W. A. Benjamin, Massachusetts, USA, 1973.

[Lam2] T. Y. Lam, Orderings, valuations and quadratic forms, Regional Conference Series in Mathematics 52, American Mathematical Society, Providence, USA, 1983.

[Lam3] T. Y. Lam, Introduction to Quadratic Forms over Fields, Graduate Studies in Mathematics, vol 67, AMS, 2004.

[Lam4] T.Y Lam, Ten Lectures on Quadratic Forms over Fields in Conference on Quadratic forms, Queens Papers in Pure Appl.46 (1977), 1-102. 
[Lam5] T. Y. Lam, A first course in noncommutative rings, Second edition, Graduate Texts in Mathematics 131, Springer-Verlag, New York, 2001.

[Lens] H. W. Lenstra, A normal basis theorem for infinite Galois extensions, Nederl. Akad.Wetensch. Indag. Math 47 (1985), 221-228.

[LS] T.Y.Lam, M.K. Siu, $K_{0}$ and $K_{1}$, An Introduction to Algebraic K-Theory, Amer. Math. (1975), p.329-364.

[Mar1] M. A. Marshall, A reduced theory of quadratic forms, Queen's Papers in Pure and Applied Mathematics 46 (1977), 569-579.

[Mar2] M. A. Marshall, Abstract Witt Rings, Queen's Papers in Pure and Applied Mathematics 57, Queen's University, Ontario, Canada, 1980.

[Mar3] M. A. Marshall, Spaces of Orderings and Abstract Real Spectra, Lecture Notes in Mathematics 1636, Springer-Verlag, Berlin, Germany, 1996.

[Meara] T. O'Meara, Introduction to Quadratic Forms, Springer, 1963.

[Merk] A. Merkurjev, On the norme residue symbol of degree 2, Dokladi Akad. Nauk. SSSR 261 (1981) $542-547$.

[Men] J. Mennicke, Finite factor groups of the unimodular group, Ann. of Math., 81 (1965), $31-37$.

[Mil1] J. Milne, Class field theory, course notes, 2011.

[Mil2] J. Milne, Fields and Galois theory, course notes, 2008.

[Miln1] J. Milnor, On axiomatic homology theory, Pacific J. Math.12 (1962), 337-341.

[Miln2] J. Milnor, Whitehead torsion, Amer. Math. Soc.72 (1966), 358-426.

[Miln3] J. Milnor, Algebraic K-theory and quadratic forms, Invent. Math. 9 (1970), 318-344.

[Miln4] J. Milnor, Introduction to Algebraic K-theory, Annals of Math. Studies 72, Princeton Univ. Press, 1971.

[Miln5] J. Milnor, On simply connected 4 manifolds, Symp. Topologia Algebraica, México, 122128.

[MH] J. Milnor, D. Husemoller, Symmetric Bilinear Forms, Springer-Verlag, 1973.

[Min1] H. Minkowski, Grundlagen für eine Theorie der quadratischen Formen mit ganzzahligen Koeffizienten, Mémoires présentés par divers savants a l'Académie des Sciences de l'institut National de France, Tome XXIX, No. 2., 1884

[Min2] H. Minkowski, Untersuchungen über quadratische Formen. Bestimmung der Anzahl verschiedener Formen, die ein gegebenes Genus enthält. Königsberg 1885; Acta Mathematica 7 (1885), 201-258.

[Min3] H. Minkowski, Über positive quadratische Formen, J. Reine Angew. Math. 99 (1886), $1-9$. 
[Min4] H. Minkowski, (Letter to Hurwitz), Über die Bedingungen, unter welchen zwei quadratische Formen mit rationalen Koeffizienten ineinander rational transformiert werden können, J. Reine Angew. Math. 106 (1890), 5-26, Ges. Abh. I, 219-239.

[Mon] J. P. Monnier, On Lam's conjecture concerning signatures of quadratic forms, Arch. Math. 75 (2000), 198-206.

[Mor] P. Morandi, Field and Galois Theory, Springer-Verlag, New York, 1996.

[Morel1] F. Morel, Théorie de l'homotopie et motifs I, preprint (1995).

[Morel2] F. Morel, Voevodsky's proof of Milnor's conjecture, Bull. Amer. Math. Soc., 35 (1998), 123-143.

[MLan1] S. Mac Lane, Homology, Springer-Verlag, Berlim, 1963.

[MLan1] S. Mac Lane, Categories for the Working Mathematician, Springer-Verlag, Berlim, 1971.

[Mrn] H. L. Mariano, The Galois Group of pre-Special Groups, em preparação.

[MV] F. Morel, V.Voevodsky, $\mathbb{A}^{1}$-homotopy theory of schemes, Publ. Math. I.H.E.S., 90 (1999), 45-143.

[MS] J. Mináč, M. Spira, Witt rings and Galois groups, Annals of Mathematics 144 (1996), $35-60$.

[MSu] A. Merkurjev, A. Suslin, K-cohomology of Severi-Brauer Varieties and the Norm Residue Homomorphism, Izv. Akad.Nauk SSSR 46 (1982), 1011-1046.

[NJac1] N. Jacobson, Basic Algebra II, Freeman, first edition, 1980.

[NJac2] N.Jacobson, Basic Algebra I, Freeman, second edition, 1985.

[NJac3] N. Jacobson, Finite dimensional algebras over fields, Springer-Verlag, 2010.

[OVV] D.Orlov, V.Vishik, V.Voevodsky, Motivic cohomology of Pfister quadrics and Milnor's conjecture on quadratic forms, preprint.

[Pfi1] A.Pfister, Darstellung von -1 als Summe von Quadraten in einem Körper, J.London Math. Soc. 40 (1965), 159-165.

[Pfi2] A. Pfister, Multiplikative quadratische Formen, Arch. Math. 16 (1965), 363-370.

[Pfi3] A. Pfister, Quadratische Formen in beliebigen Körpern, Inventiones Mathematicae 1 (1966), 116-132.

[Pfi4] A. Pfister, Quadratic forms with applications to Algebraic Geometry and Topology, London Math. Soc., Lect. Notes Series 217, Cambridge University Press, 1995.

[Pfi5] A. Pfister, On the Milnor Conjectures: History, Influence, Applications, Jber. d. Dt. Math.-Verein. 102 (2000), 15-41.

[Pont] L. S. Pontryagin, Topological groups, Translated from the second Russian edition by Arlen Brown, Gordon and Breach Science Publishers, Inc., New York, 1966. 
[Qui1] D. Quillen, Cohomology of groups, International Congress of Mathematics, Nice, 1970.

[Qui2] D. Quillen, On the cohomology and K-theory of the general linear groups over a finite field, Ann. of Math. 96 (1972), 552-586.

[Qui3] D. Quillen, Higher algebraic K-theory I, Lecture Notes in Math. 341 (1973), 85-147.

[Rib] L. Ribes, Introduction to Profinite Groups and Galois Cohomology, Queen's Papers in Pure and Applied Mathematics 24, Queen's University, Ontario, Canada, 1970.

[Ros] J. Rosenberg, Algebraic K-Theory and its Applications, Springer-Verlag, New York, 1994.

[Rot1] J. Rotman, Advanced Modern Algebra, American Mathematical Society, Providence, 2010 .

[Rot2] J.Rotman, An Introdution to Homological Algebra, Springer, 2002.

[Row] L. Rowen, Ring Theory, Academic Press, Inc. 1991.

[RZ] L. Ribes, P. Zalesskii, Profinite Groups, A Series of Modern Surveys in Mathematics 40, Springer-Verlag, Berlin, German, 2000.

[SIon1] J. P. Serre, P. Ion, Local Fields, Springer-Verlag, New York, 1979

[SIon2] J. P. Serre, P. Ion, Galois Cohomology, Springer-Verlag, New York, 1997.

[SBirk] S.Mac Lane, G .Birkhoff, Algebra, Macmillan, first edition, 1967.

[Serre1] J. P. Serre, Local Fields, Springer-Verlag, 1979

[Serre2] J.P. Serre, Galois Cohomology, Springer-Verlag, 2002.

[Sch1] W. Scharlau, Quadrastiche Formen und Galois Cohomologie, Invent. Math. 4 (1967), 238-264.

[Sch2] W. Scharlau, Quadratic and Hermitian forms, New York, Springer-Verlag, 1985 .

[Sch3] W. Scharlau, On the History of the Algebraic Theory of Quadratic Forms, preprint (1999).

[SLan] S. Lang, Algebra, Springer-Verlag, New York, 2002.

[Srin] V. Srinivas, Algebraic K-Theory, Progress in Math, Vol 90, Birkhäuser, Boston, Basel, Berlin, 1991.

[Sta] J. Stallings, Whitehead torsion of free products, Ann. of Math. 82, 354-363.

[SV1] A. Suslin, V. Voevodsky, Relative cycles and Chow sheaves, preprint (1994).

[SV2] A. Suslin, V. Voevodsky, Bloch-Kato conjecture and motivic cohomology with finite coefficients, preprint (1995).

[SV3] A. Suslin, V. Voevodsky, Singular homology of abstract varieties, Invent. Math. 123 (1996), 61-94. 
[Tate1] J. Tate, Symbols in Arithmetic, Actes Congrès Intern. Math. Tome 1 (1970), 201-211.

[Tate2] J. Tate, Relations between $K_{2}$ and Galois cohomology, Invent.Math. 36 (1976), $257-274$.

[Voe1] V. Voevodsky, The Milnor conjecture, preprint (1996).

[Voe2] V. Voevodsky, Homology of schemes II, preprint (1994).

[Voe3] V. Voevodsky, Triangulated categories of motives over a field, preprint (1995).

[Voe4] V. Voevodsky, Cohomological operations in Motivic cohomology, preprint (1995).

[Wad] A. R. Wadsworth, Merkurjev's elementary proof of Merkurjev's theorem, in Applications of algebraic K-theory to algebraic geometry and number theory, Part I, II (Boulder, Coloquium., 1983), vol. 55, Contemp. Math., no. II, Amer. Math. Soc., 1986, p. 741-776.

[Waer] B.L. Van Der Waerden, Algebra, Ungar, seventh edition, 1973.

[We1] C. Weibel, An introduction to homological algebra, Cambridge University Press, 1997.

[We2] C. Weibel, An introduction to Algebraic K - Theory, Graduates Studies in Mathematics, vol 145, AMS, 2010.

[Whi1] J. H. C. Whitehead, Nuclei and m-groups, Proc. London Math. Soc. 45 (1939),243-327.

[Whi2] J. H .C. Whitehead, Simple homotopy types, Amer. J. Math. 72 (1950), 1-57.

[Witt] E. Witt, Theorie der quadratischen formen in beliebigen köpern, J. Reine Angew. Math. 176 (1937), 31-44. 